TIUE TMUS ROLL OF THE BISIIOPRIC OF WINCHESTER, $1208-1200$ 


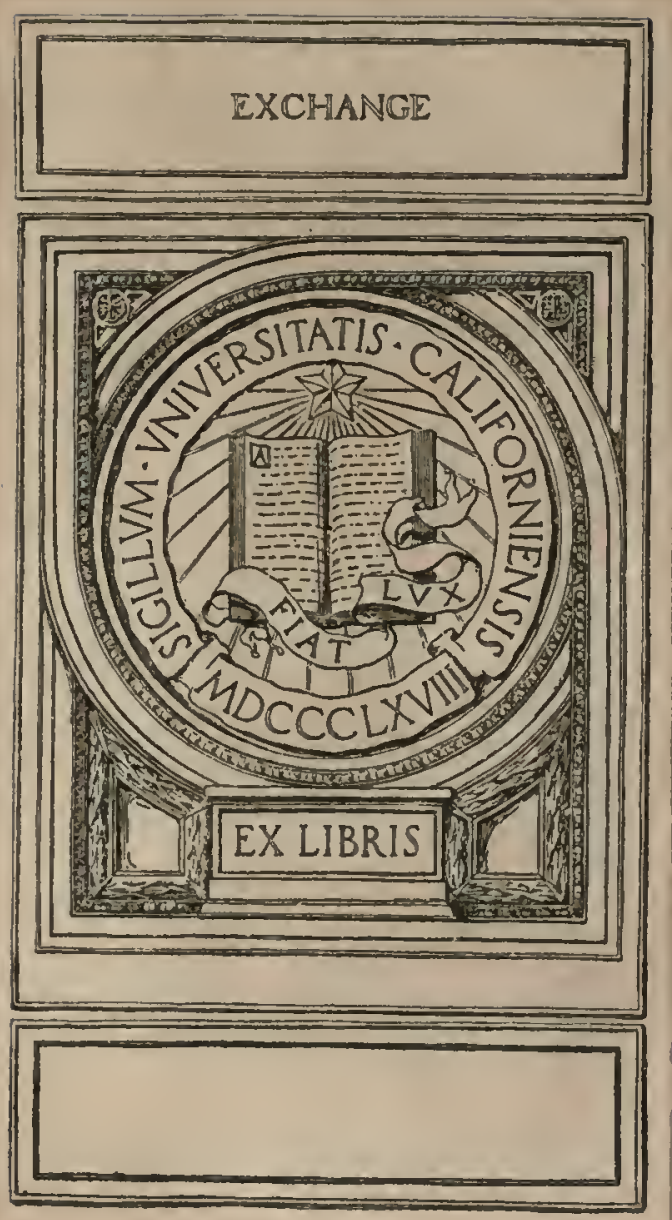


1
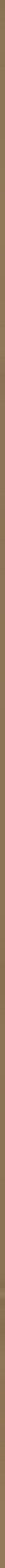

i.

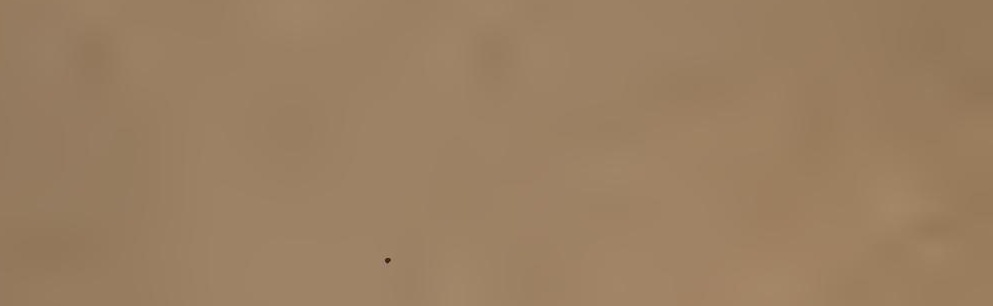

1.

1 

30

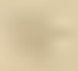




\section{Digitized by the Internet Archive}

in 2008 with funding from Microsoft Corporation 



\section{STUDIES IN ECONOMICS AND POLITICAL SCIENCE.}

A Series of Handbooks by Writers connected with the London School of Economics and Political Science.

EDITED BY PROFESSOR W. A. S. HEWINS, M.A.,

Director of the London School of Economics and Political Science.

PUBLISHED BY

P. S. KING \& SON, Orchard House, 2 and 4, Great Smith Street, Westminster.

Uniform cloth. Crown $8 v o$ ( $7 \frac{1}{2}$ in. by 5 in.) unless otherwise stated.

I. THE HISTORY OF LOCAL RATES IN ENGLAND. The substance of five lectures given at the School in November and December, 1895. By Edwin Cannan, M.A., Balliol College, Oxford; Lecturer at the School. 1896 ; 140 pp. 2s. 6d. net.

2. SELECT DOCUMENTS ILLUSTRATING THE HISTORY OF TRADE UNIONISM. I.-The Tailoring Trade. By F. W. GALTON. With a Preface by SIDNEY WEBB, LL.B. $1896 ; 242$ pp. 5s. net.

3. GERMAN SOCIAL DEMOCRACY. Six lectures delivered at the School in February and March, 1896. By the Hon. Bertrand Russell, B.A., Fellow of Trinity College, Cambridge. With an Appendix on Social Democracy and the Woman Question in Germany by Alys Russell, B.A. 1896; 204 pp. 3s. 6d. net.

4. THE REFERENDUM IN SWITZERLAND. By M. SimoN Deplotge, University of Louvain. With a Letter on the Referendum in Belgium by M. J. VAN DEN HEUVEL, Professor of International Law in the University of Louvain. Translated by C. P. Trevelyan, M.A., Trinity College, Cambridge, and edited with Notes, Introduction, Bibliography and Appendices, by Lilian Toms, of Girton College, Cambridge, Research Student at the School. 1898; x. and 334 pp. 7s. 6d. net.

5. THE ECONOMIC POLICY OF COLBERT. By A. J. Sargent, M.A., Senior Hulme Exhibitioner, Brazenose College, Oxford; and Whately Prizeman, Trinity College, Dublin, 1897 ; Lecturer at the School. 1899 ; viii. and 138 pp. 2s. 6d. net.

6. LOCAL VARIATIONS IN WAGES. (The Adam Smith Prize, Cambridge University, 1898.) By F. W. LAwrence, M.A., Fellow of Trinity College, Cambridge. 1899 ; viii. and $90 \mathrm{pp}$., with Index and 18 Maps and Diagrams. Quarto, $11 \mathrm{in.}$ by $8 \frac{1}{2}$ in. 8s. 6d. (Published by Longmans, Green, \& Co., 39, Paternoster Row, London.)

7. THE RECEIPT ROLL OF THE EXCHEQUER FOR MICHAEL. MAS TERM OF THE THIRTY-FIRST YEAR OF HENRY THE SECOND (rr85). A unique fragment transcribed and edited by the Seminar in Palæography and Diplomatic under the supervision of the Lecturer, HUBERT HALL, F.S.A., of H.M. Public Record Office. With Thirty-one Facsimile Plates in Collotype, and Parallel Readings from the contemporary Pipe Roll. 1899 ; folio. Very few copies are left.

8. ELEMENTS OF STATISTICS. By ArThur L. Bowlex, M.A., F.S.S., Lecturer in Statistics at the London School of Economics and Political Science; appointed Teacher of Statistics in the University of London; author of "Wages in the United Kingdom in the Nineteenth Century." 500 pp. Demy 8vo, cloth, 40 Diagrams. Second edition; 1901. Price 10s. 6d. net.

9. THE PLACE OF COMPENSATION IN TEMPERANCE REFORM. By C. P. SAxger, M.A., late Fellow of Trinity College, Cambridge; Barrister-at-Law. viii. and 136 pp. 1901. Price 2 s. 6 d. net.

ro. A HISTORY OF FACTORY LEGISLATION, I802-I901. By B. L. Hutchins and A. Harrison, B.A., D.Sc., London. With a Preface by Sidoney WEBB, LL.B. 1903 ; 372 pp. Demy 8vo, cloth. 10s. 6d. net.

II. THE PIPE ROLL OF THE EXCHEQUER OF THE SEE OF WINCHESTER FOR THE FOURTH YEAR OF THE EPISCOPATE OF PETER DES ROCHES (1207). Transcribed and edited from the original Roll in the possession of the Ecclesiastical Commissioners by the Seminar in Palæography and Diplomatic under the supervision of the Lecturer, HUBERT HALL, F.S.A., of H.M. Public Record Office. With a Frontispiece giving a Facsimile of the Roll. 15s. net.

12. SELF-GOVERNMENT IN CANADA, AND HOW IT WAS ACHIEVED: THE STORY OF LORD DURHAM'S REPORT. BY F. BRADSHAw, B.A., Senior Hulme Exhibitioner, Brasenose College, Oxford. 1903. Demy 8vo, cloth, $424 \mathrm{pp}$. 10s. fid. net.

I3. A HISTORY OF THE COMMERCIAL AND FINANCIAL RELATIONS BETWEEN ENGLAND AND IRELAND FROM THE PERIOD OF THE RESTORATION. By Alice EFfie Murray, D.Sc., London, Former Student Girton College, Cambridge; Research Student London School of Economics. Demy 8 vo, cloth, 486 pp. 10s. 6 d. net. (In the press.) 
STUDIES IN ECONOMICS AND POLITICAL SCIENCE.

Edited By W. A. S. HEWINS, M.A.,

Direolor of the London Sehool of Economies and Political Seionce.

\section{THE PIPE ROLL \\ OF THE \\ BISHOPRIC OF WINCHESTER. \\ $1208-1209$.}




\section{DEPARTMENT OF ADVANCED HISTORICAL STUDY.}

THE critical use of Historical Sources and the Method of Historical Composition have formed, from the first, an essential feature of the course of instruction provided at the School of Economics. In addition to the supervision afforded by the respective seminars, classes have been also held since Lent Term, 1896, for special instruction in Palrography, Diplomatic, and the Classification and Bibliograplyy of Historical Sources. In connection with these classes a seminar has also been held for the purpose of practical instruction and exercise in the technical process of transcribing, extending and editing historical documents. These exercises have already resulted in the publication of the Receipt Roll of the Exchequer for Michaelmas Term of the thirty-first year of Henry the Second (1185), and the Pipe Roll of the Exchequer of the See of Winchester for the fourth year of the Episcopate of Peter des Roches (1207), edited by the class, in the series of publications issued by the School. This seminar has the further object of enabling research students to become acquainted with the contents of public archives and libraries by means of organised visits of inspection.

In the Summer Term of 1902 the instruction given at the School in the department of higher historical teaching was materially enlarged by the temporary endowment of a "Creighton" Lectureship in the "Study of Historical Sources." This was the result of a movement set on foot by the President of the Royal Historical Society and other distinguished scholars forming a "Committee for furthering Advanced Historical Study in London." The offer of the committee to defray the stipend of a lecturer for a period of three years was accepted by the Governors of the School, and the "Creighton" lectures in Historical Sources were forthwith arranged.

At the same time Mr. Passmore Edwards generously offered to provide an equal endowment for a second Lectureship in higher Historical Studies, and his offer having, been accepted both by the Committee for furthering Advanced Historical Studies and by the Governors of the School, a "Passmore Edwards" lecturer was appointed for the purpose of continuing the systematic instruction in Palæography and Diplomatic, for which no adequate provision could be made from the "General Purposes Fund" at the disposal of the School. The technical subjects of the Classification and Bibliography of Historical Sources, which had hitherto been combined in the former courses on Paliegraply and Diplomatic, were specially recognised by the University of London in the appointment of a Teacher in Early Economic Sources, and a further course of lectures and a seminar have been established in this department.

Economic History, which occupies such an important place both in the general scheme of Historical Studies and in the Curricnla of the University of London, is represented by a two.years' course: of lectures on "Modern Economic History," which is co-ordinated with seviral: other courses, embracing "The Economic Position of the Great P.owers?" "The Orgarisation and Structure of Modern Industry and Commerce," "Foreign" "Tride," "Currency," etc., "Historical Geography," "French Commercial History," and elaborate courses on modern European History, Political and Economic.

In the Historical Section are also included several independent or subsidiary courses, such as the "History of Political Ideas," in Europe and the United States, the History of the English Poor Laws, the Constitutional History of England and her Colonies, Constitutional Law and History, "The Financial Systems of England, France and Germany" and "The Economic Position of England," Foreign Trade with the Far East, and "French Commercial History."

It will be evident from the above analysis of the work in the Historical department of the School that it affords perhaps unequalled facilities for the study of Mediæval and Modern History in its Political, Constitutional, Economic and Social bearings, as well as for the definite and scientific pursuit of Historical research. 

$x+5$

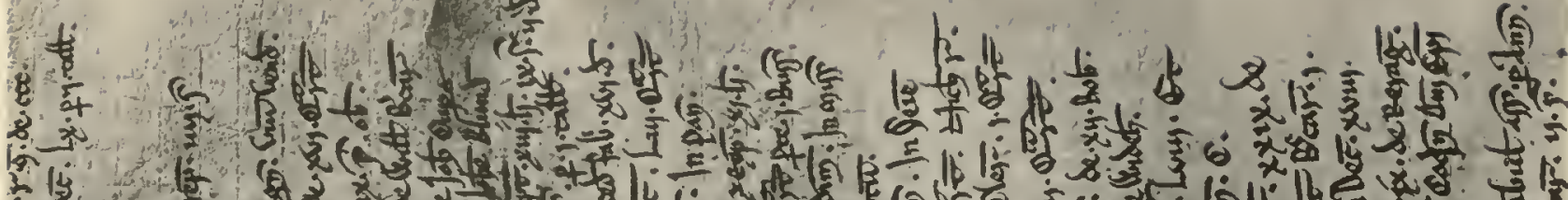

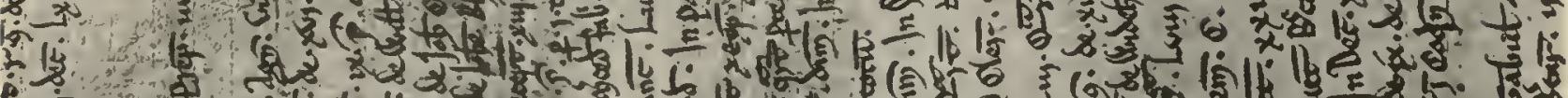
f-

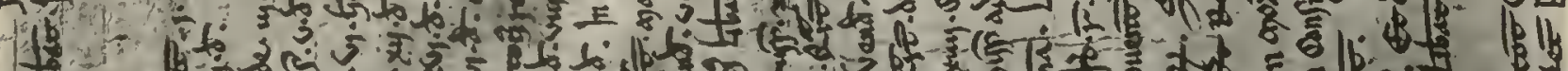

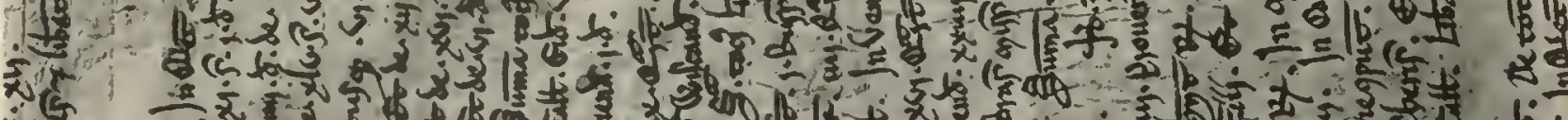

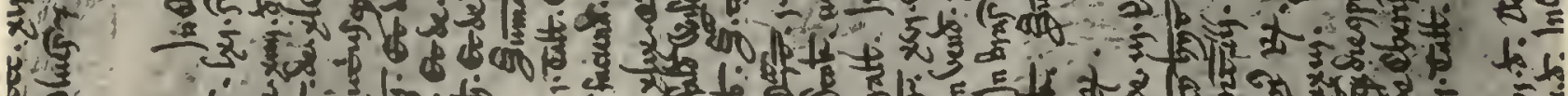
$5:$ : 5 (a)

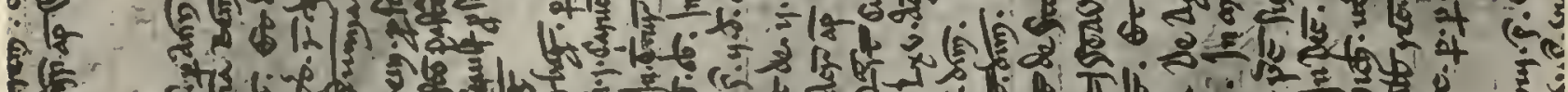
f.

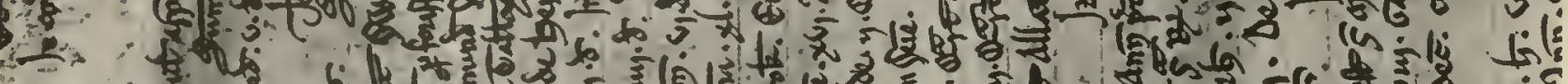

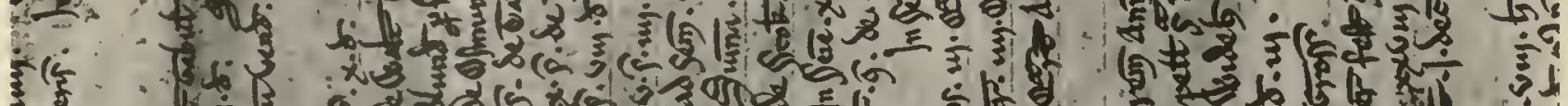

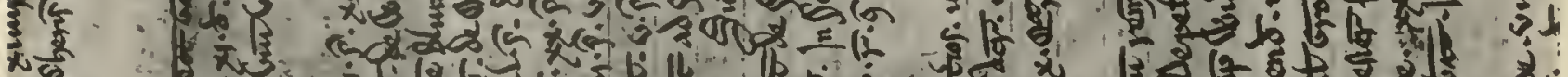

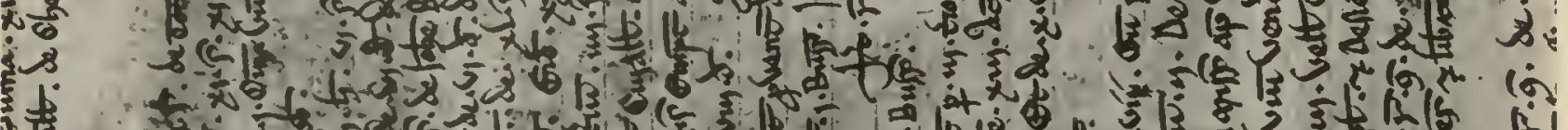
办

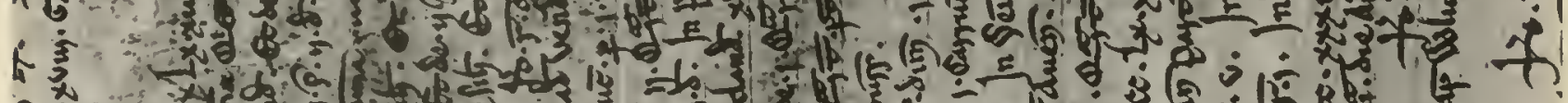

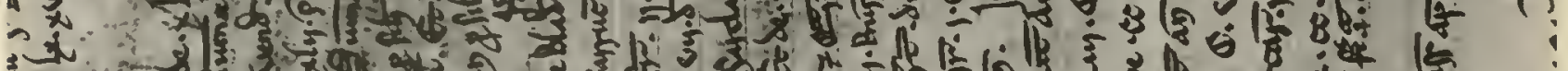

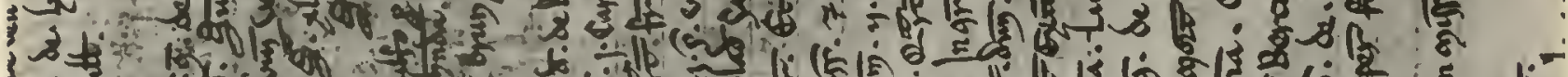

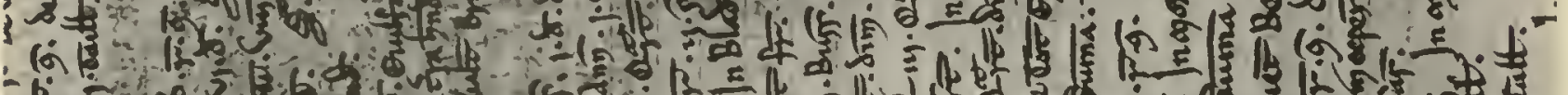

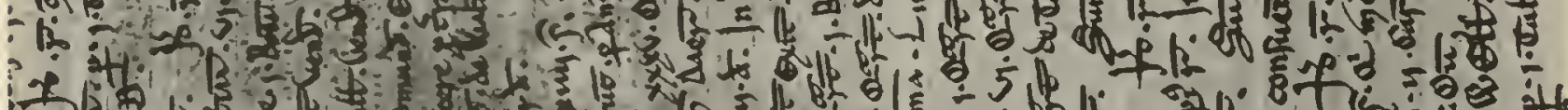

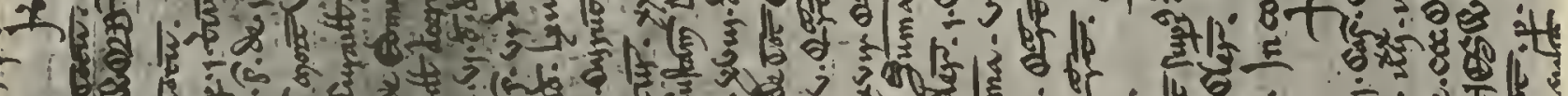

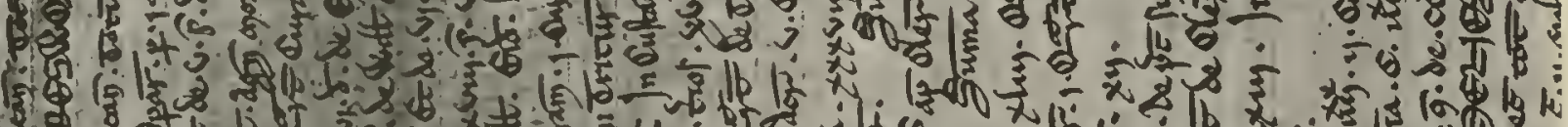
X

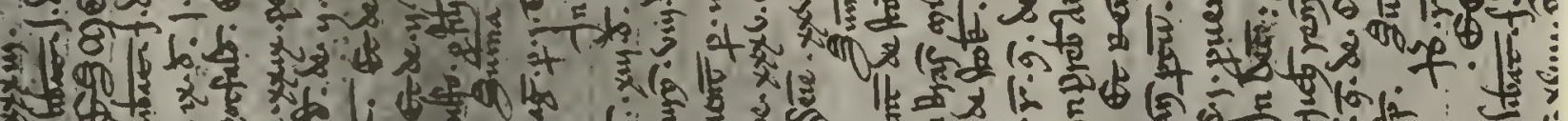

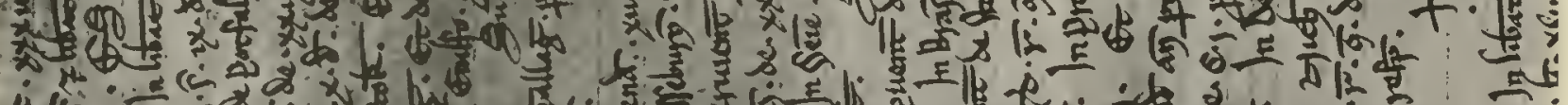
1. :

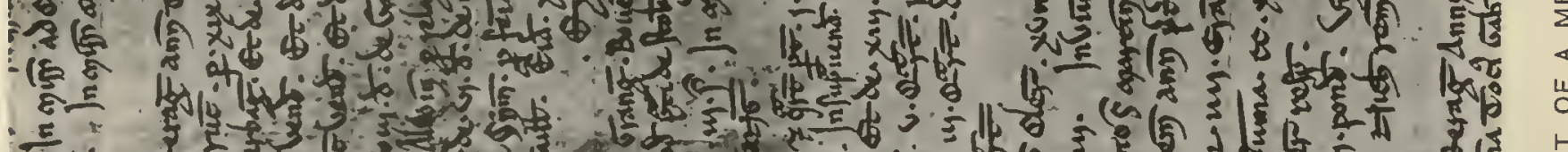

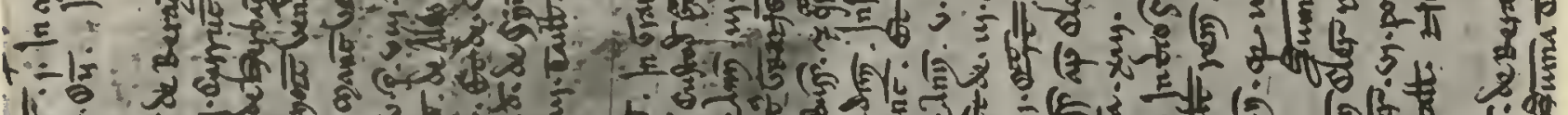
100 1. of

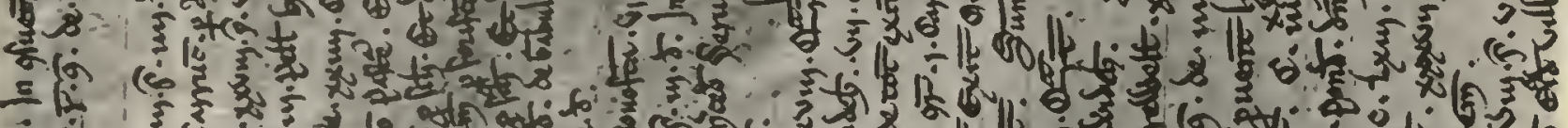

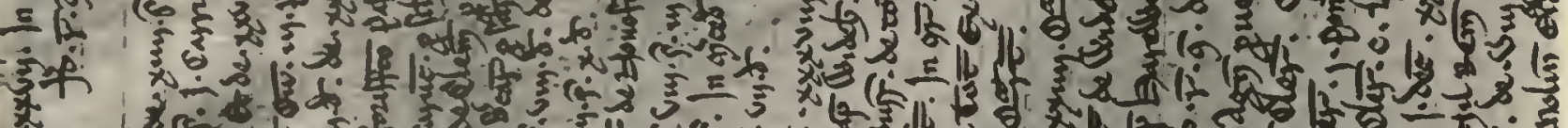

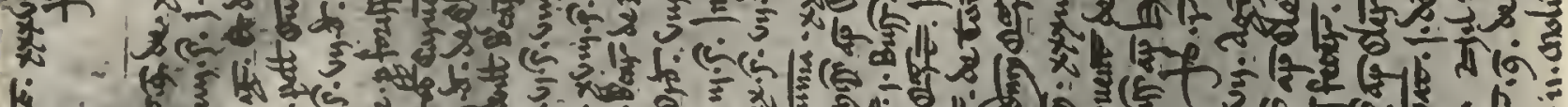

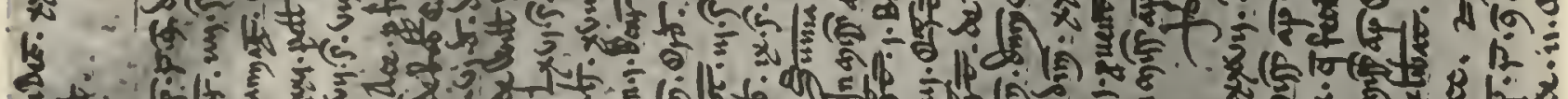

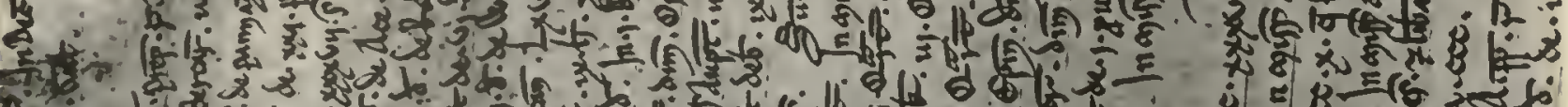

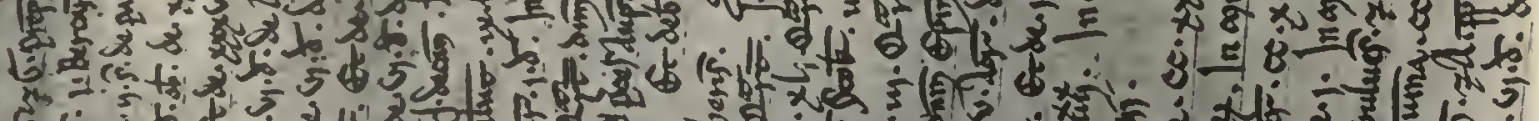
年

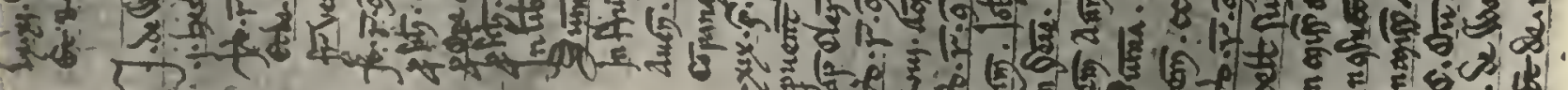
(1) $x^{2}+\frac{1}{7}$ 


\title{
THE PIPE ROLL
}

OF TEE

\section{BISHOPRIC OF WINCHESTER}

For the Fourth Year of the Pontificate of

Peter des Roches, I208-1209.

TRANSCRIBED FROM THE ORIGINAL ROLL AMONGST THE RECORDS OF THE ECCLESIASTICAL COMMISSIONERS, EXTENDED AND EDITED, WITH AN INTRODUCTION, INDEX, AND GLOSSARY,

\section{BY THE \\ Elass in Palacographr}

OF THE LONDON SCHOOL OF ECONOMICS AND POLITICAL SOIENCE (UNIVERSITY OF LONDON)

\author{
UNDER THE SUPERVISION OF THE LECTURER, \\ H UBERT HALL, F.S.A. \\ (OF H.M. PUBLIC RECORD OFFICE) \\ (Appointed Teacher of the University of London in "Early Economic Sources.")
}

London:

PUBLISHED BY P. 8. KING \& SON, ORCHARD HOUSE, WESTMINSTER FOR

THE LONDON SCHOOL OF ECONOMICS AND POLTTICAL SCIENCE

1903. 


$$
\begin{gathered}
f M C .254 \\
.35
\end{gathered}
$$




\section{CONTENTS.}

Facsimile of the Roll - _ _ _ _ _ _ _ _ _ _ _ - Frontispiece.

Intropucrion

vii

Appandix (Itinerary of the Bishop and King, 1208-9; Balunce Sheet; Tables of Crops and Stock of the Winchester Manors, 1208-9)

Rotulus Compoti Anni Quarti Pontificatus Domini Petri Wratoniensis Episcopi

\begin{tabular}{|c|c|c|c|c|c|c|c|c|c|}
\hline & & & & Page & & & & & $P_{a g}$ \\
\hline Waltham & - & - & - & 1 & Ecclesia de N & Menes & - & - & 46 \\
\hline Clera - & - & - & - & 5 & Hameledona & - & - & - & 48 \\
\hline Widehae & - & - & - & 8 & Craweleia & - & - & - & 50 \\
\hline Esmereswords & & - & - & 10 & Twiforda & - & - & - & 52 \\
\hline Ecbeneswella & & - & - & 11 & Stokes & - & - & - & 55 \\
\hline Brictewella & - & - & - & 13 & Edborebiria & - & - & - & 57 \\
\hline Harewella & - & - & - & 15 & Suwerke & - & - & - & 60 \\
\hline Wittene & - & - & - & 16 & Alresforde & - & - & - & 60 \\
\hline Duntona & - & - & - & 20 & Prevet - & - & - & - & 63 \\
\hline Overtona & - & - & - & 23 & Tantona & - & - & - & 63 \\
\hline Ferham & - & - & - & 25 & Rimtona & - & - & - & 72 \\
\hline Weregrave & - & - & - & 28 & Cnoel - & - & - & - & 73 \\
\hline Biterne & - & - & - & 30 & Ministerium & Wintoni & & - & 76 \\
\hline Wicumba & - & - & - & 32 & Faleleia et 0 & $\mathrm{ra}$ & - & - & 79 \\
\hline Meredona & - & - & - & 34 & Funtel - & - & - & - & 80 \\
\hline Fernham & - & - & - & 37 & Cheritona & - & - & - & 80 \\
\hline Suttona & - & - & - & 40 & Beworda & - & - & - & 82 \\
\hline Menes & - & - & - & 44 & Walda - & - & - & - & 83 \\
\hline
\end{tabular}

Glossary - 



\section{INTRODUCTION.}

THE Document which is described and printed in the following pages is Classification preserved amongst the Records of the Ecclesiastical Commissioners which of the Roll. have been deposited in the Public Record Office since the year 1890. The modern official reference is to Ecclesiastieal Commission, Various, Bdle. 22, Roll 159270, but in the older official lists of the Commissioners' archives the Roll bearing this number headed a long series of Rent Rolls of the Bishopric of Winchester extending from the year 1207 to 1455, with numerous gaps for the earlier period. ${ }^{1}$ The Roll itself, like others of the series, once bore the 17th century press-mark, "1207. John $8^{\circ}$. Roach $4^{0 . "}$ which has been replaced by a later official endorsement. The period thus assigned to it was derived from an erroneous calculation of the pontifical date. ${ }^{2}$

There are no existing means of tracing the life-history of these Official manorial Records, or of realizing their earlier official environment. Like custody. the strong castle and stately palace in which they were once compiled and preserved, they serve to recall the princely state and fiscal establishment to which they owed their inception and execution in the days when the Norman rule was yet remembered in the land. Unlike the records of the Royal Exchequer, upon which they were largely based, these Rolls have not descended to us with any direct official traditions of their early custody, but from our knowledge of their later vicissitudes ${ }^{3}$ we have much cause for thankfulness that so large a number came into the careful custody of the Ecclesiastical Commissioners." Fortunately, the Roll whicb has been printed in the following pages had escaped serious injury, and the skilful repair which it has undergone since its transfer to the Public Record Office still further facilitated the task of its transcription.

The Roll in its present state consists of 13 parchment membranes of The Palæothe average length of 24 inches and the average width of 13 inches. The graphy. last membrane, however, contains only 17 lines of writing, and the spare parchment has long since been cut away.

The several membranes, which are now mounted on guards, were formerly secured by being loosely stitched at the top; but it would appear from other specimens of the class to which the document belongs that they were originally secured at the head by leather thongs passed between two pairs of eyelet holes at a slight distance apart. The lower extremity of

1178 rolls exist for this period of 248 years, but of these several are the second parts of year rolls.

2 See p. viii.

3 Somerset Arch. and Nat. Hist. Soc. Trans, xviii., 84.

4 It may be of interest to mention that, on the occasion of a recent visit to Winchester for the purpose of inspecting the Cathedral archives, by the kind permission of the Dean and Chapter the present Editors were enabled, through the courtesy of their custodian, the Rev. F. T. Madge, to inspect a Compotus or Pipe Roll of the 4tlı year of Bishop Athelmar (120̈3), which is missing in the series preserved in the Public Record Office, on behalf of the Ecclesiastical Commissioners. The whole of the Accounts of this troublous Pontificate are now missing, for reasons which may perhaps be explained by the fiscal relations between the Bishopric and the Convent referred to below (p. xi., n. 1). 
each membrane appears to have been shaped to a broad point, on which bands were laced for the purpose of securing the membranes in the form of a roll. ${ }^{1}$ To the lower extremity of the last membrane a flap of stiff parchment was formerly attached, which served as an outer cover, and the greater part of this cover has still been preserved, and bears traces of the title of the Roll in a contemporary hand. ${ }^{2}$

At one time the consecutive numbers of the membranes were written in Roman figures at the apex of each membrane. Above them and along the whole width of the lower margin, the names of the manors are engrossed in Gothic capitals, with some minuscule letters intermixed. These, like the titles of the counties engrossed on the Exchequer Pipe Rolls, were evidently intended to serve as a means of ascertaining the position of a particular manor before unrolling the membranes; but, unlike the Exchequer Rolls, the titles are written on the front and not on the back of the membrane. Moreover, the entries are continued from membrane to membrane, so that the Roll has not the appearance of being composed of a number of rolulets, each consisting in the case of the Exchequer Records of two consecutive membranes. Thus the transition to book form in the middle of the 15th century was easily accomplished, the series of "Rent Rolls" being continued from the year 1458 as a new series of "Rentals" in vellum covers, still termed "Pipæ," and agreeing in all respects with the ancient enrolments."

The penmanship of these episcopal accounts bears a marked resemblance to the stately hand of the Exchequer Pipe Rolls for the same period, though the characters are distinctly smaller and the writing is more compressed. The spacing of the entries, however, shows the same arrangement in both cases. As in many MSS. of the period, capital and uncial letters are still occasionally introduced in the middle of a word.

The fact that these inrolments were merely copies of original accounts will perhaps explain the numerous erasures and the sometimes unintelligent forms noticed in the following pages. Again, the addition of the individual sums is even more careless than that which is usually found in mediæval accounts, and in some cases the inconsistencies found almost excite a suspicion of fraudulent intent.

The system of contraction employed by the scribe resembles that observed in the contemporary Exchequer Rolls. In the official orthography of the Episcopal Roll provincialisms and Gallicisms are of rare occurrence, and phonetic forms are confined to a few vowel sounds, suggesting, with other indications, that the original Rolls were visually transcribed and were not dictated to the copyist. Few archaisms of any interest occur. The Old English character "đ" has a distinct value. "W" has the value of "uu," as in "wlpes" and "Wlveseia"; but it also stands for "v," as in "wellera." Variants in spelling, especially of proper names, are exceptionally rare, though some pronounced forms are to be seen in the Index. Not a few grammatical errors may be found, and certain irregular forms of syntax appear to be used without much regard for consistency.

1 'These elongated extremities with their patterned holes have been almost entirely cut away in the Roll before us.

2 "Annus quartus Episcopi Petri."

3 This series is, however, separately elassified in the official arrangement. There is a gap of three years between the two series. 
Such formulas, however, as "In missis," "In venditis," "In mortuis," etc., may be regarded as purely technical, and their presenee forms a characteristic feature of these manorial accounts. Of the frequent omission of the conjunction "et" mention is made elsewherc."

The document, of which the outward appearance is described above, is The Diplo-

in the nature of an enrolment of the annual accounts rendered by the local offecrs of the bishopric of Winchester in respect of the receipts, issues, and stock of the episcopal manors in several counties.

Formula.-'I'he form of these accounts is preeisely similar to that of the typical manorial Compotus Rolls of a later period, with the exception that the statements of receipt and expenditure are couched in the formal style of the Exehequer Pipe Rolls. As usual the account contains three parts-the Charge, the Discharge, and the Schedule or Inventory of Stock. Each of these in turn contains several speeific itcms, which are entered in regular order, with the usual distinction between the Arrears and Current Account and the Receipts and Expenditure respectively. The normal construction may be tabulated as follows:-

\section{THE MANOR (or MINISTRY). NAMES OF OFFICERS ACCOUNTING.}

The Charge.

I. The Receipt (Recepta):

(1) The Arrears (Reragium) of former Accounts (if any).

(2) The Relditus manerii, ${ }^{3}$ here found in the shape of Gafol (Gabulum assisum), Rents of assize (Redditus assisi), or increments thereof (Incrementum); less :

(a) Acquittances or rebates (Quietancia) in favour of manorial servants ;

(b) Deficiencies (Defectus) for which the accountant is not liable.

(3) The Exitus manerii, or produce realized; usually subdivided into (a) uncultivated products (from pannage down to wild fruits and honey) and live stock, $(b)$ grain and dead stock, including the issues of the mills.

(4) The Purchasia, or issues of the manorial courts and other franchizes together with the Tallage or Aid.

The Discharge.

Total of the whole Receipts forming the Accountant's charge.

I. The Delivery (Liberatio) stating the several sums paid over to the Lord on account of the above receipts, and specifying the liability in respect of which it is paid, with the form of payment. ${ }^{5}$

II. The Expense (Expensa) following in the case of the more important manors the later subdivision into the Expensa Necessaria, or upkeep of the implements, buildings and wages of labour or allowances; and the Expensa Forinseca, or household and personal expenses of the Lord and his retinue.

\section{Total of the Delivery and Expense forming the Discharge.}

Balance of the Charge and Discharge.

1 Infra, p. xxxvi.

"The "ministry" of Winchester and church of Meon sre trested in the sane way in every year, and in other Rolls the Scutage of the bishopric frequently appears as a distinct accouut.

This does not appear as a marginal heading till a later date.

- Correspondiug to the later term Perquisita Curix.

5 It is very noticeable that in the later accounts of the 13th century the "Liberatio" was used in an entirely different sense, namely, the payments out made at the exchequer to officers and servants. Thus the formula read: "In libelatione in thesauro apud Wolvesey per manus domini thessurarii," \&c. At this later date slso the Liberatio followed the Expensa. The ase of this form of entry in the present scconnt seems wo suggest the organization of a Receipt department of the Wolvesey exchequer (cf. infra, p. xi, n. 7). 
Schedule A.-The Grain Account (Exitus Grangix) according to the several crops with the following formula of Account for the manor or mill :-

Charge :-

(1) Bulk remaining from last year (Remanens anno proterito).

(2) Bulk of this year's produce (De toto exitu hoc anno).

(3) Bulk of griin transported (De allutis, receptis, proventis) or bought (emptis) or acquired (de cheriset).

Discharge :-

Total (for each crop). ${ }^{1}$

(1) Bulk expended in seed per acre (In semine-acrarum).

(2) Bulk transported (missis, liberatis).

(3) Bulk expended in allowances (In corredio, consuetudine, precariis, liberatione, donis, expensis) or provision (In probenda, pascendis, pastu, cibo, pane, potagio, cervisia).

(4) Bulk sold (In venditis).

(5) Bulk remaining on hand (Remanens).

Total and State of Account (for each crop). ${ }^{1}$

Schedule B.-The Stock - Account (Instaurum) according to the several species in the following formula:- -

Charge:-

(1) Stock remaining from last year (Remanens anno praterito).

(2) Increase of stock during the present year (De proventis hoc anno; de exitu).

(3) Stock purchased (De emptis) or transferred (De proventis) or acquired (de testamento, purchasiis, decima, cheriset, consuetudine, redditu, pannagio, herbagio, potfalda, inventis).

Discharge :-

Total (for each species).

(1) Stock rendered as tithe (In decimis).

(2) Stock expended in allowances (In corredio, consuetudine, elemosina, donis, expensis, precariis, bederipa, medsip, companagio) or provision (In liberatis, occisis, missis ad lardarium).

(3) Loss of stock by natural causes (In mortuis, devoratis).

(4) Stock sold (In venditis) or transported (In missis, liberatis).

Total and State of Account for each species.

Schedule C. - The Larder Account (if distinct), the Salt Account, the Cellar Account, and the account of fruit and honey (if any).

\section{Note as to final State of the Account (if required).}

The above formula is consistently preserved throughout the Roll as well as during the succeeding period; but in certain instances, such as the manor of Privet and the submanors of Taunton, variations occur in conformity with local requirements, whilst a few simple variants are employed in other parts of the account.

Date.-It has been already observed that the date of this account has been incorrectly assigned to the year $1207,{ }^{2}$ and it would appear from internal evidence that it may be attributed to the fiscal year 1208-1209. The exact period covered by the account is, however, a matter of considerable importance in view both of its references to persons and political events, and also of its value for the illustration of certain economic conditions.

In the present case we might naturally expect that the pontifical date would be computed from September to September, since Godfrey de Luci died on the 11th or 12th September 1204, and Peter des Roches, although he did not receive the temporalities till March 1206, was conse-

\footnotetext{
1 The total is not always given.

${ }^{3} \mathrm{p} \mathrm{v}$, supra.
} 
crated on the 25th September 1205. Therefore the fourth year of this prelate should extend from 25th September 1208 to 25th September 1209, unless we had reason to suppose that the mere fact of his pre-occupation of the see with all its profits in virtue of royal mandates ${ }^{1}$ during the preceding year. had led the scribe to ignore the date of consecration for fiscal purposes. ${ }^{2}$

There is, however, another method of computing the period of these accounts from year to year; for whatever may have been the general, practice in this respect, ${ }^{3}$ the fact that the revenues of the See of Winchester were administered in connexion with an exchequer at Wolvesey would seem to indicate that the procedure of the royal Exchequer was followed in this as in other particulars.

The Bishop's Exchequer.-It has been usual to compare the Bishop of Winchester's exchequer at Wolvesey with the royal Exchequer at Westminster, and it has been generally assumed that the annual audit of the episcopal revenues is reeorded in the long series of stately muniments which still bear the title of "Pipe Rolls."

As no critical examination of the episcopal archives has ever been attempted, we must not attach a greater importance to this eursory description than it was meant to bear. With fuller opportunities for a diplomatic study of their contents it can, we believe, be shown that the series of inrolments, of which the earliest surviving specimen is printed in the following pages, does not correspond in any true sense with the Pipe Rolls of the royal Exchequer. The latter are primarily concerned, it is true, with the farms of the royal demesnes, and equally record the casual revenue of the Crown, together with the annual disbursements made by due authority. Similar informatiou is also contained in the "Pipe Rolls" of the bishopric; but, for all that, the classification of the two species must follow different lines. The Exchequer Pipe Roll is, in fact, something more than a balance sheet of the territorial profits and perquisites of the Crown, for every source of royal revenue passed through this capacious conduit to fill the coffers of the Ireasury. Now it will be evident at once that this purpose was not served by the existing episcopal account rolls, which take no cognizance of any temporal source of revenue external to the manors of the See or of any spiritualities whatsocver. And yet we cannot doubt that these sources of revenue were brought to aecount, just as they duly appear in the "states of account," which are fitfully preserved in later ecclesiastical and monastic registers. ${ }^{5}$

1 Pat. 6 John, m. 8 (21st September 1204); Close 7 John, m. 16 (19th August 1205).

2 In spite of.these grants, the profits of the See for the year ending 29th September 1205 were rendered to the Crown at the Exchequer (Pipe Roll, 7 John, Hants). It is possible, however, that the intention of the grants was only to effect an assignment to the Bishop elect in connection with his extensive pecuniary dealings with the Crown.

3 The ancient practice of the Frankish Curia, from which the form of our own accounts is immediately derived, prescribed a fiscal year extending from Christmas to Christmas, a period which, coinciding with the Christian and the civil years, has been chiefly favoured by western natious. It was even largely used for the accounts of ministers of the Crown, which were not audited at the Exchequer itself.

4 An exception to this might seem to be found in the case of the Scutages accounted for in several of these Rolls, but it must be remembered that this was merely a customary exaction in the case of sub-tenants.

${ }^{6}$ e.g. Canterbury (Hist. MSS. Comm. ix. Appx. i.), and Winchester itself (Hampshire Record Society, Registers of Sandale and De Asserio). 
At the same time it might certainly appear somewhat improbable that a whole series of such records compiled in the veritable form of the Ex. chequer Pipe Rolls should have entirely disappeared. Moreover, we have good reasons for believing that a simpler method of account was practised in the case of other seignorial establishments, ${ }^{1}$ and this we know, from later accounts of the bishopric of Winchester itself, took the form of a periodical audit of the manorial accounts by experts employed for the occasion. $^{2}$

The real explanation of the matter probably is that the bishopric of Winchester occupied an exceptional position in respect of its clerical establishment during the whole of the 12th and the early part of the 13th century. The proximity of the administrative centre at Wolvesey to the ancient royal Treasury and the official position of the great prelates who flourished during the whole of this period, ${ }^{3}$ would lave facilitated if it did not actually induce an assimilation of clerical practice to that of the royal Exchequer.

Subsidiary Documents.-From an examination of the few isolated and fragmentary account rolls preserved in the archives of the Dean and Chapter of Winchester, and amongst the Records of the Court of Exchequer, we might conclude that the Roll before us represented a certain stage in the preparation of the manorial accounts of the bishopric for the purpose of the annual audit. In the first place there was the Compotus Roll, or "particulars" of the account presented by the local serjeants and the bailiff for every manor. This Compotus Roll ${ }^{4}$ in turn was compiled from several distinct manorial accounts, of which specimens of the grange and stock accounts during the 13th century have survived, ${ }^{5}$ whilst the separate existence of others, such as a Rotulus Redditualis and an estreat roll of fines of court, is fairly certain. ${ }^{6}$

Conventual Records. - It is interesting to find that this first draft of the account was made in precisely the same form as the inrolments with which we are concerned, and, curiously enough, the whole of this evidence is confirmed by the survival of certain muniments relating to the estates of the Prior and Convent of Winchester. ${ }^{7}$ From the almost identical methods employed by the clerical establisliment of the Prior's exchequer we may fairly conjecture that they used the style of the Bishop's

1 The Palatinates of Durham, Lancaster, and Ely, which afford no trace of any sessions of an Exchequer for fiscal purposes.

2 The expenses of these audits are given in 15 th century accounts of the bishopric of Winchester and the Duchy of Lancaster.

${ }^{3}$ In the case of Richard of Ilchester, cf. Dialogus de Scaccario, p. 77, "Datus est ei (Episcopo Wintoniensi) locus ad latus Thesaurarii, ut, scilicet, scripturæ rotulorum et hiis omnibus cum eo intenderet."

4 Ministers' Accts. Gen. Series, 1141, and Eccles. Comm. "Various."

${ }^{5}$ A mongst the fragments of the accounts of Bishops' Temporalities connected with the See of Winchester, specimens of the following subsidiary accounts, dated for the most part in the 13th century, can be identified, viz. rolls setting out sales of stock, loss of stock by murrain, flockmasters' accounts, sales of forage, wages of manorial officers, grange and stock accounts, rough drafts of bailiffs' accounts.-(Ministers' Accounts, General Series, 1141-1143.)

6 In the later period a roll of arrears seems to have been compiled every year by a clerk specially employed at Wolvesey for that purpose. No trace of this, Lowever, survives.

7 This can easily be verified by an examination of the Compotus Roll of 1248 , still preserved at Winchester, and of other manorial records of the church which are so well described in Mr. Baigent's edition of the Crondal Manor Rolls for the Hampshire Record Society. 
exchequer at Wolvesey ${ }^{1}$ but at the same time we find no trace amongst these conventual muniments of a ceremonious audit or fiscal establishment. On the other hand, we have a Rental or Customary dating back to $1221{ }^{2}{ }^{2}$ and other indications that the normal practice of the analysis of income and expenditure in a mouastic precedent book was resorted to in the place of any independent attempt to follow the practice of the royal Exchequer to its climax in the composition of a great revenue roll.

This, then, is the real nature of the muniments with which we are here concerned. They are the official inrolments of a long series of accounts of manorial officers, specimens of which still survive in their original form from the beginning of the 14th century. The system here observed is, therefore, precisely similar to that employed for the official inrolment of ministers' accounts in the Pipe Office of the Royal Exchequer, ${ }^{3}$ and this similarity of practice favours the hypothesis that besides these inrolments of manorial accounts, actual Pipe Rolls recording the state of the whole episcopal revenue were compiled in the exchequer at Wolvesey.

Establishment of the Bishop's Exchequer.-Indeed, if this were not the case it would seem difficult to explain the allusions made in this and other accounts to an offieial who clearly acts as, and is elsewhere entitled, the Bishop's Treasurer, to an Exchequer and Treasury, to the departments of a Marshal," and a Constable ${ }^{5}$ to a "scriptorium" or "camera clericorum," to clerks, serjeants, and messengers, and to the regular provision of parchment at Wolvesey. ${ }^{6}$ Moreover, we have notices in the text itself of sessions of this episcopal Exchequer at which the Bishop presided on certain occasions in person, whilst adjournments from term to term are recorded in the precise form of King's Courts at Westminster. Finally, we liave a distinct reference in the present account to a further record which is actually styled "Magnus Rotulus," this being the contemporary designation of the so-called "Pipe Rolls" of the royal Exchequer."

The Great Roll. - That the record here referred to was not a preceding account roll of the same series, ${ }^{8}$ nor even a mere Rotulus Redditualis such as may be found in ecclesiastical and monastic registers from the 13th century onwards is proved, not only from the marginalia of this account, but to some extent also by the external evidence of the Exchequer Pipe Rolls themselves. Here the Accounts of the custody of the bishopric during vacancy have preserved several headings which presumably occurred in the episcopal rolls which must have been used for

1 It will be remembered that the famous dispute between the Bishop and Convent in 1253 concerned the jurisdiction claimed by the Wolvesey exchequer (Ann. Winton. s. a.).

2 Crondal Manor Rnlls, p. 83.

3 L.T.R. Foreign Accouvts.

4 It was apparently his business, in addition possibly to the custody of tallies, writs, aud prisoners, to supervise the collection of the Scutage and the service of the Bishop's knights.

5 The Constable in a slightly later period seems to have paid the liveries of the household just as his namesake in the royal Curia is represented as doing in the reign of Henry II., and this would imply that a "Liberate" Roll of some sort was in use.

6 cf. Dialogus de Scaccario (Clar. Press) p. 81, where the Sacristan of the cathedral church of Winchester is represented as supplying parchment for the royal Exchequer.

7 p. 56. "Præterea liberavit, anno præterito, iijs. iijd. plusquam recepit ; et ei computatur per Maguum Rotulum." In addition to this "Great Roll" there must evidently have been \& "Rotulns Receptarum " to check the Receipts allowed in these accounts under the heading "Liberatio " (cf. "The Receipt Roll of the Exchequer, 31 Henry II." previously published in this serles).

8 The technical reference to these seems to have been (us in the case of the Exchequer records) to a "rotulus anni prateriti, tertii," etc. 
the purpose in hand.' Amongst these we find the "Terræ datæ," the "Liberationes constitutæ servientium maneriorum," and the "Elemosyn constitutæ per maneria Episcopatus," the "Firma anni præteriti" and the "Nova firma." 2 The occurrence of these distinctive terms as early as the year 1172 would, moreover, seem to indicate an antiquity for this fiscal procedure which can searcely be measured by existing records. ${ }^{3}$ But at least we have evidence that the series of inrolled manorial accounts with which we are immediately concerned existed for an earlier period than the year $1208,{ }^{4}$ together with numberless allusions to questions ' of account going back to the days of Bishops Godfrey and Richard, and even to the time of Henry of Blois himself. ${ }^{5}$

An explanation of the use of the formulas of the royal Exchequer by the episcopal Scriptorium followed' in turn by the conventual staff has already been suggested from the close proximity of the two establishments at an earlier date ${ }^{6}$ and the official relations of Peter des Roches and his predecessors with the royal Exchequer. When we add to this fact the personal interest displayed by these great statesmen in the administration of their estates, ${ }^{7}$ it is scarcely to be wondered if they made full use of the administrative model familiar to them.

Manorial Records.-One other consideration is suggested by the survival of this venerable series of rolls, namely, the antiquity of the system of compiling manorial accounts in the form that is so familiar to us in a later period. That such accounts were compiled in a very similar style on behalf of the temporal and spiritual princes of Western Europe from the beginning of the 9th century at least, we know from surviving examples, but we have here the means of tracing baek the actual form in general use in this country in the middle of the 13th century to the closing years of the 12th; and we have also allusions to the same form at least as early as the middle of the reign of the first Angevin king. ${ }^{8}$ Moreover, the form referred to clearly implies the co-existence of another manorial record, the prototype, if not the actual model of the Court Roll, surviving specimens of which date as a class

1 That they were so used in a later period is proved by several iuteresting remains preserved amongst the accounts of Bishops' 'Temporalities.

2 Pipe Roll, 18 H. 2, Southn.; ef. also the subsequent accounts during racancy recorded in the Pipe Rolls, 1 Ric. 1. and 7 John.

a ef. the account of the bishopric of Lincoln in the Pipe Roll, 31 Hen. 1., which agrees with the forms of later accounts during vacancy for other churches than Winchester, which latter church alone possesses the distinctive formulæ above referred to.

1 In Roll 155278 (1222) arrears of 18 years past (1204) are brought to account; ef. pp. 73 and 78 infra, which give references to an account of 1205 .

5 Pipe Roll, 18 H. 2.

- The Winchester account rolls preserve several indications of these relations under the heading of the Ministerium of Winchester.

7 William Giffard's activity and Henry of Blois' ambition herein are well known. The latter, moreover, according to one of his bingraphers, left his manors well stocked and was an example to all in the administration of his estates (Gerald. Cambr. (Rolls) VII., 49). Whether Riclard of llchester and Goulfrey de Luci were, equally capable (ef. Stubbs' Oxford Lectures, p. 128) is so clear in the face of the evidence of the episcopal and royal accounts (cf.pp. 76-77 and Pipe Roll, 7 John Southt); but Peter des Roches not only continued to take a leading part in the administration of public affairs, but left at his deatlı in 1238 his manors cultivated to the highest point, an example followed by most of his successors (cf. Registers of Sandale and De Asserio).

8 'That such accounts were in use as early as the year 1170 is evident from the allusions. in a contemporary and original inquisition in which default in a bailiff's "compotus," and also in the several items of the inventory of stock and the "exitus grangiz" are duly "sppreciated" (Red" Book of the Exchequer (Rolls), p. cclxxvii.). 
only from the last years of the 13th century. ${ }^{1}$. To these two classes of manorial records may perhaps be added most of the subsidiary accounts of the "Compotus" itself, namely, the inventories of grain and stock which reappear as schedules to the latter document, and the statement of rents and services which was in turn derived from inquests and surveys of immemorial antiquity.2

In the present account many incidental notices occur, as might be Historical expected, of the Bishop's establishment as well as his public and private life, and these are supplemented by several historical allusions of more general interest.

In addition to the fiscal references cited above, we have others to the Bishop's wardrobe (peripatetic like the King's), to his household and kitchen, and to his princely hospitality. The cost of a dinner at Farnham, where the Bishop entertained the justiciary, ${ }^{3}$ William Brewer, and the Chancellor, is charged as $4 s .8 d$.

There are also many payments and allowances in connection with hunting and falconry, some of which are connected with royal visits, and the forester, verderer, and warrener are familiar figures in the manorial economy. On one occasion the. Bishop buys hawks, ${ }^{4}$ and on another he buys or sells dogs at St. Giles' fair, whilst, as a result of the royal hunting, he incurs, the cost of salting the King's venison, ${ }^{6}$ for which he perhaps recoups himself by selling 71 foxskins from Waltham to a Winchester tradesman. ${ }^{7}$ That wolves were still in evidence in Hampshire is clear from the entry under forest ponies devoured by these marauders. ${ }^{8}$

In connection with hunting accurs one of the few direct contributions of our Roll to the history of the perind. This is found in the references to the severe drouglit of the summer of 1209 , in the shape of payments for deepening wells and the substitution of cows' milk for that of the ewes in certain manors, "propter nimiam siccitatem." Apart from its economic importance, the incidental record of the occurrence of this drought is of considerable interest, owing to the fact that it does not seein to be mentioned by any medirval historian. It is also possible that this plienomenon may explain an obscure passage in the chronicle of John of Oxnead, ${ }^{10}$ where we read that in this year the king ordered the hedges to be burnt down and the fences to be levelled in all the forest districts in England, leaving the forest beasts free to feed on the crops.

Now this transaction is taken by the Rolls editor to refer to an edict

1 The reference is of course to the Purchasic or issues of the manor courts estreated into the bailiff's accounts (cf. Manorial Pleas, Selden Soc., p. xv.), and the inquisition of 1170 sbove referred to (Red Book, p. cclxxvii.). It is scarcely too much to say that the Compotus Roll of 1209 enables us to reconstruct the contemporary Court Roll with the help of existing local forms of a later date.

2 These may be traced back in the case of the bishopric of Winchester to the beginning of the 10th century (Cart. Sax. 612,618,622), and through continental examples to the 6th century.

${ }^{3}$ p. $39,1.4$.

p. $38,1.64$.

s p. $35,1.51$.

${ }^{6}$ p. $71,1.45$.

7 p. 5, 1. 16. In the Year Roll of 1210-11 we find that the King sent deer from Hereford to replenish the Bishop's depleted herd at Taunton.

${ }^{8}$ p. 36, 1. 31. At Rimpton, the whole hen-roost was cleared by foxes (p. 73).

9 pp. 37, 50, 82 . Similar notices occur in the Prior's "Compotus" Roll for the year 1248 (Crondal Manor Rolls, p. 54, sq.). ,

10 Rolls Ed., p. 112. 


\section{( niv)}

for the "free pasturage of cattle," but this could not in any case be the sense of the passage, since "bestia" is the technical expression for "beasts of "the forest," 1 and the act imputed to the king seems to be one of tyranny and not of munificence. It is not unreasonable to suppose, however, that the expedient was resorted to by the Crown to save the deer from starvation, owing to the failure of herbage; and it is interesting to find, though the fact is unnoticed in the only surviving Chancery Roll for the period, a writ despatched at this time from the king to the Bishop "pro defensa boscorum." " Perhaps, too, the drought may account for the considerable value of manorial herbage in this year, and payments for the home farms, as well as for the numerous fines "pro pastura" in the issues of the courts.

Such local notices as those which refer to the lepers of Farnham ${ }^{3}$ and the 'fire at the Bishop's palace of Marwell ${ }^{4}$ have some interest, and in this connection attention may be called to the survey of the arms of the hundred of Sutton, ${ }^{5}$ to the lighting of a beacon upon Quantock in Somersetshire, ${ }^{6}$ and the issue of the "new charter of liberties" in favour of the church of Winchester. ${ }^{\text {? }}$

As a source of historical reference, this Roll possesses a peculiar value as a means of supplementing, however slightly, the meagre notices which have survived of the movements of the Court between May and October of the year 1209. It is well known that, for some unexplained cause. the great series of Chancery Rolls are wanting for this and the two following years, ${ }^{\mathrm{s}}$ and this void in the existing materials for a royal progress and its incidents has been partially supplied by the survival of a Wardrobe Account ${ }^{9}$ for the 11 th year of the reign, and kindred records ${ }^{10}$ for the succeeding period. Now an examination of the first of these supplementary enrolments will show that it is chiefly coneerned with the movements of the king and the affairs of State, which seem to have been largely transacted by the Bishop of Winchester who was in attendance during the greater part of the period between the Ascension and Miehaelmas. Thus the information which our Roll 'supplies respecting the engagements of the Bishop, throws considerable light upon those of his royal master, in addition to supplementing the published itinerary of the Court in several particulars, though, unfortunately, the nature of 'the account does not enable us to fix the dates of certain of these notices. An itinerary showing the Bishop's movements and relations with the Court during the period of this aecount, is appended to the Preface.

The names of notable persons which occur in the Roll will be found in the Index."

1 Pleas of the Forest (Selden Soc.), p. x.

2 p. 68.

p. $38,1.62$.

p. 53.

5. 42,1.27. At Rimpton it seems to have been the custom to give a feast to the suitors of the hundred Court (pp. 72, 73).

6. 68, 1. 43. A beacon is also charged for at Witney (p. 18, 1. 1.)

7 p. 17, 1. 43.

s The Close Rolls break off in May, 1208, and the Patent and Charter Rolls in May, 1209.

- Rotulus Misæ (Record Commission).

10. Rotuli de Liberate et Præstitis.

11 Further notices of most of these will be found in the Rotulns Misæ for this date, and in the Chancery Rolls of the preceding years. 
It will be seen from the Table of Contents that the manors included The Episcoin this Account are thirty-seven in number, reckoning the manors of pal Manors. Fawley and Ower, the manor and church of East Meon, and the Ministry of Winchester itself, as fiscal units. As this list obviously does not represent the full rent roll of the bishopric, it appeared to the Editors that some explanation of the discrepancy should be attempted. To present a complete list of the possessions of the See in the year 1208 would scarcely come within the scope of the present edition, although the task of tracing these possessions from tho ninth eentury to the thirteenth has been essayed for the purpose of a preliminary investigation of the subject. But even when the full extent and position of the episcopal holdings have been ascertained, we are by no means justified in assuming that the accounts of certain manors should have appeared in the rent roll for a given year. In the first place it must be remembered that the distinction between the possessions of the Bishop himself and those of the Prior and Convent is, as in so many other cases, excecdingly obscure. Again, it will be found from a careful examination of the rent rolls for a subsequent period that the classification of the several manors is somewhat artificial, and that a hamlet which appears elsewhere as an independent estate may be included here in the account of the capital manor. Thus, in the present account no distinction is made, as in subsequent rolls, between Old and New Alresford, or between High Clere and Burgh Clere, whilst Waltham St. Lawrence and North Waltham are probably included in the accounts for the manors of Wargrave and Overton respectively. Other hamlets which held an independent position in the rolls of a later period cannot be distinguished under the principal manors, and in more than one case are certainly not included therein. Finally, we must bear in mind the possibility of the loss of one or more membranes of the existing record, a mutilation which can clearly be proved to have taken place in the case of more than one of the following year rolls.

Therefore after every allowance has been made, it will be evident that certain recognised manors which duly appear in subsequent accounts are omitted from this roll. ${ }^{1}$ It can also be shown that other manors which were, presumably, at this time in the Bishop's possession, are not represented in any of the accounts for the period.

These omissions do not, on the whole, amount to very much, but an explanation of their existence is still to be found. Possibly the manors in question were temporarily alienated from the possessions of the See, or were let to farm under conditions that precluded the necessity or possibility of their inclusion in the present series of accounts.

It is true that even in the case of a farmed manor the seignorial dues would have to be accounted for, and tliree such manors are, in fact, brought to account in this very roll. It may be suggested, however, that the "Perquisites" of a manor that was not actually in the lord's hand were not infrequently collected and rendered in the account of the tenant as farmer, ${ }^{2}$ and that this account would have been included in the general account of the

1 Amongst the noticenble omissions under this heal are the manors of Calborne in the Isle of Wight, Bentley in Hampshire, and Iringhoe in Bucks. A possible explanation of these omissions which might be found in the compilation of the accounts at irregular intervals does not seem applicable to the present case.

2 Cf. Cart. Rames. (Rolls) II. 244. 
temporalities and spiritualities of the See, which must have been rendered at the Bishop's Exchequer in another form of inrolment. On the whole, therefore, it seems probable that the intention of the auditors at Wolvesey was to enter such manors as were occasionally at farm by way of a schedule to the regular series of bailiffs' accounts, in much the same way as certain foreign accounts were appended as a schedule to the earlier Pipe Rolls of the Royal Exchequer, and in both cases these entries were frequently appended to the local accounts instead of being entered on a separate membrane. ${ }^{1}$

Local Notices. - The variations which have been noted in the titles of the Episcopal Manors are not perhaps sufficient to cause any real difficulty in their identification, in spite of the fact that the order of enrolment is perfectly haphazard, and that there are few indications of local identity in the body of the account. The principal manors of the See are easily recognised, but several difficulties have been encountered in the identification of tithings and field names.

Amongst the casual topographical notices to be found in these manorial accounts the enumeration of the tithings of various townships is of some value at this early date. Local archæologists will naturally be interested in the notices which oecur here of the episcopal seats at Farnham, Taunton, and Winchester itself. To these may be added several references to the residences at Waltham, Clere, Marwell, Downton, Mardon, and Southwark.

Geographical Distribution.-Of the thirty-five manors accounted for in

Means of Communication. the roll, twenty-two lie in Hampshire. The rest are scattered here and there in the neighbouring counties, Southwark, Adderbury, and Taunton being the most distant on the east, north, and west respectively. The Hampshire manors fall into two main geographical divisions. The first, comprising sixteen out of the twenty-two, lies in the south-east of the county, along the upper and lower Itchen and its estuary, and in the chalk uplands to the east and west, forming an irregular circle round Winchester and Bishop's Waltham. The second, a compact geographical and economic group, lies in the high chalk downs on the northern border, with its centre in Bishop's Clcre. ${ }^{2}$ The isolated manor of Overton lies between the two. The external manors, scattered as they are, may also be considered as falling into two or three groups. Four manors ${ }^{3}$ lie in the basin of the middle Thames, and have special economic relations with Southwark. 'Two others ${ }^{4}$ situated on the tributaries of the upper 'Thames form outposts, as it were, of the estate to north and north-west. ${ }^{5}$ Farnham, on the River Wey, lies on the road between Southwark and Winchester. Finally, the three Wiltshire manors, ${ }^{6}$, with Rimton in Somerset, form geographically a chain of connection between Winchester and the important but isolated episcopal borough of Taunton.

Intermanorial Communications.-Although these manors collectively form a single economic whole, the internal management is, as a rule,

\footnotetext{
1 They are entered on the top margin of the roll for the sixth year. In the fourth year only three are entered, Fonthill, Fawley, and Ower. An interesting inventory of the leased stock of the first-named manor, as well as of that found in the manor of Ebbesborne (Bishopston), on its resumption, appears in the roll for the sixth year. We also learn incidentally from later rolls that other manors, previously omitted in these accounts, had been farmed, and amongst these are Bentley, Ivinghoe, and Combe Florey. From the Rimpton account in the present roll we find that this manor hidl been previously at farm.

2 See "group" No. 1.

3 Harwell, Brightwell, Wargrave, Wycombe.

4 Adderbury, Wituey. $\quad{ }^{5}$ See the section on travellers. ${ }_{6}^{6}$ Downton, Fonthill, Knoyle.
} 
entirely independent. There is ample evidenee, however, of a casual interchange of grain and stock, and in a few cases it is possible to distinguish certain manorial groups, the distinctive feature being the rendering of the several accounts by one and the same serjeant, though by different reeves. These groups are collected as indicated in the following table.

Group,

1. Clere

\begin{tabular}{lll|c} 
Woodhay & - & - & - \\
Ashmansworth & - & - & Johannes \\
de \\
Itchingswell - & - & - & Welton.
\end{tabular}

2. Alresford

Privet ${ }^{1}$

Cheriton

Wield

Beauworth

3. Brightwell

Harwell
Serjeant.

\section{REMARKS.}

Clere is to some extent the centre of the group for the sale of produce and the supply of the bishop, but each manor is worked independently in respect of grain. In the stock account, however, there are traces of specialisation and consequent interchange in the different generations of sheep.

The manors are worked independently. Alresford sells part of the surplus produce of all. The bishop is supplied at Sutton directly from each manor.

The grain is accounted for and sold separately, each sending a quantity to London (i.e., Southwark) for sale. The stock shows the same specialisation as in the Clere group.

Though each sells separately, they are apparently worked in combination, the sale of large quantities of produce being thereby facilitated. The stock of oxen and sheep is also managed jointly without apparent specialisation.

Worked independently, but Stoke assists Twyford in the supply of the bishop at Marwell. A comparison of the accounts shows the bookkeeping to be distinct. Stoke ignores the agency of 'Twyford (indicated there) in the receipt of bacon from Wolvesey.

Fawley and Ower are practically submanors, accounted for by Johannes as "serviens de Biterne." The expenses of the latter and the reeve of Bitterne visiting 15 times are charged in the Fawley and Ower accounts.

Markets.-The Roll affords very little evidence or illustration of the function of the special market in the manorial economy. Even within a group whose chief village (e.g. Clere and Alresford) is a market, it appears that the sale of a good deal of the produce takes place in each manor separately. In the isolated manors there is no evidence whatever of the

I In later Rolls Privet and Wield are combined, Still later Privet is a sub-m ınor of Wield. 
method of sale, or of the disposal of the produce accounted for as sold. In the case of Brightwell and Hurwell only does it appear from a record of - the cost of carriage, that a portion of the wheat sold ( $\frac{1}{4}$ and $\frac{1}{3}$ respectively) was despatched by water to London, and a comparison with the later Rolls shows that this sale was carried out by the agency of the Southwark centre. ${ }^{1}$

Special Centres.-The following are the chief Centres for the receipt or supply of special commodities :-

1. Bitterne, which supplies salt to Downton, Farnham, and Sutton.

2. Southampton, the port of entry for foreign goods, e.g. millstones and wine. The latter is imported in bulk and packed for distribution by the Bitterne tenants.

3. Fareham appears to have been a depôt for wine, which forms a separate item in the "Instaurum."

Relations with Headquarters.-The needs of the bishop and his household are supplied by the manors, either by delivery at the various episcopal residences, or by direct contributions to his maintenance from the manors at or near which he may be staying on his journeys. With the exception of the regular supply of hens and eggs to Wolvesey, and of pigs for the larder, these contributions are of such a casual nature that they appear to be a kind of oceasional purveyance resembling the ancient Pastus, rather than the payment of a stated due--the amount supplied in kind simply reducing the total sum received from the sale of produce.

The episcopal seats draw their supplies for the most part only from the manors near at hand: Thus, Waltham receives supplies from 9 manors, all in South-east Hampshire. Wolvesey from 13 (6 being fiscal contributions), all in Hampshire. Downton from 5 manors rather more distant, ${ }^{2}$ and Sutton almost wholly from the Alresford group. Southwark has direct relations with the manors of the middle Thames basin-Brightwell, Harwell, Wargrave, and Wycombe.

External Communications.-The following instances of transport to or from places outside the bishopric appear in the Roll.

Downton and Knoyle send supplies to the neighbouring royal seats of Cranborne and Gillingham respectively. Farnham, Downton, and Clere receive rams from Lincolnshire; Knoyle, wool from the same county; Southwark, ${ }^{3}$ timber from St. Leonard's Forest; Twyford, a horse from Devizes; Witney, horses from Cardiff, and Mardon and Clere, mares from Wales; Taunton receives wine from Exeter, spears from Wales, venison from Bridgwater, and a cart from Bristol, whilst Sutton charges for the carriage of a consignment of wool to Beaulieu and back, and Witney for the carriage of arms and provisions to and from Tewkesbury.

Routes.-In respect of routes, the Roll furnishes little information. Corn from Brightwell and Harwell goes to London by water. So, naturally, do the consignments of wine from Portsmouth to Fareliam, and from Southampton to Bitterne, whilst presumably the timber from Kingston and the

1 'The later Rolls give rather more evidence of special markets. An account for the Forum de Alresford appears in 1211 , as also does an account for the granges at Southwark, showing that a very large proportion of the grain received from the Thames manors was disposed of by sale.

2 This manor is isolated, aud was visited more than once by King and bishop.

3 In addition to this entry, references to "Loudon" under Brightwell, Harwell, Wycombe, and Wargrave evilently refer to the manor of Southwark. 
brushwood from Wargrave would have been similarly conveyed. The most interesting entry in this connection is the charge for the carriage of wool from Bishops Sutton to Beaulieu and back "per aquam," i.e. by the Itchen navigation, the canal made by Bishop Godfrey de Luey a few years earlier.

Cost of Carriage.-As a rule transport and the moving of stock forms part of the labour service due from the manors. The few records of payments for carriage refer to exeeptional commodities such as wine, ${ }^{1}$ millstones, wool, and in one instance salt; or to distant places-London, Tewkesbury, Beaulieu; or to carriage for the casual pleasure of the bishop. ${ }^{2}$ In the account for the church of Meon, the collection of the tithe and the transport of the produce to Winchester and Marwell appear to have been outside the obligations of the bishop's tenants. The cost of carriage, as might have been expected, is somewhat high. A tun of wine from Southampton to Waltham costs $1 s$. $2 d$. To Witney the cost is $6 s$., and to Marwell $1 s$. $7 \frac{1}{2} d$. From Portsmouth to Fareham by water the charge is only $6 d$., and from Southampton to Bishops Sutton, where the Itchen navigation could be used, 1s. $6 d$. The corn sent from Brightwell and Marwell to London costs $2 d$. a quarter, and a millstone from Southampton to Winchester 18.

Travellers. - The visits of several of the bishop's officers to the various manors are recorded, but the entries are so often undated, that they afford no evidence of any systematic visitation or of the movements of the bishop's household. There are also, here and there, clarges for the maintenance of occasional travellers, notably at the outlying manor of Witney, which was visited by King and bishop, and which seems to have been a starting-point for journeys to the North and West (York, Lincoln, Northampton, Cardiff, Tewkesbury). Harwell entertains the bishop's knights on their way back from the Scotch expedition; ${ }^{3}$ Clere the men who carried the bishop's harness to Gloucester, and to Witney after he had left; and Southwark the "Milites de Ispania."

On the manors of the Bishop of Winchester the conditions of employ- The Mament appear to be somewhat primitive. The necessary ploughing, mowing, norial Ecoand reaping were almost entirely performed by the tenants as a condition of their tenure, so that the relation of the tenants to their lord determines the relation of most of the labourers to their employer. Money wages are paid, but to a small extent, and by far the greater part of the agricultural work is performed by the tenants by way of service.

The payments of the tenants to their lord are made in (1) money: (2) kind, (3) service, and (4) as a mark of subjection to the lord's jurisdiction.

1. Payments in Money. - The clice payment made in money is the "land-gafol" (gabulum). In two of the Berkshire manors, however, and also at Southwark "rents of assize" (redditus assisi) are found. In normal years these charges furnished more than a third of the total revenue from all sources. Here the proportion is somewhat less owing to the

1 The transport of wine from Exeter or Topsham to Taunton at a uniform rate of $2 s$. per cask, appears to have been one of the customary services of that manor. (Somerset Arch., and Nat. Hist. Soc. Trans. xviii., 82.)

2 e.g. presents to the King's daughter, venison, etc.

3 Cf. under Adderbury, where knights of the bishop are present "in crastino Assumptionis" (August 16). Cf. Ann. Winton. "Facta sunt hrec (the Scotch experlition) meusibus Augusti et Septembris." 
incidence of the Tallage. The system by which it increased and decreased appears from the details of the "incrementum" and "defectus."

The Incrementum.-The incrementum refers to the increase of rent in the year of the account only; next year it is added to the gross amount of the gabulum, which thus continuously increases.' Sometimes new houses are built (at Brightwell, a new fulling mill), and for these in future years rent is paid which increases the gabulum. ${ }^{2}$ But a more considerable addition arises from the cultivation of waste land. At Cheriton rent is now first paid for two encroachments, ${ }^{3}$ while at Witney $£ 211 s$. $1 d$. in this one year forms a permanent inerease "de assarto." "

Another cause of an increase of the gabulum was the letting of the demesne land at a money rent. At Witney $12 s, 6 d$. more is paid this year because some of the demesne land had been so let. $^{5}$

The Defectus.-The decrease in the gabulum may be due to causes operating over a long period of time, as can be well seen in the accounts of the Ministerium Wintonice. But the most important decrease arises from the enlargement of the demesne. Thus at Clere one piece of land taken in reduces the gafol by $64 s$; ; another portion of 335 acres by $37 s$., whilst at Otterford also the rent is reduced "pro dominico elargando ": and in most of the manors both large and small pieces of land appear as "tractæ in dominium." "These deficiencies also did not arise in one year, but in a series of years dating probably from the beginning of the accounts. These entries, therefore, show that there was then little tendency to decrease the amount of land held in the lord's own hand, either for pleasure or profit. In one case, however, we find a holding "quae modo operatur et non solebat." s

Firma burgi.-Besides the regular gabulum, Taunton and Farnham paid a "firma burgi" and Wallingford a "burgagium." " This means that the sums due from the burgesses of these towns were farmed for a lump sum, unless the town was "in the lord's hand."

Firma. - In the case of three manors "farms" are paid to the lord," and in several other cases part of the produce of the manors is farmed. Fawley, indeed, seems to have been only a sheep farm. ${ }^{11}$. Sheep are also farmed at Hambledon, ${ }^{12}$ and their milk at Menes. ${ }^{13}$ Rimpton seems to have been lately at farm. ${ }^{14}$

On most of the manors mills also are let at farm, ${ }^{15}$ though in others they are kept in the lord's hand, and have their own profit-and-loss account. ${ }^{16}$

Besides the gabulum, rent was paid for the use of certain demesne lands entered in the accounts as Terra Locate. At Waltham, Bitterne, and Sutton the lands so let were rented at about $2 d$. per acre. ${ }^{17}$

I Thus at Waltham the gross gabulum is as follows:-1208, 46l. 7 s. $2 \frac{1}{2} d_{\text {. ; }} 1210,46 l$. 11s. $4 \frac{1}{2} d$.; $1211,46 l .17$ s. $4 \frac{1}{2} d$. ; 1290, 75l. 3s. 11 d.

2 At both Downham and Alresford there were new markets; in the former town ten places were newly assized.

${ }_{6}^{3}$ p. $80,1.20 . \quad$ p. 16, 1. 34. $\quad 5$ This expedient was as yet rare.

${ }^{6}$ p. $64,1.11 \quad{ }^{7}$ p. $1,1.68 ;$ p. $11,1.47 ;$ p. $55,1.50$, \&c. $\quad$ s p. $26,1.10$.

9 New Alresford does not figure in the present account.

${ }^{10}$ p. $80,11.7$ to 10 . See supra, p. xvi., n. 1.11 p. $79,1.40$.

12 p. $48,1.51$. 31s. $0 \frac{1}{2} d$. for 155 sheep seems to be at the rate of nearly $2 \frac{1}{2} d$. a sheep.

${ }_{13}$ p. $44,1.20$. 4l. $2 s$. $6 d$. for milk of 412 sheep.

14 p. $72 . \quad{ }^{15}$ p. 30, 1. 34, \&c. 16 pp. 64, 67.

17 pp. 1, 30, and 41. At Menes $3 d$. an acre was paid, p. 44, 1. 23. 
The usual payments, Herbage and Pannage, for the use of pasture by eattle and sheep and for feeding the swine in the woods, occur in every manor.

In addition to these various forms of rent a "tallage" is paid in nearly every manor, but at Taunton a "seutagium" takes its place. This tallage was exceedingly heavy, amounting to little less than the net gabulum. Probably its occurrence in this year is due to the heavy national taxation of the year 1207.

Akin to the tallage is the sum paid "de redditu annuo" at Wargrave. This seems to have been a regular annual payment over and above the rent.

Local Dues.-Other payments made to the lord were the "hundred penny," and sums paid in several places "de telonio tabernarii." 1 There were also the inevitable "scotales," from which at Sutton as much as $3 l$. $10 s$. $8 \frac{1}{2} d$. was received.

Peter's pence was presumably paid to the bishop in his ecelesiastical character. It occurs in the accounts of Witney, Downton, Wycombe, and Rimpton.2

The money payments made by the tenants are thus very considerable in number and amount. The payments in kind are less important.

2. Payments in Kind.- "Church scot" (Cheriset) was another payment made to the bishop as representative of the Church. The payment was made partially in money at Downton, Sutton, and Knoyle, ${ }^{3}$ but it was usually paid in grain ${ }^{4}$ and in poultry. ${ }^{5}$ Payments in kind were also made "de redditu." At Waltham $2 \mathrm{lb}$. of eummin were due, ${ }^{6}$ two thousand eggs at Clere, ${ }^{7}$ and hens at Witney ${ }^{8}$ and other places. At Downton also fortyseven sheep were rendered "de eonsuetudine herbagii." "

A more important obligation was connected with the lord's fold (Potfalda). 'The sheep of the tenants were obliged to lie on the lord's land so that his land and not theirs was enriched by the manure. In several manors money is given by the tenants for the relaxation of this privilege. ${ }^{10}$

Multure.-Another right largely exercised by the mediæval lord was that of obliging his tenants to grind their corn at his mill. There is little trace of any payment for the relaxation of this burden in this Roll. At Holway a fine is paid "pro moltura," "11 and in the accounts of some of the mills we hear of payments as "moltura" which at Flodstoc amounted to 39s.6d. " in denariis." 12

A payment is made at Bishops' Hull to be quit of the Bishop's beer, ${ }^{13}$ and this may indicate that the Bishop claimed a monopoly of brewing as well as of grinding corn.

3. Payment in Service.-But apart from the payment of rent or dues as above, the tenants also rendered service to the lord, either as manorial officers or as ordinary labourers, in a specified manner.

Manorial Officers.-In nearly every manor several manorial officers are found. These may be divided into three classes:-(1) Those who held land

\footnotetext{
${ }^{1}$ p. 15 , Harwell ; p. 16, Witney ; p. 32, Wycombe. Here there is also a payment "de thelonio lini."

${ }^{2}$ pp. 16, 20, 32, and 72. ${ }^{3}$ pp. 20, 41, 74. $\quad{ }^{4}$ pp. 3, 10, 12, 15, 45, 49.

5 p. $58 \mathrm{sq}$.

${ }^{8}$ p. 20.

6 p. 5.

? p. 22.

"p. 65, 1. 7.

${ }^{7}$ p. 8.

${ }^{10}$ pp. 41, 9, 5, 44, 10, \&c.

${ }^{13}$ p. $65, \mathrm{~L} .45$.
} 
and were rewarded for their services by abatements in rent ${ }^{1}$ (quietanciox), and sometimes also by payments in kind or money (consuetudines); (2) those who were probably household servants and were paid principally by small salaries (stipendia) and by allowances in grain (corredia); and (3) the more important officials who received much higher salaries (liberationes).

To the first class belong the reeve and the typical manorial officers. Besides the propositus there is sometimes a bedellus, or foreman, usually in connection with a sub-manor.

Forestarii are present in the forest manors and were presumably manorial officers. ${ }^{2}$ In any case tliey received an abatement of rent.

In nearly all the manors ${ }^{3}$ there are many ploughmen (carucarii). These apparently ploughed every day except Saturday, and if they ploughed on Saturday they were paid for so doing. Thus, at Stokes, eight ploughmen receive abatements of rent, and eight are paid for ploughing on eight of the Saturdays between Easter and Michaelmas. On the remaining sixteen Saturdays they receive no payment because they ploughed their own lands."

On most of the manors there were also swineherds, cowlierds, oxherds, haywards, shepherds, and a certain number of operarii, all of whom must have held land, as they were paid by quietancioe. Other officers more rarely mentioned are the grangiarius ${ }^{5}$ or berebrettus, ${ }^{6}$ special guardians for the corn, for the lambs, or for the cows; ${ }^{7}$ one manor has a "parcarius" 8 and besides a special man to care for the horses. At Downton, we find a plot of land held for the special service of repairing the bridge. ${ }^{9}$

The most necessary work which was not agricultural was that of the smith. In many manors he received an abatement of rent, ${ }^{10}$ but in others he was paid in money. ${ }^{11}$ Thus, at Farnham, the smith shoed a certain number of the horses by custom ${ }^{12}$ and was paid for shoeing the rest. In addition, the ironwork of ploughs, carts, and harrows was paid for, ${ }^{13}$ as well as the iron about the mills. ${ }^{14}$

Consuetudines.-The manorial officers occasionally received perquisites (consuetudines) as part of the reward of their labour. Thus, the swineherd receives a pig, the shepherd a lamb or fleece, ${ }^{15}$ the ploughmen have barley and cheese, ${ }^{16}$ the forester has a pig, ${ }^{17}$ and the smith a lamb. ${ }^{18}$ Payments to the ploughmen are also made in money ${ }^{19}$ (as at Harwell and Brightwell).

1 At Crawley, we are told, four ploughmen, one smith, one swineherd, two shepherds, and thirteen workmen pay no rent because "se defendunt per opera sua" (p. 50).

2 This is more doubtful in the case of the verderers who also occur.

3 Wycombe and Farnham are exceptions.

p. 56, 1. 47. At Waltham the seven ploughmen on twenty eight Saturdays in sowing time earned $1 \frac{1}{2} d$. a day; at the second ploughing they worked twenty-two Saturdays and earned $1 d$. a time.

5 Waltbam, Farnham, Ferham.

6 Overton, Sutton, Adderhury, Beauworth, Cheriton, and Knoyle.

7 Overton, Wargrave.

- Mardon.

9 p. $20,1.16$.

${ }^{10}$ Clere, Fareham, Fernham, Sutton, Hambledon, Crawley, Twyford, \&c.

1 Overton, Downton, Wargrave, Wycombe, Mardon, Ashmansworth.

12. p. $38,1.38$. $\quad$ p. $2,1.30$.

14 p. 2, 1. 45 .

${ }^{15}$ Overton, Woodhay, and Ashmansworth.

${ }^{16}$ p. $22,1.13$, and p. $23,1.13$.

${ }^{17}$ p. $19,1.52 . \quad{ }^{18}$ p. $22,1.66$, and p. $51,1.49$.

${ }^{19}$ p. $13,1.41$ p., 151.28. 
The Stipendiaries.-The class below the above receive stipendia in lieu of remission of rent." At Waltham they consist of seven oxherds, three carters, a gardener, a cowherd, and a dairymaid, and they are there styled famuli. The men receive an annual salary of $3 s$., the dairymaid $2 s$, and like salaries are paid elsewhere. Besides their salary they received allowances in grain (corredia), generally from six to six and a half quarters, and from this it would seem that barley formed the staple bread of the poor on these manors, varied by curall and mancorn. Taunton is, however, an exception, wheat being used on nearly all the sub-manors there. Occasionally, but not often, these servants also receive consuetudines. This was especially the case with the dairymaid, who usually received a cheese as part of her reward. ${ }^{2}$

"Liveries."-The third class of manorial servants comprises those who received liberationes. These were not so much manorial workers as special agents of the lord. Amongst these would be a great officer like the constable of Taunton, ${ }^{3}$ and lesser people like the warreners of Waltham and the serjeants who had charge of groups of manors." The ordinary payment for these seems to be a salary of $£ 30 s$. 10d. a year, or twopence a day. ${ }^{5}$

Besides these local officers there were others who visited the manors at special times for particular services. Such are the knights and others who survey the forests, ${ }^{6}$ the verderers who visit Clere and Witney, the huntsman in charge of $\operatorname{dogs}^{8}{ }^{8}$ fishermen, ${ }^{9}$ and officials who prepare for the coming of the bishop and look after his kitchen. ${ }^{10}$

The Seneschal.-At the head of the manorial officers was the seneschal or steward.11

Operarii.-From the ranks of the tenants also came the labourers who performed the agricultural work in most of the manors; but as there is no separate account of the "opera" at this date, we have only occasional information as to their number and services. Among them were the operarii who receive quittances on the various manors. At Farnham one operarius worked every day in autumn and every other day for the rest of the year, nine worked every other day throughout the year, and three worked in September.

Virgatarii.-At Witney forty virgatarii receive quietancice because of autumn work, and ten for winter work. 'Ten find five ploughmen for the whole year except Saturday, and two find a ploughman for quarter of the year. At Brightwell twenty-two virgatarii found fifteen men who worked every day except Saturday from the feast of SS. Peter and Paul until Michaelmas. At Brightwell and at Adderbury there were also cottagers (cotagii). But we only hear of these people because they receive quietanciae.

1 Probably most of these were farm servants.

2 Clere, Ashmansworth, Itchingswell.

${ }^{3}$ p. $68,1.17$; p. $72,1.43$.

"p. 2, 1.50. Also William Widecoc, who was probably a falconer; a cook and porters at Taunton (p. 68), a gardener at Southwark (p. 60), \&c.

s Waltham, Witney, Overton, Fareham, Wargrave, \&c.

p. $39,1.14$; p. $75,1.7$.

${ }^{8}$ p. $35,1.52$.

${ }^{9}$ p. 31. 1. 25.

${ }^{7}$ p. $6,1.54$; p. $17,1.63$.

10 Witney, p. 17, 11.47 and 48.

11 p. $6,1.55$; p. $24,1.42$; p. 50 , 1. 59 ; p. $61,1.48$; p. $53,1.60$; p. $76,1.46$; p. $34,1.43$. There are officials mentioned who may have acted as stewards or sub-stewards, chief among whom is Robert Bassett. 
Of the tenants who worked and received no quietancice there is little record. We hear of a provision of seed on the lord's part for the villeins in some of the sub-manors of Taunton. But besides the regular "works" the payments on account of precarice and bedripa seem to show that these were general and well attended. On most of the manors there were precarice in winter, in Lent, and in autumn, and there was also a precaria for the mowing of the great meadow.

Commutation.-One of the most remarkable features of the Roll, so far as it relates to labour, is the comparatively slight mention made of money paid in lieu of service. For thirty-one of these manors the amount received by the bishop by way of commutation for service is only $\$ 1017 s .3 d$. The very gradual extension of this practice of paying money instead of rendering service is one of the most interesting features of the accounts during the next hundred and forty years. The practiee gradually makes its appearance in nearly all the manors and slowly increases, but even in 1348 it does not reach a very considerable amount.

Wages.-But besides the work done by the tenants for their lord, some work is paid by money wages. At Privet and Wield most of the labour is performed in this way, whilst in many of the manors threshing and winnowing is paid for in money. In most cases, however, the wages bill does not exceed $£ 210$ s. The wages paid varied considerably; at Waltham men are paid $1 \frac{1}{2} d$. a day and women $\frac{3}{4} d$., and this seems about the usual rate for a day's work." Especially low wages were paid at Farnham, and much higher ones at Wycombe. ${ }^{2}$

There is little information as to the wages of artisans. Two sawyers at Taunton earned at the rate of about $1 s .5 d$. a week eaeh. Carpenters in Southwark earned $3 d$. a day, but wages would naturally be higher near London.

This account indicates the existence of manufactures by its mention of fulling mills. They existed in three manors, ${ }^{3}$ and a new one was built at Brightwell and had already begun to be profitable.".

With regard to the poor also the accounts afford certain information. The bishop frequently gives alms, though there are not instances on every manor, but only occasional gifts to individuals. ${ }^{5}$ Thus from Waltham he gives six bacons to the sick, ${ }^{6}$ from Cheriton he sends a quarter of wheat to a certain woman at Winchester. ${ }^{7}$ From Witney, Farnham, and Sutton alms and provisions are given to the lepers, ${ }^{8}$ and at Farnham money to a hermitage, ${ }^{9}$ and wheat to the monks of Winchester. ${ }^{10}$

Besides this, at Rimpton, a feast seems to have been given at the lord's expense; the whole Hundred was entertained at Christmas and flesh and grain were provided for their entertainment. ${ }^{11}$

1 Sowing at Rimpton costs $2 d$. a day, threshing $1 d$. At Bitterne two men and one boy obtain $2 s$. for six days.

2 At Farnham the rate for both winnowing and threshing wheat was $1 \frac{1}{2} d$. a quarter; at Wycombe $2 \frac{1}{2} d$. for thre日hing only. The average rates were as follows:-Threshing wheat and mancorn $2 d$. per quarter; threshing barley about $1 d$. per quarter. Ploughing at Woodhay and Overton costs $2 d$. an acre.

4 p. 13, 1. 55. The mill at Brightwell cost $£ 948.4 d$.

- In the Pipe Roll of 1172 during vacancy we read of "Elemosynæ constitutæ per maneria."

${ }^{8}$ p. $5,1.9 . \quad{ }^{7}$ p. $81,1.8 .{ }^{8}$ p. $19,1.62$; p. $38,1.63 ;$ p. $43,1.51$.

9 Of Ichulla, p. $38,1.63$.

10 p. 39, 1. 34. Bacon also is given in alms at Farnham, p. 40, 1. 49.

p. $73,1.20$; p. $72,1.56$, etc. 
4. The Lord's jurisdiction.-Besides the agricultural services owed by the tenants, they also owed service at the lord's Court, and the fines they there paid formed a profitable source of revenue. Many of these fines arc connected with the possession of land. Heavy fines are often paid for heriots and for reliefs ${ }^{1}$ as well as for admission or to keep possession." Other fines are connected with agricultural service; for failure to attend the lord's work, ${ }^{3}$ or for doing it in an incompetent manner." But perhaps most of the fines are for trespasses of various kinds, especially on pasture. ${ }^{5}$ A few concern the keeping of order, breaches of the peace, ${ }^{6}$ and making false claims." Some are from the whole luundred or tithing, sometimes for concealment ${ }^{8}$ of offences, and at other times for an offence not stated, probably non-appearance. ${ }^{9}$ Others relate to the disabilities of the villein status. Payment is constantly made for marrying a daughter, ${ }^{10}$ sometimes for marrying a sister, ${ }^{11}$ and fines are exacted for dealing in cattle without leave, ${ }^{12}$ or for licence to leave the manor. ${ }^{13}$ At Itchingswell a man is fined because his brother left the precaria without licence. ${ }^{14}$

From the Balance Sheet given in the Appendix it will be seen Crops. that the sale of corn provided one of the most important sources of the Episcopal revenue, being equal to about one-half of the net gabulum, and nearly 16 per cent of the total receipts. In addition to the usual grain crops of wheat, barley, and oats, most of the manors cultivated also a fourth crop, consisting either of mixed corn ("mancorn" or mestilo) or of rye (silligo or sigalum). Mestilo seems to liave been the Berkshire, Buckinghamshire, and Oxfordshire name for the crop which was more generally known as "mancorn," i.e., "mang-corn," for each term indicates a mixture. In no instance do both terms appear in the same grange account, and in the case of Wargrave the quantity sold is entered as mestilo in the grange account, and as "mancorn" in the sale account. In most cases it probably consisted of wheat and rye, but only slight indications are given of the nature of the mixture.

Rye by itself seems to have been grown as an equivalent crop in the Surrey, Oxfordshire, and Somersetshire manors, and in one casc only, viz., the Church of Meon, do both ryc and mixed corn appear in the same account. However, it is doubtful whether what is called silligo or sigalum was not itself a mixture, for in the case of Adderbury a sinall quantity of mestilo bought for seed is entered under rye in the grange account, and in the case of the Taunton mills the produce of ryc is entered in the sale account as rye and barley, presumably a mixture of these grains.

It is curious that there is no mention of drageum, a mixture of barley and oats, which is of frequent occurrence in medixeval manorial aceounts. It is also wortlyy of note that no barley was grown in the Somersetshire manors in the previous year, and that in the current year only a few acres were sown with this grain, possibly by way of experiment.

1 p. 44, et passim.

${ }^{4}$ p. $74,1.27$; p. $65,1.49$.

7 p. $45,1.14$; p. $65,1.17$.

2 p. $65,1.26$, et passim.

${ }^{5}$ pp. 52, 53 .

8 p. $65,1.33,41,47$, and p. 66 .

$$
\begin{aligned}
& { }^{3} \text { p. 44, 1. } 41 . \\
& \text { " Pro mellea,"etc. } \\
& 9 \text { p. 47, 1. } 16 .
\end{aligned}
$$

8s. is paid when a daugliter married " extra hundredum" (p. 28, 1. 27).
11 p. $28,1.49$.
12 p. $6,1.20$.
13 p. $17,1.4$
14 p. $12,1.6$ 
The grain referred to as curaillum or curallum appears to have been small corn of wheat. Its price was comparatively low, and it was never used for seed, except in one case, when it was mixed with " mancorn." I It was generally accounted for separately, but in some cases it was included with the wheat, ${ }^{2}$ barley, and "mancorn" " respectively, although in the last it.was stated to be small corn of wheat.

Harwell, Brightwell and a few other manors derived a certain amount of wheat from the custom of Churchscot, and at Fareham and Bishop's Stoke a small quantity of rye and barley, respectively, was derived from the same source.

In addition to the cereals, the leguminous crops of peas, beans, and vetches were occasionally grown, but in comparatively small quantities, the largest area tilled being nine acres of peas at Alresford. Beans were planted in the garden at Downton ${ }^{5}$ and apparently both beans and peas in the new apple-orchard of the Church of Meon (East). ${ }^{6}$

An analysis of the grain crops is given in an Appendix, which shows in tabular form for each manor the total produce of each crop, the number of acres sown, the quantity used for seed, the quantity sold, and the average price per quarter. The following is a summary of the whole, with the addition of corresponding figures for small corn and legumes, the quantity consumed boing calculated from the difference between the total produce and the sum of the quantities sown and consumed or sold :-

\begin{tabular}{|c|c|c|c|c|c|c|c|c|}
\hline- & & Wheat. & Mized Corn. & Rye. & Barley. & Oats. & Small Corn. & Legumes. \\
\hline Total produce in quarters & - $\quad-$ & 3,679 & 915 & 310 & $1,750 \frac{8}{4}$ & $7,507 \frac{1}{2}$ & 382 & 80 \\
\hline Acres sown & $\cdot$ & 6,838 & $1,685 \frac{1}{2}$ & 547 & $1,891 \frac{1}{2}$ & 7,317 & - & $61 \frac{1}{2}$ \\
\hline Quantity sown in quarters & - & $1,589 \frac{1}{2}$ & $460 \frac{1}{2}$ & $101 \frac{1}{4}$ & $689 \frac{3}{4}$ & $3,402 \frac{1}{2}$ & - & $21 \frac{1}{8}$ \\
\hline Bushels per acre & - & 1.86 & $2 \cdot 2$ & $1 \cdot 5$ & $2 \cdot 9$ & $3 \cdot 7$ & - & $2 \cdot 0$ \\
\hline Quantity sold in quarters & - & $1,767 \frac{3}{8}$ & $356 \frac{5}{8}$ & $119 \frac{3}{4}$ & 488 & $1,274 \frac{1}{8}$ & $232 \frac{5}{8}$ & $28 \frac{3}{4}$ \\
\hline Price per quarter & - & 2s. $9 \frac{1}{2} d$. & 1s. $9{ }_{4}^{3} d$. & 1s. $10 \frac{1}{2} d$. & $1 s, 11 d$. & 1s. $0 \frac{1}{2} d$. & $1 s .7 d$. & $1 s .7 d$. \\
\hline Quantity consumed in quarters & - & 323 & 98 & 91 & $543 \frac{1}{2}$ & 2,831 & $149 \frac{3}{8}$ & $30 \frac{1}{8}$ \\
\hline
\end{tabular}

The most interesting fact revealed by these figures is the extremely small yield of the various crops. Assuming that the total acreage and quantity sown were approximately the same as in the preceding year, we get the remarkable result that the yield per acre of wheat was only 4.3 bushels, mixed corn 4.3 bushels, rye 4.5 bushels, barley $7 \cdot 4$ bushels, and oats 8.2 bushels. It should, lowever, be noted that in the case of Downton the produce of mixed corn was 50 quarters less than the quantity sown and sold, owing perbaps to a clerical error in the account. In all other cases the two sides of the account are either exactly or approximately equal. It is also curious that, with the exception of oats in a few of the manors, there was hardly any corn in hand either at the beginning or the end of the year. However, it may be that the corn in hand was not always entered, for at Taunton it is stated that at the end of the year there were 65 quarters of oats remaining in the grange, as well as two stacks of the same grain and two stacks of wheat, although the whole produce of the year had
1 p. $7,1.6$.
p. $81,1.10$.
2 p. 33, 1. 45.
${ }^{5}$ p. $22,1.29$
3 p. $31,1.40$.
${ }^{6}$ p. $48,1.11$. 


\section{(. xxvii )}

been disposed of in seed, eustoms, provender, and sale. At Wield, too, a stack of wheat, containing about 50 quarters, was sold for $£ 8$, but was not accounted for in the grange account.

Another interesting feature indicated by the above figures is the comparatively small amount consumed, espeeially of wheat and mixed corn. Less than 9 per cent. of the former, and only a little more than 10 per cent. of the latter were actually used on the manors, and the percentages of rye, barley, and oats were $30,31 \frac{1}{2}$, and $37 \frac{3}{4}$ respectively; the large percentage of oats is chiefly due to provender for the horses of offieers and distinguished visitors, the amount allowed on such exceptional occasions being apparently one bushel for each horse daily. The oxen were probably fed on sheaves (garba) during the winter. ${ }^{1}$ All the cereals except wheat, were occasionally used for feeding the Bishop's or the King's dogs, and all were apparently malted and used for brewing beer for the boon works, scotales, \&c. The wheat was used mainly in making bread for the Bishop and his officers, but was occasionally consumed at the boon works or given in customary allowances to certain servants.

Barley was chiefly disposed of in allowanees to various labourers, but was used also in making bread for the boon works, and in feeding pigs in winter. Of the small corn, about 61 per cent. was sold, and the remainder consumed in a similar manner to the barley. The peas and vetches were used chiefly for feeding pigs in winter.

Corn was measured both by the strike bushel (estrica) and the heaped bushel (hopa), but in adding them up to form the totals, no apparent distinction is made between them and eight of each are reckoned as equivalent to a quarter.

Wild Growths and Forage.-In addition to the proceeds from the customs of pannage and herbage, which occur in nearly every manor, there are occasional sales of nuts (nuces), underwood (busca), rushes (juncus), stubble (stipula), flax (linum), haulm (culmus), reed (arundo), meadow grass (herba), pasture, twigs (coperones), dead wood (morbois), and briars (bruera). Hay and forage were frequently bought for the oxen and horses, and at Adderbury a large quantity of forage was sold. In the latter manor, and at Southwark there are records of sales of apples, and there were apple orchards also at Meon and Marwell.

Mills.-About fifty mills are enumerated, and of these about one-half were let to farm at a fixed annual rent, while the remainder were kept in hand, an income being obtained from them by sale of the produce. Four are stated to have been fulling mills, and it is believed they are the earliest recorded in this country. They were situated at Bishop's Waltham, Brightwell, Bishop's Sutton, and Alresford. The first and third were let for $20 s$. and $11 s$. $1 d$. respeetively. The second, which had been newly made for $9 l .4 s$. $4 d$., was retained in hand, the produce for a part of the year being sold for 16d. At Alresford the rent of the mili is apparently ineluded in the gabulum, but the large sum of $8 s .3 d$. was paid for collecting stubble for it, possibly for the purpose of roofing it. All the other mills were probably com mills driven by water power. At Fareham there was a "sea mill" (molindinum maris), which may have been either a tide mill or a floating mill operated by the currents. A

\footnotetext{
${ }^{1}$ p. $42,1.60 ;$ p. $81,1.22 ;$ p. $82,1.53$.
} 
millstone for this mill cost $25 s .4 d$., including carriage from Southampton, and slates for roofing it were bought for $9 \frac{1}{2} d$. The price of millstones varies from 36s. to $13 s$. $4 d$. The river mills seem to have been of the usual type. Each apparently had a pair of horizontal stones, of which the upper was supported by a bail (bila) on an iron spindle working in a footstep ( $i n k \alpha)$, and was driven by lantern and pin gearing from the wooden spindle of the water wheel. This wheel was carried by arms (brachii) and provided with buckets (alvi), and it was supplied with water by a shoot (alico) from the mill-pond (stagnum). The pond was sometimes stocked with fish, and then served also as a fish-pond (vivarium). At Brightwell young fish (pisciculi) were bought for stocking the fish-pond, and at two of the Winchester mills fish were sold and small amounts were paid for repairing the fisheries. At Taunton 15 sticks of eels were obtained from the fish-pond and mills, and at Twyford 1,100 eels were sold for 9s. $2 d$. At Taunton, too, payment was made, in connection with the Borough mill, for repairing three fishing-nets.

The produce of the mills, which was doubtless derived as toll from the tenants, consisted chiefly of wheat and mixed corn, but in most cases malt (braseum) and meal (gruellum) also were sold, and in the Taunton mills the former is separated into two different qualities (braseum and contrabraseum). The average prices of these mill products were: Wheat, $2 s .11 d$. per quarter; mixed corn, 1s. $4 \frac{1}{2} d$. ; meal, 2s. 10d.; malt, 2s. $4 d$.; and malt of inferior quality, 1s. $2 d$.

A molindinum ad braseum is mentioned (at Twyford), and it will be observed that malt is a usual product of the mills. As this staple of brewing is not mentioned in any other connection, it is perhaps possible to infer a combination of milling and malting. The function of the crapestre (later crupestre) mill at Winchester is rcferred to in the Glossary.

Buildings. - The occasional references in the Roll to the various farm and other buildings are not sufficient to enable a definite pieture of each homestead to be formed, but a consideration of the whole series of entries gives some indication of the nature and arrangement of the buildings of a typical manor. These entries occur under the heading Expensa, and relate almost entirely to roofing and repairing the various houses, ${ }^{1}$ but a few instances of new houses furnish some information as to their prime cost.

It is probable that all the buildings were in most cases situated around the courtyard (curia), which, however, appears to have been complete in itself, for it was surrounded by a hedge and a ditch ${ }^{2}$ and entered by one or more doors. The ditch was often made at considerable expense, that at Wield costing $8 s .1 d$., and that at Burghclere 5s. 6d. At Downton the yard was called a barton (berlona), and a ditch of 22 perches was made in it at the rate of $3 \frac{1}{2} d$. per perch. A door to the same barton cost $1 s$. It is likely that all the sheds for animals opened into this barton or court, for the dung produced was stored in it until it was required to be spread on the land.

The dwelling-house, which in most manors consisted merely of the usual hall and chamber (aula and camerc or thalamus) probably occupied one side of the court, and near it or adjoining it stood the dairy, which was in some cases a very simple structure of wood, for a new one at Witney cost only $3 s$.

$$
{ }^{1} \text { p. } 31,1.15 . \quad \text { p: } 31,1.16 ; \text { p. } 6,1.37 .
$$




\section{( $\operatorname{xxix~)~}$}

In the larger manors the larder and kitchen were, no doubt, placed at the same side. The kitchen, too, was often of wood, and, as at Bitterne, was liable to be burnt, but a new one, which was made for the Church of Meon at a cost of $59 s .3 \frac{1}{2} d$., must have been of a more substantial character. The chief larder seems to have been at Downton, but there were others at Bishop's Waltham, Witney, and Farnham. Under Downton, too, there are entries relating to a pantry, a buttery, and a cellar.

The house at Marwell, near Twyford, which was used by the bishop as a residence, was much more elaborate than the ordinary manor houses. ${ }^{1}$ The chamber had been burnt, and it was rebuilt and other parts repaired at a cost of over 10l. This chamber had walls wholly or partially of stone and a timbered roof, and it was furnished with the unusual convenience of a window. The cloisters and the porch of the hall were tiled, and leaden gutters and a chimney were also provided. A chapel is recorded at this place, at Highclere, and at Bitterne.

The grange seems to have been a large building for storing corn in the sheaf, for several of the manors had also a granary for storing grain. Both were surrounded by a ditch. ${ }^{2}$ Some of the granges must have been substantial stone buildings. The great grange at Mardon cost the large sum of $4 l .5 s .9 \frac{1}{2} d$., while a granary at Cheriton was built for 2øs. In three other instances the cost of making a new grange was only about $10 s$.

The sheep-shed (bercaria) was also in many manors an important structure. At Twyford a new one was made for 29s. $7 d$., but in other cases a new one cost only $12 s .8 d ., 8 s .4 d$., and $6 s .2 d$. respectively. This shed, like the court, was enclosed by a hedge and a ditch. The former was formed either with pales and twigs (pala and ramilla), ${ }^{3}$ or with brushwood (clostura). ${ }^{4}$ At Alresford six hedges were made for $2 s$. The ditch varied in length in different manors from 114 to 21 perches, but it was dug at the uniform rate of $3 d$. per perch. The building itself was roofed either with tiles or straw, walled with boards, and provided with a door, which in one case cost $1 s^{5}$

There are fewer entries relating to the cattle-shed (bovaria), and no new one was built, but it was probably similar to the sheep-shed. However, it is possible that in some cases the building was dispensed with, and its place taken by an open enclosure (prasepe boum). ${ }^{6} \quad$ The hedge was made of poles (virga):

Nearly every manor has entries relating to the fold ( falda), which was made of wattled hurdles. At Mardon 36 hurdles were made and 52 wattled for $2 s .1 d$., while in other cases hurdles were bought at the rate of three or four a penny.

Occasional references are found to a stable (stabula), a dovecot (columbaria), a poultry-house (gallinaria), a pigstye (porcaria), a cart-house, and a pound (punfalda). At Downton a new dovecot was built for $22 s .11 \frac{1}{2} d$., and at Bishop's Sutton a poultry-house was made for $2 s .3 d$., and a pigstye for $1 s .6 d$.

Implements.-In each manor there are entries of payments made for the cost of 'ironwork for the ploughs and carts, and for shoeing the horses, but the amounts vary so much that no useful deduction can be

\footnotetext{
1 A few years later painter was employed to decorate this residence. $2.82,1.37$.

${ }^{3}$ p. 53, 1. 41. ${ }^{1}$ p. $82,1.36 .{ }^{5}$ p. $50,1.54 .{ }^{6}$ p. $15,1.37 .{ }^{7}$ p. $80,1.53$.
} 
derived from them. The plough was obviously a very simple contrivance, and the cost was very small indeed, for the woodwork (mairemium) was provided by the lord, and the carpenter and smith were in most cases hired at a fixed annual wage. Thus, in separate entries we find the cost of making six new ploughs "before the carpenter came" $1 s$. ; of making one plough $1 . d$. ; of making three ploughs and plough-wheels $6 d$. ; and of making three new ploughs $6 d$. The price for a pair of plough-wheels seems to have been about $3 \frac{1}{2} d$., but this doubtless included the ironwork as well.

The carts were much more elaborate, and were of two distinct kinds, viz., the ordinary wooden cart, costing from $1 s .6 d$. to $3 s .8 d$, and the iron-bound cart, having also its wheels bound with iron, costing from 6s. $6 d$. to $11 s$. $1 d$. One such cart, and three additional pairs of wheels, were bought at Bristol for $38 s$. $8 d$. A pair of ordinary cart-wheels eost 1s. In some cases the term biga or karrus is used instead of the more general careta, but there does not appear to be any essential difference between them. However, it is possible that the biga was of lighter construction than the careta, for three "for marling" were bought for $7 s$., a new one bound with iron cost only 5s. $7 d$., and two pairs of wheels were bought for 3s. $7 d$. The karrus, on the contrary, may have been heavier, for at Taunton seven pairs of wheels were bought at an average price of $3 s$. $3 \frac{1}{2} d$. The plaustrum used for marling at Bitterne was possibly a waggon with four wheels.

Other implements are only mentioned occasionally. Harrows were bought for $2 d$. each; baskets (corbella) for carrying corn, etc., $2 \frac{1}{2} d$. ; another "bascat," $1 d$; ; a seedlip, $1 d$.; a winnowing-fan (vannus), 14d.; other fans (ventorii) for cleaning corn, $6 d$. and $7 d$.; a reaping-hook, 1 s. $1 \frac{1}{2} d$.; a bushel measure, $2 d_{\text {.; }}$ three sieves, $2 d$; a bucket or trough for watering the oxen, 4d.; a "bokett" and cord for drawing water from the well, $4 d$.; a tun for cider, $1 s$. ; locks, about $2 \frac{1}{4} d$. ; picks, $5 \frac{1}{4} d$. ; a gardening spade and hoe, $4 \frac{1}{2} d$. Sacks cost from $4 d$. to $9 d$., and canvas for making them $2 d$. per ell.

Stock.

The Instaurum, generally the last section of the manorial account, deals with the various branches of the live stock and their profits, together with their store of meat, wine and cider, honey and fruit. These are arranged in a definite order beginning with the three principal classes of the live stock, namely, (1) horses, (2) cattle, (3) sheep, followed by notices of (4) wool, and (5) cheese. The position of the remaining items is subject to many variations, but the pigs always precede the poultry, and the larder with the inventory of the cellar (if any) come near the end.

Each section is treated on a common plan, setting forth the remainder (if any) of stock or goods from last year, the changes taking place during the year, and finally, the result, showing the quietus, superplus, or defectus, as the case may be. The account also refers back, sometimes expressly, as in the case of hides, wools, woolfells, and cheese, sometimes incidentally, as in the case of stock bought or sold, to the earlicr portion of the manorial account, particularly to the Exitus Manerii and Expensa. It is thus possible to verify the totals in many instances. 
The totals of the live stock are generally more accurate than those of the hides, wool-fells, etc. Totals are frequently right in gross, but wrong in detail. Many mistakes of this kind appear, which in most cases admit of correction when compared with the supplementary evidence of the related sections. The defects are not all of them favourable to the bailiffs, in which case we could reasonably suspect fraud.

Space forbids a detailed consideration of the valuable evidence contained in the thirty-three stock accounts of the Roll. In the Appendix will be found two general tables, one specifying the numbers and distribution of each kind of stock among the several manors, showing the additions made during the year, by way of produce and acquisition, as well as the losses incurred by murrain and other causes. The second table shows the profits arising from the various kinds of stock, including hides, wool-fells, cheese, etc., produced and sold in the several manors during the same period.

The manorial stock is treated of here in the following order:(1) Equine class (including asses), (2) Bovine class, (3) Ovine class (including goats), (4) The Dairy, (5) Porcine class, (6) Poultry, (7) The Larder, Kitchen, and Salt-store, (8) The Cellar, (9) Miscellaneous (Nuts, Cummin, etc.).

Horses.-The usual terms of stallion (stalo), mare (equa), and colt (pullus), or filly (pullana) occur, and there is a further distinctive nomenclature from the particular work performed. The avrus for the plough, and the carectarius for the cart. The riding horse (palefridus) and the sumpter-horse (summarius) are also incidentally mentioned in connection with the Bishop's Itinerary. ${ }^{2}$

Under the name equus silvestris the famous forest pony of Hampshire is apparently referred to. Eighteen Welsh mares were received in Clere and Mardon during the year. In both cases they were drafted into the forest pony stock.

About fourteen per cent. of the whole number of horses was lost by murrain. The hides are carefully accounted for, and are for the most part sold. Four were dealbated for local manorial purposes. ${ }^{3}$ The cost of dealbating a hide was $4 d$. The highest price received for a horse-hide is 13d. at Adderbury, which is exceptional, the average price being about $6 d$. Asses were kept at Waltham and Witncy.

Cattle.-The cattle stock may be divided into two classes: (1) The active class, including $(a)$ the parent or producing stock-the taurus and vacca; (b) the working or non-producing stock-the boves. (2) The store cattle (otiosa animalia) comprising the vituli, the annales, and the bovetti and geniculae of all ages.

The latter class is sometimes technically called the "instaurum" or "store," by which the active class are supplemented during the year. Geniculae is the name by which heifers of all ages, above one year, are known until they are added to the vaccae. Similarly boviculi is the term applied to bullocks of all ages above one year prior to their advancement to the boves; the younger ones arc termed bovetti juniores, in contradistinction to the bovetti prolixioris or provectioris aetatis.

1 cf. pp. 8, 81, 82, where 21 not 16 gallinae were sent from Beauworth.

" On p. 60 we have the phrase "equos molindum."

${ }^{3}$ р. $70,1.38$. 
The boves form the most numerous section of the cattle stock, and take an important part in the working of the manor. The majority were employed in agricultural operations, and others were fattened for the larder, ${ }^{\mathrm{I}}$. etc. Quantities of hay were bought in some manors for the oxen, ${ }^{2}$ in addition to the ordinary provender of corn, and in some instances expense was incurred for the special provision of water. ${ }^{3}$ All the cattle (161) "bought" in the Roll are boves, some of them expressly to relieve the plough-teams." As eompared with last year, the number remaining is 64 less. The number sold is perhaps unusually large, some of the oxen sold being old and infirm ("veteres et debiles"). ${ }^{5}$ The number of calves produced is a little more than half the number of cows remaining last year. About forty per cent. were barren, and besides this, some died ante foetum. ${ }^{6}$ The amount and value of cheese and butter produced and sold will be found stated elsewhere. ${ }^{7}$

A little more than ten per cent. of the entire cattle died. With the exception of nine (eight of which are calf skins) the hides are all accounted for. Calf-skins (once termed pelles) were of little value $;^{8}$ they were sometimes sold at a penny each. The average price of the ox-hide was about 18d. Hides of slain (occisi) are apparently of a higher value than those of the murrain (mortui) oxen.

Sheep.-The division of the sheep flock is parallel to that of the cattleherd. The active class including (1) the parent or producing stock-the hurtardus and the ovis matrix; and (2) the non-producing stock-the multones and hoggetti.

Hoggetti are yearling lambs, before their transfer (after shearing) from the Instaurum to the respeetive flocks. When distinguished as to sex, the males are called masculi, the females gerciae.

The multones and hurtardi are sometimes classed together (p. 82). Eighteen multones and 22 hurtardi from Lincolnshire, were introduced into the flocks of Clere, Downton, and Farnham. The term cuillardus is used in one instance as an alternative for multo. ${ }^{9}$

Only the oves matrices were expected to bear lambs. From the evidence of the account it is clear that a ewe produced only one lamb, a single exception occurring at Adderbury. Some of the gerciae oceasionally bear lambs. These are always aceounted for separately, and we find no effort on the part of the bailiffs to check their number as in the case of the oves matrices, where all changes taking place "ante partum" and "post partum" are carefully noted. Hogget-lambs tended to weaken the stock, and the twelve produced at Alresford all died. A custos agnorum ${ }^{10}$ during the lambing season is sometimes referred to in the Roll. Hay was bought ${ }^{11}$ and eorn used ${ }^{12}$ in some of the manors for the ewes and lambs.

About three and a half per cent. of the produeing floek were barren, and nearly fifty lambs were lost by abortion. A tenth of the lambs produced

" "In bobus pascendis" frequently appears in the Exitus Grangiae. Oat sheaves were often used for this purpose; e.g. p. $42,1.60 ;$ p. $81,1.23 ;$ p. $82,1.53$.

2 e.g. p. $72,1.35$; p. $74,1.37$; sometimes straw (foragium), p. 42, 1. $15 ;$ p. $49,1.18$.

3 cf. supra, p. xiii.

${ }^{5}$ p. 16, J. 54 ; p. $41,1.15 ;$ p. $61,1.7$.

7 p. 49 , I. 20 ; p. $50,1.57$; p. $82,1.30$.

${ }^{8}$ p.,9, 11. 3, 58.

11 p. $53,1.44$.

p. $68,1.3$.

6 p. 34, 1. 15.

8 p. $71,1.2$.

${ }_{10}$ p. $6,1.45$; p. $29,1.5$; p. $54,1.39$.

22 p. $75,1.23$. 
of course went as tithe. ${ }^{1}$ Several were also given in customary payments to servants of the manor. Mutton was chiefly used during the harvest season, in contrast to the beef and pork consumed in the autumn and winter.

As elsewhere, the flocks wero made use of for fortilizing purposes. The practice of folding is frequently referred to. ${ }^{2}$ It was one of the chief duties of the shepherd to see that all the sheep lay in the fold over night, so that the land might benefit from the compost. Gilbert, the shepherd at Wargrave, was amerced $6 d$. for neglect of this. ${ }^{3}$

It has been previously noted that in some few cases sheep were let " at farm" as was the milk of more than four hundred ewes at Meon." Other items of profit derived from the sheep flock were the receipts for wool and wool-fells sold.

Wools are divided into vellera grossa (or crispa) and vellera agnina. To check the amount produced, all changes oecurring in the stock "ante tonsionem" and "post tonsionem" are noted. Sixty-eight small lambs (subtiles agni) in Crawley were left unshorn. Stray wool to the value of $6 s$. was collected at Clere. Wool was sold by weight. The shepherd usually had a fleece, and in three cases the dairymaid. In the section of the Expensa there are usually some interesting details of the canvas and thread bought, and the sarpelers made to contain the large wool, as well as of the sacks purchased to contain the lock-wool.

The total loss by murrain for the whole flock is equal to twenty per cent.; that of the lambs alone is about thirty-seven per cent.

Goats.-Goats appear in Bitterne and Wicombe and were all disposed of during the year. The he-goat is termed a "buke" and the she-goat a matrix capra, the kids being known as capreoli.

The Dairy.-The profits of the dairy appear among the items of the Exitus Manerii. The incidental costs (custus daerice) connected therewith, e.g., the purchase of tins, pans, pots, jars, salt, etc., will be found in the Expensa. The Instaurum gives the number of cheeses produced and their disposal by way of customary payments, sale, etc. Only nine of the manors sell butter during the year, the total value being 10s. $4 d$.

Compared with the previous year there is a decrease of nearly three hundred in the number of cheeses left over. The production under this head was doubtless affected by the drought in this year previously referred to. As usual cheeses were chiefly made from May to Michaelmas. Some were made during the winter season from October to February, and during the season from February to May (ante ablactationem), when most of the milk was used in the rearing of the young calves.

Pigs. - The pig and poultry classes form the more transient section of the live stock. They are short-lived, and reach their maturity and accomplish their function at a much earlier age than the horse, ox, and sheep. This has its marked effeet both on the nomenclature and on the form of account.

1 At p. 46 we have a singular instance of the tithe assessed on the number left after subtracting the loss by murrain, with a tithe of the fells of the number that so died. Other unusual tithe assessments appear on p. 58, 1. 43-44.

2 Cf. p. 9, 1.29, et passim.

3 p. $28,1.44$.

Here the Church took its tithe from the money-rent (cf. pp. 48, 49). 
Omitting the parent stock (verres and sus), the rest of the porci fall into two divisions, those above (super) and those helow (infra) the age of one year. Litters (of which more than one was occasionally produced by each sow during the year) ${ }^{1}$ contain the porcelli, sometimes termed porcelli juniores, to distinguish them presumably from those of the previous litters, porcelli separati. These latter are sometimes termed hogetti, and are thus distinguished from the porci superannati, though the term porcelli is in many instances indiscriminately used to include all those infra annum. The porci superannati are differentiated as to sex by the words masculi and femella, sometimes found as maelli and suella. As distinguished from the young sows (juniores sues or suella) the old sows (veteres sues) are called domina sues.

No pigs, it will be noticed, were sold. The porci were usually fattened during the winter ${ }^{2}$ for the larder. Sucking pigs were received by the porcarius and were in great demand at the bishop's table.

Poultry.-Fowls and geese are the only species of poultry recorded. Little attention was apparently paid to the rearing of young fowls, the greater part received during the year coming from the custom of churchseot. They were mostly consumed by the bishop's household and the local officers. 'The surplus, after satisfying local requirements, was generally sent to the kitchen at Wolvesey. Here most of the eggs were also sent. Eggrs are loosely accounted for. Fowls appear in 29 manors, but eggs only in 10, and in several of these are the product of customary dues. Only one dovecote, at Downton, is mentioned in this Roll. ${ }^{3}$ Wildfowl (aves) appear to have been rendered at Christmas by the tenants of one manor. ${ }^{4}$

The Larder.-The principal larders with their dependent manors have been referred to above. ${ }^{5}$ They supplied the bishop's household with bacon and becf during the year, and furnished similar foods to labourers during the autumn and other boon works. Venison was cured both at Waltham and Winchester.

Nearly five pounds worth of lard (unctum porci) was sold from the larders of Waltham, Witney, Downton, and Farnham. At Taunton, tallow was sold with the hides.

The incidental expenses connected with the larder include salt for the curing of the flesh. ${ }^{6}$ Drink and food were given to the male and female workers in addition to their money stipend.

The Cellar. - Wine and cider, the extraordinary and ordinary drink of the household and official class, appear in the Instaurum accounts of seven manors. Southampton and Exeter were the respective ports of supply for the Hampshire and Somerset manors. Cider was produced at home. In East Meon $8 \frac{1}{2} d$. is paid for making a tun of cider. Wine and cider, as may be seen from evidence contained in other sections of the Roll, were largely used in the "scotales."

Beer, which is not referred to in the Instaurum, was largely consumed by the workers in the larder.

\footnotetext{
1 p. $40,1.43$.

p. $79,1.44$.

${ }^{5}$ p. 10.

2 e.g., p. 3, 1. 51 ; p. 14, 1. 13.

3 p. 21, 1. 14.

6 See Glossary 8. v. Sulcitium, Eschina, and Salsigia.
} 
Fruit.-Nuts were grown on several manors, and were apparently included in the Instunrum when not disposed of during the year. Six quarters remained from last year at Meon. During this year nuts were

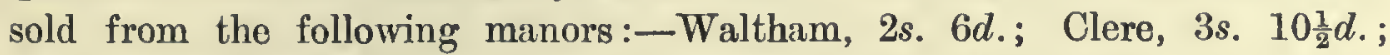
Itchingswell, $2 s$. ; Witney, 15s.; Bitterne, 6s. 6d.; Meon, 10s.; Hambledon, $2 s$.; Taunton, $2 s .9 \frac{1}{2} d$. ; and Wield, $1 s$. 4d. Total value, 2l. 11s. The Taunton account includes the dues received for "nutting." The average price of nuts per quarter is about $1 s .6 d$.

The total value of honcy sold was 8 s. 11d. and it was received at Waltham, $2 \frac{1}{2} d_{\text {. }}$; Farnham, $4 s$.; Clere ("de consuetudine"), $2 s$. ; Bitterne ("de purchasiis"), 2s. $0 \frac{1}{2} d$; and Alresford, $8 d$.

A new orchard was planted at Meon during the year, and the old one at Marwell was cleaned and trimmed. Apples were sold at Southwark, 15s.; and at Alresford, $2 s .3 \frac{1}{4} d$.

It has been already stated in the Prospectus of this Edition that Method of the work of transcribing and extending the Text, together with the preparation of the Introduction, Index, and Glossary, have been entirely performed by the students of the class in Palrography, Diplomatic and Historical Sources of the London School of Economics, under the supervision of the Lecturer. 'This, indeed, must be regarded as an essential feature of an undertaking for which the only merit claimed lies in the value of the practicable experience thus acquired in several of the auxiliary branches of listorical study. It will, however, be easily understood that the exigencies of an academic course have imposed strict limitations on the scope of the Editor's researches. The present edition represents, in fact, merely the "practical work" of the weekly seminar, the application of the lecture courses of the past session on the "Seignorial position of the Church in the Middle Ages" to a selected thesis-the manorial systcm observed in the case of a single church in a given year. The end kept in view throughout the above course was the equipment of the students for the comparative study of the manorial economy in all its bearings throughout the period indicated, and specialization might have been attcmpted in any other direction for which opportunity served. The subject selected seemed, however, deserving of attention on account of the comparative neglect of this unique series of manorial records. At the same time, although the end has been held to justify the means, it must be admitted that the plan of such an Edition, however systematic in its method, leaves much to be desired in respect of the performance. Great difficulty, for example, has been experienced in securing uniformity in respect of the extension of the 'lext, and of the procedure employed for the purpose of an analysis of its economic evidence. The systematic and uniform extension of mediæval official documents is virtually an unknown practice in this country, whilst the number of alternative forms which may be found in medireval glossaries increases the difficulty of co-operation by independent workers, and this difficulty has been enhanced in the case of the present MS. by the vagaries of the scribe.

The method pursued by the Editors may be briefly described as follows:-

1. The Text has been extended throughout as a matter of principle, to aroid the primitive expedient of "Record type" on the one hand, and the 
unsightly devices of italics and square brackets on the other. ${ }^{1}$ The system of extension followed is that which appeared to be indicated by a close observation of the habits of the scribe. The original spelling has been preserved in the Text, but obvious errors have been indicated in the footnotes. The habitual omission of the conjunction "et" has been merely indicated by the punctuation. The arrangement and spacing of the original account have been followed as far as practicable, and much pains have been taken to reproduce the general features of the MS. without resorting to typographical imitation. Roman figures have been preserved, if only to enable the mediæval system of numeration to be represented in the Text. The capitalization and punctuation of the MS. have, however, been modernized, and the orthography of certain letters has been also modernized, in accordance with the practice of the Rolls Series. ${ }^{2}$

2. The Introduction, Indices, and Glossary have been assigned, like the Text itself, to groups of students working independently, guided to some extent by the general course of lectures on Manorial Sources, and by a special course of lectures on historical composition and criticism. In the first place an attempt has been made to describe the official classification and custody of the MS., its palæographical and diplomatic characteristics and its historical and economic interest. In the Index of Places the identifications have been given to the best of the Editors' abilities, according to the admirable method of the Rolls series of mediæval calendars; but, following the practice of English editions, this process has not been applied to the names of persons, these being for the most part of very little interest. Space could not be found for a full Glossarial Index, but the recurrent terms of the account have been discussed at some length in the Introduction, where they are illustrated by references to the Text itself.

The publication in a separate form of the sources, both manuscript and printed, which have been collected and utilised for the purpose of this Edition, is contemplated at a later date; but the Editors wish to take the present opportunity of gratefully acknowledging the courtesy of the Ecclesiastical Commissioners and the assistance and encouragement which they have received from the Director of the School, from the Authorities of the Public Record Office and British Museum, and from the Committee for furthering Advanced Historical Studies in London. They also wish to express their obligations to the Printers (Messrs. Eyre and Spottiswoode).

The following members of the Class co-operated generally in the present Edition :-Rev. H. Birt (O.S.B.), F. Bradshaw, M. M. Calthrop, M. E. Carter, R. P. Chope, E. F. Churchill, F. G. Davenport, L. Drucker, J. Greaves, H. Hadley, C. Hogg, E. M. Leonard, E. A. Lewis, H. R. Maynard.

Of the above, Mr. H. R. Maynard is responsible for the section on Manorial Communications, Miss E. M. Leonard for that on the Manorial Revenues and Services, with some assistance from Miss F. G. Davenport;

I Doubtful extensions, particularly of proper names, are given in square brackets. Extensions not attempted are indicated by an apostrophe.

2 It must not, however, be supposed that this implies an expression of opinion as to the correctness of the form in question. The students are equally instructed in the method which is now more general, but which renders an historical text practically as useless as "Record type" to the uninitiated. 
Messrs. R. P. Chope and E. A. Lewis have written the sections on Manorial Crops and Stock respectively, and have compiled the accompanying Tables. Mr. Chope has also compiled the General Balance Sheet. The Palæographical and Diplomatic descriptions of the Record were drafted by the Lecturer. The Index of Persons has been compiled by Miss M. E. Carter, and that of Places by Miss H. Hadley and Miss L. Drucker, and they are also respectively responsible for the information concerning Persons and Places in the Introduction. The first half of the Glossary (A-K) has been contributed by Mr. E. F. Churchill, and the remaining letters by Mr. F. Bradshaw.

June 1903. 

APPENDIX

TO

P R E F A C E. 


\section{APPENDIX TO PREFACE.}

APPENDIX I.-Itinerary of Peter des Roches, Bishop of Wixciester, 29 Sept. 120829 SePt. 1209, in conNexion with the Itinerary of King John. ${ }^{1}$

The places printed in italins indivate that the bishop's presence at the date given is not mentioned in the Roll, but is inferred from that of the king.

BISHOP (COMPOTUS ROLL).

1208.

Oct. 15 Witney.

Nov. 6 Downton.

"11 Downton.

25 Witney. ${ }^{2}$

Dec. 31 Waltham.

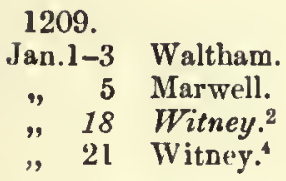

Mar.12 Witney.2 " $29^{5}$ Waltliam. April 1 Adderbury.
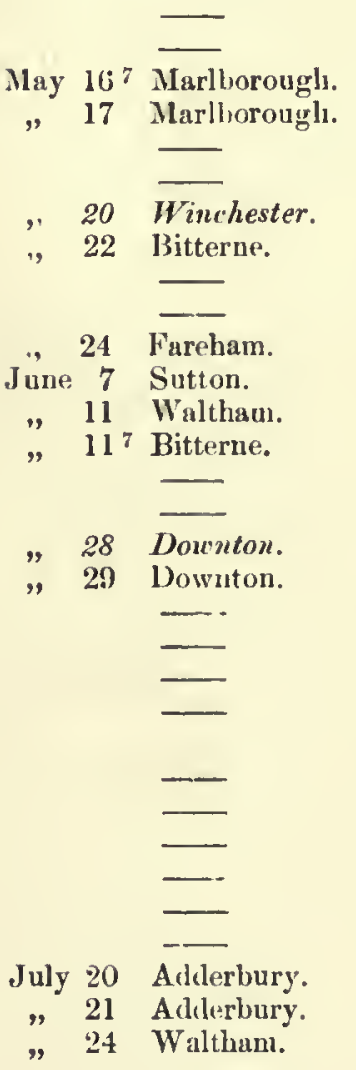

") Adilerbury

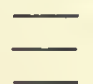

KING (COMPOTUS ROLL). BISHOP (MISA ROLL). 1208.

Oct. 15 Witney.

Nov. 6 Downton.

, 11 Downton.

1209.

Mar. 12 Witney.
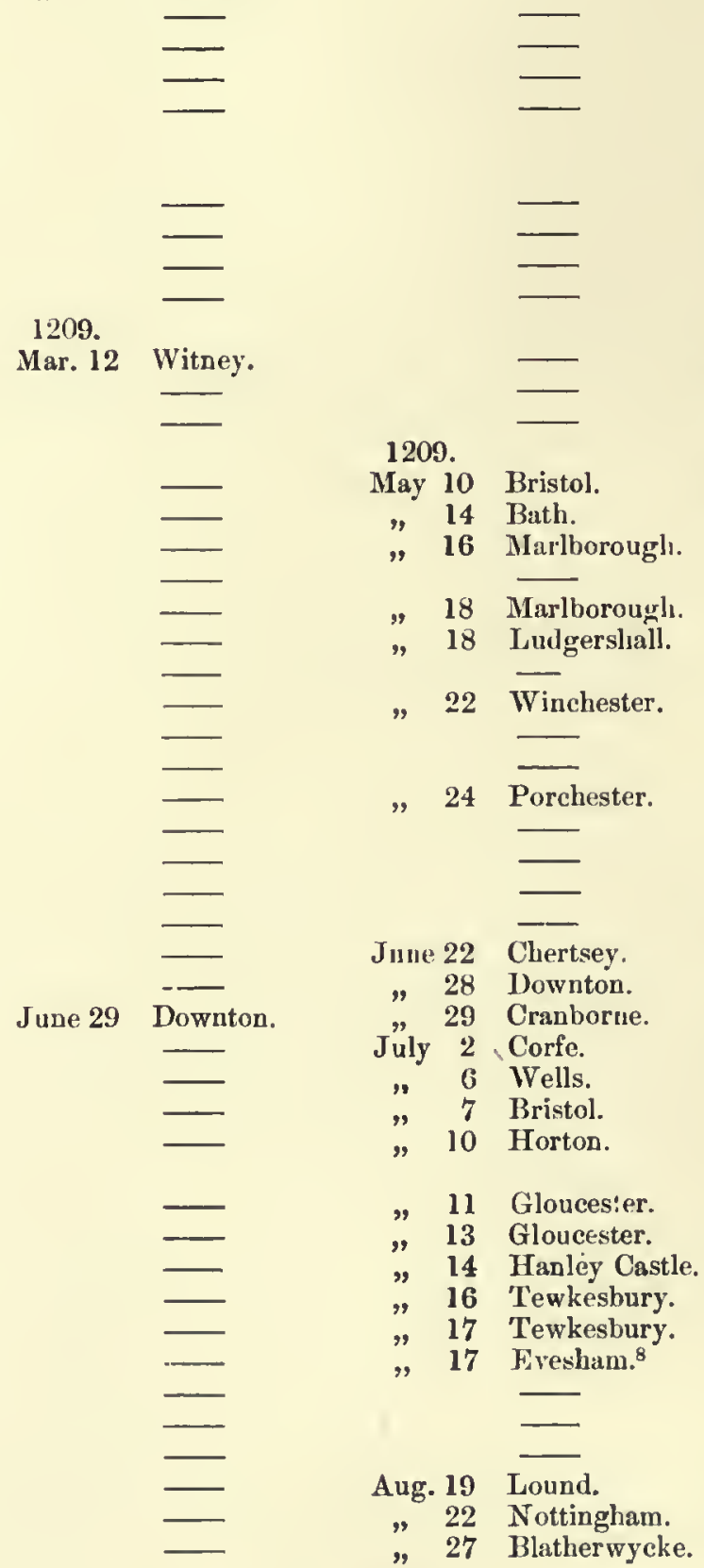

KINO (HARDY).

$120 \mathrm{t}^{\circ}$

Oct. 20 Oxford.

Nov. 6 Cranborne. ${ }^{2}$

" 8 Clarendon.

, 12 Christchurch

" 14 Gillingham.2

" 19 Cranhorne. ${ }^{2}$

1209.

Jan. 1 Winchester. $\left.{ }^{2}{ }^{3}\right)$

\begin{tabular}{|c|c|c|}
\hline & & 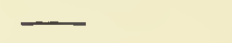 \\
\hline June & & Westminster. \\
\hline 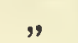 & 22 & Chertsey. \\
\hline & 28 & Downton. \\
\hline & 29 & Cranborne. ${ }^{2}$ \\
\hline July & 2 & Corfe. \\
\hline$"$ & 6 & Wells. \\
\hline ", & 7 & Bristol. \\
\hline$"$ & 10 & $\begin{array}{l}\text { Horton, Glouces } \\
\text { ter. }\end{array}$ \\
\hline " & 11 & Gloucester. \\
\hline & 13 & Gloucester. \\
\hline & 14 & Hanley Castle. \\
\hline & 16 & Tewkesbury. \\
\hline & 17 & 'Tewkesbury, \\
\hline & 17 & Evesham. ${ }^{\theta}$ \\
\hline & & - \\
\hline & 19 & Lound. \\
\hline & $\begin{array}{l}22 \\
27\end{array}$ & Nottingham. \\
\hline & & Blatherwycke. \\
\hline
\end{tabular}

" 18 Witney.

" 21 Tewkesbury.

Mar. 13 Witney.

$$
\begin{aligned}
& \text { May } 10 \text { Bristol. } \\
& \text { " } 14 \text { Bath. } \\
& \text { " } 16 \text { IIarlborough. } \\
& \text { " } 17 \text { Marlborough. } \\
& \text {, } 18 \text { Marlborongh. } \\
& \text { " } 18 \text { Isudgershall. } \\
& \text { " } 20 \text { Winchester. } \\
& \text {, } 22 \text { Winchester. } \\
& \text {, } 22 \text { Southampton. } \\
& \text { " } 23 \text { Soutbainpton. } \\
& \text { " } 24 \text { Porchester. }
\end{aligned}
$$

1 From the Winchester "Compotne" Roll compared with the paralle] notices in the Chancery Misa Roll of 1209 (Kecord Commission) and in the Patent linlls (Hardy "Introduction to the Patent Rolls")

2 The Roll does not confirm the presence of either hishop or king on these dates, but nndated notices occur of the presence of the bishop at Witney and Crauborne; of the king at Gillingham; and of bishop and king together at Winchester, Farnbam, and Bishop's Sution.

3 The birth of Prince Richard at Winchester on this date is not alluded to in the Roll.

4 The Roll mentions the carriage of arms and provisions betwcen Witney and Tewkesbury, but no date is given.

s Easter. 5 Whitsuntide. 
1209

BISHOP (ROLL).

Aug. 29 Witney.

Sept. 1 Adderbury.

, 15 Downton.

26 Knoyle.

" 29 Knoyle.

Taunton, ${ }^{2}$ Meon, Clere, Overton, Clewer, Wargrave, Reading, Fareham, Twyford, Marwell.

Farnham.

Sutton.

Cranborne.

Wolvesey.
1209.

\author{
KING (ROLL).
}

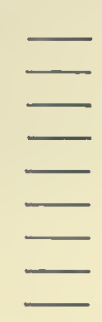

BISHOP (MISE ROLL). 1209.

12)

Sept. 13 Marlborough. Sept. 13 Marlborough.

- " 17 Cranborne.

" 20 Iorehester.

" 23 Gillingham.

\section{Undated Entries.}

Farnham.

Sutton.

Crunborne, Nov. 6-19 (1208), June 29 and Sept. 17 (1209).

Winchester.

Winchester (Jan. 1 and May 20, 22).

Gillingham.

Gillingham (23 Sept.).

1 The Rol] does not confirm the presence of either bishop or king on these dates, but undated notices occur of the presence of the bishop at Witney and Cranborne; of the king at Gillingham; and of bishop and king together at Winchester, Farnham, and Bishop's Sutton.

2 Taunton eharges for the maintenance of the bishop's household, awaiting the arrival of the bishop and the king; but neitber in the Roll nor in IIardy's itinerary is there any evideace of the date or aelual occurrence of the visit. An attempt has also been made, though without sueeess, to identify the dulivery of the "tun of good wine" which the coutemporury Pipe. Roll records as the fioe assessed upon the bishop for failing "to remind the king ubout giving a girdle to the Countess of Albewurle." (Pipe Roll, 11 John, Southampton). 
APPENuiX II. - Balance Sheet of the Manorial Revenue of the

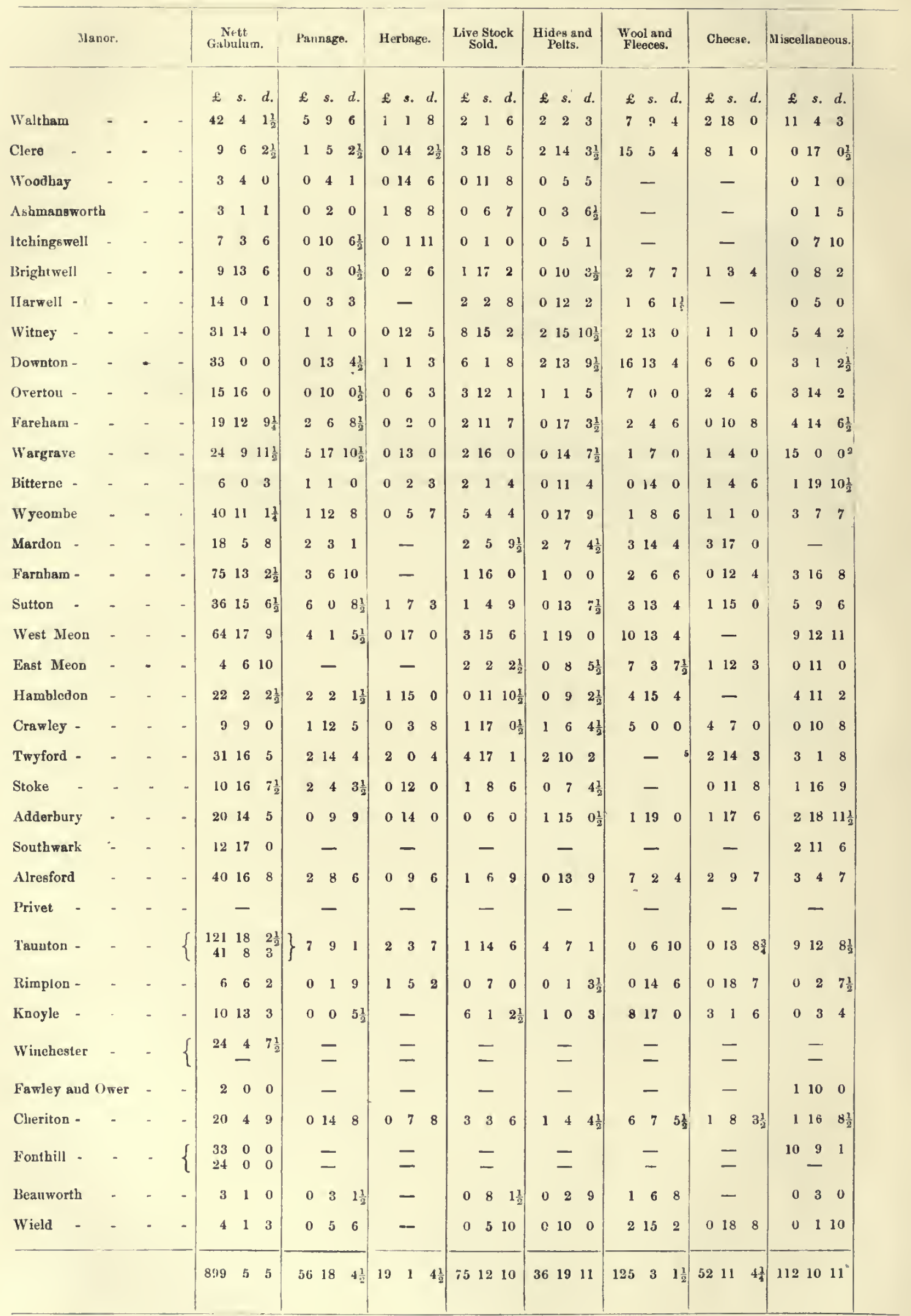


APPENDIX III.-Table Showing the Rate of Production and Frices of

\begin{tabular}{|c|c|c|c|c|c|c|c|c|c|c|c|c|c|c|c|c|c|c|c|}
\hline \multirow{2}{*}{\multicolumn{2}{|c|}{ Manor. }} & & \multicolumn{9}{|c|}{ WHEAT. } & \multicolumn{8}{|c|}{ MAYCORE OR RYR.! } \\
\hline & & & \multicolumn{2}{|c|}{$\begin{array}{c}\text { Total } \\
\text { Produce. }\end{array}$} & \multirow{2}{*}{$\frac{\begin{array}{c}\text { Acres } \\
\text { Sown. }\end{array}}{551}$} & \multicolumn{2}{|c|}{$\begin{array}{l}\text { Quantity } \\
\text { Sown. }\end{array}$} & \multicolumn{2}{|c|}{$\begin{array}{l}\text { Quantity } \\
\text { Sold. }\end{array}$} & \multicolumn{2}{|c|}{$\begin{array}{l}\text { A verage } \\
\text { Price } \\
\text { per qr. }\end{array}$} & \multicolumn{2}{|c|}{$\begin{array}{l}\text { Total } \\
\text { Produce. }\end{array}$} & \multirow{2}{*}{$\begin{array}{c}\text { Aores } \\
\text { Sown. } \\
-\end{array}$} & \multicolumn{2}{|c|}{$\begin{array}{l}\text { Quantity } \\
\text { Sown. }\end{array}$} & \multicolumn{2}{|c|}{$\begin{array}{l}\text { Quantity } \\
\text { Sold. }\end{array}$} & $\begin{array}{l}\text { Average } \\
\text { Price } \\
\text { per qr. }\end{array}$ \\
\hline Waltham & - & - & $\begin{array}{l}\text { qrs. bu } \\
207 \frac{\frac{1}{2}}{}\end{array}$ & $\begin{array}{c}\text { bus. } \\
1\end{array}$ & & $\begin{array}{l}\text { qrs. } \\
112 \frac{1}{2}\end{array}$ & $\begin{array}{c}\text { bus. } \\
-\end{array}$ & $\begin{array}{l}\text { grs. bu } \\
20 \frac{1}{2}\end{array}$ & & & & $\begin{array}{l}\text { qrs. bu } \\
-\end{array}$ & & & $\begin{array}{c}\text { qre. bu } \\
-\end{array}$ & & qrs. bu & us. & $\begin{array}{l}\text { s. } d . \\
-\end{array}$ \\
\hline Clere - & - & - & 127 & 3 & 372 & 63 & - & 74 & - & 2 & 8 & $116 \frac{1}{2}$ & 1 & 218 & $45 \frac{1}{2}$ & - & 49 & 1 & $14 \frac{1}{2}$ \\
\hline Woodhay & - & - & 52 & - & 84 & 17 & - & $44 \frac{1}{2}$ & - & & $9 \frac{1}{2}$ & $4 \frac{1}{2}$ & 1 & 56 & $16 \frac{1}{2}$ & - & $53 \frac{1}{2}$ & - & 15 \\
\hline Asbmanswor & & - & 36 & - & 11 & $9 \frac{1}{2}$ & $1 \frac{1}{4}$ & 16 & - & 2 & 10 & 54 & 1 & 73 & $19 \frac{1}{2}$ & - & 24 & - & $16 \frac{3}{4}$ \\
\hline Itchingswell & - & - & 58 & - & 112 & 22 & - & 35 & - & 2 & 8 & 12 & 1 & 24 & 5 & - & 1 & - & 18 \\
\hline Brightwell & - & - & $262 \frac{1}{2}$ & - & 282 & 62 & - & 202 & 3 & 2 & 7 & - & - & - & - & - & - & - & - \\
\hline Harwell & - & - & 110 & - & 182 & 41 & - & 76 & - & 2 & 9 & 15 & - & 7 & 3 & - & - & - & - \\
\hline Witney - & - & - & $188 \frac{1}{\frac{1}{3}}$ & - & 417 & 82 & - & 50 & - & & $3 \frac{5}{4}$ & - & - & - & - & - & - & - & - \\
\hline Downton & - & - & $221 \frac{1}{2}$ & 1 & 351 & 117 & - & 110 & 1 & & $10 \frac{1}{2}$ & 29 & 1 & 156 & 53 & 1 & 27 & - & 20 \\
\hline Overton & - & $\cdot$ & $34 \frac{1}{2}$ & 1 & 42 & 13 & - & $17 \frac{1}{2}$ & 1 & 3 & $1 \frac{3}{4}$ & 99 & 1 & 208 & $a 60$ & - & $14 \frac{1}{2}$ & 1 & $18 \frac{3}{4}$ \\
\hline Fareham & - & - & 126 & - & 262 & 64 & - & $55 \frac{1}{2}$ & - & & $6 \frac{3}{4}$ & - & - & - & - & - & - & - & - \\
\hline Wargrave & - & - & 17 & - & 47 & 14 & - & 10 & - & & $7 \frac{1}{2}$ & 220 & - & 355 & $89 \frac{1}{2}$ & - & 111 & 1 & $2 \quad 1 \frac{1}{4}$ \\
\hline Bitterne & - & - & 4 & - & 16 & 4 & - & - & - & - & - & $* 2$ & 2 & 9 & 2 & 2 & - & - & - \\
\hline Wycombe & - & - & $201 \frac{1}{2}$ & - & 156 & $78 \frac{1}{2}$ & - & 116 & - & & $5 \frac{1}{2}$ & $50 \frac{1}{2}$ & - & 21 & $10 \frac{1}{8}$ & - & - & - & - \\
\hline Mardon - & - & - & $169 \frac{1}{2}$ & - & 566 & 90 & - & $28 \frac{1}{2}$ & - & & 8 & 13 & - & 26 & 6 & - & - & - & - \\
\hline Farnham & - & - & $168 \frac{1}{2}$ & 3 & $142 \frac{1}{2}$ & $22 \frac{1}{2}$ & 1 & $85 \frac{1}{2}$ & - & & $10 \frac{1}{2}$ & $* 30$ & 2 & 208 & 21 & - & $5 \frac{1}{2}$ & - & 30 \\
\hline Sutton - & - & - & 91 & 2 & 242 & $66 \frac{1}{2}$ & - & 42 & - & & $6 \frac{3}{4}$ & $31 \frac{1}{2}$ & - & 66 & $19 \frac{1}{2}$ & - & 7 & - & 17 \\
\hline West Meon & - & - & 200 & 2 & 479 & $90 \frac{1}{2}$ & - & $100 \frac{1}{2}$ & 2 & & $10 \frac{1}{2}$ & - & - & - & - & - & - & - & - \\
\hline East Meon & - & - & 235 & 2 & 80 & 16 & - & 207 & - & & 9 & $\left\{\begin{array}{c}10 \\
* 8 \frac{1}{2}\end{array}\right.$ & $\overline{2}$ & I & - & $\overline{-}$ & $\begin{array}{l}10 \\
8 \frac{1}{2}\end{array}$ & $\overline{2}$ & $\begin{array}{ll}1 & 8 \\
2 & 4\end{array}$ \\
\hline Hambledon & $\cdot$ & - & $31 \frac{1}{2}$ & - & 110 & $23 \frac{1}{2}$ & 1 & 9 & - & 2 & $6 \frac{1}{2}$ & $15 \frac{1}{2}$ & - & 44 & $12 \frac{1}{2}$ & - & 3 & - & 18 \\
\hline Crawley & - & - & $66 \frac{1}{2}$ & - & 90 & 26 & - & $61 \frac{1}{2}$ & - & & $6 \frac{1}{6}$ & - & - & - & - & - & - & - & - \\
\hline Twyford & - & - & $123 \frac{1}{2}$ & - & 212 & 92 & - & 39 & - & 2 & 10 & - & - & - & - & - & - & - & - \\
\hline Stoke - & - & - & $105 \frac{1}{2}$ & - & 224 & $24 \frac{1}{8}$ & - & - & - & - & - & - & - & - & - & - & - & - & - \\
\hline Adderhury & - & - & 65 & - & 152 & 31 & - & 34 & - & 2 & 2 & ${ }^{*} 166 \frac{1}{2}$ & 1 & 180 & 40 & - & $104 \frac{1}{2}$ & - & 16 \\
\hline Alresford & - & - & $96 \frac{1}{2}$ & - & 168 & 48 & - & 53 & - & 2 & 9 & $75 \frac{1}{2}$ & - & 101 & $33 \frac{1}{2}$ & - & 43 & - & 20 \\
\hline Privet - & - & - & $54 \frac{1}{2}$ & - & 62 & $15 \frac{1}{2}$ & - & $2 \frac{1}{2}$ & - & 2 & 8 & 39 & - & 39 & $13 \frac{1}{2}$ & - & 2 & - & 20 \\
\hline Taunton & - & - & 392 & 1 & 757 & 182 & 1 & 190 & - & 3 & $7 \frac{1}{4}$ & $* 80 \frac{1}{2}$ & 1 & 122 & 31 & - & - & - & - \\
\hline Rimpton & - & & $18 \frac{1}{2}$ & - & $60_{2}^{3}$ & 15 & - & $7 \frac{1}{2}$ & - & 2 & 6 & $* 21$ & 3 & 28 & 7 & - & 1 & - & 24 \\
\hline Knoyle - & - & - & 37 & - & $76 \frac{3}{2}$ & $16 \frac{1}{2}$ & - & 13 & - & 3 & 0 & 45 & 2 & 143 & $b 25 \frac{1}{2}$ & - & - & - & - \\
\hline Cberiton & - & - & $103 \frac{1}{2}$ & - & 240 & $60 \frac{1}{2}$ & - & $35 \frac{1}{2}$ & - & 3 & 0 & $31 \frac{1}{2}$ & - & 74 & $23 \frac{1}{2}$ & - & - & - & - \\
\hline Beauworth & - & 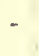 & 47 & - & 103 & $24 \frac{1}{2}$ & - & 18 & - & 2 & $9 \frac{1}{2}$ & 9 & - & 9 & $2 \frac{1}{2}$ & - & - & - & - \\
\hline Wield & - & & 26 & - & $158 \frac{1}{2}$ & 45 & - & 13 & - & 3 & 2 & 45 & - & $65 \frac{1}{2}$ & $21 \frac{1}{2}$ & - & 11 & - & 28 \\
\hline Tot: & & & 3679 & - & 6838 & 1589 & $4 \frac{1}{4}$ & 1767 & 3 & 2 & $9 \frac{2}{2}$ & $\left\{\begin{array}{r}914 \frac{2}{2} \\
* 309 \frac{1}{2}\end{array}\right.$ & $\begin{array}{l}3 \\
3\end{array}$ & $\begin{array}{c}1685 \frac{1}{2} \\
547\end{array}$ & $\begin{array}{l}460 \frac{1}{2} \\
101\end{array}$ & $\begin{array}{l}1 \\
2\end{array}$ & \begin{tabular}{|l|}
556 \\
$119 \frac{1}{2}$
\end{tabular} & $\begin{array}{l}3 \\
2\end{array}$ & $\begin{array}{rr}1 & 9 \frac{3}{4} \\
1 & 10 \frac{1}{2}\end{array}$ \\
\hline
\end{tabular}

1 Kye is distinguished by an asterisk. 


\section{$(\mathrm{xlv})$}

Cereals in the Manors of the Bishopric of Winchester for the Year 1208-1209.

\begin{tabular}{|c|c|c|c|c|c|c|c|c|c|c|c|c|c|c|c|}
\hline \multicolumn{8}{|c|}{ BABLET. } & \multicolumn{7}{|c|}{ Oats. } & \multirow{2}{*}{ Remarks. } \\
\hline $\begin{array}{l}\text { Total } \\
\text { Produce }\end{array}$ & & $\begin{array}{l}\text { Acres } \\
\text { Sown. }\end{array}$ & $\begin{array}{l}\text { Quant } \\
\text { sown }\end{array}$ & & $\begin{array}{l}\text { Quanti } \\
\text { Sold. }\end{array}$ & & $\begin{array}{c}\text { A verage } \\
\text { Price } \\
\text { per qr. }\end{array}$ & $\begin{array}{r}\text { Tota } \\
\text { Produ }\end{array}$ & & $\begin{array}{l}\text { Acres } \\
\text { Sown. }\end{array}$ & $\begin{array}{l}\text { Quantity } \\
\text { Sown. }\end{array}$ & & $\begin{array}{l}\text { Quautity } \\
\text { Sold. }\end{array}$ & $\begin{array}{l}\text { Average } \\
\text { Price } \\
\text { per q. }\end{array}$ & \\
\hline $\begin{array}{c}\text { grs. bu } \\
99\end{array}$ & $\begin{array}{l}\text { us. } \\
-\end{array}$ & 148 & $\begin{array}{l}\text { qrs. } \\
40 \frac{1}{2}\end{array}$ & bus. & $\begin{array}{c}\text { qrs. b } \\
-\end{array}$ & & $\begin{array}{l}\text { s. } d \text {. } \\
-\end{array}$ & $\begin{array}{l}\text { qrs. } \\
477\end{array}$ & $\begin{array}{r}\text { bus. } \\
3 \frac{1}{2}\end{array}$ & 498 & $\begin{array}{l}\text { qrs. bus } \\
241 \frac{1}{2}\end{array}$ & & $\begin{array}{cc}\text { qrs. bus. } \\
12 & -\end{array}$ & $\begin{array}{ll}s . & d . \\
0 & 8\end{array}$ & \\
\hline $21 \frac{1}{2} 1$ & 1 & 37 & $12 \downarrow$ & - & - & - & - & $174 \frac{1}{2}$ & 2 & 242 & 74 & - & $-\quad-$ & - & \\
\hline 8 & - & 4 & $1 \frac{1}{2}$ & - & $6 \frac{1}{2}$ & - & 14 & 89 & - & 71 & 41 & - & - & - & \\
\hline $6 \frac{1}{2}$ & - & 13 & $4 \frac{1}{2}$ & - & - & - & - & 53 & - & $85 \frac{1}{2}$ & 34 & - & - & - & \\
\hline - & - & - & - & - & - & - & - & 19 & - & 72 & 24 & - & - & - & \\
\hline - & - & 12 & $3 \frac{1}{2}$ & - & - & - & - & $98 \frac{1}{2}$ & 3 & 60 & 29 & - & $42-$ & 10 & \\
\hline - & - & - & - & - & - & - & - & 39 & 3 & 17 & $8 \frac{1}{2}$ & - & $22 \frac{1}{2} \quad 1$ & 10 & \\
\hline $73 \frac{1}{2}$ & - & 96 & 31 & 1 & 1 & - & 16 & $334 \frac{1}{2}$ & - & 212 & 90 & - & $16-$ & 10 & \\
\hline $178 \frac{1}{2}$ & - & $102 \frac{1}{2}$ & $49 \frac{1}{2}$ & - & $91 \frac{1}{2}$ & - & $2 \quad 1 \frac{1}{4}$ & 311 & 2 & $221 \frac{1}{3}$ & $110 \frac{1}{2}$ & - & 12 & 10 & \\
\hline $32 \frac{1}{2}$ & - & 48 & 15 & - & $17 \frac{1}{2}$ & - & 19 & 104 & 1 & 198 & $69 \frac{1}{2}$ & - & - & 10 & $a+8$ qrs. of wheat. \\
\hline 23 & - & 46 & 18 & - & - & - & - & $182 \frac{1}{2}$ & 2 & 200 & $125 \frac{1}{2}$ & - & - & - & \\
\hline $46 \frac{1}{2}$ & 1 & 40 & 13 & 1 & $31 \frac{1}{2}$ & - & $111 \frac{1}{4}$ & 472 & 2 & 320 & 149 & - & 232 & $10 \frac{1}{2}$ & \\
\hline $25 \frac{1}{2}$ & 1 & 22 & 11 & - & - & - & - & 39 & 2 & 70 & 35 & - & - & - & \\
\hline 40 & - & 14 & 7 & - & - & - & - & $256 \frac{1}{2}$ & 3 & 215 & $143 \frac{1}{2}$ & - & 26 & 10 & \\
\hline 67 & 1 & 111 & 36 & - & - & - & - & $106 \frac{1}{2}$ & 3 & 432 & 89 & - & 16 & 011 & \\
\hline $26 \frac{1}{2}$ & - & 33 & 8 & - & - & - & - & 306 & - & 349 & $115 \frac{1}{2}$ & - & - & $\begin{array}{ll}0 & 9\end{array}$ & \\
\hline $65 \frac{1}{2}$ & - & 98 & 30 & - & $12 \frac{1}{2}$ & - & $19 \frac{1}{4}$ & $100 \frac{1}{2}$ & - & 306 & $98 \frac{1}{2}$ & - & - & - & \\
\hline 163 & 2 & 230 & $46 \frac{1}{2}$ & - & 33 & - & $\begin{array}{ll}2 & 0\end{array}$ & 590 & 1 & 600 & $318 \frac{1}{2}$ & - & 5 & 10 & \\
\hline 182 & - & 10 & 4 & - & $138 \frac{1}{2}$ & - & $111 \frac{1}{2}$ & $477 \frac{1}{2}$ & - & 120 & 70 & - & $22 \frac{1}{2}$ & 10 & \\
\hline 76 & - & 80 & 23 & - & 6 & - & $110 \frac{1}{2}$ & 112 & - & 170 & 50 & - & - & 10 & \\
\hline $147 \frac{1}{2}$ & - & 173 & 57 & - & $12 \frac{1}{2}$ & - & $\begin{array}{ll}2 & 0 \frac{1}{2}\end{array}$ & 201 & - & 286 & 104 & - & - & - & \\
\hline $106 \frac{1}{2}$ & - & 114 & 50 & - & $17 \frac{1}{2}$ & - & $2 \quad 4 \frac{3}{4}$ & 266 & 4 & 235 & 131 & - & 44 & $12 \frac{1}{4}$ & \\
\hline 66 & $0 \frac{1}{2}$ & 85 & $17 \frac{1}{2}$ & - & $33 \frac{1}{2}$ & - & $15 \frac{3}{4}$ & $85 \frac{1}{2}$ & 1 & 160 & $41 \frac{1}{2}$ & - & - & - & \\
\hline 40 & - & 22 & 5 & - & 30 & - & 14 & 167 & 2 & 88 & $41 \frac{1}{2}$ & - & 74 & $10 \frac{1}{2}$ & \\
\hline 132 & - & 131 & $52 \frac{1}{2}$ & - & 55 & - & 20 & 205 & - & 165 & 110 & - & 105 & $1 \quad 0 \frac{1}{4}$ & \\
\hline $11 \frac{1}{2}$ & - & 11 & 4 & - & - & - & - & $148 \frac{1}{2}$ & - & 107 & $5.8 \frac{1}{2}$ & - & 29 & $10 \frac{3}{4}$ & \\
\hline- & - & 7 & 2 & 1 & - & - & - & 1397 & 3 & $964 \frac{1}{2}$ & 609 & - & 505 & 10 & \\
\hline- & - & 4 & 2 & - & - & - & - & 42 & - & 50 & 33 & - & 2 & 07 & \\
\hline $18 \frac{1}{2}$ & - & 38 & $8 \frac{1}{2}$ & - & - & - & - & $93 \frac{1}{2}$ & 2 & 195 & 66 & - & - & - & $b+6 \frac{1}{2}$ qrs. of wheat. \\
\hline 72 & - & 124 & 41 & - & - & - & - & 250 & - & 264 & 132 & - & - & - & \\
\hline 22 & - & 30 & $12 \frac{1}{2}$ & - & $1 \frac{1}{2}$ & - & 20 & $86 \frac{1}{2}$ & - & 117 & 51 & - & - & - & \\
\hline - & - & 8 & 3 & - & - & - & - & $218 \frac{1}{2}$ & - & 2261 & $113 \frac{1}{2}$ & - & 95 & 12 & \\
\hline 17.50 & $6 \frac{1}{2}$ & $1891 \frac{1}{b}$ & $689 \frac{\mathrm{l}}{2}$ & 2 & 488 & - & 111 & 7507 & $3 \frac{1}{2}$ & 7317 & $3402 \frac{1}{2}$ & - & 1274 & $10 \frac{1}{2}$ & \\
\hline
\end{tabular}


appendiX IV.-Table Showing the Produce and Value of Live Stock, Hides,

MichaeluMas 1208 to

\begin{tabular}{|c|c|c|c|c|c|c|c|c|c|c|c|c|c|c|c|c|c|c|c|c|c|c|}
\hline \multirow{4}{*}{\multicolumn{3}{|c|}{$\begin{array}{c}\text { LIVE STOCK - } \\
\text { NAMR OP MANOR. }\end{array}$}} & \multicolumn{5}{|c|}{ EQTINR CLAss. } & \multicolumn{5}{|c|}{ Bovim Class. } & \multicolumn{5}{|c|}{ Oving Class. } & \multicolumn{5}{|c|}{ Poncink Chass. } \\
\hline & & & \multirow{3}{*}{ 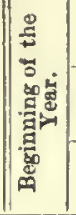 } & \multicolumn{4}{|c|}{ During the Year. } & \multirow{3}{*}{ 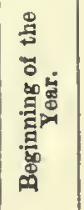 } & \multicolumn{4}{|c|}{ During the Year. } & \multirow{3}{*}{ 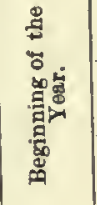 } & \multicolumn{4}{|c|}{ During the Year. } & \multirow{3}{*}{ 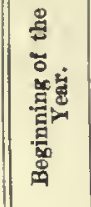 } & \multicolumn{4}{|c|}{ During the Year. } \\
\hline & & & & \multicolumn{2}{|c|}{ Increaso. } & \multicolumn{2}{|c|}{ Decrease. } & & \multicolumn{2}{|c|}{ Increase. } & \multicolumn{2}{|c|}{ Decrease. } & & \multicolumn{2}{|c|}{ Increase. } & \multicolumn{2}{|c|}{ Decrease. } & & Inere & ease. & Decr & rease. \\
\hline & & & & Pr. & $M i$. & Mu. & Mi. & & Pr. & Mi. & Mu. & Mi. & & Pr. & $\mathrm{Mi}$. & Mu. & Mi. & & Pr. & Mi. & Mu. & Mi. \\
\hline Waltham & - & - & 49 & 4 & - & 7 & 35 & 188 & 20 & 19 & 34 & 3 & 920 & 370 & 16 & 178 & 138 & 76 & 67 & - & 3 & 37 \\
\hline Clere - & - & - & 15 & 5 & 9 & - & 11 & 140 & 29 & 一 & 5 & 30 & 1,164 & 480 & 357 & 576 & 320 & 80 & 39 & - & 8 & 17 \\
\hline Woodhay & - & - & - & - & - & - & 一 & 31 & - & 5 & 1 & 13 & 383 & - & 102 & 25 & 19 & - & 一 & - & - & - \\
\hline Ashmanswort & & - & - & - & - & - & - & 12 & - & 1 & - & 1 & 281 & 237 & 104 & 53 & 247 & 一 & - & - & - & - \\
\hline Itchingswell & - & - & - & - & - & - & - & 20 & - & 11 & 1 & - & 253 & 244 & 2 & 68 & 185 & - & - & - & - & - \\
\hline Brightwel1 & - & - & 9 & 1 & - & - & 2 & 49 & 4 & 1 & 2 & 4 & 200 & 181 & 116 & 60 & 154 & 30 & 47 & - & 2 & 36 \\
\hline Harwell - & - & - & 1 & - & - & - & - & 24 & - & 2 & 1 & - & 305 & - & 89 & 67 & 211 & 14 & - & - & - & 14 \\
\hline Witney - & - & - & 15 & - & 1 & 5 & - & 80 & 16 & 4 & 13 & 3 & 683 & 144 & - & 259 & 304 & 284 & 56 & 53 & 46 & 237 \\
\hline Dornton & - & - & 2 & - & - & - & - & 144 & 13 & 19 & 23 & 1 & 1,764 & 750 & 71 & 173 & 179 & 124 & 29 & 19 & 9 & 86 \\
\hline Overton - & - & - & 11 & 2 & 1 & 1 & 1 & 58 & 3 & 1 & 3 & 16 & 775 & 282 & - & 140 & 98 & 35 & 15 & - & 3 & 12 \\
\hline Fareham & - & - & 2 & - & 1 & 1 & - & 41 & 1 & 11 & 7 & 5 & 283 & 138 & - & 25 & 59 & 65 & 22 & - & $\tau$ & 40 \\
\hline Wargrave & - & - & 10 & - & - & - & - & 95 & 10 & 4 & 8 & 12 & 190 & 57 & - & 54 & 9 & 68 & 48 & 427 & 29 & 35 \\
\hline Bitterne - & - & - & 2 & 1 & 15 & 2 & 10 & 34 & 10 & 27 & 8 & 1 & 90 & 90 & 100 & 61 & 82 & - & - & - & - & - \\
\hline Wycombe & - & - & 11 & - & 1 & - & 2 & 60 & 5 & 9 & 9 & 13 & 212 & 78 & - & 74 & 40 & 50 & 65 & 12 & 39 & 30 \\
\hline Mardon - & - & - & 43 & 7 & 63 & 34 & 29 & 101 & 14 & 24 & 28 & 6 & 581 & 215 & - & 173 & 89 & 92 & 43 & - & 6 & 77 \\
\hline Farnham & - & - & 16 & - & 28 & 1 & 2 & 79 & 10 & 20 & 6 & 16 & 498 & 212 & 9 & 190 & 45 & 158 & 83 & 16 & 4 & 158 \\
\hline Sutton - & - & - & 2 & - & 1 & - & 1 & 83 & - & 3 & 4 & 22 & 495 & 284 & 86 & 86 & 308 & 66 & 53 & 85 & 15 & 12 \\
\hline West Meon & - & - & 20 & - & 1 & 一 & 1 & 105 & - & 17 & 3 & 19 & 1,276 & 454 & 20 & 493 & 37 & 56 & 55 & 20 & - & 3 \\
\hline East Meon & - & - & 一 & - & - & - & - & 34 & - & 4 & 2 & - & - & - & 239 & 77 & - & - & - & - & - & - \\
\hline Hambledon & - & - & 5 & - & 1 & 2 & 1 & 21 & 一 & 5 & - & - & 533 & 160 & - & 135 & 28 & 43 & 20 & 8 & 13 & 2 \\
\hline Crawley & - & - & 10 & - & - & - & - & 59 & - & 6 & 4 & 20 & 1,063 & 456 & 48 & 219 & 114 & 34 & 23 & - & 7 & 1 \\
\hline Twyford & - & $\cdot$ & 10 & 4 & 8 & 1 & - & 106 & 2 & 4 & 2 & 12 & 1,627 & 638 & - & 328 & 104 & 42 & 22 & 一 & 3 & 2 \\
\hline Stoke - & - & - & - & - & - & - & - & 120 & 23 & 5 & 13 & 9 & - & - & 一 & - & 一 & 40 & 24 & - & 9 & 1 \\
\hline Adderbury & - & - & 12 & 1 & - & - & - & 27 & 5 & 1 & 5 & 2 & 265 & 92 & - & 142 & 11 & 34 & 20 & 一 & 3 & 2 \\
\hline Southwark & - & - & - & - & - & - & - & - & - & - & - & - & - & - & - & - & - & - & - & 104 & - & 4 \\
\hline Alresford & - & - & 3 & 1 & 1 & - & 2 & 95 & 8 & 3 & 2 & 7 & 889 & 331 & - & 195 & 65 & 55 & 46 & 83 & 5 & 10 \\
\hline Privet - & - & - & 3 & - & - & - & - & -- & - & - & - & - & - & - & - & - & - & - & - & - & - & - \\
\hline Taunton. & - & - & 28 & 7 & 6 & 3 & 12 & 240 & 11 & 37 & 22 & 26 & 251 & 36 & 1 & 163 & 3 & 20 & 54 & 3 & 7 & \\
\hline Rimpton & - & - & - & - & 2 & - & - & 5 & 一 & 22 & - & 1 & - & 79 & 83 & 11 & 21 & - & - & - & - & - \\
\hline Knoyle - & - & - & 3 & - & 5 & - & - & 79 & 8 & 10 & 7 & 10 & 1,048 & 406 & - & 266 & 228 & 27 & 23 & 一 & 5 & 1 \\
\hline Cheriton & - & - & 3 & - & 2 & - & - & 67 & - & 12 & 17 & 5 & 591 & 299 & 239 & 229 & 88 & 47 & 48 & - & 17 & 2 \\
\hline Beauworth & - & - & 1 & - & - & - & - & 26 & - & 4 & 2 & 1 & 132 & - & - & 2 & 10 & - & - & - & - & - \\
\hline Wield & - & - & 10 & - & 1 & 1 & 3 & 33 & - & 2 & 1 & 2 & 331 & {$[170]$} & - & 163 & 16 & - & - & - & - & - \\
\hline Tota & & - & 296 & 32 & 146 & 58 & 112 & 2,253 & 178 & 307 & 233 & 284 & 17,083 & 6,833 & 1,682 & $4,68.5$ & 3,233 & 1,540 & 902 & 842 & 240 & $1,53:$ \\
\hline
\end{tabular}


Cheese, Butter, and Wool in the Manors of the Bishopric of Winchester, Michaelmas 1209.

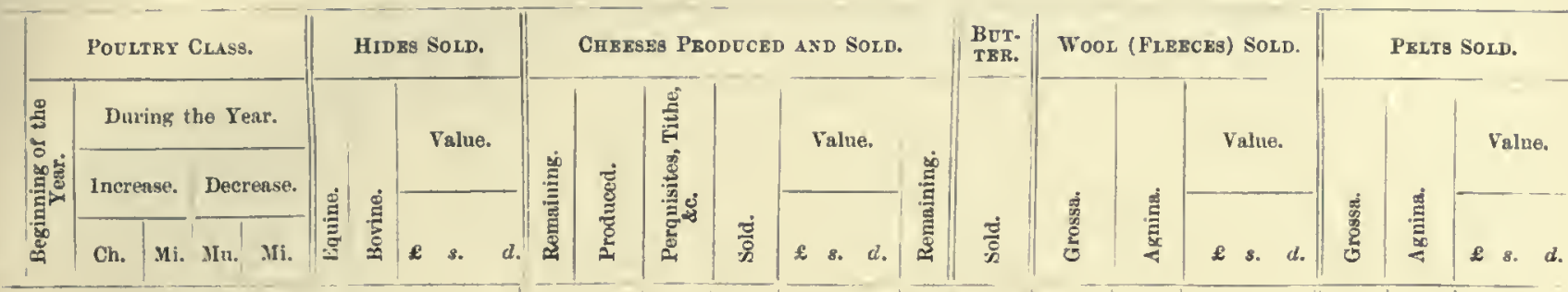

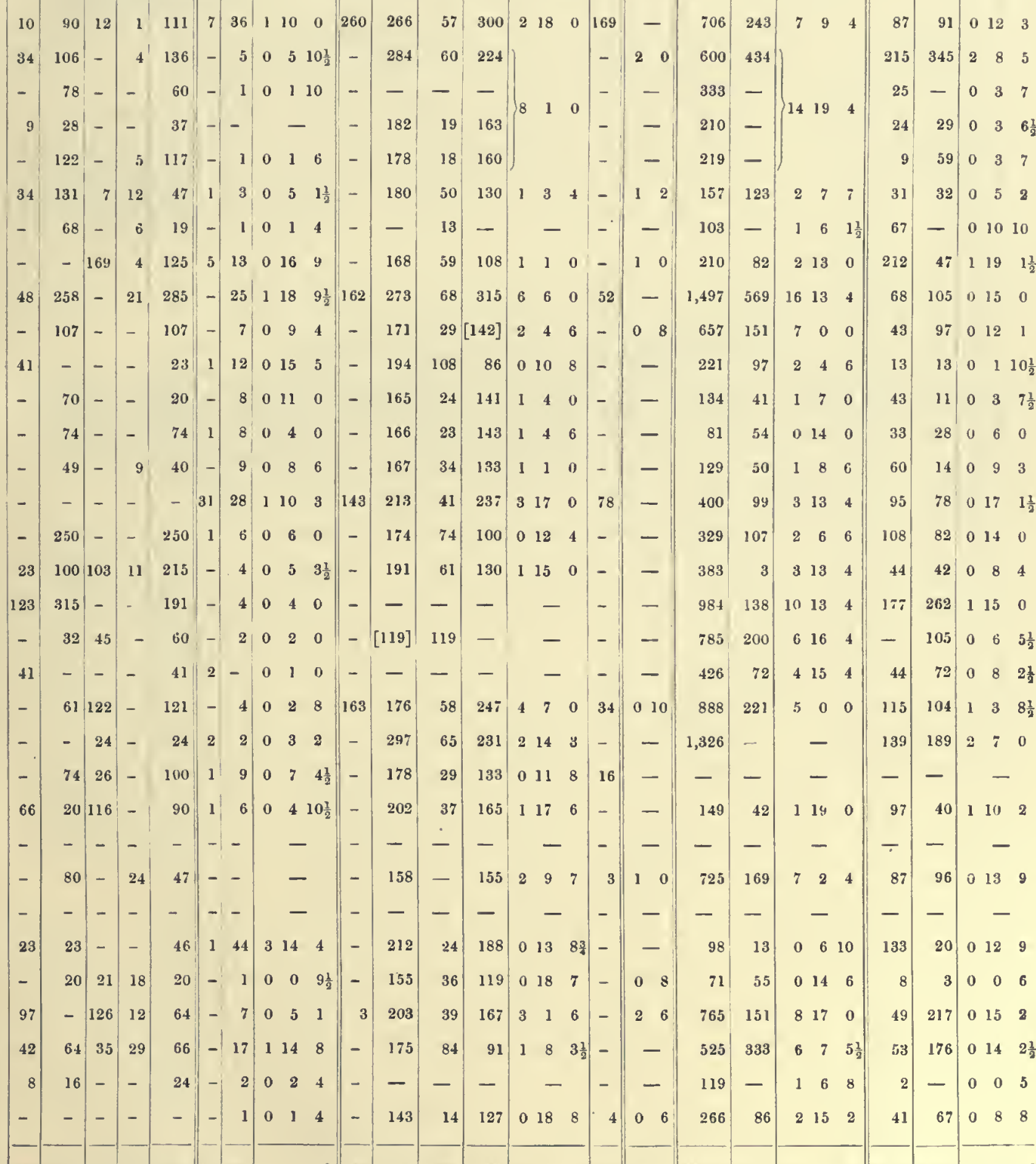




\section{( xlviii )}

appendiX V.-Table Showing the State of the Live and Dead Stock in the above Manors during the same Period.

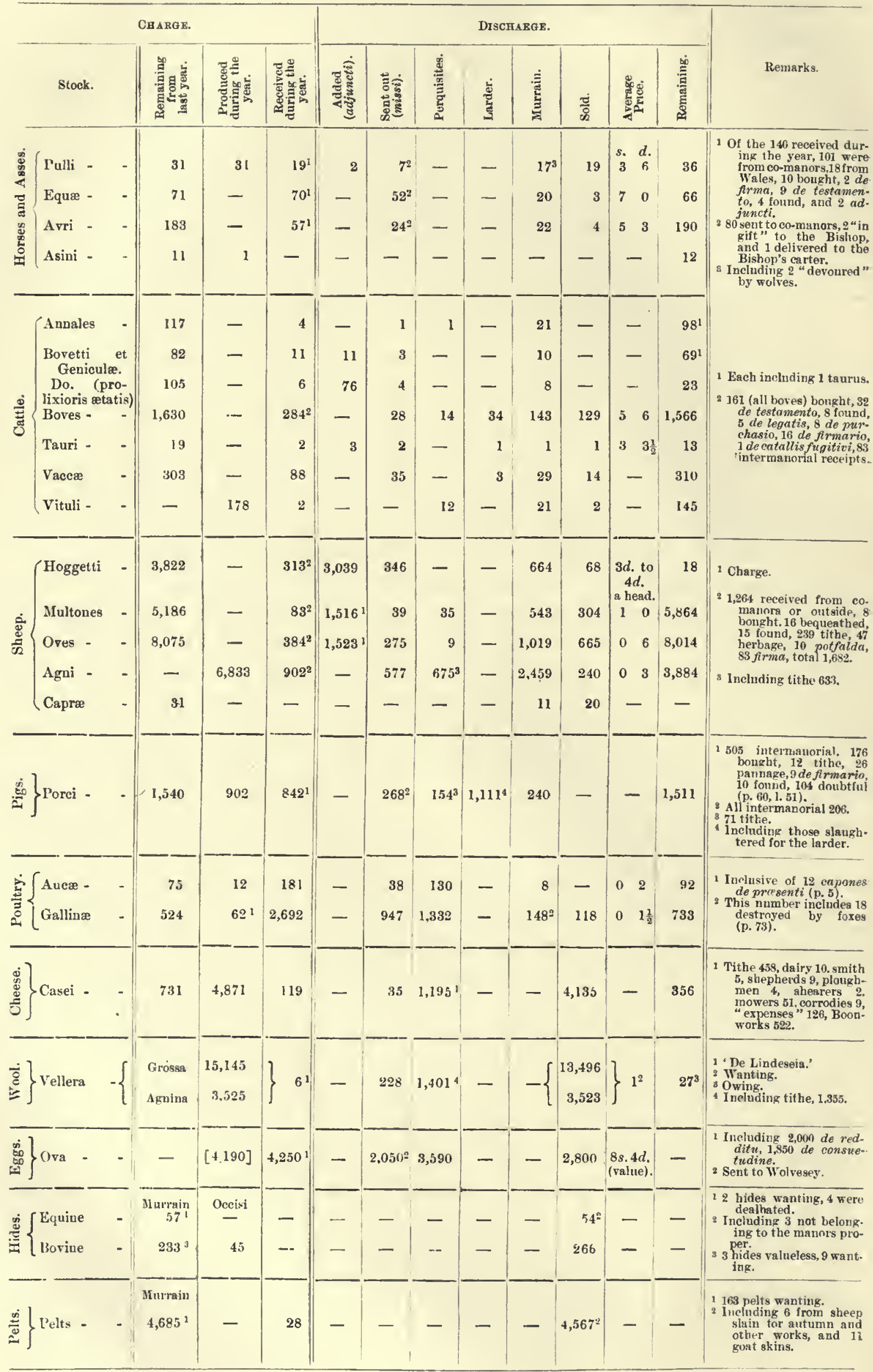




\section{ROTULUS COMPOTI}

ANNI QUARTI PONTIFICATUS DOMINI PETRI WINTONIENSIS EPISCOPI 



\section{ANNUS QUARTUS ROTULUS COMPOTI.}

\section{WALTHAM 1}

M. 1. Walteros de Parfit et Adam præpositus reddunt compotum de xlvjl. vijs. ij l. ob. de gabulo assiso. Et de ijs. de Henrico villatæo de incremento gabuli de terra Bal. Et de ijs. de incremento gabuli Michaelis Hudi, pro viij acris. Summa xlvjl. xjs. ijd. ob.

In quietanciis $\mathrm{j}$ præpositi, $\mathrm{j}$ bedelli, per annum, $\mathrm{x} s$. In quietanciis $\mathrm{j}$ liaiwardi, $\mathrm{j}$ bercariorum, $\mathrm{j}$ fabri, $\mathrm{j}$ porcarii, per annum, ixs. vjd. In quietanciis vij carrucariorum, xiiijs. In quietanciis $\mathrm{x}$ operariorum, $\mathrm{xxs}$, $\mathrm{j}$ operarii cum biga sua, iijs., ij operariorum, ijs., ij grangriariorum, iijs. Summa lxijs. vjd.

In defectu terrarum tractarum in dominium, xxijs. viijd. In defectu terræ Osberti de Langel[ega], viijd. . In defectu terre Ricardi Baconis, vjd. In defectu terræ Henrici filii Azonis, vjd. In defectu terræ Walteri de Swanemere, iijd. Summa xxiiijs. vijd.

${ }^{2}$ Summa tam quietanciarnm quam defectuum, iijl. vijs. jd.

$$
\text { Et remanent xlijl. iiijs. iij ob. }
$$

Idem reddunt compotum de vl. ixs. vjd. de pannagio. Et de xxjs. viijd. de herbagio. Et de iijs. vjd. de juncco vendito. Et de xviijs. de vj bobus vivis venditis. Et de xxijs. de xxxiij ovibus vivis venditis, pro qualibet, viijd. Et de lx.xiiijs. vijd. de iiij tonellis vini venditis. Et de xxxs. de coreis xxix boum, $\nabla$ vaccarum, ij annalium, iij equarum, iij pullorum mortuorum venditis. Et de $x$ viijd. de $\mathbf{v j}^{3}$ agnis vivis venditis. Et de vjs. iijd. de pellibus xxv ovium, xlij multonum, $x x$ hogg[ettorum]. Et de vjs. de pellihus iiij ${ }^{x x} \cdot x j$ agnorum mortuorum venditis. Et de xxviijs. de uncto cexiij porcorum vendito, quia porcarius habuerat consuetudinem ix porcorum. Et de $x \mathrm{v} d$. de exitu $x$ porcorum. Et de ijs. rle $x l$ parvis caseis factis in hyeme. Et de vijl.ixs. iijd. de $\mathrm{v}$ ponđeribus grossæ lanæ et agninæ, unde iij mainardi fuerunt de lana agnina. Et de lvjs. pro octo capitibus casei venditis de reragio anni præteriti. Et de vjs. $x d$. pro xlj acris locatis hoc anno, pro singula acra ijd. Et de cvijs. iiijd. pro busca vendita de parco. Et de ijs. vjd. ob. de nucibus venditis. Et de $\mathrm{ij} d$. ob. pro j galone mellis vendito. Summa xxxijl. vjs. vjd.

Idem reddunt compotum de xlvs. pro $\mathrm{xx}$ quarteriis et dimidio frumenti venditis. Et de viijs. pro zij quarteriis ivenæ venditis. Et de vjs. de iij quarteriis ordei, de blado Nicolai occisi. Et de vs. viijd. de $v$ quarteriis dimidio, ij bussellis avenæ, ejusdem Nicolai occisi, venditis. Summa lxiijs. viijd.

Idem reddunt compotium de xijs. pro molendino de Kaldecote. Et de xs. pro molindino de Matykesforda. Et de viijs. pro molindino Edwardi Frogge. Et de xxs. pro molindino full[er]atico. Summa ls.

Idem reddunt compotum de xliijs. pro xxj quarteriis et dimidio mancorni venditis de molindino quod est in manu domini Episcopi. Et de iiijs. pro $\mathrm{j}$ quarterio frumenti et dimidio venditis. Et de $x v j s$. ijd. pro $\mathrm{v}$ quarteriis dimidio brasei venditis. Summa lxiijs. ijd.

De Purchasiis.-Idem reddunt compotum de xijd. de testamento Coleswini. Et de iiijs. de Willelmo Duc pro fine terræe. Et de xijd. de Henrico filio Acelini. Et de ijs. de filio Godwini de Parkehu[r]ste. Et de xijd. de filio Moisis. Et de xijd. de filio Paviæ. Et de vjd. de Waltero Prestes[man]. Et de vjd. de Ricardo Portereve pro lege. Et de vjd. de Alexandro Skegge pro simili. Et de vjd. de Osberto de Curderigge. Et de ijs. de Henrico de Upham pro filia sua maritanda. Et de j-marca de Brando pro veredicto. Et de ijs. de Henrico Vilur pro terra. Et de dimidia marca de vidua Edwardi pro molindino. Et. de dimidia marca de Willelmo Spiringe. Et de zviijd. de Basilla pro fine terræ. Et de xijd. de Petro Leverici. Et de vjd. de Ricardo filio Radulfi pro pastura. Et de xijd. de Henrico filio Radulfi pro simili. Et de vjd. de Willelmo Ruffo pro simili. Et de jis. de Rogero Bor pro simili. Et de vjd. de vidua Ricardi Gileberti pro simili. Et de vjol. de Ricardo Coco pro simili. Et de vjd. de Edwardo Mallori pro mellea. Et de vjd. de

50 Waltero de Holemore pro simili. Et de vjd. de Edmundo de molindino pro simili. Et de vj . de Willelmo de molindino pro simili. Et de dimidia marca de Thoma de Hestona. Et de dimidia marca de Thoma de Hou. Et de vjd. de Willelmo Ruffo pro aratura. Et de vjd. de Pagano de Estona pro simili. Et de xviijd. de Ricardo Prinne propastura. Et

The titles of the several manors are also written in large capitals at the bottom of each membrane.

An orasure in MS.

oj is repeated in MS. 
de ijs. vjd.de Willelmo filio suo pro simili. Et de xijd. de Willelmo Vise pro simili. Et de vja. de Willelmo Bus pro simili. Et de vja. de Alwino Hudi pro simili. Et de dinidia marca de vidua Cotere pro falso clamore. Et de ijs. de Roberto Poche pro simili. Et de xijd. de Wudeman pro simili. Et de vjd. de Ada Swede pro porcis. Et de xijd.de Radulfo de Parco pro pastura. Et de vs. de Ada Cune pro simili. Et de vjd. de uxore Osberti de Curderigge pro falso clamore. Et de vjd. de Giliberto Com[ite] pro pannagio. Et de vjd. de Rogero Speleman pro simili. Et de vjd. de Roberto Turbin pro lege. Et de iijs. de filio Simonis de Wintonia pro fine terræ. Lit de vj $\iota$. de Ricardo Coco pro pannagio. Et de dimidia marca de Gunnilda de Cuderigge pro filia sua maritancla. Et de vs. de Emma de Vule pro simili. Et de xijd. de Ricardo gardinario pro tonsura. Et de vjd. de Rogero 10 Dolfin pro simili. Et de vjd. de Osberto de Cuderigge pro simili. Et de xijd. de Jolianne Udelini pro simili. Et de xijd. de Ricardo Coco pro motura. Et de xijd. de Ada Cune pro lege. Et de xijd. de Ermisio de Frithe pro simili. Et de vjd. de Roberto Gosei pro falso clamore. Et de in respectu ${ }^{1}$ de Radulfo de Felda pro tonsura. Et de vjd. de Moyse de Parkeurste. Et de vjd. de Stephano de Heche. Et de xijd. de Ada Wuluini pro pastura. 15 Et de vjd. de Johaune de Aqua pro simili. Et de vjd. de Osberto Albo pro simili. Et de vjd. de Willelmo Malherbe pro simili. Et de xijel. de Willelmo Cappe pro lege. Et de xijd. de Roberto Colesueni. Et Ce vjd. de Nicolao Pig pro miur'.1 Et de ijs. de Godwino de Parkeurst pro filia sua maritanda. Et de ijs. de Willelmo parmenter pro simili. Et de iijs. viijl. de vidua de Fonte pro falso clamore. Et de zviijd. de Johanne 20 jro simili. Et de xviijd. de Hervico pro simili. Et de vjd. de filio Gunteri pro simili.

Summa vjl. xs. Et de tallagio xll.

Sumına summarum exxixl. xvjs. vd. ob. ${ }^{2}$

Liberatio.-In liberatis J[ohanni] decano de termino Natalis, vijl. xs. Eidem, de termino Paschæ, cs. Eidem, de termino Sancti Johannis, vjl. xs. Eidem, de termino Sancti 25 Michaelis, xiiijl. Eidem, de vino lx.xiijjs. vijd. Eidem, de reragio casei venditi, lvjs. Eidem, de lana vendita, vijl. ix. iijd. Eidem, de ovibus venditis, xxijs. Eidem, de ferro vendito, xljs. Eidem, de tallagio, xxxvijl. xjs. Eidem, de purchasiis, lx.viijs. Summa tocius liberationis, iijj ${ }^{x x} . x j l$. vs. $x j d$.

Expensa.-In ferramentis vij carrucarum, iij avrorum per annum, xxxvs. $x d$. ob. In viij 30 carrucis de novo parandis, vj paribus rotarum ad idem, ij herciis parandis, ijs. ijd. ob. In ij paribus rotarum bigis emptis, iijs. vijd. In iij bigis emendis, axandis, xjd. In paronis, paronellis, sellis, bacis, clutis, capistris, xvd. ob. In stipendiis iij hominibus, iiij feminabus, ad parandum lardarium de Waltham, per $\mathrm{x}$ dies, vijs. vjd.; singulo ' homini, in die, iij ob., et singulæ feminæ, ijj quadrantes. In 35 ix sextariis cervisiæ ad sulcit[ium], vs. iijd. $u b$. In thalamo et turri et cæteris domibus emendandis et cooperiendis, iijs. $\mathrm{x} d$. In $\mathrm{j}$ besca, $\mathrm{j}$ hoa, emptis ad opus gardinarii, iijd. ob. In $\mathrm{j}$ sera empta ad lardarium, iij ol. In $\mathrm{ij}$ saccis emptis, xijd. ob. In precariis hyemalibus et quadragesinalibus de clx.x carrucariis de Waltham xiijs. ijd. In precariis hyemalibus et quadragesimalibus de lx.xv carrucariis de Drokeneforda, vjs. iijd. In aratura vij 40 carrucariorum de $x x$ viij sabbatis ${ }^{2}$ ad seminationem, xxiijs. vj $d$., cuilibet illorum ill sabbato, iij $o b$. In araturil vij carrucariorum pro xxij sabbatis ad warectam, xijs. xd., cuilibet illorum $\mathrm{j} d$. in sabbato. In stipendiis vij bovariorum, iij carettariorum, $\mathrm{j}$ gardinarii, $\mathrm{j}$ vaccarii, per annum, xxxvjs. In stipendio $\mathrm{j}$ daiæ, per annum, ijs. In custo daeriæ, ijs. $\mathrm{j} d$. In j mola empta, xxviijs. vd. In ferramento molindini empto, utensilibus parandis et domo 45 molindini cooperienda, ijs. $v d . o b$. In xvj bobus enptis, iijjl. xviijs. vd. In xxxvj picheris et ollis emptis ad opus domini Episcopi, xijd. In ij cuvis lavator[iis] de novo parandis, xijd. In vj tonellis ligindis et reparandis ad ciceram inponendam, viijd. In precariis auptumnalibus pro M. M. ccc. allecis emptis, vs. jd. In xij sextariis cervisia emptis ad idem, vijs. In liberatione servientium de Waltham, per annum, lxs. xd. In liberatione 50 Adæ et Walteri warennariorum, $\mid x s$. $x d$. In liberatione Willelmi Widecoc, per annum, xlvs. vijd. $o b$., quolibet ${ }^{3}$ die, iij $o b$. In liberatione Giliberti Tegulatoris et sociorum suorum, per ix septimanas, vj dies, xxxiijs. vijd. ob. In expensis magistri Roberti Basset et magistri Alani, quando fuerunt infirmi, xvjs. vijd. In expensis magistri Alani, per se, et carrettariorum domini Episcopi a crastino Paschæ usque proximam diem Veneris ante 55 Pentecosten, xxijs. In expensis Roberti de Ellestede cum ix hominibus suis a crastino Sancti Lucæ usque Caput Jejunii,,$^{5}$ per xvij septinanas, xx8. vijd. In expensis magistri Roberti Basset multociens supervenientis pro negotiis villæ, xxvijs., per ix tallias. In cariandis ij tonellis vini, de dono Roberti de Turneham, de Portesmutha usque Farram, per aquam, xijd. In cariandis ij tonellis vini de Hamptona usque Walthan, ijs. iiijd. In 60 claudendis iiij ${ }^{\mathrm{xx}}$.xij perticis, v $l$. vijs. iijd., pro qualibet pertica, xiijd. In vanandlis xxxvj quirteriis frumenti ad semen, xviijd. In furniendo pane canum, xijd., per Episcopum. In $\mathrm{j}$ tunello empto ad ciceram inponendam, xijd. Summa xxxvl. vs. ijd.

Summa tam liberationis quam expensæ, cxxvjl. xs. ja." Et debent lxvs. iijd ob.

Sic in MS, 2 Sabbatorum in MS. '2qualibet in MS. "Singuli in MS. 'Junii in MS. 
Exitus Grangiø.-Iden reddunt compotum de ecvij quarteriis dimidio, j bussello de totu exitı frumenti, et de dimidıo quarterio iiij bussellis' de consuetudine churset. Et de v quarteriis allatis de Merewella. Et de iiij quarteriis allatis de Stokes. Summa tocius frumenti, ccxvij quarteria v busselli. In semine Dlj aerarum, exij quarteı ia dimidiun. In pane domini Episeoli, lxj quarteria vij busselli, ${ }^{1}$ per vij tallias. In pane Roberti de Ellestede et hominum suorum, xj quarteria vj busselli. In pane magistri Alani et lıninum suorum, iiij quarteria dimidiun. In expensis magistri Roberti Basset et magistri Alani, yuando fuerunt infirmi, ij quarteria, per ix dies. In supravenditis, $x x$ quarteria vimidium. Debet. $^{2} \quad$ Summa, cexij quarteria v busselli. ${ }^{3} \quad$ Et debet $\mathrm{v}$ quarteria. Et preciata sunt xjs, viijd. ${ }^{2}$

Idem reddunt compotum de Ixvj quarteriis de toto exitu curalli. In suibus et purcellis pascendis in hyeme, $v$ quarteria. I" pane ad precarias auptummiles, vj quarteri:ı. In eorrudio vij bovariorum, $\mathrm{ij}$ carretariorum, $\mathrm{j}$ gardenarii, $\mathrm{j}$ vaccarii, xxix quarteria. In pane ad pascendun canes, liberato Willelmo Widecor, $x \times v j$ quarteria, scilicet ad leporarios domini Episcopi, per annum, et canes domini Regis qui venerunt ad festum Saneti Jaeoli. Et quiet[i sunt]. Summa, $1 \times$ vj. $^{3}{ }^{3}$

Idem reddunt compotum de iij $^{x x}$.xix quarteriis de toto exitu ordei. Et de $x x v j$ quarteriis receptis de Hameledona. Summa, exxv quarteria. In semine cxlviij acrarum, xl quarteria dimidium. In corredio prædictorum famulorum, xlvj quarteria. In eorredio daiæ, vj quarteria dimidium, per annum. In precariis auptunıalibus, vj quarteria. In consuetudinibus bercarii, porearii, wiscatoris faldæ, iiij quarteria dimidium. In pastı canum domini Episcopi, Jiberata Willelmo Widecoc, xviij quarteria dimidium. In cibo j herciatoris in hyeme et Quadragesima, per xxiij septimanas, iij quarteria. Summa, cxxv. ${ }^{3}$ Et quicti sunt.

Idem reddunt compotum de xliiij quarteriis avenæ remanentibus anno prætr-rito, de quibus v quarteria dimidium fuerunt vendita. Et de cccelx.xvij quarteriis iij bussellis dimidio de toto exitu avenæ hoc anno. Et de lxviij quarteriis allatis de Menes, per ij tallias. Et de xlvij quarteriis allatis de Hameledona. Et de xlvij quarteriis allatis de Forrefelda, per $\mathrm{j}$ talliam. Et de $\mathrm{x}$ quarteriis allatis de Biterne. Et de $v$ quarteriis allatis de Farham. Suınma De iijj ${ }^{x x}$.xiij quarteria ij busselli dimidius. In semine ecciiij $\mathbf{x x}$ acrarum et $x$ viij acrarum, cexlj quarteria dimidium. In præbenda domini Episcopi, missa apud Merewellam, $x v$ quarteria, per $j$ talliam. In præbenda domini Episcopi in vigilia Sancti Jacobi, $\mathbf{x}$ quarteria. In præbenda domini Episcopi ad Pascham, xxxiij quarteria. In præbenda domini Episcopi, missa apud Biterne die Vencris ante Trinitatem, x quarteria. In præbenda domiui Episcopi in manerio de Walthan, iiijxx quarteria $\mathrm{j}$ bussellus. In præbenda domini Episcopi, vigilia Natalis Sanetæ Mariæ, per iiij dies, xxj quarteria dinidium, $\mathrm{j}$ bussellus. In præbenda Roberti de Ellestede, ad vj equos, per xvij septimanas, xxij quarteria. In pastu xvij canum suorum, xij чuarteria, per prædictum terminum. In præbenda Willelmi de Sorewilla, j quarterium. In præbenda Henrici pistoris supervenientis multociens, ij quarteria iij buselli. In præbenda magistri Alani et carretari[orum] domini Episcopi, per vij septimanas post crastinum Paschx, $x x v$ quarteria dimidium, $\mathrm{j}$ bussellus dimidius. In præbenda magistri Alani et magistri Roberti Basset quando fuerunt infirmi, ij quarteria. In jastu canum Gervasii venatoris, per totum annum, iiij ${ }^{x}$.xiij quarteria. In pastu cæeterorum eanum qui sunt in custodia Willelmi Widecoe, Adæ Walteri, xxiiij quarteria. In præbenda magistri Roberti Basset multociens ibidem venientis, per annum, pro negotiis manerii, xij quarteria liıidium, v busselli. In præbenda boum, ab Epiplıania usque Iuventionem Sanctæ Crucis, per xvij septimanas, xlij quarteria. ${ }^{3}$ In præhenda iij avrorum, xvj quarteria. In præbenda Eustachii de Greinvilla et Johannis de Mara, j quarterium dimidium, quando venerunt de Euere. In supravenditis, xij quarteria. Et renanent in granario, xx quarteria. Sumuia, Delx.xviij quarteria, iij busselli dimidius.

Idem reddunt compotum de iij quarteriis le toto exitu vesciæ. In senin'; $i j$ acrarum, dimidium quarterium. In porcellis paseendis in hyeme, diunidium quarterium. In corredin prædictorum famulorum, iij quarteria. Nichil remantet. Et quieti sunt.

Idem reddunt compotum de xxviij quarteris dimidio mancorni de toto exitu moliulini. In pastu priedictorum canum, vij quarteria. In supravenditis, $\mathrm{xxj}$ quaıteria dimidium.

Idem reldunt compotum de $\mathrm{j}$ quarterio dimilio frunenti de toto exitu molindini. Et de vj quarteriis dimidio brasei. In supravenditis, totum.

60 Instaurum.-Idem reddunt compotum de iij avris, j pullo, remanentibus anıo preterito. In mortuo, $\mathrm{j}$ pullus. Et remanent iiij avri.

Idem reddunt compotum de $\mathrm{xl}$ equabus remanentibus anno præterito, $\mathrm{j}$ stalone. Summa xlj. In missis apud Bitterne xiij. In missis apud Fernham xvij equæ, ij pull[anæ] de anno præterito. Summa xxxviij. In mortuis iiij equæ, ij pull[anæ]. ${ }^{3}$ Et remanent $j$ stalo, ${ }^{6}$ ij pull[ani] ij annorum.

Idem reddunt compotum de iij pull[anis] qui provenerunt de equabus prædietis loce auno. In missis apud Feruham, totum.

1 vij busselli interlineated. The number should he 3.
Sic in MS.
$2 \mathrm{~A}$ later eury in MS.

3 Stalon' in .IS. 
Idem reddunt compoturn de $\mathrm{iij}$ asivis remanentibus anno præterito. Et de $\mathrm{j}$ pullo qui provenit de prædictis asinis. Onuses remanent.

Idem reddunt compotum de ciij bubus ${ }^{1}$ remanontibus anno præterito. Et de vij de ociosis junctis annalibus. Et de xvj postea emptis. Et de iij de testamentis Edwardi Frogge et Matillis de Wisterhulla et Ricardi Duc. Summa cxxix boves. In vivis venditis vj. In mortuis xxiiij. In occisis ad lardarium v. Summa xxxv. Et remanent iiij ${ }^{x x}$.xiij; ; de quibus Radulfus habet in custodia sua viij.

Idem reddunt compotum de xliij vaccis remanentibus anno præterito. Et de ix geniculis junctis de ociosis annalibus. Summa lij. In mortuis v. Et remanent xlvij vaccæ.

Idem reddunt compotum de xvij annalibus provectioris ætatis remanentibus anno præterito. Summa xvij. In adjunctis bobus, vij. In adjunctis vaccis, ix geniculæ. Summa xvj. Et remanet $\mathrm{j}$ taurus.

Idem reddunt compotum de $\mathrm{xxv}$ annalibus qui fuerunt vituli anno præterito. Summa xxv. In mortuis, $i j$. Et remanent xxiij; unde $x$ masculi, $j$ taurus, xij geniculæ.

Idem reddunt compotum de $\mathrm{xx}$ vitulis proventis de prædictis vaccis, quia cæeteræ fuerunt steriles. Summa xx. In decimis, ij. In mortuis, iij. Summa v. Et remanent $\mathrm{xv}$ vituli.

Idem reddunt compotum de ccclxv ovibus remanentibus anno præterito, et de $x$ ovibus de Nicolao qui fuit occisus. Et de iijj $^{x}{ }^{1 i j}$. gerciis junctis. Summa Dlviij. In missis apud Biterne ad festum Sancti Martini, lviiij. ${ }^{2}$ In venditis, xxxiij. In mortuis ante partum xiij. In mortuis post partum et ante tonsionem, vj. In mortuis post tonsionem, vj. Summa cviij. Et remanent ccccl. ${ }^{2}$

Idem reddunt compotum de cciiij ${ }^{x}$ iiij multonibus remanentibus anno præterito. Et de $\mathrm{j}$ de Nicolao. Et de lx.xiij masculis junctis. Summa ccclviij. In mortuis ante 25 tonsionem, xxx; post tonsionem, xij. Summa.xlij. Et remanent cccxvj.

Idem reddunt compotum de clx.xj hogg[ettis] remanentibus, anno præterito agnis. Et de $\mathbf{v}$ de Nicolao. Summa clx. $x \mathbf{v j .}$ In mortuis ante tonsionem, $\mathbf{x x}$. Et remanent clvj; de quibus iijxiij sunt gerciæ, junctæ ovibus; lx.xiij masculi, juncti multonibus. Et nichil remanet.

Idem reddunt compotum de ccclx.x agnis proventis de prædictis ovibus, quia aliæ fuerunt steriles, et iiij fecerunt aborsun. In consuetudine $\mathrm{j}$ bedelli, $\mathrm{j}$ bercarii, $\mathrm{ij}$. In consuetudine fabri, $\mathrm{ij}$. In decimis, $x \times x v j$. In vivis venditis, vj. In mortuis ante separationem, lxix. In mortuis post separationem et ante tonsionem, xij. In mortuis post tonsionem, x. Summa cxxxvij. Et remanent ccxxxiij.

Idem reddunt compotum de Dcciiijxxiiij velleribus grossæ lanæ. In decimis, lx.xviiij. In supravenditis, Dcc. $v j$, quæ fecerunt $v$ pondera et terciam partem ponderis; cum ccxliij velleribus agninis. Summa Dcciiij ${ }^{x}$ iiij.

Idem reddunt compotum de cclx caseis de reragio anni præteriti. Et de xl caseis factis in hyeme de vaccis. Et de xij factis antc ablactationem. Et de ccxiij qui 40 inceperunt fieri $x v j^{\text {to }}$ Kalendas Maii, et desierunt in festo Sancti Michaelis. Et fecerunt per xlvij dies binos caseos, scilicet ab octaro Kalendas Junii usque vjto Idus Julii. Summa Dxxvj casei. In decimis xxvj. In consuetudine fabri, ij. In consuetudine bercarii et falcatoris prati, ij. In precariis auptumnalibus, iij. In liberatis Reginaldo sauser[io] ad fladones domini Episcopi, xij, qui facti erant ante ablactationem. In liberatis 45 de coquina, iij. In liberatis Johanni de Dispensa, vij. In corredio senescalli, $j$. In expensis Eustachii de Greinvilla et Johannis de Mares, j caseus. In supravenditis xl, de parvis caseis factis in hyeme, de vaccis. In supravenditis, cclx, qui fecerunt viij capita, de reragio anni præteriti. Summa ccel[vij] casei. Et debent clxix caseos, qui fecerunt vj capita. Precium xls.

Idem reddunt compotum de lxix porcis remanentibus anno præterito. Et de lxvij porcis qui provenerunt de suibus hoc anno. Et de vij porcis remanentibus de Tantona. Summa cxliij porci. In decimis, vj. In consuetudine porcarii, $j$. In lardario de Waltham, xxx. In mortuis, iij. Summa xl. Et remanent ciij; de quibus vj sunt sues, ij verres, xliij porci superannati, lij hogetti.

Idem reddunt compotum de cxxxiiij sextariis dimidio, iij ambris salis, de reragio anni prateriti. Et de clij sextariis salis de Bursed[ona], de gabulo hujus anni. Summa cciiij ${ }^{\mathrm{x}} \mathrm{vj}$ sextaria dimidium, iiij ambræ. In expensis daeriæ hoc anno, vij sextaria iij ambra. In expensis domini Episcopi, in coquina, $\mathrm{j}$ sextarium, liberatum coco. In carne salienda, lard[ario], xxviij sextaria $j$ ambra. In venacione salienda, $x$ sextaria iij ambræ. 60 In missis apud Wlveseiam, liberatis Johanni decano, iiijxix sextaria salis, per $j$ talliam. Summa cxxxvj sextaria j ambra. Et remanent cl sextaria iij ambræ.

Idem reddunt compotum de $\mathrm{l} x$ baconibus, de reragio anni præteriti. Et de $\mathbf{x x x}$ porcis occisis ad lardarium de Waltham, de instauro. Et de liij poreis de Tantona. Et de xxx porcis de Menes. Et de xxiij porcis de Tuiforda. Et de xvj porcis de Cheritona. Et de 65 $\mathrm{Xx}$ porcis de Hameledona. Et de xxxvij porcis de Ferram. Et de xiij porcis de Stokes.

M. 1d. Summa cciiijxxij porci. In missis apud Ferham et Menes et in expensis apud Wiltham, lv bacones. In expensis domini Episcopi, apud Suttonam Dominica ija post 
Trinitatem, xvj bacoues. In expensis domini Episcopi apud Waltham, lij bacones, de veteri carne. In expensis domini Episcopi contra Pascham, xij bacones. In expensis domini Episcopi ad festum Sancti Barnabæ, missis Philippo de Croperie, xvj bacones. In expensis domini Episcopi, liberatis Nicholao de coquina ad Natalem Sanctæ Mariæ, ix bacones. In expensis magistri Roberti Basset et magistri Alani quando fuerunt infirmi, $\mathrm{j}$ baco $\mathrm{xx}$ eschinæ. In expensis magistri Alani per se, iij bacones $\mathrm{xl}$ eschinæ. In expensis Roberti de Elestede, Ix eschinæ. In liberatione Roberti de Hales, xx eschinæ, et sulcit[ium] de clx pricis. In expensis precariarum auptuınnalium, $\mathrm{j}$ baco et carnes $^{\mathrm{I}} \mathrm{v}$ boum. In expensis Eustachii et Johannis de Mara, dimidium baconem. In dono infirmis, vj bacones. Summa clx.xi bacones et dimidium. Et remanent cx bacones dimidius.

Idem reddunt compotum de $\mathbf{x}$ gallinis de reragio anni præteriti. Et de xij caponibus de præsenti. Et de $\mathrm{iijj}^{\mathrm{xx}} \times \mathrm{x}$ do churiset. Summa cxij. In missis apud Merewellam ad expensam clomini Episcopi in vigilia Epiphaniæ, iiijxx.xvj, per $\mathrm{j}$ talliam. In expensis senescalli, iij. In expensis magistri Alani, xij capones. In mortuo, j. Summa cxij. Et quieti sunt

Idem reddunt compotum de $1 \mathrm{x} \cdot \mathrm{xj}$ pellibus wulpium liberatis J[ohanni] fabro de Wintonia per dominum Episcopum.

Idem reddunt compotum de vj tomnellis siceræ de recepta. In potu Roberti de Ellestede, iij tonnelli. In liberatis Danieli Pincernæ, iij tonelli.

Idem liberaverunt apud Wlueseiam, ij libras simini de redditu.

Et debet ${ }^{1}$ cxixs. vjd. ob. Solvit et quietus est. ${ }^{2}$

\section{CLERA}

Johañes DE Woltona et Michaelis præpositus reddunt composum de xixl. xvijs. viijd. de reragio anni tercii. Et de xviijl. vjs. vijd. ob. de gabulo assiso per annum. Et de xj $l$. de incremento gabuli hoc anne. Et de $x s$. de molindino ejusdem villø. Et de xijd. de molindino de Sandelforde. Summa tocius gabuli xviijl. xviijs. vjd. $o b$.

In quietanciis præpositi de Burclera, præpositi de Alta Clera, per annum, viijs. viijd. In quietanciis ij haiwardorum de eisdem villis, vs. In quietanciis ij bercariorum de eisclem villis, iiijs. iiijd. In quietanciis $\mathrm{x}$ carrucuriorum de Burclera, per annum, ij per $\mathbf{j}$ terminum, xxixs. iiijd. In quietanciis iiij carrucariorım apud Altan Cleram, per annum, $\mathrm{ij}$ per $\mathrm{j}$ terminum, ixs. In quietancia $\mathrm{j}$ forestarii, iijs. In quietauciis $\mathrm{j}$ fabri, $\mathrm{j}$ porcarii, iiijs. In quietanciis ij operariorum, per annum, iijs. In quietancia Osmundi Sutere, xijd. In quietanciis xij operariorum de Hauecleia, per annum, xlviijs. Summa vl. xvijs. iijd.

In defectu gabuli cccxxxv acrarum retractarum in dominium, $x \times x v i j s$. In $x \times i i j$ acris de Alta Clera, tractis in dominium, $x d$. In defectu terræ Wulwini de Cruce tractæ in dominium, iijs. In defectu crofti Roberti Wixi tracti in dominium, xijd. In defectu $\mathrm{x}$ acrarum Rogeri Malpetit tractarum in dominium, $x \mathbf{x} d$. In defectu $\mathrm{x}$ acraruın Blakemanni Cheffinges tract:trum in dominium, ijs. vjd. In defectu iij acrarum Thumae Parmenter tractarum in dominium, xijd. In defectu terræ Symonis clerici tractæ in dominium, $x x d$. In defectu iij acrarum Eadwini de Pund tractarum in dominium, xijd. In defectu crofti Osmundi Dust tracti in dominium, xijd. In defectu gabuli de Sages tractæ in dominium, xjs. iijd. In defectu gabuli Rogeri Bule, ijs. ${ }^{3} \quad$ Summa Ixiijjs. ${ }^{3}$

Summa tam quietantiarum quam defectuum, ixl. xvjd. Summa remanens ixl. xvijs. ij $d$. ob.

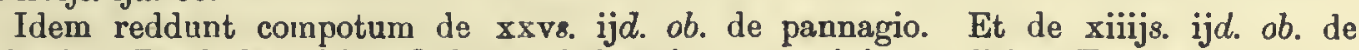

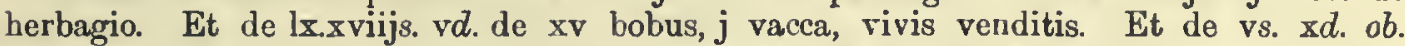
de iij coriis boum, $\mathbf{j}$ corio vituli anno præterito $\mathbf{j}$ anno præsenti mortuis venditis.

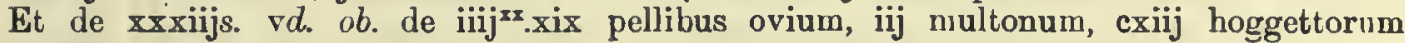
mortuorum venditis. Et de xiijs. xjd. ob. de ccexlv pellibus agnorum mortuorum venditis. Et de ijs. de consuetudine mellis. Et de iijs. $\mathbf{x} d$. ob. de nucibus venditis. Et de ijs. de potfalda. Et de xxxijd. de via injusta. Et de ijs. de butiro vendito. Et de viijl. xijd. de $x$ xiij capitibus casei venditis. ${ }^{3}$ Et de xiijl. xijs. viijd. de $x$ ponderibus grossæ lanæ venditis. Et de $x x v j s$. viijd. de $\mathrm{j}$ pondere lanæ agninæ vendito. Et de iijs. vjd. de Mecc ovis venditis. Et de vjs. de lana quæ cecidit de ovibus. Et de xijd. de norbois. Summa xxxijl. xvs. vjd.

Idem reddunt compotum de ixl. xvijs. vjcl. de $l x$.xiij quarteriis frumenti venditis. Et de xxxvs. vd. ob. de xvj quarteriis dimidio curalli venditis. Et de lxixs. de xlix quarteriis mancolni venditis. Et de $\mathrm{xx} d$. de $\mathrm{v}$ estricis fabarum venditis.

Summa $x \vee l$. iijs. vijd. ob.

60 De Purchasiis. - Idem reddunt compotum de vijd. de Alwino de Faccumba pro pastura. Et de xijd. de Waltero de Faccumba pro simili. Et de vjd. de Acere pro simili. Et de xijd. de Willelmo Citte pro simili. Et de vjd. de Rogero Dipres pro simili. Et de vjd. de Willelmo pro simili. Et de vjd. de Radulfo de Mora pro simili. Et de vjd. de Reginaldo Parvo pro simili. Et de xijd. de Willelmo pro terra Eilsi. Et de

\footnotetext{
1 Sic in MS. 2 The last four words are a later entry in another hand. 3 On an erasure in MS.
} 
xijd. de Randulfo de Stokes pro pullo capto in pastura. Et de vjd. de Willelmo Hadewolf pro pastura. Et de vjd. de Reginaldo Parvo pro bobus receptis sine licentia. Et de vjd. de eodem quia non venit ad opera domini. Et de vj.., de Symone Brun pro simili. Et de vjd. de Alwino quia celavit capellam. Et de xijd. ${ }^{1}$ de Ricardo Billinge q[uia] de Acere pro plegagio. Et de xviijd. de Alfredo Palmerio pro femina sua maritanda in terra domini Episcopi. Et de xviijd. de Ald' vidua pro terra sua hakencla. Et de vjd. de eadem pro pastura. Lt de ijs. de Alwino pro terra dimittenda. Et de xviijd. de Matildi vidua pro pastura. Et de vjd. de Alwino Symonis pro simili. Et de vjd. de Willelmo Buric pro mala aratura. Et de vjd. de Edwino Novo pro pastura. Et de ijs. de Waltero Cole pro forisfacto bosci. Et de vjd. de Johanne Juvene pro simili. 10 Et de vjd. Ricardo Cittel pro pastura Et de xijd. de Godwino Notte pro simili. Et de vj[d.] de Waltero Cupere pro mala aratura. Et de vs. de Waltero Forestario ut daret filiam suam extra terram domini Episcopi. Et de vjd. de Ricardo de Gerstona pr" melle. Et de vjd. de Matildi pro falso clamore. Et de ijs. de Ricardo de la Gerstone pro forisfacto bosci. Et de xijd. de Godwino Notte pro pastura. Et de xiijs. iiijd. de Acerre pro 15 teria habenda. Et de zxs. de Willelmo Gerardi pro terra sua habenda. Et de xijd. de Alwino de Forde pro mala custodia blarli. Et de vjd. de vidua Hadewolf pro simili. Et de vjd. de vidua Rogeri Blundi pro pastura Et de iijs. de Nicolao Ruffo pro blado stulte capto. Et de xijd. de Acerre de Helle pro simili. Et de xijd. de Godwino Ruffo et Culping pro pastura. Et de xijd. de Osmundo constabulario pro opere. Et de xijd. 20 de Hedewolf et de Hernebi quia non duxerunt carnem domini Episcopi. Et de xijd. de Walkelino pro opere auptumnali. Et de xijd. de Hugone Bubbe pro terra dimittenda et pro falso clamore. Summa lx.xvjs. $x d$.

Idem reddunt compotum de vijl. de tallagio de Burclera. Et de iiijl. xs. de Alta Clera pro simili.

\section{Summa tocius receptæ, $\mathbf{l}$.xiijl. [iijs. ijd. $]^{2}$}

Liberatio.--In liberatis J[ohanni] decano vijl. xviije. jd., de gabulo, per iiij tallias. Eidem, de tallagio, xjl. xs., per ij tallias. Eidem, de blado vendito, viijl. vijs. iijd., per j talliam. Eidem, de lana vendita, xiijl. iijs. vjd., per j talliam. Eidem, de exit[ibus], vijl. ixs. Summa liberationis, zlix $l$. vijs. $x d$.

Expensa.-In ferramentis vij carrucarum per annum, j carrucæ per dimidium annum, xxjs. ixd. Et in $\mathrm{j}$ carreta ferro ligata et $\mathrm{ij}$ carretis emendis, bacis, parunis, nncto, et aliis paramentis caretarum, xijs. viijd. In custo daeriæ, vjs. vjd. In iij serruris emptis, xjd. ob. In $\mathrm{j}$ sellop, $\mathrm{j} d$. In $\mathrm{j}$ nova grangia paranda et cooperienda et $\mathrm{j}$ bercaria paranda, apud Burclerim, $x l s$. $i j d$. In $\mathrm{j}$ domo facta de novo apud Altam Cleram, in 35 daeria, bercaria, cooperiendis et emendis, et $\mathrm{j}$ fenestra ad Capellam, iiijs. ijd. $u b$. In furagio empto, xvs. In fossato faciendo circa curiam de Burciera, vs. vjd. In pane, cervisia, companagio, ad precarias liyemales, et pane et companagio ad $\mathbf{v}$ precarias auptumnales, xxxvjs. viijd. ob. In sarpeleriis ad lanam ponderandam, vjs. xjd. In saccis, iijs. In mercede illius qui debuit facere puteum. ${ }^{3}$ In triturandis iijj ${ }^{x i i i j}$ quarteriis frumenti, 40 xvj quarteriis dimidio curailli, ex quarteriis mancorni, ccj quarteriis avenæ, xxvj quırteriis ordei, xlvs. iij $a b$. In metendis iij ${ }^{\mathbf{x}}$.xvij acris dimidia, xvjs. iijd. In consuetudine falcatorum de Burclera et de Rycroft, viijd. In corredio illorum qui braciaverunt cervisiam in auptumno, ixd. In mercede custodis bladi, xxxijd. In stipendiis ij caretariorum, j servientis, per annum, ixs. In stipendio custodis agnorum, xiijid. In stipendio daiæ, 45 ijs. iijd. In ij faldis wiscandis, $\mathrm{x} d$. In liberatione Johannis servientis, lxs. $\mathrm{x} d$. Summa xiiijl. xiijs. jd.

In corredio Stepnani, Petri, qui venerunt apud Cleram in crastino Sancti Jacobi et perendinaterunt usque diem Jovis post festum Sancti Egidii, scilicet per xl dies, xs. In corredio Gregorii Capellani et Willelmi Bastardi et caretariorum quando duxerunt 50 harnasium domini Episcopi apud Glocestriam, xxxijd. In corredio magistri Thomæ de Heli qui remansit per $\nabla$ dies, iijs. iijd. In corredio Willelmi Bastardi, Petri Juvenis, qui remanserunt retro dominum Episcopum et duxerunt harnasium domini Episcopi apud Wittenciam, ijs. In corredio viridarii, vjs. iijd. In corredio Gibbc, hominis marescalli, qui remansit infirmus per xxxj dies, ijs. vjd., præcepto Episcopi et senescalli. In corredio 55 Pelehaste qui remansit infirmus per $x \times x v$ dies, $x \times x d$. In corredio liibhe qui fugavit corvos de parco, pracepto domini Episcopi, a die Lunæ post festum Sincti Gregorii usque festum Sanctorum Nerei et Achillei, scilicet per xlv dies, viijs. iijob. In mercede vij hominum qui fugaverunt corvos de parco, iijs. viijd. In corredio senescalli per iiij vices, vjs. iijd. ob. per iiij tallias. In corredio G. de Acland, ixd. Summit xlviijs.

Summa tocius expensæ, xvijl. xiij $[d .]^{4} \quad$ Summa tocius liberationis et expensæ, lxvjl. viijs. xjd. Et $\operatorname{debet}^{5}$ vjl. xiiijs. iijd.

Exitus Grangiæ.-Idem reddunt compotum de cxvj quarteriis iij bussellis de toto exitu frumenti de Burclera, et de $x j$ quarter'is de toto exitu de Alta Clera, et de v quarteriis allatis de

\footnotetext{
I $E t$ de riid. repeated in MS.

2 'The margin of the membrane is mutilated.

Space appears to have been left in the MS. For the cost of this entry.

- The margin of the meubrape is mutilated.

${ }^{5}$ Sic in MS.
} 
Echeneswella, et de $v$ quarteriis allatis de Esmeresworda. Summa cxxxvij quarteria dimidiuı, $\mathrm{j}$ estrica. In semine cccxvj acrarum apud Burcleram, lvj acrarum apud Haltam Cleram, Ixiij quarteria. In venditis, lx.xiiij quarteria. In consuetudine falcatorum prati de Bureleri, iij estricœ. Summa, exxxvij quarteria iij estricæ.

Idem reddunt compotum de xix quarteriis dimidio de toto exitu curalli de Burelera, et de ij quarteriis dimidin de Alta Cleri. Summa, xxij quarteria. In semine mixto cun miucorno, v quarteria. In corredio servientium, dimidium quarterium. In supravenditis, xvj quarteria dimidium. Summa, xxij quarteria.

Idem reddunt compotum de liij:quarteriis j bussello de toto exitu ínancorni de Burclera, et de Ixij quarteriis dimidio de Alta Clera, et de v allatis „de Echeneswella, et de $\mathbf{j}$ quarterio dimidio allatis de Esmeresworda. Summa, exxiij quarteria $\mathrm{j}$ bussellus. In semine cxvij acrarum apud Burcleram, cj acrarum apud Altam Cleram, xlv quarteria dimidium. In corredio ij carrucariorum, $\mathrm{j}$ servientis, per annum, $\mathrm{xv}$ quarteria dimidium, $\mathrm{j}$ bussellus. In corredio hıminum qui fugaverunt corvos de parco, jussu domini Episcopi, $v$ quarterii $j$ bussellus. In debilibus porcis, porcellis pascendis, v quarteria. In braseo, ij quarteria dimidium. In supravenditis, xlix quarteria $\mathrm{j}$ bussellus. Summa, cxxiij quarteria dimidium, ${ }^{1} \mathrm{j}$ bussellus.

Iden reddunt compotum de $\mathrm{xxj}$ quarteriis, dimidio, $\mathrm{j}$ bussello, de toto exitu ordei, et de j quarterio dimidio allatis de Esmeresworda. Summa xx[iij] [quar]teria, ${ }^{2} \mathrm{j}$ bussellus. In semine xxxvij acrarum, xij quarteria dimidium. In corredio daiæ, iiij quarteria. In corredio custodis agnorum, dimidiun quarterium $\mathrm{j}$ bussellus. In braseo, vj quarteria dimidium. Summa, [xxiij] quarteria $j$ bussellus.

Idem reddunt compotum de cxxix quarteriis dimidio de toto exitu avenæ de Burclera, et de xlv quarteriis j estriea j hopa de Alta Clera, et de xviij de Echeneswella, et de xviij ventis de Esmeresworda et cle xxxviij ventis de Widehaia. Summa, cexlviij quarteria, dimidium, $\mathrm{j}$ estlica, $\mathrm{j}$ hopa. In semine clx.xxj acrarum apud Burtleram, Ixj acrarum apud Altam Cleram, Ix.xiiij quarteria. In præbenda donini Episcopi, iijj ${ }^{\mathbf{x}}$.xiij ${ }^{2}$ quarteria dimidium $\mathrm{j}$ estrica per iij taillias. In præbenda senescalli per annum, ij qunrteria dimidium In præbenda Eustachii de Grenvilla, Galfridi de Cauz, j quarterium, dimidium, j estrica. In præbenda Gregorii Capellani, W[illelmi] Bastardi, earrettariorum domini Episcopi, quando duxerunt harnesium apud Gilocestriam, ij quarteria. In præbenda T[homæ] de Heli, per $\mathrm{v}$ dies, j quarterium dimidium, iij l.opæ. In præbenda W[illelmi] Bastardi, P[etri] Juvenis, multor'un aliorum remanencium retro Episcopum, et duxerunt ${ }^{3}$ harnesium apud Witteneiam, ij quarteria. In præbenda $\mathrm{j}$ equi domini Episcopi, a die Lunæ ante festum Sancti Gre rorii, usque Pentecosten, ij quarteria. In præalenda viridari[orum], ij quarteria, limidium. In præbenda P[etri] Russ[inol], dimidium quarterium. In missis ipud Merlelvergum, ad opus domini Épisc pi, xvij quarteria, per j talliam. In missis apud Wulveseiam et liheratis Johanni decano, xviij quarteria, per $\mathrm{j}$ talliam. In præbenda iij caretariorum, per annum, $\mathrm{xxxj}$ quarteria. Summa, cexlviij quarteria, dimidium, j estricum, j hopa.

Idem reddunt compotum de $\mathrm{j}$ quarterio, dimidio, $\mathrm{j}$ estrica, de totı exitu fabarum. In venditis, v estricre. In semine, dimidium quarterium. Nichil remanet.

Instauram.-Idem reddunt compotum de xiiij avris remanentibus anno præterito. Et de $\mathrm{j}$ adjuncto de instauro. Summa xv. In missis apur Cnoel, j. Et remanent xiiij.

Iden reddunt compotum de ix equabus receptis de Wallia. In missis apud Fernham, omues, per $\mathrm{j}$ talliam.

Idem reddunt compotum de $\mathrm{j}$ pullo $\mathrm{ij}$ annorum. Et de $\mathrm{v}$ proventis hoe anno. In adjunctis avris, $\mathrm{j}$. Et remanent $\mathrm{v}$.

Idem reddunt compotum de iijix. $x$ bobus remanentibus anno præterito. Et de vij adjunctis de instauro. Et de ij de testamentis Hadewolf et Edwi. Et de ij anno præterito tauris. Et de xij de Widehaia. Summa cxiij. In mortuis, iij. De coriis superius respondent. In vivis venditis, xv. De preeio superius respondent. In miksis apud Echeneswellam, $x j .{ }^{4}$ In missis apud Widehaiam, ij. Summa xxxj. Et remsnent iiijxxij.

Idem reddunt compotum de xxviij vaccis, ij tauris, remanentibus anno præterito. Et de $\mathrm{v}$ adjunctis de instauro. Summa xxxv. In adjunctis bobus, ij tauri. In vendita j vacca. Et remanent xxxij.

Idem reddunt compotum de vij hovettis, $v$ geniculis remanentihus anno præterito. In adjunctis bobus, vij bovetti. In adjunctis vaccis, $v$ geniculæ. Et nihil remanet.

Idem reddunt compotum de $\mathrm{j}$ bovetto, anno præterito annali. Et ibi remanet.

Idem reddunt compotum de vij annalibus, anno præeterito vitulis. In mortuo, $\mathbf{j}$. De corio superius respondent. Et remanent $v j$, unde $j$ masculus, $v$ femellæ.

Idem reddunt eompotum de $\mathrm{xv}$ vitulis proventis hoc anno, quia xiij vaccæ erant steriles. In decima j. In mortuo j. De corio superius respondent. Et remanent xiij.

Idem reddunt compotum de Dliiij rvibus remanentibus anno præterito. Et de ccl adjunctis de instauro. Et de $\mathrm{j}$ inventa. Summa Decev. In missis apud Biterne, l, per $\mathrm{j}$ talliam, in termino Sancti Martini. In missis apud Harewellam, in termino Saneti Martini, xiiij, per $\mathrm{j}$ talliam. In mortuis ante partum ix. De pellibus superius respondent.
Dimidium is interlineater.
2 'The inembrane is injured by damp in several places
3 Sic in MS.
- The last entry is interlinealed. 
In mortuis post partum et ante tonsionem, iijjx. $x^{1}$ De pellibus superins "respondent. De agnis habent respondere. Post tonsionem xj. De pellibus, well[eribus] superius respondent. Summa clx.xiiij. Et remanent Dexxxj.

Idem reddunt compotum de xviij multonibus proventis de Lindeseia. Et de xliiij adjunctis de instauro. Summa Ixij. In mortuis post tonsionem, iij. De pellibus, vell[eribus] superius respondent. In missis apud Echeneswellam, ij. In missis apud Esmereswordam, j. Et remanent lvj.

Idem reddunt compotum de Dex hoggettis, anno præterito agnis. In adjunctis ovibus, cci. In adjunctis multonibus, xliijj. In missis apud Widehaiam, post tonsionem, c. In missis apud Esmereswordam, prist tonsionem, c. In mortuis ante tonsionem 10 iiijxx.xvj. De pellibus superius respondent. Post tonsionem, xx. De pellibus, vell[eribus] superius respondent. Et nichil remanet.

Idem reddunt compotum de cccclx.xj agnis proventis hoc annoquia xj erant steriles. Et de ix proventis de hoggettis. Et de ciiij ${ }^{x j}$ proventis de Esmeresworda. Et de clvij proventis de Echeneswella. Summa, Dccexviij. In decimis xlvij. In consuetudine 15 bercarii, j. In mortuis, cccxxxj ante tonsionem. Post tonsionem, xvj. De pellibus superius respondent. In venditis, v. De precio superius respondent. Summa ccce. Et remanent ccccxviij.

Idem reddunt compotum de Dcccxxij velleribus grossis. In decimis, xcij. In consuetudine $\mathrm{ij}$ bercariorum, ij. In liberatis Petro Neuelo, ccxxviij, quæ fecerunt 20 ij pondera vj claves. In venditis Dc, quæ fecerunt iij pondera. De precio superius respondent. Nichil remanet.

Idem reddunt compotum de ccexxxij velleribus grossis, receptis de Widehaia, qum fecerunt iij pondera. De precio superius respondent. Et de ccxix receptis de Echeneswella quæ fecerunt $j$ pondus dimidium. De precio superius respondent. Et de ccx 25 receptis de Esmeresworda, quæ fecerunt $\mathrm{j}$ pondus dimidium. Summa Mccclxi, ${ }^{2}$ quæ fecerunt $x$ pondera. De precio superius respondent.

Idem reddunt compotum de cccexxxiiij velleribus agninis, quæ fecerunt $j$ pondus. In venditis totum. De precio superius respondent.

Idem reddunt compotum de ciiij ${ }^{\mathbf{x}} \mathbf{v}$ caseis qui inceperunt fieri die Dominica post. 30 Annunciationem Sanctæ Mariæ, et desierunt die Sancti Michaelis, utraque ${ }^{2}$ die computata Et de iijj ${ }^{x x}$.xix caseis de vaccis qui inceperunt fieri die Sancti Mathæi Apostoli, secundo die, usque ad extremum diem Maii. Post illum diem usque diem Sanctæ Mariæ Magdalenæ quaqua ${ }^{2}$ die deinceps miscebatur lac cum lacte ovium. Summa cciiijxiiij casei. In decimis xxviij. In consuetudine daiæ $\mathrm{j}$. In missis apud Witteneiam ix, per j tailliam. 35 In corredio senescalli, $\mathrm{j}$. In corredio Gregorii capellani et aliorum, $\mathrm{j}$. In corredio domini Regis, iij. In liberatis Roberto de Hales $v$, per talliam. In precariis auptumnalibus, $x$. In consuetudine falcatorum prati apud Burcleram, $j$. In corredio Petri Juvenis, $j$. Summa $1 x$. Et remanent cexxiiij, qui faciunt xj capita; et clxiij recepti de Esmeresworda, qui fecerunt vj capita. Et clx recepti de Echeneswella, qui fecerunt vj capita. 40 Summa tocius ${ }^{2}$ Dxlvij, qui fecerunt xxiij capita. De precio superius respondent.

Idem reddunt compotum de iijj $^{\mathrm{xx}}$ porcis remanentibus anno præterito. Et de $\mathrm{xxxix}$ proventis hoc anno. Summa cxix. In decimis iij. In consuetudine porcarii, $\mathrm{j}$. In missis apud Duntonam, ix per $\mathrm{j}$ talliam. In missis apud Wlveseiam et lilieratis Johanni decano iij, per $j$ talliam. In mortuis, viij. In expensis viridari[orum], $j$. Summa xxv. 45 Et remanent iijox.xiiij; ${ }^{2}$ unde $i j$ sunt verres, xij sues, $x x$ vij masculi super annum, $x x j$ femellæ super annum xj hogetti masculi, xv femellæ, iij porcelli.

Idem reddunt compotum de cvj gallinis receptis de cheriset. Et de xxxiiij de reragio. Summa, cxl gallinæ. In missis apud Wlveseiam, et liberatis Johanni decano, cxxiiij. In mortuis, iij. In expensis viridariorum xij. Et nichil remanet.

Idem reddunt compotum de $M M$. ovis de redditu. In missis apu'! Wlieseian et liberatis Johanni decano, cc, per $\mathrm{j}$ talliam. In expensis viridari[orum], ccc dimidium. In expensis senescalli, dimidium centenum. In supravenditis Mecc. De precio superius respondent.

Idem reddunt compotum de carne bovis, et de iij partibus bovis, remanentibus anno 55 præterito. In expensis viridari[orum], $\mathrm{j}$ quarterium. In precariis auptumnalibus, vj.

Ilem reddunt compotum de sliij baconibus receptis de Mlerlebergo. In expensis domini Episcopi et hominum suorum, xvj. Et remanent xxvij, unde habent respondere.

\section{WIDEHAE.}

JohanNes DE WeLTONa et Nicolaus præpositus reddunt compotum de iiijl. iijs. $\mathrm{x} d$. de toto gabulo assiso per annum. Summa iijl. iiijs. $x d$. In quietancia j præpositi, per annum, ijs. iiijd. In quietanciis $\mathrm{j}$ haiwardi, $\mathrm{j}$ bercarii, iijjs. In quietanciis iiij carrucariorum, per annum, viijs. In quietancia $\mathrm{j}$ forestarii, $v s$. In quietanciis $\mathrm{ij}$ carrucariorum, per $\mathrm{j}$ terminum, xijd. In quietancia $\mathrm{j}$ operarii, per $\mathrm{j}$ terminum, vjd. Summa xxs. $\mathrm{xd}$. i5 Summa remanens lxiiijs. 
Idem reddunt compotum de iiijs. jd. de pannagio. Et de xiijjs. vjd. de herbagio. Et de $x i j d$. de potfalda. Et de $\mathrm{xxd}$. de corio $\mathrm{j}$ bovis mortui vendito. Et de $\mathrm{xj}$. viij . Ie xiiij cuillardis vivis venditis, post tonsionem. Et de iijs. vijd. de $x x v$ pellibus multunum venditis. Summa $\mathrm{x} \times \mathrm{x} \times \mathrm{vj}$. $\mathrm{vjd}$.

Idem reddunt compotum de vjl. iiijs. vd. ob. de xliiij quarteriis rlimidio frumenti venditi.. Lt de ixs. vjd. de iiij quarteriis dimidio curalli venditis. Et de lx.xv.. vjd. de lvj quarteriis' dimidio ntancorni venditis. Et de viijs. vjd. ob. de vj quarteriis dimidio ordei venditio. Sumua $\mathrm{x} l$. $\mathrm{x}$ viijs.

Summa utriusque xijl. xiiijs. vjd.

10 De Purchasiis. - Idem reddunt con potum de vjd. d Reginaldo de Cumba pro pannagio concelato. Et de vjd. de Godwino Blakemanno pro forisfacto bosci. Et de vjd. de Alvredo de More pro simili. Et de xijd. de Willelmo haiwardo pro simili. Et de xijd. le Hugone Albo pro simili. Et de xijd. de Gocelino pro simili. Wit de ijs. de ij lıominibus de WestWidehae quia seminaverunt super defensam. Et de xviijd. de Petro de Dene et de Gervasio et de Turrien et de xvijjl. de Nicolao de Estwicho pro forisfacto pasturæ. Et de vjd. de Rogero Blakemanno, quia non venit ad summonitionem. Et de vjd. de Osmundo de Stanhulle pro forisfacto pusturæ. Et de vjd. de Seiva vidua quia non venit ad opera domini. Kit de ijs. de Gilberto pro terra et uxore habenda." Et de vjd. de Osmundo pro falso clanore. Summa xiijs. vjd.

Idem reddunt compotum de iiijl. xs. de tallagio. Summa tocius receptæ, xxjl. ijs.

Liboratio.--In liberatis Johanni decano, de gabulo, lxiiijs., per iiij taillias. Eidem, de tallagio, iiijl. xs., per $\mathrm{j}$ talliam. Eidem, viijl. $x s$. , per $\mathrm{ij}$ tallias; scilicet de blado vendlito. Eidem, xjs. viiijd., de hurtardis venditis. Summa xvjl. xvs. viijd.

Expensa.-In ferramentis ij carrucarum, per annum, $\mathrm{j}$ carrucæ, per $\mathrm{j}$ terminum, iiijs. $x d$. In terrura avrorum, $i j d$. In triturandis lij quarteriis frumenti, vj quarteriis dimidio curalli, lx.xj quarteriis dimidio mancorni, iiij ${ }^{x x} i x$ quarteriis nvenæ, viij quarteriis ordei, xxiiijs. vjd. In mercede illorum qui cooperierunt grangiam, bercariam, xvjd. In aratura $\mathrm{v}$ acrarum, $\mathbf{x} d$. In falda wiscanda, $\mathbf{v} d$. In stipendiis servientium, vjd. In consuetudine falcatorum prati, ijs. In pane, cervisia, companagio ad precarias hyemales; pane, companagio ad precarias auptumnales, ixs. vd. In tribus bobus emptis, $x x j$ s. jd. In cariando $\mathrm{j}$ dolium vini a Bitterne usque Cleram, iijs. vjd. Summa lxixs. vijd.

Summa tocius liberationis et expensæ, $\mathrm{xxl}$. vs. iijd. Item vs. viijd. computati sunt cis quos ${ }^{1}$ solverunt in anno præterito. Et debet $^{2}$ xjs. $\mathrm{j} d$. Postea solvit et quietuvit decanum per $\mathrm{j}$ tailliam. ${ }^{3}$

35 Exitus Grangiø.-Idem reddunt compotum de lij quarteriis de toto exitu frumenti. Et de ij quarteriis de cheriset. Et de vij quarteriis dimirlio proventis de Esmereswordi. Summa lxj quarteriı dimidium. In semine iiijxiiij acrarum, xvij quarteria. In venditis, xliiij quarteria dimidium. Summa Ixj quarteria dimidium.

Idem reddunt compotum de iij quarteriis dimidio ${ }^{2}$ de toto exitu mancorni. In scmine lvj acrarum, xvj quarteria dimidium. In liberatione cujusdam 'anuli, per xv septimanas, j quarterium dimidium. In venditis, liij quarteria dimidium."

Idem reddunt compotum de viij quarteriis de toto exitu ordei. In semine iiij acrarum, $\mathrm{j}$ quarteriunı dimidium. In venditis, vj quarteria dimidium. Summa, viij quarteria.

Idem reddunt compotum de iijjxix quarteriis de toto exitu avenæ. In semine $1 x . x j$ acrarum, xlj quarteria. In missis apud Cleram, xxxviij quarteria. In missis apud Merlebergum, in vigilia Pentecostes, ad præbendam domini Episcopi, $\mathbf{x}$ quarteria. Summa, iijzxix quarteria.

50

Instaurum.-Idem reddunt compotum de xxxj bobus ${ }^{4}$ remanentibus anno præterito. Et de ij proventis de Clera. Et de iij emptis. Summa xxxvj. In missis ad Cleram, xij. In mortuo $\mathrm{j}$; de corio superius respondent. In misso apud Esmereswordam, j. Summa xiiij. Et remanent xxij.

Idem reddunt compotum de ccciij ${ }^{x x} i i j$ unultonibus remanentibus anno præterito. Et de ij proventis de Kismeresworda post tonsionem. Et de $\mathrm{C}$ proventis de Clera post tonsionem, anno præterito agnis. Summa ccciiij ${ }^{x \mathbf{v}} \mathrm{v}$. In missis apud E£meresworda, iij oves quae erant inter multones ante torsionem. In mortuis ante tonsionem, ix; de pellibus superius respondent. Post tonsionem, $x v j$; de pellibus, velleribus superius respondent. In venditis post tonsionem, xiiij; de precio et velleribus superius respondent. In consuetudine falcatorum prati de Burclera post tonsionem, $\mathrm{j}$; de vellere superius respondent. In consuetudine falcntorum prati de Sidemantona post tonsionem, $\mathrm{j}$; de vellere supra respondent. Summa xliij. Et remanent cccexlj.

1 Quam in MS. $\quad$ Sic. in MS. $\quad{ }^{3}$ Later entry in MS. Bov' in MS. 
Idem reddunt compotum de ccelx.xj velleribus grossis. In decimis, xxxvij. In consuetudine bercarii, j. In missis ad Cleram, cccxxxiij.

Idem r'eddunt compotum de lxxviij galliuis de cheriset. In missis apud Wlveseiam et liberatis Johanni decano, lx, per ij tallias. Et remanent $x$ viij gallinæ.

Idem reddunt compotum de ML ovis. In missis apud WIveseiam et liberatis Johanni decano, totum, per $\mathrm{j}$ tailliam.

\section{ESMERESWORDA}

J[oHANnEs] DE Woltona et G[alfridus] præpositus reddunt compotuin de xiiijs. iiijd. ob. de reragio anni tercii. In liberatis Johanni decano, totum.

Idem reddunt compotum de lx.xiijs. de toto gabulo assiso, per annum. In 10 quietanciis j præpositi iiijs., j heiwasdi, j bercarii iijs., j caırucarii, per annum, j carrucarii, per xix septimanas, ijs. ix $d$. , j operarii, per j terminum, vjd.

Summa quietanciarum xjs. xjd. ${ }^{1} \quad$ Summa rem:ınens lxjs. jd.

Idem reddunt cumpotum de ijs. de pannagio. Et de xxviijs. viijd. de lerbagio. Et de $x$ vijd. de potfalda. Et de vs. de $\mathrm{j}$ bove vivo vendito. Et de $\mathrm{j}$ ove viva vendita, $\mathrm{vd} .15$ Et de xiiijd. de iij agnis vivis venditis. Et de $x x x d$. ob. de $x x j$ pellibus ovium, iij pellibus hoggettorum mortuorum venditis. Et de xijd. de xxix pellibus agnorum mortuorum venditis. Summa xlijs. ijd. ob.

Idem reddunt compotum de xlvs. $v d$. de $x v j$ quarteriis frumenti venditis. Et de xxxvijs. vijd. de xxiiij quarteriis inancorni venditis. Et de iijs. $x d$. de ij quarteriis curailli 20 venditis.

\section{Summa iijl. vjs. xd. Summa utriusque vjl. ixs. ob.}

De Purchasiis.-Idem reddunt conıpotum de vjd. de Ace pro forisfacto pasturæ. Et de vs. viijd. de gabulo de Stokes. Et de vjd. de Edmundo Eiulfo ${ }^{1}$ pro simili. Et de vjd. de Waltero Swein pro simili. Et de xijd. de Willelmo bercario pro simili. Et de vjd. de 25 Roberto carucario pro simili. Et de vjd. de Alwino pro relaxacione legis. Et de ijs. de Willelmo Acere pro terra habenda. Et de ijs. de Johanne Blundo pro forisfacto pasturæ. Et de xvjd. de Johanne Cupere pro opere auptumnali. Et de vjd. de Clemente pro forisfacto pasturæ. Et de vjd. de Edmundo Eiulfo ${ }^{1}$ pro simili. Et de vjd. de Waltero Brun pro simili. Et de vjd. de Osmun lo Seiulf pro simili. Et de vjd. de Johanne 30 Blundo pro simili. Et de vjd. de Willelmo bercario pro simili. Et de xijd. de Symone pro femina maritanda. Summa xviijs. vjd.

Idem reddunt compotum de xls. de tallagio. Summa tocius receptæ, xiijl. ixs. ijd. $o b .^{1}$

Liberatio.-In liberatis Johanni decano, lxvjs. viijd., de gabulo, per iiij taillas. Eidem, xls., de 35 tallagio, per $\mathrm{j}$ talliam. Eidem, lxiijjs. jd., de blado vendito. Eidem, xxs., de herbagio, per j talliam. Eidem, viijs., per j talliain. Summa liberationis, ixl. xviijs. $x d$.

Expensa.-In ferramentis j carrucæ, per annuın, $\mathrm{j}$ carrucæ, per $\mathrm{j}$ teruninum, iiijs. viijd. In $\mathrm{j}$ caruca facienda, $\mathrm{j} d$. In mercede fabri, xvjd. In ferrura avri, $\mathrm{j} d$. In $\mathrm{j}$ hercaria de novo facta, vjs. ijd. In grangia, boveria cooperiendis, xiijd. In triturandis $\mathrm{xxxv}$ quarteriis 40 frumenti, ij quarteriis curalli, vs. iiijd. In triturandis xlix quarteriis mancorni, liij quarteriis avenæ, vj quarteriis dimidio ordei, viijs. iijd. In mercede custodis prati de Stokes et de Usseburne, viijd. In custamento daeriæ, ijs. vijd. In pisis emןtis ad semen, vjd. $o b$. In falda wiscanda, vd. In pane, companagio, ad precarias auptumnales, iijs. In mercede servientis, per anuum, iijs. In mereede $\mathrm{j}$ servientis, per iij terminos, $\mathrm{x} v \mathrm{iijd}$. 45 In blado sarclando, xviijd. Summa, xls. ijd. ob.

Summa tocius liberationis et expensæ, zjl. sixs. ob. Et debent ixs. vijd.

Exitus Grangiø.-Idem reddunt compotum de xxxv quarteriis, de toto exitu frumenti. Et de $\mathrm{j}$ quarterio provento de Stokes. Et de ij quarteriis, $\mathrm{j}$ bussello, et quarta parte $\mathrm{j}$ busselli, proventis de cheriset. Summa xxxviij quarteria, $\mathrm{j}$ bussellus et quarta pars ${ }^{1}$ busselli. 50 In semine $\mathbf{x x v}$ acrarum, $\mathbf{v}$ quarteria, $\mathrm{j}$ bussellus et quarta pars ${ }^{2} \mathrm{j}$ busselli. In semine xvj aerar um apud Stokes, iij quarteria dimidium. In missis apud Cleram, v quarteria. In missis apud Widehaiam, vij quarteria dimidium. In supravenditis, xvj quarteria. Sunma xxxvij quarteria dimidium ij busselli. ${ }^{1}$

Irlem reddunt compotum de ij quarteriis curalli. In venditis totum.

Idem reddunt compotum de xlj quarteriis, j bussello de toto exitu mancorni. Et de xiij quarteriis proventis de Stokes. Summa liij quarteria, dimidium, $\mathrm{j}$ bussellus. ${ }^{.}$In semine lxv aerarum, xvj quarteria dimidium. In semine viij aerarum apud 'Stokes, iij quarteria. In corredio $\mathrm{j}$ carrucarii, per annum, $v$ quarteria $\mathrm{j}$ bussellus. In braseo misso apud 
Cleram, j quarterium. ${ }^{1}$ In corredio j carrucarii, per iij terminos, iij quarteria dimidium. In venditis xxiiij quarteria. Nichil remanet.

Idem reddunt compotum de iij quarteriis de toto exitu ordei. Et de iij quarteriis dimidio, proventis de Stokes. Summa vj quarteria dimidium. In semine xiij acrarum, iiij quarteria dimidium. In braseo misso ad Cleram, j quarterium dimidium. Johanni, per dominum Episcopurn, dimidium quarterium. Summa vj quarteria.

Idem reddunt compotum de xliij quarteriis, de toto exitu avenæ. Et de $x$ quarteriis allatis de Stokes. Summa liij quarteria. In semine iiij ${ }^{x \mathbf{x}}$ acrarum dimilliæe, $x x x i i i j$ quirteria. In missis apud Cleram, xviij quarteria. In præbenda avrorum, j quarterium. Summa liij quarteria.

Instaurum.-Idem reddunt compotum de xij bobus remanentibus anno præterito. Et de $\mathrm{j}$ provento de Widehaia. Summa xiij. In vivo vendito, j. Et remanent xij.

Idem reddunt compotum de celx.xvij ovibus remauentibus anno præterito. Et de iij proventis de Widehaia. Summa cciijjx. In missis apud Harewellam xxxvj, in termino Sancti Martini. In vendita ante partum, j. De precio, superius respondent. In mortuis ante partum, ij. De pellibus, supra responilent. le agnis habent respondere. Summa lviij.' Et remanent cexxij.

Idem reddunt compotım de iij nultonibus remanentibus anno præterito. Lit de cj ${ }^{1}$ proventis de Clera. Summa cv. In missis apud Widehaiam, ij. In mortuis, iij. Et remanent $\mathbf{c}$.

Idem reddunt compotum de ccxxxvij agnis proventis boc anno, quia iij erant steriles. In decimis, xxiij. In consuetudine bercarii, $j$. In vivis venditis, iij. De precio superius respondent. In nortuis, xxix. De pellibus, superius respondent. In missis a pud Cleram, ciiij ${ }^{x x}$. Sunma cexxxvij. Nichil remanet.

Idem reddunt complotum de ccxxxiiij velleribus grossis. In decimis, xxiij. In consuetudine bercarii, $\mathrm{j}$. In missis apud Cleram, cex quae fecerunt $\mathrm{j}$ pondus dimidium, unde Clera respondet.

Idem reddunt compotum de ciiij ${ }^{x} \mathrm{jj}_{\mathrm{j}}$ caseis, qui inceperunt fieri, $\mathrm{j}^{\mathrm{a}}$ die Aprilis, et desierunt festo Sancti Michaelis, utraque ${ }^{2}$ die computata. In decimis, xviij. In consuetudine daiæ, $j$. In missis apud Cleram clxij, qui fecerunt vj pondera, unde Clera respondet. :umma ciiijxij casei.

Idem reddunt compotum de xxviij grallinis receptis de cheriset. Et de ix de reragio. In missis apud Wulveseiam, et liberitis Jolianni decano, xxxvij per $\mathrm{j}$ tallian. Nichil remanet.

Idem reddunt compotum de cce ovis. In nissis apud Wlveseiam, et liberatis Johanni decano ce, per ${ }^{2} \mathrm{j}$ talliam. Liberata in coquina domini Episcopi, c ova. Summa ccc. Nil remanet.

\section{ECHENESWELLA.}

J[ohannes] DE Woeltona et Adam præpositus reddunt compotum de viijs. vd. ob. de reragio anni tertii. In liberatis Jobanni decano totum per $\mathrm{j}$ talliam.

Idem reddunt compotum de viijl. viijs. vjd. de toto gabulo assiso per annum. Et de ixs. vjd. de ij molindinis ejusdem villæ. Summa tocius gabuli viijl. xviijs. cum ij molindinis.

In quietanciis præpositi, baiwardi, per annum, vs. viijd. In quietancia bercarii, ijs. In quietanciis iiij carrucariorum, per annum, ij carrucariorum, per iij terminos, xiijs. In quietanciis ij operariorum per dimidium annum, ijs. . Summa xxijs. viijd.

In defectu terræ Willelmi de Bosco tractæ in dominium, ijs. iijd.

Summa tam quietanciarum quam defectuum, xxv8.

Summa remanens, vijl. xiijs.

Idem reddunt coupotum de $\mathbf{x}$. vjd. ob. de pannagio. Ft de xxiijd. de herbagio. Et de xxviijd. de potfalda. Et de ijs. pro opere auptumnali. Et de xviijd. de corio $\mathrm{j}$ bovis mortui vendito. Et de xijd. de iij agnis vivis venditis. Et xixd. de is pellibus ovium mortuarum venditis. Et de ijs. de lix pellibus agnorum mortuorum venditis. Et de ijs. de nucibus venditis. Et de xviijd. de De ovis venditis. Summa xxvjs. iiijd. $o b$.

Idem reddunt compotum de iijl. xiijs. vjd. de $x \times x v$ quarteriis frumenti venditis. Et de vijs. vjd. de iij quarteriis curalli venditis. Et de $x \times d$. de $\mathrm{j}$ quarterio mancorni vendito. Summa vl. ijs. viijd.

De Purchasiis.-Idem reddunt compotum de xviijd. de Reginaldo molindinario quia frater ejus discessit de parco sine licentia. Et de vjd. de Roberto Mop pro forisfacto pasturæ. Et de vjd. de Galfrido Wibbe pro simili Et de vjd. de Edmundo de Chnihtebrigge pro simili. 
Et de vjd. de Ricardo Purcel pro simili. Et de vjd. de Reginaldo Turnur pro simili. Et de vjd. de Willelmo Blundo pro simili. Et de vjd. de Willelmo Bolt pro simili. Et de vjd. de Godefrido de Echeneswella ${ }^{1}$ pro simili. Lt de vjd. de Waltero de Cnihtebrigge pro simili. Et de vjd. de Ricardo molindinario pr" falso clamore. Et de xijd. de Waltero WIfo quia wulneravit canem Gregorii. Et de vjd. de Ricardo Purcel pro stultiloquio. Et de vjd. de Galfrido Wibbe quia discessit de precariis sine licentia. Et de vjd. de Roberto Mop pro relaxatione legis. Et de iijjs. de Waltero Wlvrici, Edgaro fabro, Edgaro Nigro, Reginaldo molindinario pro mala custodia bladi. Et de vjd. de Willelmo Ret, Ricardo de Holme, quia non venerunt ad opera domini. Et de xijd. de Rogero Turnur pro mala custodia bladi. Et de vjd. de Ace pro simili. Et de vjd. de Alfredo pro simili. Sum- 10 ma xvs. vjd. ${ }^{2}$

Idem reddunt compotum de vjl. de tallagio. Summa tocius, $x x l$. $x$ vijs. vjd. ob.

Liberatio.-In liberatis Johanni decano vjl. xvjs. de gabulo, per iij taillias. Eidem, vjl. de tallagio, per $\mathrm{j}$ tailliam. Eidem, lx.xixs. de blado vendito, per $\mathrm{j}$ talliam. Summa xvjl. xvs.

Expensa.-In ferramentis ij carrucarum per annum, j carrucae per iij terminos, vjs. jd. In iij carrucis faciendis et rotis carrucarum, vjd. In ostio parando ad grangiam, xvjd. In clatis ad faldam; viijd. In ferrura avrorum, iijd. In herciandis xxiij acris in Quadragesima, xvjd.; pro xij acris in hyeme, xiijd. ob. In falda wiscanda, vd. In mercede illius qui carinavit oves, $x v j d$. In custo daeriae, iijs. iiijd. In ducendo fimo super $\mathbf{v}$ acras, iiijs. ijd. 20 In triturandis xlvj quarteriis frumenti iiij quarteriis mancorni, viijs. ijd. In $\mathbf{x v i i j}^{3}$ quarteriis avenae emptis ad semen, xvjs. Summa lxiijis. vjd. ob.

Summa tocius liberationis et expensæ, xixl. xixs. vjd. ob. Et debet, ${ }^{3}$ xviijs. Solvit, quietus est. ${ }^{4}$

Exitus Grangiæ.-IIdem reddunt compotum de lviij quarteriis de toto exitu frumenti. Et de 25 iiij quarteriis de cheriset. Summa lxij quarteria. In semine cxij acrarum, $x$ xij quarteria. In missis apud Cleram, v quarteria. In venditis, $x \times x v$ quarteria. Summa lxij quarteria. Quietus.

Idem reddunt compotum de iij quarteriis dimidio de toto exitu curalli. In corredio $\mathrm{j}$ herciatoris, dimidium quarterium. In supravenditis, iiij quarteria. Summa iiij 30 quarteria dimidium.

Idem reddunt compotum de xij quarteriis $\mathrm{j}$ bussello de toto mancorno. In semine xxiiij acrarum, v quarteria. In missis apud Cleram, v quarteria In consuetudine bercarii, haiwardi, $\mathrm{j}$ quarterium, $\mathrm{j}$ bussellus. In supravendito, $\mathrm{j}$ quarterium. Summa xij quarteria $\mathrm{j}$ bussellus.

Idem reddunt compotum de xix quarteriis de toto exitu avenae. Et de xxiiij $^{3}$ quarteriis enptis. Summa xliij quarteria. In semine lx.xij acrarum, xxiiij quarteria. In missis apud Cleram, xviij quarteria. In præbenda avrorum, $\mathrm{j}$ quarterium. Summa xliij quarteria. Nichil remanet.

Instaurum.-Idem reddunt compotum de $x x$ bobus remanentibus anno præterito. Et de $x j 40$ proventis de Clera Summa xxxj boves. In mortuo, j. De corio superius respondent. Et remanent $x x x$.

Idem reddunt compotum de ccliij ovibus remanentilıs anno praterito. In mortuis ante partum, vij. De pellibus supra respondent. Post partum et ante tonsionem, ij. De pellibus, well[eribus], supra respondent. Summa ix. Et remaneut ccxliiij.

Inem reddunt compotum de $\mathrm{ij}$ multonibus proventis de Clera. Omnes remanent.

Idem reddunt compotum de ccxliiij agnis proventis hoc anno, quia ij erant steriles. In decimis, xxiiij. In consuetudine bercarii, $j$. In vivis venditis, iij. De precio superius respondent. In mortuis, lix. De pellibus supra respondent. In missis apud Cleram, clvij. Summa ccxliiij. Nichil remanet.

Idem reddunt compotum de ccxliiij velleribus grossis. In decimis, xxiij. In consuetudine bercarii, j. In missis apud Cleram, ccxix. Summa ccxliiij. Nichil remanet.

Idem reddunt compotum de clx.xviij caseis qui inceperunt fieri die Dominica post festum Sancti Ambrosii et desierunt die Sancti Michaelis, utraque ${ }^{3}$ die computata. In 55 decimis, xvij. In consuetudine daiæ, j. In missis apud Cleram, clx. Summa clx rviij casei.

Idem reddunt compotum de cxxij gallinis de cheriset. In missis apud Wlveseiam at liberatis Johanni decano, exvij, per $\mathrm{j}$ talliam. In mortuis, v. Summa cxxij. Nichil remanet.

Idem reddunt compotunn de Dcce ovis. In missis upud Wlveseiam et liberatis Johanni decano cc, per $\mathrm{j}$ talliam. In supravenditis, Dc. 'Summa Dcce. Nichil remanet.

$$
\text { I On an erasure in the Roll. } \quad{ }^{2} \text { Ixvs, altered to xvs. } \quad{ }^{3} \text { Sic in MS. } \quad{ }^{4} \text { A later entry. }
$$




\section{BRICTEWELLA.}

Ricardus serviens et Godefridus præpositus reddunt compotum de viijl. v8. ixd. iij $q d r$. de reragio anni tercii. Solverunt et quieti sunt.

Idem reddunt compotum de xiijl. xxd. de redditu assiso cum burgagio de Wallingefordia. In quietancia $\mathrm{j}$ præpositi, v8. In quietanciis $\mathrm{j}$ porcarii, $\mathrm{j}$ heiwardi, j bercarii, vjs. vjd. iij $q d r$.; singulis, ijs. ijd. j $q d r$. In quietanciis vij carrucariorum, xvs. iijd. iij $q d r$.; singulis, ijs. ijd. $\mathrm{j} q d r$. In quietanciis vij cotagiorum pro opere tocius anni, xvs. ijjd. iij $q d r$; singulis, ijs. ijd. $\mathrm{j} q d r$. In quietanciis $x x i j$ virg[atariorum] pro opere auptumnali qui inveniunt $x v$ homines uperantes singulis diebus, exceptis Sabbatis, a festo Sancti Petri et Pauli usque festum Sancti Michaelis, xxvs. ijd. ob. In quietancia Rogeri Kinedoit, vd. ob. pro opere auptumnali. Summa lxvijs. $x d$. j $q d r$.

In defectu terræ quam Andreas de Wallingefordia tenuit in minu domini Regis, iijd. iij $q d r$.

Summa utriusque, lxviijs. ijd. Summa remanens, ixl. xiijs. vjd.

Idem reddunt compotum de viijl. xjs. vd. ob. de exitu molındini molentis. Et de xvjd. de exitu molindini foleratici. Summa viijl. xijs. ixd. ob.

Idem reddunt compotum de iijs. ob. de pannagio. Et de ijs. vjd. de herbagio. Et de vijs. de stipula vendita. Et de xxxiijs. iiijd. de clvij velleribus grossis venditis quæ fecerunt vj petras, unde xiiij ficiunt pondus. Et de xiijs. iijd. de cxxiij velleribus agninis venditis quæ fecerunt vj petras, unde xiiij faciunt pondus. Et de xxiijs. iiijd. de cxxx caseis qui fecerunt iij pondera et terciam partem ponderis. Et de xiiijd. de butiro vendito. Et de iijjs. de $\mathrm{j}$ avro vendito. Et de $\mathrm{xs}$. de $\mathrm{ij}$ bobus vivis venditis. Et de $x x s$. vd. de $x \times x v$ oribus vivis venditis. Et de ijs. ixd. de ix agnis vivis venditis. Et de vs. iij $o b$. de coriis $\mathrm{j}$ avri, ij boum,' $\mathrm{j}$ vacce venditis. Et de iijs. iiijd. de $\mathbf{x x x j}$ pellibus grossis venditis. Et de $\mathrm{x} x d$. de $\mathrm{xxxij}$ pellibus agninis venditis. Summa vjl. $x j s$. xjd.

Idem reddunt compotum de $x x v j l$. $x d$. de ccij quarteriis iij bussellis frumenti venditis. Et de xlijs. iiijd. de xlij quarteriis avenæ venditis. Et de viijs. ixd. de vij quarteriis dimidio pisarum venditis. Summa xxviijl. xjs. xjd.

30 De Purchasiis.-Idem reddunt compotum de viijs. de vidua Ricardi pro fine terræ. Et de xijd. de Thoma pro mala warda. Et de rjd. de Arnaldo pro pace habenda. Et de xxs. de Roberto Ruffo pro terra de Hungerhulle habenda. Et de liijs. iiijd. de Willelmo Tederici pro terra patris sui habenda. Et de xiijs. iiijd. de Thoma de Makene pro simili. Et de vjs. viijd. de Randulfo Palmer pro filia sua maritanda. Et de xs. de Willelmo Mentor pro bona pace. Et de ijs. de tota thedinga pro siuili. Et de vjd. de Rogero Kinedoit pro scapiis. Et de vjd. de Randulfo cle Mortona pro manutenemento. Et de vjd. de Rogero de Kulneham pro simili. Summa rxvjs. iijjd.

Idem reddunt compotum de $x v l$. de taillagio. Sumina summarum lx.xiiijl. vjs. vd.ob.

Liberatio.--In liberatis Johanni decano cs. viijd.ob. de gabulo. Eidem de blado venditoxijl. xvs. 40 M. $2 d$. Eidem de tallagio xvl. Summa xxxijl. xvs. viijd. ob.

Expensa.-In ferramentis iiij avrorum, $v$ carrusarum, xs. In consuetudine carrucarii, ijs. vjd. In precariis araturæ, scilicet $\mathrm{xxxv}$ carrucariorum, iijs. ob. In $\mathrm{xvj}$ quarteriis ordei emptis, $\mathbf{x x s}$. iiijd. In $\mathrm{j}$ bove empto, vs. In hartis et aliis necessariis ad carrucas, ijs. viijd. In $\mathrm{j}$ careta empta, iijs. viijd. In Nortmede falcandn, $x v d$. In precariis auptumnalibus, $x v 8$. ixd. oh. In metendis $\mid x . x$ acris, $x v s$. In $i j$ saccis emptis, viijd. In paronis, capistris, et alio harnasio ad caretas, corbellis, cribris, et aliis minutis expensis, v8. iijd. ob. In sale, pressura, pottis, pannis, et aliis custis daeriæ, $\mathrm{jj}$. $\mathrm{x} d$. In stipendio daiæ, iijs. In triturandis liij quarteriis dimidio frumenti, xxvs. vijd. In triturandis lxxij quarteriis avenæ, vjs. In clatis ad faldam, ijs. In herciatura, ijd. ob. In grangia reparanda, xijs. $\mathrm{x} d$. ob. In purtandis 1 quarteriis frumenti apud Londoniam per aquam, viijs. iiijd. In expensis senescalli, xxjs. xjd., per $\mathrm{v}$ tallias. In liber, tis Ricardo servienti. lxs. xd. Summa xjl. xixs. vd. ob.

In aula, camera, et aliis domibus reparandis, xs. vijd. In perficiendo molindino molenti, et stagno emendando, $x l$. xiijs. $x d$. In j batillo empto et alio reparando, xiijs. vd. In $\mathrm{j}$ molindino foleratico facto de novo ixl. iiijs. iiijd. In pisciculis ad vivarium instaurandum vjs. iijd. In pane $R$ [icardi] servientis de anno praterito.: Summa xxxijl. ${ }^{3}$ xviijs. iijd. ob.

Summa tocius liberationis et expensæ, lxvl. xiiijs. Et debet ${ }^{4}$ viijl. xijs. vd. ob.

Exitus Grangiæ.-Idem reddunt compotım de cclxij quarteriis dimidio de toto exitu frumenti. In semine cciiijxij acrarum, lxij quarteria. In Nortmede falcando, iij busselli. In precariis arruræ, ij quarteria. In precariis allptumnalibus, v quarteria. In supravenditis, ccij quarteria iij busselli. Quietus est. ${ }^{4}$

\footnotetext{
1 Bobus in MS.

3 On an erasure in MS 
Idem reddunt compotum de $\mathrm{xx}$ quarteriis curalli. In corredio $\mathbf{v j}$ servientium totum. Quietus.

Idem reddunt compotum de xvj quarteriis ordei venditis. ${ }^{2}$ In semine xij acrarum, iij quarteria dimidium. In corredio prædictorum servientium, xj quarteria dimidium. In cibo porcarii, $\mathrm{j}$ quarterium. Quietus.

Idem reddunt compotum de iij $^{x x} . x v i i j$ quarteriis dimidio, iij bussellis de toto exitu avenæ. In semine lx acrarum, xxix quarteria. In præbenda tam avrorum quam boum, xj quarteria per estimacinnem. In præbenda senescalli per prædictas $v$ taillias, vj quarteria iij busselli. In equo domini Episcopi perendinantis per xj septimanas, $x$ quarteria dimidium. In supravenditis, xlij quarteria. Quietus.

Idem reddunt compotum de xiiij quarteriis dimidio, $\mathrm{j}$ bussello de toto exitu pisarum. In semine ix acrarum, ij quarteria $\mathrm{j}$ bussellus. In porcis pascendis per hyemem, $\mathbf{v}$ quarteria. In supravenditis, vij quarteria dimidium. Quietus.

Instaurum.-Idem reddunt compotum de viij avris, $\mathrm{j}$ pullo $\mathrm{ij}$ annorum, remanentibus anno præterito. Et de $\mathrm{j}$ pullo hujus anni. Summa $\mathrm{x}$. In vendito $\mathrm{j}$; de precio superius 15 respondent. In mortuo $j$; de corio superius respondent. Summa ij. Et remanent vij avri, j pullus.

Idem reddunt compotum de $\mathbf{x x x r}$ hobus, $\mathrm{j}$ tauro, remanentibus anno præterito. Et de $\mathbf{j}$ adjuncto. Et de $\mathbf{j}$ postea empto. Summa xxxvij boves, $\mathbf{j}$ taurus. In vivis venditis $\mathrm{ij}$; de precio superius respondent. In mortuis $\mathrm{ij}$; de coriis superius respon- 20 dent. Summa iiij. Et remanent $x \times x$ xij boves, $j$ taurus.

Idem reddunt compotum de $v j$ raccis remanentibus anno præterito. Et de j adjuncta de instauro. Summa vij. In occisa ad precarias auptumnales, j; de corio superius respondent. Et remanent vj vaccæ, $j$ taurus.

Idem reddunt compotum de $\mathrm{j}$ bovettn, $\mathrm{j}$ genicula, remanentibus anno præterito. 25 In adjunctis bobus et vaccis, omnes. Nichil remanet.

Idem reddunt compotum de $\mathrm{j}$ bovetto remanente, anno præterito, annali. Omnes remanent.

Idem reddunt compotum de iiij annalibus remanentihus, anno præterito vitulis. Omnes remanent; unde ij sunt maschuli, ij femellæ.

Idem reddunt compotum de iij vitulis hujus anni quia ij vaccæ fuerunt steriles. In decinis, $\mathrm{j}$. Et remanent iij vituli, unde $\mathrm{j}$ masnulus, ij femellæ.

Idem reddunt compotum de cj ovibus remanentibus anno præterito. Et de iiij adiunctis de instauro. Et de cxvj receptis de. Harewella. Summa ccxxj. In venditis ante partum, $\mathbf{x x \times v}$. De precio superius respondent. In consuetudine messepe 35 post tonsionem, $\mathrm{j}$. In consuetudine Nortmede post tonsionem, $\mathrm{j}$. In mortuis ante partum, ij; ante tonsionem, ix; post tonsionem, xj. De pellibus superius respondent. Summa lxij. Et remanent clix.

Idem reddunt compotum de xxviij multonibus, $j$ hurtardo, remanentibus anno præterito. In missis apud Harewellam, omnes. Nichil remanet.

Idem reddunt compotum de lx.x hogettis remanentibus, anno præterito agnis. In adiunctis ovibus, iiij. In mortuis ante tonsionem, vj. In missis apud Harewellam, lx, unde habent respondere. Summa lx.x.

Idem reddunt compotum de clx.xvij agnis proventis de ovibus hoc anno, quia iij fuerunt steriles. Et de iij proventis de hogettis. Summa ciiij ${ }^{\mathrm{x} j}$. In 45 decimis, zviij. In consuetudine bercarii, j. In supravenditis, ix. De precio superius respondent. In mortuis ante tonsionem, xxx; post tonsionem, ij. De pellibus superius respondent. Summa lx. Et remanent cxxj agni.

Idem reddunt compotum de clx.xv velleribus grossis. In decimis, $x v i j$. In consuetudine bercarii, $\mathrm{j}$. In supravenditis, clvij, quæ fecerunt $\mathrm{j}$ pondus. Nichil 50 remanet.

Idem reddunt compotum de cxxiij velleribus agninis. In supravenditis, totum. De precio superius respondent.

Idem reddunt compotum de ciijjx caseis qui inceperunt fieri vigilia Sancti Ambrosii, et desierunt die Sancti Michaelis utraque die computata. In decimis, xviij. In consuetudine messupe, $\mathrm{j}$. In consuetudine Nortmede, $\mathrm{j}$. In precariis auptumnalibus, $x v$. In precariis de Harewella, xiij. In expensis senescalli, ij. In supravenditis, exxx, qui fecerunt iij pondera et terciam partem ponderis. Summa ciiijxx.

Idem reddunt compotum de $j$ verre, vij suibus, vij porcis masculis remanentibus 60 anno præterito porcellis, et $\mathrm{x} v$ porcis remanentibus, anno præterito porcellis. ${ }^{1}$ Summa xxx. In occisis, ij. De carne habent respondere. In mortuis, ij. In liberatis servienti de Witteneia, xxiiij per $\mathrm{j}$ talliam. Summa xxviij. Et remanent $\mathrm{j}$ verres, j suis. ${ }^{2}$

Idem reddunt compotum de xlvij porcellis proventis hoc anno. In decimis, 65 iiij. In consuetudine porcarii, j. Summa v. Et remanent xli ij; unde $\mathrm{j}$ est $\operatorname{tr}[\mathrm{ua}]$. 
Idem reddunt compotum de xxvj aucis remanentibus anno præterito. Et de vij de purchasiis. Summa xxxiij. Omnes reunaneut.

Idem reddunt compotum de viij gallinis remanentibus anno præterito. Et de cxxxviij de eleriset hujus anni. Summa clx.xviij. ${ }^{1}$ In defectu terræ quam persona tenuit, viij gallinæ. In defectu aliarum minutarum terrarum, xvj. In mortuis, xij. In expensis senescalli, xv. Item in expensis eiusdem, viij. Summa lix. Et remanent iiijas 1 gallinæo.

In expensis domini Episcopi, $\mathrm{j}$ tonellum siceræ de instauro.

\section{HAREWELLA.}

R[ICARDUS] serviens et G[odefridus] præpositus reddunt compotun de xvl. ixs. jd. de redditu assiso in Harewella. In quietancia $\mathrm{j}$ præpositi, vs. In quietanciis $\mathrm{j}$ haiwardi, j bercarii, vj carrucariorum, xxiijs., singulis, iijs. Summa xxixs. Summa remanens xiiijl. jd.

Idem reddunt compotum de $\mathrm{x} v \mathrm{j}$ s. ijjd. ob. de exitibus molindini. Idem reddunt compotum de iijs. iijd. de pannagio. Et de xviij $l$. de teloneo tabernarum. Eit de iij8. vjd. de stipula vendita. Et de xxvjs. iij ob. de ciij velleribus grossis venditis, quæ fecerunt xj petras, unde xiiij faciunt pondus quarterium. Idem redflunt compotum de xlijs. viijd. de $x l v i j$ oviluus, $x x j$ multonibus, $x x v i j$ hogettis, vivis venditis. Et de xvjd. de corio $\mathrm{j}$ bovis vendito. Et de $\mathbf{x} s$. et $\mathbf{x} d$. de lxvij pellibus grossis venditis. Summa iijl. ixs. ijd. ob.

Idem reddunt compotum de $x l$. ixs. xjd. de lx.xvj quarteriis frumenti venditis. Et de xxijs. ixd. de xxij quarteriis dimidio, j bussello avenæ venditis. Summa xjl. xijs. viijd.

De Purchasiis.-Idem reddunt compotum de xijd. de Roberto Hunwini pro mellea. Et de vs. de tota thedinclıa pro brna ${ }^{2}$ pace. Et de iiijl. de Milone pro fine terræ. Et de vjd. de Stephano pro concordia. Summa iijl. vjs. vjd.

Liberatio,-In liberatis Johanni decano xxiiijl. vs. per talliam. Et de tallagio xvjl. Summs xll. vs. ${ }^{3}$

Expensa.-In ferramentis j avri, iij carrucarum, vije. viijd. In consuetudine præpositi, fabri, haiwardi, $\mathrm{j}$ l:ercarij, vj carrucariorum, ijs. vjd. In companagio ij servientium, iiijs. iiijd. In carrucis, hartis, [et] aliis necessariis, ad illas, iijj. iijd. ob. In j caretta ferrata et apparatu ad illam, xjs. $\mathrm{j}$. In triturandis cxx quarteriis frumenti, $\mathrm{xv}$ quarteriis mancorui, xxxix quarteriis dimidio $\mathrm{j}$ bussello avenae, xxiijs. $v d$. In toto blado illo ventilando, ijs. vijd. In feno empto, vs. ixd. In feno cariand", viijd. ob. In ij precariis auptumnalibus, xxvs. ob. In $\mathbf{x}$ acris dimidia metendis iijs. $\mathbf{x} d$. In blado cariando, $\mathrm{j} j$. $\mathrm{xj} d$. In blado tassando, ijs. ix $d$. $c b$. In expensis Thomae de Pincernaria qui praefuit custodiæ bladi in auptumno, per iiij septimanas, vs. In prato falcando, vjs. $x d$. In clatibus ad faldam, virgis ad praesepe houm, iijs. vjd. In $\mathrm{j}$ bove empto, vijs. ixd. In cribris, corbellis, et aliis minutis expensis, iijs. iijd. ob. In cariandis xxvi quarteriis frumenti apud Londoniam, per aquam, iiijs. iiijd. In feno cariando, viijd. $a b$. In cibo porcorum ductorum apud Duntonam ad lardarium, iijd. In herciandis lxix acris, ijs. iijd. In $\mathrm{j}$ falce empta, xiijd. ob. In expensis senescalli, $\mathrm{x} x d$, per $\mathrm{j}$ talliam. In expensis magistri Petri Russinol per $\mathrm{j}$ noctem, ijs. In expensis militum domini Episcopi, xjs. ixd. ob., in

redditu de partifus Scocia. Summa vijl. xjs. ixd. ob. ${ }^{\prime}$ Et debent lxvijs. xjd. ob. Et præterea $x d$. de v velleribıs multonum. Summa tocius, lxviijs. ixd. $o b$.

Exitus Grangiæ.-Idem reddunt compotum de cx quarteriis de toto exitu frumenti. Et de xiiij quarteriis de cheriset hoc anno. Summa cxxiij quarteria. In semine ciiij $x x_{i j}$ acrarum xlj quarteria. In precariis auptummalibus tam de anno præterito quan de hoc anno, vij quarteria. In supravenditis Ix.xvj quarteria. Quietus.

Idem reddunt compotum de xv quarteriis de toto exitu mancorni. In semine vij acrarum ijj quarteria. In corredio ij servientium per tıtum annum, x quarteria dimidium. In cibo porcolum, j quarterium dimidium. Quietus.

Idem reddunt compntum de xxxix quarteriis iij bussellis de toto exitu avenæ. In semine xvij acrarum, viij quarteria rlimidium. In præbenda $\mathrm{j}$ avri, boum, vj quarteria. In fræbendi Thomæ in auptumno, j quarterium. In præbenda magistri Petri Russinol, dimidium quarterium. In præbenda senescalli, dimilium quarterium ij busselli. In supravenditis, xxij quarteria dimidium j bussellus. Summa xxxix quarteria iij busselli. 
Instaurum.-Idem reddunt compotum de $\mathrm{j}$ avro remanenti. Et remanet $\mathrm{j}$.

Idem reddunt compotum de xxiiij bobus remanentibus anno præterito. Et de $\mathrm{j}$ de purchasio. Et de j postea empto. Summa xxvj. In mortuo j. De coreo superius respondent. Et renianent $\mathrm{xxv}$ boves.

Idem reddunt compotum de ccvij ovibus remanentibus anno præterito. In venditis ante partum, xlvij. De precio superius repondent. In missis apud Brictwellam ante partum, cxvj. In mortuis ante partum, xlij. De pellibus superius respondent. Et remanent $\mathrm{ij}$, quibus adduntur xxxix gerciæ. Sunma $x \mathrm{j}_{.}{ }^{1}$ Omnes remanent.

Idem reddunt compotum de xlviij multonibus, $j$ hurtardo remanentibus anno præterito. Et de xxviij multonibus, j hurtardo proventis de Brictwella. Summa, Ix.xviij multones, ij hurtardi. In venditis ante tonsionem, $x x j$. De precio superius respondent. In mortuis ante tonsionem, vij multones, $\mathrm{j}$ hurtardus; post tonsionem, iiij. De pellibus superius respondent. Et remanent xlv multones, $\mathrm{j}$ hurtardus. Eisdem adduntur xxx de instauro. Summa, $1 \mathbf{x} . x v$ multones. Omnes remanent.

Idem reddunt compotum de xlix hoggettis remanentibus, anno præterito agnis. Et de 15 Ix proventis de Brictewella. Summa cix. In vivis venditis ante tonsionem, xxvij. De precio superius respondent. In mortuis ante tonsionem, xiij. De pellibus superius respondent. Summa $x$. In adjunctis ovibus xxxix. In adjunctis multonibus, xxx. Et nichil remanet.

Idem reddunt compotum de exv velleribus grossis. In decimis, $x j$. In consuetu- 20 dine bercarii, $\mathrm{j}$. In supravenditis ciij, qui fecerunt $x \mathrm{j}$ petras.

Idem reddunt compotum de xiij caseis proventis de Brictewella. In precariis auptumnalibus, totum.

Idem reddunt compotum de xiiij porcis remanentibus anno præoterito. In missis in lardarium de Duntona, totum.

Idem reddunt compotum de Ixviij gallinis de cheriset. In expensis magistri Petri Russinol, iiij. In expensis senescalli, v. In expensis Ricardi servientis pro transmeante, x. In mortuis, vj. Summa xxv. Et remanent xlijj.

Summa tocius debiti, lxviijs. ixd. ob. Post compotum solverunt. Et quieti sunt.

\title{
WITTENE.
}

RICARDUS serviens et Alexander præpositus reddunt compotum de xijl..$^{3}$ xvijs. $x d . o b$. de reragio anni tercii et secundi. Solverunt xvijs.

Idem reddunt compotum de $\mathrm{xll}$. vs. viijd. de gabulo assiso in Uppelanda, unde ljs. jd. de assartis, xijs. vjd. de incremento hujus anni super dominicum assisum. In quietanciis ij præpositorum $\mathrm{j}$ porcarii, $x v 8$. In quietanciis $\mathrm{x}$ virgatariorum terræ qui inveniunt 35 v carrucarios per totum annum præter sabbata, 1s.- scilicet, singulis virgatariis, vs. In quietanciis ij virgatariorum qui inveniunt $\mathrm{j}$ carrucarium per quartam partem anni, ijs. vjd.-scilicet, singulis virgatariis, $\mathrm{xvd}$. In quietanciis $\mathrm{x}$ virgatariorum pro opere hyemali, xxvs. - scilicet, singulis, ijs. vjd. In quietanciis xl virgatariorum pro opere auptumnali, 1s.-scilicet, singulis virgatariis, $x v d$. Summa vijl. ijs. vjd.

In defectu terræ Eustachii de Hulle, xijs. In defectu terræ Reginaldi de Sanforda, xiijs. iiijd. In defectu terræ bercarii tractæ in dominium, ijs. In defectu terræ haiwardi tractæo in dominium, ijs. Summa xxixs. iijd.

Summa quietanciarum et defectuum, viijl. xjs. viijd. ${ }^{1} \quad$ Summa remanens, xxxjl. xiiijs.

Idem reddunt compotum de xxjs. de pannagio. Et de iijs. vjd. de residuis denariis 45 Sancti Petri. Et de xijs. vd. de herbagio. Et de ijs. viijd. de herba vendita in prato. Et de viijs. de piscatura. Et de vd. de thelonio tabernarum. Et de $x \times x$. vijd. de trenchea facta in bosco. Et de $\mathbf{x} v s$. de $\mathbf{x}$ quarteriis nucium venditis. Et de iijs. de terra locata. Et de xxs. de quadam veteri domo vendita. Et de xxjs. de iij ponderibus casei venditis. Et de xijd. de butiro vendito. Et de xlvs. iiijd. de ccx velleribus grossis 50 venditis, quæ fecerunt $\mathrm{j}$ pondus et terciam ${ }^{1}$ partem ponderis. Et de vijs. viijd. de iiijxx.ij velleribus agninis venditis. Et de $\mathbf{x x s}$. de iij ponderibus uncti porcini venditis. Summa, $x l$. xjs. vijd.

Idem reddunt compotum de ixs. viijd. de ij bobus veteribus vivis venditis. Et de iijs. de coriis $\mathrm{v}$ avrorum mortuorum venditis. Et de xijs. xjd. de coriis vij boum ij annaliunı 55 mortuorum venditis. Et de $x d$. de coriis iiij vitulorum mortuorum venditis. Et de cxviijs. vjd.de ccxxxiij ovibus vivis venditis pro infirmitate. Et de ls. de l multonibus vivis venditis pro simili. Et de ijs. de vj hoggettis vivis venditis pro simili. Et de xxxvjs. iijob. de pellibus cxiiij ovium, iv multonum, xliij hoggettorum mortuorum venditis. Et de iijs. de xlvij pellibus agninis venditis.

Summa xjl. xvijs. ob. Summa utriusque, xxijl. viijs. vijd. ob.

Idem reddunt compotum de cxvs. vjd. de l quarteriis frumenti venditis. Et de xviijd. de $\mathrm{j}$ quarterio ordei vendito. Et de xvjs. de xvj quarteriis avenæ venditis. Et de xvjd. de $\mathrm{j}$ quarterio pisarum vendito. Summa vjl. xiijjs. iiijd.

Summa totalis exitus et bladi venditi, xxixl. ijs. xjd. ob.

.

0


De Purchasiis.- Idem reildunt compotum de Hugone Fent xls. pro fine terræ. Et de xxxs. de Alexandro præposito pro terra dimittenda et pro licentia ædificandi super assartun. Et de lxvjs. viijd. de Hardekille pro aucis stulte retentis. Et de iiijs. de Ricardo Grant pro filia sua maritanda. Et de xs. de Galfiido molindinario pro licentia abeundi. Et de vjs. viijd. de hominibus de Credebrigge pro falso clamore. Et de xijd. de Thoma filio Bald wini pro simili. Et de xijd. de Rogero filio Hildæ pro bosco. Et de xijd. de Radulfo filio Hardekil pro simili. Et de ijs. de Waltero fabro pro terra habenda. Et de xijd. de Jacobo pro terra dimittenda. Et de ijs. de Hardekillo pro filia maritanda. Et de iijs. de Eva Dunna pro licentia ædificandi super assartum. Et de iijs. de Galfrido Geile pro simili. Et de iijs. de Ricardo 10 Grant pro simili. Et de xviijd. de Symone Grant pro besco. Et de vjd de Ricindo Kena pro simili. Et de ijs. de Waitero de Cherdesleia pro assarto. Et de ijs. de Pagano pro simili. Et de xijd. de Roberto Kena pro simili. Et de vjs. viijd. de Henrico filio Hugonis pro terra habeldi. Et de viijs. cle Radulfo filio Edwini pro dome facienda super assartum. Et de vlijs. de Gervasio Geile pro simili. Et de xijd. de Arnalde Grant pro surcisa. Et de vjd. de Galfrido Geile pro simili. Et de vjd. de Johanne filio Govilda pro sinili. Et de vjd. de Davide Kene pro simili. Et de vjd. de Radulfo filio Agnetis pro simili. Et de xijd. de Randulfo molindinario pro simili. Et de vjd. de Ricardo Dulere pro simili. Et de vjd. de Aiwardo pro simili. Et de vjd. de Roberti filio Godivæ pro simili. Et de ijs. de Willelmo flio Gerardi pro simili. Et de ijs. de Waltero de Wica pro bosco. Et de ijs. de Symone Grant pro pastura. Et de xs. de thedinga de Uppelanda pro pace habenda. Et de xxs. de Burgo pro simili. Et de xiijs. iiijd. de Willelnı filio Gerardi pro terra habenda. Et de xiijs. iiijd. de Rogero Juvene et sociis suis pro pastura. Et de vjs. de operariis pro blado stulte capto. Et de vjs. viijd. de Walkelino porcario pro terra recuperanda. Summa xiiijl. iiijs. $\mathbf{x} d$.

Idem reddunt compotum de $\mathrm{xvl}$. vjs. viijd. de tallagio de Uppelanda. Summa tocius receptæ, præter reraginm, iiijxx.xl. viijs. $v d . o b$.

Liberatio.-In liberatis Johanni decano xxxjl. xiiijs. de gabulo per iiij taillias. Eidem, iiijl. vijs. vjd. de blado vendito per ij taillias. Eidem, xxxiijs. iiij $l$. de lana vendita per j tailliam. Eidem, xxixs. viijd. per j tailliam. Eidem, vijl. iijs. vjd., de purchasiis, per $\mathrm{j}$ tailliam. Eidem, xiiijl. de tallagio per j tailliam.

Expensa. - In ferrameutis viij avrorum, ixs. vjd. In custo carrucarum, herciarum, xxjd. In M. 3. precuriis lxiiij carrucarum, vs. vjd. In decasu pannagii, ijs. jd. In iij bobus emptis, $\mathrm{xxvj}$ s. ixd. In $\mathrm{j}$ careta ferro reparanda, $\mathrm{xxd}$. ob. In paronis, capistris, cribris, corbellis et aliis minutis expensis, ijs. vjd. In $\mathrm{j}$ tripide empta, iiijd. ob. In $\mathrm{j}$ sacco empto, ixd. In iij serruris emptis, iijo. In sale, pressuris, pottis, pannis, et alio custo daeriæ, iijs. vjd. In triturandis l quarteriis frumenti, $\mathrm{xxx}$ quarteriis ordei, l quarteriis avenæ, xiijs. xjd. al. In mercede xij famulorum, per dimidiun annum, xiijs. vjd. In blado sarclando, xviijd. In $\mathrm{j}$ grangia de novo tacta, $x$ s. vjd. In $\mathrm{j}$ daeria de novo facta, iijs. In grangia et aliis domibus co-operiendis et reparandis, $x$ viijd. In expensis senescalli per ij taillias, vs. ijd. In liberatione Ricardi servientis, lxs. xd. Summa viijl. iiijs. viijd. ob.

In expensis magistri Ricardi de Pavilli circa festum Sanctæ Fidis per v dies, ijs. vjd. In acquietancia hospicii Horderi Wintoniæ, G. de Aucl[and], euntium apud Norhamptonam pro libertate novæ cartæ, iiijs. ob. In expensis G[alfridi] de Cauz et Thedbaldi Balistarii venientium cum armis domini Regis, xijd. In Fulcone veniente cum duobus equis domini Episcopi circa festum Sancti Lucæ per ij dies, iijd. In expensis Reginaldi salsarii et Walteri de Coquina per preceptum domini Episcopi per xv dies, iijs. ixd. In expensis Radulfi Bastardi preparantis hospicium domini Episcopi per ij dies, vjd. In portandis avibus apud Wintoniam quas dominus Episcopus misit filiæ domini Regis, xijd. In expensis Roberti nuncii et sociorum suorum ducentium equas a Cardif usque Witteneiam, viijd. In porta vivarii et serrura eiusdem, xijd. In sale ad lardarium et cervisia ad sulcid[ium] et toto custo lardarii, xxxiiijs. xjd. In carbonibus cariandis apud Oxoneford ad opus nepotis domini Episcopi, xiijjd. In domibus reparandis, xijd. In expensis Petri, nepotis domini Episcopi, euntis apud Lincolniam, xiijs. ixd. ob. per R[icardum] de Wikewano. In expensis Baldwini de Cumin[ora] proxima die Mercurii ante Purificationem per breve'domini Episcopi, vijs. $x d$. In expensis falconarii domini Episcopi circa festum Sancti Lucæ, per iiij dies, iiijs. ijd. ob. In expensis magistri Alani et magistri Ricardi de Pavilli circa Purificationem, per xv dies, xxvs., per breve domini Episcopi. In expensis Nicolai Ace post Purificationem, per vj dies, xijd. In ij garderobis factis pro domino Rege, xxixs. xjd. In magna porta emendenda, iiijd. In expensis $\mathrm{j}$ hominis Widonis de la Jaile per $x$ ij dies, ijs. In viridariis facientibus regardum forestæ, xvjs. vjd. ob. In expensis $\mathrm{j}$ hominis Hamonis coci domini Regis perhendinantis cum j equo ad festum Sancti Lucæ, per lxij dies, vjs. viijd. In expensis Andreæ Clerici de Cancellaria et fratris sui ad Pascha Florida, xviijd. In expensis Galfridi de Cauz, Radulfi Bastardi, in festo Sancti Marci, vjd. In expensis Eustachii de Greinvilla ad Ascencionem, per ij dies, xijd. ab. In expensis G. de Aucl[and] in 
Pentecoste, viijd. In uno rogo facto, $x l s$. vjd. In expensis Jobannis filii Hugonis et senescalli sui, ijs. viijd. In rotulandis armis domini Episcopi, xijd. In armis portandis apud Theukesbiriam, vs. In reportandis apud Witteneiam baconibus missis apıd Teokesbiriam, $x v d$. In vj furaturis agnellis emptis per E[ustachium] de Greinvilla, xxvjs. viijd. In expensis J[oliannis] de Mara per $\mathrm{j}$ diem, viijd. In $\mathrm{ij}$ tonellis vini cariandis ab Hamp- 5 tona usque Witteneiam, per senescallum, xijs. In expensis G[alfridi] de Cauz et Dionisii de Camera, per viij dies, iijs. iijjd. ob. In expensis archidiaconi Huntingdoniæ et magistri $\mathrm{A}[\mathrm{lani}]$, xxijd. In $\mathrm{j}$ leporario domini Regis per dimidium annum et $\mathrm{j}$ leporario per totum annum, xvjs. In $\mathrm{j}$ leporario domini Episcopi per iij septimanas, vjd. In expensis forestarii, ijs. vjd. In $\mathrm{j}$ plumbo et tripode empto pro domino Rege, vjs. viijd. In viij 10 aucis emptis ad opus eiusden, $\mathrm{xvj} d$. In $\mathrm{xxx}$ pullis emptis, xiiijd. In $\mathrm{j}$ fossato facto circa terram adjacentem novo molendino per preceptum domini Episcopi, xiijs. iiijd. Summat xiiijl. xixs. iijd.

Summa tocius expensæ, xxiijl. iijs. xjd. ob. Summa tocius liberationis et expensæ, iiijxiijl. xs. xjd. ob. Et debet xviijl. xviijs. iiijd. ob. computatis xijl. $x d$. $o b$. de reragio 15 anni tercii.

Sxitus Grangiæ.-Idem reddunt compotum de ciiij ${ }^{x x}$ viij quarteriis dimidio de toto exitu frumenti. In semine cccxvij acrarum, iijuxij quarteria. In expensis domini Episcopi, vj quarteria per $\mathrm{j}$ tailliam. In expensis magistri A[lani] et magistri $\mathrm{R}$ [icardi] de Paveilli, iij quarteria. In precariis hyemalibus, ij quarteria dimidium. In consuetudine medsipe, 20 dimidium quarterium. In pratis falcandis, $\mathrm{j}$ quarterium dimidium. In precariis auptumnalibus, iij quarteria. In consuetudine porcarii, dimidium quarterium. In conredio xij servientium, per annum, xxxvij quarteria dimidium. In conredio porcarii custodientis porcos emptos, $\mathrm{j}$ quarterium. In supravenditis, l quarteria. De precio superius respondet. In dono domini Episcopi passori de Babileia, ${ }^{1}$ j quarterium. Quietus est.

Idem reddunt compotum de lx.xiij quarteriis dimidio de toto exitu ordei. In semine iiijx.xvj acrarum, xxxj quarteria. In conredio prædictorum servientium, xxxvij quarteria dimidium. In pascendis xij catulis domini Regis, per ix septimanas, iiij quarteria. In supravendito, j quarterium. De precio superius respondet. Quietus est.

Idem reddunt compotum de cccxxxiiij quarteriis dimidio de toto exitu avenæ. In 30 semine cexij acrarum iij] $\times x$ quarteria. In præbenda domini Episcopi circa festum Sancti Egidii, vj quarteria dimidiım, ij busselli per $\mathrm{j}$ tailliam. In præbenda domini Regis et Episcopi die Jovis ante festum Sancti Luc , xxxvij quarteria dimidium, per j tailliam. In præbenda donini Episcopi in festo Sanctæ Agnetis, per ij dies, xvij quarteria j bussellus, per $\mathrm{j}$ tailliam. In præbenda domini Regis in crastino Sancti Gregorii, iiijx.iiij quarteria 35 iij busselli per $\mathrm{j}$ tailliam. In præbenda domini Episcopi in Decollatione Sancti Johannis, xij quarteria, per $\mathrm{j}$ tailliam. In præbenda magistri $\mathrm{R}$ [icardi] de Paveilli circa festum Sanctæ Fidis, per v dies, ij quarteria dimidium per $\mathrm{j}$ tailliam. In præbenda G[alfridi] de Cauz et Teodbaldi balistarii, in eodem tempore, ij quarteria. In præbenda ij equorum domini Episcopi, per ij dies, per Fulconem custodem eorum, dimidium quarterium. In 40 præbentla equi Hamonis coci domini Regis perhendinando, per lxij dies, iij quarteria dimidium. In præbenda R[adulfi] Bastardi preparantis hospicium domini Episcopi circa festum Sanctæ Katerinæ, per ij dies, dimidium quarterinm. In præbenda magistri P[etri] Russ[inol] in vigilia Sancti Andreæ, dimidium quarterium. In præbenda Petri nepotis donnini Episcopi, vj busselli. In præhenda Baldewini de Cumin[ora] proxima dis Mercurii 45 ante Purificationem, ij quarteria dimidium. In prabenda falconarii domini Episcopi in festo Sanctæ Luciæ, j quarterium dimidium. In præbenda magistri A[lani] et R[icardi] de Paveilli circa Purificationem, per xvj dies, xv quarteria. In præbenda Nicolai Ace, per iij dies, dimidium quarterium. In equo Eudonis de la Jaile perhendinando per xvij dies, ij quarteria. In præbenda viridari[orum], iiij quarteria. In præbenda Andreæ de Cancel- 50 laria clerici et fratris sui, per $\mathrm{j}$ diem, $\mathrm{j}$ quarterium. In præbenda $\mathrm{G}$ [alfridi] de Cauz et $\mathrm{R}$ [adulfi] Bastardi redeuntium de Eboraco, dimidium quarterium. In præbenda E[ustachii] de Greinvilla ad Ascensionem, per iij dies, $\mathrm{j}$ quarterium dimidium. In præbenda G. de Aucland in Pentecoste, dimidium quarterium. In perhendinando $\mathrm{j}$ equo caretæo domini Episcopi, per $x l$ dies, iij quarteria. In præbenda sencscalli, Johannis filii Hugonis, 55 et sociorum suorum per $\mathrm{j}$ noctem, $\mathrm{j}$ quarterium. In præbenda J[ohannis] de Mara, dimidium quarterium. In præbenda G[alfridi] de Cauz et Dionisii de Camera per viij dies, ij quarteria. In præbenda archidiaconi de Huntingdonia et magistri $A[$ lani], per j diem, jj quarteria. In præbenda senescalli, iij q[uarteria], per ij taillias. In prælenda viij avrorum, xviij quarteria. In supravenditis, xvj quarteria. De precio superius respon- 60 dent. Summa ccexxxiiij ${ }^{1}$ quarteria dimidium. Et debet j quarterium.

Idem reddunt compotum de iiij quarteriis de toto exitu pisarum. In porcellis pascendis per hyemem, iij quarteria. In supravendito, $\mathrm{j}$ quarterium. De precio superius respondent. Summa iiij quarteria.

Instaurum.-Idem reddunt compotum de vij avris remanentibus anno præterito, et de j fortuitu 65 invento. Summa viij. In mortuis, v. De precio superius respondent. Et remanent iij. 
Idem reddunt compotum de viij asinis remanentibus anno præterito. Omnes rem[anent. ${ }^{1}$

Idem reddunt compotum de xlvij bobus remanentibus anno præterito. Et de ij adjunctis. Ev de iiij postea emptis. Summa liij. In mortuis, vij. De coriis superius respondent. In vivis venditis $\mathrm{ij}$. De precio superius respondent. Summa ix. Et remanent xliiij.

Idem reddunt compotum de $x v$ vaccis remanentibus anno præterito. Et de $j$ adjuncta de instauro. Summa xvj. Omnes remanent.

Idem reddunt compotum de $\mathrm{j}$ genicula remanente anno præterito. In adjuncta vaccis, $j$. Nichil re[1nanet. ${ }^{1}$

Idem reddunt compotum de $\mathrm{ij}$ bovettis prolixioris atatis remanentibus anno præterito. Et de ix junioribus remanentibus, anno proterito annalibus. Et de vj annalibus remanentibus, anno præterito vitulis. Sımma xvij. In mortuis, ij bovetti juniores. In adjunctis bubus, ij. De coriis superius respondent. Summa iiij. Et remanent xiij; unde vij juniores, scilicet, ij masculi, v femella, vj annales.

Idem reddunt compotum de $x$ vj vitulis proventis de vaccis hoc anno. In decimis, $\mathrm{j}$. In mortuis, iiij. De pellibus superius respondent. Et remanent $x j$; unde $v$ sunt in:sculi, vj femellæ.

Idem reddunt compotam de ccexj ovibus remanentibus anno præterito. Et de xxj adjunctis de instauro. Summa cecexxxij. In supra-venditis, cexxxiij. De precio superius respondent. In mortuis ante partum et tonsionom, xxij. Post partum et ante tonsionem, iijjx. Post partum et post tonsionem, xij. De pellibus superius respondent. Summa ccexlvi[j. $]^{\prime}$ Et remanent iijijxv.

Idem reddunt compotum de ciijixvj multonibus remanentibus anno præterito. Et de xvj adjunctis. Summa ccij. In supravenditis 1 ante tonsionem. ${ }^{2}$ De precio superius respondent. In mortuis ante tonsionem, xxxiiij. De pellibus superius respondent. Post tonsionem, xxj. [le pellibus superius respondent. Summa cr. Et remanent iij ${ }^{\mathrm{Ix}}$.xvij.

Idem reddunt compotum de $i^{1 i j}{ }^{x z} v j$ hoggettis remanentibus, anno præterito agnis. In adjunctis ovibus, $x \times j$. In adjunctis multonibus, $x v j$. In mortuis ante tonsionem, xxiiij. Post tonsionem, xix. De pellibus superius respondent. In supravenditis ante tonsionem, vj. De precio superius respondent. Summa iiij ${ }^{x x}$ vj. Nichil remanet.

Idem reddunt compotum de cxliiij agnis proventis de ovibus hoc anno, quia $v$ fuerunt steriles, vij feccrunt aborsum. In decimis, xiij. In consuetudine bercarii, $j$. In mortuis ante separationem, xlvij. Et remanent iijj $^{\mathrm{x}}$.ij; unde $\mathrm{xl}$ sunt masculi, xlij femellæ.

Idem reddunt compotum de cexxxiiij velleribus grossis. In decimis, xxiij. In consuetudine bercirii, $\mathrm{j}$. In supravenditis ccx, quæ fecerunt $\mathrm{j}$ pondus et terciam partem ponderis; unde superius respondent. Summa cexxxiiij. Quiet[us est.]

Idem reddunt compotum de iijjxij velleribus agninis. In supravenditis, totum. Quietus.

Idem redilunt compotum de clxviij caseis, qui inceperunt fieri xvij Kalendas Maii et desierunt fieri in die Sancti Michaelis, utraque die computata. In decimis, xvj. In dignerio domini Regis, ij. In expensis domini Episcopi, ix. In missis nepoti domini Episcopi, ij. In expensis senescalli, $j$. In expensis magistri $A$ [lani] et $R$ [icardi] de Pavilli, ij. In expensis archidiaconi Huntindoniæ, j. In expensis E[ustachii] de Greinvilla, $\mathrm{j}$. In consuetudine daiæ, $\mathrm{j}$. In pratis falcandis, iiij. In precariis auptumnalibus, xviij. In bladis sarclandis, ij. In supravenditis, cviij, qui fecerunt iij pondera. De precio superius respondent. Summa clxviij.

Idem reddunt compotum de cciiijxiiij porcis remanentibus anno præterito. Et de xxiiij reccptis de Brichtewella. Et de xx receptis de Edborbiria. Et de ix proventis de pannagio. Summa ccexxxvij. In nccisis in lardario, ccxxx. De carne habent respondere. Et de exitu superius respondent. In consuetudine forestarii, $\mathrm{j}$. In mortuis, xlvj. Summa cclxxvij. Et remanent $1 x$.

Idem reddunt compotum de lvj porcellis proventis hoc anno; de suibus de instauro, in decimis, v. In consuetudine porcarii, j. Summa vj. Et remanent 1.

Summa omnium remanentium tam porcorum quam porcellorum, cx.

Idem reddunt compotum de cexxx baconibus de porcis de instauro occisis. In liberatis Rogero Wacelino, iijj $^{x x}$.xvj jer j tailliam. In expensis magistri A[lani] et $R$ [icardi] de Paveilli, v, per $\mathrm{j}$ tailliam. In expensis Baldwini de Cumin[ora] dimidium baconis. In missis nepoti domini Episcopi, ij. In liberatione servientis de Edborebiria, v. In expensis viridarii, ij. In expensis $\mathrm{E}$ [ustachii] de Greinvilla, dimidium. In precariis auptumnalibns, viij. In pratis falcandis, ij. In elemosina Episcopi leprosis, vij. Et remanent cij.

Idem reddunt compotum de exitu cexxx porcorum. In liberatis $R$. salcerio exitum de ciiij ${ }^{x} x$ porcis. In precariis hiemalibus, $x l$. In liberatis domino Episcopo, $x$ porci saginati qui fecerunt $\mathrm{j}$ pondus.

Idem reddunt compotum de viij aucis emptis. Et de xxxij de purchasiis. Summa xl. In expensis domini Episcopi, xxiiij. In expensis archidiaconi Huntindoniæ, iij. In expensis senescalli, iij. Summa xxx. lit remanent $\mathrm{x}$.

\footnotetext{
1 The rargin of the membrane is mutilated. $\quad$ Interlineated in MS. $\quad{ }^{3}$ Sic in M 3.
} 
Idem reddunt compotum de lij gallinis receptis de redditu. Et de xlvij receptis de Edborbiria. Summa iiij ${ }^{x x}$ xix. In expensis domini Episcopi liiij, per j tailliam. In expensis magistri Alani et $\mathrm{R}$ [icardi] de Pavilli, $x v$, per $\mathrm{j}$ tailliam. In expensis Baldwini de Cumin[ora], $x$. In expensis viridari[orum], xij. In expensis senescalli, iiij. In mortuis, iiij.

Idem reddunt compotum de xxx pullis cmptis. Omnes remanent.

Summa tocius debiti xviijl. xviijs. iiijd. ob. Solverunt post compotum vjl. xvijs. vjd. Et debentur de tempore Oliveri, xijl. $x$ d. ob. ${ }^{1}$

\section{DUNTONA.}

W[rletmus] filius Giliberti et Gocelmus præpositus et Ailwardus berebr[ectus] reddunt compotum de vijl. xijs. xjd. de reragio anni præteriti. Solverunt et quieti sunt. Et de 10 xxxvl. ijs. ijd. de gabulo assiso. Et de xijd. de incremento gabuli pro $\mathrm{j}$ parco quod ${ }^{2}$ Willelmus de Widintona tenuit pro nichilo. Ett de ijs. vjd. de incremento gabuli pro dimidia virgata terræ quam Jacobus Oisel tenuit sine servitio. Et de xixs. pro xix placitis assisis in foro novo. Et de xs. de incremento gabuli pro $\mathrm{x}$ placitis de novo assisis in foro hoc anno. Summa tocius gabuli, xxxvjl. xiijjs. viijd.

In quietancia $\mathrm{j}$ præpositi, vs. In quietanciis pro ponte reficiendo, vs.; $\mathrm{j}$ forestarii, iiijs.; ij haiwardorum de Duntona et Wika, iiijs.; $\mathrm{j}$ haiwardi de Widintona, $\mathrm{xxd}$. ; xiiij carucariorum de Duntona, Wike, et Nuntona, per annum, xxviijs.; ij carucariorum de Widintona, per annum, iiijs. iiijd.; ij carucariorum per dimidium annum, ijs.; j porcarii, j bovarii, j vaccarii, per annum, vjs. ; iij bercariorum de Wika, Bereforda, et Nuntona, per 20 annum, vjs.; $\mathrm{j}$ bercarii de Widintona, per annum, $\mathrm{xxd}$. ; iij operariorum, per annum, viijs. Summa quietanciarum, lx.xiiijs. viijd. Summa remanens, xxxiijl.

Idem reddunt compotum de xiijs. iiijd. ob. de pannagio. Et de xxjs. iijd. de herbagio. Et de xiijd. de hominibus qui dant cheriset. Et de vs. iijob. de residuo denariorum Sancti Petri. Et de lx.xiiijs. de xij bobus vivis venditis. Et de xxxviijs. iiijd. de 25 xlvj ovibus vivis venditis. Et de xlvijs. viijd. de xxvij multonibus, $x$ hurtardis, vivis venditis. Et de $x x x v i i j s$. $i x d$. $o b$. de coriis $x x j$ boum, iij vaccarum, $\mathbf{j}$ annalis mortuorum venditis. Et de viijs. de pellibus xxxij ovium, xiiij multonum, xxij hoggettorum, mortuorum venditis. Et de vijs. de cv pellibus agnorum mortuorum venditis. Et de $\mathrm{xxxiijs.} \mathrm{pro} \mathrm{j}$ tonello dimidio vini ad scotallum. Et de xxijs. de ij ponderibus uncti de 30 ciiij $x x . x v$ porcis occisis ad lardarium. Et de $x v j l$. xiijs. iiijd. de xj ponderibus grossæ lanæ, i pondere dimidio de lana agnina vendita. Et de vjl. vjs. de xviij capitibus casei venditis. Summa xxxviijl. viijs. xjd. $o b$.

Idem reddunt compotum de $x v l$. xvjs. vijd. de cx quarteriis j estrica frumenti venditis. Et de lx.xvijs. de xxxviij quarteriis dimidio curalli venditis. Et de liijs. viijd. de xxvij 35 quarteriis mancorni venditis. Et de $\mathrm{ix} l$. xijs. xjd. de iijjx ${ }^{\mathrm{x}} \mathrm{xj}$ quarteriis dimidio ordei venditis. Et de xijs. de xij quarteriis avenæ venditis. Et de xijs. de vj quarteriis fabarum venditis. Et de iijjs. vijd. de ij quarteriis dimidio, $\mathrm{j}$ estrica pisarum venditis. Summa xxxiijl. viijs. ixd.

De Exitu Molindini.-Idem reddunt compotum de xxvijs. vd. de viij quarteriis dimidio, 40 j estrica frumenti venditis. Et de vl.iiijs. ixd. de xlvj quarteriis dimidio, j estrica mancorni venditis. Et de ljs. iij $d$. de xxvj quarteriis dimidıo brasei venditis. Et de viijs. ixd. de iiij quarteriis $\mathrm{j}$ estrica, dimidia gruelli venditis.

Summa ixl. xijs. iijd. ${ }^{3}$

De Purchasiis.-Idem reddunt compotum de xijd. de Ernaldo filio Comitis pro terra patris sui 45 habenda. Et de xijd. de Ricardo Mai pro lege relaxata. Et de xijd. de (Usberto Peche pro simili. Et de iiijs. de vidua Anketilli pro fine terræ. Et de vjs. viijd. de Willelmo filio Selidæ pro terra Emmæ habenda. Et de vs. pro filiastra Bren maritanda. Et de vs. de Radulfo filio Edithæ pro terra Jacobi hahenda. Et de xijd. de Ada de Roxi pro forfengo animalium suorum. Et de xijd. de Ailet pro lege relaxata. Et de xs. de 50 Waltero pro filia sua maritanda. Et de iiijs. de testamento Juonet. Et de vjs. viijd. de testamento Siwaldi. Et de ijs. de Ingelram de Wika pro forisfacto pasturæ. Et de xijd. de Rogero Belfiz pro simili. Et de xijd. de Helia de Wika pro simili. Et de vjd. de Radulfo Trapel pro simili. Et de xijd. de Ricardo Haver pro simili. Et de iiijs. de Roberto Rad[ulf] pro simili. Et de ijs. de Willelmo Dal pro simili. Et de xviijd. de 55 Bernardo Cave pro simili. Et de xviijd. de Edwardo Biliz pro j acra non seminata. Et de vjs. viijd. de Ricardo Hereber to ${ }^{2}$ pro bosco. Et de ijs. de Ricardo Glide pro pastura. Et de xijd. de Roberto de Lane pro sursisa. Et de xijd. de Ada Scalc pro simili. Et de xijd. de Waltero molindario pro simili. Et de vjd. de Stephano Capra pro virga. Et de xviijd. de Willelmo Birctmar pro blado. Et de ijs. de Galfrido bercario pro bosco. Et 60 de $x i j d$. de Jordano fitio Normanni pro mellea. Et de vjd. de Norman pro plevina. Et de xijd. de Gileberto Pig pro simili. Et de xijd. de Ricardo parmentario pro sursisa Et de xijd. de Willelmo Alani pro bosco. Et de xijd. de Willelmo Ruffo de Stanlinche

\footnotetext{
1 The pussage printed in italics is a later entry in the Roll. Sic in. MS. ${ }^{3}$ Underlined in MS.
} 
pro bosco. Et de xijd. de Wulvrieo Haver pro pastura. Et de ijs. de Nicolao Albo pro simili. Et de xijd. de Rorero Wlgar pro simili. Et de ijs. de Ricardo Sprot pro simili. Et de xxs. de Symone Baus pro misericordia. Et de ijs. de Willelmo de Cruce pro pastura. Et de vjd. de Ernaldo Crunc pro simili. Et de vjd. de Ricardo Grime pro simili. Et de ijs. de Ricardo Brun pro simili. Et de vjd. de Waltero Burel pro hosco. Et de xijd. de Koberto Grant pro blado. Et de vjs. viijd. de Galfrido Frawinc pro simili. Et de xijd. de Willelmo piscatore pro pastura. Et de vjd. de Ernaldo Breu' pro mellea. Et de vjd. de Ernaldo carucario pro sursisa. Et de vjd. de Rogero 'Tessæ pro simili. Et de vjs. viijd. de thedinga de Cherlet[ona]. Et de iijs. de thedinga de Duntona. Et de v8. [de] thedinga de Wika. Et de iiijs. de Boterham. Et de xviijd. de thedinga de Widentona. Et de vjs. viijd. de thedinga ecclesiæ. Et de ijs. de Radulfo de Wika pro filia sua maritanda. Et de $x$ s. de Helia de Wika pro filia sua maritanda. Et de iiijs. de Willelıno filio Osgod pro terra Walteri Cumhichan habendit. Et de ijs. de Walkelino de Nuntona pro relaxatis operibus auptumnalibus. Et de xijd. de Rogero Tessæ pro simili. Et de ijs. de Waltero Hale pro simili. Et de xijd. de Ailwardo de Nuntona pro simili. Et de xijd. de Willelmo bercario pro simili. Et de xijd. de Beruardo Dugrel pro simili. Et de xviijd. de Norman pro simili. Et de xijd. de vidua Pep pro simili. Et de xijd. de Godwino Peche pro simili. Et de vjd. de Rieardo de Fraxino pro simili. Et de v8. de Seman Pute pro mellea. Et de iijs. de Odone pro falso clamorc. Et de xijd. de Elia Fis pro lege relaxata. Et de ijs. de Rogero Mabon pro terra patris sui habenda. Et de ijs. de Roberto Coppe pro terra sua. Et de xxs. de hominibus villæ pro relaxacione emptionis mediæ bladi.

\section{De tallagio, $x l l$. Summa tocius cum taillagio, clxvl. $x$ vijd. ob.}

25 Liberatio.- In liberatis J[ohanni] decano, de gabulo Natalis, vijl. xs., per j talliam. Eidem de gabulo Paschæ, viijl., per $\mathbf{j}$ talliam. Eidem de gabulo Sancti Johannis, viijl., per $\mathrm{j}$ talliam. Eidem de gabulo Sanetı Mirhaelis, viijl. xs., per $\mathrm{j}$ talliam. Eidem de blado vendito, xxvjl., per ij tallias. Eidem de blado, purchasiis et caseis, $x x l . x v j s . x d$. Eidem de lana, xvjl. xiijs. iiijd., per j talliam. Eidem de tallagio, xxxixl., per j talliam.

Expensæ.-In ferramentis viij carucarum per annum, $\mathbf{j}$ carucæ per dimidium annum, $x x x i j s . x d$. M. 3d. In ferrura ij avrorum per annum, $\mathrm{ij} s$. viijd. In rotis ad carucas, $\mathrm{ij} s$. $\mathrm{ixd}$. In vj carucis factis de novo, xijd., ante adventum earpentarii. In mercede fabri, per annum, viijs. vjd. In $\mathrm{j}$ biga ferro ligata novi empta, $v s$. vijd. In rotis emptis ad $\mathrm{j}$ caretam ad fimum extrahendum, xijd. In paronis, paronellis, bacis, clutis, eapistris, xiiijd. In ij cordis emptis, iijd. In ij saccis emptis, viijd. In $v$ serruris emptis ad grangiam, xjd. In ij portis parandis ad hercariam, ijs. In $\mathrm{j}$ porta ad bertonam, xijd. In acero, sepo emptis et fusis reparandis ad molindinum, per annum, vjs. $x d$. In $\mathrm{j}$ mola empta ad molindinum, xxiijs. In $\mathrm{j}$ porta paranda versus molindinum, xijd. In carne paranda in lardario, iijs. In cervisia empta ad sulcit[ium], ijs. $\mathrm{j} d$. In fossandis clviij perticis, xxxijs. xjd., pro qualibet pertica ijd. ob., circa pasturam in marisco. In columbario facto novo, xxijs. xjd. ob. In seeando $\mathrm{c}$ bordos ad planchendum ultra dispensariam et boteleriam, vjs. iijd. In clavis emptis ad bordandam ultri celariam, xvjd. In claudendo circa gardinum cum duabus portis parandis, vjs. vijd. ob. In fodiendo in gardinis, vijs. vd. In precariis hyemalibus de lv carucis, ixc. ijd. In precariis Quadragesimalibus de xlix carucis, viijs. vjd. In spargendis $v j$ acris de fimo, vjd. In triturandis xxiiij quarteriis frumenti apud Mcredonam ad semen, vs. In ventilandis eisdem, vijd. In vannandis xxxvj quarteriis frumenti ad semen, iijs. ixd. In triturandis ciiij.x ${ }^{x i j}$ quarteriis frumenti, $x \times x i j s ., p r o$ quolibet quarterio, $\mathrm{ij} d$. In triturandis $\mathrm{xx}$ quarteriis mancorni, $\mathrm{ij} s . \mathrm{vjd}$. In triturandis xlij quarteriis ordei, iijs. vjd. In tritırandis liij quarteriis avenæ, ijs. ijd. ob. In attrahendo gravcli ad pontem et calceam, xiiijd. In custo daeriæ, scilicct, sale, iij tinis, pannis et ollis, vjs. $\mathrm{x} d$. In xvij bobus emptis, $\mathrm{v} l$. xiijs. In sarclandis exl acris, $v s . \mathrm{x} d$. In stipendiis ij carctariorum, $\mathrm{j}$ bovarii, per annum, ixs. In stipendio $\mathrm{j}$ carpentarii, ${ }^{2}$ per annum, vjs. viijd. In stipendio daiæ, ijs. vjo. In consuetudine falcatorum prati de Nuntona, vjd. In octo ovibus emptis, viijs. In stipendio $\mathrm{j}$ bovarii de Nuntona, xijd. In cariandis ij tonellis vini per Walterum Locarde, in termin" Sancti Martini, viijs. ijd. In cariandis ij tonellis vini de Sudhamptona usque Duntonam per senescallum, iijs. vjd., in festo Sancti Laurentii. In fossandis xxij perticis in bertonam, vjs. vd., pro qualibet pertica, iijd. $a b$. In corredio Roberti de Lurdona qui erat infirmus, per xxj dies, eum homine suo, vs. iijd. In corredio Sewali qui custodiebat ij equos domini Episcopi per iij septimanas, $x x j d$. In corredio $R[$ ogeri] Wacel[ini], per ij vices, qui fecit exonia domino Regi apud Clarendonam, iijs. ixd. per ij taillias. In corredio mavistri R[oberti] Basset, per iij itinera, ixs. iijd. $o b$. In liberatione Willelmi filii Giliberti, lxs. xd. In xxx ulnis de cauevacio emptis ad imponendam lanam, et $\mathrm{ij}$ saccis parandis ad curiam, v8. In viij ovibus emptis. cum agnis, viijs.

Summa xxvl. xxiijd. Summa tam liberationis quam expensæ, clixl. xijs. jd.

Et debet vl. ixs. iiijd. ob. 
Exitus Grangiæ.-Idem reddunt compotum de cexxj quarteriis dimidio j estrica de toto exitu frumenti ; et de xxiiij quarteriis allatis de Meredona. Summa, cexlv quarteria dimidium jestrica. In semine ceclj acrarum, exvij quarteria. In pane domini Episcopi, xviij quarteria dimidium liberata Johanni de Dispensa per iij taillias. In supravenditis ex quarteria j estrica.

Idem reddunt compotum de xxxviij quarteriis dimidio de toto exitu curailli. In supravenditis totum.

Idem reddunt compotum de $x x^{1}{ }^{1}$ quarteriis $j$ estrica de toto exitu mancorni. In semine clvj acrarum, liij quarteria $\mathbf{j}$ estrica. In pane ad iij precarias auptumnales, ix quarteria. In supravenditis, xxvij quarteria.

Idem reddunt compotum de clx.xviij quarteriis dimidio de totu exitu ordei. In semine cij acrarum dimidiæ, xlix quarteria dimidium. In consuetudine carucarum, $\mathrm{j}$ quarterium. In consuetudine fimorum extrahendorum, ij quarteria. In corredio ij caretariorum, ${ }^{2}$ j carpentarii, $\mathrm{j}$ bovarii, $\mathrm{j}$ daiæ, per annum, xxxij quarteria dimidium. In porcis pascendis in hyeme, $\mathrm{ij}$ quarteria. In supravenditis, $\mathrm{iij}^{\mathrm{xx}} . \mathrm{xj}$ quarteria dimidium. 15 Quietus est.

Idem reddunt compotum de cccxj quarteriis ij bussellis de toto exitu avenæ. In semine cexxj acrarum dimidiæ, cx quarteria dimidium. In præbenda domini Episcopi et domini Regis, per plures vices, cxxxj quarteria dimidium, ij busselli, per $\mathrm{v}$ taillias. In præbenda Rogeri Wacel[ini], ij quarteria dimidium iij busselli. In præbenda magistri 20 Roberti Basset, iij quarteria dimidium $j$ bussellus. In præbenda ij equorum domini Episcopi et $j$ equi Ricardi de Marisco, per $v$ septimanas, $v$ quarteria dimidium ij buisselli. In præbenda ij equorum domini Episcopi qui fecerunt moram per xvj noctes apud Duntonam, iiij quarteria. In missis apud Cnoel, ${ }^{3}$ xviij quarteria. In præbenda $\mathrm{j}$ equi Roberti de Lurdune, per iij septimanas, j quarterium dimidium. In præbenda 25 ij carettariorum, vij quarteria ij busselli. In supravenditis, xij quarteria. Et remanent xiiij quarteria $\mathrm{j}$ estrica.

Idem reddunt compotum de vj quarteriis dimidio de toto exitu fabarum. In plantatione in gardino, dimidium quarterium. In supravenditis, vj quarteria. Quietus est.

Idem reddunt compotum de iiij quarteriis j estrica de totu exitu pisarum. In semine 30 vj acrarum, $\mathrm{j}$ quarterium dimidium. In supravenditis, ij quarteria dimidium, $\mathrm{j}$ estrica. Quietus est.

Idem reddunt compotum de iiij quarteriis de totu exitu vesciæ. In porcis pascendis in hyeme, totum. Quietus est.

Instaurum.-Idem reddunt compntum de ciiij bobus remanentibus anno præterito. Et de 35 ij junctis de otiosis ${ }^{4}$ animalibus. Et de j de testamento Roberti Coppe. Et de xvij emptis. Summa cxxiiij. In vivis venditis, xij. In mortuis, $x x j$. Summa xxxiij. Et remanent iiij ${ }^{2 x} . x j$ boves.

Idem reddunt compotum de ij avris remanentibus anno præterito. Omnes remanent.

Idem reddunt compotum de xix vaccis remanentibus anno præterito. Et de vij 40 junctis de otiosis ${ }^{3}$ animalibus, et de $\mathrm{j}$ inventa. Summa xxvij. In mortua, j. In occisis ad opus domini Episcopi deferatis apud Craneb[urnam], ij. Summa iij. Et remanent xxiiij vaccæ.

Idem reddunt compotum de vij geniculis, ij juvencis masculis, remanentibus anno præterito. In adjunctis vaccis, vij geniculæ. In adjunctis kobus, ij masculi. Summa ix.

Idem reddunt compotum de xij annalibus remanentibus anno præterito. In mortuo, j. Et remanent $x j$; de quibus v sunt geniculæ, vj masculi.

Idem reddunt compotum de xiij vitulis proventis hoc anno de vaccis, quia ceteræ fuerunt steriles. In decimis, $\mathrm{j}$. Et remanent xij.

Idem reddunt compotum de Dccclviij ovibus remanentibus anno præterito. Et de 50 xlvij ovibus de consuetudine herbagii, post partum et ante tonsionem. Et de viij emptis ante partum. Et de cxxxvij gerciis junctis de hoggettis. Summa ML. In vivis venditis in termino Sancti Martini, xlvj. In mortuis ante partum, $\mathrm{xx}$. In mortuis post partum et ante tonsionem, xij. Summa lx.xviij. Et remanent Dccclx.xij oves.

Idem reddunt compotum de Diiij ${ }^{x}{ }^{i i i j}$ multonibus remanentibus anno præterito. Et 55 de clxiij nnasculis junctis de hoggettis. Et de xvj hurtardis de Lindeseia, qui venerunt per fratrem Walterum ante tunsionem. Summa Declx.iij. In vivis venditis in termino Sancti Martini, xxvij multones, $x$ hurtardi. In liberatis hominibus de Eblesburna, ante tonsionem, xx, per breve senescalli. In mortuis ante tonsionem, xiiij. Summa lx.xj. Et remanent Dciiij ${ }^{x x}$.xij multones.

Idem reddunt compotum de cccxxij hoggettis remanentibus, agnis anno præterito. In mortuis ante tonsionem, xxij. Et remanent ccc; unde cxxxvij sunt gerciæ, junctæ uvibus, clxiij masculi, juncti multonibus.

Idem reddunt compotum de Decl agnis proventis de ovibus hoc anno, quia $x \mathrm{x}$ fuerunt steriles, et $x \times x$ fecerunt aborsum. In consuetudine fabri, ij, bercariorum, iij. In 65 decimis, lx.xiij. In mortuis ante tonsionem, ev. Summa ciiij ${ }^{x j}$. Et remanent Dlxix agni. 
Idem reddunt compotum de M.D.clxiiij velleribus grossis, unde xvj tuerunt de hurtardis de Lindeseia. In decimis, clxiiij. In consuetudine iij bercariorum, iij. In supravenditis, mecciiij ${ }^{x x} . x v i j$ vellera cum $x v j$ velleribus de Lindescia, qua fecerunt xj pondera.

Idem reddunt compotum de Dlxix velleribus agninis. In supravenditis, totum, quæ fecerunt $\mathrm{j}$ pondus dimidium.

Idem reddunt compotum de exxxviij caseis de reragio anni præteriti. Et de xix parvis caseis. Et de $\mathbf{v}$ de majoribus de reragio anni prateriti. Et de cclx.xiij caseis, qui inceperunt fieri viij Idus Aprilis, et desierunt in festo Sancti Michaelis, utroque ${ }^{1}$ die computato. Et fecerunt jer iiij ${ }^{x x} \times x_{j}$ dies binos caseos-scilicet, a quinto Kalendas Maii usque vigiliam Sancti Petri ad Vincula, utroque ${ }^{1}$ die computato. Surnma cccexxxv casei. In decimis, xxvij. In consuetudine bercarii, falcator[um] prati de Nuntona, ij. In consuetudine carucarii, iij. In precariis auptumnalilus, $x$. In expensis domini Episcopi in coquina, $\mathrm{ij}$, per $\mathrm{j}$ tailliam. In supravenditis, cxxxviii casei qui f'ecerunt $\mathrm{x}$ capita, de reragio anni præoteriti. In supravenditis clx.xvij casei, qui fecerunt xviij capita in hoc anno. In expensis domini Regis et domini Episcopi in festo Sancti Leonardi et Sancti Martini, xix de parvis caseis, $\mathbf{v}$ de majoribus, $d \epsilon$ reragio anno præeterito. Et remanent lij parvi casei qui faciunt $\mathrm{j}$ caput.

Idem reddunt compotum de cxxiiij porcis remanentibus anno præterito. Et de xxix, qui provenerunt de suibus. Summa cliij porci. In decimis, ij. In mortuis, ix. In occisis arl lardarium, iiij ${ }^{x x}$ iij. Summa iij $^{x x} . x v$ porci. Et remanent lviij porci. Item xix porcelli. Summa tocius, lx.xvij porci.

Idem reddunt compotum de xlviij gallinis de reragio anni præteriti. Et de cclviij gallinis de cheriset. Summa cccvj. In expensis domini Episcopi in festo Sancti Martini, xxxvj, per $\mathrm{j}$ talliam. In expensis ejusdem in festo Sancti Leonardi, evj, per $\mathrm{j}$ tailliam. In expensis domini Regis et Episcopi in festo Apostolorum Petri et Pauli, cxxxj gallinæ, per ij taillias. In corredio Rogeri Wacel[ini], viij. In corredio magistri Roberti Basset, iiij. In mortuis, xxj. Summa cccvj gallinæ. Quietus est.

Idem reddunt compotum de cclx.xiij gallinis, xxvij estikis anguillarum, iiij porcellis, liberatis ad expensas domini Regis et Episcopi.

De Lardario.-Idem liberaverunt ad expensas domini Episcopi, carnem ij vaccarum allatarum apud Craneburnam.

Idem reddunt compotum de xiij baconibus, reragii anni præteriti. Et de $\mathbf{v}$ bobus j quarterio de veteri carne boum de reragio, anni præteriti. Et de iijjxiij porcis de Duntona. Et de lx.xj porcis de Meredona. Et de $\mathrm{x}$ porcis de Overtona. Et de ix porcis de Clera. Et de xiiij porcis de Harewella. Et de vij porcis de Cnoel. ${ }^{2}$ Summa, ccviij porci, et carnes $\mathrm{v}$ boum, et $\mathrm{j}$ quarterium. In expensis domini $\mathrm{E}_{\mathrm{j}}$ iscopi in festo Sincti Martini, viij bacones. In expensis ejusdem in festo Sancti Leonardi, $\mathrm{x} v \mathrm{ij}$ bacones, carnes $\mathrm{v}$ boum, et $\mathrm{j}$ quarterium bovis. In expensis ejusdem in crastino Sanctre Crucis, liberati Nicolao coco, xxvij bacones. In expensis domini Episcopi, liberatis eidem coco apud Cnoel, die Sabbati ante festum Sancti Michaelis, xv bacones. In expensis ejusdem et domini Regis, in festo Apostolorum Petri et Pauli, 1 bacones. In corredio magistri R[oberti] Basset ad festum Omnium Sanctorum, dimidium baconem.' In corredio ejusdem die Mercurii et Jovis ante Pentecosten, $\mathrm{j}$ baconem. ${ }^{1}$ In missis apud Cnoel ar precarias auptumnales, vj bacones. In iij precariis auptumnalibus apud Duntonam, ix bacones dimidius.

Summa, cxxxiiij bacones. Et remanent lx.xiiij bacones.

Idem reddunt coinpotum de schinis, salcigis et sulcit[io] prædictorum porcorum. In expensis domini Regis et domini Episcopi, in festo Sancti Leonardi, totum. Nichil remanet.

\section{OVERTONA.}

Baldewinus de WitTeneia et W[illelmus], præpositus, reddunt compotum de xlixs. $o b$., de reragio anni tercii. Et de lijs. vjd. de vij capitibus dimidlio casei, de reragio anni tercii. Et de xviijd. de iij caseis venditis de reragio. Summa vl. iijs. ob.

Idum reddunt compotum de $x i x l$. ijs. de gabulo, per annum. In quietancia $\mathrm{j}$ præpositi, per annum, vs.; j berbrecti, ij haiwardorum, per annum, ixs.; j porearii, j vaccarii, ij bercariorum, per annum, xijs.; vij carrucariorum, per annum, xxjs.; ij operariorum, vjs.; iij custodum bladi, per dimidium aunum, iiijs. vjd.; ij custodum, per j terminum, xviijd.; j custodis agnorum, per annum, iijs. Summa lxijs.

In defectu terræ fabri, per annum, iijs. In defectu terræ Pilvorki, xijd. Summa iiijs. Summa tam quietanciarum, quam defectuum, lxvjs. Summa remanens, xvl. xvjs.

Idem reddunt compotum de $v l$. $x s$. de tallagio. Et de $x s . o b$. de pannagio. Et de vjs. iijd. de herbagio. Et de viijs. de $\mathrm{j}$ avro vivo vendito. Et de lviijs. iiijd. de 1 multonibus vivis venditis. Et de xijd. de $\mathrm{v}$ hogettis vivis venditis. Et de iijjs. ixd. 
de xvij agnis vivis venditis. Et de ixs. iijd. de v coreis houm, ij vaccarum mortuarum, venditis. Et de vs. vijd. de xvj pellibus ovium, xj pellibus multonum, xvj pellibus de hogettis mortuis venditis. Eu de vjs. vjd. de iiij ${ }^{x x}$.xvij pellibus, agninis mortuis venditis. Et de ljs. de relaxacione precariarum hyemalium. Et de xliiijs. vjd. de vj capitibus casei venditis. Et de $\mathrm{x}$ marcis, de $\mathrm{v}$ ponderibus grossæ lanæ. Et de dimidia marca, de lana agnina vendita. Lt de ijs. de fratre Roberti, pro pastura vendita. Et de xxs. vjd. de scotallo ciceræ. Et de viijd. de butiro vendito. Summa xxiijl. xviijs. vd.

Idem reddunt compotum de lijs. $\mathrm{jj} d$. de xvij quarteriis dimidio, $\mathrm{j}$ estrica, frumenti venditis. Et de xxvs. iijd. de xiiij quarteriis dimidio, $\mathrm{j}$ estrica, mancorni venditis. Et de xxxs. ixd. ${ }^{1} o b$. de xvij quarteriis dimidio, ordei venditis. Et de ijs. de ij quarteriis 10 avenæ venditis. Summa vl. ixs. ijd. ob.

De Purchasiis.-Idem reddunt compotum de Jolıanne Cokerel pro discessione. Et de vjs. viijd. de Cristino de Nodwaltham pro discessione. Et de ijs. de Ada haiwardo pro pastura. Et de iijs. de Willelmo Teler pro simili. Et de xijd. de Galfrido Peni pro simili. Et de vjd. de Alano Merwon pro simili. Et de ijs. de Roberto Pallet pro simili. Et de vjd. de 15 Johanne Gervasii pro simili. Et de ijs. de Waltero Prat pro simili. Et de vjd. de Willelmo Baldicot pro simili. Et de iijs. de Alfredo Bere pro simili. Et de vjd. de Thoma molindario pro simili. Et de vjd. de Edwardo molindario pro simili. Et de xijd. de Ricardo de Linche ${ }^{2}$ pro simili. Et de vjd. de Willelmo Steorre pro lege. Et de vjs. de thedinga de Overtona. Et de iijs. de thedinga de Dene. Et de xs. de willata 20 de Waltham pro veredicto. Et de vm. de Ricardo de Thadelega pro auxilio. Et de iijs. de Gocelino pro pastura. Et de vs. de Roberto de Thadel ga jro maritagio. Et de vjd. de Alfredo Bere pro mellea. Et de vjd. de Galfrido Gos pro simili. Et de xijd. de Osberto de Alresforde pro bosco. Et de vjd. de Gileberto quia noluit reddere servicium pastoris. Et de xijd. de Giliberto pro falso clamore. Ei de ijs. de Alfredo Bere pro mellea. Et de 25 iiijs. de Ivone pro terra Johannis Cokerel habenda. Et de xijd. de Hereberto pro terra Sidegivæ habenda. Et de xijd. de Henrico.

Summa vijl. viijs. xd. Summa tocius receptæ, lijl. xjs. vjd. ob. Excepto reragio.

Liberatio.-In liberatis J[ohanni] decano xvl. xijs. iiijd. de gabulo, per iiij taillias. Eidem iiijl. ixs. vjd. de reragio, per j tailliam. Eidem vl. xs. de tallagio, per j tailliam. Eidem 30 xlixs. de blado vendito, per $\mathrm{j}$ tailliam. Eidem vjl. xs. de lana vendita, per $\mathrm{j}$ tailliam. Eidem $\mathrm{xx} s$. de sicera, per $\mathrm{j}$ tailliam. Eidem, lviijs. iiijd. de multonibus venditis, per j tailliam. Eidem xxxviijs. vjd. de caseo vendito, per j talliam.

Summa xxxvl. xviijs. viijd.

Expensa.-In ferramento iiij carucarum, 'x avrorum, per annum, xvijs. $x d$. In mercede fabri 35 pro reparacione ferramenti iijj carucarum, per annum, xs. viijd. In rotis carucarum et aliis paramentis rarucarun, per annum, ijs.ob. In jaramentis bigæ fractæ, $\mathrm{x} d . o b$. In expensis precariarum hyemalium et quadragesimalium, iijj. vjd. In $\mathrm{j}$ avro empto, iiijs. vjd. In clatis ad faldam et uiscatura, vjs. vjd. In custo daeriæ, pressura, pannis, et aliis custamentis, iijj. xjd. In blado sarclando, vs. ob. In prato falcando de Overtona 40 et de 'Thadelega, iijs. ijd. In stipendio carettarii, bovarii. per annum, vjs. In stipendio daiæ, ijs. In corredio senescalli, per $\mathrm{v}$ vices, xiijs. ijd. ob. In grangiis et cæeteris domibus reparandis et cooperiendis, xxiijd. In fossatis circa bercariam et Luvegerstonam, vs. ixd. In porcharia paranda, iiijd. In ostio herchariæ faciendo, iiijd. In arura $\mathrm{xxj}$ acrarum, iijs. vjd. In $\mathrm{j}$ trancheia ad closturam, $\mathrm{ij} s$. In comis, iijob. In iiij quarteriis frumenti 45 emptis ad semen, xiijs. vjd. In dimidio quarterio fabarum empto ad semen, $x d$. $o b$. In porta berchariæ et $\mathrm{j}$ tum . . ${ }^{3}$ iijd. In triturandis ij sextariis frumenti, xiijd. In muro punfaldæ faciendo, xjd. In saccis lanæ, iijs. $\mathrm{j} d$. In $\mathrm{j}$ corda ad caretam, iijob. In chiphis, vjd. In dignerio Johannis de Seles, iijs. xjd. In liberatione servientis, lxs. $x d$.

Summa ixl. iijd. ob.

Summa tocius liberationis et expensæ, xliiijl. xviijs. xjd. ob. Et debet vijl. xijs. vijd. Unde W[illelmus] serviens debet xxxiiijs. iiij $d$.

Exitus Grangiæ.-Idem reddunt compotum de xxxiv quarteriis dimidio j estrica de toto exitu M. 4. frumenti, et de iiij quarteriis emptis. Summa xxxviij quarteria dimidium, $\mathrm{j}$ estrica. In semine xlij acrarum, xiij quarteria. In mixto cum semine mancorni, viij. In supravenditis, xvij quarteria dimidium, $\mathrm{j}$ estrica.

Idem reddunt compotum de iijj $^{x x}$.xix quarteriis $\mathrm{j}$ estrica de toto exitu mancorni. In semine ceviij acrarum, lxviij quarteria dimidium, unde viij sunt de frumento, et ex his supra reddunt compotum. In precariis hyemalibus, iij quarteria dimidium. In 60 precariis quadragesimalibus, $\mathrm{j}$ quarterium. In hominibus qui dant cheriset, $\mathrm{j}$ quarterium.

1 ixd. interlineated in MS. $\quad$ Ricardo de an interlineation. $\quad$ The margin of the Roll is mutilated. 
In porcis pascendis, iij quarteria. In liberatione $\mathrm{ij}$ servientium, per annum, xiij quarteria. In liberatione daiæ, ij quarteria dimidium. In supravenditis, xiij quarteria dimidium, j estrica.

Idem reddunt compotum de xxxij quarteriis dimidio de toto exitu ordei. In semine xlviij acrarum, $x v$ quarteria. In supravenditis, $x$ vij quarteria dimidium.

Idem reddunt compotum de ciiij quarteriis $\mathrm{j}$ estrica de toto exitu avenæ. In semine ciiij ${ }^{x x}$.x viiij acrarum, lxix quarteria dimidium. In præbenda domini Episcopi, iij quarteria iij hopæ, per $\mathrm{j}$ tailliam. In probenda senescalli, $i$ ij quarters iij hops. ${ }^{1}$ In præbenda $x$ avrorum, per annum, xxj quarteria dimidium. In bobus, iiij quarteria dimidium. In supravenditis, ij quarteria.

Instaurum.-Idem reddunt compotun de $\mathrm{xj}$ avris remanentibus alıno praterito et de $\mathrm{j}$ empto. Summa $x \mathrm{ij}$. In mortuo $\mathrm{j}$, undo dealbatum est corium. In vivo vendito, $\mathrm{j}$. De precio superius respondent. Et remanent $x$ avri.

Idem reddunt compotum de j pullo provento hoc anno. Et remanet.

Idem reddunt compotum de xxxvj bobus remanentibus anno praterito et de ij adjunctis de instauro. Summa xxxviij. In occisis in termino Sancti Martini, iij. De coriis et carne superius respondent. In mortuo, j. De corio superius respondent. Et remanent xxxiij.

Idem reddunt compotum de xviij vaccis, $\mathrm{j}$ tauro, remanentibus anno præterito. Et de $\mathrm{j}$ fortuitu inventa. Summa xx. In missis apud Bitterne vj, per talliam. ${ }^{2}$ In missis apud Stokes, $v$, per $\mathrm{j}$ talliam. In mortuis, $\mathrm{ij}$. De coriis superius respondent. Et remanent vj vaccæ, $\mathrm{j}$ taurus.

Idem reddunt compotum de ij bovettis remanentibus anno præterito. In adjunctis bobus ij.

Idem reddunt compotum de $\mathrm{j}$ annali remanente, anno præterito vitulo. In misso apud Bitterne $\mathrm{j}$, per $\mathrm{j}$ tailliam.

Idem reddunt compotum de iij vitulis hujus anni, quia iij vacco fecerunt aborsum. Ibi remanent.

Idem reddunt compotum de cclxviij ovibus remanentibus anno præterito et de vij de reragio; qui ita remanent adhuc. Et de iijj $^{x x} . x j$ adjunctis de instauro. Summa ccclxvj. In mortuis ante partum, iiij De pellibus superius respondent. Post partum et ante tonsionem, vij. De pe:libus superius respondent. Post tonsionem, v. De pellibus superius respondent. Summa xvj. Et remanent cccxlvj. ${ }^{2}$

Idem reddunt compotum de cciiij ${ }^{x}$.xiij multonibus remanentibus anno præterito. Et de iiij ${ }^{x x} . x v$ adjunctis de instauro. Summa ccciij ${ }^{x x} \cdot x^{x j}{ }^{2}$ In mortuis ante tonsionem, $x$. De pellibus superius respondent. Post tonsionem, j. De pelle superius respondent. In venditis post tonsionem, I. De precio superius respondent. Summa lxj. Et remanent cccxxxj. ${ }^{2}$

Idem reddunt compotum de ccvij hogettis remanentibus, anno præterito agnis. In adjunctis ovibus, iiij $^{x x} \cdot x j$. In adjunctis multonibus, ${ }^{1 i i j}{ }^{x x} \cdot x v$. In mortuis ante tonsionem, xv. De pellibus superius respondent. In mortuo post tonsionem, $j$. De pelle superius respondent. In venditis post tonsionem, v. De precio superius respondent.

Idem reddunt compotum de cclviij agnis proventis hoc anno, quia vj oves erant steriles. Et de xxiiij agnis proventis de hogettis hoc anno. Summa cciijux $x_{j j}$. In decimis, $x x v$. In consuetudine bercarii, $j$. In venditis, $x v i j$. In mortuis $i i j \mathbf{x x} . \mathbf{x v i j}$; scilicet ix post tonsionem. De pellibus superius respondent. Et remanent cxlv agni.2

Idem reddent cumpotum de l)cexxxij velleribus grossis. In decimis lx.xiij. In consuetudine ij bercariorum, ij. In supravenditis, dclvij. De precio superius respondent.

Idem reddunt compotum de clj velleribus agninis. In venditis, totum, quod fecit j quarterium. De precio superius respondent.

Idem reddunt compotum de clx.xj caseis, qui inceperunt fieri in vigilia Pasclıæ et desierunt in die Exaltationis Sanctæ Crucis, utruque die computato. In decimis, xvij. In consuetudine bercarii, $d i i \rightsquigarrow, i j$. In precariis auptumnalibus, $x$. In venditis, $v^{\mathbf{x x}} \cdot \mathrm{xviij}$.

Idem reddunt compotum de $\mathbf{x x x v}$ porcis remanentibus auno præterito. Et de xv proventis hoc anno. Summa $\mathrm{l}$. In decimis, $\mathrm{j}$. In consuetudine porcarii, $\mathrm{j}$. In mortuis, iij. In missis apud Duntonam, $x$, per $j$ talliam. Et remanent xxxv. Unde j verres, $x i j$ sues, $x$ superannati, vj hogetti, vij porcelli.

Idem reddunt compotum de cvij gallinis receptis de cheriset. In liberatis J[olianni] decano totum, per $\mathrm{j}$ tailliam.

Idem reddunt compotum de iij carnibus ${ }^{2}$ boum. In expensis precariarum auptumnalium, totum. Quietus est. Et debet viijl. iiijs. viijd. $o b$.

\section{FERHAM.}

W[ILLELMUS] DE DAMARTIN et G[alfridus] præpositus et W[illelmus] grangiarius reddunt computum de iijl. xvs. ixd. de reragio anni præteriti. Quietus est.2

1 In prebenda . . . hops is a marginal insertion in another band. 2 sic in MS. 
Idem reddunt compotum de xxij'. xiijjs. vjd. jqdr. de gabulo assiso. Et de xijd. de Stephano Oisel per j acram dimidiam de incremento gabuli hoc anno. Et de iijj. $a b$. de incremento gabuli Herevei pro ${ }^{1}$ iij partibus $\mathrm{j}$ acræ. Et de vjd. de incremento gabuli Galfridi de Porta pro j acra. Et de iijl. de incremento gabuli Thomæ de Solio pro dimidia acra. Et de iijob. de incremento gabuli Galfridi Pur pro iiij parte $\mathrm{j}$ icræ. Summa, xxijl. xvje. ixd. jqdr. Summa tam reragii quam gabuli, xxvijl. xijs. vjd. jqdr.

In quietancia G[alfridi] præpositi, vs.; $\mathrm{j}$ grangiarii, $\mathrm{j}$ porcarii, iijs.; $\mathrm{j}$ bercarii, iijs.; $\mathrm{j}$ fabri, iijs. vjd.; vj carucariorum, per annum, $\mathbf{x}$ viijs. ; $\mathrm{x}$ operariorum, per annum, xxs. Summa liiijs. vjd.

In defectu gabuli terræ quæ fuit Fulconis, tractæ in dominicum, iijs. In defectu terræ 10 Anketilli, tractæ in dominicum, ijs. vjd. In defectu terræ Ingulfi, tractæ in dominicum, xijd. In defectu terræ Ricardi de Bello Monte, quæ modo operatur et non solebat, ijs. In defectu terræ Hawis[iæ], tractæ in dominicum, xijd. Summa, ixs. vjd.

Summa tam quietanciarum quam defectuum, lxiijjs. Et reminet xix $l$. xijs. ixd. jqdr.

Idem reddunt compotum de xlvjs. viijd. ob. de pannagio. Et de ijs. de herbagio. Et 15 de xlvjs. de xxiiij ovibus vivis venditis in termino Sancti Martini. Et de iijs. iiijd. de xvj agnis vivis venditis. Et de $x v s$. vd. de coriis $x i j$ boum, $j$ avri, venditis. Et de ijs. iijd. de iij hurtardis vivis venditis. Et de $\mathbf{x x d . ~ o b . ~ d e ~ p e l l i b u s ~} x \mathrm{j}$ ovium, $\mathrm{j}$ multonis, j hoggetti, iij agnorum mortuorum venditis. Et de xxxvijs. vjd. de j pondere dimidio grossæ lanæ vendito. Et de vijs. de $\mathbf{v}$ petris lanæ agninæ vendita. ${ }^{2}$ Et de $x s$. pro j 20 capite dimidio casei vendito. Et de viijd. de $\mathbf{x} v j$ caseis parvis venditis. Et de xiijs. $\mathrm{x} d$. de coperonis venditis in bosco, unde mairemum ${ }^{3}$ tractum fuit apud Merewellam. Et de iiijl. viijd. $o b$. de $\mathrm{v}$ tonellis vini venditis. Summa xjl. xvijs. iijob.

Idem reddunt compotum de vijl. ijs. iijd. de lv quarteriis dimidio frumenti venditis. Et de $x v j$ s. jd. de $x \mathrm{j}$ quarteriis dimidio curalli venditis. Et de vs. iiijd. de ij quarteriis silliginis venditis. Et de $x x j$ s. de xxviij quarteriis de veteri avena venditis. Et de iijs. de iij quarteriis vesciæ venditis. Summa ixl. viijs. viijd.

De Exitibus Molindinorum.-Idem reddunt compotum de xxxvjs. $x d$. de xiiij quarteriis dimidio frumenti venditis. Et de $v l$. iijs. ijd. de lij quarteriis dimidio mancorni venditis. Et de $x s$. de iiij quarteriis gruelli venditis. Et de ixs. de vj quarteriis brasei venditis. 30 Summa viijl.

De Purchasiis.-Idem reddunt compotum de vl. iijs. viijd. de purchasiis.

Idem reddunt compotum de $x x l$. de tallagio. Summi summarum cum tallagio, lx. xiiijl. xvijd. iijgdr. ${ }^{4}$

Liboratio.-In liberatis J[ohanni] decano, de termino Natalis, xixl. vijs. ijd. per iij tallias. 35 Eidem, de blado vendito, $x l$. xs., per j tailliam. Eidem, de tallagio, xixl. vjs. vijd. Eidem, sl. xiijs., per j tailliam.

Summa tocius liberationis, lixl. xvjs. $x d .{ }^{4}$

Expensa...-In ferramentis iij carucarum, $\mathrm{ij}$ avrorum, per annum, xs. vijd. In rotis, jugis ad idem, viijd. In custamento daeriæ, ijs. iijd. ob. In mercede ij herciatorum, xxd. In blado 40 vanando, $x i j d$. In precariis hyemalibus de liiij carucariis, pro companagio, vjs. $x d$. In fimis extrahendis de curia ad fimandum vij acras, iijs. vjd. In bladosarclando, xviijd. In pratis falcandis, xijd. In $\mathrm{j}$ pari ${ }^{3}$ rotarum ad caretam, xijd. In paramentis caretarum, $v d$. In aula, thalamo, et cæteris domibus emendandis et cooperiendis, ijs. viijd. In clavis emptis ad idem, $x d$. In $v j$ bobus emptis, xxxvjs. $x d$. In $\mathrm{j}$ avro empto, vjs. vjd. In 45 expensis magistri R[oberti] Basset per iijj taillias et totidem vices, xvijs. ixd. In liberatione Willielmi de Danmartin, lxs. $x d$. In $\mathrm{j}$ quarterio dimidio ordei empto ad semen, ijs. vjd. In ferramentis iij molindinorum, xijs. $x d$. In $\mathbf{j}$ mola empta ad molindinum maris cum cariagio de Hamptona, xxvs. iiijd. In sclata empta ad molindinum maris cooperiendum, ixd. ob.

Summa ixl. Ivijs. iiijd. Summa liberationis et expensæ, lxixl. xiiijs. jd. Et debet iiijl. vijs. iiijd. iijqdr.

Exitus Molindinorum.--Idem reddunt compotum de xiiij quarteriis dimidio frumenti. In supravenditis totum. Et de iij quarteriis gruelli. In supravenditis totum. Et de vj quarteriis brasei. In supravenditis totum. Et de iijj ${ }^{x x} . \mathrm{xv}$ quarteriis mancorni. 55 In corredio vj bovariorum, $\mathrm{j}$ porcarii, per annum, $\mathrm{j}$ herciatoris, per xxiiij septimanas, $j$ per $x v j$ septimanas, xlij quarteria dimidium. In supravenditis, lij quarteria dinidium.

1 pro is interlineated. 2 'The last two words are marked for transpesition as printed.
${ }_{3}^{3}$ paria in MS. 
Exitus Grangiø.-Idem rediunt com otum de cxxvj quarteriis de toto exitu frumenti. In semine cclxij acrarum, Ixiiij quarteria. In pane ad precarias hyemales, ij quarteria dimidium. In pane ad precarias auptumnales, iiij quarteria. In supravenditis, lv quarteria dimidium.

Idem reddunt compotum de xiij quarteriis dimidio de toto exitu curailli. In porcis pascendis, $j$ quarterium dimidium. In custodi agnorum, dimidium quarterium In supravenditis, $x j$ quarteria dimidium.

Idem reddunt compotum de $\mathrm{ij}$ quarteriis silliginis de cheriset. In supravenditis totum.

Idein reddunt compotum de xxiij quarteriis de toto exitu ordei. Et de xij quarteriis allatis de Hameledona. Et de $\mathrm{j}$ quarterio dimidio emptis. Summa xxxvj quarteris dimidium. In semine $x l v j$ acrarum, $x$ viij quarteria. In corredio prædictorum servientium, vij quarteria. In iij precariis suptumnalibus, $\mathbf{x}$ quarteria. In braciat[ura] ad precarias hyemales, $\mathrm{j}$ quarterium dimidium.

Idem reddunt compotum de iijj.xix quarteriis dimidio de veteri avena, de reragio anni præteriti. In liberatis Johanni decano de Wlveseia, lxiiij quarteria iij buisselli, per $\mathrm{j}$ talliam. In braciat[ura] ad precarias hiemales, iij quarteria. In supravenditis, xxviij quarteria. In amissione per cariagium in ventil[atione], iij quarteria $\mathrm{j}$ bussellus.

Idem reddunt compotum de ciiij ${ }^{x i j}$ quarteriis dimidio $\mathrm{ij}$ bussellis de toto exitu avenæ hoc anno. ${ }^{1}$ In semine cc ${ }^{2}$ acrarum cxxv quarteria dimidium. Iu liberatis $\mathrm{P}[$ etro] Ace, ad opus domini Episcopi in die Sanctæ Trinitatis, per ij noctes, xxiij quarteria ij busselli, per $\mathrm{j}$ talliam. In missis apud Waltham, $\mathrm{v}$ quarteria, per $\mathrm{j}$ talliam. In præbenda senescalli, iij quarteria dimidium, iij busselli, per iiij tallias et per totidem vices. In præbenda G. de Aclanda die Veneris post Purificationem Beatæ Mariæ, v busselli, per j talliam. In præbenda boun a vigilia Natalis Domini usque ad xviij Kalendas Maii, per cxj $^{3}$ noctes, $x^{3}{ }^{3}$ quarteria. In præbenda ij avrorum a festo Sancti Martini usque ad xviij Kalendas Maii, scilicet per clv nortes, v quarteria dimidium.

Idem reddunt compotum de $\mathrm{ij}$ quarteriis de toto exitu pisarum. In semine iij acrarum, $\mathrm{j}$ quarterium. In conredio prædictorum servientium, $\mathrm{j}$ quarterium.

Idem reddunt compotum de iiij quarteriis dimidio, de toto exitu vesciæ. In semine $\mathrm{v}$ acrarum dimidix, $\mathrm{j}$ quarterium dimidium. In supravenditis, iij quarteria.

Instaurum.-Idem reddunt compotum de $\mathrm{ij}$ avris remanentihus anno præterito. Et de $\mathrm{j}$ postea empto. Summa iij. In mortuo, j. Et remanent ij.

Idem reddunt compotum de xlj bobus remanentibus anno præterito. Et de vj postea emptis. Et de ij de testamento Hugonis Piscatoris et Bretelli de Rethereiate. Summa xlix. In occisis in lardario, v. In mortuis, vij. De coriis superius respondent. Summa xij. Et remanent xxxvij.

Idem reddunt compotum de $\mathrm{j}$ vacca de testamento Rogeri Serich. Et de $\mathrm{j}$ vitulo provento de eadem vacca. Et de $j$ annali masculo de testamento Thomæ Withonde. Et de $\mathrm{j}$ annali femella fortuitu inventa. Summa $\mathrm{j}$ vacca, $\mathrm{j}$ annalis masculus, $\mathrm{j}$ annalis femella $\mathrm{j}$ anni, et $\mathrm{j}$ vitulus. Omnes remanent.

Idem reddunt compotum de clxj ovibus remanentibus anno præterito. Et de xlvij gerciis adjunctis. Summa ccviij. In mortuis ante partum, vj. In mortuis post tonsionem, v. De pellibus superius respondent. In supravenditis xxiiij in termino Sancti Martini. De precio superius respondent. In consuetudine bercarii, j. Summa xxxvj. Et remanent clx.xij.

Idem reddunt compotum de $\mathbf{x}$ multonibus remanentibus anno præterito. Et de lxiiij masculis adjunctis. Summa lx.xiiij. In vivis venditis, iij. De precio superius respondent. In precariis auptumnalibus, j. De pelle superius respondent. Summa iiij. Et remanent lx.x multones.

Idem reddunt compotum de exij hoggettis remanentibus, agnis anno præterito. In mortuo ante tonsionem, j. Et remanent cxj; unde xlvij sunt gerciæ, junctæ ovibus ; lxiiij masculi, juncti multonibus. Nichil remanet.

Idem reddunt compotum de cxxiij agnis proventis de ovibus hoc anno, quia iij fuerunt steriles, ij fecerunt aborsum. Et de xv agnis proventis de gerciis, quia cæateræ fuerunt steriles. Summa cxxxviij. In decimis, xij. In consuetudine bercarii, $j$. In vivis venditis, $x v j$. De precio superius respondent. In mortuis ante tonsionem, $x j$. In mortuis post tonsionem, $\mathrm{ij}$. De pellibus superius respondent, Summa xliij. ${ }^{2}$ Et remanent iijj $^{x x} \cdot \mathrm{xv}^{2}$ agni.

Idem reddunt coinpotum de ccxlviij velleribus grossis. In consuetudine bercarii, $\mathrm{j}$. In decimis, xxiiij. In supravenditis, ccxxj, quæ fecerunt $j$ pondus dimidium. Et debet ij vellera. Valent iij ob.

Idem reddunt compotum de $\mathrm{iij}^{\mathbf{x x}} . \mathrm{xvij}$ velleribus agninis. In supravenditis, totum, quæ fecerunt $\mathrm{v}$ petras.

Idem reddunt compotum de ciijux xiiij caseis qui inceperunt feri xiijto Kalendas Aprilis, et desierunt in festo Sancti Michaelis, utroque die computato. In decimis, xix. In consuetudine bercarii, $\mathrm{j}$. In liberatis in coquina domini Episcopi, $\mathrm{j}$, per $\mathrm{j}$ talliam. In 
liberatis P[etro] Juveni in dispenea domini Episcopi, ij per $\mathrm{j}$ talliam. In iij precariis auptumnalibus, iiij ${ }^{\mathbf{x}} v$. In supravenditis, lx.x, qui fecerunt $\mathrm{j}$ caput dimidium. In supravenditis, xvj parvi casei. Et quietus est. ${ }^{1}$

Idem reddunt compotum de $\mathrm{lxv}$ porcis remanentibus anno præterito. Et de $x x i j$ proventis de suibus. Summa iiij ${ }^{x x}$ vij. In decimis, ij. In consuetudine porcarii, $\mathrm{j}$. In mortuis, vij. In liberatis ad lardarium de Waltham, xxxvij. Summa xlvij. Et remanent $x l$, de quibus vj sunt sues, $\mathrm{j}$ verres, $x x$ suellæ, xiij masculi.

Idem reddunt compotum de $\times x \times v$ gallinis, vj aucis remanentibus anno præterito. In liberatis in coquina domini Episcopi, xxijj ${ }^{2}$ per $\mathrm{j}$ talliam. Et remanent vj aucæ, xij gallinæ. Idem liberaverunt in coquina domini Episcopi carnes $v$ boum, per $\mathrm{j}$ tailliam.

Idem reddunt compotum de $\mathrm{v}$ tonellis vini remanentibus anno præterito. Et de ij receptis de Hamptona per llanum Danielis. Et de ij de dono R[oberti] de Turneham. Summa ix. In liberatis pincernæ domini Episcopi, iij. Sed sciendum est quod $R$ [obertus] de Turneham potavit in vigilia Ascensionis et die feræ diuidietatem ij tonellorum. In supravenditis, $\mathbf{v}$ tonelli. Nihil remanet.

Summa tocius debiti, iiijl. vijs. vjd. ob.

Solverunt post compotum et quieti sunt de toto.

\section{WEREGRAVE.}

W[rletelyos] DE Cantoc et Willelmus præpositus reddunt compotum de iijl. xvjs. ijd. de reragio anni præteriti. Solverunt et quieti sunt. Et de xxvl. xs. $x d$. ob. de gabulo assiso. Et de jd. de incremento gabuli Radulfi [de] Frida[m]. Summa xxvl. xs. xjd. ob.

In quietancia $\mathrm{j}$ præpositi de Weregrave, xviijd.; $\mathrm{j}$ præpositi de Walthan, ijs.; j præpositi de Culnam, xijd.; j forestarii de Weregrave, iijs.; j forestarii de Waltham, ijs.; j forestarii de Warefelde, ijs.; iiij carucariorum de Weregrave, iij carucariorum de Waltham, iiij carucariorum de Culnham, vjs. per annum; $\mathrm{j}$ porcarii, $\mathrm{j}$ bercarii, $\mathrm{j}$ custodis 25 vaccarum, per annum, xviijd.

Summa quietanciarum xixs. Et remanent xxiiijl. jxs. xjd. ob.

Idem reddunt compotum de $x l$. de redditu annuo. Idem reddunt compotum de exvijs. $x d$. $o b$. de pannagio. Et de ziijs. de herbagio. Et de lvjs. de ix bobus, ij vaccis, vivis venditis. Et de xjs. de coreis $\mathrm{v}$ boum, iij vaccarum mortuorum venditis. Et de 30 iijs. vijd. $o b$. de pellibus $x \times v$ ovium, viij multonum, $\mathbf{x}$ hoggettorum, $x \mathrm{j}$ agnorum mortuorum venditis. Et de xxvijs. de j pondere grossæ lanæ et agninæ venditis. Et de xxiiijs. de iij capitibus casei venditis. Et de cs. de relaxacione operationum. Summa xvijl. xijs. vjd.

Idem reddunt compotum de xxvjs. iijd. de $\mathbf{x}$ quarteriis frumenti venditis. Et de 35 xjl. xiiijs. ijd. ob. de cxj quarteriis, j estrica mancorni venditis. Et de lxjs. iijd. de xxxj quarteriis dimidio ordei venditis. Et de $x j l$. xixs. $x d$. de ccxxxij quarteriis avenæ venditis. Et de vjs. de iiij quarteriis dimidio pisarum venditis.

Summa xxviijl. vijs. ${ }^{1}$ vijd. $o b$.

De Purchasiis.-Iden reddunt compotum de xls. de Roberto de Waltham pro fine terræ. Et 40 M. 4d. de vjs. viijd. de Osberto filio Baldwini pro simili. Et de iijs. de Symone Lep pro simili. Et de $x l s$. de Stephano de Cruce pro sepe de Pillingebere asportata. Et de xxs. de thedinga pro communi misericordia. Et de vjd. de Symone Kemelif pro mellea. Et de vjd. de Giliberto bercario quia oves suæ jacuerunt extra faldam Episcopi. Et de xxjd. de Osberto de la Lake pro defectu operis. Et de zijd. de Radulfo Ruffo pro simili. Et 45 de vjs. viijd. de Radulfo filio Godæ ut possit discedere liber. Et de iiijs. de Willelmo de Strode pro filia sua maritanda. Et de iijs. de Ernolfo pro simili. Et de ijs. de Radulfo Lep pro simili. Et de xijd. de Henrico Cres pro lege relaxata Et de iijs. de Osberto filio Baldwini pro sorore sua maritanda. Et de xijd. de Radulfo de Frid[am] pro lege relaxata. Et de $x l$. iiijs. de Osberti de Hurl[ega] pro terra de Widegate.

Summa xvjl. xviijs. jd.

Idem reddunt compotum de $x l l$. de taillagio.

Summa summarum, cum taillagio, cxxxvijl. xs. ijd.

Liberatio.-In liberatis J[ohanni] decano de duobus terminis, xxiiijl. xjs. ixd. ob. Eidem de blado vendito, dono annuo, et purchasiis, lvjl. vs. $x d$. per ij tallias. Eidem de taillagio 55 xll. per j tailliam.

Summa, cxxl. xvijs. vijd. ob.

\footnotetext{
Sic in MS. SOn an erasure in MS, 3 A later marginal insertion in another band.
} 
Exponsa.-In ferramentis vj carucarum per annum, xxijs. ixd. In rotis ad carrucas, xijd. In mercede fabri de Waltham, iijs. In ferrura iij carettariorum, vij avrorum, per annum, vs. viijd. In ij paribus rotarum ad caretas de Waltham et Culnham, ijs. In paronis, paronellis, sellis, bacis, clutis et axandis ${ }^{1}$ cum uncto, $x$ viijd. In rotis parandis ad karros ex mairemio Episcopi, xijd. In custode agnorum per dimidium annum, ijs. In ij grangiis factis ex novo apud Weregrave et Culnham, xixs. vjd. In ij serruris emptis ad grangiam, iijd. In vij quarteriis frumenti emptis ad semen, xxjs. In iij quarteriis mestilonis emptis ad semen, $x s$. In custo daeriæ, ijs. $x d$. In stipendiis iij servientium et daix, xijs. In iiij precariis auptumnalibus, xvs. $x d$. In liberatione W[illelmi] de Cantoc, lxs. $x d$. In conredio Mathæi Pernin qui remansit infirmus, ijs. ijd. In conredio Udonis in auptumno a vigilia Sanctæ Mariæ Magdalenæ usque Decollationem Sancti Johannis per xxxix dies, sibi et homini suo, ixs. ixd.; singulis diebus, iijd. In ferrura equi sui, iijd. In pisce empto ad opus dunini Episcopi deferato apud Cliuar[ain], iijs. Is expensis magistri R[oberti] Basset per $v j$ itinera, xliiijs. vijd. $o b$. In lxv porcis emptis iiijl. $x j 8 . v d$.

Summa, xvjl. xijs. vjd. ob.

Summa tocius liberationis et expensæ, cxxxvijl. xs. ijd. Quietus est.

Exitus Grangiø.-Idem reddunt compotum de $\mathbf{x}$ quarteriis dimidio de Walthara, et de vj quarteriis dimidio de Culnham de toto exitu frumenti. Et de vij quarteriis frumenti emptis ıpud Weregrave ad seınen. Summa, sxiiij quarteria. In semine xxiij acrarum apud Weregrave, xvj acrarum apud Waltham, viij acrarum apud Culnham, xiiij quarteria. In supravenditis, $x$ quarteriı.

Idem reddunt compotum de cij quarteriis dimidio, iij bopis de Weregrave, lx.xij quarteriis, $\mathrm{j}$ estrica, iiij hopis de Waltham, xliiij quarteriis dimidio, $\mathrm{j}$ estrica, $\mathrm{j}$ hopa de Culnham, de toto exitu mestilonis. Et de iiij quarteriis emptis. Summa ccxxij quarteria dimidium, jestrica, iij hopæ. In semine, cxxxv acrarum de Weregrave, iijj ${ }^{x} v$ acrarum de Waltlıam, cxxxv acrarum de Culnham, iijjxix quarteria, dimidium. In consuetudine falcatorum prati de Weregrave, Waltham et Culnham, ij quarteria, $\mathrm{j}$ estrica, ij hopæ. In conredio iij servientium et daiæ per annum, $x x$ quarteria, dimidium, $\mathrm{j}$ estrica, j hupa. In supravenditis, exj quarteria, j estrica.

Idem reddunt compotum de $x$ xiiij quarteriis, $j$ estrica de Weregrave, vij quarteriis de Waltham, xv quarteriis dimidio de Culnham de toto exitu ordei. Summa xlvj quarteria dimidium, $\mathrm{j}$ estrica. In semine viij acrarum de Weregrave, $\mathrm{x}$ acrarum de $W$ altham, xxij acrarum de Culnham, xiij quarteria, $\mathrm{j}$ estrica. In porcis pascendis, ij quarteria. In supravenditis, $x \times x j$ quarteria, dimidium.

Idem reddunt compotum de cclxj quarteriis, ij hopis de Weregrave, iiijzx xiij quarteriis de Waltham, cxviij quarteriis de Culnham de toto exitu avenæ. Summa cccc.lx.xij" quarteria, ij hopæ. In semine cxxv acrarum de Weregrave, iij ${ }^{x x}$ acrarum de Waltham, cxv acrarum de Culnham, cxlix quarteria. In jræbenda domini Episcopi apud Weregrave et Cliuar[an! J et Radingian, Ix.xiij quarteria, j estrica, iij hopæ, per iiij tallias. In præbenda magistri $R$ [oberti] Basset, per vj itinera, vj quarteria dimidium, iij lıopæ. In $\mathrm{j}$ equo Mathæi Pernin, dimidium quarterium. In præbenda Odonis in auptumno, ij quarteria dimidium. In præbenda iij caretariorum, vijj quarteria. In supravenditis, ccxxxij quarteria.

Idem reddunt compotum de iij quarteriis de Weregrave, ij quarteriis de Waltham, de toto exitu pisarum. Summa vj quarteria. In semine ij acrarum de Waltam ${ }^{2}$ dimidium quarterium. In porcis pascendis $\mathrm{j}$ quarterium. In supravenditis, iij quarteria dimidium.

50

Instaurum.-Idem reddunt compotum de $x$ avris remanentibus anno præterito. Omnes remanent.

Idem reddunt compotum de lxiiij bobus remanentibus anno præterito. Et de $\mathrm{j}$ bove de testamento Baldwini Ov[er]gig. ${ }^{2}$ Et de ij junctis de ociosis annalibus. Summa lxvij. In vivis venditis, ix. In mortuis, v. Summa xiij. Et remanent liij boves.

Idem reddunt compotum de ij tauris remanentibus anno præteritu. Omes remanent.

Idem reddunt compotum de $x x$ vaccis remanentibus anno præterito. Et $d e j d e$ testamento Baldwini Lep. Et de ij fortuitu inventis. Et de $\mathrm{j}$ genicula juncta eisdem. Summa xxiiij vaccæ. In vivis venditis, ij. In mortuis, iij. Et remanent xix vaccæ.

Idem reddunt compotum de ij bovettis, $\mathrm{j}$ genicula anno præterito annales. In junctis bobus $\mathrm{ij}$. In juncta vaccis $\mathrm{j}$ genicula. Nichil remanet.

Idem reddunt compotum de vj annalibus anno præterito vituli. Umnes remanent, unde $\mathrm{ij}$ sunt geniculæ, iij masculi.

Idem reddunt compotum de $\mathbf{x}$ vitulis proventis hoc anno de vaccis quia cæteræ fuerunt steriles. In decimis, j. Et remanent ix vituli. 
Idem reddunt compotum de iijaiij ovibus remanentibus anno præterito. In mortuis ante partum, xxv. Et remanent lix nves. Eisdem junctæ sunt xix gerciæ. Summa utriusque, lx.xviij matrices oves.

Idem reddunt compotum de lv multonibus remanentibus anno præterito. In mortuis ante tonsionem, viij. In consuetudine falcatorum prati, post tonsionem, iij vivi. Et remanent xliiij multones. Eisdem juncti sunt $x x i j$ masculi. Summu utriusque, lxvj multones.

Idem reddunt compotum de lj hoggettis remanentibus, anno præterito agnis. In mortuis ante tonsionem, viij. In mortuis post tonsionem, ij. Et remanent $\mathrm{xlj}$; unde xix fuerunt gerciæ, junctæo ovibus, xxij masculi, juncti multonibus. Et, nichil remant.

Idern reddunt compotum de lvij agnis proventis hoc anno, quia ij fuerunt steriles. In consuetudine bercarii, $\mathrm{j}$. In decimis, $v$. In mortuis ante tonsionem, $x$. In inortuo post tonsionem, $\mathrm{j}$. Et remanent $\mathrm{xl}$ agni.

Idem reddunt compotum de cxlix velleribus grossis. In clecimis xiiij. In consuetu. dine bercarii, $\mathrm{j}$. In supravenditis exxxiij cum xlj velleribus agninis, quæ fecerunt 15 j pondus.

Idem reddunt compotum de clsr caseis qui inceperunt fieri xiiij Kalendas Maii et desierunt in festo Sancti Michaelis. In decimis, xvj. In consuetudine falcatorum prati, iij. In expensis senescalli, $\mathrm{j}$. In precariis auptumnalibus, iiij. In supravenditis, cxli casei, qui fecerunt iij capita.

Idem reddunt compotum de lxviij porcis remanentibus anno præterito. Et de xvij de pannagio. Et de xlviij provenientibus de suibus. Et de cclvij provenientibus de Londonia. Et de iiij ${ }^{x x}$ viij provenientibus de Wicumba. Et de lxv emptis. Summa Dxliij porci. In decimis, iiij. In consuetudine porcarii, j. In mortuis, xxix. In missis apud Wintonian ad lardarium de Wlveseia, ccxxv porci. In missis apud Londoniam 25 ad lardarium, cxx porci. Summa, ccclx.xix porci. Et remanent $\mathrm{iij}^{\mathrm{xx}}$.xix porci; unde $x$ sunt veteres sues, ij verres, $x l v i j$ juniores sues, $x l$ maelli. Summa remanens, clxiiij.

Idem reddant compotum de lx.x gallinis de cheriset. In expensis domini Episcopi, $\mathbf{x v}$ In expensis senescalli, $v$. Et remanent 1 gallinæ.

\section{BITERNE.}

JoHANNEs serviens et Azo præpositus reddunt compotum de cviijs. viijd. ob. de reragio. Solvunt et quieti sunt.

Idem reddunt compotum de vjl. vijs. iijd. de gabulo assiso. Et de vs. de firma parvi molindini. Summa vjl. xijs. iijd.

In quietancia j præpositi, per annum, iijs. ; j carucarii, per annum, iijs. Summa vijs. Summa remanens vil. vs. iij $d$.

Idem reddunt compotum de xxjs. de pannagio. Et de ijs. iijd. de herbagio. Et de vjs. vjd. de nucibus venditis. Et de xijs. ijd. de lx.xiij acris terræ locatæ. Et de xxiiijs. vjd. de cxliij caseis venditis, qui fecerunt iij pondera dimidium. Et de xxjs. de 40 $\mathrm{xxxj}$ ovibus $\mathrm{x}$ multonibus vivis venditis ad Natalem; ij oves quas bercarius occidit si sanæ fuissent. Et de $x s$. de $\mathbf{x x x}$ hoggettis permanentibus agnis anno præterito. Et de $x$. iiijd. de $X v$ matricibus capris, $j$ buke, iij capriolis venditis. Et de xiiijs. de iiijaxj velleribus grossis liiij velleribus agninis qui fecerunt dimidium pondus, unde xviij libræ fuerunt lana agnina. Et de iijs. de coriis iiij vaccarum, ij bovettorum, ij vitulorum, $\mathrm{j}$ pulli, venditis. 4 . Et de iijs. vijd. de pellibus xxvj ovium, iij multonum, iiij hoggettorum. Et de ijs. vd. de pellibus xxviij agnorum. Et de xvjd. de pellibus iiij matricum caprarum, iij bukes, iiij capriolorum venditis. Et de vs. iiijd. de liiij ${ }^{1}$ gallinis de cheriset venditis. Et de xiijs. ixd. de $\mathrm{j}$ tonello et quarta parte $\mathrm{j}$ tonelli vini pessimi vendito. Et de viijs. vd. de iiij quarteriis dimidio mancorni de molindino venditis. Et de ijs. ob. de melle de purchasiis in 50 bosco.

\section{Summa viijl. ijs. vijd. ob.}

De Purchasiis.-Idem reddunt compotum de $x 8$. de Johanne filio Ricardi Baldewini pro fine terræ. Et de xijd. de widua Edwardi Grossi pro simili. Et de v8. de filio Siwardi pro uxore capienda. Et de vjd. de Edwardo Bas pro simili. Summa xvjs. vjd.

Idem reddunt compotum de vjl. de taillagio. Summa purchasiorum cum taillagio, vjl. $\mathrm{xvjs.}$ vjd.

Summa summarum, xxjl. iijs. iijd. ob.

Liberatio.-In liberatis Johanni decano de termino Natalis, xxxs. Eidem, de termino Paschæ, xls. Eidem, de termino Sancti Johannis, xxiiijs. Liberata Johanni] decano, de termino 60 Sancti Michaelis, $x x x j$ s. iijd. $^{2}$ Eidem, de purchasiis et aliis exitibus, xvijs. $o b$. Eidem, de vino, xiijs. ixd. Eidem, de taillagio, cs.

Summa xijl. xvjs. ob. 
Expensæ.-In ferramento $\mathrm{j}$ carucæ per annum, $\mathrm{j}$ carucæ per $\mathrm{v}$ septimanas. In ferrura ij avrorum, ixs. ob. In carncis, rotis, jugis parandis, ixd. In precariis hyemalibus de xij carucariis, ijs. In stipendiis $\mathrm{j}$ bovarii, $\mathrm{j}$ vaccarii, $\mathrm{j}$ bercarii, per annum, ixs.; dais, per unnum, ijs. In custo dacriæo, xiijd. ob. In iiij quarteriis ordei ad opus servientium, iiijs. ijd. In iij criblis emptis, ijd. In mercede ij herciatorum a Purificatione Besta Mariæ usque Hockedaye, $\mathrm{x} v \mathrm{j} d$. In custo marlae et $\mathrm{j}$ plaustro de novo parando ad marliudum, $\mathrm{xx} d$. In $\mathrm{j}$ careta empta ad idem, ijs. In jugis ad ij plaustra parandis, iijd. In iij caretis quæ venerunt de ${ }^{1}$ Ivingeho emendandis, ij plaustris axandis, iiij poch[iis] parandis ad idem, xvijd. In clutis, mincto, et ferrura ij caretarum ad marlandum, a festo Sanctæ Barnabæ usque festum Sancti Michaelis, vs.jd. In stipendiis ij caretariorum ad idem per eundem terminum, iijs. In corda empta ad plaustra, caretas pro feno et blado cariando, iijjd. ob. In prato claudendo, xijd. In pratis falcandis, levandis, vjs. vjd. In feno, blado, tassandis iu grangia et $\mathrm{j}$ mullone feni facienda, xijd. In blado claudendo, xviijd. In iij parvis domibus et bercariis emendandis et cooperiendis, $x v d$. In calce cmpta, $x v j d$. In magnis domibus et claustris comperiendis de sclatis, scilicet, ij hominibus, $\mathrm{j}$ garcioni, per vj dies, ijs. In sepe claudenda circa curiam, iiijd. In corio equi dealbando, iiijd. In coquina combusta reparanda, $\mathrm{ij} s$. $\mathrm{ijd}$. In capella extra curiam cooperienda, $\mathrm{ijs}$. iijd. In triturandis $\mathrm{ij}$ quarteris frumenti, iijd. In triturandis ij quarteriis ij busellis de silligine, vjd. In triturandis vij quarteriis dimidio ordei, $x v d$. In triturandis $x x x i j$ quarteriis avene, $\mathrm{ijs}$. In ferrura molindini reparanda, $x v d$. In warra molindiui, $x d$. In liberatione Johannis servientis, per annum, lviijs. ijd., quia recepit liberationem suam ad feriam Wintoniæ per xvj dies. In custo $\mathrm{j}$ tonelli vini per P[etrum] Russ[inol], j tonelli per Danielem pincernam, ducendorum de Hamptona usque Biternam per aquam, xvjd. In xv tonellis vini carcandis, ligandis, barıandis, selendis, apud Hamptonam, per præceptum senescalli, iijjs. vjd. In expensis Walteri piscatoris et hominis sui et ij hominibus ${ }^{2}$ domini Episcopi ad piscandum in vivario de Biterna pest Epiphaniam, per ij dies, xijd. In expensis Roberti de Ellestede cum viij hominibus suis in festo Sancti Martini per iij dies, in festo Sanctæ Andrex per ij dies, festo Sanctorum Fabiani et Sebastiani per $\mathrm{v}$ dies, xiijj. ob. In expensis magistri R[oberti] Basset in festo Sanctæ Andreæ, per ij dies, iijs. vijd. ob. In expensis ejusdem die Jovis et Veneris ante mediam Quadragesimam, iijs. vd. ob. In expensis ejusdem in primo festo Sancti Swithini, per ij dies, iijs. vd. ob. In expensis ejusdem die Lunæe et Martis ${ }^{2}$ post Assumptionem Beatæ Mariæ, iijj. vjd. ob. In expensis ejusdem die Sabbati ${ }^{3}$ ante festum Sancti Michaelis, ijs. xjd.

Summa viijl. viijs. iiijd. Summa tocius liberationis et expensæ, xxjl. iijs. vd.

35 Exitus Grangiæ.-Idem reddunt compotum de iiij quarteriis de toto exitu frumenti. "In semine zvj acrarum, totum.

Idem raddunt compotum de $\mathrm{ij}$ quarteriis $\mathrm{ij}$ bussellis de toto exitu silliginis. In semine ix acrarum, totum.

Idem reddunt compotum de $x x v$ quarteriis dimidio, $\mathrm{j}$ bussello de toto exitu ordei. Et de $\mathbf{v}$ quarteriis dimidio curailli. Et de vj quarteriis ordei allatis de Merewella. Et de iiij quarteriis ordei emptis. Summa xlj quarteria $\mathrm{j}$ bussellus. In semine xxij acrarum, $x j$ quarteria. In conredio $\mathrm{j}$ bovarii, $\mathrm{j}$ vaccarii, $\mathrm{j}$ bercarii, $\mathrm{j}$ daiæ, per annum, xxiij quarteria dimidium, iij busselli. In conredio ij marl[atorum] a festo Sanctæ Barnabæ usque festum Sancti Michaelis, scilicet, per xv septimanas, ij quarteria dimidium ij bosselli. ${ }^{2}$ In conredio ij herciatorum a Purificatione Sanctæ Mariæ, silicet, per $x$ septimanas, ij quarteria. In pastu canum Roberti de Ellestede, dimidium quarterium. Summa xlj quarteria j bossellus. ${ }^{2}$

Idem reddunt compotum de iij quarteriis dimidio mancorni de toto exitu molindini. In supravenditis, totum.

Idem reddunt compotum de zxviij quarteriis dimidio vj bussellis avenæ remanentibus anno præterito. Et de xxxix quarteriis ij bussellis de toto exitu avenæ hoc anno. Sumua lxviij quarteria dimidium. In semine lx.x acrarum, xxxv quarteria. In præbenda iij avrorum dum herciaverunt in hyeme et quadragesima, iij quarteria dimidium. In præbenda ij equorum dum marlaverunt a festo Sanctæ Barnabæ usque festum Sancti Michaelis, iij quarteria dimidium, ij busselli. In missis apud Walthau ad præbendam domini Episcopi, x quarteria. In præbenda domini Episcopi circa festum Sanctæ Barnabæ apud Biterne, iij quarteria j bussellus. In præbenda senescalli, pluries, iij quarteria iij busselli. In præbenda Roberti de Ellestede, per x noctes, vj quarteria ij busselli. In canibus suis, dimidium quarterium. Summa, lxviij quarteria dimidium. Nichil remanet.

60 Instaurum.- - Idem reddunt compotum de ij avris remanentibus anno præterito. Et de ij missis de Ivingeho. Sunıma iiij. Omnes remanent, et $\mathbf{j}$ pullus bujus anni.

Idem reddunt compotum de xiiij bobus remanentibus anno præterito. Et de ij de testamento Azonis Albo et Edwardi Gosse. Et de j de testamento magistri Willelui Gutte de Falel[eia]. Summa xvij. Omnes remanent.

Idem reddunt compotım de ix vaccis remanentibus anno præterito. Et de ij geniculis junctis. Et de ix, j tauro, missis de Suttona. Et de iij de Overtona. Summa xxv. In mortuis, iiij. Et remanent $\mathrm{xx}$ vaccæ, $\mathrm{j}$ taurus.

\footnotetext{
2 Repeated in MS. $\quad$ Sic in MS. S Sabbato in MS. This sum is inserted in the margin.
} 
Idem reddunt compotum de viij annalibus rewanentibus anno præterito. Et de.ij geniculis proventis de Overtona. Et de iij geniculis, $\mathrm{j}$ boviculo proventis de Suttona. Summa xiiij. In adjunctis vaccis, ij. In mortuis, ij. Summa iiij. Et remanent $x$, unde vj sunt geniculæ, iiij boviculi iij annorum.

Idem reddunt compotum de iij annalibus remanentibus anno præterito vitulis. Et de iij proventis de Suttona. Et de j de Overtona. Summa vij. In mortuis, ij. Et remanent $\mathrm{v}$; unde iij sunt masculi, ij femellæ ij annoruแ.

Idem reddunt compotum de $\mathbf{x}$ vitulis proventis hoc anno de vaccis. In decimis, $\mathrm{j}$. Et remanent ix vituli.

Idem reddunt compotum de xliij ovibus remanentibus anno præterito. Et de 10 1 proventis de Waltham. Et de l proventis de Clera. Sumına cxliij. In mortuis ante partum, $x x$. In mortuis post tonsionem, vj.. In supravenditis, $\mathbf{x x x j}$, et ij bercarius occidit. Summa lix. Et remanent iiijxxiiij oves.

Idem reddunt compotum de xiij inultonibus remanentibus anno præterito. In mortuis ante tonsionem, iij. In vivis venditis, $x$. Nichil remanet.

Idem reddunt compotum de xxxiiij hoggettis remanentibus anno præterito agnis. In mortuis ante tonsionem, iiij. In supravenditis, $\mathrm{xxx}$. Nichil remanet.

Idem reddunt compotum de iijix.x agnis proventis hoc anno de ovibus. In decimis, ix. In consuetudine bercarii, $j$. In mortuis ante separationem, $x x v j$. Post tonsionem, ij. Summa xxxviij. Et remanent lij agni.

Idem reddunt compotum de xix matricibus capris, et iiij bukes remanentibus anno præterito. Summa xxiij. In mortuis, vij. In supravenditis, xvj. Summa xxiij. Nichil remanet.

Idem reddunt compotum de viij capriolis remanentibus. In ulortuis, iiij. 'In supravenditis, iiij. De precio superius respondent. Nichil remanet.

Idem reddunt compotum de iijjx. $x$ velleribus grossis. In decimis, ix. In supravenditis, iiijxx quæ fecerunt dimidium pondus, cum liiij velleribus agninis quæ fecerunt xviij libras.

Idem reddunt compotum de clxvj caseis, qui inceperunt tieri proxima die Veneris post Kulendas Maii, et desierunt die Sancti Michaelis. In decimis, xvj. In consuetudine feni, 3 bladi tassandorum, et feni cariandi, v casei. In expensis senescalli, $\mathrm{j}$. In supravenditis, cxliiij qui fecerunt iij poudera dimidium.

Idem reddunt compotum de xiij equis silvestribus, proventis de Waltham. In mortuis, ij. In missis apud Meredonam, $\mathrm{x}$. Et remanet $\mathrm{j}$ duorum annorum.

Idem reddunt compotum de lr.xiiij gallinis proventis de cheriset. In expensis sene- 35 scalli, $x$. In supravenditis, lxiiij. Idem reddunt compotum de cclvij sextariis salis remanentibus anno præterito. In missis apud Duntonam, xv sextaria. In missis apud Fernham, x sextaria. In missis apud Suttonam, iij sextaria. In daeria, ij. Summa xxxj. Et remanent ccxxvj sextaria salis. Idem liberaverunt apud Menes iij $\mathbf{M}$. de azais, per ij taillias. In domibus et capella recooperiendis, iij M. dimidium.

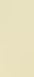


xxxiiij ovium, xj multonum, xv hoggetiorum, xiiij agnorum mortuorum venditis. Et de xviijd. de xxiiij gallinis venditis. Et de vjs. vjd. de capris venditis. Et de vjd. de coriis ij bovettorum mortuorum venditis. Summa viijl. vijs. $v d$.

Idem reddunt compotum de $x l$. de iijj. quarteriis frumenti venditis ; singulis quarteriis, $x \times x d$. Et de iijl. iijs. de xxxvj quarteriis frumenti venditis; singulo quarterio, ijs. iijd. Et de $x x v j s$. de $x x v j$ quarteriis avenæ venditis.

$$
\text { Summa xvl. xs. }
$$

Idem reddunt compotum de lviijs. de lana et caseis venditis anno præterito. Et de xxviijs. vjd. de cxxix velleribus grossis venditis, et de l velleribus agninis quæ non perfecerunt $j$ jondus. Et de xxjs. de cxxxiij caseis, qui fecerunt iij capita.

\section{Summa $v l$. vijs. vjd.}

De Purchasiis. - Idem reddunt compotum de v8. de Henrico de Merre pro fine terræo. Et de ijs. de Ernaldo de Lorteburne pro simili. Et de xijs. de Johanne Rege pro simili. Et de $\mathbf{s}$. de Roberto filio Leuevæ pro simili. Et de vs. de Willelmo filio Alvrici pro simili. Et de xxs. de tota thedinga. Et de ijs. de Cristin[a] Ruff[i] pro fine terræ. Et de xijd. de Reginaldo Iver pro simili. Et de zijd. de Alvet vidua Warneri pro simili. Et de xijd. de widua Warini de Holme pro simili. Et de xijd. de Willielmo filio Edit pro misericordia. Summa lxs.

Idem reddunt compotum de $x x l$. de tallagio. Summa cum tallagio, xxiijl. Summa tocius receptæ, iijox.xijl. xvjs. jqdr.

Liberatio.- In liberatis J[ohanni] decano per $j$ talliam de gabulo Natalis, viijl. Eidem, de termino Paschæ, viijl. vs., per j talliam. Eidem, de termino Sancti Johannis, vijl. xv8., per j talliam. Eidem, de termino Sancti Michaelis, xijl. viijs., per j talliam. Eidem, de blado vendito, $x j l . x s$. xjd., per $\mathrm{j}$ talliam. Eidem, de lana et caseis venditis, de reragio, lviijs., ${ }^{1}$ per $\mathrm{j}$ tallian. Eidem, de tallagio, $x \times l$., per ij tallias.

$$
\text { Summa } l x . x l \text {. xvjs. xjd. }
$$

Exponsa.-In ferramentis iij carucarum, xij avrorum, per annum, $x l s$. In viij rotis ad carucas, xijd. In stipendio $\mathrm{j}$ fabri, viijs. In stipendiis viij bovariorum, ij carettariorum, $\mathrm{j}$ berebr[ecti], per dimidium annum, xxijs. In stipendiis $\mathrm{j}$ daiæ, $\mathrm{j}$ porcarii, $\mathrm{j}$ vaccarii, ij herciatorum, vs. In consuetudine prati, xijd. In $\mathrm{j}$ nova domo, xijs. iijd. In precariis auptumnalibus, xiijs. In claudendo circa pasturam, ijs. In $\mathrm{j}$ careta empia, $x$ viijd. In clutis, uncto, ad carros et caretas, xijd. In viij bobus emptis, lvjs. ijd. ob. In iij corbellis, $\mathrm{xd}$. In custo daeriæ, iijs. ijd. ob. In triturandis clx.xv quarteriis frumenti, xxxvjs. vd. ob. In eisdem ventilandis, iijs. vijd. ob. In triturandis xxvij quarteriis mestellonis, iijjs. vjd. In eisdem ventilandis, vjd. ob. In triturandis xviij quarteriis ordei, xviijd. In eisdem ventilandis, iijd. ob. In triturandis ccvj quarteriis avenæ xvijs. ijd. In eisdem ventilandis, . ijs. $x d$. In elegendo frumentum ad semen, iijs. In expensis senescalli, $x s . \mathbf{v} d$. In $\mathrm{j}$ bussello empto, $\mathrm{ijd}$. In $\mathrm{ij}$ picois emptis, $\mathrm{x} d$. $o b$. In blado empto ad porcos pascendos, ijd. In vj quarteriis mestellonis emptis ad semen, ixs. In ferramentis ad trublas, ijd. In corredio vicecomitis, v8. In liberatione $\mathrm{R}[\mathrm{icardi}]$ servientis, lxs. $\mathrm{x} d$. Summa xvjl. iiijs. viijd. ob.

$$
\text { Summa tam liberationis quam expensæ, iijj }{ }^{x x} \text { vijl. xixd. ob. Et debet }
$$
cxiiijs. iijd. iijqdr.

Exitus Grangiæ.-Idem reddunt compotum de ccj quarteriis dimidio de toto exitu frumenti. Et de xij quarteriis dimidio de toto exitu curailli. In semine clvj acrarum, lx.xviij quarteria dimidium In consuetudine prati, $j$ quarterium. In precariis auptumnalibus, vj quarteria. In supravenditis, exvj quarteria. In corredio servientium, xij quarteria dimidium.

Idem reddunt compotum de 1 quarteriis dimidio de toto exitu mestellonis. Et de vj quarteriis emptis ad semen. In semine $x \times j$ acrarum, $x$ quarteria dimidium. In corredio servientium, xlvj quarteria.

Idem reddunt compotum de $\mathrm{xl}$ quarteriis de toto exitu ordei. acrarum, vij quarteria. In porcis, porcellis pascendis, iij quarteria. In semine xiiij vientium, $\mathrm{xxx}$ quarteria.

Idem reddunt compotum de vj quarteriis pisarum. In semine, ij quarteria ij busselli. In corredio servientium, iij quarteria dimidium, $\mathrm{j}^{2}$ bussellus.

Idem reddunt compotum de cclvj quarteriis dimidio, iij bussellis de toto exitu avenæ. In semine ccxv acrarum, cxliij quarteria dimidium. In præbenda domini Episcopi, xiij quarteria dimidium, iij busselli. In præbenda senescalli, vj quarteria dimidium. In præbenda G. de Aclanda, dimidium quarterium $j$ bussellus. In præbenda G[alfridi] de Calc[eto] dimidium quarterium. In præbenda vicecomitis, v busselli. In præbenda xij avrorum, a festo Sancti Thomm usque festum Sancti Dunstani, xxxviij quarteria. In præbenda. boum, a festo Sancti Thornæ usque Pentecosten, xxvij quarteria. In supravenditis, xxvj quarteriä. ${ }^{2}$ 
Instanrum.-Idem reddunt compotum de $x j$ avris remanentibus anno præteritn. Et de $j$ de testamento Algeri de Dunlee. Summa xij. In venditis ij. . Et remanent $x$.

Idem reddunt compotum de $x \times x v j$ bobus remanentibus anno præterito. Et de viij emptis. Summa xliiij. In mortuis, iijj. In venditis, xij. Summa xvj. Et remanent xxviij boves.

Idem reddunt compotum de viij vaccis, $\mathrm{j}$ tauro, remanentibus anno præterito. Et de $\mathrm{j}$ vacca de testamento viduæ Thedrici. Summa $\mathrm{x}$. In mortuis, iij. In supravendito, j taurus. De precio superius respondent. Summa iij. Et remanent vj vaccæ.

Idem reddunt compotum de iij bovettis $\mathrm{ij}$ annorum et dimidii remanentibus anno præterito. Et de iij geniculis ejusdem ætatis remanentibus. Et de iij boviculis 10 remanentibus, anno præterito annalibus. Et de iij geniculis. Et de ij annalibus. ${ }^{1}$ Summa $x v$. In mortuis, $\mathrm{j}$ bovettus $\mathrm{ij}$ annorum, et $\mathrm{j}$ genicula $\mathrm{ij}$ annorum. Summa $\mathrm{ij}$. Et, remanent xiij.

Idem reddunt compotum de $\mathrm{v}$ vitulis proventis de vaccis prædictis, quia $\mathrm{j}$ fuit sterilis, iij fuerunt mortui ante fœtum. Et remanent $\mathbf{v}$ vituli; unde iijj sunt masculi, 15 j femella.

Idem reddunt compotum de cviij ovibus remanentibus anno præterito. Et de xxiiij adjunctis ovibus de instauro. Summa cxxxij. In venditis ante partum et tonsionem, viij. In mortuis ante partum et tonsionem, xxx. In mortuis post tonsionem et partum, iij. Summa xlij. Et remanent $\mathrm{iiij}^{\mathrm{xx}} \mathrm{x}$ oves.

Idem reddunt compobum de $\mathrm{xxxv}$ multonibus remanentibus anno præterito. In adjunctis multonibus de stauro, $x \times x$. Summa lxv. In venditis ante tonsionem, ij. In venditis post tonsionem, $\mathrm{xx}$. In consuetudine prati post tonsionem, ij. In mortuis ante tonsionem, $x j$. Summa xxxv. Et remanent $x \times x$.

Idem reddunt compotum de lxix hoggettis remanentibus, anno prǽterito agnis. In 25 adjunctis ovibus, xxiij. In adjunctis multonibus, $\mathrm{xxx}$. In mortuis ante tonsionem, $\mathrm{xv.}$ Summa lxix. Nichil remanet.

Idem reddunt compotum de lx.xviij agnis proventis hoc anno, unde xj sunt proventi de geniculis. ${ }^{2}$ In decimis, vij. In consuetudine bercarii, $j$. In mortuis ante tonsionem, xiiij. Et remanent lvj agni.

Idem reddunt compotum de cxlvj velleribus grossis. In decimis, xiij. In consuetudine bercarii, j. In venditis, cxxxix. Et debent ij, precium iijd.

Idem reddunt compotum de l,velleribus agninis quæ non fecerunt $j$ pondus.

Idem reddunt cumpotum de clxvij caseis qui inceperunt fieri die Jovis proxima post festum Sancti Tiburcii et desierunt in festo Sancti Michaelis. In decimis, xvj. In 35 consuetudine prati, $\mathrm{ij}$. In expensis senescalli, $\mathrm{ij}$. In precariis auptumnalibus, $x$ iiij. In venditis, cxxxiij, qui fecerunt iij capita.

Idem reddunt compotum de 1 porcis remanentibus anno præterito. Et de $\mathrm{x}$ porcis inventis. Et de ij porcis proventis de Ivingeho. Et de lxv proventis de prædictis porcis. Summa cxxvij. In missis apud Lundoniam ad lardarium, et in decimis, vj. In 40 mortuis, xxxix. Et remanent lviij, unde xvij sunt masculi, xlj femellæ.

Idem reddunt compotum de xlix gallinis proventis de cheriset. In expensis senescalli, xvj. In mortuis, ix. In venditis, xxiijj. Summa xlix. Nichil remanet.

Summa tocius debiti, cxiiijs. iiijd. iijqdr. Item iiijd. de lana.

\section{MEREDONA.}

R[rcardus] serviens, Robertus præpositus, reddunt compotum de $x \times x i i j j$. ixd. de reragio anni præteriti. Solverunt et quieti sunt. ${ }^{3}$

Idem reddunt compotum de xxjl. xviijd. de totn gabulo assiso. In quietanciis j præpositi, j bedelli, vs.; ij bercariorum, iijs. ixd.; j equiciarii, iijs. vjd.; $\mathrm{j}$ haiwardi, xvd.; 50 $\mathrm{j}$ porcarii, ijs. vjd.; j parcarii, iiijs. iiijd.; viij carucariorum per annum, $\mathbf{x}$., cuilibet $\mathbf{x} \mathrm{v}$. ; $\mathrm{x}$ operariorum per annum, xijs. vjd., cuilibet $\mathrm{xvd}$.

Summa $x$ lijs. $x d$.

In defectu gabuli terræ Willelmi filii Reginaldi, iijs. In defectu terræ quæ fuit Seiwæ tractæ in dominium, vs. In defectu terræ Philippi et Ricardi de Marisco tractæ in 55 dominium, iiijs. Summa defectuum, xiijs.

Summa tam quietanciarum quam defectuum, lvs. $\mathbf{x} d$.

Summa remanens, xviijl. v8. viijd.

Idem reddunt compotum de xliijs. jd. de pannagio. Et de $x v j s$. de $\nabla$ bobus vivis venditis. Et de xiiijs. ijd. de xiiij coriis boum mortuorum venditis. Et de vjs. vjd. de 60 coriis vj vaccarum, ij annalium mortuorum venditis. Et de $v d$. de coriis $v$ vitulorum anni præteriti. Et de ixs. ijd. de coriis vij avrorum, xxiij tam equorum quam pullorum silvestrium mortuorum venditis. Et de exvijs. $v d$. de viij pullis silvestribas vivis venditis. Et de ixs. vijd. ob. de pellibus liij ovium, xvij multonum, zxv hoggettorum mortuorum venditis. Et de vijs. vjd. de pellibus lxxviij egnorum mortuorum venditis. Et de ijs. iijjd.ob. 65

1 Annul' in MS. . ' 3 Sic in MS. for gerciis. . ' Solverunt . . . sunt is an interliveation. 
de xix agnis vivis venditis. Et de lvjs. de exliij caseis anni,prøteriti venditis, qui fecerunt viij pondera. Et de $\mathbf{x} x j$ s. de iiijxx ${ }^{x i i i j ~ c i s e i s ~ a n n i ~ p r æ s e n t i s ~ v e n d i t i s, ~ q u i ~ f e c e r u n t ~ i i j ~ p o n-~}$ Jera. Et de lx.xiijs. iiijd. de cccc velleribus grossis qui fecerunt ij pondera dimidium. Et $d \theta^{1}$ iijj $^{x z} . x v j$ velleribus agninis quæ fecerunt quartam partem ponderis, venditis.

Idem reddunt compotum de lx.xvs. iiijd. de xxviij quarteriis dimidio frumenti venditis. Et de ijs. de j quarterio curalli vendito. Et de xiijs. viijd. de xvj quarteriis avenæ anni præteriti venditis. Summa iijl. xijs.

De Purchasiis.-Idem reddunt compotum de ijs. de Waltero de Fraxine pro terra habenda. Et de iiijs. de Ivone Gardre pro simili. Et de vs. de Rogero de Pichemora pro simili. Et de xijd. de Wimarko pro tilia sua maritanda. Et de vjs. viijd. de Rogero Rabb[er]e. pro simili. Et de ijs. de Widone de Anfelda pro falso clamore. Et de xijd. de Ricardo eapellano pro simili. Et de xijd. de Nicolao de Emnolt pro simili. Et de xijd. de Leverico de Stocepunda pro simili. Et de xijd. de Roberto Swenge pro simili. Et de ijs. de Wineman Fox pro simili. Et de ijs. de Nicolao Hoppere pro simili. Et de ijs. de Ricardo Bricwini pro sursisa. Et de vjd. de Cupping Oute pro mellea. Et de xijd. de Johanne Rundet pro terra habenda. Et de ijs. de Laurencio pro filia sua maritanda. Et de xijd. de Jordano Fichet pro mellea. Et de iiijs. de Ricardo Palmer pro falso clamore. Et de vs. de Ada do Hiltonebrich pro filia sua maritanda. Et de iijs. de Waltero de Felda pro sinili. Summa xlvijs. ijd.

Idem reddunt compotum de xiiijl. xvijs. iiijd. de tallagio.

Summa tocius cum tallagio, liiijl. vijs. ixd.

Liberatio.-In liberatis J[ohanni] decano, xvjl., per j talliam. Eidem, xiiijl. xvs. iijob., per $\mathrm{j}$ talliam.

Expensa.-In ferramentis vij carucarum per annum, $\mathrm{j}$ carucæ per $\mathrm{j}$ terminum, $x x v i i j s$. ixd. In ferrura xij avrorum per annum, ixs. ijd. ob. In mercede fabri per annum, vs. vjd. In xij paribus rotarum, vj carucis et jugis factis de novo, vjs. viijd. In careta empta, iijs. vjd. In $\mathrm{j}$ careta ferro ligata empta, ixs. viijd. In sellis, bacis, panellis, paronis, paronellis, tam ad caretas quam ad carucas, ijs. vijd. In ij caretis axandis et multociens reparandis, clutis ad easdem, xviijd. In precariis hyemalibus de cxlj carucis, xvs. ixd. ob. In triturandis lxvj quarteriis frumenti, xiijs. ixd., siugulo quarterio ijd. ob. In triturandis viij quarteriis ordei, xijd. In claudendo clx.xviij perticas inter frumentum et boscum, xviijs. vjd. ob. In fossando lx.xviij perticas inter avenam et boscum et pasturam, vjs. vd. In claudendo circa ordeum, xiijd. In ij carpentariis conductis ad faciendum novam bercariam, iijs. In operariis conductis ad claudendum parietes et ad walluram faciendam, iijs. iijd. In prædicta bercaria cooperienda, ijs. In fossato xxxij perticarum circa prædictam bercariam, viijs., per singulam perticam iijd. In carpentariis conducendis ad bovariam separandam vs. ixd. In claudendo prædictam bovariam, xvd. II predicta bovaria et $\mathrm{j}$ grangia cooperienda, ${ }^{\mathrm{ijj}}$. vjd. In xviij acris marlandis, xixs. vjd. In marla spargenda per xxxvj acras, iijjs. iijd. In sartando $i j$ acras, $x$ viijd. In $j$ sccure, et aliis utensilibus domus reparandis, vij d. In xxxvj clatis factis ad faldam, lij clatis wiscandis ad idem, ijs. jd. In ccclxviij acris bladi serclandis, xiiijs. In custo daeriæ, vs. iijd. ob. In stipendio daiæ, per annum, ijs. In carpentariis ${ }^{2}$ conductis ad faciendum magnam

45 grangiam de novo in tasca, xlijs. viijd. In faciendı v M. lattarum et in carpentariis ad lattandum prædictam grangiam, xjs. viijd. In xxj miliariis clavorum ad lattas, xjs. iiijd. ob. In cl clavis plancheret emptis ad idem, iijs. vd. In parietibus prædictæ grangiæ faciendis, iiijs. iijd. In faciendo ostium grangiæ in tascha, iijs. In bordis enptis ad idem, xvjd. In clavis, gunnis, emptis ad idem, ijs. In j sera empta ad idem, xijd. In eadem grangia cooperienda, vs. In corredio Galfredi de Akinges, xvija. $o b$. In corredio Roberti Basset, multociens, ibidem, xixs. In corredio Gervasii sociorumque ejus cum canibus dounini Episcopi ad feriam Sancti Egidii ibidem, ijs. In aula et camera cooperienda, ${ }^{1}$ xijs. iijd. In iij miliariis ce[n]dularum faciendis ad idem, iiijs. vjd. In clavis emptis ad idem, vjd. In viij carrucariis pro aratura sabbatorum, per annum, xliijs. In iiij precariis auptumnalibus, xxxixs. vjd. In solidis vj bovariorum, per annum, xxjs. In solidis ij caretariorum, per annum, vjs. In solido $\mathrm{j}$ vaccarii, per anuum, $\mathrm{ij} s$.

Summa xxjl. xixs. Summa tam liberationum quam expensarum, lijl. xiijjs. iijob.

Et debet ${ }^{1}$ xxxiijs. vijd. ob.

Exitus Grangiæ.-Idem reddunt compotum de clxix quarteriis dimidio de toto exitu frumenti. In semine Dlxvj acrarum, iij $^{x x} . x$ quarteria. In missis apud Craweleiam ad semen, xxvj quarteria. In missis apud Duntonam ad semen, xxiij quarteria. In missis apud Wlveseiam, $j$ quarterium. In supravenditis, xxxviij quarteriandimidium. Quiet[i sunt]. 
Idem reddunt compotum de xij quarteriis de toto exitu curalli. In corredio servientium domus, $x j$ quarteria, pro defectu ordei. In supravenditis, $j$ quarterium. Quiet[i sunt].1.

Idem reddunt compotum de xiij quarteriis de ${ }^{2}$ toto exitu mancorni. In semine $\mathbf{x x v j}$ acrarum, vj quarteria. In corredio servientium domus, vij quarteria. Quiet[i sunt]. ${ }^{1}$

Idem reddunt compotum de lxvij quarteriis $\mathbf{j}$ hopa de toto exitu ordei. Et de xxij quarteriis receptis de Craweleia. Summa iijjxix quarteria $\mathrm{j}$ hopa. In semine cxj acrarum, xxxvj quarteria. In corredio iij bovariorum, ij caretariorum, $\mathrm{j}$ vaccarii, per annum, xxvij quarteria dimidium. In corredio $j$ bovarii per xxix septimanas, iij quarteria dimidium, $\mathrm{ij}$ hopæ. In colredio $\mathrm{j}$ bovarii per xlij septimanas, $\mathrm{v}$ quarteria 10 vj hopæ. In corredio daiæ, per annum, $v$ quarteria iiij hopæ. In porcis pascendis $\mathrm{x}$ quarteria. In consuetudine bercarii, ${ }^{3} \mathrm{j}$ quarterium. In consuetudine porcarii, dimidium quarterium. Quiet[i sunt. $]^{1}$

Idem reddunt cumpotuı de xliiij quarteriis dimidio viij hopis avenæ remanentibus anno præterito. Et de cvj quarteriis dimidio iij hopis de toto exitu avenæ hoc anno. 15 lit de viij quarteriis receptis de Craweleia. Summa clix quarteria dimidium, $v$ hopæ. In semine cccexxxij acrarum, iijixix quarteria. In missis apud Wlveseiam, viij quarteria. In præbenula equorum domini Episcopi ibidem, viij quarteria. In præbenda $\mathrm{v}$ caretarioruın domini Episcopi ibidem, dimidium quarterium, per j noctem. In præbenda $\mathrm{R}$ [oberti] Basset, vj quarteria. In præbenda G[alfridi] de Akinges, iiij hopæ. In præ- 20 benda xvj avrorum per dimidium annum, xij avrorum per totum annum, xxxij quarteria. In suprarenditis, $x v j$ quarteria. Quiet[i sunt].

Instaurum.-Idem reddunt compotum de xxj avris remanentibus anno præterito. Et de $\mathrm{j}$ de testamento Galfridi Blechc. Et de $\mathrm{j}$ adjuncto de instauro. Et de $\mathrm{j}$ qui venit de Wlveseia. Summa xxiiij. In missis apud Alresfordam, ij. In missis apud Norwaltham, iij. In 25 mortuis, vij. Summa xij. Et remanent xij.

Idem reddunt compotum de xij equabus, $x$ equis et pullis silvestribus remanentibus anno præterito. Et de $x \times x j$ equis, xj pullis qui venerant de Waltham. Et de v equabus, iiij equis, j pullo qui venerant de Biterna. Et de ix equabus, quæ venerant de Wallia. Summa iij ${ }^{x}$ iij. In missis apud Fer[n]ham, xiij equæ, ij pulli. In mortuis, xiij equæ, 30 xij pulli. In devoratis a lupis, ij pulli. In venditis ad festum Sancti ${ }^{4} \longrightarrow$, viij. In adjunctis avris, j. Summa lj. Et remanent $x \times x i j$, unde $x x i x$ sunt equæ, iij equi.

Idem reddunt compotum de vij pullis proventis hoc anno. Omnes remanent.

Idem reddunt compotum de lxiiij bobus remanentibus anno præterito. Et de ij adjunctis de instauro. Et de $\mathrm{j}$ de catallis Willelmi fugitivi. Et de $\mathrm{j}$ de testameıto 35 Walteri de Emnolt. Summa lxviij. In vivis venditis, v. In mortuis, xiiij. Et remanent xlix.

Idem reddunt compotum de $x i j$ vaccis remanentibus anno præterito. $E t$ de $i j$ tauris. Et de vij vaccis adjunctis de instauro. Et de $\mathrm{xv}$ vaccis, $\mathrm{j}$ tauro, receptis de Craweleia. Summa xxxiiij vaccæ, iij tauri. In mortuis, vj. Et remanent xxviij vaccæ, iij tauri.

M. 5d. Idem reddunt compotum de iij bovettis, $\mathrm{j}$ genicula iij annorum. Et de vj bovettis, iiij geniculis $i j$ annorum. Et de vj geniculis qui venerant de Wlveseia. Summa $\mathbf{x x}$. In adjunctis bobus, $\mathrm{ij}$. In adjunctis vaccis, vij. In mortuis, iij. Et remanent viij, unde $\mathrm{v}$ sunt bovetti, iij geniculæ ij annorum.

Idem reddunt compotum de ix annalibus. In mortuis, v. Et remanent iij, unde 45 iij sunt femellæ, j masculus.

Idem reddunt compotum de xiij vitulis proventis hoc anno. In decima, j. Et remanent xiij, unde $\mathrm{v}$ sunt femellæ, viij masculi.

Idem reddunt compotum de ccxlij ovibus remanentibus anno præterito. Et de lxviij junctis de instauro. Summa cccx. In mortuis ante partum, xv. In mortuis post 50 partum et ante tonsionem, xxxiij. In mortuis post tonsionem, v. Summa liij. Et remanent cclvij.

Idem reddunt compotum de ciiij.xx.xiij multonibus remanentibus anno præterito. In mortuis ante tonsionem, xiij. In mortuis post tonsionem, iiij. Summa xvij. Et remanent ciiij ${ }^{x} j$.

Idem reddunt coinpotum de cxlj hoggettis remanentibus annu præterito agnis. In missis apud Craweleia, xlviij. In mortuis ante tonsionem, $x \times 5$. Summa lx.xiij. Et remanent lxviij quæ sunt gerciæ matricibus ovibus junctæ.

Idem reddunt compotum de ecxv agnis qui provenerunt de ovibus hoc anno, unde xij fuerunt steriles. In -decimis, $x \times j$. In consuetudine bercarii, $\mathrm{j}$. In vivis venditis, 60 xix. In mortuis ante tonsionem, lx.xv. In mortuis post tonsionem, iij. Summa cxix. Et remanent iiijx.xvj.

Idem reddunt compotum de cccexlvij velleribus grossis. In decimis, xliiij. In consuetudine ij bercariorum, j daiæ, iij. In supravenditis, cecc vellera, quæ fecerunt ij pon: dera dimidium. 
Iden reddunt compotum de iiijx ${ }^{x i x}$ velleribus agninis, quæ fecerunt iiijtam partem ponderis. In venditis totum.

Idem redunt compotum de cxliij caseis remanentibus anno præterito. Et de clx caseis, qui inceperunt fieri $x^{\circ}$ Kalendas Maij et desierunt iiij Kalendas Uctobri, utroque die computato. In decimis, xvj. In consuetudinibus fabri, bercarii, daim, iij. In precariis auptumnalibus, xvij. In supravenditis, ccxxxvij. Summa cclx.xiij. Et remanent $x \times x$.

Idem reddunt compotum de liij caseis qui inceperunt fieri $\mathrm{ix}^{\circ}$ Kalendas Junii et desierunt Idibus Julii, utroque die computato quia lac vaccarum fuit tunc mixtum cum lacte ovium, quod penc defecerat proper nimiam siccitatem. In decimis, $v$. Et remanent xlviij.

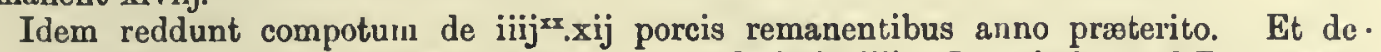
xliij de exitu eorum. Summa cxxxv. In decimis, iiij. In missis apud Duntonam ad festum Sancti Martini, lx.xj. In missis apud Wolveseiam, j. In consuetudine porcarii, $\mathrm{j}$. In mortuis, vj. Summa iijjxiij. Et remanent lij, unde $\mathrm{j}$ verres, ix sues, $\mathrm{xx}$ porci, xxiij porci et porcelli juniores.

Idem reddunt compotum de $\mathrm{x}$ baconibus receptis de Wlveseia. In precariis auptumnalibus totum exp[enditur].

Idem reddunt compotum de cccc ovis. In missis apud Wlveseiam et liberatis Johanni decano, totum.

Summa tocius debiti xxxiijs. vijd. ob. Unde solvit post compotum, xjs. vjd. Et xxijs. iijd. ob. sunt super Willelmum Wudesman qui terminum habet usque ad Sanctum Hilarium, per Episcopum. ${ }^{2}$

\section{FERNHAM.}

G[alfridus] DE MoneTs et Edmundus et Ricardus ${ }^{2}$ et Hugo præpositus de Sela reddunt compotum de xvjl. xjs. vd. ob. de reragio anni præteriti.

G[alfridus] de Moneta et Hugo et Ricardus præpositus reddunt compotum de lx.xvijl. xjs. de toto gabulo assiso de Huppel[anda] hoc anno. Et de xs. iijd. de molindino de Elestede. Et de xiijs. $x d$. de xxiij acris, $j$ pertica dimidia, ij purroc, $j$ mesagio. Et de xvjs. ixd. de lxvij acris. Et de iijs. vjd. de vij perchis assisis hoc anno. Summa incrementi, xliiijs. iiijd. Et de vijl. de tirma Burgi. Sumına iiij ${ }^{x x} v j l . x v s . ~ i i j j d$.

In quietanciis $\mathrm{j}$ grangiarii apud Fernham, $\mathrm{j}$ præpusiti apud Sele, xs. ; j bedelli, ijjs.; j fabri, ijs. vjd.; iiij bovariorum apud Fernham, ij bovariorum apud Sele, xijs.; ij bercariorum, $\mathrm{j}$ vaccarii, $\mathrm{j}$ porcarii, viijs. ; $\mathrm{j}$ operarii cotidie in auptumno et altera die per totum annum, iijs. iijj. ob. In quietanciis ix operariorum altera die in auptumno et per totum annum factum, iij operariorum in Septembri, xiss. viijd. ob.; $\mathrm{j}$ operantis per $\mathrm{j}$ terminum, iijs. jd. ; j operarius altera die per $\mathrm{j}$ terminum, $\mathrm{xjd}$. $\mathrm{ob}$.

Summa lxiijs. vijd. ob.

In defectu terræ Ricardi de Londonia, vjl. vs. $x d$. In defectu terræ de Senellinges, xijs. iijd. In defectu $\mathrm{j}$ virgatæ terræ apud Chert et apud Tilefordam, vs. In defectu terræ de Sele tractæ in dominium, iiijs. ijd. In defectu $\mathrm{j}$ curtellagii tracti in dominium, xijd.

Summa vijl. viijs. iijd. Summa tain quietanciarum quam defectuum, $\mathbf{x} l . x j s . \mathbf{x} d$. ob. Summa remanet, lx.xvjl. iijs. vd. $a b$.

Idem reddunt compotum de $1 x v j s . x d$. de pannagio. Et de $x x i x s$. de vj bobus, ${ }^{3} j$ vacca, venditis. Et de vijs. de $x x$ ovibus infirmis venditis. Et de sxvjs. de ij ponderibus, iiij clavis minus, uncti venditis. Et de iiijs. de melle vendito. Et de xlijs. de $\mathrm{j}$ pondere dimidio et quarta parte ${ }^{4}$ ponderis vellerum grossorum venditis. Et de iijs. vjd. de cvij velleribus agninis venditis. Et de xijs. iiijd. de $\mathrm{j}$ pondere dimidio et quarta parte ${ }^{4}$ ponderis casei venditis. Et de vjs. de coreis $\mathrm{v}$ boum, $\mathrm{j}$ avri, $\mathrm{j}$ vituli venditis. Et de Xs. vjd. de xxxix pellibus multonum, xxvij pellibus ovium, xlij pellibus hog[ettorum] venditis. Et de iijs. vjd. de iiij ${ }^{x i j}$ pellibus agninis venditis. Et de xlvjs. viijd. de vino vendito.

\section{Summa xijl. xviijs. iijd.}

Idem reddunt compotum de $x j l$. vjs. vjd. de $l x, x v$ quarteriis dimidio frumenti venditis, singulo quarterio pro iijs. Et de $\mathrm{xxs}$. de $\mathrm{x}$ quarteriis frumenti venditis, singulo quarterio ijs. Et de xvjs. vjd. de $\mathbf{v}$ quarteriis dimidio silliginis venditis, singulo quarterio iijs. Et de iiijs. vjd. de vj quarteriis avenæ venditis, singulo quarterio ixd. Et de ijs. de ij quarteriis pisarum venditis. 
De Purchasiis.-Idem reddunt compotum de xijd. de Waltero Barat pro misericordia. Et de j marca de Johanne de Ivingeho pro fine terræ. Et de dimidia marca de Willelmo Cunan pro simili. Et de xijd. de Willelmo Stut pro simili. Et de iijs. de vidua Hevede pro simili. Et de $v \varepsilon$. de Osberto tilio Edwini pro simili. Et de ij marcis de Osberto molindinario pro molindino habendo. Et de jjs. de Edwino filio Godwini pro fine terræ. Et de xviijd. de Ricardo de Londonia pro pannagio. Et de vs. de Radulfo filio Gocelini pro simili. Et de vs. de filiastr[o] Lucæ pro simili. Et de ijjs. de Roberto filio Ricardi pro simili. Et de ijs. de Hugone Boilat pro simili. Et de ijs. de Hugone Grom pro simili. Et de xls. de Willelmo caretario pro fine terræ. Et de ijs. de Turb[erto] pro filia sua maritanda. Et de iijs. de Galfrido molindinario pro fine terræ. Et de vs. de vidua 10 Walteri pro fine et relevio. Et de dimidia marca de Willelmo molindinario pro simili. Et de vs. de Osberto pro uxore ducendo. Et de xviijd. de Galfrido molindinario pro simili. Et de iijjs. de Roberto et Martino pro fine terræ. Et de ijs. de Fluri pro simili. Et de iijs. de Roberto de Hulla pro simili. Et de xijd. de Ricardo tilio Edwini pro simili. Et de ijjs. de vidua Roberti pro simili. Et de xijd. de divisa Johannis filii Hugonis. Et 15 de jjs. de filio Johannis pro fine terræ. Et de dimidia marca de Ricardo cimentario pro simili. Et de iijs. de Willelmo filio Eilrici pro simili. Et de iijs. de Edmundo de Bacsete pro simili. Et de iijs. de Ricardo de Quercu pro simili. Et de ij marcis de filiastr[o] Edmundi pro agno stulte detento. Et de ij marcis de Willekin de Runewika pro terra matris suæ havenda. Et de dimidia marca de Edmundo præposito pro balliva relaxata. 20 Et de $\mathbf{j}$ marca de Margareta pro fine terræ. Et de $\mathbf{x} s$. de Willelmo de Wile pro simili. Et de vjd. de Alexandro pro simili. Et de vjd. de Ricardo filio Hugonis pro simili. Et de ijs. de Galfrido de Bacsete pro sorore sua maritanda. Et de ijs. de Willelmo Edwin[i] Kinc pro simili. Et de ijs. de Willelmo de Punfalda. Et de ijs. de Ada filio Sewari pro uxore ducenda. Et de $\mathrm{j}$ marca pro curtilagiis habeudis. Et de ijs. de Lucia vidua pro 25 saisina terræ. Et de iijs. de Philipno de Dokenesfelda pro mellea. Et de vs. de Roberto de Bosco pro filia sua maritanda. Et de viijs. de Alueva de Marisco pro filia sua maritanda extra hundredum. Et de iiijs. de Andrea de Chert pro filia sua maritanda. Et de xvijjd. de Willelmo filio Sewari pro fine terræ. Summa xvl. ijd.

Idem reddunt compotum de $l x l$. de tallagio.

Summa summarum clx.xvijl. xjs. iiijd. ob.

Liberatio.-In liberatis J[ohanni] decano xxxiijl. ixs. Dominæ Iseut de Laci, per magistrum $\mathrm{J}[$ ohannem] de Lond[onia], per dominum Episcopum, xxxiijl. vjs. viijd., per breve domini Episcopi. Mariscallo domini Episcopi lxijs. Dionisio de Camera, xxxl. Jordano clerico, xxijl. Ricardo cementario, ad opus de Wintonia, iiij marcas, per dominum Episcopum. Summa cxxiijl. xjs.

Expensa.-In ferramentis v carucarum, rotis, et aliis necessariis ad carrucas, per annum, xxxiijs. ixd. ob. In ix avris ferrandis, per annum, quia faber ferrat ij de consuetudine, xijs. iijd. ob. In paronis, paronellis, sellis, capistris, ad avros, ijs. ixd. ob. In xiij bobus emptis, lxxviijs. In iij avris emptis, xxiijs. iiijd. In xij quarteriis ij hopis frumenti 40 emptis ad semen, $x l_{8}$. $x d$., singulo quarterio iijs. iijjd. In $\mathrm{j}$ careta ferrata facta de novo, viijs. vjd. In precariis hyemalibus et quadragesimalibus de cv carucariis, $x s$. ixd. ob. In fossato facto circa bladum, ijjs. viijd. ob. In clutis, sepo, savono, ad caretas, xviijd. $o b$. In mercede iiij bovariorum apud Fernham, ij bovariorum apud Sele, xvs. In mercede $\mathrm{j}$ caretarii per annum, iijs. In mercede $\mathrm{j}$ caretarii, per iij terminos, ijs. In mercede 45 ij herciatorum apud Fernham, $\mathrm{j}$ apud Sele, $x$ viijd. In iiij caretariis locatis apud Menes qui tulerunt ix quarteria dimidium ordei apud Fernham, xviijd. In triturandis, ventilandis, cix quarteriis frumenti, xiijs. vijd. ob.; xij quarteriis silliginis, xviijd.; $\mathrm{xvij}$ quarteriis ordei, $x v i j d$. ; ccxlv quarteriis aven $x, \mathrm{x} s$. $\mathrm{jj} d$. ob. In triturandis et ventilandis xxij quarteriis frumenti, xij quarteriis silliginis apud Sele, iijs. iijd.; vij quarteriis 50 ordei, vijd.; l lvij quarteriis avenæ, ijs. iijjd. ob. In puteo munclando, ijs. iijjd. In cribulis, corbellis, sedlepes emptis, ixd. ob. In corda empta, ijd. ob. In ponte ante aulam emendando, iijd. In grangia de Sele suppodienda et emendenda, iiijs. vjd. In sulfure empto ad medicinandum oves, iijd. In veteri coquina emendenda et cooperienda, xvs. ixd. ob. In stabula emendanda, xiijd. ob. In custo daeriæ, ijs. iijd. ob. In blado 55 sarclando, xiiijd. ob. In pratis falcandis, viijs. ixd. ob. In precariis auptumnalibus lijs. vijd. In feno empto, ixs. iiijd. In liberatis G[altrido] de Moneta, lx.xs. Summa xxijl. ijs. ixd.

Expensa facta pro domino Episcopo. - In cariagio $x$ quarteriorum salis de Biterna usque Fernham ad lardarium, vjs. viijd. In cumpanagio et cervisia facientium lardarium, 60 iijs. vijd. ob. In mercede facientium lardarium, vs. vjd. In cervisia ad sulc[itium], $\mathbf{x} d$. In elemosinis domini Episcopi, inclusa de Ichulla, per annum, iiijs. iiijd.; leprosis de Fernham, iijjs. iijjd. In lorica Rolandi, xiiijd. ob. In portando breve domini Episcopi apud Meredonam, iijd. In $\mathrm{j}$ muta facta de novo, xviijd. In expensis hostoriorum, falconum, vjs. vijd. ob. In portando breve domini Episcopi Nicolao Haringe, vjd. In 65 
expensis Willelmi ${ }^{1}$ euntis ad dominum Episcopurn, xijd. In expensis G. de Aclanda die Sancti Romani, xvjd. In expensis R[oberti] Basset pluries, per talliam, xvjs. iijob. In expensis Justiciarii, Comitis de Clara, R[eginaldi] de Cormhulla, per G[alfridum] de Calc[eto], lvjs. In dignariis domini Episcopi, Justiciarii, Willielmi Briweri, Cancellarii domini Regis, iiijs. viijd. In expensis Reg[inaldi] de Valletorta, iiijd. ob. In expensis Nicolai Haringe, xixd. ob. In clerico Savarici de Mauliun [et] vicecomitis de Tuars, v8, iijd. ob. per breve domini Episcopi. In expensis custodis palefredorum domini Regis, a vigilia Omnium Sanctorum, usque festum Sancti Hyllarii, xiijs. $x d$. ob. In expensis militis venientis propter cirfaucones domini Regis, vjd. In expensis Mathiø et hominis sui, per xxviij dies, vijs. iijd. In expensis Radulfi Bastardi, per xvj dies, ijs. viijd. In expensis Abbatis de Biledona, $x d$. $a b$. In prisonibus ducendis apud Wlveseiam et iterum apud Fernham, xviijd. In dono domini Episcopi Gileberto forestario, xxs.; Heliø de Breuile, jm.; Osberto forestario, ijs. In expensis magistri Thomæ de Eli, xvjd. ob. In expensis milit[um] vis(c)orum ${ }^{2}$ forestæ, viijs. ixd. $a b$. In expensis Mathiæ, per iij septimanas, iiijs. viijd. In expensis Walteri piscatoris, piscantis in vivario de Fernham, xijd. ob. In mercede juvandi eidem, xvd. In expensis Gervasii, Willelmi Widecoc, iijs. iijd. ob. In expensis G. de Acl[anda], viijd. In perdonis domini Episcopi de taillagio Willel mi caretarii Nota quod iiij $^{2}$; Goding, vjd.

Fpiscopus Summa $x l$. xs. ijd. ob. . Summa utriusque expensæ, xxxijl. xijs. xjd. ob. Summa $20 \mathrm{w}_{\mathrm{W} \text { [illelmo] }}$ tam liberationis quam expensæ, clvijl. iijs. xjd. ob. Et debet $x \times x l$. vijs. vjd. Unde super [de] Clere Willelmum de Clera sunt, vijl." ... . . postuland postuland

Exitus Grangiø.-Idem reddunt compotum de cxliij quarteriis ij hopis de toto exitu frumenti de Fernham. Et de $x x v$ quarteriis dimidio, j hopa de toto exitu frumenti de Sele. Et de xij quarteriis ij lopis emptis. Summa ciiijxj quarteria $j$ hopa. In semine 25 cxiij acrarum dimidiæ de Fernham, xxix acrarum apud Sele, xxij quarteria dimidium, $\mathrm{j}$ hopa. In pane facto ad opus domini Episcopi, iij quarteria. In pane facto ad opus Justiciarii, Comitis de Clara, ij quarteria dimidinm. In consuetudine servientium contra Natalem et Pascham, $\mathrm{j}$ quarterium dimidium. In precariis hyemalibus, quadragesimalibus, iij quarteria dimidium. In pane facto fac[ientibus] lardarium, vj hopæ.

30 . In blado sarclando, $\mathrm{j}$ quarterium. In pratis falcandis, ij quarteria. In precariis auptumnalibus, viij quarteria dimidium. In corrediis bovarii, caretarii, herciatoris, frumento mixto cum ordeo, xxiiij quarteria. In expensis G[alfridi] de Moneta, xiiij quarteria ij hopæ. In dono' domini Episcopi sanctimonialibus de Wintonia, xij quarteria. In supravenditis, iiij ${ }^{\mathrm{xx}} \mathrm{v}$ quarteria dimidium.

Idem reddunt compotum de iij quarteriis de toto exitu curailli de Fernham. Et de j quarterio de curallo de Sele. Summa $\nabla$. In porcis pascendis, totum.

Idem reddunt compotum de xij quarteriis de toto exitu silliginis de Fernbam. Et de xviij quarteriis ij hopis de Sele. Summa $\mathrm{xxx}$ quarteria ij hopæ. In semine clviij acrarum apud Felnham, 1 apud Sele, xxj quarteria. In corrediis bovarii, caretarii herciatoris, iij quarteria dimidium ij hopæ. In venditis $\nabla$ quarteria dimidium.

Idem reddunt compotum de xvij quarteriis dimidio ij hopis, de toto exitu ordei de Fernham. Et de viij quarteriis dimidio ij hopis de Sele. Et de ix quarteriis dimidio, de Muenes. Summa exxvj quarteria. In semine $x x$ acrarum apud Fernham, xiij acrarum apud Sele, viij quarteria. In corrediis bovarii, caretarii, herciatoris, sxij quarteria dimidium $\mathrm{j}$ hopa. In porcis pascendis, $\nabla$ quarteria iij bopæ.

Idem reddunt compotum de cexlviij quarteriis dimidio ij hopis de toto exitu avenæ de Fernham. Et de lvij quarteriis ij hopis de Sele. Summa cccvj quarteria. In semine cciiij ${ }^{x x}$ vij acrarum apud Fernham, lxj acrarum apnd Sele, cxv quarteria dimidium. In præbenda domini Episcopi pluries, per tallias, iijü.xiij quarteria iij hopæ. In præbenda Justiciarii, Comitis de Clara, R. de Cornhulla, $x \nabla$ quarteria. In præbenda palefridi domini Regis, ix quarteria. In præbenda Mathiæ, $v$ quarteria dimidium $\mathrm{j}$ hopa. In præbenda caretariorum domini Episcopi per $\mathrm{j}$ noctem, iij hopæ. In præbenda pastoris domini Episcopi per ij vices, vj hopæ. In præbenda Willelmi de Sorevilla per $\mathrm{j}$ noctem, iiij hopæ. In præbenda J [ohannis] de Mara, $\mathbf{v}$ hopæ. In præbenda R[ogeri] Wacelini $R$. Cerarii civitatis Wintoniæ, v hopæ. In præbenda G[alfridi]de Cauz, ij hopæ. In præbenda W[illelmi] Bastardi per iij noctes, G[alfridi] de Cauz per j noctem, fratris W[alteri] et magistri Ailwardi, j quarterium $\mathbf{v}$ hopæ. In præbenda clericorum, Savarici de Mauliun, et Vicecomitis de Tuars, j quarterium. In præbenda Roberti Basset pluries, vij quarteria dimidium. In præbenda avrorum, xj quarteria dimidium. In pressura facta, ij bopæ. In porcis pascendis, iij quarteria dimidium. In præbenda G[alfridi] de Moneta, xxxiij quarteria. In venditis, vj quarteria.

Instaurum.-Idem reddunt compotum de xij avris remanentibus ano præterito. Et de iiij M. 6. postes emptis. Summa xvj. In donis domini Episcopi, ij. In mortuo, j. Et 
Idem reddunt compotum de lv bovettis remanentibus anno præterito. Et de j adjuncto, anno præterito tauro. Et de vj proventis de testamento Edwini, Willelmi, Roberti, Turg[isii]. Et de xiij postea emptis. Summa lx.xv. In donis domini Episcopi, viij. In mortuis, v: In venditis, vj. Et remanent lvj. Unde dicunt quod W[illelmus] de Clarn debet iij.1

Idem reddunt compotum de xiiij vaccis, $\mathrm{j}$ tauro, remanentibus anno præterito. Et de j vacca de testamento Sweini. Summa xvj. In adjunctis bobus, $\mathrm{j}$ taurus. In vendita, $j$ vacca. Et. remanent xiiij vaccæ et $j$ taurus. ${ }^{2}$

Idem reddunt compotum de $\mathrm{j}$ tauro $\mathrm{ij}$ annorum, iij bovettis ejusdem æatatis, iiij geniculis, anno praterito annalibus. Et omnes remanent.

Idem reddunt compotum de $\mathbf{x}$ vitulis proventis hoc anno de prædictis vaccis quia

iiij fuerunt steriles. In decimis, $j$. In mortuo, $j$. Et remanent viij.

Idem reddunt compotum de cexlij ovibus remanentibus anno præterito., Et de $\mathrm{j}$ fortuitu inventa. Et de $\mathrm{ij}$ quæ fuerunt loco multonum. Et de xliij adjunctis de instauro. Summa cciiijxy viij. In venditis, xx. In mortuis ante partum, vj. In 15 inortuis post partum et ante tonsionem, xxj. Summa xlvij. Et remanent ccxlj.

Idem reddunt compotum de cx multonibus remanentibus anno præterito. Et de vj hurtardis proventis de Lindeseia. Et de lxj adjunctis. Summa clx.xvij. In mortuis, sxxix. "In missis apud Sele, ij gerciæ. Et remanent ${ }^{3}$ cxxxvj.

Idem reddunt compotum de cxlvj hoggettis, anno præterito agnis. In mortuis ante 20 tonsionem, xlij. In adjunctis ovibus, xliij. In adjunctis multonibus, lxj. Nichil remanet.

Idem reddunt compotum de ccxij agnis proventis hoc anno, quia vij oves fuerunt steriles. In decimis, $x x j$. In consuetudinibus $i j$ bercariorum, $i j$. In mortuis iiijxziiij. Et remanent cvij agni.

Idem reddunt compotum de ccclxvij velleribus grossis. In decimis, xxxvj. In consuetudinibus $\mathrm{ij}$ bercariorum, ij. In venditis, ccexxviij vellera quæ fecerunt $\mathrm{j}$ pondus dimidium et quartam partem ponderis.

Idem reddunt compotum de clx.xiiij caseis, qui inceperunt fieri $\mathrm{v}$ Idus Aprilis et desierunt fieri in festo Sancti Michaelis. In decimis, xvij. In consuetudine bercarii, 30 daiæ, ij. In expensis domini Episcopi, v.j. In corredio $\mathrm{R}$ [oberti] Basset, ij. In blado sarclando, iij. In pratis falcandis, ix. In precariis auptumnalibus, xxviij. In expensis G[alfridi] de Mon[eta], vj. In venditis, c qui fecerunt $\mathrm{j}$ pondus dimidium, quartam partem ponderis.

Idem reddunt compotum de $\mathrm{j}$ verre, xij suibus, xvij hoggettis, cxxviij porcis super- 35 annatis. Et de viij porcis de pannagio. Et de $\mathrm{j}$ porco de firmario de Beneti[ega]. Et de $\mathrm{j}$ verre, $\mathrm{j}$ sue proventis de Lnndonia. Summa clxix. In missis apud Menes, $\mathrm{j}$ verres, $\mathrm{j}$ sus. In mortuo, $\mathrm{j}$. In occisis ad lardarium, cxxxj. In corredio hominum domini Episcopi, iij. In expensis Justicarii, Comitis de Clara, ij. Et remanent xxix superannati.

Idem reddunt compotum de lix porcellis proventis de omnibus suibus. Et de 40 $v$ proventis de Londonia. Summa lxiiij. In decinis, v. In expensis domini Episcopi et domini Regis, vij. In consuetudine porcarii, j. Et remanent lj.

Idem reddunt compotum de xxiiij porcellis postea proventis. In decimis, ij. In missis apud Menes, iiij. In mortuis, iij. Summa ix. Et remanent $x v$. Summa tocius remanentis $\mathrm{iiji}^{\mathrm{xx}} . \mathbf{x} \mathrm{v}^{4}$

Idem reddunt compotum de ccl gallinis, proventis de bedeleria manerii, expensis.

Idem réddunt compotum de MD ovis. Expensis per Galfridum.

Idem reddunt compotnu de cxxxj haconibus. In corredio domini Episcopi, xxij. In corredio militum visorum forestæ, $j$. In elemosinis domini Episcopi, $j$. In precariis auptumnalibus, ix dimidius. In expensis G[alfridi] de Moneta, ij dimidius. In corredio 50 venatorum, dimidius. In expensis, Justiciarii, Comitis de Clara, iij. ' Et remanent iiij ${ }^{x x} . x j$ bacones.

Idem reddunt compotum de $\mathbf{x v}$ equis silvestribus receptis de Meredona. Et de ix equabus receptis de Clara. Istæ remanent.

Summa tucius debiti $x x l$. vijs. vd. Unde vijl. $x s$. jd. sunt de tempore W[illelmi] de 55 Clara ${ }^{5}$ et $\mathrm{v}$ boves xij multones superius computantur.

\section{SUTTONA.}

G[AI.FRIDUS] serviens et Hugo et W[illelmus] præpositi reddunt xiiijl. iijs. vijd. de reragio anni præteriti. Et quieti sunt.

Idem reddunt compotum de xljl. vijs. vjd. ob. de gabulo assiso de Suttona et Stanforda. 60 Et de vjd: de incremento gabuli Stephani fabri de $j$ mesagio. Et de xijd. de incremento gabuli Rogeri de Huvercompe de $\nabla$ acris quas Agnes de Surlonde tenuit. Summa xljl. ixs. $o b$.

In quietanciis $\mathrm{j}$ præpositi, vs. ; $\mathrm{j}$ berebr[etti], iijs. ; j bedelli, j fabri, $\mathrm{j}$ porcarii, $\mathrm{j}$ bercarii, xijs.; viij carrucariorum, per annum, xxiiijs.; ij carrucariorum, per dimidium annum, 66

\footnotetext{
1 iij on an erasnre. ${ }_{3} \mathrm{Et}$ de $l_{x j}$ adjunctis $\therefore . \quad$. Temanent is on an erasnre.

4 The word compotum has been erased. … remanent is on an erasnre.
} 
iijs.; ij operariorum, per annum, vijs. vjd.; iij operariorum, per annum, ix8. vjd ; ij operariorum, per annum, vjs.; ij operariorum, per dimidium annum, iijs. ; xij operariorum pro opere auptumnali, xijs. Summa iijl. vs.

In defectu gabuli terræ Kogeri militis, Roberti Blakemanni, tractæ in dominium, vs. vjd. In defectu gabuli terræ Pliilippi molindindarii tractæ in dominium, ijs. In defectu gabuli terræ Galfridi Hose datæ canonicis de Mertona in excambio pro dampno novi vivarii, per Godefridum Episcopum, xijd.

Summa viijs. vjd. Summa tam quietanciarum quam defectuum, iiijl. xiijs. vjd.

Summa remanens xxxvjl. xvs. vjd. ob.

Idem reddunt compotum de cvijs. iijd. ob. receptis de molindino molente de Alresforda. Et de xjs. $\mathrm{j} d$. de molindino foleratico.

Summa cxviijs. vd. ob.

Idem reddunt compotum de vjl. viijd. ob. de pannagio. Et de xxijs. iijd. de herbagio. Et de xxiijd. de cheriset. Et de vs. de consuetudine herbagii de Ferendona. Et de iiijs. vjd. de ij debilibus bobus venditis. Et de vs. iijd. ob. de curiis iiij boum mortuorum venditis. Et de vijs. de j equa viva vendita. Et de vijs. vijd. de xlj agnis vivis venditis. Et de vs. viijd. de $\mathrm{j}$ vacca, $\mathrm{ij}$ vitulis vivis venditis. Et de viijs. iijjd. de pellibus $x x v i i j$ ovium, xvj hoggettorum, xlij agnorum mortuorum venditis. Et de lx.xiijs. iiijd. de ij ponderibus dimidio, iiij parte ponderis grossorum vellerum venditis. Et de xxxvs. de v capitibus casei venditis. Et de iiijs. de potfalda relaxata. Et de xxviijs. vjd. de operibus relaxatis hoc anno. Et de lx.xs. viijd. ob. de $\mathbf{v}$ tonellis vini expensis in scotallo, unde iij tonella venerant de Wlveseia, ij de Waltham. Et de iiijs. iiijd. ob. de xxj acris terræ locatis hoc anno. Summa $\mathrm{xxl}$. iijjs. jd.

Idem reddunt compotum de cvijs. iijd. de xlij quarteriis frumenti venditis. Et de xjs. de vij quarteriis mancorni venditis. Et de xxijs. ijd. de xij quarteriis dimidio urdei venditis. Summa vijl. vjd.

De Purchasiis.-Idem reddunt compotum de iijs. de thedinga de Stanforda. Et de ijs. de thedinga de Biketnna. Et de iijs. de thedinga de Suttune. Et de ijs. de Willelmo de Biketona pro forisfacto bosci. Et de ijs. de Rogero fabro pro simili. Et de xijd. de Henrico Hastiler pro simili. Et de vjd. de Ricardo Taverner pro simili. Et de xijd. de Hugone Kitier pro simili. Et de xijd. de Roberto de Cruce pro simili. Et de ijs. de Ricardo Bole pro simili. Et de xijd. de Waltero filio Avice pro simili. Et de iijs. de Ricardo de la Sole pro mellea. Et de vjd. de Edmundo pro simili. Et de vjd. de Ricardo genero Philippi pro simili. Et de vjs. viijd. de Waltero de Done pro simili. Et de sijd. de Willelmo molindinario pro blado retento. Et de vjd. de Tedbaldo pro simili. Et de ijs. de Willelmo Palmer pro pastura. Et de iijs. de Philippo molindinario pro simili. Et de xijd. de Ricardo filio Roberti pro terra Gervasii injuste arata. Et de vjd. de Hervi pro mellea. Et de vjd. de Serlone Hud pro sursisa. Et de ijs. de Henrico de Hatelinca pro simili. Et de vja. de Waltero de Gaterigge pro simili. Et de xijd. de Ada filio Aviciæ pro simili. Et de ijs. vjd. de Hugone de Puteo pro falsuclamore. Et de xijd. de Hugone filio Nutricis pro sursisa. Et de xijd. de Willelmo de Ramescunnba pro simili. Et de vjd. de Henrico de Havercompe pro simili. Et de ijs. de Hugone de la Le pro mellea. Et de vjd. de Rogero de Havercompe pro pasturia. Et de xijd. de eodem pro terra sua. Et de vjd. de Rogero Mensel pro simili. Et de vjd. de Willelmo de Prato pro simili. Et de vjd. de Hunfrido Drake pro simili. Et de vjd. de Giliberto vaccario pro simili. Et de vjd. de Radulfo de Flode pro simili. Et de vjd. de Hugone Worvesur pro simili. Et de vjd. de Radulfo Colegrim pro simili. Et de vjd. de Godmundo Ferliche pro simili. Et de vjd. de Rogero de la Tee pro simili. Et de vjd. de Johanne Leverich pro simili. Et de v8. de Willelmo filio Ainolfi pro terra habenda. Et de vjd. de Willelmo Com[ite] pro simili. Et de vjd. de Stephano fabro pro simili. Et de xijd. de Serlone Hud pro simili. Et de ijd. de Willelmo Cobbe pro simili. Et de xijd. de Keginaldo Hud pro misericordia. Et de xij. de Roberto Hundesher pro mellea. Et de xijd.de Radulfo filio Haiwardi de Alresforda pro forisfacto bosci. Et de xijd. de Godefrido de Cruce pro mellea. Et de xijd. de Willelmo de Prato pro simili. Et de xiijs. iiijd. de Ricardo filio Roberti pro fine terræ. Et de vjd. de Olivero pro lege relaxata. Et de xxs. de Rogero filio Ailwini pro fine terræ. Et de vjs. viijd. de Willelmo, fratre suo, pro simili. Et de ijs. de Willelmo caprario pro mellea. Et de xijd. de Malgero de Stanforda pro falso clamore. Et de vjd. de Ada, filio suo, pro simili. Et de vjl. de Godwin de Fontc pro simili. Et de xiijs. iiijd. de Reginaldo de Stanforda pro fine terræ. Et de vjs. viijd. de Reginaldo Dolbohe pro simili. Et de xijd. de Ricardo de Fonte pro terra lıabenda. Et de xijd. de Ricardo Bello, quia non fecit domum in terra Episcopi, quam facere debuit. Et de iijs. de Johanne de Havercompe pro pluribus excessibus. Et de ijs. de Ricardo telario pro terra habenda. Et de ijs. de Willelmo Covenant pro simili. Summa vijl. iijs. ijd.

Idem reddunt compotum de $\mathrm{xxxl}$. de tallagio.

Summa cl. xxiijs. iijd. ob.'

Idem receperunt vijl. ${ }^{1}$ de Johanne decano, ad facienda opera. Summa tocius receptæ cxiiijl. $x \times j d$. 
Liberatio.-In liberatis Johanni decano, xxxjl. vijs. ijd. per v tallias.

Expensa.-In ferramentis $\mathbf{v}$ carucarum et rotis ad eas, xiiijs. viijd. In ferramentis ij avrorum, vjd. In iij paribus rotarum ad carettas de mairemio Episcopi, xviijd. In clutis, sellis, paronis, paronellis, cordis et eisdem axandis, $x v i j d$. ob. In iij corbellis emptis ad bladum portandum, vijd. ob. In sera empta ad ostium daeriæe et ponte emendando ante daeriam, vd. In bercaria reparanda et tegulanda, xijd. ob. In iijbus pirtibus j quarterii fabarum emptis ad plantacionem in gardino, xxijd. In gardino fodiendo, $\mathbf{x} x d$. In clatibus ad faldam, $x v d$. In $\mathrm{j}$ novo gallinario faciendo, ijs. iijd. In nova porcaria facienda, xviijd. In precariis hyemalibus, quadragesimalibus de iijixix carucis, xiijs. $x d$. In mercede ij famulorum in curia per annum vjs. In mercede daiæ, xviijd. In custo 10 daeriæ, xxjd. In MD lattıs emptis et parandis, et clavis ad magnam grangiam, et in mercede carpentarii eam parantis, xs. iiijd. $o b$. In eadem grangia tegulanda, iijs. In precariis auptumnalibus, xviijs. $j d$. In ponte reparando in capite calciæ magni vivarii, xijd. In portauda lana usque Bellum Locum et iterum reportanda per aquam, xijd. In furagio ${ }^{1}$ empto ad opus boum, vs. In furagio triturando in grangia canonicorum 15 ad opus boum, et ad alia negotia curn, iiijs. viijd. In liberatione Philippi servientis, lxs. $x d$. Summa vijl. $x v j s . x d .^{2}$

Expensa Forinseca.-In aula, camera clericorum, coquina, stabula emendandis, xiijd. In terra portanda in cameram clericorum ad aerain dessiccrndam, ijs. In gutteria facienda ad eandem cameram. In bordis emptis, ad bordandum supra ostium ejusdem cameræ, xviijd. 20 In ij pontibus reparandis ante $\mathrm{ij}$ portas curiæ, iijs. iiijd. In ij plumbis emptis ad coquinam Episcopi, iijs. viijd. In $x v$ porcis emptis ad larderium Episcopi, xxxjs. vjd. Iı j leporario cum catellis suis pascendis, per exvj dies, iijs. $x d$. In portando $\mathrm{j}$ tonello vini de Surhamptona usque Suttonam, xviijd. In ix . . . . ${ }^{3}$ emptis ad expensam domini Episcopi, iijs. In $\mathrm{xx}$ caponibus, iiijxij gallinis, emptis ad expensam domini Episcopi et 25 domini Regis, viijs. vijd. In corredio G. de Acl[anda] pluries, iiijs. iiijd., per ij t[allias]. ${ }^{3}$ In corredio magistri $R$ [oberti] Basset pluries, $x \times x s$. jd., per $\mathrm{v}$ tallias. In curredio Silvestri clerici ipsius, quando prævidit arma hominum per hundredum de Suttona, vijd. ob. In j nova grangia facienda. . . . . In ijj gutteriis taciendis et missis apud Wlveseiam, per præceptun senescalli, ijs. In corredio fratris Walteri bercarii pluries, xijd. In novo 30 servario faciendo juxta granarium, xxxijs. iij ob. In [portandis]. ${ }^{3}$. . ij tonellis vini de Wlveseia usque Suttonam, ijs. iij d.

Summa ix $l$. xjs. iijd. ${ }^{5}$ Summa tocius expensæ, xvijl. viijs. jd. ${ }^{5}$

Item. Expensa facta apud Alresfordam de . . ..$^{3}$ perticiend' et furno et fabrica et calcia reficienda, xlvl. xvijs. vjd.ob., de quibus dominus Episcopıs debet audire compotum. 35 Aulivit postea et pacatus est. ${ }^{6} \quad$ Summa tam liberationis quam expensæ in omnibus

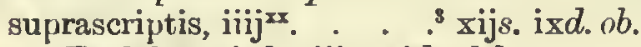

Et debet $x i x l$. viijs. $x j d$. ob.?

Exitus Grangiæ.-Idem reddunt compotum de xx quarteriis ij bussellis frumenti proventis de cheriset. Et de iij $^{x x} . x j$ quarteriis $i j$ bussellis de toto exitu frumenti. Summa cxj 40 quarteria dimidium. In semine cexlij acrarum, $\operatorname{lxv}\left[\mathrm{j}\right.$ quarteria dimidium]. ${ }^{8}$ In precariis auptumnalibus, iij quarteria. In supravenditis, xlij quarteria.

Idem reddunt compotum de xxxj quarteriis dimidio de toto exitu mancorni. In semine lxvj acrarum, xix quarteria dimidium. In corredio, ij . . . . ${ }^{8}$ per annum, iii) quarteria. In corredio daiæ, $\mathrm{j}$ quarterium. In supravenditis, vij quarteria.

Idem reddunt compotum de lxv quarteriis dimidio de toto exitu ordei. In semine iiij ${ }^{\mathrm{xx}} . \mathrm{xviij}$ acrarum, $\mathrm{xxx}$... . . In consuetudine porcarii et custodis agnorum, ij quarteria. In precariis auptumnalibus, vij quarteria. In corredio ij servientium, ix quarteria. In corredio daiæo, ij quarteria dimidium. In porcis, porcellis pascendis ij quarteria dinidium. [In supravenditis $]^{8} \mathrm{xij}$ quarteria dinidium.

Idem reddunt compotum de $\mathrm{c}$ quarteriis dimidio de toto exitu avenæ. Et de iiij ${ }^{x} \cdot x_{i i i j}$ quarteriis allatis de Cheritona. Et de $\mathrm{xxvj}$ quarteriis allatis de B[eworda]. ${ }^{\mathrm{s}}$ Et de xvj quarteriis allatis de Walda. Summa cexxxvj quarteria dimidium. In semine cccvj acrarum, $\mathrm{iijj}^{\mathrm{xx}} . \mathrm{x}$ viij quarteria dimidium. In præobenda domini Episcopi apud Suttonam, xxix quarteria dimidium . . ${ }^{8}$ per iij tallias. In prabenda magistri $R$ [oberti] Basset, pluries, $v$ quarteria dimidium per $\mathrm{v}$ tallias. In præbenda $\mathrm{G}$, de Acl[anda], v busselli per ij tallias. In præbenda G[alfridi] do Cauz et Danielis Pincernæ, dinnidium quarterium. In præbenda fratris Walteri, dimidium . . . . ${ }^{8}$ In præbenda iij avrorum, $x v$ quarteria. In bobus pascendis in garbis, xj quarteria. Summa clxjquarteria, dimidium. Et remanent lx.xv quartcria. 
[Instau]rum.-Idem reddunt compotum de $\mathrm{ij}$ avris remanentibus anno præterito. Et de $\mathrm{j}$ qui venit de curia Episcopi. Et de $\mathrm{j}$ equa fortuitu inventa. Summa iij. In vendita, j equa. Et remanent iij avri.

Illein reddunt compotum de lxvj bobus remanentibus anno præterito. In vivis venditis, ij. De precio superius respondent. In mortuis, iiij. De coriis superius respondent. Summa vj. Et rcmanent lx.

Idem reddunt compotum de ix vaccis, $\mathrm{j}$ tauro, iij bovettis, iij annalibus remanentibus anno proterito. Et de $\mathrm{j}$ vacea de testamento Roberti filii Ailwini. Et de ij vitulis forte inventis. Sunma $x \mathbf{x}$. In missis apud Biterne, ix vaccæ, $\mathrm{j}$ taurus, iij bovetti, iij anniles. In supravenditis, $\mathrm{j}$ vacca, ij vituli. Summa $x \mathrm{x}$. Nichil remanet.

Idem reddunt compotum de cciij ${ }^{x x} . \mathbf{x}$ ovibus remanentibus anno præterito. Et de jiijxxviij adjunctis de instauro. Summa ccelx.xviij. In consuetudine bercarii, j. In mortuo ante partum, $\mathrm{j}$. In mortuis post partum et ante tonsionem, xv. In mortuis post tonsionem, xij. Summa xxix. Et remanent cccxlix.

Idem reddunt compotum de viij cuillardis, iij multonibus remanentibus anno præterito. Et de lx.xij adjunctis. Surnma iiijxiij. Omnes remanent.

Ilem reddunt computum de ciijjxx xiijj hoggettis remanentibus anno præeterito agnis. In mortuis ante torsionem, xvj. De pellibus superius respondent. In adjunctis multonilius, Ix.xij. In adjunctis ovibus, iijjxziij. Summa clx.xvj. Et remanent $x$ viij.

Nota quod dorninus Episcopus condonavit post compotum de prascriptis ovibus, xiiij matrices oves, iij multones, ij hoggetti et exitum illarum. Scilicet, xiiij hogetti et xvj agni, et xviij velleru grossa, quia probatum erat quod prodicta xiiij matrices, et $i i j$ multones, et ij hoggetti expensi erant in precariis auptumnalibus pontificatus domini Wintoniensis. ${ }^{1}$

M. 6d. Idem reddunt compotum de ceiiijxiiij agnis proventis hoc anno de præedictis ovibus, quia iiij fuerunt steriles. Et de iiijxxvj proventis de Aweltona. Summa ccclx.x. In decimis, xxvj. In consuetudine bercarii, $j$. In vivis venditis, $x l j$. De precio superius respondent. In mortuis ante tonsionem, xlij. De pellibus superius respondent. In missis apud Cheritonam, cexxxix. Summa ccexlix. Et remanent xxj.

Idem reddunt compotum de cccexliiij ${ }^{2}$ velleribus grossis. Et de iij velleribus agninis. Summa ceccslviij. ${ }^{2}$ In decimis, xlij. In consutudine bercarii, $j$. In supravenditis, ccciijjxiij, cum iij velleribus agninis, quæ fecerunt ij pondera dimidium et quartam partem ponderis. Et debent xviij vellera. ${ }^{3}$

Idem reddunt compotum de ciij ${ }^{x x} . x j$ caseis qui inceperunt fieri iij die ante Annuneiacionem Beatæ Maria, et desierunt die Sancti Michielis utroque die computato. In decimis, xix. In consuetudine fabri, $\mathrm{j}$. In expensis domini Episcopi, vj. In expensis magistri R[oberti] Basset, $\mathrm{j}$. In precariis auptumnalibus, $x \mathbf{x} x \mathbf{i i j}$, qui fecerunt $\mathrm{j}$ caput. In supravenditis, $\operatorname{cxxx}$, qui feceruut $\mathrm{v}$ capita.

Idem reddunt compotum de lxvj porcis remanentibus anno præterito. Et de liij de exitu illorum. Et [de] xv emptis. De precio superius respondent. Et de lx.x proventis de Clera. Summa cciij. In decimis, v. In consuetudine porcarii, j. In missis apud Wlveseiam et liberatis J[ohanni] decano, cij. In mortuis, xv. Summa cxxiij. Et remanent iiij ${ }^{x x} . j$, unde $x x$ sunt sues, $j$ verres, $x x v$ porci superannati, xiiij juniores dimidii anni, $x x j$ porcelli.

Idem reddunt compotum de xxiij aucis remanentibus anno præterito. In expensis domini Episcopi, xviij, liberatis $K$ [ogero] Wacel[ino] apud Wlveseiam. In mortuis, v.

Idem reddurt compotuin de iij]xiij gallinis, $\mathbf{x}$ caponibus emptis. Et de c gallinis de cheriset. Summa cciij. In liberatis J[ohanni] decano apud Wlvesciam, 1 per $\mathrm{j}$ talliam. In elemosina domini Episcopi leprosis de Sancta Maria Magdalena, xx gallinæ. In expensis domini Regis et domini Episcopi, xx capones, xvij gallinæ liberatæ Nicolao de Coquina, per $\mathrm{j}$ talliam apud Wlveseiam. In liberatis G[alfrido] de Cauz, xlviij gailinæ ix he[bdomadarum] per ij tallias. In expensis domini Regis, $\mathrm{xl}^{2}$ gallinæ, sine tallia, apud Suttonan. In mortuis, viij.

Idem reddunt compotum de viij baconibus reecptis de Wlveseia per manum J[ohannis] decani. In precariis auptumnalibus, omnes.

Summa tocius debiti xixl. viijs. xj.$^{2} \circ b$. unde xiijl. xiijs. viijd. $o b .{ }^{4}$ est manifeste debitum ; præterea j marea de fine Rogeri præpositi. ${ }^{5}$

Not[andum] quod Episeopus condonavit post compotum Ricardo de Widehaie xxiiijs. xjd. de debito præscripto, quia probatum erat quod illos expendidit dum fuit serviens apud Suttonam. Item condonavit de præscripto debito Phillippo de Croperie lx.vjs. iiijd., quia probatum fuit quod illos expendidit in servicio domini Episcopi apud Chiritonam et Suttonam anno secundo pontificatus domini Petri Wintoniensis. Item Episcopus relaxavit Rogero de Haverecamp xxiijs. de tempore Ricardi de Widehaie de eodem debito post compotum pro j marca qua finivit. Summa cxiiijs. iijd.

1 A later addition in a clerical hand in the lower margin of the roll. Et debent rviij. velleru is a later cntry in the roll in au official band.

An official correction on an erasore in the roll. 
Solverunt post compotum et quieti sunt per totuı, præter marcam de fine Rogeri præpositi.

Item debent $x$ viij hoggettos, xviij agnos, xviij vellera de xviij ovibus quæ supercomputati sunt eis ad duo compotos præteritos, ut dicunt.

Idem reddunt compotum de Dxl ovis liberatis. In coquina totum et quieti sunt. ${ }^{1}$

\section{MENES.}

G[Ar.Fridus], Audelinus, servientes, et R[icardus] et Thomas præpositi reddunt xxiijl. xiijs. vjd. ob. de reragio anni præteriti. Et quieti sunt.

Idem reddunt compotum de lx.xiiijl. $x j s . x d$. $n b$. de gabulo assiso cum incremento gabuli hoc anno de xijd. In quietancia $\mathrm{j}$ bercarii, $v$. In quietanciis $\mathrm{j}$ fabri, $\mathrm{j}$ porcarii, 10 iij bercariorum, xvij carrucariorum, lvijs. vjd. Summa lxijs. vjd.

In defectu terrarum tractarum in dominium, xxjs. vijd. ob. In defectu terræ de Ricch[ereombe] datæ canonicis de Novo Loco, cs. In defectu molindini de Capella tracti in dominium, xs. Summa vjl. xje. vijd. $o b$.

Summa tam quietanciarum quam defectuum, ixl. xiiijs. iij ob. Summa remanens 15 lxiiijl. xvijs. ixd.

Idem reddunt compotum de iijl. xvijd. ob. de pannagio. Et de xvijs. de herbagio. Et de xxiijd. de potfalda. Et de lxs. iijd. de xviij bobus vivis venditis. Et de xvs. ijd. de $x \times v j$ ovibus vivis venditis. Et de $x l$. xiijs. iiijd. de viij ponderibus lanæ venditis. Et de iijl. ijs. vjd. pro lacte cccexij ovium ad firmum. Et de iijs. de iij coriis boum 20 mortuorum venditis. Et de xvijs. vjd. de pellihus lvj ovium, xxxix multonum, iiij ${ }^{\mathrm{xx}} \mathrm{ij}$ hogettorum mortuorum venditis. Et de xvijs. vjd. de celxij pellibus agnorum mortuorum venditis. Et de ixd. de ij acris locatis hoc anno. Et de xvs. ixd. pro opere relaxato. Et de $x s$. de vj quarteriis nueium venditis. Et de iijl. ijs. de iiij tonellis vini venditis. Summa $\mathrm{xxxl}$. xixs. ijd. ob.

Idem reddunt compotum de xiiijl. xs. iiijd. de c quarteriis dimidio, ij hopis frumenti venditis. Et de viijs. de iiij quarteriis silliginis venditis. Et de lxvjs. de xxxiij quarteriis ordei venditis. Et de vs. de v quarteriis avenæ venditis. Summa xviijl. ixs. iijd.

Idem reddunt compotum de xxxvijs. ixd. de toto exitu molendini novi.

De Purchasiis.-Idem reddunt compotum de iijs. de Gocelino de Bord[ene] pro filia sua maritanda. Et de xijd. de Roberto Stigandi pro simili. Et de vjd. de Thoma Blundo pro simili. Et de xiijs. iiijd. de Willelmo de Privet pro fine terræ. Et de xiijs. iiijd. de Willelmo fratre pro terra de Roden[haın]. Et de vjs. viijd. de Willelmo carter pro terra de Oxeneburne. Et de ijs. de Roberto filio Willelni carter pro terra patris sui. Et de xs. 35 de Rogero molindinario pro molindino. Et de ijs. de Roberto de Ramesd[ene] pro filia sua maritanda. Et de xiijs. iiijd. de Gocelino de Strode pro veredicto. Et de xijd. de Stephano Blakeman. Et de liijs. iiijd. de hundredo. Et de vjd. de Ricardo Rufto pro fine terræ. Et de xxs. de uxore Adæ de Ecclesia pro fine terræ. Et de xxvjs. viijd. de Waltero de Holewei pro simili. Et de xiijs. iiijd. de Willelmo de Efforda pro filia sua maritanda. Et de xijol. de Stephano Blakemanno quia non aravit. Et de vjd. de Ricardo de Cumba pro pastura. Et de vjd. de Galfrido de Cumbe ${ }^{2}$ pro simili. Et de vjd. de Giliberto de Cumba pro simili. Et de vjd. de Reginaldo de Cumba pro simili. Et de vjs. viijd. ${ }^{3}$ de Edwardo de Fungh' pro lege. Et de xs. de Hugone de Halstede pro fine terræ. Et de xijd. de Roberto filio Sewardi pro simili. Et de vjd. de Galfrido Gule pro 4 lege. Et de vjs. viijd. de Galfrido Blakewine pro simili. Et de vjs. viijd. de Waltero Tedgari pro sinili. Et de vs. de Roberto Stigandi pro simili. Et de vjs. viijd. de Randulfo de Ramesdene pro simili. Et de vjd. de Theobaldo de Ramesdene pro simili. Et de vjd. de Aldr[edo] pro simili. Et de xviijs. de Ricardo Lukitale pro decima. Et de vjd. de Hugone Smart pro boseo. Et de vjs. vijd. de Thoma de Hoke pro decima. Et 50 de vjd. de Elviat Geroldi pro lege. Et de vjd. de Ricardo carp[entario] pro śimili. Et de vjd. de Waltero filio Galfridi pro simili. Et de vjd. de Ricardo Hadhewis pro simili. Et de xijd. de Thoma de Writh' pro plegio. Et de xijd. de fratre ejus pro simili. Et de vjs. viijd. de Galfrido Gosecrei pro manutenemento Episcopi, vjs. viijd. de Randuito rle Essturd' pro vidua de Frorx[felda]. Et de xviijd. de Gileberto de Cumba pro pas- 55 tura. Et de ijs. de Ricardo de Cumba pro simili. Et de xijd. de Rogero de Cumba pro simili. Et de xijd. de Galfrido de Cumba pro simili. Et de xijd. de Walkelino pro simili. Et de xijd. de Hersent pro simili. Et de vjd. de Malin pro simili. Et de vjd. de Willelmo de Bosco pro simili. Et de vjd. de 'I'loma Banne pro simili. Et de vjd. de Nicholao filio Godæ pro simili. Et de vjd. de Chacheis pro simili. Et de vjd. 60 de Wudem[an] pro simili. Et de vjd. de Hoeres pro simili. Et de xijd. de Patricio præposito pro simili. Et de vjd. de Waltero Pudd[inge] pro simili. Et de vjd. de Willelmo Hordrun pro simili. Et de vjd. de God[efrido] Gredere pro simili. Et de xijd. de Banne

1 The whole of the above entries from Notandum to the end of the account are inserted in the margin. 2 Sic in MS. 3 On an erasure in. MS. 
pro simiii. Et de vjd. de Tebaldo pro simili. Et de xijd. de Alano capellano pro simili. Et de xijd. de Patricio. Et de vjd. de Gocelino Saia pro simili. Et de vjd. de Caie pro simili. Et de vjd. de Henrico de Ilestan pro simili. Et de vjd. de Othino pro simili. Et de vjd. de Roberto Palmer pro simili. Ét de vjd. de Osberto de Mara pro simili. Et de vjd. de Cecilia de Mara pro simili. Et de xijd. de Wiilelmo filio Anketilli pro simili. Et de vjd. de Ricardo Grede pro eervisia vendita contra assisam. Et de vjd. de Banne pro simili. Et de vjd. de G[e]ldun pro simili. Et de vjd. de Goda Waldø pro simili. Et de vjd.de filio Poehe et pro simili. Et de vjd.de Radulfo de Ponte pro simili. Et de vjd. de Stephano Bole pro mellea. Et de xijd. de Waltero Tedgar pro simili. Et de vjd. de Willelmo Blake pro simili. Et de vjd. de Thoma Albo pro simili. Et de ijs. de Ricardo de Aesete pro sursisá. Et de ijs. de Willelmo et Ailwino pro simili. Et de xijd. de T'ebaldo de Sodebeche pru simili. Et de vjd. de vidua de Ramesd[enc] pro fine terræ. Et de vjd. de Jocelino Brung' pro simili. Et de vjd. de Willelmo earetario pro falso clamore.

Summa xvl. iiijs. viijd.

Idem reddunt compotum de $l l$. de tallagio.

Summa tocius lxvl. iiijs. viijd.

Summa summarun tocius receptæ, ciiij ${ }^{x} \mathbf{j} l$. viijs. viijd. ob.

Liberatio.-In liberatis Johanni deeano apud Wlveseiam, per vj taillias, tam de auxilio quam gabulo et alio exitu, exxiijl. xiijs. ob.

Expensa - In ferramento j earucæ, iijs. $x d$. In ferrura ij earuearum, xviij avrorum, vijs. vid. $o b$. In $j$ eareta ferrata, et iiij cliarum caretarum ad fimum extraheudum, xiijs. iijd. In carueis, herciis, earetis sæpius repar'andis, iijs. vjd. In clutis, uneto, [sellis ${ }^{1}$ ], panellis, et alio harnesio, ad caretas, vijs. viijd. ob. In xiij bobus emptis, iiijl. vijs. ixd. In $\mathrm{j}$ avro empto, vs. In $\mathrm{ij}$ poreis emptis, ijs. In preeariis hyemalibus, quadragesimalibus, de cexliij carueariis, Ixiiijs. viijd. In triturandis ce quarteriis frumenti, xxxiijs. iiijd. In triturandis iiij quarteriis sigali, viijd. In triturandis cl quarteriis ordei, xvs. vijd. ob. In triturandis Diiij ${ }^{x x}$ quarteriis avenæ, $x x i x s$. $x d . o b$. In $\mathrm{j}$ vanno empto, xiiijd. In vanandis $\mathrm{iij}^{\mathrm{xx}} . \mathrm{xvij}$ quarteriis frumenti, viijs. $\mathrm{j} d$. In iij saceis emptis, xviijd. In saccis emptis ad linnam, vijs. $\mathrm{ij} d$. In stipendiis, $\mathrm{j}$ caretarii, $\mathrm{j}$ haiwardi, iij carucariorum, per annum, $\mathrm{xv} s$. iiijd. In stipendiis iij earetariorum, per $\mathrm{x}$ septimanas, ad fimum extrahendun, $\mathrm{x}$ viijd. In potu et companagio ad magnam bederipam, ot ad xvj alias bederipas, $x x x v s$. ixd. In [corredio] G. de Ancl[anda] et familix, auptumno, per vj septimanas, xs. viijd. In enrredio iiij præpositorum in auptumno, viijs. viijd. In calce, clavis, emptis ad grangiam, et ipsa grangria reeooperienda, vijs. viijd. In j mola empta, xiijs. iijd. In bordis, clavis, ad ipsum molindinum et in domo molindini facienda de novo, et stagno ipsius molindini emendendo, xxiijs. In expensis senescalli pluries, xxxviijs. $x d$. In vino eariando de Walthain ad Menes, ijs. In decima casei, viijs. iijd. In liberatione Germ[ani], lxs. $x d$. Summa xxvl. xvijs. viijd.

40 Expensa neccessaria.-In $\mathrm{j}$ porta facienda de novo, eum ferramento ad eam pertinente, et $\mathrm{j}$ ponte faciendo ante ipsam portam, vijs. vijd. In eusto piscationis apud Kneppe, et in pisee dueendo ad Menes et Merewellam, xxixs. viijd. In novo vivario emendendo, xxiijs vjd. In $\mathrm{j}$ novo pomario plantando, et eireumfossando et cireulando, $\mathrm{x} v i i j s, \mathrm{x} d$. In corredio Roberti de Elestede eum earris suis, ijs. vjd. In expensis Johannis de Mara, et Johınnis de Briwes, M. de Turvile, et R[oberti] Basset, per j noetem, pro terra Eustachii de Grenvills, et in expensis prædictorum Johannis de Mare, et Eustachii de Grenvilla, itinerantium per ij adventus, xviijs.

Summa cs. jd. Sumina tocius expensæ, $\operatorname{xxx} l$. xvijs. ixd. Quitantiæ per breve senescalli vjs. viijd. Summa tocius liberationis et expensæ cum quitantiis, eliiijl. xvijs. vd. $o b$.

Grangia.-Idem reddunt compotum de ce quarteriis, ij hopis, de toto exitu frumenti. Et de iij quarteriis de eheriset. Et de $\mathrm{j}$ quarterio dimidio easu proventis. Summa eeiiij quarteria dimidium, $\mathrm{ij}$ hopæ. In semine ceeclxxix acrarum, iijj ${ }^{\mathbf{x}} . \mathrm{x}$ quartcria dimidium. In liberatis Waltero de Parfit, xiij quarteria dimidium. In supravenditis, e quarteria dimidium ij hopæ. De precio superius respondent.

Idem reddunt compotum de iiij [quarteriis] sigali casu proventis. [In] ${ }^{1}$ supravenditis, totum. De precio superius respondent.

Idem reddunt compotum de clxiij quarteriis ij hopis, de toto exitu ordei. In semine cexxx acrarum, xlvj quarteria dimidium. In liberatis G. [de] ${ }^{2}$ Moneta, apud Fernham, ix quarteria dinnidium. In liberationibus, $\mathrm{j}$ caretari, $\mathrm{j}$ hai wardi, iij caruciriorum, $\mathrm{j}$ daiæ, per annum, xxxvij quarteria dimidium. In liberationibus herciatoris, custodis agnorum, $\mathrm{ij}$ quarteria. In liberationibus ...2 caret[ariorum], per $\mathrm{x}$ septimanas ad finum extrahendum, iij quarteria dimidium ij hopæ. In pane, xvj bedriparum, xxxj quarteria. In supra- 
venditis, sxxiij quarteria. De precio superius respondent. Idem reddunt compotum de D [iij] $\left.{ }^{x x} . x\right]^{1}$ quarteriis $\mathrm{j}$ hopæ, de toto exitu avenæ. Et de $\mathrm{x}$ quarteriis receptis ie ecclesia. Summa De quarteria, j hopa. In semine De acrarum, ecexviij quarteria dimidium. In consuetudine carucariorum, xvj quarteria. [In]' bobus pascendis, xix quarteria. In præbenda $x x$ equorum cum caretariorum tum avrorum, lx.xij quarteria dimidium. In 5 præbenda $\mathrm{j}$ caretarii, ${ }^{2} \mathrm{j}$ herciatoris, a festo Sanctæ Crucis, per vj ebdomadas proximas, $\mathbf{x}$ quarteria. In præbenda sen[escalli] . . . . . . . . In præbenda domini Episcopi, apud Menes, xxviij quarteria j hopa. In probenda J. de Mare, J. de Briw[es] M de Turvilla, ij quarteria, dimidum. In præbenda Roberti de Ellestede, j quarterium. In liberatis Johanni Decano [apud Wlv]es[eiam] ${ }^{1}$ iijj ${ }^{x i i j}$ quarteria. In liberatis Waltero 10 de Parfit, xxiij quarteria. In venditis, $\vee$ quirteria. De precio superius respondent. Ide " reddunt compotum de xxvj quarteriis $\mathrm{j}$ hopa, de toto exitu [molindini. In] ${ }^{1}$ liberatione molindinarii, iij quarteria dımidium, $\mathrm{j}$ hopa. In supravenditis, xxij quarteria dimidium.

Instaurum.-[Idem reddunt compotum $]^{11}$ de ij carettariis, xviij avris remanentibus anno proterito. Et de $\mathrm{j}$ avro empto. Summa $\mathrm{xxj}$. In liberato ad caret[am] domini 15 Episcopi, $\mathrm{j}$ caretarius. Et remunent $\mathrm{xx}$.

[Idem reddunt] ' compotum de cv bobus remanentibus anno præterito. Et de iiij de legatis. Et de xiij emptis. Summa cxxij. In vivis venditis, xviij. In magna bedripa j. In mortuis, iij. Summa xxij. [Et remanent]' c.

Idem reddunt compotum de ccciiij ${ }^{x x} . x v$ ovibus remanentibus anno præterito et de viij 20 casu proventis ante partum. Et de $\mathrm{v}$ de potfalda post partum et ante tonsionem. Et de

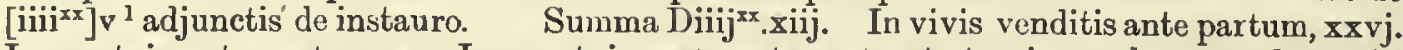
In mortuis ante partum, $\mathbf{x}$. In mortuis post partum ct ante tonsionem Ix.x; unde xxviij pelles [fuerun] $\mathrm{t}^{1}$ in consuetudine firmariorum. In mortuis post tonsiouem, iiij. Summa cx. Et remanent ccciiij ${ }^{x i}$ iij oves.

Iden recldunt compotum de ccciiij ${ }^{x x}$ j multonibus remanentibus anno [præterito]' Et de ij casu proventis ante tonsionem. Et de $\mathbf{v}$ de potfalda ante tonsionen. Et de exxviij adjunctis de instauro. Summa Dexxj. In consuetudine prati falcandi [póst] ${ }^{1}$ tonsionem iij vivi. In mag!ı bedripa, ij. In mortuis ante tonsionem, xxxiij. In mortuis post tonsionem, iiij. Summa xlij. Et remanent Dlx.xix. multones.

Idem reddunt compotum [de] cciiij ${ }^{x x} . x v$ hoggettis remanentibus anno præterito agnis. In mortuis ante tonsionem, lx. In mortuis post tonsionem, $x x i j$. In adjunctis ovibus, iiijxx ${ }^{x x}$ In adjunctis multonibus, cxxviij.

Idem reddunt compotum de ccccliiij agnis proventis hoc anno, de prædictis ovibus, quia xiij fuerunt steriles. In mortuis ante separationem cciiij ${ }^{x x}$. In decimis, $x v i j$. 35 In consuetudine ij bercariorum, ij. In consuetudine firmariorum, vij, tarde nati. In mortuis post separationem, ix. Summa cecxvj. Et remanent exxxviij agni.

Idem reddunt compotum de Miiij ${ }^{\mathrm{x}}$.xvij velleribus grossis. In decimis, cix. In consuetudine iiij bercariorum, iiij. In supravenditis, Dcccc iiij ${ }^{x x} . i i j$, quae fecerunt vij pondera dimidium et quartam partem ponderis.

Idem reddunt compotum de exxxviij velleribus agninis. In supravenditis, totum; quae fecerunt quartam partem ponderis.

Iden reddunt compotum de cciijjx. pellibus agninis. In decimis, xxviij. In supravenditis celxij.

Idem reddunt compotum de lvj porcis remanentibus anno præterito. Et de ij emptis. Et de vj receptis de Fernham. Et de lv de exitu illorum. Et de xij receptis de Ecclesia. Summa cxxxj. In liberati[s] Walter[o] de Parfit, xxx. In consuetudine porcarii, j. Summa $\mathbf{x x y}$. Et remanent c, unde ij sunt verres, $x$ sues, $x v$ suellæ, $x x v$ femellæ, xlviij porcelli separati.

Idem reddunt compotum de cxxiij gallinis remanentibus anno præterito. Et de 50 cccxv de cheriset, hoc ann\%. Summa cccexxxviij. In liberatis Johanni Decano apud Wlveseiam, clvj. In expensis senescalli pluries, et aliorum de familia Domini Episcopi, xxxv. Et remanent cexlvij gallinæ.

Idem reddunt compotum de $\mathrm{xx}$ baconibus, receptis de Wlvescia. In companagio bedriparum, xj bacones dimidius. In familia, auptumno, apud Menes, Hameldonam, 55 Frox[feldam], Stepe, v dimidius. Et remanent iij.

Idem reddunt compotum de vj quarteriis nucium remanentibus. In venditis totum.

Sumua totius debiti, xxvjl. xjs. iijd., unde vjl. xiiijs. iijd. sunt de areragio terræ Fulconis de Ed .. . . de v annis. Et ls. le auxilio terræ assignatæ Canonicis Novi Loci : et xvijs. vjd. sunt de redditu terræ de Ambr[esbiria] qui nondum erat . . . . . . 60 Summa $x l$. et ixd. Summa residui xvjl. ixs. vijd.

\section{ECCLESIA DE MENES.}

M. 7. Galfridus, Audelinus, servientes, Robertus, Thomas, et Patricius præpositi reddunt iiijl. vd. de reragio anni præteriti. Et quieti sunt. 
Idem reddunt compotum de iiijl. xvijs. iiijd. de toto gabulo assiso, cum incremento gabuli hoc anno de xijd. In quietanciis $\mathrm{j}$ præpositi iijs., iij carucariorum per annum vijs. vjd.

Summa $x s$. vjd. Et remanent iiijl. vjs. $\mathrm{x} d$.

Idem reddunt comnotum de xxjs. ijd. ob. de decima agnorum. Et de vijs. iijd. ob. de decima lanæ. Et de xxiiijs. de decima casei. Et de xxjs. de decina pullorum et vitulorum. Et de vjs. de lino vendito. Et de vjl. xvjs. iiijd. de v ponderibus lanæ et iij clavibus. Et de viijs. nij $d$. de decima dominici casei. Et de vs. de opere relıxat». Et de ijs. de coriis ij houm. Et de vjs. vd. ob. de cv pellibus agninis venditis. Summa xjl. xvijs. vjd. ob.

Idem reddunt compotum de $x x v i i j l . x s$. vijd. de cevij quarteriis fiun.enti venditis. Et de $\mathrm{x} v \mathrm{j} s$. viijd. de $\mathrm{x}^{1}$ quarteriis mancorni venditis. Et de $\mathrm{xx} s$. vd. de viij quarteriis dimidio, ij hopis sigali venditis. Et de xiijl. xiiijs. iijjd. de cxxxviij quarteriis, dimidio, ordei venditis. Et de xxijs. vjd. de xxij quarteriis avenæe et dimidio venditis. Summa xlvl. iiijs. vjd.

15 De Purchasiis.-Idem reddunt compotum de xls. de Alexandro de Biged[ona] pro fine terræ. Et de iijjs. de thedinga. Et de vjd. de Leticia pro fine terræ. Et de xijd. de Puddinge pro filia sui maritanda. Et de vjd. de eodem pro cervisia vendita contra assisam. Et de vjd. de Matildi vidua pro simili. Et de vjd. de Reginaldo Pell[ipario] pro simili. Et de vjd. de filio Saus[erii] pro simili. Et de vjd. de Radulfo Toli pro lege. Et de vjd. de Henrico de Trilest[er] pro simili. Et de vjd. de Radulfo Toli pro excambio. Summa xlixs.

Idem reddunt compotum de vjl. de tallagio. Summa tocius viijl. ixs.

Summa summarum tocius receptæ $1 \mathrm{xix} l$. xvijs. $\mathrm{x} d$. ob.

Liberatio.-In liberatis J[uhanni] decano, xvjl. xs., per j talliam. Magistro R[oberto] Basset, xls., unde habet responderc.

25 Expensa.-In ferramentis ij carucarum cum uercede fabri, viijs. vjd. In carucis reparandis, rotis, xijd. In $\mathrm{j}$ careta ferrata et iiij aliis ${ }^{2}$ caretis emptis xijs. vjd. In ferrura $\mathrm{j}$ carctæ per annum, vij aliarum ${ }^{3}$ in auptunino, viijs. viijd. In uncto, clutis, panellis, sellis, et alio harnesio, vijs. $v d$. $o b$. In stipendiis $\mathrm{j}$ caretarii $\mathrm{j}$ bovarii, $\mathrm{j}$ haiwardi, $\mathrm{j}$ daiæ per annum, iij caretariorum in auptumno, xviijs. In iiij bobus emptis, xxxijs. In securibus, volgis, beschis, et trublis emptis, xiijd. In triturandis ciiij $x^{x}$.xvj quarteriis frumenti, xxxijs. viijd. In triturandis viij quarteriis dimidio, ij hopis sigali, xvijd. ob. In triturandis clxiij quarteriis dimidio ordei, $x$ vijs. In triturandis cciiij ${ }^{x \mathrm{x}} \mathrm{ix}$ quarteriis avenæ, $\mathrm{xvs}$. $o b$. In grangiis de Menes, Hameldona, Frox[felda], Step[e] reparandis et recooperiendis, xjs. viijd. In seris iijd. $o b$. In vanandis iijoxiiij quarteriis frumenti, vijs. In iij saccis emptis, $x$ viijd. In $j$ alveo faciendo ad pobia pilinda, $x v j d$. In sicera facienda, viijd. $o b$. In companagio bedripæ, iijs. iijd. In decimis de Menes cum pertinentiis, præter Hameldonam, colligendis et intassandis, lxiijs. $x d$. In eisdem cariandis, xxxviijs. In colligendis et intassandis decimis de Hameldona, xxvjs. iiijd. In eisdem cariandis, xvijs. xjd. In liberatione magistri Ailwardi ibidem per vij septimanas, ixs. iijjd. In liberatione Ricardi Clerici apud Frox[feldam] et Davidis apud Stepe per vj septimanas, xvjs. In liberatione Silvestri et Audelini, per xlvj septimanas, liijs. $x d$. In saccis emptis ad lanam, ijs. In culmo colligendo, iijjs. $\mathrm{j} d$. In coquina facta de novo, lixs. iijd. ob. In aula et camera recooperiendis, iijjs. vijd. In cariandis cexxij quarteriis avenæ apud Wintonıam et Merewellam, xviijs. vjd. Summa xxiiijl. xiiijs. $\mathrm{x} d$.

Sumına tam liberationis quam expensæ, xlijl. iijjs. $x d$. Et debent xxvjl. xiijjs. $o b$,

Exitus Grangiæ.-Idem reddunt compotum de cexxxv quarteriis, ij hopis de toto exitu frumenti. In senine iiijxx acrarum, xvj quarteria. In cervisia auptumno, $\mathrm{j}$ quarteriuın ij hopæ. In pane ad familiam auptumno, vj quarteria dimidium. In nissis apud Waltham, iij quarteria dimidium. In supravenditis, ccvij quarteria. De precio superius respondent.

Idem reddunt coinpotum de toto exitu mancorni. In supravendito, totum. De precio superius respondent.

Idem reddunt compotum de viij quarteriis dimidio $\mathrm{ij}$ hopis de toto exitu sigali.

In supravenditis totum. De precio superius respondent.

Idem reddunt compotum de ciiij ${ }^{x}{ }_{i j}$ quarteriis de toto exitu ordei. In semine $x$ acrarum, iiij quarteria. In liberationibus, $j$ caretarii, $j$ bovarii, per annum, xiij quarteria. In libcrationibus iiij carctariorum in auptumno, iiij tassatorum, vj quarteria. In missis Waltero de Parfit, xij quarteria. In pane bedriparum, vj quarteria dimidium. In supravenditis, exxxviij quarteria dimidium. Et debent dimidium quarterium Precium xijd.

Idem reddunt compotum de cccelx.xvij quarteriis dimidio de toto exitu avenæ. Et de $\mathbf{x x}$ quarteriis brasei remanentilus anno præterito. Summa ccccxiij ${ }^{x \mathbf{x}} \cdot \mathbf{x i j}$ quarteria dimidium. In semine $\operatorname{cxx}$ acrarum lx.x quarteria. In consuetudine carucariorum ij quarteria, ij hopæ. In præbenda $\mathrm{j}$ caretarii per annum, vij per auptumnum, per vij septimanas, xxxviij quarteria. In bobus sustinendis, $x$ quarteria dimidium,

$$
1 \text { On an erasure in MS. } \quad 2 \text { Alias in MS. } \quad \text { Aliorum in MS. }
$$


ij hopæ. In præbenda vij equorum per auptumnum, scilicet et vj custodum, xlij quarteria. In præbenda $\mathrm{j}$ caretarii a festo Sanctæ Crucis per vj septimanas proximas, iij quarteria. In liberatis Johanni decano iiijxy quarteria dinidium. In liberatis præposito Manerii, x quarteria. In cervisıa, auptumno, vj quarteria. In libcratis Waltero de Parfit, cxx quarteria. In liberatis $\mathrm{P}$ [etro] Ace, liiij quarteria. In liberatis præposito de 5 Merewella, xx quarteria. In præbenda senescalli, ij quarteria. In præbenda J[ohannis] de Mara, E[ustachii] de Grenvilla, iij quarteria, dimidium. In liburatis præposito de Hameldona, iiij quarteria. In supravenditis, xxij quarteria dimidium. De precio superius respondent. Debent viij quarteria.

Idem reddunt compotum de vj quarteriis de toto exitu fabarum et pisarum. In 10 semine in novo pomario, ij quarteria dimidium. In porcis pascendis, iij quarteria dimidium. Nichil remanet.

Instaurum.-Idem reddunt compotum de xxxiiij bobus remanentibus anno præterito. Et de iiij emptis. In mortuis $\mathrm{ij}$, et remanent $\mathrm{xxvj}^{\prime}$ boves. Et remanet $\mathrm{j}$ carettarius.'

Idem reddunt compotum de cexxxix agnis proventis de decima. In mortuis ante 15 tonsionem xxxix. In mortuis post tonsionen, xxxviij. Summa lx.xvij. Et remanent clxij agni.

Idem reddunt compotum de Dcciiij ${ }^{x} v$ velleribus grossis quae fecerunt iiij pondera dimidium, iij claves. In supravenditis totum. De precio superius respondent.

Idem reddunt compotum de cc velleribus agninis quæ fecerunt dimiclium pondus. In 20 supravenditis totum. De precio superius respondent.

Idem reddunt compotum de lxxvij pellibus agnorum, et de xxviij receptis de Manerio. Summa cv. In supravenditis totum. De precio superius respondent.

Idem reddunt compotum de xij porcellis proventis de decima. In liberatis in Manerio, totum.

Idem reddunt compotum de xxxij gallinis de cheriset. In expensis domini Episcopi apud Menes, xv. Et remanent xvij.

Idem reddunt compotum de xlv aucis de decima. In missis apud Wlveseiam, xx. In expensis senescalli, pluries, J[ohannis] de Mare, E[ustachii] de Grenvilla, x. In familia, auptumno, vj. In corredio Episcopi apud Menes, ix. Nichil remant. totum.

Idem reddunt compotum de cxix caseolis de decima. In expensis in anptumno,

Idem reddunt compotum de vij tonellis siceræ remanentibus anno præterito. Et de j de hoc anno. In expensis domini Episcopi apud Menes et Waltham, vj. In fæce, dimidium. In acidato, dimidium. Et remanct, $\mathrm{j}$.

Idem reddunt compotum de $\mathrm{v}$ sextariis, dimidio salis remanentibus anno præeterito. Et de iiij receptis de Hameledona. In expensa hospicii, v sextaria dimidium. Et remanent, iiij sextaria.

Summa tocius debiti, xxvjl. xiiijs. ob. Et viij quarteria avenæ.

\section{HAMELEDONA.}

G[ALFRiDUS], Everardus, Serlo, reddunt. ${ }^{1}$

Idem reddunt compotım de xxiijl. vijs. xjd. ob. de gabulo assiso. In quietanciis j præpositi, vs., iiij carıcariorum, $\mathrm{j}$ fabri, $\mathrm{j}$ porcarii, ij bercariorum, iij nperariorum, per annum, jopersrii, per $\mathrm{j}$ terminum, xviijs. ixd. Summa $\mathbf{x}$ xiijs. ixd.

In defectu terrarum tractarum in dominium, ijs. Summa utriusque xxvs.ixd. Et 45 remanent xxijl. ijs. ijd. ob.

Idem reddunt compotum de xlijs. iij ob. de pannagio. Et de xxxvs. de herbagio. Et de $x \times j d$. ob. de potfalda. Et de ixs. iiijd. de xvj ovibus vivis venditis. Et de viijs. ijd.ob. de pellibus xij ovium, viij multonım, xxiiij hoggettorum, Ixxij agnorum mortuorum venditis. Et de $\mathrm{ij} s$. vjd. $o b$. de $\mathrm{j}$ avro vendito. Et de $\mathrm{xij} d$. de coriis $\mathrm{ij}$ avrorum venditis. 50 Et de xxxjs. ob. de firma clv ovium. Et de vs. pro relaxatione operis. Et de iiijl. xvs. iiijd. de iij ponderibus dimidio, ij clav[ibus] lanæ venditis. Et de xls. ijd. tle ij tonellis vini jbi potatis. Et de vijs. de iiij quarteriis dimidio de nucibus venditis. Et de vjs. ijd. de kablo et bruere venditis. Summa xiiijl. js. ${ }^{2}$ viij $d$. $o b$.

Idem reddunt compotum de xxijs. xd. de ix quarteriis frumenti venditis. Et de vs. 5.5 de iij quarteriis mancorni venditis. Et de xjs. iiijd. de vj quarteriis ordei venditis. Et de vjs. de vj quarteriis avenæe venditis. Summa xlvs. ijd.

De Purchasiis.-Idem reddunt compotum de xijd. de Giliberto Trenchem[an] pro mellea. Et de vjd. de Waltero clerico pro misericordia. Et rle xijd. de Roberto Crulle pro melle furato. Et de $x i j d$. de Willelmo tiliastro Willelmi Proci pro simili. Et de xijd. do Willelmo Cluus pro 60 simili. Et de rijd. de Rogero Fabro pro pastura. Et de xijd. de Awino Frere pro bosco. Et de xijd. de Ricardo de Leghe pro simili. Et de xijd. de Henrico de Hoca pro pastura. 
Et de ij marcis de iiij thedingis pro misericordia. Et de dimidia marca de Knko pro filis sua maritanda. Et de vjdl. de Ada Classn pro boseo. Et de xijd. de Alrico filio Hugonis pro simili. Et le vjd. de Willelmo forestario pro sursisa. Et de vjl. de Blankardo pro pastura. Et de xviijl. de Radulfo filio Godwini pro cervisia. Et de vs. de liicardu Grandel pro sursisa. Et de vjd. de Jocelino filio Godæo pro bosco. Et de vjd. de Rogero \igro pro via clausa. Et de ijs. de liicardo de Leghe pro plegagio. Et de xijd. de Osberto Stoct [er] pro fissato. Et de xijd. de Samuel de Mara pro bosco. Et de vjd. de Roberto filio Alfredi pro pastura. Et de xijd. de Willelmo Procus ${ }^{1}$ pro corvisia. Et de xijd. de Godefrido Fabro pro filia su» maritında. Et de vjd. de Willelno Fitil pro pastura.

Et de xls. de hominibus de Stokes pro mellea. Et de iijs. de Cattesbert. Et de ijs. de Fitil. Summa ciijs. $x d$.

Idem reddunt compotum de xxiijl. de tallagio. Summa tocius receptr, lxviijl. ijs. xj $l$.

Liboratio.-In liberatis Johanni decano de Wlveseia, per $\mathrm{v}$ tallias, xlijl. xvijs. jd.

Expensa.-In ferro et calibe ij carucarum, vijs. ixd. In ferrura iiij avrorum, iijjs. $x d . o b$. In rotis et reparatione carucarum $\mathrm{x} d$. In $\mathrm{j}$ careta cunpta, ijs. ijd. In careta reparanda, iiij $d$. In clutis, capistris, uncto, paronellis et alio hemesio, xviijd. In precariis hyemalibus, quadragesimalibus, xxixs. iij $o b$. In iij bobus emptis, xxijs. vd.ob. In $\mathrm{j}$ avro empto, iiijs. vd. In j bercarin ficta de novo, xijs. viijd. In foragio empto ad boves, ijs. iiijd. ob. In triturandis xj quarteriis frumenti, vij quarteriis mancorni, viij quarteriis ordei, iijs. viijd. In $\mathrm{j}$ alveo faciendo ad boves adaquandos, iijd. In $\mathrm{j}$ corda ad idem, iijd. In domibus recooperieudis, xijd. In stipendio caretarii, iijs. iiijd. In expensis senescalli, ijs. In viij " porcis emptis, iijs. vjd. In xiij ulnis de canevaz ad lanam, ijs. vd. In decimis caseis iijs. $\mathrm{j} d$. In companagio xviij bedriparum $\mathrm{x} s$. viijd. In ${ }^{3}$ [mercede] ij præpositorum per xviij dies, xviijd. Summa vjl. xxjd. ${ }^{1}$

In liberatione Mathæi cum homine suo, per annum, iiijl. xixs. viijd. Summa utriusque, xjl. xvijd.' Summa tocius liberationis et expensæ, liijl. xviijs. vjd. Et debet xiiijl. iiijs. $v d$.

Exitus Grangiæ.-Idem reddunt compotum de xxxj quarteriis, dinidio, de toto exitu frumenti. Et de $\mathrm{j}$ quarterio $\mathrm{j}$ hopa de cheriseto. In semine $\mathrm{cx}$ acrarum, xxiij quarteria dimidium, $\mathrm{j}$ hopa. In venditis, ix quarteria. De precio superius respondent.

Idem reddunt compotum de $\mathrm{xv}$ quarteriis, dimidio, de toto exitu mancorni. In senine xliiij acrarum xij quarteria dimidium. In venditis, iij quarteria. De precio superius respondent.

Idem reddunt compotum de lxxvj quarteriis de toto exitu ordei. In semine $\mathrm{ijij}^{\mathrm{xx}}$ acrarum, ' xxiij quarteria. In missis apud Fareham, xij quarteria. In missis ajund Waltham, xiiij quarterid. In liberatione $\mathrm{j}$ caretarii, $\mathrm{v}$ quarteria, dimidium. In poreis nutriendis, $\mathrm{j}$ quarterium. In custode agnorum, dimidium quarterium. In precariis auptumnalibus, xiiij quarteria. In venditis, vj quarteria.

Idem reddunt compotum de cxij quarteriis de toto exitu avenæ. Et de iij receptis de ecclesia. In semine clxx acrarum, l quarteria. In præbenda Mathæi per xiv septimanis, xxij quarteria, dimidium. In præbenda $\mathrm{j}$ caretarii, $\mathrm{ij}$ avroruın, $\mathrm{xij}$ quarteria dimidium. In bobus sustinendis, vj quarteria. In missis ad Waltham, xix quarteria. I 11 venditis vj quarteria. De precio superius respondent.

Instaurum.-Idem reddunt compotum de $\mathrm{v}$ avris remanentibus anno præterito. Et de $\mathrm{j}$ empto. In mortuis, ij. In vendito, $\mathrm{j}$. Et remanent iij avri.

Idem reddunt compotuin de $x x i$ bobus remanentibus anno præterito. Et de iiij, emptis. Et de j de legato Ricardi Fabri. Summa xxvj. Et remanet totum.

Idem reddunt compotum de ciiij ${ }^{x x}$ xiij ovibus remanentibus anno præterito. Et de xix adjunctis de instauro. Summa ccxij. In vivis venditis ante partum, $x \mathrm{rj}$. In mortuis ante partum, vij. In mortinis post partum et ante consionem, v. In mortuis post tonsionem, ij. Summa $\mathrm{xxx}$. Et remanent ciiiij ${ }^{\mathrm{xx}} \mathrm{ij}$ oves.

Idem reddunt compotum de cciiij ${ }^{x x} v$ multonibus reunanentibus anno præterito. Et de xij adjunctis de instauro. Sunıma cciijj ${ }^{x x} \times v^{1} i j$ multones. In mortuis ante tınsioneın, vj. In mortuis post tonsionem, ij. Et remunent cciijexix multones.

Idem reddunt compotum de lv loggettis remanentibus anno præterito agnis. In mortuis ante tonsionem, xxiiij. In adjunctis ovibus, xix. In adjunctis multonıbus, xij. Nichil remanet.

Idem reddunt compotum de rlx agnis proventis hoc anno de prædictis ovibus, quia $x$ fuerunt steriles. In mortuis ante separationem, xlviij. In decima, xj. It consuetadine, $j$. In mortuis post separationem et ante tonsionem, xxviij. In mortuis post tonsionem, xiij. Summa cj. Et remanent lix.

Idem reddunt compotum de ccclxxv velleribus grossis. In decima, xlvij. Iı consuetudine bercarii, ij. In venditis ccccxxvj vellera, quæ fecerunt iij pondera dimidium, ij claves, cum lxxij velleribus agninis.
${ }^{3}$ On an erasure in MS. 
Idem redhunt compotum de xliij porcis remanentibus anno præterito. Et de viij emptis. Et de $\mathrm{xx}$ inde proventis. Summa lxxj. In missis ad Waltham ad lardarium, $x x$. In decima, $\mathrm{ij}$. In consut tudin", $\mathrm{j}$. In mortuis, xiij. Et remanent $\mathbf{x x x v}$.

Idem reddunt compotum de viij sextariis salis. In missis ad Menes, iiij. Et remanent iiij.

Idem reddunt compotum de xlj gallinis. In missis ad Wlveseiam, totum.

Summa tocius debiti, xiiijl. iiijs. vd.

\section{CRA WELEIA.}

Ricardus serviens et Herbertus et Arnoldus præpositi reddunt xxxviijs. ijd. ob. de reragio anni præteriti. Et quieti sunt.

Idem reddunt compotum de $x l$. xijd. de toto gabulo assiso præter terras iiij carıcariorum, $\mathrm{j}$ fabri, $\mathrm{j}$ porcarii, ij bercariorum, xiij operariorum qui se defendunt per opera sua. In quietancia j præpositi, vs. In defectu gabuli terræ Strong' tractæ in dominium, ijs. In defectu gabuli terræ de Alebroc, vs., qui modo redduntur apud Meredonam.

Summa quietanciarum et defectuum xijs. Summa remanens ixl. ixs.

M. 7d. Idem reddunt conpotum de xxxijs. vd. de pannagio. Et de iijs. viijd. de herbagio. Et de xxs. de iiij bohus vivis venditis. Et de ijs. vjd. de coriis ij boum venditis. Et de ijl. de ij pellibus vitulorum, anno præterito mortuorum, venditis. Et de viijs. iiijd. (le $\mathrm{xxx}$ multonibus, veteribus ${ }^{1}$ et debilibus, vivis venditis. Et de xiijj. $\mathrm{x} d . \mathrm{ob}$. de exv pellibus ovium mortuarum venditis. Et de viijs. viijd. ob. de xlv agnis vivis venditis. Et de viijs. $x d$. de ciiij pellibus aguorum mortuorum venditis. Et de ixs. $x d$. de terra locata hoc anno. Et de $\mathrm{x} d$. de butiro vendito. Et de lxiijs. de clxiij caseis remanentibus anno præterito, qui fecerunt ix pondera, venditis. Et de xxiiijs. de iiijxx.iiij caseis hnjus, amni, qui fecerunt iij pondera dimidium, venditis. Et de cs. de cccciijjxix ${ }^{2}$ velleribus 25 urossis quæ fecerunt iij pondera inij partem ponderis, et ccj velleribus agninis quæ fecerunt dimidium pondus venditis. Summa xiiijl. xvijs. ijd.

Idem reddunt compotum de vijl. xvs. iiijd. de lxj quarteriis dimidio frumenti venditis. Et de xjs. iijd. de v quarteriis curailli venditis. Et de xxvs. viijd. de xij quarteriis dimidio ordei venditis. Et de ijs. iijd. de j quarterio dimidio pisarum venditis. Summa 30 ixl. xiiijs. vjd.

De Purchasiis.-Iden reddunt compotum de $\mathrm{xxs}$. de tota villata pro plevina hominum qui fuerunt in cippo apud Meredonam pro mellea, anno præterito. Et de xxs. de Herberto præposito pro clave dimittendo. Et de ijs. de Willelmo Palmer pro sursisa. Et de xijd. de Ivone Coch pro forisfacto bosci. Et de vjd. de Willelmo Tole pro simili. Et de xviijd. de Roberto Longo pro simili. Et de xijd. de Hugo Niwem[an] pro terra Episcopi male seminata. Et de xijd. de Odone pro simili. Et de vjd. de Sewale pro forisfacto bosci. Et de vjcl. de Tanculfo pro simili. Et de vjd. de Roberto de Insula pro simili. Et de xijd. de Ricardo de Vinea pro simili. Et de viijs. de Roberto milite pro terra habenda. Summa lvijs. vjd.

Idem reddunt compotum de ixl. xviijs. de tallagio.

Summa tocius receptæ, xlvjl. xvjs. ijd.

Liberatio.-In liberatis $\mathrm{J}$ [ohanni] decano cs. iij ob., tam de reragio anni præteriti venditionibus. Eidem de purchasiis xx8., per j talliam. Eidem de blado vendito xls., per j talliam. Eidem de tallagio ixl. xviijs., per j talliam. Eidem de termino Natalis, xlvs. jd., per j 45 talliam. Eidem de iij terminis sequentibus, vjl. xvs., per $\mathrm{j}$ talliam. Eidem ix $l$. xijd., per j talliam.

Summa tocius liberationis, xxxiijl. xijd.

Expensa.-In ferramentis iiij carrucarum, x avrorum, per annum, xiiijs. ijd. In vj paribus rotarum emptis ad carrucas; iiij caruess factis de novo; et in carucis reparandis, ijs. iiijd. 50 In $j$ careta ferrata empta, ixs. viijd. In $\mathrm{j}$ careta reparanda ixd. In $\mathrm{j}$ sella, parono, paronella emptis ad caretam, xiiijd. In capistris emptis, iij ob. In vj bobus emptis, xxxvs. ijd. In precariis hyemalibus de xiij carucis, xiijd. In fossandis $l x$ perticis circa bercariam, xvs. In sepe facienda circa bercariam, xijd. In $\mathrm{j}$ nova porta facienda ante bercariam, xijd. In porta curiæ reparanda, vijd. In parietibus daeriæ et granarii faciendis, 55 ijs. $\mathrm{j} d$. In prædicitis domibus et $\mathrm{j}$ bercaria et ij grangiis cooperiendis, vs. jd. In serruris reparandis, iiijd. In corda et utre ad puteum emptis multociens propter nimiam siccitatenı, xxd. In xxxviij clatis ad faldam, $x d$. In custo daeriæ, iijjs. $j d$. In pullis emptis contra Pentecosten, per præceptum senestalli, scilicet lxiiij pullis, iijs. va.ob. In Ix.xij acris l.ladi sarclandis, xvjd. In corredio magistri R[oberti] Basset ibidem, ixs. ob. In corredio 60 'Thomæ de Wichtona ibidem in auptumno, per v septimanas, vjs. ijd., per diem ijd. In 
canevaz empto ad imponendam lanam, xvjd.ob. In solido iiij bovariorum, per annum, xvjs. In solido $\mathrm{j}$ huiwardi, iijs. In solido $\mathrm{j}$ daia, iijs. In liberatione Roberti de Foxcote servientis, per annum, lxs. xd. Summa $x l$. iiijd.

Summa tocius liberationis et expensæ, xliiijl. xvjd. Et debent liiijs. xd.

5 Exitus Grangiæ.-Idem reddunt compotum de lxvj quarteriis dimidio de toto exitu frumenti. Et de xxvj quarteriis receptis de Meredona. Summa, iiijxx.xij quarteria dimidım. In semine iijjxx.x acrarum, xxvj quarteria. In pane precariarum auptumnalium, v quarteria. In supravenditis, ixj quarteria dimidium. De precio superius respondent.

Idem reddunt compotum de $\mathrm{v}$ quarteriis de toto exitu curailli. In venditis, totum. De precio superius respondent.

Idem reddunt compotum de cxlvij quarteriis dimidio de toto exitu ordei. In semine clxxiij acrarum, lvij quarteria. In missis apud Cumbam ad semen et ibi liberatis Roberto de Deneforde, vj quarteria. In missis apud Meredonam, xxij quarteria. In corrediis iiij bovariolum, j haiwardi, $\mathbf{j}$ daix, pel anuum, xxxix quarteria. In mereede et corredio $\mathrm{j}$ herciatoris, a festo Sancti Michielis usque Pascham, iij quarteria. In poreis pascendis, viij quarteria. In supravenditis, xij quarteria dinidium.

Idem reddunt compotum de cej quarteriis de toto exitu avenæ. In semine cciiijxxvj acrarum, ciiij quarteria. In missis apud Meredonam, viij quarteria. In missis apud Wlveseiam et ibi liberatis J[ohanni] decano, xxx quarteria, per ij tallias In missis apud Wlveseiam et liberatis Petro Ace ad præbendam Episcopi, xviij quarteria. In præbenda $\mathrm{R}$ [oberti] Basset, iij quarteria. In præbenda x avrorum, per annum, xxxviij quarteria.

Idem reddunt compotum de ij quarteriis dimidio de toto exitu pisarum. In semine iiij acrarum, j quarterium. In supravenditis, j quarterium dimidium. Quieti [sunt].

Instaurum.-Idem reddunt compotum de $\mathrm{x}$ avris remanentibus anno præterito. Omnes remanent.

Idem reddunt compotum de xxxvij bobus remanentibus anno præterito. Et de vj postea emptis. Et de ij adjunctis de instauro. Summa xlv. In vivis venditis, iiij. De precio superius respondent. In mortuis, ij. Summa vj. Et remanent xxxix boves.

Idem reddunt compotum de $x i j$ vaccis, $j$ tauro, remanentibus anno præterito. Et de iij vaccis adjunctis de instauro. Summa $x v$ vacce, $j$ taurus. Omnes remanent et sunt missi apud Meredonam.

Idem ruddunt compotum de ij bovettis, iij geniculis, remanentibus anno præterito. In adjunctis bobus $\mathrm{ij}$. In adjunctis vaccis, iij. Nichil remanet.

Idem reddunt compotum de iij vitulis remanentibus anno præterito In mortuis, ij. Et remanent ij.

Idem reddunt compotum de ccciijixx ovibus rumanentibus anno præeterito. Et de cx adjunctis de instauro. Summa Diiijx $x^{x x}$. In mortuis ante partum, $x$; post partum et ante tonsionem, viij; post tonsionem, viij. Summa xxvj. Et remanent Dlxiiij oves.

Idem reddunt compotum de cccxlv multonihus remanentibus anno præterito. Et de cxxvij adjunctis de instauro. Summa cccelxij. In vivis venditis, xx. In mortuis ante tonsionem, xxxiij. In mortuis post tonsionem, vj. Summalx. Et remanent cecexij multones.

Idem reddunt compotum de cexviij hoggettis, anno præterito agnis. Et de xlviij receptis de Meredoni. Summa cciiijxxvj. In mortuis ante tonsionem, xlix. Et remanent ccxxxvij; unde cx sunt gerciæ, junctæ ovibus; exxvij sunt mares, juncti multonibus.

Idem reddunt compotum de ccceliij agnis proventis hoc anno, et iij proventis de gerciis, quia xvij oves fuerunt steriles. In decina, xlv. In consuetudinibus $\mathrm{i}$ fabri, ij bercariorum, et custodis agnorum, iiij. In vivis venditis, xlv. In nortuis ante tonsionem iiijxx.xiij. In mortuis post tonsionem, xj. Summa ciiij ${ }^{x x} . x v i i j . \quad$ Et remanent cclviij.

Idem reddunt compotum de Decciiij ${ }^{x x} . x$. velleribus lanæ crispæ et grossæ. In decimis iiijxx.xix. In consuetudinibus ij bercariorum, daiæ, iij. In venditis, Deceiiij ${ }^{x x}$.viij, qua fecerunt ij p indera lanæ minutæ, iij pondera lanæ grossæ, et iiij partem j ponderis.

Idem reddunt compotum de ecj velleribus lanæ agrinæ, quia lxviij subtiles agni non fuerunt tonsi. In supravenditis, ccj quæ fecerunt dimidium pondus.

Idem reddunt compotuın de clx.xvj caseis, qui inceperunt fieri viijo Idus Aprilis et desierunt iiij kalendas Uctobris, utroque die computato. In decima, xvij. In consuetudinibus bercarii, fabri, daiæ, iij. In missis apud Wlveseiam, xxiiij. In precariis auptumnalibus, xiiij. In supravenditis, iiijxxiiij. Et renanent xxxiiij casei.

Idem reddunt compotum de xxxiiij por:is remanentibus anno praterito. Et de xxiij de exitu eorundem. Summa lvij. In decima, ij. In consuetudine porcarii, j. In lardario Wintoniæ, $\mathrm{xv}$. In mortuis, vij. Summa xxv. Et remanent xxxij porci.

Idem liberaverunt Radulfo Salserio apud Wlveseiam, $x x x$ pullos.

Idem reddunt compotum de lxj gallinis de cheriset. Et de lxiiij emptis. In liberatis Johanni decano, iijjx.xj. Et remanent xxxiiij, et præeterea xxviij capones de adquisitione $R[i c a r d i]$ servientis. 
Illem reddunt compotum de iij baconibus receptis de Wlvescia. In precariis auptumnalibus, totum expendid[erunt].

Summa tocius debiti, liiijs. $x \mathrm{~d}$. Solvit post compotum et quietus est per totum. ${ }^{1}$

\section{TWIFORDA.}

Alvredus DE Quinci et Herebertus præpositus reddunt vjl. xvijs. vjd. ob. de reragio 5 denariorum et caseorum. Et quieti sunt.

Idem reddunt compotum de $\mathrm{xlj} l$. $\mathrm{ij} s$. $\mathrm{vd}$. de toto gabulo assiso. Et de xls. de firma molindini de Brombrigge. Summa xliijl. ijs. vd.

In quietantia j præpositi, ij bedellorum, xvs.; iiij bercariorum, per annum, xijs.; j fabri, per annum, iijs.; j porcarii, per annım, ijjs. ; xij carucariorum, per annum, xxxıjs. ; 10 iiij carucariorum, per annum, xjs.; ij bovariorum, per annum, vjs.; viij operariorum, per aunum, xxiijjs. Snmma quietanciarum cxs

In defectu gabuli de Alebroc, vs. In defectu gabuli de Brombrigge, xlvjs. $\mathbf{x} d$. In defectu gabuli de Huppam, tracta in dominium apud Stokes, ijs. In defcetu gabuli Turberti et Crudæ, et prati præoccupati in doininium, viijs. iijd. In defectu 15 gabuli Algeri de Duddingwuda, vd. In defectu gabuli Roberti Coc, propter cursum aquæ, vjd. In defectu gabuli terræ quæ fuit Hasardi, iijs. In defectu gabuli terræ quæ fuit Radulfi de Ypre, data Waltero Romano per Petrum episcopum, xs. Summa defectuum lx.xvjs.

Summa quietanciarum et defectuum, ixl. vjs. Summa remanens, xxxiijl. xvjs. vd. 20

Idem reddunt compotum de liiijs. iij d. de pannagio. Et de xls. iiijd. de herbagio. Et de xviijs. iiijd. de inhocg. Et de lxvijs. xd. de xij bobus vivis venditis. Et de ijs. ixd. de coriis ij boum mortuorum venditis et de corio $\mathrm{j}$ avri mortui vendito. Et de vd. de corio $\mathrm{j}$ caretarii episcopi mortui vendito apud Merewellam. Et de xxixs. iijd. de xxxix multonibus debilibus venditis. Et de xxixs. vd. ob. de pellibns cxxxix 25 ovium mortuarum venditis. Et de xvjs. iiijd. de clx pellibus agnorum venditis. Et de xiijol. ob de xxix pellibus deterioribus agnorum venditis. Et de liiijs. iijd de cexxxj caseis hujus anni venditis, qui fecerunt vij pondera demidium et iijj partem ponderis. Et de ixs. ijd. de M.C. anguillis venditis. Et de xxxiiijs. ijd. de $\mathbf{j}$ scotallo. Summa xvijl. xvijs. $x$.

Idem reddunt compotun de exjs. de xxxix quarteriis frumenti venditis. Et de vijs vjd. de $\mathbf{v}$ quarteriis curalli venditis. Et de xljs. ijd.ob. de xvij quarteriis dimidio ordei venditis. Et de lijs. ijd de xliiij quarteriis avenæ venditis. Sumna $\mathrm{x} l . \mathrm{xj} s . \mathrm{x} d . o b$.

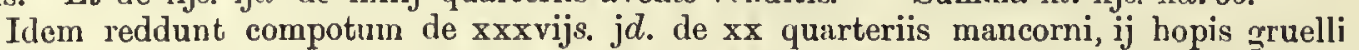
venditis, de toto exitu novi molindini. Et de xxviijs. jd. de xvj quarteriis mancorni 35 iij hopis gruelli venditic, de toto exitı molindini de Scaldeforda. Et de xxxs. iiijd. de $x v$ quarteriis dimidio, $\mathrm{j}$ estrica mancorni, et de dimidio quarterio $\mathrm{j}$ estrica, $\mathrm{j}$ hopis gruelli de toto exitu molindini de Comtona. Et de lviijs. vijd. ob. de xxx quarteriis $\mathrm{j}$ estrica mancorni et de dimidio quarterio fiumenti, de dimidio quarterio $\mathrm{j}$ bussello gruelli, et de $\mathrm{j}$ quarterio dimidio brasei, de toto exitu mulindini de Nortwiforda. Et 40 de xijs. de consuetudinibus ejusdem molindini. Summa viijl. vjs. ijj. ob.

De Purchasiis.-Idem reddunt complotum de xijd. de Nicholao 'l'riwe pro terra relaxata. Et de ijs. de Waltero de IIaistona pro terra habenda. Et de ijs. de Ricardo Fohel' pro simili. Et de vs. de Gileberto filio Agathæ pro simili. Et de iijjs. de Ricardo Simon[is] pro filia sua maritanda. Et de xijd. de Willelıo parnentario pro terra relaxata. Et de vjd. de 45 Galfrido filio Edmundi pro simili. Et de vjd. de Osmundo servienti pro simili. Ei de vj de Willelmo filio Giliberti pro sinili. Et de vjd. de Adam pro sursisa precariarun auptumnalium. Et de xijd. de vidua Proche pro simili. Et de vjd. de Hawis vidua pro simili. Et de xijd. de Edwardo 'T'riwe pro simili. Et de vjd. de Radnlfo Garliec pro simili. Et de vjd. de Adam Segar pro simili. Et de vjd. de Ricardo Synon[is] pro simili. Et de 50 ijs. de Willelmo Spraculf pro forisfacto pasturæ. Et de vjd. de Willelino Albo pro simili. Et de xijd. de vidua Ainolfi pro simili. Et de vjd. de Johanne bovario pro simili. Et de xijd. de vidua Warneri pro simili. Et de xijd. de Ricardo Hersent pro simili. Et de xijd. de Willelmo Pagel pro simili. Et de xijd. de Willelmo Dodde pro simili. Et de xviijd. de Willelmo Kier' pro forisfacto pasturx. Et de ijs. de Ivone parvo pro simili. Et de vjd. 55 de Radulfo Juniore Freke pro simili. Et de vjd. de Willelmo do Hamptesira pro forisfacto pasturæ. Et de vjd. de Radulfo filio Ailrici pro simili. Et de xijd. de Arnoldo de Colvedene pro simili. Et de vjd. de Ivone de Insula pro simili. Et de xijd. de Acone Sewade pro simili. Et de xijd. de Willelmo Craft pro falso clamore. Et de vja. de Ricardo Porth pro mellea. Et de vjd. de Waltero capistrario pro simili. Et de xijd. de 60 Bernardo fabro pro forisfacto pasturæ. Et de vjd. de Nicholas Hunwino pro sinili. Et de vjd. de Thoma Ballinge pro simili. Et de xijd. de vidua Roberti pro simili. Et de xijd. de Johanne 'Turberti pro simili. Et de vjd. de Edmundo Wilde pro simili. Et de vjd. de Greta 
pro simili. Et de xijd. de Galfrido artifice pro simili. Et de vjd. de Absalon Palmer pro simili. Et de vjd. de Ildulf prosimili. Et de xviijd. de Radulfo pro simili. Et de vjd. de Johanne Liede pro simili. Et de xijd. de Ivone de Fonte pro simili. Et de vjd. de Roberto Ingulf pro simili. Et de xviijd. de Ailwardo Stanere pro simili. Et de vjd. de Ricardo Belami pro simıli. Et de xijd. de Roberto Potere pro simili. Et de vjd. de Roberto Corkieu pro simili. Et de xijd. de Willelmo tilio Edmundi pro simili. Et de vjd. de Ricardo filio Ivonis pro simili. Et de xijd. de Osberto God[æ] pro falso clamore. Et de vjd. de vidua Rogeri pro simili. Et de vjd. de Ricardo Greh pro simili. Et de xijd. de Agatha vidua pro simili. Et de xviijd. de vidua Wlvrici pro simili. Et de xijd. de vidua Esmundi pro simili. Et de vjd. de Hugone pro forisfacto pasturæe. Et de vjd. de Ricardo filio Wlvrici pro simili. Et de xvijd. de Ingulf Poche pro simili. Et de vjd. de Osmundo pro simili. Et de vjs. viijd. de Ricardo Luwini cum tota thedinga sua. Et de xviijd. de Ricardo Bar[at] pro melle: . Et de xijd. de Ricardo de Castello pro sursisa. Et de vjs. viijd. de Willelmo Frewin[i] pro filia sua maritanda. Et de iijs. de vidua Estmundi pro simili. Et de lxvjs. viijd. de Johanne de Bro[ın]brigrge pro venacione domini Regis recepta. Et de $\mathrm{xx} 8$. de Johanne molindinario pro tonello eum vino empto fracto. Summa viijl. viijs.

Idem reddunt compotum de iijl. receptis de Johanne decano ad operarios de Merewella.

Idem reddunt compotum de xxjl.' de tallagio.

Sumına tocius receptæe ciiijl. iijd.

Isiboratio.-In liberatis Johanni decano de termino Natalis, vijl. xiijs. Eidem, de termino м. 8. Pasehre, vjl. xjs. Eidem, de termino Sancti Johannis, vijl. xs. Eiden, de termiıo Sancti Michaelis, xijl. Eidem, de tallagio, xxjl. Eidem, de blado vendito, et exitu nıolindini, xijl. xjs. per ij tallias. Eidem, de caseis venditis, xxxvjs.

Summa tocius liberationis, $1 \times i x l$. xijd.

Expensa.-In ferramentis viij carucarum, per annum, et avrorum et carettariorum, xxxs. xjd. In mercede fabri pro ferramentis carucarum per annum, vjs. viijd. ob. In viij paribus rotarum ad carncas, ijs vjd. ob. In carucis, jugis, faciendis de novo, ijs. iijd. In j careta empta et altera repar..ndi, ijjs. ijd. ob. In bacis, sellis, panellis, paronis, paronellis, clutis, corda, ad caretas, xijd. ob. In iij saccis emptis, xvijd. In iiij bobus emptis, xxijs. In ij precariis hyemalibus de lvij carncis, ixs. vjd. In $\mathrm{j}$ precaria quadragesinuali de lviij carucis, iiijs. $\mathbf{x} d$. In xlviij quarteriis frumenti vanandis, tan ad semen quan ad panem Episcopi, xviijd. In triturandis xlj quarteriis frumenti, viijs. vjd. ob. In ij ventoriis ad bladum purgandum, xijd. In consuetudine wis'atoris faldre, ijs. In mereede ij carpentariorum ad erigendam bovari:ım, et ad faciendum stabulum avrorum de novo, per vj dies, iijs In grangia et prædictis domibus cooperiendis, iiijs. ixd. ol. In $\mathrm{j}$ bercaria facta de novo, xxixs. vijd. In fossato circa predictam bercariam pro xxj perticis, vs. iijd. In mercede ij carpenturiorum pro ij bercariis de Haselega reparandis, et hostii: earundem faciendis, et presepe ovium faciendn, et parietibus claudendis, et borlis emptis ad idem, viijs. $\mathbf{x} d$. In predictis ij berciriis cooperiendis, ijs. vd. In ramillis, palis, colligendis ad claudendum circin predictas bercarias, et pro sepe facienda, vs. iijd. In bercuria de Hevenestig[ge] reparanda et cooperienda, iijs. ixd. In sulfure empto ad medieamentum ovinm, $\mathrm{j}$ l. In feno empto ad oves, xvs. In fossando circa bladuin, xvd. In ij pratis falcandi, iijs. ixd. In custo daerix, iijs. ijd. ob. In xvj carucariis pro aratura sabbatorum, per dimidium annum, xviijs. In precariis auptumnalibus, ijs. In solido j caretarii, per aunum, xviijd. In mercede ij custodum agnorum in partu ovium, per præceptum fratris Walteri, ijs. In liberatione Alvredi de Quinci, Ixs. xd. In xxxvj ulnjs dimidi $\bullet$ de caneva\% ad lanım subtilem inponendam, vjs. jd. Summı xiiijl. ob.

Exponsa facta apud Merewellam.-In parco claudendo per breve magistri R[oberti] Basset [in mercede] ca[r]pentari[orum] et hominum suorum, xxvs. iijob. In reparando ${ }^{1}$ domos, et cameris, et claustra, do Merewella, et eisdem cooperiendis, xiijs. $x d$. In calce empta ad idem, ijs. iiijd. In bordis emptis all idem, xviijd. In clavis ferreis emptis ad idem, iijs. iinjd. In kivillis emptis ad tegulam, $x d$. In tegulario et sibi adjuvantibus ad claustra et apenticium aulæ cooperienda, xxs. $\mathrm{ijj} d$. $\omega b$. In plumbatoribus ad fundandum plumbum ad goterias, ${ }^{2}$ xijd. In carpentario conducendo ad levandum cumulum altæ cameræ, et sibi adjuvantibus, xiijs. In ceclx.xv bordis emptis ad idem, xvjs. vijd. In elavis emptis ad idem, ijs. iiijd. In cariandis iiij tonellis vini de Hamptona usque Merewellam, vjs. vjd. In cariandis ij toncllis vini de Hamptona usque Fernham, per breve senescalli, vjs. In ponario gardini de Merewella mundando et suceidendo, per præce,tum magistri R[oberti] Basset, vijd. In $\mathrm{j}$ sera empta ad ostium cameræ ante capellam, iiijd. In faciendo cameram, quæ fuit combusta, tan in taseal quam in mercede et corredio carpentaiorum, iijl. ijs. ijd. In faciendo implementum muri cameræ combustæ per iij dies, iijs. iiijd. In $\mathrm{j}$ camino faciendo per ix septimanas, xxvijs. $\mathrm{ij} d$. In $\mathrm{iijj}^{\mathrm{xx}}$.xiij petris emptis ad idem, vjs. iiijd. In calce empta ad idem, iijs. ijd. In barris ferr emptis ad idem, xijd. In 
petra carianda de Bello loco usque Hamptonam, vijd. In perficiendo ostium et murum, et $\mathrm{j}$ fenestraı, in eadam camera faciendam, et eisdem dealbandis, vijs. vijd. In calce empta ad idem, ijs. In pluribus gutteriis imponendis, vjd. In clavis emptis ad idem, iijd. In expensis ij venatorum domini Regis cum $\mathrm{v}$ hominibus suis, et 1 canibus ibidem perhendinantibus, a festo Sancti Thomæ Apostoli usque Caput Jejunii, xxjs. jd. In 5 expensis magistri R[oberti] Basset multociens ibidem, xxxjs. vijd.

Summa tocius expensæ apud Merewellam, xvi. iiijd. ob.

Expensa Molindini.-In ferro empto ad fusas, inkas, et bilas faciendas ad novum molindinurn, et molindinum de Scaldeforde, et molindinum de Cumtona, iiijs. iijd. In mercede fabri, xijd. In bordis emptis ad alvos faciendos, et rotas novi molindini reparandas, $x d$. In 10 mercede carpentarii, ad idem, iijd. In j mola empta ad prædictum molindinum, xxjs. In mercede et corredio, carpentari[orum] facienti[um] novum molindinum ad braseum, apud Scaldeforde, xiiijs. In bordis emptis ad idem, xviijd. In clavis ferri emptis ad idmu, xd. In hordis emptis ad alvos et rotas molindini de Cumtona reparandis, xvd. In mercede carpentarii eundem facientis, vjd. In clavis ad ilem iiijd. In stagno ejusdem molindini 15 opturandi et emendandi, iijs. Summa xlviijs. ixd.'

Expensa Molindini de Nortwiforde.-In ferro empto ad fusas, inkas et bilas reparandas, iijs. iiijd. $o b$. In mercede fabri, vjd. In $\mathrm{j}$ mola empta ad prædictum molindinun, xxvijs. ijd. Summa xxxjs. ob. Summa expensarum omnium molindinorum, lx.xixs. ixd. $o b$.

Summa tocius expensæ xxxiijl. ijd. ob.

Summa tocius liberationis et expensæ, cijl. xiiijd. ob. Et debent, xxxixs. $a b$.

Exitus Grangiæ.-Idem reddunt compotum de exxiij quarteriis dimidio de toto exitu frumenti. Et de lxviij quarteriis receptis de Stokes ad seinen. Summa ciiijxx.xj quarteria dimidiun. In semine ccxij acrarum, iiijxx.xij quarterin. In missis aput ${ }^{1}$ Waltham et, ibi liberutis 25 $\mathrm{A}[\mathrm{rnoldo}]^{2}$ præposito ad panem Episcopi, v quarteria. In pane ad opus venatorum domini Regis apud Merewellam, cum canibus suis, vij quarteria dimidium. In capellanis de Wintonia, de dono domini Episcopi, xlj quarteria. Willelmo Widecoc, per breve magistri $\mathrm{R}$ [oberti] Basset, ij quarteria. H. Balistario per breve magistri Roberti Bassett, iij quarteria. Waltero Romano de dono domini Episcopi, j quarterium. In supravenditis, xxxix 30 quarteria. De precio superius respondent.

Idem reddunt compotum de $\mathrm{x}$ quarteriis de toto exitu curailli. In pane ad canes domini Regis per viij septimanas, v quartcria. In supravenditis, v quarteria. De precio superius respondent.

Idem reddunt compotum de evj quarteriis dimidio, de toto exitu ordei. In semine 35 exiiij acrarum, I quarteria. In missis apud Biterne et ibi liberatis Johanni de Stonham, vj quarteria. In corredio $\mathrm{j}$ caretarii, $\mathrm{j}$ daiæ, per annum, $\mathrm{x}$ quarteria viij honæ In corredio $\mathrm{j}$ herciatoris per dimidium annum, $\mathrm{ij}$ quarteria dimidium. In corredio ij custodum agnorum in partu ovium, dinidium quarterium, iij hopæ. In porcis pascendis. iiij quarteria. In pane ad $\mathrm{v}$ precarins auptumnales, xij quarteria dimidium. In pane ad 40) canes domini Regis, iij quarteria. In supravenditis, xvij quarteria dimidium.

Idem reddunt compotum de celxvj quarteriis iiij hopis, de toto exitu avenæ. Et de $\mathrm{xx}$ quarteriis receptis de Meıes. Summa cciijj ${ }^{\mathrm{x}} \mathrm{vj}$ quarteria iij hopæ. In senine cexxxv acrarum, cxxxj quarteria. In pane ad canes domini Regis per viij septinanas, xxij quarteria. In corredio hominis magistri Roberti de Pavilli, per præcoptum senescalli, 45 j quarterium. In missis apud Wlveseiam et liberatis Johanni Decano. xxxiiij quarteria, per ij tallias. In præbenda iij venatorum domini hegis apud Merewellam, p'er viij septimanas, xviij quarteria dimidium. In præbenda $\mathrm{R}$ [oberti] Basset ihidem, vj quarteria dimidium, iij hopæ. In præbenda Hugonis bercarii, $x v$ quarteria. In præebenda ij aviorum per annum, viij quarteria. In consuetudine ij bercariorum pro pressura, ij quarteria. 50 In farina servienti[un1] per annum, iij quarteria. In supravenditis, xliiij quarteria. De precio superius respondent.

Instaurum.-Idem reddunt compotum de iij avris remanentibus anno præterito. Et de $\mathrm{j}$ qui venit de Divisis. Et de $\mathrm{j}$ qui venit de Wlvescia. Sunma v. In mortuo, $\mathrm{j}$. Et remanent iiij.

Idem reddunt compotum de e bobus remanentibus anno præterito. Et de iiij postea

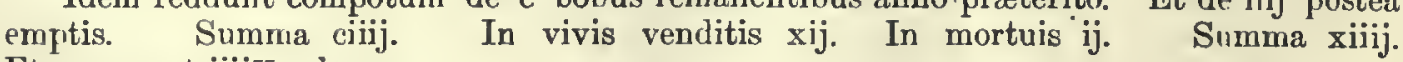
Et remanent iiij ${ }^{\mathrm{xx}} \mathrm{x}$ boves.

Idem reddunt compotum de $i j$ vaccis, $j$ tauro, iij annalibus remanentibus anno præterito, unde $\mathrm{j}$ est taurus adjunctus vaccis. Et de ij vitulis anni præsentis. Omnes 60 remanent. 
Idem redd'ınt compotum de iij equabus silvestribus. Et de $i j$ quæ venerunt de Wlveseia. Et de iij pullis anni præteriti. Et de iij pullis anni præsentis. Summa xij.' Omnes remanent.

Idem reddunt compotum de Dclx.x ovibus anno praterito. Et de ciijjxix adjunetis de instauro. Sunuma Decelix.

In mortuis ante partum, $x x j$. In mortuis post partum et inte tonsionem, xlij. In mortuis post tonsionem, xiiij. Summa lx.xvij. Et remanent Dcciiij ${ }^{x} \mathrm{ij}$ oves.

Idem reddunt compotum de Dlx.x multonibus remanentibus anno præterito. Et de clx.xj adjunctis de instauro. Summa Decxlj. In vivis venditis ad festum Sancti Martini, xxxix. In mortuis ante ton $\times$ ionem, xxiij. In mortuis post tonsionem, xij. Summa lx.xiiij. Et remanent Dclxvij multones.

Idem reddunt compotum de ccciijex vij hoggettis, anno præterito agnis. In mortuss ante tonsionem, xxv. In mortuis post tonsionem, $\mathrm{ij}$. In adjunctis ovibus, ciiijsxix. In adjunctis inultonibus, clx.xj.

Idem reddunt compotum de l/cxxiiij agnis proventis hoc anno de ovibus, unde xxv fuerunt steriles. Et de xiiij proventis de gerciis. Summa Dexxxviij. In decima, lxiij. In consuetudine ij bercariorım, ij. I moruis ante separationen, elx. In mortuis post separationem, xxix. Summa ccliiij. Et remanent ccciiijxx agni, iiij."

Idem reddunt compotum de $M$ cccclx.xvij velleribus lanæ crispæ. In detinis, exlvij. In consuetudine iij bercariorum, iij. In supravenditis Mccexxxij, eum v velleribus hurtardorum qui sunt de Waltham, quæ fecerunt ix pondera. Liberatt[um] Petro Neuelin totum.

Iden reddunt compotum de cciiij ${ }^{x x}$.xvij caseis qui inceperunt fieri iij Nonas Maii et desierunt iiij Kalesidas Octobris; et fecerunt quaternos caseos per xxxvj dies; binus per xlij dies. In decina, xxix. In consuetudine $\mathrm{ij}$ bercariorum, ij. In expensis senesealli apud Merewellam, j. In precariis auptumnalibus, xxxiij. In supravenditis, cexxxj, qui fecerunt vij pondera dimidium et quartam partem capitis. Summa cciiijxx.xvij casei.

Idem reddunt compotum de xlij porcis remanentibus anno præeterito. Et de xxij proventis de instauro. summa lxiij. In consuetudine portarii, $j$. In missis ad lardarium de Waltham, xxiij. In mortuis, iij. Summa xxvij. Et remanent xxxvij, unde $j$ est verres, iiij sues, $x$ viij porci superamnati, xiiij sunt guellæ juniores.

Idem reddunt compotum de xxiiij gallinis proventis de Stokes. In corredio venatorum, totum. Quietus est.

Idem reddunt compotum de xxj baconibus receptis de Wlveseia. In unissis apud Stokes, vj. In precariis auptumnalibus, $x$. In corredio venatorum, v. Quietus est.

Iden reddunt compotum de viij tonnellis siceræ proventis de Merewella. Et de j provento de Stokes. Summa ix. In dono domini Episcopi H. balistario, j tonnellus. In potu venatorun domini Regis ibidem, ij. In scotallo, iij. In liberatis pincernæ domini Episcopi apud Merewellam, iij. Nichil remanet.

Summa tocius debiti $\mathrm{xxxixs.ob.} \mathrm{Solverunt} \mathrm{post} \mathrm{compotum} \mathrm{et} \mathrm{quieti} \mathrm{sunt} \mathrm{per} \mathrm{totum.}$

\section{STOKES}

Alvredus de Quincr et Henricus præpositus reddunt compotum de ij capitibus casei, et de pondere $x x$ librarum remanentium anno præterito. Solvunt et quieti sunt.

Idem reddunt enmpotum de xiijl. xs. vjd. $v b$., de toto gabulo assiso. Et de xijs. de ineremento gabuli hujus anni de molindino et terra Henrici de Stokes. Et de xviijd. de ineremento grabuli Achardi, de quadam terra quæ fuit de lominico. Summa xiiijl. iiijs. ob. In quietancia j præpositi, per annum, iiijs.; $\mathrm{j}$ hedelli, $\mathrm{j}$ fabri, $\mathrm{j}$ vaccarii, per annum, vjs.; viij carucariorun, $\mathrm{j}$ bovarii, per annum, xviijs.; $\mathrm{jj}$ operariorum, per annum, iijs. Sumna xxxijs. In defectu terræ Ricardi Calvi, ixs. In defectu longi prati tracti in doninium, vijs. In defectu Westfeldæ tractæe in doninium, vijs. In defcctu terræ Picoti tractæ in dominium, xiiijd. In defectu terræ Edrici et Willelmi tractæ in dominium, ijs. vjd. In defertu terræ Nicolai tractæ in dominium, xijd. In defectu [terræ] Rieardi Juvenis tractæ in dominium, $x v j d$. In defectu molindisi Henriei, dum fuit in ınanu domini Episcopi, per $\mathrm{j}$ terminum, vs. vd. In defectu gabuli de piscatoria ex præcepto domini Episcopi, iijs. hoe anno. Summa xxxvs. vd.

Summa quietanciarum et defectuum $\mid x v i j[s$.$] vd. Sumıia remanens x l$. xvjs. vijd. $o b$.

Idem reddunt compotum de xliiijs. iijd. ob. de pannagio. Et de xijs. de herbagio. Et de xxviijs. vjd. de iiij bobus iij vaccis vivis venditis. Et de vs. $\mathrm{x} d . \mathrm{ob}$. de coriis iiij boum $\mathrm{j}$ vaceæ mortnorum venditis. Et de $\mathrm{xj} d$. de coriis $\mathrm{ij}$ bovetturum ij genicularun mortuorum venditis. Et de vijd. de corio j equi, qui venit de Wlveseia, mortui, vendito. Et de 
xxvjs. ixd. de scotallo de sicera. Et de xjs. viijd. de ij capitibus casei venditis. Summa vjl. xs. vijd.

Idem reddunt compotum de xlixs. ijd. ob. de xxxiij quarteriis dimidio ordei venditis.

Idem reddunt compotum de xviijs. vijd. de molindino de Stokes, dum fuit in manu domini Episcopi.

De Purchasiis.-Idem reddunt compotum de ijs. de Giliberto de Brombrigge pro forisfacto bosci. Et de xijd. de Ailwardo Spure pro simili. Et de ijs. de Picoto pro simili. Et de xviijd. de Gunhilda de Brombrigge pro simili. Et de vjd. de Edwardo Ballinge pro simili. Et de vjd. de Gunt[er] pro simili. Et de vjd. de Hugone de Pila pro simili. Et de xviijd. de Alvredo Peio pro simili. Et de xviijd. de vidua Sevari. Et de xviijd. de Godwino 10 Trewe pro simili. Et de vjd. de Godefrido Pote pro simili. Et de xijd. de 'l homa de Brombrigge. Et de xijd. de Willelmo de Brombrigge pro forisfacto prati. Et de vjd. de Willelmo Ingulf pro forisfacto bosci. Et de vjd. de Ricardo Sewardi pro forisfacto piscat[urm]. Et de vjd. de Ada de Legha pro forisfacto bosci. Et de vjd. de Serlone Pote pro pastura. Et de vjd. de Willelmo Dom pro simili. Et de vjd. de Edrico pro sinili. 15 Et de vjd. de Edierlo pro simili. Et de vjd. de Radulfo de Colebroc pro simili. Et de ijs. de vidua de Cnavehulle pro forisfacto prati. Et de iijs. de Algero de Bikebroc pro pastura. Et de vjd. de Roberto Dom pro simili. Et de vjd. de Ricardo Haruldi pro veteri gario for[e]st[æ]. Et de xijd. de Galfrido de Sleate pro falsa plevina. Et de vjd. de Johanne filio Udelini pro pastura. Et de vjd. de (ialfrido Ailmeri pro sursi-a. Et de 20 xijd. de Hereberto Cobhe pro simili. Et de iijs. de Edwardo de Sleate pro simili. Et de vjd. de Henrico Pote pro pastura. Et de xijd. de Willelmo de Cnolle pro falso clamore. Et de vjd. de Algero gardin[ario] pro forisfacto bosci. Et de vjd. de Ricardo Vegrel pro sursisa. Et de $\mathrm{x}$ ijd. de Galfrido Franc[isco] pro simili. Et de xviijd. de Willelmo Ricardi pro simili. Et de xijd. de Waltero de Bikebroc pro simili. Et de xijd. de 25 Osberto de Coleoroc pro pullo Willelmi Odonis. Et de vs. de Henrico et de tota thedinga pro domo male edificata. Et de vjs. de vidua de Cnavehulle pro tilia sua maritanda. Et de vs. de Willelıno Kinge pro simili. Et de ijs. de Edredo pro uxore capienda. Et de ijs. de Thoma de Brombrigge pro simili. Et de xijd. de Willelmo Mercer pro simili. Et de xijd. de Roberto de Bertona pro simili. Et de $\mathrm{j}$ marca de filio Galtridi de Quabbe ${ }^{1} 30$ pro veredicto hundredi. Et de xs. de Galfrido de Alebroc pro simili. Et de x marcis de Henrico pro fine molindini. Summa $x l$. xvjs. viijd.

Idem reddunt compotum de $x v l$. de tallagio.

Summa summarum tocius receptro, xlvjl. xvijs. jd.

Liberatio.-In liberatis J[ohanni] decano de reragio casei, xiiijs. Summa per se.

In liberatis eidem, de termino Natalis, liijs. Eidem, de termino Paschæ, liijs. Eidem de termino Sancti Johannis, liijs. Eidem, de termino Sancti Michaelis, lvs. Eidem, de blado vendito, xxxs. Eidem, de fine molindini, $\mathrm{x}$ marcis per $\mathrm{j}$ tailliam. Eiclem, de exitu manerii, cs. Eidem, de tallagio, xvl. Summa xxxviijl. xvijs. iiijd.

Expensa.-In ferramentis iiij carucarum, $\mathrm{jj}$ avrorum, per annum, vs. iijjd. In parandis v carucis; 40 vj paribus rotarum emptis ad idem, ijs. vjd. In careta empta, ijs. viijd. In careta ferrata empta ex novo, vjs. vjd. In sellis, baciis, paronis, clutis, capistris, cordis, xiijd. ob. In precariis hyemalibus de xxvj carrucariis, iiijs. iiijd. In precariis quadragesimalibus de xxxiij carucariis, ijs. ixd. In arura viij carucarum $p[e r]^{2}$ viij sabbata, a Pascha usque festum Sancti Michaelis, vs. iijjd, quia arraverunt terras suas per xvj sabbata. In grangiis et 45 ceteris domibus emendandis et cooperiendis, $\mathrm{ijs}$. ixd. In iij portis emendandis et fossato. . . ando ${ }^{2}$ circa boscum, iijs. In stipendiis caretariorum, iijs. In stipendio daiæ, per annum, ijs. In custode vitulorum et herciatore per annum, $\mathrm{xvjd.}$ In stipendio $\mathrm{j}$ porcarii, xijd. In custo dacriæ, $\mathrm{ijs}$. $\mathrm{j} d$. In $\mathrm{D}$ [allecis] [e]mptis ${ }^{2}$ ad $\mathrm{ij}$ precarias auptumnales, xvd. In falcatione longi prati, xijd. In falcatione de Brantmede, viijd. In vj caponibus 50 emptis, et missis apud Ferham $^{3}$ ad opus domini Episcopi, xijd. In $x x$ [pullis] ${ }^{2}$ emptis, et missis ibidern ad opus ejusdem $x d$. In expensis magistri R[oberti] Basset, per iiij tallias xviijs. In $\mathrm{j}$ mola empta, xxxvjs. In ferramento molindini, $\mathrm{ij} s$. xjd. ob. In molindino reparando, xxxviijs. [Summa ${ }^{2} l$. vs. vd.

Summa expensæ et liberationis, lxvjl. ijs. ixd. Præterea liberavit anno præterito, 55 iijs. iijd. plusquam recepit, ${ }^{1}$ et ei conıpntantur per Magnum Rotulum. [Summa]" liberationis et expensæ, xlvjl. vjs. cum, iijs. ${ }^{1}$ prædictis. Et debent, ${ }^{1} \mathrm{xj} s . \mathrm{j} d$.

Exitus Grangiø.-Idem reddunt compotum de $\mathrm{cv}$ quarteriis dimidio de toto exitu frumenti.

M 8d. In semine ccxxiiij acrarum, xxiiij quarteria dimidium. ${ }^{1}$ In missis apud Waltham ad opus domini Episcopi, iiij quarteria. In missis ibidem, et liberatis Henrico pistori ad 60

1 Sic in MS.

The right hand margin of the roll is mutilated.

3 Apparently aitered from Fernham in MS. 
opus dumini Episcopi, viij quarteria. In missis apud Twifordam, ad semen, Ixviij quarteria. In pane ialcatorum prati, $j$ quarteriusu. Et quiet[i sunt].

Idem reddunt compotum de lxv quarteriis, viij bussellis dimidio, de toto exitu ordei. Et de ij quarteriis dimidio, ij boissellis ${ }^{1}$ dimidio, de cheriset. Et de ij quarteriis dimidio curailli. Summa, $\mathrm{lx} \times \mathrm{x}$ quarteria dinidium, $\mathrm{j}$ bussellus.

In semine $\mathrm{iij}{ }^{x z} v$ acrarum, xvij quarteria dimidium. In cibo $\mathrm{j}$ caretarii, $\mathrm{j}$ daim, per anuum, $\mathbf{x}$ quarteria viij busselli. In cibo $j$ berciatoris et custodis vitulorum, iij quarteria, iij bosselli. In pane ad vj precarias auptumnales, vj quarteria. In supravenditis, xxxiij quarteria dimidium.

Idem reddunt compotum de $x j$ qualteriis avenæ remanentibus anno præterito. Et de iijj ${ }^{x x} \mathbf{v}$ quarteriis dimidio, $\mathrm{j}$ hussello, de toto exitu avenæ hoc anno. Summa, iiij ${ }^{2 x}$.xvj quarteria dimidium, j bossellus. In semine clx acrurum, xlj quarteria dimidium. In missis apud Wlveseiam, et liberatis $\mathrm{J}$ [ohanni] decano, vij quarteria, per $\mathrm{j}$ talliam. In liberatis P[etro] Ace, xxxj quarteria, per iij tallias. In præbenda magistri R[oberti] Basset, iij quarteria dimidium, $\mathrm{j}$ bussellus. In probenda $\mathrm{ij}$ avrorum, iij quarteria dimidium. In consuetudine servientium ad farinam, $j$ quarterium. Summa iij ${ }^{x z} v i j$ quarteria dimidium, $j$ bussellus. Et remanent ix quarteria.

Idem reddunt compotum de dimidio quarterio de toto exitu fabarum. Et de dimidio quarterio, $\mathrm{j}$ bussello, de toto exitu vesciæ. In porcis pascendis, totum.

Instaurum.--Idem reddunt compotum de xlvij bobus, remanentibus anno præterito. Et de vj adjunctis de instauro. Summa, liij.

Summa, viij. Et remancnt $x l v$ boves.

Idem reddunt compotum de $x \times x$ ij vaccis, $\mathrm{j}$ tauro, remanentibus anno præterito. Et de ij adjunctis de instauro. Et de ij de Weregrave. Summa, xxxvj vaccæ, j taurus. In vivis venditis, iij. In mortua, $j$. Et remanent $x \times x i j$ vaccæ, $j$ taurus.

Idem redilunt compotum de vij bovettis, ij geniculis, remanentibus anno præterito. In mortuo, $\mathrm{j}$. In adjunctis bobus, vj. In adjunctis vaccis, $\mathrm{ij}$. Nichil remanet.

Idem reddunt compotum de $x v$ animalibus remanentibus anno præterito. Et de iij geniculis de Weregrave. Summa xviij. In mortuis $j$, et ij geniculæ. Et remanent Xv; unde vij sunt bovetti, viij geniculæ iij annorum.

Idem reddunt compotum de xvj annalibus remanentibus, anno præterito vitulis. Omnes remanent; unde viij sunt bovetti, viij geniculæ ij annorum.

Idem reddunt compotum de xxiij vitulis proventis de prædictis vaccis hoc anno. decimis, ij. In mortuis, iiij. Summa vj. Et remanent xvij vituli.

Idem reddunt compotum de ciiij ${ }^{x \mathbf{j}}$ caseis de reragio anni præteriti. In supravenditis, totum, et fecerunt ij capita.

Idem reddunt compotum de xiiij caseis factis in hyeme. Et de xiiij factis a primo nonas Aprilis usque ad vj nonas Maii ; quia fecerunt per ij dies, $\mathrm{j}$ caseum. Et de cl caseis qui inceperunt fieri die Inventionis Sunctæ Crucis, et desierunt die Sancti Michaelis, utroque die computato. Summa clx.xviij casei. In decimis, xvij. In consuetudinibus falcatorum ij prat[or]um, ij. In precariis magni prati, ix. In expensis senescalli, $j$. In supravenditis, cxxxiij, qui fecerunt ij capita. Et remanent xvj casei; precium, xijd.

Idem reddunt compotum de $\mathrm{xl}$ porcis remanentibus anno præterito. Et de $\mathbf{x x i i i j}$ proventis de suibus. Summa lxiiij. In decima, j. In consuetudine porcarii, j. In mortuis, vij porci, ij porcelli. In missis apud Waltham ad lardarium, xiij. Summe xxiiij. Et remanent $x l$; unde iiij sunt sues, $\mathrm{j}$ verres, xxviij superannati, vij porcelli.

Idem reddunt compotum de Ix.xiiij gallinis proventis de cberiset. In liberatis Johanni decano apud Wlveseiam, l gallinæ. In liberatis Henrico de Merewella, ad opus venatorum, xxiiij gallinæ. Idem liberaverunt $\mathrm{R}$ [ogero] Wacelino ad opus domini Episcopi apud Ferham, vj capones, $x x$ pullos de emptis.

Idem reddunt compotum de ovis, quia male custodita erant. Appreciata, xviijd.

Idem reddunt compotum de vj baconibus receptis de Johanne decano de Wlveseia. In precariis auptumnalibus totum exp[enditur].

Idem liberaverunt Nicolao de coquina domini Episcopi, apud Waltham, ccl anguillas de termino Natalis.

Idem liberaverunt apud Merewellam, $\mathrm{j}$ tonellum siceræ, precium, viijs.

Idern liberaverunt, apud Tuifordam, xj mullones feni de longo prato ad opus domini Episcopi et ad opus ovium, precium, xxxs.

Summa tocius debiti, xiijs. vijd. Solvit post compotum et quietus est. ${ }^{1}$

\section{EDBOREBIRLA.}

Rogerus serviens et Ricardus præpositus reddunt compotum de lxvjs $\mathrm{x} d$. de reragio anni præteriti. Solvit vjs. per $\mathrm{j}$ talliam, et quietus est quantum ad eum pertinet.2

Idem reddunt compotum de xxiiijl. xiiijd. de gabulo assiso, unde, vjs. vijd. sunt de incremento anni iiijti. In quietantiis $j$ præpositi, $v \varepsilon_{0} ; j$ berebr[ecti], iujs.; $j$ fabri

1 A later entry in MS.

2 Sic in MS. 
j hercarii, viijs.; viij carrucariorum, xxjs.; xvij virgatariorum et dimidii, pro opere auptumno, xxvjs. iijd., scilicet singulo xviijd. ; iij cotagiorum dimidii, iijs. vjd. Summa lxvjs. ixd. Summa remanens, $\mathbf{x x l}$. xiijs. vd.

Idem reddunt compotum de ixs. ixd. de pannagio. Et de xiijjs. de herbagio. Et de

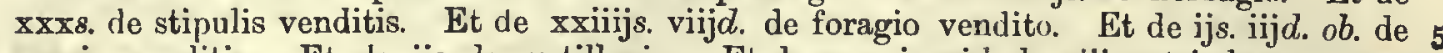
pomis venditis. Et de ijs. de curtillagio. Et de $x \times x v j s$. vjd. de xiij petris lanæ grossæ venditis. Et de ijs. vjd. de xlij velleribus agninis venditis. Et de xxxvjs. vjd. de v ponderibus, iij petris, casei venditis. Et de xijd. de xxiiij caseis vaccinis venditis. Et de vjs. de $\mathrm{j}$ huve vivo vendito. Et de $\mathrm{ij} s$. ixd. de $\mathrm{j}$ corio bovis vendito. Et de xiijd. de

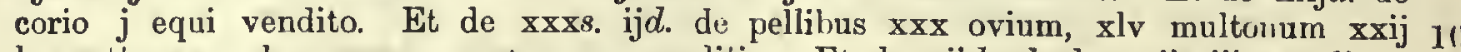
hoggettorum, xl agnorum mortuorum venditis. Et de xijd. ob. de coriis iij anualiun, ij vitulorum venditis. Summa $x l$. $x i j d$.

Idem reddunt compotum de lx.xiij[l.] viijd. de xxxiiij quarteriis frumenti venditis, scilicet singulo, xxvjd. ${ }^{2}$ Et de ixl. iijs. vd. de ciijj quarteriis dimidio sigali venditis. Et de xls. de $x \times x$ quarteriis ordei venditis. Et de lxvijs. iiijd. de lx.xiiij quarteriis 15 aveur venditis. Summa xviijl. iiijs. vd.

De Purchasiis._-Idem reddunt compotum de vjs. viijd., de heriet Osberti clerici. Et de xls. de relevo ${ }^{1}$ Willelmi filii ejus. Et de vs. de heriet Matildis viduæ. Et de xxs. de relevio Willelıi filii ejus. Et de vjs. viijd. de Willelmo Tragis pro nxore cıpienda. Et de vjd. de heriet Willelmi filii Ailwini. Et de vs, de heriet Roberti filii Gervasii. Et de ijjs. vjd. 20 de Ailwino filio Willelıni pro terra patris sui habenila. Et de vjd. de heriet Aliciu viduæ. Et de vjs. viijd. de Ricardo molindinario pro manutenemento. Et de xijd de Willelmo clerico pro filia sua maritanda. Et de iijjs. de Niculao de Witham pro pann[o] stulte capt[o]. Et de vjs. viijd. de Michaele Merefreins pro terra habenda. Et de iijs. de Randulfo Merefreins pro fine. Et de iijs. de Willelmo Tut pro terra habenda. Et de x8. 25 de tedinga pro pace hahenda. Et de vjd. de Ricardo filiu Reginaldi. Et de vjd. de Roberto filio Lewini pro pastura. Et de xijd. de Ricardo heiwardo pro melleil. Et de vjd. de Ada Siveken pro manutenemento. Et de vjd. de Waltero filio Reginaldi pro sinili. Et de vjs. viijd. de Ricardo filio Gonnildæ pro fine terræ. Summa vjl. xjs. xd.

Idem reddunt compoturn de xviijl. de tallagio.

Summa purchasiorum cum tallagio, xxiiijl. xjs. $x d$.

Summa tocius receptre, Ix.xiijl. xjs. viijd.

Liberatio.-In liberatis Johanni Decano, de gabulo et exitu, xxvijl. viijs. ixd. ob. per iij taillias. Eidem de blado vendito, xjl. xs. per iij tallias. Eidem de caseis venditis, xviijs. vjd. per j talliam. Eidem de purchasiis iiijl. xiijs. per j talliam. Eidem de 35 taillagio, xvjl. vs. per j talliam. Summa, Ixjl. xvs. iijd. ob.

Expensa.-In ferramentis ix avrorum, per annum, ijs. In consuetudine carıcarum proestuvariis carucarum præter ferrum, ijs. In ferramento carucarum, iijjs. vjd. In $\mathrm{j}$ bove empto, x8. vjd. In iij quarteriis mestellonis emptis ad semen, vjs. In hernesio caretarum, vjd. ob. In custo daeriæ, ijs. In sacco empto, vjd. In domo emendanda, ijs. ob. In virgis, et clatis 40 ad faldam, xxiijd. In lavandis et tondendis clxix ovibus, $x l$ agnis, $x j d$. In sagimine et sulphure ad easdem, ixd. In decima iij vitulorum, $\mathrm{j}$ pulli, ijd $o b$. In decima pellium ovium mortuorum, ijs. In expensis senescalli, ixs. vd. ob., per iij tallias. In liberatione Ricardi servientis, lxs. $x d$. In expensis Ricardi de Gray et Reineri, a die Jovis Paschæ usque diem Martis proximam ante Pentecosten, xijs. ixd. In expensis G. de Acl[anda] die 45 Mercurii proxima ante Pentecosten, iijj. In expensis militum domini Episcopi in crastino Assumptionis, iijs. ob. In marscalleia summarii, et duorum equorum domini Episcopi, xviijd, ob. In venatione ducenda apud Wintoniam, xiijd. ob.

Summa, vjl. vjs. $\quad$ Summa tocius liberationis et expensæ, lxviijl. xvd. ob. Et debent, cxs. iiijd. ob.

Exitus Grangiø.--Idem reddunt compotum de lxv quarteriis de toto exitu frumenti. In semine clij acrarum, xxxj quarteria. In supravenditis, sxxiiij quarteria. Summa lxv. Et quietus est.

Idem reddunt compotum de clxvj quarteriis dimidio $\mathrm{j}$ bussello, de toto exitu sigali. Et de iiij emptis. Summa clxix quarteria dimidium j bussellus. In semine c.iijjx acrarum, xl quarteria. In supravenditis ciiij quarteria dimidium. In precariis auptumnalibus, iij quarteria, dimidium. In liberatione caretarii, haiwardi, per totum annum, xiij quarteria. In liberatione $\mathrm{j}$ vaccarii, $\mathrm{j}$ herciatoris, $\mathrm{j}$ porcarii, per totum annum, ij quarteria dimidinm. In liberatione $\mathrm{j}$ daiæ, $\mathrm{v}$ quarteria $\mathrm{j}$ bussellus. Summa clxix quariteria dimidium, $\mathrm{j}$ bussellus. Et quietus [est].

Idem reddunt compotum de xl quarteriis de toto exitu ordei. In semine xxij acrarum, v quarteria. In supravenditis, $\mathbf{x x x}$ quarteria. De precio superius respondent. 
In braciatura ad precarias anptumnales, $\mathrm{j}$ quarterium. In victu porcorum per hyemem, iiij quarteria. Sumına $x l$ quarteria, ct quiet[i sunt].

Idem reddunt compotum de c.lx.vij quarteriis, ij bussellis de toto exitu avenæ. In semine iiijxzviij acra'um, xlj quarteria dimidiun. In præbenda domini Episcopi in festo Sancti Egidii, vij quarteria ij busselli. In præbenda ejusılen die Mercurii in Pascha, ix quarteria dimidiun, iij busselli. In præbrn la j equi W[illelıni] de Sancto Max[entio] per $\mathrm{xx}$ dies, ante festum Suncti Michnelis, ij quarteria. In præbenda Magistri Petri Russinol circa festum Sincti Andreæ, iij bosselli. In præebenda illırım qui duxerunt venationem apud Wintonianı, dimidiun quarteriuı. In præbenda Ricardi de Gray et Reineri a die Jovis Pascha, usque diem Martis proximam ante Pentecosten, vij quarteria dimidium. In præbenda j summarii domini Episenpi a die Jovis in septimana Paschæ, usque diem Veneris proximam ante festum Sancti Barnabæ; et in alio equo ejusdem a Sabbat, in septimana Paschæ, usque dicm Veneris proximam ante Penlecosten, v quarteria. In præbenda domini Episcopi, die crastino post ${ }^{1}$ festum Sanctæe Margaretæ, ij busselli. In præbenda Magistri Radulfi de Wikewane in nuptumno, ij busselli. In probendn G. de Aclanula, iij bosselli. In præbenda senescalli, per iij tallias, ij quarteria, dimidium, j bossellus. In præbenda avrorum xij quarteria. In braciatura ad precarias auptumnales iiij quarteria. In supravenditis, Ix.xiiij quarteria. De precio superius respondent. Summa clxvij quarteria. Et debent ij bosselli, appreciati iijd.

20 Instaurum.-Idem reddunt compotum de ix avris remanentibus anno præterito. Et de iij pullis remanentibus. Et de $\mathrm{j}$ pullo provento hoc anno de instauro. Summa xijj. Et remanent ix avri, iij pulli ij annorum, j pullus $\mathrm{j}$ anni.

Idem reddunt compotum de xvij bobus remanentibus anno præterito. Et de j postea empto. Summa xviij. In precariis anptumnalibus $j$. De corio superius respondent. In supravendito j. De precio superius respondent. Summa ij. Et remanent xvj.

Idem reddunt compotum de $\mathrm{v}$ vaccis, remanentibus anno præterito. Omnes remanent.

Idem reddunt compotum de $v$ annalibus remanentibus, anno præterito vitulis. In mortuis iij. Et de coriis superius respondent. Summa iij. Et remanent ij.

Idem reddunt compotum de v vitulis proventis hoc anno. In mortuis ij. De coriis superius respondent. Summa ij. Et remanent $i i j$, unde ij sunt masculi et $j$ femella.

Idem reddunt compotum de ciij ovibus remanentibus anno præterito. Et de xxj adjunctis. Summa, cxxiiij. In mortuis ante partum et tonsionem, xiiij. Post partum et ante tonsionem xvj. Post tonsionem j. Summa xxxj. Et remanent iiijxx.xiij.

Idem reddunt compotum de cxiij multonibus remanentibus anno præterito. Et de vj adjunctis. Summa cxix. In consuetudine messoris $j$ vivus. In mortuis ante tonsionem xliiij. Post tonsionem j. De pellibus superius respondent. Summa xlvj. Et remanent lx.xiij.

Idem reddunt compotum de xlix hnggettis remanentibus anno præeterito agnis. In mortuis ante tonsionem xxij. De pellibus superius respondent. In adjunctis ovibus $\mathbf{x x j}$. In adjunctis multonibus vj. Et nichil remanet

Idem reddunt compotum de iiijxx agnis proventis hoc anno de ovibus, quia $\mathbf{j}$ peperit gemellos. Et de ij proventis de gerciis. Summa iijjxx.xij. In decima, ix. In consuetudine bercarii, j. In mortuis ante tonsionem $\mathrm{xl}$, post tonsionem, iij. De pellibus superius respondent. Summa liiij. Et remanent xxxviij agni.

Idem reddunt compotum de clxviij velleribus grossis. In decima, xvij. In consuetudine bercarii, $j$. In supravenditis, cxlix, quæ fecerunt xiij petras, unde xiiij faciunt $j$ pondus. Summa clxvij. ${ }^{1}$ Et debet $^{1} \mathrm{j}$, precium, iijd.

Idem reddunt compotum de xlij velleribus agninis. In supravenditis, totum.

Idem reddunt compotum de xxiij caseis factis de vaccis post festum Sancti Michaelis. Et de clx.xviij caseis hujus anni, qui inceperunt fieri in octavis Paschæ, et desierunt in festo Sancti Michaelis, utroque die computato. Sumna ccij casei. In decimis, xvij. In consuetudine daiæ, $\mathrm{j}$. In consuetudine carucariorum, $\mathrm{j}$. In consuetudine messipe, $\mathrm{j}$. In domino Episcopo, $\mathrm{j}$, in festo Sanctre Margaretæ. In militibus domini Episcopi in crastino Assumptionis, $\mathbf{j}$. In precariis auptumnalibus, xv. In supravenditis clxv casei.

Idem reddunt compotum de xxxiiij porcis remanentibus anno præterito. In missis apud Witteneiam, xx. In mortuo, j. Summa xxj. Et remanent xiij.

Idem reddunt compotum de $\mathrm{xx}$ porcellis proventis hoc anno de instauro. In decima, ij. In consuetudine præpositi, j. In mortuis, ij. Summa v. Et remanent $x v$. Summa utriusque remanentis, xxviij.

Idem reddunt compotum de vj aucis remanentibus anno proterito. Et de xij de exitu illorum. Et de $x x$ de purchasiis. Summa xxxviij. In militibus domini Episcopi. xij. Et remanent, $x \times v j$. 
Idem reddunt compotum de $\mathrm{l} x$ gallinis remanentibus anno præterito. Et de $\mathrm{xx}$ de cheriset. Et de $x x$ de purchasiis. Summa, c. In missis apud Witteneiam xlvij. In liberatis Galfrido de Cauz, v. Summa lij. Et remanent xlviij.

Idem reddunt compotum de xvj caponibus de redditu. Et de xxviij de purchasiis. Sunma xliiij. In liberatis Galfrido de Cauz, xij. In militibus domini Episcopi, iij. Summa xvj. Et remanent xxviij.

Idem reddunt compotum de $\mathrm{xx}$ pullis de exitu instauri. In militibus, $x$. Et remanent $x$.

Idem reddunt compotum de $\mathrm{v}$ baconibus receptis de Witteneia. In precaliis auptumnalibus, iiij. In Ricardo de Grey et Reinerio, j. Nichil remanet.

Summa tocius debiti, cum reragio anni præteriti, viijl. xjs. viijd. ob. Unde cxs. $x d . o b$. est manifestum debitum $l x s . \mathrm{x} d$. de tempore Oliveri.

\section{SUWERKE.}

J[OHANNES] serviens reddit compotum de receptis a festo Sancti Petri ad Vincula; scilicet de xls. de Episcopo. Et de xls. de Abraham Judæo. Et de xxs. de Henrico de Sancto 15 Albano. Et de Xxs. de pratis. Et de xijs. de vj quarteriis de exitu molindini vendito. Et de $x s$. vjd. de vino Radulfi vendito, per manum Willelmi Clopparde. Et de xxs. de vinis venditis Arnolfo vinitario. Et de vjl. xvijs. rle redditu termini Sancti Michaelis. Et de vjs. de Ros. ${ }^{1}$ Et de pomis, xvs. In defectu iij placitorum iijs. Summa xvjl. $\nabla j d .^{2}$

Expensa.-In duabus civeris ${ }^{3}$ cum rotis xjd. In terra ducenda ad kaium $x d$. Item pro eodem, M. 9. $x d$. Ir vj navatis terræ ad kaium, xxvijd. In iiij navatis gravelli ad idem, xiijd. Item pro terra ferenda ad idem, ij $d$. ob. In sepo ad molindinum, iiijd. In pisce ad vivarium xxd. In pisis metendis, iijs. viijd. In eisdem ligandis et cariandis, xxviijd. In ij batellis gravelli, vjd. In seruris ad ostium, ijd. In paliciis emendantis circa vivarium, ijd. In 25 br[evi] deferendo apud Weregrave, iiijd.' In expensis W'illelmi Baratte a die Jovis ante festum Sancti Petri ad Vincula usque diem Martis post festum Sancti Laurentii, xxvjd. In lais parandis, $x d . o b$. In expensis $\mathbf{j}$ equi a die Mercurii proxima post festum Sancti Laurentii usque diem Sabbati proximam ante festum Sanctæ Crucis, iijs. vjd. In $\mathrm{j}$ garcione custodiente ipsum equum per vj septimanas, vs. In avena et brennio ad iij equos a die 30 Jovis ante festum Sancti Petri ad Vincula usque in crastino Sancti Laurentii, iijs. vjd. In iiij navatis terræ ad breccam, xxvijd. In iiij hominitıas ad faciendum breccam per iiij dies, xld. In iij navatis terræ, xvjd. ob. In mairemio ad breccam, ijs. vjd. In terra ad opturandum molindinum, $v d$. In $\mathrm{j}$ carpentario per $\mathrm{ij}$ dies, vjd. In renovando terram ad molindinum, xxjd. In custo gardini, xvjd. In $\mathrm{j}$ quarterio de bordis ad molindinum, 35 $\mathrm{ij} d . a b$. In clavis, $o b$. In $\mathrm{j}$ carpentario, iijd. In ferramento ad molindinum, xixd. In sepo, iijd. In $\mathrm{j}$ bascat jd. In ij operariis, iijd. In drasco ad equos molindini, xviijd. In ferrura eorundem, xxj $d$. In gutteriis ponendis, $\mathrm{ijs}$. In $\mathrm{j}$ carpentario, ijd. In gravello ad breccam, xjd. In j carpentario per ij dies, vjd. In expensis P[etri] Ace, vs. ixd. ob. In expensis iij equorum remanentibus post regem, xix $d$. ob. In arrura xij acrarum 40

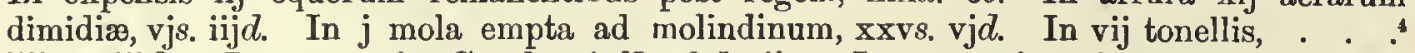
iiijs. viijd. In expensis G. de Acl[anda], ijs. In expensis militum de Ispania, xvijs. vjd. In expensis $J$ [ohannis] servientis, $x s$. iij $d$. In expensis gardinarii, vijs. ixd. In Harefango, xvd. ob. In liberat[is] Monach[is], iiijl. In expensis Magistri R[oberti] Basset, ijs. Ir. custo mairemii venientis de foresta Sancti Leonardi ad molindinum et 45 carpentario ad evellandum mairemium, xiijs. $x d$. In planchis sicandis, $x x j s$. In fustis sullevandis, ijs. vjd. In mairemio ducto de foresta Sancti Leonardi usque Dorkinges, xs. Item in mairemio ducto de eodem foresta usque Kingestonam, xxijs. vjd. In algeo faciendo, iiijs. ijd. In rungis factis ad molendinum, vjd.

Summa tocius expensæ, xiijl. xijs. viijd. Et debet $x x v i j s . x d$.

Idem reddunt compotum de ciiij porcis remanentibus post decessum Radulfi Cerarii In expensis domini Episcopi, xliiij porci. Et remañent, lx.

\section{ALRESFORDE.}

Henricus ra Martre et Osbertus præpositus reddunt compotum de xxxiijl. xiijjs. iiijd. ob. de reragio anni præteriti. Et de xlvs. de lana agnina vendita. Solvunt et quieti 55 sunt.

Idem reddunt compotum de xxvjl. xixs. viijd. de toto gabulo assiso apud Alresforde. Et de iiijd. de incremento hujus anni. Et de xviijl. ijs. viijd. de novo foro. Summa xlvl. ijs. viijd. In quietanciis $\mathrm{j}$ præpositi, vs.; $\mathrm{j}$ haiwardi, iiijs.; ij bercariorum de Alresforde et de Haiwode, vjs.; vj carucariorum, per annum, xviijs.; ix operariorum, 60 xxvijs., j fabri, j porcarii, j bovarii, j vaccarii, xijs.; j præpositi de Prevet,iijs.; j haiwardi, xijd.; j carucarii, iijs. Summa lxxixs.

1 Sic on an erasure of rosis in MS.

2 Sic in MS.

${ }^{3}$ Cuũs in MS.

t Blank in MS. 
In defectu terræ Reinerii tractæ in dominium, ijs. In defectu terræ Haroldi, iijs. In defectu terræ Ricardi Piscatoris, ijs. Summa vijs.

Summa quietanciarum et defectuum, iiijl. vjs. Summa remanens $x l$. xvjs. viijd.

Idem reddunt compotum de xlviijs. vjd. de pannagio. Et de ixs. vjd. de herbagio. Et de vjl. xiijs. iiijd. de $\mathrm{v}$ ponderibus grossæ lanæ venditis. Et de ixs. de vij clavis lanæ agninis venditis. Et de ixs. de iij bohus veteribıs ${ }^{2}$ venditis. Et de viijs. de iij vaccis venditis. Et de vs. $\mathrm{x} d$ de $\mathrm{x}$ multonibus vivis venditis. Et de $\mathrm{xij} d$. de ij multonibus venditis. Et de ijs. xjd. de $\mathrm{xx}$ agnis vivis venditis. Et de vijs. vjd. de pellibus $\mathrm{xxxj}$ ovium, xxxv multonum, xxj hoggettorum mortuorum venditis. Et de vs. vjd. de iiijxxiiij pellibus agnorum mortuorum venditis. Et de vs. iijd. de pastura vendita. Et de iijs. jd. de caseis venditis, qui provenerant de vaccis. Et de iiijs. de veteri careta vendita. Et de xlvs. vjd. de vj ponderibus dimidio casei venditis. Et de xxvjs. iijd. de xxxv quarteriis avenæ de Ferham venditis. Et de xxiiijs. de ij tonellis vini venditis. Et de xijd. de butiro vendito. Et de iijs. iiijd. de Dcce ovis venditis. Et de viijd. de melle invento vendito. Et de ixd. de xij pellibus agninis proventis de hoggettis adjunctis venditis. Summa xvijl. xv8.

Idem reddunt compotum de xxxvjs. de xij quarteriis frumenti venditis. Et de vl.ixs. iiijd. de xlj quarteriis frumenti venditis. Et de iiijl. vjs. de xliij quarteriis mancorni venditis. Et de cxs. de lv quarteriis ordei venditis. Et de iiijl. xxd. de lxx quarteriis avenæ venditis. Et de iijs. de j quarterio dimidio pisarum venditis. Et de iijs. iiijd. de ij quarteriis visciarum venditis. Summa $\mathrm{xxjl}$. ixs. iiijd.

De Purchasiis. - Idem reddunt compotum de xiijjs. de Roberto de Medestede pro terra habenda. Et de ijs. de Symone Juveni pro filia sua maritanda. Et de xijd. de Benedicto filio Edwini pro terra patris sui habenda. Et de iijs. de Martino flio Ernaldi pro uxore ducenda. Et de xls. de Unfrido de Wodeforde quia celavit venditicionem equi domini Episcopi. Et de vjs. viijd. de Guihut de Haiwode pro terra habenda. Summa lxvjs. viijd.

Idem reddunt compotum de xxxjl. de taillagio.

Summa tocius receptæ, cxiiijl. vijs. viijd.

Liberatio.-In liberatis J[ohanni] decıno de termino Natalis, lxijs. per i tailliam. Eidem, de 30 termino Paschæ ixl. ixs. iiijd., per j talliam. Eidem, de termino Sancti Johannis, lxiijs. jd. per i talliam. Eidem, de termino Saneti Michilelis, xviijl. xs. per ij. tillias. Eidem, de blado vendito, cs. Eidem, de lana vendita, vjl. xiijs. iiijd. Eidem, de exitu manerii, ixl. viijs. iijob. Eidem, de taillagio, xjl. xiijs. jd. Iterum eiden, ls. Summa lxixl. viijs. xjd. ob.

35 Expensa.-In ferramento iij carrucarum, per annum, j carrucæ, per j terminum, iiijs. In ferrura ij equorum caretariorum, per annum, xixd ob. In caretis axandis et reparandis, clutis, uncto et alio hirnesio empto ad caretas, xjd. In nova careta empta, viijs. iiijd. In vj præsepibus factis in bercaria, ijs. In sepibus emendandis et clostura coliganda ad idem, xixd. In tribus saccis emptis, xijd. In iij paribus rotarum factis ex maremio domini episcopi, xijd. In carucis et rotis empti - ad carucas, xiijd. In blado claudendo apud Haiwode, iiijs. In custo daeriæ, iijs. viijd. In triturand is et ventilandis xxiij quarteriis frumenti, iijs. iijd. ob. In triturandis et ventilandis ix quarteriis ordei, xid. In triturandis et ventilandis xxxij quarteriis avenæ, $\mathrm{xxd}$. In sarclandis el acris apud Alresforde et Haiwode, iiijs. In precariis hyemalibus de xxxij carucis, vs. iiijd. In quadragesimalibus precariis de xxxvj carrucis. vjs. In cecc allecis emptis ad precarias auptumnales, xvid. In xxj ulnas de canevacio emptis ad lanam per proceptum senescalli, iijs. vjd. In filo empto ad sagenam refieiendam et stipendio et corredio hominis eim reparantis, xiijd. $o b$. In trahenda sagena per iiij vices, iijs. $x d$. In stipendiis ij servientium per annum vjs. In stipendio daiæ, per annum, ijs. In expensis Henrici la Martre quando venit tenere hundredum, per plures vices, iijs. In companagio $\mathrm{j}$ hominis Henrici La Martre custodis curiæ, per annum, ijs. In grangia cooperienda, vjd. In ij bobus emptis, xiiijs. In stipula coligenda ad molindinum foleraticum, viijs. iijd. In liberatis præposito de Prevet ad bladum metendum, xxixs. Summa vjl. vs, xjd. ob.

Summa liberationis et expensæ lx.xvl. xiiijs. xjd. Et debent xxxviijl. xijs. ixd.

55 Exitus Grangiæ.-Idem reddunt compotum de iiij ${ }^{x x}$.xvj quarteriis dimidio de toto exitu frumenti. Et de iiij quarteriis dimidio receptis de Prevet. Summa cj quarteria. In semine clxviij acrarum, xlviij quarteria. In venditis, liij quarteria; de precio superius respondent.

Idem reddunt compotum de vij quarteriis de toto exitu curailli. In præbendi ij avrorum, $\mathrm{i}$ quarterium. In præbenda $\mathrm{ij}$ equorum caretarum, iiij quarteria. In porcis pascendis, ij quarteria. 
Idem reddunt compotuw de $\mathrm{Ix} . \mathrm{xv}$ quarteriis dimidio de toto exitu mancorni, cum iij quarteriis pisarum mixtis eidem. Et de xij quarteriis dimidio receptis de Prevet. Summa iiijaxviij quarteria. In semine cj acrarum, xxxiij quarteria dimidium. In corredio $\mathrm{ij}$ servientium, vij quarteria. In corredio $\mathrm{j}$ daiæ, $\mathrm{ij}$ quarteria. In corredio custrodis agnorum, per dinidium annum, $\mathrm{ij}$ quarteria dimidium. In venditis, xliij quarteria.

Idem reddunt compotum de exxxij quarteriis de toto exitu ordei. In semine cxxxj acrarum, lij qnarteria limidium. In missis apud Prevet, iij quarteria. In rorredio ij servientium, vj quarteria. In corredio daiæ, iij quarteria. In jurcis pascendis in hyemr, ij quarteria. In consietudine porcarii, $\mathrm{j}$ quarterium dimidium. In iii precariis auptumnalibus, viij quarteria. In venditis, lv quarteria.

Idem reddunt compotum de ccv quarteria de toto exitu avenæ. Et de xxxviij receptis de Ferham. Summa ccxlinj quarteria. In semine clxi acrarum, cx quarteria. In missis apud Suttonam et liberitis $\mathrm{P}$ [etro] Ace, viij quarteria, per j tailliam. In præbenda $\mathrm{ij}$ carettariorum, per annum, vj quarteria. In præbencla ij uvrorum, per $\mathrm{j}$ terminum, $\mathrm{ij}$ quarteria. In præbenda boum, ix quarteria. In porcis pascendis in hyeme, per senes- 15 callum, iij quarteria. In venditis, cr quarteria.

Idem reddunt comp tum de ix quarteriis de toto exitn pisarum. In semine ix acrarum, ij quarteria dimidinı. In mixtis cum mancorno, iij quarteria. In porcis pascendis, $\mathrm{j}$ quarterium. In missis apud Cherionam, $\mathrm{j}$ q"arterinm. In venditis, $\mathrm{j}$ quarterium dimidium.

Idem reddunt compotun de iij quarteriis de exitu viciarum. In semine iij acrarum, $\mathrm{j}$ quarterium. In venditis, ij quarteria. Quietus est.

Instaurum.-Idem reddunt compntum de iij equis remanentibus anno præterito. Et de j recepto de Walda. Summa iiij. In missis ad Curiam domini Episcopi, per breve domini Eustachii de Grenvilla, ij. Et remanent ij.

Idem reddunt compotum de lv bohus remanentibus anno præterito. Et de ij postea emptis. Et de vj adjunctis. Et de $\mathrm{j}$ de Hunfrido de Wodeforde. Summa lxiiij boves. In mortur, j. In misso apud Waldam, j. In venditis, iij. Summa v. Et remanent lix.

Idem reddunt compotum de $\mathrm{xxj}$ vaccis, $\mathrm{j}$ tauro, remanentibus anno præterito. Et 30 de ij adjunctis. Summa xxiiij. In venditis $i \mathrm{ij}$. Et remanent $\mathrm{xx}$ vacco, et $\mathrm{j}$ taurus.

Idem reddunt comprtum de $v j$ boviculis, ij geniculis, $x$ annalibus, remanentibus anno præeterito. Summa xviij. In adjunctis bobus, vj. In adjunctis vaccis, ij. Ec remanent $x$ annales; unde vj sunt masculi, iij femellæ.

Idem reddunt compotum de viij vitulis proventis hoc anno. In mortuo, j. Et remanent vij.

Idem reddunt compotum de cccxxxv ovibus iij hurtardis remanentibus anno præterito. Et de iiij ${ }^{x x}$ adjunctis. Summa cccexviij. In mortuis ante partum, viij; post partum et ante tonsionem, xviij; post ton 4 inem, v. Summa xxxj. Lit remanent 40 ccciiij ${ }^{x x} \times x$ cum iij hurtardis.

Idem reddunt compotım de cceiij ${ }^{x x}$ multonibus remanentilus anno præterito. Et de Ix.x adjunctis. Summa cecel. In venditis arl f'stum Sancti Martini, xij. In mortuis ante tonsionem, xxiij. In mortuis post tonsionem, xij. Sumnı xlvij. Et remanent cccciij.

Idem reddunt compotum de clx.xiiij hoggettis remanentibus, anno præterito agnis. In moltuis ante tonsionem, $x x j$. In adjunctis ovibus, iiij ${ }^{x x}$. In adjunctis multonibıs, Ix.x.

Idem reddunt compotum de cc.xix alnis proventis hujus anni, quid viij oves erant steriles. In mortuis ante tonsionem, iiij ${ }^{\mathrm{xx}} . \mathrm{xvj}$. In consuetudime bercarii, $\mathrm{j}$. In decima, xxxij. In venditis, $\mathrm{xx}$. Et remanent . . . .

Idem reddunt compoum de $\mathrm{xij}$ agnis proventis de hoggettis adjunctis. In mortuis, comnes. De pellibus superius respondent.

Idem reddunt compotum de Deccx velleribus grossis. In decimis iijax. In consuetudinibus $\mathrm{ij}$ bercariorum, ij vellera. In venditis, Dccxxv, quæ tecerunt $\mathrm{v}$ pondera. Et debent iij vellera, precium $v j d$.

Idem reddunt compotum de cixix velleribus agninis qua fecerunt vij clavos. In venditis, totum.

Iden reddunt compotu , de clviij caseis, qui inceperunt fieri festo Sancti Marci Ewangelistæ, et desierunt in festu Sancti Michaelis. In venditis, clv, qui fecerunt vj pondera dimidium. Et debent iij casei ; precium vjd.

Idem reddunt compotum de lv porcis remanentibus anno præeterito. Et de iij $^{x \times} \mathrm{iij}$ emptis. Et de xlvj de exitu illoru . . Summa ciiij ${ }^{x}$ iiij. In decimis, iiij. In colsuetudine porcarii, j. In nissis apud Wlveseiann, c. In mortuis, v. Summa cx. Et remanent lx.xiiij; unde vij sunt sues, $\mathrm{j}$ verres, xxv porci superannati, xlj porcelli hujus anni.

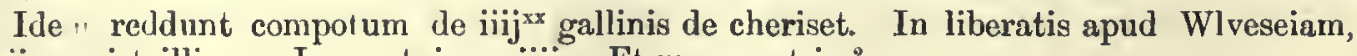
xlvij, per $\mathrm{j}$ tailliam. In mortuis, xxiiij. Et remanent ix."

\footnotetext{
1 A later insertion in another hand; the figures have been partly erased.
}

2 Et remanent ix is a later entry in another hand. 
Idem reddunt compotım de Dece ovis le consuetudine. In venditis, totum.

Idem reddunt computum de vij baconibus receptis de Wolvesei^. In præcariis auptumnalibus, totum. Idem , blitus est iij quarteria avenæ, appreciati iijs.

Summa tocius debiti, xxxviijl. xvjs. $\mathbf{x} d$.

\section{PREVET}

H[enricus] la Martre et Robertus de Brichtewella et. Symon fuvenis, redilunt compotum de xxixs. recoptis de Alresforda. Et de xlviijs. viijd. de receptis de Walda. Suınua lx.xvijs. viijd.

Idem reddunt compotum de vjs. viijd. de $\mathrm{jj}$ quarteriis dimidio frumenti venditis. Et de iiijs. de ij quarteriis mancorni venditis. Et de ijs. de ij quarteriis curailli venditis. Et de xxxs. vijd. de xxix quarteriis avenæ venditis. Summa xliijs. iijd.

$$
\text { Summa tocius receptro, vjl. xjd. }
$$

In ferramento $\mathrm{j}$ carrucæ per annum, $\mathrm{j}$ carrucæ per $\mathrm{j}$ terninum, $\mathrm{ij}$ s. $\mathrm{ij} d$. In mercede fabri, xjd. In ferrura ij avrorum, per annum, $v$ avrorum per $\mathrm{j}$ terminum, $\mathrm{x} v \mathrm{j} d$. In rotis emptis ad carrucas, iijd. In triturandis et ventilandis liiij quarteriis dimidio frumenti, xs. ijd. ob. In triturandis et ventilandis xxxix quarteriis mancorni, vijs. iijd. ob. In triturandis et ventilandis $\mathrm{x}$ quarteriis $\mathrm{j}$ estrica orlei, xijd. ob. In triturandis et ventilandis exlviij quarteriis avenæ, $\mathbf{x} s . \mathbf{i x d}, a b$. In sarenlandis cexix acris, vijs. vjd. In blado metendo, cariando et tassanilo, lxixs. vd. ob. In stipendiis iij servientium per $\mathrm{j}$ terminum, ijs. iijd. In stipendiis $\mathrm{ij}$ servientiuın per $\mathrm{j}$ terminum, $\mathrm{xviij} d$. In stipendio $\mathrm{j}$ herciatoris per $\mathrm{j}$ terminum, vjd. In stipendio $\mathrm{j}$ servientis per ij terminos, $x v i i j d$. In grangia emendenda, $\mathbf{v} d$. In fimo extrahendo de curia, $\mathrm{v} d$.

Sunma vl. xvijs. vjd. ob. Et debent iijs. iiijd. ob.

Exitus Grangiæ.-Idem reddunt compotun de liij quarteriis dimidio de cxitu frumenti. In semine lxij acrarum, xv quarteria dimidium. In missis apud Alresfordan, iiij quarteria dimidium. In missis apud Waldım, xxxij quarteria. In venditis, ij quarteria dimidium.

Idem reddunt compotum d" iiij quarteriis curailli. In præbenda avrorum, ij quarteria. In venditis, ij quarteria.

Idem reddunt compotum de xxxix quarteriis de exitu mancorni. In semine xxxix acrarum, xiij quarteria dimidium. In missis apud Alresfordam, xij quarteria dimidium. In missis apud Waldam, $x j$ quarteria. In venditis, $i j$ quarteria.

Idem reddunt compotum de xj quarteriis dimidio de exitu ordei. Et de iiij quarteriis receptis de Alresforda. Summa, xv quarteria dimidium. In semine xj acrarum, iiij quarteria. In corredio iij servientium per $\mathrm{j}$ terminım, iij quarteria dimidiuı. In corredio ij servientium per xiij septimanas, iij quarteria. In corredio $\mathrm{j}$ herciatoris per $\mathrm{j}$ teruninum, $\mathrm{j}$ quarterium. In corredio $\mathrm{j}$ servientis per dimidium annum, iij quarterii.

Idem reddunt compotum de $\mathrm{e}[\mathrm{xlviij}]^{1}$ quarteriis dimidio de toto exitu avenæ. In semine cvij acrarum, liij quarteria dimidium. In missis apud Waldam, lij quarteria. In missis apud Mresfordam, ıij quarteria. In præbenda $\mathrm{j}$ carretarii, per xxx septimanas, v quarteria. In præbenda vj avrorum .. . ${ }^{1}$ quarteria. In venditis, xxix quarteria.

[Instaurum]. ${ }^{1}$ - Idem reddunt compotum de iij equis remanentibus anno præterito. Umnes remanent.

\section{TANTONA.}

M. 9d. WILlelmus IDE SOREWELLA reddit compotum de xxixl. xiijjs. vijd. de reragio anni præateriti. Solvit et quietus est.

Præterea solvit vj marcas de fine Prioris de Tantona. Et j marcam de fine Roberti de Lega. Et quietus est.

Idem reddit compotum de cxxixl. ob. de toto gabulo assiso in Uppelande. Et de xxs. vijd. de incremento gabuli hoc anno, cum quibusdam firmis, et cum turlingo terræ de Bruges. Et de xlvjs. vijd. de gabulo issiso de La Mulelande. Summa cxxxijl. vijs. ijd.ob.

Quietanciæ.-In quietaneiis apud Hol[weiam] vj carucariorum, per annum, vijs. vjd.; vj opera. riorum, vijs. vj l.; j bedelli, vjd.; $\mathrm{j}$ fabri, per annum, xvd.; xiij aliis operariis, ${ }^{2}$ iijs. iij $l$. A pud Corf. In quietanciis ij carucariorum, $\mathrm{j}$ bedelli, vij operariorum, per annum, $\mathbf{x} x$. Apnd Pond[esfordau]. In quietanciis ij carueariorum, $\mathrm{j}$ bedelli, per annum, iijs. ixd.; vj operariorum, vijs. vj $\boldsymbol{i}$. ; iiij aliis operariorun, $\mathrm{ij} s$. vjd. Apud Trindle. In quietanciis ij carucariorum, j bedelli, iijs. ixd.; vij operariorum, xxjd. Apud Hulle. In quietanciis ij carucariorum, $\mathrm{j}$ bedelli, per annum, iijs. ixd. ; viij operariorum, ijs. Apud Stapl|e]. In quietanciis ij carucariorum, j bedelli, per annum, iijs. ixd.; vıj operariorum, xijs. iijd. Apud 
Ful[fordam]. In quietanciis ij carncariorum, $\mathbf{j}$ bedelli, $v s$. vjd.; vj operariorum, xijs. Apud King[estonam]. In quietanciis $\mathrm{j}$ bedelli, ijs. vjd.; $\mathrm{j}$ carucarii, iijs.; vj operariorum, vjs. Apud Otrifordam. In quietanciis ij carucarioruu, j operarii, iijs. ixd. Apud Watel[egam]. In quietanciis operacionum ex antiquo, vjs. vijd.

Summa vjl. xjs. iijd.

Defectus.-Apud Hol[weiam]. In defectu terræ Swift tractæ in dominicum, xijd. In defectu terræ Roberti de Lega, viijs. vjd. Apud Stapl[e]. In defectu ij bordell[orum] pro paupertate, xviijd. Apud Nail[esburnam]. In deiectu ij bordell[orum] tractorum in dominium, ijs. Apud Ful[fordım]. In defectu curtillagii tracti in dominium, vjd. Apud King[estonam]. In defectı terræ et molindini Roberti Manselli, iiijs. vijd. ob., quod R[icardus], 10 Episcopus, reddidit ei ante mortem suam. Apud Otrifordam, pro dominico elargando, vjs. vijd. $o b$. In defectu terræ de Pikestona, datæ B. filio G[alfridi] in escambio de Corf, Nail[esburna] et Ful[forda] per R[icardum], Episcopum, xlvs. Apud Fidoc. In defectu terræ redditæ Jordano de Fidoc, iiijs. xjd. In defectu terræ de Bruges per W[illelmum] Briwerre, iijs. Summa lx.xvijs. viijd.

Summa tam quietanciarum quam defectuum, $x l$. ixs.

Summa remanens exxjl. xviijs. ijd. ob.

Burgus.-Idem reddit compotum de xljl. viijs. iijd. de firma burgi hoc anno.

Firma Molendinorum.- - Idem reddit compotum de $\mathrm{j}$ marca de firma molindini de Corf, quod Willelmus tenet. Et de cs. de firma molindin[orum] de Pipmerustre ${ }^{1}$ et Trindl[e] quod 20 Willelmus de Swafam tenet. Et de lxs. de firmal molindin[orum] de Ful[forda] et King[estona] quod Ada clericus tenet. Et de xxxs. de firma molindin[orum] de Otriforda et Watel[ega] cum terris adjacentibus, de ijs., quas Roiertus Samson tenet. Et de xijd. de terra adjacente molindino de Hulle, quam Edwardus de inolindino tenet. Summa xl. iiijs. iiijd.

Exitus Molindin[orum] sub Castello et Langefordæ.-Idem reddit eompotum de iiijl. xvs. iiijd. de xxx quarteriis frumenti venditis. Et de $x \times x j s$. viijd. de $x j$ quarteriis gruelli venditis. Et de xxixs. vijd. de Xv quarteriis, ij hopis silliginis et ordei venditis. Et de xlviijs. de $\mathrm{xvj}$ quarteriis hrasei venditis. Et de xlijs. viijd. de xxxvj quarteriis contrabrasei venditis. Summa xijl. vijs. iijd.

Exitus Molindini de Tabrigge et Vivarii.-Idem reddit compotum de lxijs. viijd. de $\mathrm{x}$ vij quarteriis frumenti venditis. Et de xlvijs. iiij $l$. de xiiij quarteriis gruelli venditis. Et de xxvijs. $x d$. de $x v$ quarteriis silliginis et ordei venditis. Et de xxiiijs. de viij quarteriis brasei venditis. Et de xviijg. ijd. de $\mathrm{xv}$ quarteriis dimidio contrabrasei venditis. Summa ixl.

Exitus Molindini de Hulle.-Idem reddit compotum de xxxvijs. de ix quarteriis dimidio, $\mathrm{j}$ hopa frumenti venditis. Et de $\mathrm{xxxjs.}$ viijd. de $\mathrm{x}$ quarteriis gruelli venditis. Et de viijs. $x d$. de iij quarteriis dimidio sigali et ordei venditis Et de ijs. vjd. de $\mathrm{j}$ quarterio dimidio brasei venditis. Summa iiijl. Summa molindinorum cum firma, $x \times x v l . x j s . v i j d$.

[Exitus Manerii.]-Idem reddit compotum de vijl. ixs. jd. de pasnagio Berth[onæ] hujus anni. 40 Et de xlijs. ijd. de toto hundred peni ${ }^{2}$ Berth[onæ] hujus anni. Et de xliijs. vijd. de herbagio Berth[onæ] hujus anni. Et de xxxiiijs. vjd. de ij equabus, $x$ pullis, vivis venditis. Et de xlvs. viijd. de coriis xxiiij boum, $\mathrm{j}$ tauri, et sepo eorundem venditis, et carne $^{3}$ misso in lardarium. Et de xxviijs. viijd. de coriis $\mathrm{j}$ equæ, vj boum, $\mathrm{j}$ tauri, ij vaccarum, iiij genicularum, $\mathrm{j}$ bovetti, vannlilium mortuorum, venditis. Et de xijs. ixd. de pellibus iiijix.xviij 4 ovium, ij castr[atorum], $\mathrm{j}$ multonis, $\mathrm{xlij}$ hoggettorum, $\mathrm{xx}$ agnorum, mortuorum, venditis. Et de ijs. xjd. de residuo denariorum Sancti Petri hoc anno apud Corf, Nail[esburnam], Ful[fordam]. Et de ijs. ixd. ob. de denariis nuccandi ct nucibus venditis. Et de vs. de culıno et arundine venditis. Et de xiijs. viijd. iijgdr. de ciiijjx viij caseis venditis. Et de vjs. $\mathbf{x} d$. de iiij ${ }^{\mathrm{xx}} . \mathrm{xviij}$ velleribus grossis, $\mathrm{xiij}$ velleribus agninis, venditis. Et de vjl. $\mathrm{xv} v . \mathbf{x} d .50$ de scotallis domini Episcopi factis hoc anno. Summa xxvjl. iijs. vjd. jqdr.

Idemu reddunt compotum de $\mathrm{xxxjl.} \mathrm{iiijs.} \mathrm{x} d$. de ciiij ${ }^{\mathrm{x} x} \cdot \mathrm{x}$ quarteriis trumenti venditis Et de ixl. ixs. viijd. jqdr. de exxij quirteriis, j hopa, curailli venditis. Et de xxvl. iijs de Dv quarteriis avenæ venditis. Summa lxvl. xvijs. vjd. jqdr.

De Purchasiis.-HoL[weia]. Idem reddunt compotun de iiijs. de Rogero Cochel pro filia sua 55 danda. Et de ve. de Ada Barda pro terra quæ fuit Roberti Broche. Et de xijd. de Willelmo filio Reginaldi pro terra quæ fuit Gileberti Ordrich. Et de vs. de Chilwino pro mellea. Et de iiijs. de Godefrido de Stoclı[es] pro filia sua danảa. Et de xijd. de Waltero Chet pro simili. Et de ijs. de Rogero Durc pro fine terræ. Et de ijs. de Ordrich de Ristona de legato. Et de ijs. de Rogero Ang[erin] pro tilia sua danda. Et de xvs. de Benedicto de 60 Mora pro præfectura dimittenda. Et de jm. de Durando de Pola pro simili. Et de xs. de uxore Thomæ de Galinant pro terra douini sui retinenda. Et de äimidia marca de Rogero 
Fleca pro terrn patris sui dimidianda. Et de $x 8$. de Ricardo Cole pro j acra prati habenda. Et de xijd. de vidua Roberti Longi pro fine terræ. Et de xvs. de Mauricio de Stoch[es]. pro fulso dicto. Et de dimidia marca de Willelmo Vis pro manutenemento. Et de iijs. de Giliberto de Soredich pro j acra prati habenda. Et de xijd. de Radulfo de Pola pro prato ${ }^{1}$ habendo. Et de ijs. de Cristina vidua Edrici pro fine terræ. Lit de ijs. de Thoma Luch' pro visu terræ habendo. Et de dimidia marca de Radulfo carettario pro falso dicto. Et de xijd. de Algar de Sernege pro moltura. Et de iiijs. de Emma vidua de Halnake pro filia sua danda. Et de $\mathbf{x}$. de Roberto filio Edivæ pro terra patris sui habenda. Et de xijd. de vidua Algar pro terra habenda. Et de iiijs. de Roberto de Lega clerico pro namiis contra legem detentis. Et de xs. de Rogero filio Gileberti pro vidua et terra quæ fuit Edrici. Et de dimidia marca de Johanne de Vexgrave pro vidua et terra Godwini habenda. Et de dimidia marca de Rogero Poda pro mellea. Et de xijd. de Mauricio de Stokes pro bosco. Et de xviijd. de Edwardo de Haidona pro simili. Et de vjd. de Eustachio Ridere pro assisa cervisiæ. Et de vjd. de Radulfo Trai pro simili. Et de vjd. de Galfrido Bux pro simili. Et de vjd. de Ada Beringer pro simili. Et de ijs. de Roberto Orảrich pro terra patris sui habenda. Et de ijs. de Kicardo filio Heliæ Palmer pro terra quæ fuit Ordrici. Et de ijs. de Thoma Luche pro falso clamore. Et de iijjs. de Albrea vidua de Sireforda pro filia sua danda. Et de ijm. de Randulfo de Tabrigge pro advenis illicito hospitatis. Summa $x l$. ijs. $x d$.

20 Conf. Idem redidit compoturn de iijs. de Waltero de Corf pro filia sua danda. Et de jm. de Ada filio Willelmi pro terra quæ fuit Edivæ viduæ. Et de xijd. de Willelmo de Swafham pro j ferlinga terræ de assarto. Et de iijs. de Wlvrico de King[estona] pro terra et filia Simonis de Corf. Et de xijd. de Herman de Corf pro iij acris de assarto. Et de iijs. de Yleberto pro filia sua datia sine licencia. Et de ijs. de Willelno de Chilcapad[er]e pro falso dicto. Et de xviijd. de Ada tilio Levivæ pro terra et filia Gunnildæ. Et de ijs. de Waltero filio Levivæ ut non capiat[ur] terra hoc anno. Summa $x \times x s . x d$.

PundL[esforda]. Idem reddit compotum de $\mathrm{j}$ marca de Stephano de Sevenhac pro filia sua danda. Et de ijs. de Almar de Dudelestona pro simili. Et de ijs. de Ada Fatte et Gautero Budde pro confirmacione terræ R[oberti] le Lega. Et de vs. de Geraldo de Porta pro warranto non habito. Et de xxs. de Oswardo de Lega pro concelamento. Et de iijs. de Jordano de Cumba pro assarto. Et de ijs. de Rogero Cherda pro sorore sua danda. Et de $x$ viijd. de Roberto Smalbroch pro terra habenda. Et de $x x s$. de hundredo de Pundl[esforda] pro concelamento. Et de iijs. de Ada Watte pro escambio terræ Oswardi de Lega. Et de xijd. de Fugello pro curtillagio habendo. Et de vs. de Rogero de Foleforda pro filia sua danda. Et de vs. de Henrico de Dudelestona pro terra babenda. Et de vs. de Rogero Chelm'2 pro homine non habito ad hundredum. Et de dimidia marca de Ada de Chicapadere pro namiis injuste detentis, Et de xijd. de Willelmo Fabro pro terra quam J. de Voxgr[ave] tenuit. Et de vs. de Hegin' de Ful[forda] pro bove empto contra defensam. Summa cs. vjd.

Trindel[E]. Idem reddit compotum de ijs. de Philippo de Cochage pro mellea. Et de ijs. de thedinga Lifig de Trindl[e] pro concelamento. Et de xijs. de Roberto de Depeforda pro sursisa. Et de dimidia marca de Herevi pro vidua Algar habenda. Summa xxijs. viijd.

Houl. Idem reddit compotum de vs. de Lifeg carettario pro falso dicto. Et de vjd. de Ada Bissop ut quietus sit de dominico Episcopi usque festum Sancti Michaelis. Et de iijs. de Rogero de Veneis ut quietus sit de cervisia Episcopi. Summa viijs. vjd.

Stapl[E]. Idem reddit compotum de ijs. de vidua Ricardi Gore pro tine terræ. Et de Petru filio Palmerii pro terra habenda. Et de ijs. de thedinga Hugonis de Stapl[e] pro concelamento. Et de xijd. de Radulfo de Stana et Ada pro assisa fracta. Et de xxs. de Hugone Longo pro mala custodia bladi. Et de iiijs. de Ricardo de Apsle pro prato quod habuit habendo. Et de iiijs. de Willelmo de Witemor pro mellea. Et de xijd. de Ada de Doddehulle pro sursisa. Summa xxvs.

Natl[esburna]. Idem reddit compotum de ijs. de Ricardo Bule pro mellea. Et de iijs. de Ailwardo Peche pro arura. Et de ijs. de Sweta pro mellea. Et de $x s$. de berth[ona] de Nail[esburna] pro blado domini male triturato. Summa xvijs.

FuL[ForDA]. Idem reddit compotum de $\mathrm{j}$ marca de Matilli de Torra pro manutenemento. Et de vs. de Sewoldo de Torra pro fine terræ. Summa xviijs. iiijd.

KIng[Estona]. Idem reddit compotum de vs. de Reginaldo de molindino pro sursisa. Et de ijs. de Geroldo Capellano pro manutenemento habendo. Et de ijs. de Reginaldo de molindino pro concelamento. Et de vjs. de filio Juliani de Marisco pro mellea. Et de $\checkmark s$. de Ada Clerico pro firma molindini retinenda per $v$ annos. Summa xxs.

$$
{ }^{1} \text { prata in MS. }{ }^{2} \mathrm{Sic} \text { in MS. }
$$


OrRIForde. Idem redilit compotum de iijs. de Johanne Casse pro filia sua danda. Et de vjs. de Willelmo de Watel[ega] pro filia sua danda. Et de iijs. de Warino de Huppehulle pro simili. Et de xijd. de Ricardo Bresa pro stultiloquio. Et de ijs. de Alvredo . . . .1 pro mellea. Et de xijd. de Ada filio Hugonis, pro simili. Et de xviijd. de Ada Casse pro concelamento. Summa xviijs. vjd.

Mulelande. Idem reddit compotum de iijs. de Roberto de Cruce pro pastura. Summa iijs.

Chippel[EGa]. Iden reddit compotum de ijs. de Thoma de Chippel[ega] pro concelamento. Summa ijs.

Hundredum. Idem reddit compotum de ijs. de Germano de Nortona pro concelamento.

Forinsecum. Et de xijd. de thedinga de Lidiardo Sancti Laurencii pro simili. Et de 10 dimidia marca de Nortona et Bradeforda, pro plevina. Et de jjs. de thedinga de Talanda, pro concelamento. Et de ijs. de thedinga de Bageterge pro simili. Et de . . . de Nortona pro respecto² habendo. Et de ijs. de Osberto de Hola pro lege relaxita. Et de ijs. de thedinga de Lidiardo Sancti Laurencii, pro concelamento. Et de iijs. de thedinga de Langeforda pro concelamento. Et de xijd. de thedinga de Ijidiardo Puch[erdona] 15 pro simili. Et de vjd. de Ricardo de Willa pro sursisa, quia non venic ad fiftedai cum secta hundredi. Et de xijd. de Albreda vidua de Chipel[ega] pro lege relaxata. Et de xs. de Radulfo præposito de Nortona pro waranto non habito ad diem. Et de xs. de Rogero præposito de Bradeforda pro simili. Et de xijd. de thedinga de Nidgehidin Thomæ de Flur[i] pro concelamento. Et de ijs. de thedinga de Bradeforda pro simili. 20 Et de ijs. de thedingin de Nortona pro simili. Et de xijd. de thedinga de Hache pro simili. Et de xijd. de thedinga de Hed[felda] pro simili. Et de xijd. de thedinga de Lidiardo Punch[erdona] pro simili. Et de xijd. de thedinga de Ordena ${ }^{3}$ pro simili. 'Et de vjd. de thedinga de Occumba pro simili. Et de xijd. de thedinga de Cudeluest[ona] pro simili. Et de xijd. de thedinga de Nigehida Monachorum pro simili. Et de xijd. de thedinga de 25 Lidiardo Sancti Laurencii pro simili. Et de j marca de Martino de Wid[i]ela pro sursisa. Et de ijs. de Thoma de Lidiardo Sincti Laurencii pro defalcando filium Ricardi de Willa. Et de ijs. de Martino de Wid[i]ela pro manutenemento domini habendo. Summa iiijl. iijs. Summa totalis xxviijl. ijs. ijd.

Idem reddit compotum de viijl. $x s$. de scutagio.

Summa tocius receptæ, cccxxvijl. xjs. iijd.

[Expensa]. - ArUi) Berth[ONAM] [DE H]OL[weIA]. ${ }^{3}$ In ferramento avrorum, iijd. In precariis hyemalibus et quadragesimalibus de $\mathrm{iijj}^{\mathrm{xx}}$.xviij carucariis dimidio, viijs. ijd. $a b$. In cibo fabri, bedelli, iiij carucariorum, ij bovariorım, xvij operariorum, de consuetudine Natalis et Paschæ, ijs. vijd. In consuetudine prati falcandi, xviijd. In prato falcando quod 35 Robertus consueverat falcare, $\mathrm{ijs}$. In consuetudine bedripæ, ijs. vijjd. In carucis, parandis karris axandis et reparandis, cum unctura, [et] ${ }^{1}$ saccis emptis, ijs. vjd. ob. In $\mathrm{ij}$ paribus rotarum ad karros, vjs. ijd. In custo daeriæ, $\mathrm{xxv} d$. ob. In ferura equi caretarii et caretæ, et ferramentis $\mathrm{x}$ caretarum axandis cum [unctura]' ad idem, iijs. ixd. $a b$. In solido $\mathrm{j}$ earetarii, per annum, iijs. iijd. In solidis $\mathrm{ij}$ bovariorum, per annum, vs. In solidis 40 j vaccarii, j daiæ, per iij terminos, iiijs. vjd. [In] $]^{1}$. . . ad Berth[onam] pro defectu feni, iijd. ob. In lvij sabbatis, vijs. iijab. Summa lijs. jd.

M. 10. APUd CoRF. In ferramentis avrorum, iijd. In mercede fabri, $\mathrm{xx} d$. In precariis hyemalibus et quadragesimalibus de $\mathrm{x}$ carucariis dimidio, $\mathrm{x}$, . ob. In cibo bedelli, ij carucariorum, de consuetudine Natalis et Paschæ, vjd. In consuetudine prati falcandi, vjd. In com- 45 panagio bedripa, vd. In carrucis parandis, karris axandis, cum unctura, xiiijd. In solidis ij bovariorum, vs. In $\mathrm{x} v \mathrm{ij}$ sabbatis, $\mathrm{ij} s$. iij ab. Suınma xijs. vjd.

Apud PundL[Esfordam]. In ferrura ij avrorum, vijd. ob. In inercede fabri per annum, ijs. In precariis hyemalibus et quadragesimalibus de lx.xviij carucariis dimidio, vjs. vjd. ob. In consuetudine prati faleandi, $x d$. In companagio bedripe, ix $d$. In cibo bedelli, ij caru- 50 cariorum, $\mathrm{x}$ operariorum de consuetudine Natalis et Pasehæ, $\mathrm{x} \mathbf{v} d$. In carucis parandis, karris axandis et reparandis, cum unetura, et $\mathrm{j}$ mensura empta, $x$ vijd. In solidis ij hovariorum per annum, vs. In solidis j porcarii, per annum, ijs. vjd. In xxxvj sabbatis, iiijs. vjd. Summa xxvs. vd.

APUd TRINDL[E]. In ferrura avrorum, vjd. In mercede fabri, ijs. In precariis hyemalibus et 55 quadragesimalibus de $\mathrm{xxix}$ carucariis, $\mathrm{jj} s . \mathrm{v} d$. In consuetudine prati falcandi, vjd. In companagio bedripæe, vjd. In cibo bedelli, ij carucariorum, de consuetudine Natalis et Pascha, vjd. In cibo vij operariorum, ij ancillarum, de eadem consuetudine, viijd In

\footnotetext{
1. The margin of the membrane is mutilated. ${ }^{2}$ Sic in MS. ${ }^{3}$ Sic for Cedena (i.e. Cheddon).
}

4 The words Idem reddit compotum have been partially erased at the boginning of the line. 
carucis parandis, carris axandis, et reparandis, cum unctura et jugis emptis, xvjd. ob. In $\mathrm{j}$ pare rotaruin ad karrum, iijs. In foragine delata de Punul[esforda] ad Berth[onam], iijd. In solidis ij bovariorum, per anuum, vs. In xxxvj sabbatis, iiijs. vjd. Summa xxjs. ijd. ob.

ApUD HoLle. In ferrura avrorum, iijd. In mercede fabri per annum, ijs. In precariis hyemalibus et quadragesimalibus de $\mathrm{xvj}$ carrucariis, $\mathrm{xvj} d$. In consuetudine pratorum de Hulle et Medbroc falcandorum, xviijd. In companagio bedripæ, vjd. In cibo bedelli, ij carucariorum, de consuetudine Natalis et Paschæ, vjd. In cibo viij operariorum de eadem consuetudine, viijd. In carucis parandis, karris axandis, cum unctura, ixd. In $\mathrm{j}$ pare rotarum ad karrum, $\mathrm{xl}$. In solidis $\mathrm{ij}$ bovariorum, per annum, v8. In sabbatis, ijs. iijd. Summa xvijs. xjd. ob.

Aprd Stapl[E]. In ferramentis avrorum, iijd. ob. In mercede fabri, ijs. In precariis hyemalibus et quadragesinalibus de xlix carucariis, iijs. $\mathrm{j} d$. In consuetudine prati de Mulelanda falcandi, viijd. In companagio bedripæ, ixd. In besca ad waterletam de consuetudine, ijd. In cibo bedelli, ij carucariorum, de consuetudine Natalis et Paschre, rjd. In cibo $\mathrm{xx}$ operariorum de eadem consuetudine, $\mathrm{xxd}$. In carucis et jugis parandis, karris axandis et reparandis, cum unctura, saccis, semellis, emptis, $x x i j d . o b$. In $\mathrm{j}$ pare rotarum ad karrum, iijs. vjd. In solidis ij bovariorum, per annum, vs. In xxxvj sabbatis, iiijs. vjd. Summa xxiiijs. xjd.

Apud Nail[esburnam]. In ferrura avrorum, $j[d$.$] . In mercede fabri, xijd. In precariis$ hyemalibus et quadragesimalibus de xj carucariis, xjd. In consuetudine prati falcandi, iiijd. In consuetudine bedripæ, vjd. In consuetudine cibi j carucarii, Natalis et Paschæ, ijd. In cibo bedelli, iij operariorum, de eadem consuetudine, iijd. In carucis parandis, karris reparandis, cum unctura, herciis, capistris, semellis, emptis, viijd. In pare rotarum ad karrum, iijs. vjd. In solidis $\mathrm{j}$ bovarii, per annum, ijs. vjd. In xiiij sabbatis, xxjd. Summa xjs. ixd.

APUD FULFORDAM. In ferrura avrorum, ijd. In precariis hyemalibus et quadragesimalibus de vij carucariis dimidio, vijd. $o b$. In consuetudine prati falcandi, iiijd. In consuetudine waterletæ, iijd. In consuetudine bedripæ. vijd. In cibo fabri, $\mathrm{ij}$ carucariorum, de consuetudine Natalis et Paschro, vjd. In cibo bedelli, v operariorum, de eadem consuetudine, vjd. In carucis jugis parandis, karris axandis et reparandis, cum unctura, xiijd. In $\mathrm{j}$ pare rotarum ad karrum, iijs. vjd. In solidis ij bovariorum, per annum, vs. In xix sabbatis, iijs. iij ob. Summa xvs. ixd.

Apud Kinaestonam. In ferrura et mercede fabri, ixd. In precariis liyemalibus et quadragesimalibus de xxiiij carucariis, $\mathrm{ijs}$. In companagio bedripæe, vijd. In cibo beclelli, $\mathrm{j}$ carucarii, de consuetudine Natalis et Paschæ, iiijd. In cibo $v$ operariorum de eadem consuetudine, $v d$. In karrucis, jugis, parandis, karris axandis et reparandis, cum unctura, vjd. In solidis $\mathrm{j}$ bovarii, $\mathrm{ij}$ s. vjd. In solidis $\mathrm{j}$ bercarii per dimidium annum, $\mathrm{xijd}$. In wiscatura empta ad faldam wiscandam, iiijd. In xj sabbatis, xvjd. ob. Summa $\mathrm{x} s . \mathbf{v} d . o b$.

Apun Otrifordam. In ferramentis ij carucarum, xviijd. In ferrura avrorum, iijd. In mercede fabri, per annum, ijs. viijd. In precariis hyemalibus et quadragesimalibus de xiiij carucariis dimidio, xiijjd. $o b$. In vj acris araudis ad tramesium, ijs. vjd. In consuetudine prati falcandi, iijd. In consuetudine bedripæ, xiijd. In cilo ij carucariorum, de consuetudine Natalis et Paschae, iijd. In cibo $\mathrm{j}$ operarii, de eadem consuetudine, jd. In carucis parandis, rotis jugis emptis ad iclem, karris axandis et reparandis, cuin unctura, semine avenæ triturando, ponte ante portem reparando, sella et mensura emptis, iijs. jd. In falda facienda, iijd. In blado metendo et messoribus ibidem conductis, $j \mathrm{~m}$. In solidis ij bovariorum et custodis curiæ, per annum, vijs. vjd. In solidis custodis ociorum animalium, per dimidium annum, xviijd. In xiiij sabbatis, xxjd. Summa xxxviijs. vd. $o b$.

Summa tocius expensæ herthonarum, xjl. ixs. vjd.

Expensa Molindini de Burgo.-In mercede fabri, vs. jd. In ferramentis ad idem, iijs. In clavis, bordis, vijs. iiijd. In unctura, vs. In faciendis ij rotis de novo, iijs. In iij kidellis et filo ad idem et aliis reparandis, burochio vivarii faciendo, seris emptis, ve. ijd. ob. In iiij molis emptis, cum attructu, xlvijs. ijd. Summa lx.xvjs. ixd. ob.

Expensa Molindini de Hulle.-In mercede fabri, xvjd. ob. In ferro empto, iijd. ob. In bordis, clavis, xvjd. In unctura, vijd. In $\mathrm{j}$ rota de novo ficta et arclia reparanda, $x \times d$. In ij molis, cum attractu, xiijs. $x d$. Summa xixs. jd.

$\because$ Summa expense molindorumi, tam de Burgo quam de Uppęlandi, iijj. $x v s . x d . o b$. 
In pastura de Crich acquietanda, ijs. vjd. In xlix quarteriis frumenti emptis ad semen, $\mathrm{x} l$. In ij quarteriis $\mathrm{j}$ hopa ordei emptis ad semen, iiijs. $\mathrm{xj} d$. ob. In ij quarteriis pisarum emptis ad semen, xld. In xxxij bobus emptis ad carucas relevandas, xjl. xixs. vjd. In iij porcis emptis, xvjs. In lxvj soccis emptis ad carucas, xxs. In xij frustis aceri emptis ad idem, xs. In exxix esperductis dimidio emptis ad idem, xxxijs. xjd. In 5 semine curtillagii et eodem faciendo, xxjd. ob. In gurlis et pergamine, xiijd. In coriis ij equarum dealbandis ad hernesium caretæ, viijd. In lardario faciendo de xxiiij bobus, i tauro, ijs. In grangia et aliis domibus castelli cooperiendis et reparandis, sclatis emptis et clavis ad idem; portis similiter reparandis, vertevellis, clavis, ad idem; cordis ad lovarium, xviijs. iiijd. In coquina elarganda et cooperienda et reparanda, muro faciendo, 10 clavis, bordis, sclatis emptis ad idem, gutteriis aulæ reficiendis et fundendis, plumbo empto ad idem, $x x j s$. ijd. In mercede et cibo braciator[um], iij scotallorum domini Episcopi, iiijs. ijd. Summa xxviijl. xviijs. vd.

In expensis constabularii et familiæ domus, qui fuerunt ad mensam a xxviij die Septembris usque in $\mathrm{xx}$ diem Decembris, utroque die computato, scilicet per xij ebrlo- 15 madas, cxiijjs. $x d . a b$. In liberatione constabularii, a xxj die Decembris usque in xxviij diem Septembris utroque die computato, scilicet per cciiij ${ }^{x x} \mathrm{ij}$ dies, xiijil. ijs. In liberatione W[illelmi] coqui, W. de Ferham N. de Hattingel[ega] W[illelmi] de Swofham, per eundem terminum, ix $l$. viijs. In liberatione $\mathrm{N}$. janitoris, per eundem terminum, xxiijs. vjd. In liberatione $R$. janitoris, a xv die Novembris usque in xxviij diem Septembris, 20 utroque die computato, scilicet, per cccxviij dies, xxvjs. vjd. In liberatione Waite, a xxj die Decembris usque in $\mathrm{x}$ diem, utroque die computato, scilicet, per $\mathrm{xx}$ ebdomadas, $\mathrm{x} s$. In solidis Waite per dimidium annum, ijs. vjd. In liberatione Hugonis Bissop pro trituratione bladi apud Pundl[esfordam] per xxviij dies, ijs. iiijd. In forura W[illelmi] de Ferham, N. de Hattingelega, W[illelmi] de Swofham, xijs. In solidis ij janitorum, per 25 annum, $x x s$. Summa $x \times x i i j l . x \times d . o b$.

In expensis constabularii et W[illelıi] Coqui et Thomæ de Fluri, apud Bristolliam, contra Natalem, dimidia marca. In $\mathrm{nu}[\mathrm{n}]$ ciis missis ad dominum Episcopum pro defensa boscorum, et in aliis missis a domino ne avena sua venderetur, ix $d$. $o b$. In $\mathrm{j}$ batillo empto ad vivarium, $x s$. Summa xvijs. $v d$. $a b$.

In expensis familiæe domini Episcopi contra adventum domini Regis et domini Episcopi per iij dies, xviijs. vjd. $o b$. In expensis domini R[oberti] de Turnehain, quando venit de Pictavia, et equis ad opus suum locandis, xiijs. ijd. In expensis R[icardi] de Marisco et W[illelmi] de S[ancto] Maxentio, per ij dies, iiijs. iiijd. In hospitio vicecomitis Sumersetæ, per $\mathrm{j}$ noctem, xviijs. In liberatis eidem pro consuetudine hundredi, $\mathrm{xl}$. In c hastis 35 allatis de Wallia, et in nunciis missis ibidem bis pro eodem, ijs. vjd. In venacione domini Regis salanda et siccanda, missa de Bruges apud Tantonam, xijd. In ij tonellis vini emptis apud Exoniam, et in eisdem barrandis, stringendis, et cariandis usque Tantonam, iiijl. iijjs. In furfure ad arma, xijd. Summa ixl. ijs. vjd. ob.

In novo muro perficiendo. In cementariis, per $x$ ebdomadas, lx.xvjs. vjd. In operariis 40 facientibus cementum et in auxiliis cementariorum, per $\mathrm{x}$ ebdomadas, ad idem, lxijs. iijd. In ciiijxij mensuris minutæ petræ attrahandæ ad idem, lxs. viijd. In $\mathrm{j}$ rogo facto hoc anno super Kantoch, et in calce removenda, ct sursum ponendis de $\mathrm{ij}$ foveis, lijs. ijd. $o b$. In eciiij ${ }^{x \mathrm{x}} \mathrm{j}$ quarteriis calcis attrahendis, xxxijs. iij ob. In $\mathrm{j}$ careta ferro ligata cum harmesio, et iij paribus rotarum ad caretas, ferro ligatis, emptis apud Bristolliam, propter 45 operationem, xxxviijs. viijd. In ferrura ij caretarum, et in equis ferrandis, $x \times v d$. $a b$. In sellis, paronis, paronellis, clutis, bacis, carettis axandis et uncto ad idem, et iij pelis ferro ligandis, iijs. ixd. In solidis j caretarii, per annum, et alterius per xij ebdomadas propter operationem, iijs. ixd. In $\mathrm{j}$ equo empto ad caretam, propter operationem, xijs. In vij acris assartandis, lx.xjs. ijd. ob. In ponte apud Hulle de novo faciendo, iijs. iijd. $a b .50$ Summa $x x l$. xizs. vd. $a b$.

In mairemio sternendo, scapliando, ad novum pontem, et in planclis secandis, ferro, uncto, emptis, ligaminibus ferri arl idem faciendis, xxxiijs. vjd. $o b$. In carpentariis ad novum pontem faciendum in tasca, $1 \mathrm{x}$. In $\mathrm{ij}$ secatoribus per $\mathrm{v}$ ebdomadas $\mathrm{j}$ diem dimidium, qui secaverunt plancas ad pontem, xiijjs. $x d$. ob. In novo muro, et pressorio 55 cooperiendis, iiijs. xjd. ob. In gaholla reparanda nova porta, hostiis stabulæ perficiendis, columbaria reparanda, clavis ad idem, xs.ixd. ob. In vertevellis, elavis, ij seris ad novam portam faciendis, carbonibus ad idem; vertevellis, seris, ad gahollam; aliis portis castelli reparandis, vertevellis ad idem, clavis, xxxvs. In xxiiij ferris ad lanceas, viij ligonibus, ij hoiis, planis ad novum fossatum faciendis, et aliis reparandis; $x v$ hoiis et ligonibus ad 60 terram assartandam faciendis et illis reparandis, xvijs. vjd. In novo fossato facto ante novum murum, et serv[at]orium, et fossato inter molam et molindinum reparandis; in minatoribus per xvij ebdomadas, $\mathrm{xvjl}$. $\mathrm{iij}$ s. $\mathrm{xj} d . \mathrm{ob}$. In operariis portantibus terram sursum de fossato cum hoccis; et operabantur ibidem cum minatoribus per xvij cbdomadas, xxijl. iiijs. iijob. Summa xlvijl. vs. ixd.

Summa tocius expensæ, clvijl. $x s$. viijd. ob. Summa tocius liberationis et expensæ, cclvijl. xs. viijd. ob. Et debent lxxl. vjd. ob. Præerea de Simone de Fluri xiiij $m$. 
Exitus Grangiø.-Idem reddit compotum de ccciifjxxij quarteriis $j$ hopa de toto exitu frumenti. Et de xlix quarteriis de emptis. Summa ccecxlj quarteria, $\mathrm{j}$ hopa. Apud Hol[weiam]. In semine cexiij acrarum, liij quarteria ij hopæ. Apud Corf. In semine xlj acrarum, x quarteria ij hopæ. Apud Pund[lesfordam]: In semine cxxvij acraram, xxv quarteria iij hopæ. Et de semine villanorum xxvj acre dimidia. Apud Trindl[e]. In semine lxvj acrarum, xv quarteria dimidium. Apud Hulle. In semine lxx acrarum, xvij quarteria dimidium. Apud Stapl[e]. In semine cxxvj acrarum, xxx quarteria dimidium ij hopæ. Apud Nail[esburnam]. In semine xxx acrarum, vij quarteria dimidium. Apud Ful[fordam]. In semine xxx acrarum, vij quarteria dimidiun. Apud Otrifordam. In semine liiij acrarum, xiiij quarteria dimidium. Suınma acrarum Dcclvij acræ. Et præterea de semine villanorum xxvj acre dimidia. Summa seminis ciiij ${ }^{x} \mathrm{ij}$ quarteria j hopa.

Apud Hol[weiam]. In consuetudine bedripæ et feni congregandi, iij quarteria ij hopæ. Apud Pund[lesfordam], iij hopæ. Apud Trindl[e], ix hopæ. Apud Hulle, ix hopæ. Apud Stapl[e], $x$ hopæ. In braseo ad scotalla domini Episcopi, xxj quarteria. Summa xxviij quarteria $\mathrm{j}$ hopa.

Apud Hol[weiam]. In cibo carettarii, per annum, iij quarteria dimidium. In cibo ij bovariorum, vij quarteria. In cibo vaccarii ibidem, per annum, iij quarteria dimidium. In cibo dominæ a xvj die Februarii usque xxviij die Septembris, scilicet, per xxxij ebdomadas, ij quarteria. Apud Pundl[esfordam]. In cibo ij bovariorum, per annum, vij quarteria. In cibo $\mathrm{j}$ porcarii, iij quarteria dimidium. Apud Otrifordam. In cibo ij bovariorum et custodis curiæ, ix quarteria. In cibo custodis ociorum animalium per xviij ebdomadas, ix hopæ. In ciluo $\mathrm{j}$ carretarii, per annum, pro operatione, et alterius pro eodem, a vij die Julii usque in festum Sancti Michaelis, scilicet pro xij ebdomadis, iij quarteria $\mathrm{ij}$ hopæ. In venditis ciiij ${ }^{x x} \times \mathrm{x}$ quarteriis. De precio superius respondet. Summa cibi sine venditis, xl quarteria dimidium, iij hopæ. Et remanent ij tassi frumenti.

Idem reddit compotum de cxxiiij quarteriis dimidio, iij hopis, de toto exitu curailli. In braciatura ad scotalla domini Episcopi, ij quarteria dimidium, ij hopæ. In venditis cxxij quarteria $\mathrm{j}$ hopæ. De precio superius respondet.

M. 10d. Idem reddit compotum de iij ${ }^{x x}$ quarteriis dimidio $\mathrm{j}$ hopa de toto exitu sigali. Apud Hol[weiam]. In semine xix acrarum, v quarteria. Apud Hulle. In semine xviij acrarum, iiij quarteria dimidium. Apud Stapl[e]. In semine xij acrarum dimidix, iij quarteria j hopa. Apud Nail[esburnan]. In semine $x$ acrarum dimidiae, ij quarteria v hopæ. Apud Ful[fordam]. In semine $x \times x v$ acrarum, viij quarteria vj hopæ. Apud King[estonam]. In semine xxv acrarum, vj quarteria ij hopæ. Apud Otrifordam. In semine ij acrarum, vj hopæ. Summa acrarum, cxxij. Summa seminis, xxxj quarteria.

Apud Nailesburnam. De consuetudine bedripæ, j quarterium. Apud Fulfordam, ix hopæ. Apud Kingestonam, ix hopæ. Summa iij quarteria ij hopæ. Apud Corf. In cibo ij bovariorum, per annum, vij quarteria. Apud Trindl[e]. In cibo ij bovariorum, vij quarteria. Apud Hulle. In cibo ij bovariorum, vij quarteria. Apud Stapl[e]. In cibo ij bovariorum, vij quarteria. Apud Nailesburnam. In cibo $\mathrm{j}$ bovarii, iij quarteria dinidium. Apud Fulfordam. In cibo ij bovariorum, vij quarteria. Apud Kingestonam. In cibo $\mathrm{j}$ bovarii, iij quarteria dimidium. In cibo $\mathrm{j}$ bercharii per $\mathrm{xxx}$ ebdomadas, $\mathrm{xv}$ hopæ. Apud Otrifordam. In cibo $\mathrm{j}$ bovarii, $\mathrm{j}$ quarterium dimidium. In cibo dais per $\mathrm{x}$ ebdomadas, $v$ hopæ. In cibo custodis ociorum animalium per iiij ebdomadas, ij lıpæ. In consuetudine Waterletæo de Fulforda, j hopa. Summa xlvj quarteria iij hopre.

Idem reddit compotum de $\mathrm{ij}$ quarteriis $\mathrm{j}$ hopa ordei de super-emptis. Apud Hol[weiam]. In semine iiij acrarum dimidiæ, $x j$ hopæ. Apud Pundlesfordam. In semine ij acrarum dimidiæ, vj hopæ. Summa acrarum, vij. Summa seminis, ij quarteria j hopa.

Idem reddit compotum de Mcciiij ${ }^{x x} \times$ vij quarteriis $\mathrm{iij}$ hopis de toto exitu avenæ. Apud Holweiam. In semine cev acrarum, cxiiij quarteria. Apud Corf: In semine xliiij acrarum, xxviij quarteria. Apud Pundesfordam. In semine cxxiiij acrarum, lx.x quarteria. De semine villanorum, xxvj acræ dimidia. Apud Trindl[e]. In semine

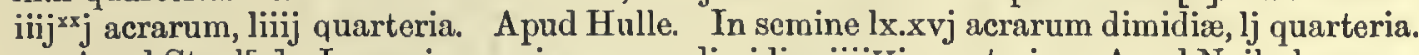

Apud Stapl[e]. In semine cxxj acrarum dimidiæ, iiij ${ }^{x j}$ quarteria. Apud Nailesburnam. In semine lx.xviij acrarum, lij quarteria. A pud Fulfordam. In semine lx.xj acrarun dimidiæ, xlviij quarteria. Apud Kingestonam. In semine lviij acrarum, xxxv quartaria. Apud Otrifordam. In semine $\mathrm{cv}$ acrarum, lx.xvj quarteria. Sumina acrarum, Decc.lx.iij acræ dimidia. Et præterea de semine villanorun xxvj acræ dimidia. Summa seminis, Deix quarteria. Notandum quod dederunt pro ventilatione avenæ, ixs, qui non computantur eis.

A pud Corf. In consuetudine bedripa, iij quarteria. Apud Pundlesfordan, iiij quarteria. Apud Otrifordam. iiij quarteria. Apud Hulle. In consuetudine Waterletæ, ij quarteria. Apud Stapl[e]. ij quarteria. Summa xv quarteria.

In præbenda equorum constabularii, Willelmi Coqui $N$. de Hattingelega, per annum,

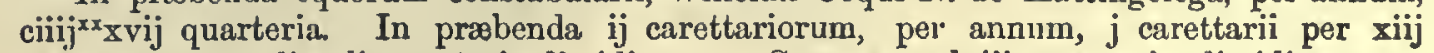
ebdomadas et $v$ dies, $l j$ quarteria dimidium. Summa cexlviij quarteria dimidium. 
In prebenda xxxix equorum domini Roberti de Tornehan qui verit de Pictavia per j noctem, iij quarteria dimidium iij hopæ. In præbenda xx equorum Vicecomitis Sumersete et, Dorsete per $\mathrm{j}$ noctem, ij quarterin dimidium. In præbenda viij equorum R[icardi] de Marisco et W[illelmi] de Sancto Maxentio, per ij noctes, ij quarteria. In præbenda equorum familiæo dorsini Episcopi pro adventu domini Regis et domini Episcopi per ijj noctes, 5 $\mathbf{x}$ quarteria dimidium. Summa xix quarteria dinidium iij hopæ. In venditis, Dv quarteria. Et remanent in grangia per æestimationem, lxv quarteria. Et remanent $i j$ tassi avense.

Idem reddit compotum de ij quarteriis pisarum desuper emptis. Apud Trindle: In semine viij acrarum, ij quarteria. Summa acrarum, viij. Summa seminis, 10 ij quarteria.

Exitus Molendinorum sub CASTELio ET LAngeforde. Idem reddit compotum de xliij quarteriis $\mathbf{v}$ hopis de toto exitu frumenti. In expensis constabularii et servientium dum fuerunt ad mensam, xij quarteria. In cibo molindinarii, xijj hopæe. In supravenditis, $x x x$ quarteria

Idem reddit compotum de $\mathrm{e}^{\mathrm{l}}$ ij quarteriis, $\mathrm{v}$ hopis de toto exitu gruelli. In cibo molindinarii de Langeforda, xiij hopæ. In supravenditis, xj quarteria.

Idem reddit compotum de $x v$ quarteriis, $i j$ hopis de toto exitu sigali. In supra venditis, totum. Et de xvj quarteriis de toto exitu brasei. In supravenditis, totum. Et de xxxvj quarteriis de toto exitu contrabrasei. In supravenditis, totum.

Exitus molindini de Tabrigge et Vivarii. ${ }^{2}$ - Idern reddit compotum de xvij quarteriis, vij hopis de toto exitu frumenti. In cibo molindinarii, vij hopæ. In supravenditis, xvij quarteria. Et de xiiij quarteriis, vj hopis de totu exitu gruelli. In cibo molindinarii, vj hopæ. In supravenditis, xiiij quarteria. Et de xv quarteriis de toto exitu sigali. In supravenditis, totum. Et de viij quarteriis de toto exitu brasei. In supravenditis, totun. Et de xv 25 quarteriis, dimidio de toto exitu contrabrasei. In supravenditis, totum.

Exitus molindini de Hulle.-Idem reddit compotum de $\mathrm{x}$ quarteriis, dimidio, de toto exitu frumenti. In cibo molindirarii, vij hopæ. In supravenditis, ix quarteria, dimidium, $\mathrm{j}$ hopa. Et de $v$ quarteriis, vj hopis de toto exitu gruelli. In cibo molindinarii, vj hopæ. In supravenditis, $x$ quarteria. Et de iij quarteriis dimidio de toto exitu sigali. In supra- 30 venditis, totum. Et de $\mathrm{j}$ quarterio, dimidio, de toto exitı brasei. In supravenditis, toturn.

Instaurum.-Idem reddit compotum de $\mathrm{ij}$ avris, xvij equabus remanentibus anno præterito Et de iij equabus proventis de testamento Godwini de Holweia, Thomæ Fride, Ruberti de Sireforda apud Hol[weiam] hoc anno. Et de j equa kimelinga apud Hulle hoc anno. 35 Et de j equo ad cartam de empto. Summa iij avri, xxj equæ. In venditis, ij equæ vivæ. De precio superius respondet. In mortuis, iij equa. De corio $\mathrm{j}$ respondet; et coria ij equarum dealbata posita sunt ad hernesium caretarum. Summa v equæ. Ett remanent iij avri, xvj equx.

Idem reddit compotum de $\mathrm{j}$ pullo iij annorum. Et de iij pullis $\mathrm{ij}$ annorum. Et de 40 $\mathrm{v}$ lactentibus, remanentibus inno proterito. Et de $\mathrm{j}$ proveniente de testamento Walteri Swein apud Otrifordam hoc anno. Et de vij proventis de predictis equabus hoc anno. Summa xvij. In venditis, $x$ pulli. Et remanent, vij, unde $j$ de ij annis, vj de hoc anno. Idem reddit compotum de ciijj ${ }^{\mathrm{xx}} \times \mathrm{iij}$ bobus, ij tauris, remanentibus anno prætes ito. Et de iij de testamento Roberti Longi, Edrici de Galmatona, Algari de Sernehege apud 45 Hol[wei:III] lıoc anno. Et de $\mathrm{j}$ de testamento Edivæ viduæ apud Curf hoc anno. Et de $\mathrm{j}$ de testamento Ricardi Gore apud Stapl[e] hoc anno. Et de $\mathrm{j}$ bovetto de instauro adjuncto bobus hoc anno. Et de xxxij de emptis hoc anno. Summa cexxxij boves, ij tauri. In missis in lardarium ibiden, xxiiij boves. De coriis superius respondet. De carne habet respondere. In mortuis, vj boves, $\mathrm{j}$ taurus. De coriis superius respondet. In missis 50 apud Rimtonam, $\mathrm{j}$ bos. Summa xxxj boves, $\mathrm{j}$ taurus. Summa remanens, ccj boves, i taurus.

Iden reddit compotum de $x i j$ vaccis, $j$ tauro remanentibus anno præterito. In adjunctis vaccis, $j$ genicula. Summa xiij vacce, $j$ taurus. In nissis ibiden in lardarium, $\mathrm{j}$ taurus. In mortuis, ij vaccæ. De coriis superius respondet.

Idem reddit compotum de $\mathrm{j}$ genicula iij annorum, vj geniculis, vij bovettis $\mathrm{ij}$ annorum remanentibus anno præterito. Summa xiijj. In mortuis, iij geniculæ iij annorum. De coriis superius respondet. In adjunctis vaccis, j genicula. In adjunctis bobus, $\mathrm{j}$ bovettus. Summa vj. Et remanent $\mathrm{ij}$ geniculæ, vj bovetti $\mathrm{ijj}$ annorum.

Idem reddit compotum de iij geniculis, $v$ boviculis annalibus remanentibus anno 60 præterito. Summa vij. In mortuo, j boviculus. 1)e corio superius respondet. Et remanent iij geniculæ, iiij boviculi, ij annorun!.

Idem reddit compotum de ix annalibus remanentibus, anno præterito vituli. In mortuis, v. 'De coriis superius respondet. Et remanent ij boviculi, ij geniculæ j anni. 
Idem reddit compotum de $x j$ vitulis proventis de predictis vaccis hoc anno. In mortuis, iij. De coriis nichil recepit. Et remanent viij, unde iiij masculi, iiij femella.

Idem reddit compotum do clj ovibus remanentibus anno præterito. Et de j. kimelinga apud Otrifordam hoc anno. Et de ix adjunctis. Sumna clxj. In mortuis ante partum et tousionem iijj $^{x x} \times x^{\prime i j j}$. De pellibus superius respondet. Et remanent lxiij.

Idem reddit compotum de iij cıstratis, xxvij multonibus, remanentibus anno præterito. Et de xis arjunctis de instauro. In mortuis, $i j$ castr[ati] $\mathrm{j}$ multo ante tonsionem. De pellibus superius respondet. Et remanent $\mathrm{j}$ castr[atus], $x \mathrm{lvj}^{1}$ multones.

Idem reddit compotum de lx.x hoggettis remaneutibus, anno præterito agnis. In mortuis ante tonsionem, xlij. De pellibus superius respondent. In adjunctis ovibus ix, multonibus, xix. Nichil remanet.

Idem reddit compotum de xxxvj agnis proventis de prædictis ovibus hoc anno, quia $x$ viij erant steriles. In decima, iij. In mortuis ante tonsioncm, $x x$. Summa xxiij agni Et remanent xiij ugui.

Idem reddit compotum de cix velleribus grossis. In decimis, $x$. In consuetudine bercarii, j. Summa $x j$. In suprivenditis, iiij ${ }^{x x} \times$ viij vellera quæ fecerunt iiij petras, et xiij vellera agnorum qua feccrunt iijtam partem $\mathrm{j}$ petræ. De precio superius respondet.

Idem reddit compotum de cexij caseis; unde, a xxix die Septembris usque in v diem Octobris utroque die computato', scilicet per vij dies, facti fuerunt vij casei, singulis diebus 1 singuli casei. Et a vj die Octobris usque in $\mathrm{x}$ diem Novembris, utroque dic computato, scilicat per xxxvj dies, facti fuerunt xviij casei secunda die. Et a xvj die Februarii usque in ij die Maii, utroque die computato, ${ }^{\prime}$ scilicet per lx.xvj dies, fact: fuerunt xxxviij casei secunda die. Et a iij die Maii usque in xxviij diem Septembria, utroque die computato, scilicet per cxlix dies, facti fuerunt cxlix casei, singulis dicbus singuli casei. Summa ccxij casei. In deeima, xxj casei. In expensis constabularii et familiæ domus dum fuerunt ad mensam, iij casei. Summil xxiiij casei. I' venditis, ciiij ${ }^{2 x}$ viij casei, qui fecerunt ij capita, dimidium, iiij petras. De precio superius respondet.

Idem reduit compotum de vij suibus, $\mathrm{j}$ maello xij porcellis remanentibus anno præterito. Et de iij maellis emptis. Summa xxiij. In mortuo, j sues.' Et remanent xiij sues, ix maelli superannati.

Idem reddit compotum de liiij porcellis proventis de prædictis suibus hoc anno. In decima, v. In consuetudine porcarii, j. In nortuis, vj. Summa, xij. Et remanent, xlij.

Idem reddit compotum de xxxiiij bobus, ${ }^{2} \mathrm{j}$ tauro, missis ibidem in lardarium hoc anno. In expensis R[icardi] de Marisco et W[illelmi] de Sancto Maxentio, iijta pars j carnis. In expensis familiæ domini Episcopi expectantis adventuın domini Regis et domini Episcopi, iijta pars j carnis. In expensis familiæ domus dum fuerunt ad mensam, iiijta pars $\mathrm{j}$ carnis et totum sulcit[itium]. In expensis constabularii, iiijta pars $\mathrm{j}$ carnis. De sepo sinul cum corio respondet. Sumıa, caro $j$ bovis et totum sulcit[ium]. Et remanet caro $x$ xiij boun, $\mathrm{j}$ tauri.

Idem reddit compotum de vij quarteriis dimidio salis remanentibus anno præterito. In lardario et in anguillis salandis et in venatione domini Regis salanda, $x$ quarteria dimidium. In expensis constabularii, ij quarteria. Nichil remanet.

Idem reddit compotum de $\mathrm{xv}$ estikis anguillarum proventis de vivario et molindino hoc anno. In decima, j estika. In expensis constabulurii et familiæ dun fuerunt ad mensam, xiiij estika. Nichil remanet.

Idem reddit compotum de xxiij gallinis remanentiijus anno præterito. Et de xxiij proventis de cheriset, de Nailesbourne et Fulforla hoc anno. Summa xlvj. In constabulurio et familia dum fuerunt ad mensam, totun. Nichil remanet.

Idem reddit compotum de xliij soccis, iiij xxiij esperductis et dimidio, vj frustis ceri remanentibus anno præterito. Et de lxvj soccis, cxxix esperductis, xij firustis aceri, emptis hoc anno. Summa cix socci ccxiij esperducta, xviij frusta aceri. In expensis berthonarum, xliiij socci. In xv hoiis factis ad assartun, $x$ socci. In xxiiij ferris ad lanceas, iiij socci. Summa lviij. Et remanent lj. In expensis berthontrum, cxx esperducta. In $\mathrm{v}$ vertivellis, $\mathrm{ij}$ seris et clavis ar novam portam, $\mathrm{xxxj}$ esperducta. In ligonibus et hoiis et planis ad novum fossatom faciendum, vj esperducta. In vertevellis, haspis, clavis ad gahollan faciendam, iij esperducta. In vertevellis, clavis ad alias portas castelli faciendas, ij esperducta. In ligaminibus ferri et clavis minutis et grossis arl portam, iij esperducta. In expensis dumus, ij esperducta. Summa clxvij. Et remanent xlvj esperducta. In expensis berthonarum, xij frusta aceri. In hoiis faciendis, ij. Summa xv. Et remanent iij frusta aceri.

Idem reddit compotum de vj scntis, iij linceis, $\mathrm{j}$ talevaz, xviij capellis, unde xij ferrei, j cum visera, vj de corio, viij fausarz, vj hathis, ij targis, xj balistis, MMM quarellis, $\mathrm{x}$ lanceis remanentibus anno præterito. Et de c hastis proventis de Wallia. Et de xxiiij ferris ad lanceas factis ibidem hoc anno. Totum reminet. 
Idem reddit compotum de ij toncllis vini remanentis anno præterito. Et de ij tonellis vini [emptis] ${ }^{1}$ apud Exoniam. In expensis domini Roberti de Torneham, Ricardi de Marisco, Willelmo de Sancto Maxentio, vicecomitis Sumersetæ, et familiæ domini Episcopi apud Tantonam expectantiun adventum domini Regis et domini Episcopi [per iij] ${ }^{1}$ dies, j tonellus vini. Et remanent iij tonelli vini.

M. 11.

\section{RIMTONA.}

Willelmus de Sorewelda reddidit xxiijs. viijd. $o b$. de reragio anni tercii. Et quietus est. Idem reddit compotum de iijl. ixs. jd. de toto gabulo assiso. In quietancia j præpositi, per annum, iijs. In quictancia j forestarii ijs. In quietanciis ij carucariorum $\mathrm{j}$ bovarii, per annum, vjs. In quietancia $\mathrm{j}$ bercarii, ijs. In quietanciis iij operariorum, 10 per j terminum, xviijd. Summa quietanciarum, xiijs. vjd. Summa reddibilis Ix.xiijs. vjd.

Idem reddit compotum de xxxijs. iijd. receptis de firmario, liberatis eidem pro c ovibus in firma sua. Et de xviijs. iiijd. receptis de firmario, pro defectu wareti et grangia co-operienda et emendatione daerize. Summa, ljs. viijd.

Idem reddit compotum de xiijs. de firma molindini, cum dimidia virgata terræ adjacente molindino, quod Hugo carpentarius tenet. Summa, xiijis.

Idem reddit compotum de xxjd. de pannagio hoc anno. Et de xxvs, ijd. de herbagio. Et de vijs. de xij agnis vivis venditis. Et de xxiijd. ob. de residuo denariorum Sancti Petri hujus anni. Et de ixd. ob. de corio $\mathrm{j}$ annalis, misso ibidem in expensis hospitii, pro 20 consuetudine in hundredo convivando. Et de xvijs. vijd. de cxix caseis venditis. Et de viijd. de butiro vendito. Et de xjs. vjd. de lx.xj velleribus grossis venditis. Et de iijs. de lv velleribus agninis venditis. Et de vjd. de pellibus viij ovium, iij agnorum, venditis. Summa, Ixxs. xjd.

Idem reddunt compotum de xviijs. $x d$. de vij quarteriis, $i j$ hopis frumenti venditis. 25 Et de ixd. de dimidio quarterio de curaillo vendito. Et de ijs. iijj. de j quarterio sigali vendito. Et de xiiijd. de ij quarteriis avenæ venditis. Summa, xxijjs. jd.

Do Purchasiis.-Idem reddit compotum de vjs. viijd. de Willelmo de Marisco pro terra et vidua quæ fuit Henrici Durc habenda. Summa, vjs. viijd. Summa tocius receptæ, xijl. xjd.

Liboratio.-In liberatis Johanni decano iijl. per j tailliam.

Expensa.-In ferramentis ij carucarum, et ferrura avrorum, per annum, iijs. ixd. In mercede fabri, hoc anno, iijs. vjd. In ovis, carne, caseis emptis ad precarias hyemales, iiijs. In consuctudine prati falcandi, xiijd. $a b$. In carucis parandis, karris axandis, et reparandis, semella, corbella, seris, mensura, tripode, ventorio, emptis, iijs. xjd. In custo daeriæ ijs. $\mathrm{x} d$. In feno empto hoc anno ad opus boum, iijjs. In $1 \mathrm{x}$ acris dimidia serclandis, vs. vijd. $o b$. 35 In $\mathrm{j}$ tasso frumenti et alio avenæ faciendo et cooperiendo, xviijd. In coopertura colligenda ad grangiam, xvjd. In mola attrahanda ad molindinum, $x d$. In hostiis grangiæ, vertevellis, gungis ad idem, muro, porta circa curiam faciondis, iijjs. ijd. In solido $\mathrm{j}$ bovarii, custodis curiæ et l'asturæ, per annum, et $\mathrm{j}$ herciatoris per xviij ebdomadas, viijs. iijd. Summa xlvs. ijd. ${ }^{2}$ In vj quarteriis frumenti emptis ad semen, xvjs. $x d . o b .40$ In ij quarteriis ordei emptis ad semen, ijs. ixd. ob. In camera et granario de novo faciendis, bordis, clavis, vertevellis, gungis, fenestris, et hostiis faciendis, coopertura attrahenda, domibus cooperiendis, xiijs. iij $o b$. In expensis constabularii per v vices pro negotiis manerii sæpius emersis, iiijs. $x d$. In liberatione $W[i l l e l m i]$ concilii ibidem, pro terra seminanda, a $x x$ die Novembris usque in ix diem Jalıurii, utroque die computato, 45 scilicet per $\mathrm{lx}$ dies, $\mathrm{xs}$. In liberatione Roteri pro trituratione seminis avenæ et terra seminanda a xxvj die Februalii usque in die xxix diem Marcii, utroque die computato, scilicet, per xxxij dies, xxxijd. In liberatione Petri Coci a $x$ die Augusti usque in ix diem Septembris, utroque die coruputato, scilicet per $\mathrm{xl}$ dies, vs. In liberatione carpentarii pro molindino faciendo in tasca, vs. In xliiij bordis, cccl clavis, bordis ad iden, iijs. viijd. 50 Summa, ]xiiijs. xjd. ob. ${ }^{2} \quad$ Summa tocius expensæ, cxs. iijob. Summa liberationis et 'expensæ, ixl. xs. iijob. Et $\operatorname{debet}^{3} l s$. ixd. ob.

Exitus Grangiæ.-Idem reạdit compotum de xviij quarteriis dimidio, de toto exitu frumenti. Et de vj quarteriis frumenti desuper emptis ad semen. Summa xxiiij quarteria dimidiun. In semine Ix acrarum dimidiæ, xv quarteria. Summa acrarum, lx dimidia. 55 Summa seminis, $x v$ quarteria. In expensis hospitii pro hundredo convivando de consuetudine die Natalis, j quarterium. In braseo ad idem, de eaden consuetudine, j quarterium. Summa, ij quarteria. In venditis, vij quarteria dimidium. De precio superius respondet.

Idem reddit compotum de $\mathrm{j}$ quarterio dimidio de toto exitu curailli. In braseo ad prædictum hundrcdun, $\mathrm{j}$ quarterium. In vendito, dimidium quarterium. De precio 60 superius respondet.

1 The margin of the membrane is mutilaled. See supra, p. 70, 1. 5.

Sic in MS.

3 Debent in MIS. with the $n$ marked for deletion. 
Idem reddit compotum de xxj quarteriis iij hopis, de toto exitu siliginis. In sumine xxviij acrarum, vij qunrteria. Summa aerarum, xxviij. Summa seminis, vij quarteria. In consuetudine prati falcandi et feni congregandi, dimidium quarterium, ij hopæ. In cibo j bovarii, et eustodis curiæ, per annuı, viij quarteria, dimidiun, ij hopæ. In cibo $j$ herciatoris, per xviij ebdumadas, $\mathrm{j}$ quarterium, dimidium. In cibo daiæ, per xxvij ebdomadas, ij quarteria, iij hopæ. In vendito, $\mathrm{j}$ quarterium. De precio superius respondet.

Idem reddit compotum de $\mathrm{ij}$ quarteriis ordei emptis. In semine iiij acrarum, $\mathrm{ij}$ quarteria. Summa acrarum, iiij. Summa seminis, ij quarteria.

Ilem reddit compotum de xlij quarteriis de toto exitu avenæ. In semine l acrarum, xxxiij quarteria. Summa acrarum, 1. Summa seminis, xxxiij quarteria. In potagio servientium in Quadragesina, de consuetudine, ij quarteria. In braseo in hospitio die Natalis pro hundredo de consuetudine, j quarterium. In præbenda constabularii ibidem in adventu suo, $\mathrm{j}$ quarterium. In præbenda $\mathrm{ij}$ avrorum, per annum, iij quarteria. In venditis, ij quarteria.

15 Instaurum.-Idem reddit compotum de $\mathrm{xvj}$ bobus receptis de firmario. Et de $\mathrm{j}$ de testamento Henrici Durc. Et de j misso de Tantona. Summa xviij. Et remanent $x$ viij.

Idem reddit de ij equabus receptis de firmario. Omnes remanent.

Idem reddit compotum de iij vaccis receptis de firmario. Omnes remanent.

Idem reddit compotum de $\mathrm{v}$ annalibus anno præterito vitulis. In expensis hospitii die Natalis in hundredo de consuetudine, caro $\mathbf{j}$ annalis. Et de corio superius respondet. Et remanent iiij annales.

Idem reddit compotum de iijux $\mathrm{ij}$ ovibus receptis de firmario. Et de c, unde superius respondet. In mortuis ante partum, iij, post partum et tonsionem, v. De pellibus superius respondet. Summa viij. Et remanent lx.xiiij oves.

Idem reddit compotum de $\mathrm{j}$ multone recepto de firmario. Et remanet $\mathrm{j}$ multo.

Idem reddit compotum de lx.xix agnis proventis hoc anno prædictis ovibus, quia iij erant mortuæ ante partum. In decimis, viij. In consuetudine bercarii, $j$. In mortuis ante tonsionem iij. De pellibus superius respondet. In venditis, xij. De precio superius respondet. Et remanent lv agni.

Idem reddit compotum de iiijxx. velleribus grossis. In decimis, viij. In consuetudine berearii, $j$. Summa ix. In venditis $\mathrm{lx} . x \mathrm{j}$ qux fecerunt dimidium pondus. De precio superius respondet.

Idem reddit compotum de lv velleribus agninis. In venditis totum, quæ fecerunt ij petras. De precio superius respondet.

Idem reddit compotum de elv caseis factis hoc anno; unde, a xxix die Marcii usque in prima die Augusti, utroque die conıputato, scilicet per cxxvj dies facti fuerunt cxxvj casei, singulis diebus singuli casei ; et a ij die Augusti usque in xxviij die Septembris, utroque die computato, seilicet per lviij dies, xxix casei facti fuerun:t secunda die. In decima $x v$. In consuetudine tercarii, $\mathrm{j}$. In consuetudine prati falcandi, $\mathrm{j}$. In consuetudine fini congregandi et mullone faciendo, $\mathrm{ij}$. In consuetudine tonsionis, $\mathrm{j}$. In iij precariis hyemalibus, v. In expensis constabularii ibidem, iij. In operariis qui fecerunt tassum frumenti et tassum avenæ, $i j$. In coopertura colligenda, ij. In grangia cooperienda, ij. In carucis parandis et reparandis, $j$. Summa, xxxvi. In venditis, exix, qui feceruut iij capita, dimidium, iij petras. De precio superius respondet.

Idem reddit compotum de iij aucis, xviij gallinis et gallis, de firmario. In deperditis, omnibus gallinis et gallis per wlpes. Et remanent $i j$ aucæ et $\mathrm{j}$ ancer.

Idem reddit compotum de $\mathrm{xx}$ gallinis proventis de cheriset. In expensis constabulari omnes. Nichil remant.

Notandum quod Willelmus de Sorewella reddit de termino Sancti Michaelis, de anno præterito, xxiijs. viijd. $o b$., de quo solebant reddi ls., et deficiunt xxvjs. iijd. ob.

Notum, quod Nicolaus de Knulle fatetur se recepisse a Willelmo Firmario de Rimptona lxjs., qui exigebantur ab eodem Willelmo ad scacarium, et prodictus Willelmus inde quietus est; et inquirendum est utrum idem Nicolaus inde respondit in compoto suo necne.

\section{CNOEL}

Mauricius serviens et Ricardus præpositus reduunt compotum de lvjs. vd. ob. de reragio anni prateriti, unde xjs. ijd. allocantur ei de reragio terræ Guher. Et residuuu reddiıit. Et quietus est."

In perdonis Eliæ Ruffo vs. viijd., per breve senescalli, de reragio xxxviij vellerum grussorum, appreciatorum iiijs., et xxxviij villerum :gninorum, appreciatorum $x \times \ell$.

Idem reddunt compotum de xiiijl. viijd. iij qdr. de toto gabulo assiso. Et de xviijd. de incremento gabuli hoc anno. Summa tocius gabuli, xiiijl. ijs. iijd.

In quietanciis j præpositi, vs.; j berebrettarii, ijs. vjd.; j bedelli, j fabri vjs.; ij bercariorum vjs.; $\mathrm{j}$ vaccarii, $\mathrm{j}$ forestarii iijs.; ix carucariorum, xiijs. vjd.; ij operariorum, per $\mathrm{j}$ terminum, xijd. Summa xxxvijs.

1 ' 1 his note is anded in an official band and is apparently the fair copy of a cursive memorandum in the margin of the roll which has been partially erased. 
In defectu terræ Goher, iijs. xjd., quæ modo est assisa. In defectu ij virgatarum $\mathrm{F}[\mathrm{r}] \mathrm{atrum}$, vjs. In defectu rimidiæ virgatæ Clateræ, xviijd. In defectu $\mathbf{j}$ croftæ ${ }^{1}$ Blakemanni, xijd. In defectu dimidiæ virga' $\rightsquigarrow$ Tettæ, xviijd. In defectu dimidiæ virgatæ Rogeri Holeweiæ, xviijd. In defectu de minuto gabulo prædictarum terrarum de termino Sancti Michaelis, xijd. In defectı virgatæ terræ Osberti Kingwde tractæ in 5 dominium, iiijs. iijd. In defectu $\mathrm{j}$ virgatæ Philippi et Galfridi de Hale, tractæ in dominium, ijs. iijd. j $q d r$. In defectu j virgatæ Johannis, tractæ in dominium, iijs. ijjd. $q d r$. In defectu crofti Galfridi de Grava, tracti in dominium, vjd. ob. In defectu $\mathrm{j}$ virgatæ Willelmi Bundi, iiijs. ijd., quæ modo est assisa. Summa xxxijs.

Summa tam quietanciarum quam defectuum, lxixs. Summa remanens $x l$. xiijs. iijd.

Idem reddunt compotum de $v d$. ob. de pannagio. Et de $x d$. de clieriset. Et de $x l s$. de vij bobus, ${ }^{2}$ ij vaccis vivis venditis. Et de lxijs. vjd. de cxxv ovibus vivis venditis in termino Sancti Martini. Et de xiiijs. viijd. de xvj hurtardis vivis venditis post tonsionem. Et de xviijd. 06. de vij agnis vivis venditis. Et de ijs. vjd. de $x \times x$ gallinis venditis. Et de vs. jd. de coriis iij boum, ij vaccarum, ij vitulorum mortuorum venditis. Et de 15 iijs. viijd. de pellibus xxvj ovium, xv multonum, ijj hurtardorum, v hoggettorum mortuorum venditis. Et de xjs. vid de pellibus ccxvij agnorum mortuorum venditis, unde cvij erant mortui post separationem, ix mortui post tonsiunem. Et de vijjl. ixs. de vj ponderibus dimidio grossæ lanæ venditis. Et de viijs. de $\mathrm{j}$ quarterio lanæ agninæ vendito. Et de lxjs. vjd. de ix capitibus casei venditis. Et de ijs. vjd. de butiro vendito. Summa 20 xxijl. iijs. ixd.

Idem reddunt compotum de xxxixs. de xiij quarteriis frumenti venditis, singulum quarterium, iijs. Summa xxxixs.

De Purchasiis.-Idem reddunt compotum de xls. de Roberto Stede, ut posset discedere liber. Et de vs. de Elia Ruffo pro pluribus excessibus. Et de ijs. de Galfrido Freke pro stulti- 25 loquio. Et de xijd. de Willelmo Ailwardi pro forisfacto pasturæ. Et de vjd. de Johanne fabro pro blado male secato. Et de ijs. de Ada Chicled[er]e et Roberto molindinario pro terra bercarii habenda per vj annos. Summa ls. vjd.

Idem reddunt compotum de vijl. de tallagio.

Summa tocius receptæ, xliiijl. vjs. vjd.

Liberatio.-In liberatis J[ohanni] decano, xiijl. vd., de gabulo assiso, per ij taillias. Eidem, vjl. $x s$. de tallagio, per $\mathrm{j}$ tailliam. Eidem, ls. vjd., de purchasiis. Summa tocius liberationis, xxijl. xjd.

Expensa.-In ferramentis v. carucarum iij avrorum, per annum ; rotis ad carucas, xxjs. viijd. In $\mathrm{j}$ ventorio empto, vijd. In $\mathrm{j}$ sacca empta, vjd. ob. In hostio aulæ faciendo ex novo, iijd. 35 In porta facienda ad cimiterium, ijjo. In faciendis $\mathrm{ij}$ domibus ad caretas et alia neccesaria inponenda, iijjs. $\mathrm{j} d$. In feno empto ad boves, $x s$. In precariis quadragesimalibus de xxxvij carucis, iijs. jd. In magna porta reparanda, ixd. In ij herciis emptis, iijd. In prisepibus parandis ad oves, vjd. In xxj clatis emptis ad faldam, vijd. In ij domibus

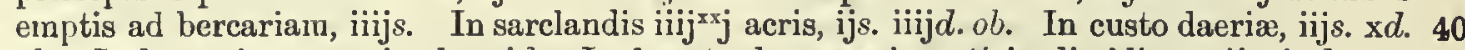
$\omega b$. In bercaria cooperienda, vjd. In fossato de cxxxvj perticis dimidia, xxijs. ixd., pro qualibet pertica, ijd. In fossato de xxiiij perticis, iijs., pro qualibet pertica, iij ob. In fossato de xiij perticis diuidio, iijs. vijd., ob pro qualibet pertica, iijd. In veteri fossato frangendo de $x l$ perticis, iijs. iijjd. In $\mathrm{j}$ careta reparanda ad bladum cariandum, vjd. In $\mathrm{j}$ equa empta ad marlandum, xjs. vjd. In iij bigis emptis ad marlandum, vijs. In 45 emendatione $\mathrm{j}$ caretæ, vd, ob. In sellis, bacis, paronis, paronellis, clutis, capistris, cingulis, et alio harnesio caretarum ad marlandum, viijs. $x d . o b$. In $j$ picois, et ij trublis, ad marlandum, vjd. In rotis an karrum ad marlandum et eisdem rotis ferrandis, ijs. vjd. Et in jequo ducendo de Edborebira, vjd. In ij acris spergendis de marla, vjd. In ferrura $\mathrm{v}$ avrorum, a Pascha usque festum Sancti Michaelis, iiijs. vd. In xiij quarteriis dimidio 50 avenæ emptis, xjs. vd. In j estrica fabarum empta ad plantandum, vd. ob. In iijjax entis emptis ad gardinum, xijs. iijd. In stipendio $\mathrm{j}$ bovarii per annum, ijs. In stipendiis ij caretariorum per dimidium annum, iijs. In mercede $\mathrm{j}$ daiæ per annum, ijs. In stipendiis ceterorum famulorum marlantium, a Pascha usque festum Sancti Michaelis, scilicet Alnatho et Willelmo Coco vjs. Et Willelmo Blakemanno et Martino, xs., et Johanni 55 Ruscel, iiijs., et j marlatori de Hockedai usque festum Sancti Petri ad Vincula, ijs.; et Warino pro corredio suo et stipendio, a die Crastino proxima post festum Sancti Aldelmi usque festum Sancti Michaelis per xvij ebdomadas, xvijs. In triturandis xxvj quarteriis dimidio frumenti, x quarteriis curailli, xliiij quarteriis mancorni, xviij quarteriis ordei, lx.xvj quarteriis avenæ, $x \times j$ s. $\mathrm{j} d$. In lxvj aucis emptis, vs. vjd. In $\mathrm{xx}$ ulnis canevaz 60 emptis ad lanam, iijs. iiijd. In liberatione Mauricii servientis, per annum, lxs. vd. In expensis Ricardi Sumeter, qui erat infirmus iiij ebdomádas, iijs. In $\mathbf{x}$ bobus emptis, lx.xjs. xjd. $\omega b$. In corredio G. de Aclanda, vjd. ob. In corredio Galfridi de Calceto, viijd. $a b$. In corredio R[oberti] Hotot, quando duxit prisonem, eundo et redeundo de 
Bristollia, ijs. vjd. In expensis magistri $\mathrm{R}$ [oberti] Basset et Willelmi de Sorewella, quando emerunt terra.n Golier, per iij dies, viijs. jd. In expensis Magistri Roberti Basset et Eustachii de Grenvilla et R[ogeri] Wacelini, iijs. iijd. ob. In corredio magistri Roberti Basset per iij itinera, quando venit per se, xijs. iiijd., per iij taillias. In corredio $\mathrm{R}$ [oberti] Bastardi, sociorunque ejus cum vj equis, et $\mathrm{R}$ [ogeri] Salserii cum sociissui, .. . equis ijs. vd. ob. In corredio Philippi de Lya, et cæterorun forestariorum ınultociens supervenientium, iijs. xjd.

Summa xirl. xiijs. vjd. Summa liberationis et expensø, xljl. xiiijs. vil. Et debent lijs. jd., unde xlvjs. vd. sunt manifestum debitum; vs. viijd. sunt super Elian præpositum, quos dicit Episcopum ei condonasse.

Exitus Grangiæ.-Idem reddunt compotum de xxxvij quarteriis de toto exitu frumenti. In M. 11d. semine lx.xvj acrarum dimidiæ, xvj quarteria dimidium. In mixtis cum semine mancorni, vj quarteria dimidium. In consuetudine seminatoris, dimidium quarterium. In consuetudine falcatoris prati, dimidium quarterium. In supravenditis, xiij quarteria.

Idem reddunt compotum de $\mathrm{xv}$ quarteriis de toto exitu curailli. In præbenda iij avrorum in hyeme, $v$ quarteria. In corredio famulorum, $x$ quarteria.

Idem reddunt compotum de xlv quarteriis, $\mathrm{jj}$ bussellis, de toto exitu mancorni. In semine cxliij acrarum, xxxij quarteria; unde vj quarteria dimidium sunt de frumento, de quibus superius respondent. In corredio ij famulorum, j bovarii, a festo Sancti Petri ad Vincula usque festum Sancti Michaelis, iij quarteria. In corredio eorundem, xiij quarteria dimidium, a festo Sancti Michaelis usque festum Sancti Petri ad Vincula, curn x quarteriis, quæ superius computantur. In corredio daiæ per eundem terminuın, $\mathbf{v}$ quarteria. In corredio bercarii, j quarterium, per senescallum, pro custodia hoggettorum. Item eidem pro custodia veterum cvium, ij busselli ; pro custodia agnorum, $\mathrm{j}$ quarteriuın; pro custodia boum in hyeme, $\mathrm{j}$ quarterium; pro c stodia c multonum, ab Hockedai usque festum Sancti Petri ad Vincula, j quarterium, ij busselli. Willelmo per $\mathrm{x}$ ebdomadas $\mathrm{j}$ quarterium ij busselli. In pane canum, $\mathrm{j}$ quarterium. In gallinis et aucis pascendis, j quarterium. In porcis pascendis, dimidium quarterium. Quietus est."

Idem reddunt compotum de xviij quarteriis dimidio de toto exitu ordei. In semine xxxviij acrarum, viij quarteria dimidium. In mercede herciatoris per dimidium annum, ij quarteria. In consuetudine præpositi, bedelli, fabri, bercarii, omnium carucariorum pro cibo Natalis et Paschæ, ij quarteria. In corredio iij marlatorum ab Octavis Paschæ usque ad Vincula Sancti Petri, vj quarteria. Quietus est."

Idem reddunt compotum de iijj $^{\mathrm{xx}}$.xiij quarteriis dimidio ij bussellis de toto exitu avenæ. Et de xiij quarteriis dimidio postea cmptis. Et de xviij allatis de Duntona. Summa cxxv quarteria, ij busselli. In semine lx.xvj acrarum in veteri campo, xxv quarteria dimidium. In semine cxix acrarum in novo ficusseiz xl quarteria dimidium. In consuetudine seminatoris, dimidium quarterium. In præbenda domini Episcopi, xxv quarteria dimidium. In præbenda senescalli et fratris Walteri in festo Omnium Sanctorum et die Veneris ante Uctavas Apostolorum Petri et Pauli, $j$ quarterium dimidium. In præbenda palefridi domini Episcopi, per vj ebdomadas, iij quarteria. In præbenda iij avrorum, vj quarteria. In præbenda G. de Aclanila, $\mathrm{ij}$ busselli. In præbenda vij avrorum marlatorum per xix ebdomadas, xxij quarteria dimidium. Quietus est. ${ }^{2}$

Idem reddunt compotum de ij bussellis de fabis emptis. In plantatione ij acrarum, totum. Quietus est.?

Instaurum.-Idem reddunt compotum de iij avris remanentibus anno proterito. Et de $\mathrm{j}$ postea empto. Et de j de testamento Eadmundi Eslac. Et de j provento de Edborebiria. Et de j provento de Clera. Et de j provento de Stokes. Summa viij avri. Omnes remanent.

Idem reddunt compotum de lij bobus remanentibus anno præterito. Et de iij adjunctis de instauro. Et de $\mathbf{x}$ postea emptis. Summa, lxv. In vivis venditis, vij. In mortuis, iij. Summa, $x$. Et remanent lv boves.

Idem reddunt compotum de xviij vaccis remanentibus anno præterito. Et de iij adjunctis de instauro. Summa xxj. In vivis venditis, iij. In mortuis, ij. Et remanent xvj vaccæ.

Ideın reddunt compotum de vj annalibus remanentibus anno præterito. In adjunctis bobus, iij. In adjunctis vaccis, iii. Nichil remanet.

Idem reddunt compotum de iij annalibus remanentibus anno praterito vitulis. Omnes remanent; unde $\mathbf{j}$ est genicula, ij bovetti.

Idem reddunt compotum de viij vitulis proventis hoc anno, quia cetere vaccæ fuerunt steriles. In mortuis ij. Et remanent vj vituli.

Idem reddunt compotum de Dlx.xiij ovibus remanentibus anno præterito; unde vi'j sunt hurtardi. Et de xlix adjunctis de instauro. Summa Dexxij. In consuetudine bercarii $\mathrm{j}$, in termino Sancti Martini. In vivis venditis in eodem termino, cxxv. In 
mortuis ante partem, v. In mortuis post partum et ante tonsionem, $\mathrm{xr}$. In mortuis ante tonsionem, $\mathrm{ij}$ hurtardi. In mortuis post tonsionem, vj oves $\mathrm{i}$ hurtardus. Summa, clv. Et remanent ccc lxvij unde $v$ sunt hurtardi.

Idem reddunt compotum de ccexliiij multonibus remanentibus anno præterito. Et de xxxix adjunctis de instauro. Summa cciiij ${ }^{x} i_{i j}$. In mortuis ante tonsionem, $v$, post 5 tonsionem, $x$. In consuetudine falcatorum post tonsionem, $j$ vivus. In vivis venditis post tonsionem, xvj. Summa, xxxij. Et remanent cccli multones.

Idem reddunt compotum de cxxxj hoggettis remanentibus, agnis anıo præterito. In adjunctis ovibus, xlix. In adjunctis ${ }^{1}$ multonibus, xxxix. In perdonis Eliæ Ruffo de reragio suo, xxxvijj, per breve senescalli. In mortuis ante tonsionem, v. Nichil 10 remanet.

Idem reddunt compotum de ceccrj agnis proventis de prædictis ovibus hoc anno, quia xxiiij fuerunt steriles et iiij fecerunt aborsum. Et viij sunt hurtardi. In decima, xxxix. In consuetudine bercarii, $j$. In mortuis ante separationem, cj. In mortuis post separationem et ante tonsionem, cvij. In mortuis post tonsionem, 1x. In venditis ante 1.5 separationem, vij. Summa, cclxirij. Et remanent cxlij agni.

Idem reddunt compotum de Dccixlvj velleribus grossæ lanæ. Et de vj de Lindesia. In decima, iiij ${ }^{x}$ iiij. In consuetudinibus ij bercariorum, $\mathrm{j}$ daiæ, iij. In supravenditis, Dcclx.v, qui fecerunt vj pondera et dimidium.

Idem reddunt compotuı de clj velleribus agninis. In supravenditis, totum, qui 20 fecerunt $\mathrm{j}$ quarterium.

Idem reddunt compotum de iij caseis de reragio anni præteriti. Et de cciij caseis qui inceperunt fieri die Annunciationis Sanctæ Mariæ, et desierunt die Sancti Michaelis, utroque die computato. Et fecerunt per xiiij dies ij casei, scilicet a die Sancti Barnabæ usque diem Sancti Johannis, utroque die computato. Summa, ccvj. In decima, xx. 25 In consuetudine falcatorum, ij. In consuetudine tonsorum, $\mathrm{j}$. In expensis domini Regis apud Gillingham, liberatis Rogeri Wacelino, ij. In expensis domini, episcopi liberatis Nicolao in coquina, $x$, per ij taillias. In expensis domini Episcopi liberatis, Petro Iuveni, ij. In expensis magistri R[oberti] Basset, in festo Omnium Sanctorum, $j$. In expensis ejusdem et Eustachii de Grenvilla et Rogeri Wacelini, j. In supravenditis, clxvij, qui 30 fecerınt ix capita.

Idem reddunt compotum de xxvij porcis remanentibus anno præterito. Et de xxiij proventis de suibus hoc anno. In decina, $\mathrm{ij}$. In consuetudine porcarii, $\mathrm{j}$. In mortuis, iij de senioribus, ij de junioribus. In liberatis arl lardarium de Dunton», vij. In expensis Domini Regis apud Gillingeham, iiij de junioribus. Summa xix. Et remanent $\mathbf{x x y}, 35$ unde vijj sunt sues, $\mathrm{j}$ verres, iiij porci superannati, xviij hoggetti.

Id $+\mathrm{m}$ reddunt conpotum de xij aucis de reragio anni proteriti. Et de lxvj postea emptis. Summa lx.xviij. In expensis domini Fpiscopi in festo Sancti Michaelis, v, liberatis Rogeri Wacelini, per $\mathrm{j}$ talliam. In expensis eidem in auptumno, xlv. In corredio $\mathrm{R}$ [oberti] Basset, E[ustachii] de Grenvilla, R[ogeri] Wacelini, vj. Item, in expensis 40 $\mathrm{R}$ [oberti] Basset, iiij. In corredio Radulfi Bastardi sociorumque ejus, iij. In mortuis, iij. Ei remanent xij.

Idem reddunt compotum de iiij $^{\mathbf{x}} v$ gallinis de reragio anni præeteriti. Et de $\mathbf{l x}$ gallinis de consuetudine forestarii et bedelli de termino Natalis et Sancti Michaelis. Summa cxlv. galline. In expensis domini Episcopi in festo Sancti Michaelis, 45 xxx gallinæ. In auptumno, v. In expensis domini Regis aput Gillingeham, viij capones. In corredio senescalli in festo Omnium Sanctor um, iij gallinæ. In exprensis senescalli et W[illelmi] de Sorewella, per iij dies, xvj gallinæ. In corredio R[oberti] de Hotot quando duxit prisonem, eundo et redeundo de Bristollia, vj gallinæ. In corredio senescalli et Eustachii de Grenvilla et R[ogeri] Wacellini, iij. In mortuis, ix. In 50 supravenditis, xxx. De precio superius respondent. Et remanent xxxiij ${ }^{2}$ gallinæ.

Idem reddunt compotum de vj baconibus proventis de Duntona. In expensis domini Episcopi ante festum Sancti Michaelis, j. In precariis auptumnalibus, v. Nichil remanet.

Idem Elias dicit Episcopum condonasse ei xxxviij agnos. Unde debet respondere cum denariis et de lana, nisi dominus Episcopus ei condonaverit.

\section{MINISTERIUM WINTONI}

Magister H. de Cardevilla reddit xijl. iijs. ijd. de reragio anni præteriti. Et quietus est.

Idem reddit compctum de xxvijl. xvjs. iijd. ob. de toto gabulo assiso. Extra Portam Orjentalem. In defectu et quietanciis et terris vastis de terra ubi mansit 60 Robertus Pache, xiiijd., quia tradita est in feodo Waltero plumbario, pro $x \times d$. per annum, per Godefridum Episcopum. Et de domibus quæ, fuerunt Reimundi redditis Gervasio in infirmitate Ricardi Episcopi, xjs. viijd., per eundem Episcopum. Et de ij terris quæ fuerunt Willelmi Suift, xijd., quia traditæ sunt in feodo Orgaro et Waltero Tichere pro ijs. per annum, per Godefridum Episcopum. Et in defectu terrarum ibidem 65 
redditarum Petro filio Reimundi, lvs, viijd. ob., per Ricardum Episenpum. In defectu j terra quam Serlo filius Payani tenuit, quæe est occupata fossato et barris civitatis, xijd. Et in terra quan Baldewinus tenuit, ijs. quia tiadita est in feodo Rogero ${ }^{1}$ Decano pro ijs. per annum, per Godefridum Episcopum.

Extra Kingate. De terra qux fuit Angeri presbyteri quæ est in manu Episcopi et wasta, iijs. Et de terra Rogeri de Insula, et de terra Nicholai Cale, et de terra Roies " viijd. quia tr.ditx sunt in feodo Gileberto H'landrensi pro xxd.per annum per Godefridum Episcopum. Et de terra magistri Hugonis medici quan Ricardus Episcopus dedit ecclesiæ Sancti Michaelis, ijs. Et de terra qus fuit Reginaldi Cobbe quæ est in manu Episcopi, xiiijd., quir locata est hoc anno pro $x d$. Et in defectu domus ubi Magister Hugo manet, quam Ricardus Episcopus dedit $\mathrm{H}$ [enrico] Cantuariæe Archiliacono, xjs. iiijd quia reddit inde de langabulo, ijs.

Et in Seftwichene. In defectu iij terrarum quas Ricardıs Medieus et Azo et Laurentius tenuerunt, redditarum J[ohanni] filio Petri in infirmitate Ricardi Episcopi per ipsum Episcopum, vjs In defectu $\mathrm{j}$ terre quæ fuit ejusdem Petri redditæ J[ohanni] prædicto filio ejus, vjd. per Ricardum Episcopum.

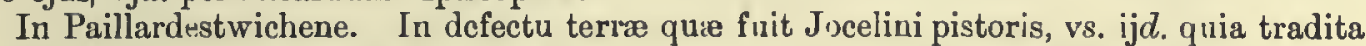
est in feodo Osberto Mariscallo, pro xviijd. per annum, per Godfridum Epis(o)pun.

Extra portam Australem. De iij terris quas Bertramus spcciarius, Willelmus et Alfredus filius Boiæ tenuerunt redditis J[ohanni] filio Petri iu infirmitate Ricardi Episcopi, per ipsum Episcopum, vjs. Et de terra Rogeri de Ticheburna quæ est in manu Episcopi, xiijd. quia locata est hoc anno pro vjd. Ricardo Aurifabro.

Ante Portam Castelli. Deterra Thomæe Oisur, vd. qure est in manu Episcopi et vasta.

Et extra Portam Orientalem. De terra Ricardi de Scotia quæ est in manu Episeopi et vasta, ijs.

Et extra Portam Borealem. De iij terris vasti- juxta fossatum qnæ sunt in mann Episcopi, $\mathrm{x} d$. Et de terra Edgari qure est in manu Episcopi et vasta, vijd. ob. Et de terra Aliciæ de Dive, lata Wil elmo filio Reginaldi, per Ricardu', Episcopum, iijjs. vj،.

Et de terra Osberti M ddere reddita Roberto Maudut, per Ricarduın Episcopım, vjd. Et in defectu ejusdem terræ, ijd., quæ est in manu Episcopis et vasta. Et de terra Ruberti Keppingr, qua est in minu Episcopi et rasta, jd Et de terra Willelni de Ponte, qua est in manu Episcopi et vasta, xiijd. Et in Flesmangerestrete, de domo quam Symon clericus tenuit, reddita J[ohanni] filio Petri in infirmitate Ricardi Episcopi, per ipsum Episcopum, xixs. iiijd. Et in Wenegerestrete, de dirno Alani de Inglesham, qua reddita est eidem per Ricardun Episcopum, vs. vjd. Et in defectu terræ Templariorum vjd., donec Episcopus inde aliud precipiat, quia est in voluntate Episcopi. Et in 'lanherestrete de terra Margaretie rle Godestre, quæ est in manu Episcopi et vasta, vjd. Et in Magno Vico, de terra quam Martinus La Martre tenuit, quæ fuit in manu Ejiseopi, modo reddita hospitali Sanctæ Crucis per Ricardum Episcopum, xiijs. iiijd. Et de terra Aliciæe de 1)ive, quam Jıhannes mercenarius tenuit, data Willelmo filio Reginaldi, per Ricardum Episcopım, iijj. Et de domn de Neubrigge, quæ fuit in manu Episcopi, data Fulconi Lepori per Ricardum Epi-copum, xvjs. iiijd. Et in Colbrokestrete, in defeetu ij terrarum ibidem, redditarum Petro filio Reimundi per Ric ırdım Episcopum, iijs. Et in Garstrete, de terra quæ fuit Arnoldi Herfast, iijd., quia tradita est in feorlo Philippo Croc, pro vjd per innum, per Godefridum Episcopum. Et in defectu ejusdem terre, quæ est in manu Episcopi et vasta, vjd. Et de terra Willelıi militis, quam Henricus de Loveraz tenuit, xijd., quia tradita est in feodo G[ilberto] de Sancta Cruce, pro ijs. per annum, per Ricardum Episcopum. Et de terra quæ fuit viduæ Picari, quæ est in manu Episcopi et vasta, vijd. Et apud Sperkefordan, in defectu terræ ubi Agene mansit, et in defectu terræ Osberti, quæ

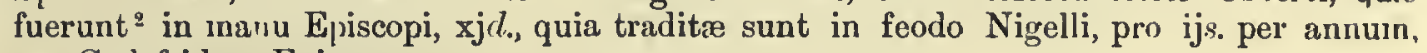
per Gollefridum Episcopum.

Summa ixl. vs. viijd. Et remanet xviijl. xs. vijd. ob.

Idem reddunt compotum de xviijs. de ministerio rotarum. Et de vjs. jd. de burgabulo extra portam Orientalem. Et de xxvd. de burgabulo extra Kingate. Et de $x d$. de burgabulo extra portam Australem. Et de xvd. de burgabulo in Grastrete. Et de $x d$. de burgabulo extra Portam Occidentalem. Et de xvd. de burgabulo extra Portam Borealem. Et de $\mathbf{x} d$. de burgabulo in Wenegerestrete. Et de $\mathbf{x} d$. de burgabulo in Tanherestrete. Et de ijs. de burgabulo apud Sperkefordam. Summa xxxiijs.

Idem reddit compotum de xxs. de aquagio molindini de hospitali Sancta Crucis. Et de xxs. de aquagio molindini Jocelini fratris Reginæ. Et de xxs. de aquagio molindini Willelmi de Mohun. Et de xxs. de aquagio molindini Abbatissæ. Summa iijl.

Do Purchasiis.-Idem reddit compotum de xijd. de Marescallo donini Regis, ut liceret ei emere terram unam extra Portam Australem. Et de xijd. de Galfrido cutellario, ut liceret ei emere eandem terram. Et de xijd. de Symone Buriman pro sursisa. Et de vjd.de Andrea Capellir pro mellea. Et de vjd. de Nigello de Sperkeforda pro simili. Et de vjd. de Walkelino de Sperkeforda pro simili. Et ile xijd. de Hereberto Spure pro simili. Et de 
viijd. de Willelmo Tapinare pro relaxata lege. Et de vjd. de filin Willelmi de Mellane pro simili. Et de ijs. de Bartolomæo de Walingefordia, ut liceret ei emere terram unam in monte Sancti Egidii. Et de vjd. de Ada caretario pro mellea. Et de vjd. de Martino carpentario pro simili. Et de ijs. de filia Nicolai Malherbe pro namio vetito. Et de vjd. de Anketillo de Monte pro relaxata lege. Et de vjd. de Aldulfo pro simili. Et de vjd. de 5 quadam femina an[te] Castellun pro simili. Et de iijjs. de Reginaldo Cobbe, ut liceret ei tenere ij terras in feodo in Monte Sancti Eqidii de domino Episcopo pro iiijs. per annum. Et de iiijs. de filio Benedicti, ut liceret ei tenere ij terras in feodo in monte Sancti Egidii de domino Episcopo pro iijjs. per annum. Et de ijs. de Briano de Garstrete, ut liceret ei tenere $\mathrm{j}$ terram in feodo in monte Sancti Egidii de domino Episcopo pro ijs. per 10 annum. Et de xijd. de Willelmo Parvo, ut liceret ei tenere dimidiam terram in feodo in monte Sancti Egidii de domino Episcopo pro xijd. per aunum. Et de iijs. de Acardo, ut liceret ei tenere ij terras ibidem in feodo pro ijis. per annum. Et de ijs. de Cristina filia Belengiri, ut liceret ei tencre unam terram ibidem pro ijs. per annum. Et de ijs. de Everarilo, ut liceret ei tenere j terram ibidem pro ijs. per aunum. Et de ijs. de Luca 15 pictore, ut liceret ei tenere $\mathrm{j}$ terram ibidem pro ijs. per annum. Et de iiijs. de Roberto de Bolonia, ut liceret ei tenere ij terras ibidem pro iijs. per annum. Et de ijs. de Alicia de Sancta Maria, ut liceret ei tenere j terram ibidem plo ijs. per annum. Et de iijs. de Ricardo Capillir, ut liceret ei tenere terram Pulcelli, extra Portam Orientalem qux fuit in manu domini Episcopi. Summa xliiijs. ijd.

Summa summarum tocius receptæ, xxvjl. viijs. ixd. ob.

Liberatio.-In liberatis Johanni decano, $x l$. ixd. ob. per iij taillias.

M. 12.

Expensa.-In expensis Magistri H[enrici] de Cardevilla, liijs. iiijd. In mercede Willelmi et Ysaac servientium, $x s$. In oblacione eurundem, iijd. In pergamine empto, vd. In 25 firma quam dominus Episcopus reddidit Ricardo de Crikelade de terragio extra Portam Orientalem, ijs. In firma domini Episcopi versus coquinarium Sancti Swithini, de terragio extra Kingate, vijd. In firma domini Episcopi versus Henricum de Garstrete, de terragio extra Portam Australem, iijd. In firma donini Episcopi versus dominum Regem, de terragio in Buckestrete, $x d$. In firma domini Episcopi versus Willelmum Beupeine, de 30 terragio extra Portam Borealem, ijs. Summa expensæ, lxixs. $\mathrm{x} d$.

Et debet, xijl. xviijs. ijd. unde super domum Sanctæ Crucis sunt, iijl. ijd.

Et notum quod, præter hoc, sunt super hospitalem Sanctæ Crucis, de iij annis præteritis, xijs. vjd. Et præterea super terram quam Gaubert et Ricardus de Balun tenent, xiijs. iiijd.

\section{MOLINDINA WINTONIE.}

ADAM BULE reddit compotum de iijl. iijd. de reragio anni prateriti de onnihus molindiıis. In liberatis Roberto de Redinges pro moltura sua anno præterito, $\mathrm{x} s$. In liberatis Adæ filio viduæ, pro simili, xs. In liberatis Henrico Swenge, pro simili, ixs. In liberatis Gocelino, pro simili, viijs. In liberatis Osberto Talun, pro simili, viijs. In liberati 40 Johanni Decano, per j tailliam, xxxvs. iijd. Summa, iiijl. iijd.

Idem reddit compotum de vjs. $x d$. de redditu assiso pertinente ad molindinum de Fonte Segrim. In defectu terræ Alexandri allutarii, viijd. Summa remanens, vjs. ijd. Idem reddit compotum de xijs. de consuetudine iiij servientium. Et de ijs. de consuetudine j garcionis. Summa xiiijs.

Idem reddit compotum de lxxs. iijd. de xxviij quarteriis $\mathrm{j}$ bussello frumenti venditis. Et de iiijd. de moltura.

Summa lxxs. viijd. Summa totalis iiijl. xs. $x d$.

Expensa.-In ferramentis ij equorum usque festum Sancti Michaelis, viijs. ijd. In feno et avena et drasca ad eosdem, xijs. viijd. ob. In cariando mairemio ad novum molindinum 50 faciendum, xvs. iijd. In carpentario ejusdem molindini ljs. vijd. In bordis ad rotam ejusdem molindini, xiiijd. In fusis ferreis ad idem, vjd. In cariagio ejusdem wolæ ar vetus molindinum ab Hamptona, xijd. In ferramentis ejusdem et martellis novis emptis et reparandis, vjd. In iiij vertevellis, iiijd. $o b$. In alicone ad idem molindinum, $x j d$. $o b$. In brachio rutæ ejusdem molindini, ijd. In bordis ad idem, xijd. ob. In clavis, iijd. In 55 fusis ligneis ad idem, vijd. In fusis ferreis ad idem, xvd. Summa, cxvs. vjd.

In moltura Osberti Talonis empta, xviijs. In moltura Alvredi pistoris empta, $\mathrm{ij} s$.

Expensæ excedunt receptam per xxiiijs. viijd. sine libcratis.

FLoDstoc. Idem reddit conpotum de xxxijs. de consuetudine servientium molindini.

Idem reddit compotum de ijs. $x d$. de j quarterio frumenti vendito. Et de sxxixs. xjd. 60 de moltura in d[enariis]. Summa totalis, lx.xiiijs. ixd. 
In liberatis Johanni decano, per $\mathrm{j}$ tailliam, ls. viijd. ob. In ferramento ij equorum per dimidium annum et $\mathrm{j}$ eorum per totum annum, vjs. $\mathrm{j} d$. In feno, avena, et drascha, et brennio ad eosdem, xs. iiijd. ob. In fusis ferreis et alio ferramento, xiijjd. In bordis ad parietes et rotas, xvijd. In tusis ligneis et alicone, viijd. In j vann', viijul. In clavis, ob. Summa expensæ xxs. ijd. Summa totalis $1 \mathbf{x} . x s . x d . o b$. Et debet, iijs. $x d$.

Bartuna. Idem reddit compotum de xs. viijd. de tirma inolindini crepestr[iæ], unde ijs. viijd. sunt de incremento anno $\mathrm{iij}$ de $\mathrm{ij}$ terminis.

Idem reddit compotum de cviijs. de consuetudine servientium molindini Bertonæ.

Idem reddit compotum de lviijs. $\mathrm{x} d$. de $x$ xiij quarteriis, $\mathrm{j}$ bussello frumenti provenientis de eodem molindino venditis.

Idem reddunt compotum de xxiiijs. de quodam prato vendito. Et de ijs. de pisce capto ibidem vendito.

$$
\text { Summa, xxvjs. Summa tocius, } x l \text {. iijs. vjd. }
$$

Liberatio.-In liberatis J[ohanni] decano, iijl. ixs. ijd.

15 [Expensa]. - In ferramento ij equorum, viijs. iiijd. In feno et avena et drascha et brennio emptis ad eosdem, xijs. viij $d$. $a b$. In carianda quadam mola ab Hamptona, xijd. In bendis ferreis emptis, vijd. In cariando mairemium ${ }^{1}$ ad stagnum eiusdem molindini, $x \mathbf{x} d$. In furis ferreis et martellis et alio ferramento, ijs. iijd. ob. In alicone et fusis ligneis, xiijd. In bordis ad rotas et alia neccessaria, xixd. In molindino tegendo, et coopertura ad iden, iijs. jd. In piscatura paranda, iiijd. In cofino empto, ijd. Summa xxxiijs. iiijd. In moltura Adæ filiæ viduæ, xxs. In inoltura Rogeri Leonis, xixs. In moltura Gocelini, xiijs. iiijd.

Summa expensæ, iiijl. vs. ijd. Sunna totalis, viijl.xiiijs. iiijd. Et debet xxixs. ijd.

Sparkeforda. Idem reddit compotum de iijs. de redditu assiso pertinente ad molindinum de Sparkeforda.

I dem reddit compotum de lijs. de consuetudine servientium eiusdem molindini.

Idem reldit compotum de xxxijs. ijd. ob. de xij quarteriis frumenti venditis. Et de iiijs. xjd. de pisce ibidem vendito. Summa iiijl. xijs. iij ob.

Liberatio.--In liberatis J[ohanni] decano, per j tailliam, xliiijs. iiijd. ob.

30 [Expensa].-In ferrameutis ij equorum, viijs. ij.. In feno, avena, drascha et brennio emptis ad eosdem, xijs. viijd. In $\mathrm{j}$ equo empto, iiijs. In hendis ferreis, vjd. In brachiis rotæ reparandis, $\mathrm{ij} d$. In fusis ferreis et martellis et alio ferramento, ijs. iijd. ob. In bordis, xjd. In alicone et fusis ligneis, viijd. $o b$. In cariando mairemium ad fundum aquæ, ijs. jd. In stagno reparando, xiiijd. $o b$. In carianda $\mathrm{j}$ mola de Segrim, et alia de Flodstoc ad molindinum de Sparkeforda, $\mathrm{ij} d$. In piscatura reparanda, ix $d$. In tectura ad molindinum et tectore, ijs. vijd. In moltura Roberti de Redinges empta, xxs.

Summa expensæ, lvjs. iijob. Summa liberationis et expensæ, cs. vjd. Et debet vjs.

\section{FALELEIA ET ORA.}

Johannes serviens de Biterne, et Willelmus de Faleleia, reddunt compotum de xiijs. de gabulo assiso. Et de xxxs. de firma cl matricium ovium. Et de ijs. de molindino, quod Briennus tenet. Summa xlvjs.

Idem reddunt compotum de xxvjs. de gabulo assiso de Ora.

\section{Summa summarum Ix.xijs. Et præterea homines de Ora debent ad Natale aves} ad valentiam, vjd.

45 Purchasia $\left[\mathrm{de}^{2}\right]$ Faleleia $\left[\mathrm{et}^{2}\right]$ Ora. -Idem reddunt compotum de dimidia marca de Odone pro terra habenda. Et de ijs. de Waltero clerico pro lege relaxata. Et de xijs. le lioberto (iitte et fratre suo, pro judicio habendo. Et de xviijd. de Roberto filio Briani pro terra habenda. Et de ijs. de vidua Odonis pro fine terræ. Et de xviijd. de Rogero filio Adam pro simili. Et de $\mathrm{j}$ marca de Willelmo Gute, quia non fecit summonitionem. Et de dimidia marca de Hugone de Tribus Aulis quia deforciavit namium. Et de dimirlia marca de Rogero de Lilleb[urna] pro judicio habendo cartæ suæ. Summa, lijs. iiijd.

Idem reddunt compotum de xxxs. de taillagio de Faleleia. Et de xls. de taillagio de Ora. Summa lx.xs.

Summa summarum, ixl. xiijs. iijjl. 
Liberatio.-In liberatis J[ohanni] decano de termino Sancti Michaelis, per manum præpositi de Faleleia, xlvjs. In liberatis eidem de taillagio, xxxs. In liberatis eidem de termino Sancti Michaelis, per manum præpositi de Ora, xxvjs. In liberatis eidem de taillagio, xls. In liberatis eidem, per manum præpositi de Biterna, $1 s$. vijd. de purchasiis. In passagio Johannis et præpositi de Biterna eundo et redeund,, per xviij vices, xixd.

\section{FUNTEL.}

Magister T[Homas] de Ehlesburna solvit xzxiijl. de firma. Et de tallagio, xijl.

$T$ [homas] sacerdos de Funtel reddit compotum de $x l$. ixs. jd. de reragio firmæ.

Idem reddit conpotum de xxiiijl. de firma hujus anni. Et de iiijl. de tallagio Summa tocius receptæ $x \times x v i i j l . i x s . j d$.

In liberatis J[ohanni] decano, per iiij taillias, xiiijl. vjs. iiijd. Eidem de reragio anvi præteriti, lixs. jd. Eidem de tallagio, lvjs. per $\mathrm{j}$ tailliam. Summa liberationis $\mathrm{x} \mathrm{x} l$. $\mathrm{v} d$. Et debet de tirma hujus anni, ixl. xiijs. viijd. Et de auxilio, sxvs. Et de reragio de tempore Nicholaæ de Kiville, ${ }^{1}$ vijl. $x s .,{ }^{2}$ quos dicit se solvisse eidem Nicholao.

\section{CHERITONA.}

H[ENRICUs] LA MARTRE, et W[illelmus] serviens, et J[ohannes] præpositi, et $R$. berebrettus, reddunt compotum de vijl. xvjd. ob. de reragio anni præteriti. Solvunt et quieti sunt.

Idem reddunt compotum de sxiijl. xiijs. ixd. de gabulo ussiso. Et de ijs. vjd. de incremento gabuli Jacobi, de dimidia virgata terræ de purprestura. Et de $\mathbf{x} x$. de 20 incremento gabuli Julianæ viduæ Noreis, de $\mathbf{x}$ acris terræ de simili. Summa, xxiijl. xiijs. ixd.

In quietanciis $\mathrm{J}$ [ohannis] præpositi vs., $\mathrm{j}$ berebretti, $\mathrm{ijs}$. vjd., $\mathrm{j}$ fabri vijs., $\mathrm{ij}$ heiwardorum iiijs., ij bercariorum iiijs., ij custodum boum iijs., i porcarii ijs., $\mathbf{x}$ carucariorum, ver annum $\mathrm{x} s ., \mathrm{z}$ operariorum, per annum, $\mathrm{xxs}$., ij operariorum, pro dimidio opere, ijs. In 25 defectu terræ R. Noreis tractæ in dominium, vjd. Summa lx.xs. Summa remanens sxl. iijjs. ixd.

Idem reddunt compotum de xiijjs. vijjd. de pannagio. Et de vijs. viijd. de herbagio Et de xixs. vjd. de $\mathrm{v}$ bobus vivis venditis. Et de xiijs. iijd. de $\mathrm{xx}$ veteribus ovibus vivis ${ }^{\circ}$ venditis in festo sancti Martini. Et de vjs. ijd. de xxxvij agnis vivis venditis. Et de 30 xs. ijd. de coriis vij boum mortuorum venditis. Et de iiijs. de terra locata, quadr[agesima] hidæ. Et de cvjs. viijd. de iiij ponderibus grossæ lanæ venditis. Et de xxs. ixd. ob. de dimidio ponderis lanæ agninæ. Et de xs. de transitu viæ. Et de xxviijs. de iiij capitibus casei venditis. Et de xiijj[s.] ${ }^{3}$ ijd.ob. de pellibus $x v j$ ovium, $x$ multonum, $\mathrm{j}$ hurtard, $\mathbf{x} \times \mathbf{j}$ hoggettoruın, clx.xvj agnorum mortuorum, venditis. Et de xxiiijs. vjd. de $\mathbf{x}$ bovettis 35 noviter mortuis. Et de iijd. ob. de vj caseis parvis venditis. Summa xiijl. xixs. xjd.ob.

Idem reddunt compotum de cvjs. vjd. de $\operatorname{xxxv}$ quarteriis dimidio frumenti venditis, singulis quarteriis, iijs. Et de xxijs. viijd. ob. de ij tonellis vini expensis in scotallo. Summa vjl. ixs. ijd. $o b$.

De Purchasiis.-Idem reddunt compotum de xls. de vidua Ailwini molindinarii pro fine terræ suæ. Et de vs. de Willelmo berebretto pro warranto. Et de $x$ s. de vidua Noreis pro $\mathrm{x}$ acris de purpestura. Summa lvs.

Idem reddunt compotum de xixl. xixs. iiijd. de taillagio. Summa utriusque, xxijl. xiiijs. iiijd. Summa tocius receptæ lxiijl. viijs. iijd.

Liberatio.-In liberatis Johanni decano per vij taillias, xlviijl. xjs. ixd. ob. Item eidem, 45 xiijs. vijd. ob.

Summa xlixl. v8. vd.

Expensa.-In ferramento $\mathbf{v}$ carucarum, $\mathbf{x} s$. In ferura iij avrorum, iijs. ijd. In $\mathbf{j}$ careta ferro ligata empta, xjs. vjd. In j pari rotarum ad caretam faciendam de mairemio Episcopi, vjd. In cordis, clutis, sepo ad caretas et ad lumen in domo in autumnno, et in capistris, xvd. ob. 50 In vj quarteriis frumenti emptis ad semen, xviijs. In bercaria tegulanda, iijs. In cxiiij perticis fossandis circa bercariam, xxviijs. vjd. In sepe facienda circa bercariam, ijs. iijd. In virgis colligendis ad præsepia boum, et eisdem faciendis, xijd. In $\mathrm{j}$ novo granario faciendo, axvs. In custo daeriæ, ijs. ijd. ob. In clatis faciendis ad faldam, ijs. ijd. In stipendiis ij servientium, per annum, vjs. In stipendio daiæ, per annum, ijs. In liberatione $\mathbf{5 5}$ W[illelmi] serventis, xvs. ijd. ob. In xj hobus emptis, lx.xvs. iiijd. In prato fossando, claudendo, vjs. viijd. In allece empto ad precarias auptumnales, vs. vd. In precariis hyemalibus et quadragesimalibus de lx.xij carucariis, xijs. In saccis emptis, xviijd. In corredio Reginaldi salsarii in auptumno, iijs. viijd. In expensis Henrici la Martre per v dies, xiijs. iijd. In prato falcando, xijd. Summu zijl. xiijs. viijd. $o b$. 
Sumına liberationis et expensa, lxjl. xixs. iijob. Et debet xxviijs. iij $o b$. Uncle xxs. vd. est manifestuı debitum, et vjs. viijd. sunt de superdemanda de Petronella de Herefordia plusquam finivit, per Henricum la Martre.

5

Exitus Grangiæ.-Idem reddunt compotum de ciij quarteriis dimidio de toto exitu frumenti. Et de vj quarteriis emptis Summa, cix quarteria dimidium. In semine cexl acrarum, lx quarteria dimidium. In corredio $W$ [illelmi] servientis, vj quarteria dimidium. In corredio Reginalıli salsarii in auptumno dimidium quarterium. In vij precariis aucumpnalibus, v quarterin dimidium. In dono domini Episcopi cuidam feminæ apud Wintoniam, $\mathrm{j}$ quarterium. In supravenditis, $\mathrm{x} \times \mathrm{xv}$ quarteria dimidiun.

Idem reddunt compotum de $x \times x j$ quarteriis dimidio de toto exitu mancorni. Et de ix quarteriis dimidis de curallo, frumento. Summa xlj quarteria. In semine $1 \mathrm{x}$.xiiij acrarum, sxiij quarteria dimidium. In precariis auptumnalibus anno præterito, vj quarteria. In precariis auptumnalibus, hoc anıo, iiij quarteria. In consuetudine unius bercarii, $\mathrm{j}$ quarterium. In cortedio $\mathrm{ij}$ serventium, vj quarteria dinidium.

Idem reddunt compotum [de] 1x.xij quarteriis de toto exitu ordei. In semine cxxiiij acrarum, xlj quarteria. In precariis auptumnalibus, $v$ quarteria, anno præterito. In vij precariis hoc anno, $\mathrm{xj}$ quarteria. In corredio $\mathrm{ij}$ servientium, vj quarteria dimidium. In curredio daiæ, iij quarteria. In consuetudine porcarii, j quarterium. In custode agnolum, $\mathrm{j}$ quarterium. In porcis, porcellis pascendis, ij quarteria dimidium.

Idem reddunt compotum de ccl quarteris de toto exitu avenæ. In semine cclxiiij acrarum, cxxxij quarteria. In missis apud Suttonam ad præbendam domini Episeopi, iiij.x.xiij quarteria. In præbenda iiij avrorum, xv quarteria. In bobus pascendi in garbis, ix quarteria.

Idem reddunt compotum de $\mathrm{j}$ quarterio pisarum allato de Alresforda. In semine $\checkmark$ acrarum, totum.

Instaurum.-Idem reddunt compotum de Ixvij bobus remanentibus anno præterito. Et de $\mathrm{xj}$ postea emptis. Et de j vacca inventa. Summa lx.xviij boves, $\mathrm{j}$ vacca. In vivis venditis $v$; unde superius respondent. In mortuis vij. De coriis superius respondent. Item, in mortuis $\mathbf{x}$; unde superins respondent. Summa, xxij boves. Et remanent lvj boves, $\mathrm{j}$ vacca.

Idem reddunt compotum de $\mathrm{ij}$ avris remanentibus anno præterito. Et de $\mathrm{j}$ qui venit de testamento Ailwini. Et de $\mathrm{j}$ qui venit de testamento Willelmi Turri. Summa, v. Omnes remanent.

Idem reddunt compotum de ecexxxvij ovibus remanentibus anno præterito. Et de xxiij adjunctis post tonsionem Summa, ccclx. In vivis venditis in festo Beati Martini, $x x$; unde superius respondent. In consuetudine $j$ bercarii, $j$. In mortuis ante partum, ix. In mortuis post partum et ante tonsionem, iiij. In mortuis post tonsionem, iij. Summa, xxxvij. Et remanent cccxxiij oves.

Idem reddunt compotum de clvij multonibus, $x$ hurtardis, remanentibus aun" præterito. Et de $x \times x v$ adjunctis post tonsionem. Summa ccij. In mortuis ante tonsionem, iiij; post tonsionem, vij. Summa, xj. Et remanent ciiij. ${ }^{x x}$.xiij multones.

Idem reddunt compotum de $i i j{ }^{x x} v i j$ hogg[ettis] remanentibus anno præterito aynis, quia vij agni subtracti erant de consuetudinibus. In mortuis ante tonsionem, xxij. In mortuis post tonsionem, iijj. In adjunctis ovibus, xxiij. In adjunctis multonibus, xxxviij. Nichil remanet.

Idem reddunt compotum de cciijij ${ }^{x x}$.xix agnis proventis hoc anno de ovibus, quia viij fuerunt steriles. Et de cexxxix qui venerunt de Suttona ante tonsionem. Summa Dxxxviij. In decima, xxix. In consuetudine bercarii, $\mathrm{j}$. In vivis venditis ante tonsionem xxxvij; unde superius respondent. In mortuis ante tonsionem, cxxxvijj. In mortuis post tonsionem, xxxviij. Summa cexliij. Et remanent cciiijx.xv agni.

Idem reddunt compotum de 1)xxvij velleribus grossis. In consuetudine ij bercariorum, ij. In supravenditis, Dxxv vellera, yuæ fecerunt iiij pondera.

Idem reddunt compotum de cccxxxiij velleribus agninis. In supravenditis totum, quæ fecerunt dimidium pondus.

Idem reddunt compotum de clx.xv caseis qui inceperunt fieri octavn die Aprilis, t.t desierunt fieri die Sancti Michaelis, utroque die computato. In decima, xvij. In consuetudine daiæ, $\mathrm{j}$. In vij precariis auptumnalibus xlix, qui fecerunt $\mathrm{j}$ caput dimidium. In missis apud Bewrdam, xvij, ad precarias, qui fecerunt quartam partem $\mathrm{j}$ capitis. In supravenditis, iij capita, et præterra vj casei venditi fuerunt, unde superius respondent.

Idem reddunt compotum de xlvij porcis remanentibus anno præterito. Et de xlviij de exitu illorum. Summa, iijj.x. $x v$. In decima, iij. In consuetudine porcarii, $j$. In missis in lardarium domini Episcopi apud Waltham et liberatis Adæ præpositi, xvj. In mortuis, xvij. Summa xxxriij. Et remanent lvij, unde vj sunt sues, j verres, xxiij porci super annum, xxvj juniores infra annum.

Idem reddunt compotum de ij aucis remanentibus anno præterito. Omines remanent.

Idem reddunt compotuiı de $x l$ gallinis remauentibus anno praterito. Et de xvj allatis de Bewrda. Et de lxiiij de cheriset. Et de xj de consuetudine. Summa, 
cxxx. In expensis domini Episcopi a jud Merewellam, lxvjl., liberatæo Nicolao de coquina per $\mathrm{j}$ tailliam. In mortuis, xxix. Et remanent xliiij.

Idem reddunt compotum de $M l$ ovis proventis de consuetudine. In expensis domini Episcopi apud Waltham Dc, liberata Nicolao de coquina per $\mathrm{j}$ tailliam. In expensis ejusdem apud Merewellam, ccccl ova, liberata eidem Nicolao per j tailliam.

Idem reddunt compotum de xiiij baconibus receptis apud Wlveseiam per manus Johannis decani. In precariis auptumnalibus, ix dimidium. In missis apud Bewordam ad precarias auptumnales, iijj dimidium.

\section{BEWORDA.}

HFnricus da MARTRE et Willelmus serviens et Gillebertus præpositus et Ricardus 10 berebrettus reddunt compotum de iiijl. ijs. vjd. de toto gabulo assiso. In quietanciis j præpositi vs., j berebretti, ijs. vjd., iij carucariorum, per annum, vjs., j bercarii, ijs., iij operariorum, per annum, vjs. Summa, xxjs. vjd. Et remanent lxjs.

Idem reddunt compotum de iijs. iijob. de pannagio. Et de ijs. iiijd. de coriis ij boum mortuorum venditis. Et de iijs. iijob. de $\mathrm{j}$ taurn vivo vendito. Et de vs. de $\mathrm{x}$ hurtardis 15 vivis venditis. Et de $v d$. de pellibus ij multonum mortuorum venditis. Et de iijs. de quodam prato vendito sub monte Sanctæ Katerinæ Et de xxvjs. viijd. de $\mathrm{j}$ pondere grossæ lanæ vendito. Summa xliijs. viijd.

Idem reddunt compotum de xlijs. de xiiij quarteriis frumenti venditis. Et de viijs. iiijd. de iiij quarteriis frumenti venditis. Et de iijs. de $\mathrm{j}$ quarterio dimidio ${ }^{1}$ ordei 20 venditis. Summa liijs. iiijd.

Purchasia.-IIJem reddunt compotum de xs. vjd. de Roberto filio Edwini pro terra patris sui habenda. Et de ijs. de Alano pro filiastra sua maritanda. Summa xijs. vjd.

Idem reddunt compotum de iiijl. viijd. de tallagio. Summa iiijl. xiijs. ijd. Summa tocius receptæ xijl. xjs. ijd.

Liberatio.-In liberatis J[ohanni] decano, per ij taillias, viijl. xjs. viijd. Item eidem, per $\mathrm{j}$ tailliam, vijs. iijd. Summa viijl. xviijs. xjd.

Exponsa.-In ferramento ij carucarum, et eisdem faciendis, et rotis ad easdem, vjs. iiijd. In ferrura $\mathrm{j}$ caretæ, $\mathbf{x v d}$. In $\mathrm{j}$ careta empta, et harnasio ad illam, iijs. xjd. In iij bobus emptis, xxvijs. vijd. ob. In $\mathrm{j}$ boketto et fune ad aquam extrahendam de puteo emptis, 30 iiijd. In $\mathrm{v}$ quarteriis frumenti eligendis ad semen, $\mathrm{xx} d$. In precariis hiemalibus et quadragesimalibus de $\mathrm{xij}$ carucis, ijs. In mercede fabri pro ferramentis ij carucarum parandis, iiijs. vjd. In iij quarteriis frumenti emptis ad semen, ixs. vijd. In solidis $\mathrm{j}$ caretarii, $\mathrm{j}$ bovarii in curiam, per annum, vjs. In allece ad pracarias auptumnales, xiijd. ab. In expensis Ricardi Salsarii in auptumno, xvii|d. In virgis colligendis ad 35 clates faciendas ad fildam, et eisdem clatibus faciendis, xiiijd. In clostura colligenda et curia claudenda, ijs. vjd. In fossatis factis circa granasium et grangiam, vjd. In granario et grangia teg[ulandis], ijs. iij $l$ Summa lx.xijs. iijd. Summa tocius liberationis et expensæ, xijl. xjs. ijd. Quietus est. ${ }^{2}$

Exitus Grangiæ.-Idem reddunt compotum de xlvij quarteriis de toto exitu frumenti. In 40 semine cijj acrasum, xxiiij quarteria dimidium. In precariis auptumnalibus, iij quarteria dimidium. In supravenditis, x viij quarteria.

Idem reddunt compotum de ix quarteriis de toto exitu mancorni. In semine ix acrarum, ij quarteria dimidium. In corredio $\mathrm{ij}$ servientium per annum, vj quarteria dimidium.

Ilem redunt compotum de xxij quarteriis de toto exitu ordei. In semine $\mathrm{x} \times \mathrm{x}$ acrarum, xij quarteria dimidium. In precariis auptumnalibas, $\mathrm{j}$ quarterium dimidium. In corredio ij servientium, vj quarteria dimidium. In supravenditis, j quarterium dimidium.

Idem reddunt compotum de iiijx vj quarteriis dimidio de toto exitu avenx. In 50 :cmine cxvij acrarum, lj quarteria. In præbenda domini Episcopi apud Suttonan, xxvj quarteria, liberata Hugoni præpositi per $\mathrm{j}$ tailliam. In præbenda avrorum, $\mathrm{v}$ quarteria dimidium. In bobus pascendis, in garbis, iiij quarteria.

Instaurum.-Idem reddunt compotum de xxvj bobus remanentibus anno præterito. Et de iiij emptis; unde superius respondent. Summa, xxx. In mortuis, ij. De coriis 55 superius respondent. In supravenditis, $\mathrm{j}$ bovettus, qui fuit taurus. Et remanent $x \times v i j$.

Idem reddunt compotum de $\mathrm{j}$ equo remanente anno præterito. Et remanet unus equus.

Idem reddunt compotum de cxxxij multonibus remanentibus anno præterito. In vivis venditis ante tonsionem, $x$ hurtardi, qui computi fuerunt multones. In mortuis 60 ante tonsionem, $\mathrm{ij}$, unde superius respondent. Et remanent cxx. 
Idem reddunt comnotuin de exx velleribus grossis. In consuetudine bercarii, $\mathrm{j}$. In supraven itis, cxix, yui fecerunt $\mathrm{j}$ pondus.

Idem reddunt compotum de xvj gallinis proventis de cheriset. Et de viij remanentibus anno præterito. In missis apud Cheritonam, omnes.

Idem reddunt compotum de $x$ vij caseis allatis de Cheritona. In cxpensis in precariis auptumnalibus omnes.

Idem reddunt compotum de iij baconibus dimidio allatis de Cheritona. In precariis auptumnalibus omnes.

Nota quod xxxiijs. iiijd. ob. de reragio anni prateriti de blado liberato lominibus de Wintonia per Magistrum Petrum Russinol, ut dicitur, est in respectu per dominum Episcopum.

\section{WALDA.}

HenRicus LA MARTRE, et R[obertus] de Brictewella præpositus, reddunt compotum de cijs. vjd. de rcragio anni prreteriti. Solvunt et quieti sunt.

Idem reddunt compotum de cxiijs. vjd. de toto gabulo assiso. Summa cxiijs. vjd. In quietanciis $\mathrm{j}$ propositi, vs., $\mathrm{j}$ fabri, $\mathrm{j}$ bercarii, vjs., iij carucariorum, per annum, ixs., $\mathrm{j}$ carucarii, per $\mathrm{j}$ terminum, ixd., iij opcrariorum, per annum, ixs. In defectu terræ Helewis viduæ, iijs. Summa xxxijs. iijd. Sumına remanens iiijl. xvd.

Idem reddunt compotum de 18 . de $\mathrm{j}$ pondere dimidio, $\mathrm{x}$ clavis lanæ grossæ venditis. Et de $v s$. ijd. de iij slavis lanie agninæ venditis. Et de xviijs. viijd. de caseo vendito. Et de vs. $x d$. de ij bobus veteribus venditis. Et de xvjd. de corio j bovis vendito. Et de iijs. de pellibus xiij ovium, xj multonum, xvij hoggettorum mortuorum venditis. Et de vs. vjd. de pellibus lxiiij agnorum mortuorum venditis, Et de vjd. de butiro vendito. Et de ijd. de pellibus iij agnorum mortuorum venditis, qui provenerunt de hoggettis. Et de xvjd. de $\mathrm{j}$ quartcrio de nucibus vendito. Et de vs. vjd. de pannagio. Summa iiijl. xvijs.

Idem reddunt compoturn de viijl. le j tasso frumenti vendito. Et de $x$ ljs. ijd. de xiij quarteriis frumenti venditis. Et de xxixs. iiijd. de xj quarteriis de mancorno vendito. Et de cxs. $x d$. de $i i j \underline{x x} . x v$ quarteriis ave 1 venditis.

Purchasia.-Idem reddunt compotum de iijjs. vjd. de Wolvrico ${ }^{1}$ pro stultiloquio. Et de ijs. de Ailwardo pro forisfacto pasturae. Et de xs. de Hugone de Duna pro bove concelato. Et de ijs. de splotgabulo. Summa xvijs. vjd.

Summa tocius recepta xxvjl. xvijs. jd.

35 Liberatio.-In liberatis J[ohanni] decano de terminis Natalis et Paschæ et sancti Johannis, xlijs. vjd., per iij taillias. Item, eidem de termino sancti Michaelis, xxxijs. xjd., per ij taillias. Item, eiden de lana vendita, xxs. Item, eidem de blado vendito, Ixs. Item, eidem, xlvs. vjd.

Summa tocius liberationis $\mathrm{x} l$. $\mathrm{xj} d$.

40 Expensa.-In ferramento iij carucarum, per annum, $\mathrm{j}$ carucr, per $\mathrm{j}$ terminum, vjs. vjd. In ferrura v avrorum, per annum, $\mathrm{jj}$ avrorum per iij terminos, $\mathrm{xxjd}$. In clutis ad caretas, $v d$. In rotis emptis ad caretas, ijs. In sellis, bacis ad caretas, vd. In saccis emptis, $v d$. In fossato circa curiam, viijs. jo. In excambitione unius avri pro meliori, iijs. iiijd. In corbella empta, ijjd. In sarpeleris emptis ad lanam, $\mathrm{x} d$. In rotis emptis ad carucils, vijd. In sera empta ad grangiam, vijcl. In rotis faciendis de maeremo domini Episcipi, vjd. In custo daeriæ, xiijjd. $o b$. In iij carucis novis, vjd. In bladis claudendis, ijs. vjd. In sarclandis cexxiiij acris, vs. iiijd. In triturandis et ventilandis xxxj quarteriis frumonti et mancorni, vs. ix $d . o b$. In triturandis et ventilandis xj quarteriis ordei, xiijd. $o b$. In triturandis et ventilandis iij ${ }^{\mathbf{x x}}$ quarteriis a venæ, vs. $\mathbf{x} d$. In bladis metendis, iijl. xiijs. ijd. In precariis hiemalibus, scilicet, $\mathbf{x}$ carucariorum, et precariis quadragesimalibus, scilicet, ix carucariorum, ijs. ijl. In allece ad precarias auptunnales, vjd. In presepibus faciendis, iiijd. In tripode empta, vjd. In stipendiis $v$ servientium, per $\mathrm{j}$ terminum, ijjs. ixd. In stipendiis iiij servientium, per iij terminos, ixs. In stipendio $\mathrm{j}$ daix, per dimidium anllum, xijd. In corio $\mathrm{j}$ avri dealbando, iiijl. In $\mathrm{j}$ bove empto, vs. vja. In liberatis præposito de Preuet, xlviijs. viijd. In liberatis Nicholao ad corredium suum, xxxvs. ijd.

Summa tocius expensæ, xijl. viijs. iijd. ob. Summa tocius liberationis et expensæ, xxijl. ixs. iijd. ob. Et debent iijl. vijs. ixd. ob.

Exitus Grangiæ.--Idem reddunt compotum de xxvj quarteriis de toto exitu frumenti. Et de xxxij quarteriis receptis de Preuet. Summa, lviij quarteria. In semine clviij acrarum dimidiæ, xlv quarteria. In venditis, xiij quarteria.

Idem reddunt compotum de $\mathrm{ij}$ quarteriis de toto exitu curailli. In præbenda avrorum, totum. Quietus est. 
Idem reddunt compotum de xlv quarteriis de toto exitu mancorni. Et de xj quartcriis receptis de Prenet. Summa lvj quarteria. In semine lxv acrarum dimidre, xxj quarteria dimidium. In corredio iij servientium, per annum, $x \mathbf{x}$ quarteria dimidıum. In corredio $\mathrm{j}$ servientis, per $\mathrm{j}$ terminum, $\mathrm{j}$ quarterium dimidium. In corredio j daiæ, j quarterium dimidium. In venditis, xj quarteria. Quietus est.'

Idem reddunt compotum de toto exitu ordei. . In semine viij acrarum, iij quarteria. In corredio iij servientium, $v$ quarteria dimidium. In corredio daix, $\mathrm{j}$ quarterium dimidium. In consuetudine bovarii, $\mathrm{j}$ quarterium. In $\mathbf{v}$ precariis auptumnalibus, iiij quarteria. Quietus est. ${ }^{1}$

Idem reddunt compotum de ccxviij quarteriis dimidio de toto exitu avenæ. Et de 10 lij quarteriis receptis de Preuet. Summa cclx.x quarteria dimidium. In semine cexxvj acrarum dimidiæ, cxiij quarteria dimidium. In præbenda $v$ avrorum, per xxvj septimanas, xj quarteria. In præbenda equi Nicholai, per ccx dies, xxvj quarteria. ${ }^{\text {" In }}$ expensis bo[um], [ix].2 In missis apud Suttunam xvj quarteria. In supravenditis, iiijx.xv quarteria. Et quietus est. ${ }^{1}$

Idem reddunt compotum de $\mathrm{j}$ quarterio pisarum. In semine $\mathrm{ij}$ acrarum, dimidium quarterium. In potagio servientium, dimidium quarterium. Et quietus est. ${ }^{1}$

Instaurum.-Idenı reddunt compotum de $x$ equis remanentibus anno præterito, et de I. 13. j cumulingo. Summa xj. In mortuo, j. In misso ad curiam per breve Eustachii de Greinvilla, j. In misso apud Alresforde, j. In misso apud Meredonam, j. Summa, iiij. 20 Et remanent vij.

Idem reddunt compotum de xxxiij bobus remanentibus anno proterito. Et de j empto. Et de j recepto de Alresforde. Summa, xxxv. In mortuo, j. In venditis, ij. In defectu debiti Gervasii de Herinodesvintho, iij boves. [Summa vj.] Et remanent xxxij boves.

Idem reddunt compotum de clx.xv ovibus remanentibus anno præterito. Et de xxiiij adjunctis hoc anno. Summa ciiij ${ }^{x x} . x_{i x}{ }^{3}$ In consuetudine $j$ fabri, $j$. In mortuo ante partum, $j$. In mortuis post partum et ante tonsionem, $x$. In mortuis post tonsionem, ij. Summa xiiij. Et remanent ciiijxx viij oves.

Idem reddunt compotum de lx.xix multonibus remanentibus anno præeterito. Et de 30 xxxvj adjunctis hoc anno. Summa, cxv. ${ }^{1}$ In mortuis ante tonsionem, lxij. In mortuis post tonsionem, iiij. Summa iiijxiiij. Et remanent iijjux vj.

Idem reddunt compotum de cciijæx.xvj velleribus lanæ grossæ. In decimis, xxix. In consuetudine bercarii, $j$. In venditis, cclxvj, quæ fecerunt $j$ pondus dimidium, $x$ claves. Et debent iij, precium, iijd. ob.

Idem reddunt compotum de iiij ${ }^{x x}$ viij velleribus lanæ agninæ quæ fecerunt iij claves. In venditis, totum, proter ij vellera, precium velleris iijob.

Idem reddunt compotum de cxliij] cnseis qui inceperunt fieri xiiij kalendas Maii et desierunt fieri vijo Idus Septembris. In decimis. xiiij. In venditis, cxxvij, unde iiijxx.xix fecerunt ij pondera et dimidim. Et xvj venditi fuerunt per se pro xiijd. Et debent 40 j de huc anno, et iij de anno præterito, qui appreciati sunt viijd.

Idem reddunt compotum de $\mathrm{v}$ baconibus receptis de Wlveseia. In expensis, omnes, in precariis auptumnalibus. Et debent iiijl. viijs. xjd. ob. unde Henricus dicit Gervasium debere iiijl. iij boves. G[ervasius] dicit contra Henricum quod injuste detinet.

$$
\text { Sic in MS. }
$$




\section{GLOSSARY.}

Aglactatio, 4 [40]. The weaning of lambs and calves. Used here to denote a period in the making of cheeses.

ACERUM, Acer. Stcel.

AcQUistanci A, 17 [43]. An acquittance, payment.

Alogvm, 60 [48]. An eel spear (cf. Prompt. Parv. s.v. algere).

Allec. Herring which was eitber red (cured with salt), or white (dried by the weather). It was apparently measured here as elsewhere, by the thou. sand or hundred, and was used, as in later times, as food for agricultural labourers.

Alico (Fr. alichon), 78 [53]. The shoot which condncted the water from the mill dam.

ALLOCARI, 73 [57]. To have allowance upon an account at the Exchequer.

ALvEUs, 49 [20]. A drinking trough 47 [35]? A hollow shaft.

AI.vU8, 54 [10]. 'l'he bnoket of the mill-wheel.

AMrRA. A dry measure of four bnshels, especially used for salt.

Apenticiom, 53 [56]. \& lean-to bnilüing, or pent house.

Appreciari. Bee Preciari.

Aqu AGIUX, 77 [60]. A water-course. Apparently a toll or due paid by external mills for the use of the stream or for passage by water.

ARATURA. The operation or customary obligation of ploughing.

A Rсн $\triangle, 67[59]$. A chest or bin.

Areqaeiva, Reragivm. The arrears of an account or debt.

Alundo, 64 [49]. A reed or rush.

Arura. Arroan, Adrora. See Aratura.

Assartare. To aggart. See Assartum.

Assarrum, 17 [11]. Woodland converted into arable by felling and grubbing trees and brushwood. Also the offence of breaking forest ground punishable by fine.

A vros (i.e. Affer), a plongh-horse.

BıCA. A hook or link of iron, apparently connected with the collar or aaddle of the dranght horge

BAco. A side or flitch of bacon ; but also the whole carcase or live auimal when referred to in connection with the larder.

BaLista, 71 [66]. An arblast or crossbow.

Barliva, 38 [20]. The office or jurisdiction of a bailiff.

BARB E civitatis, 77 [2]. The barrier, still called 'the Bars, closing the entrance to a city.

Bascat (Cornish, basced; Welsh, basged), 60 [37]. A vessel of wicker work.

Batello8, 60 [24] a barge; 13 [54] and 68 [29] a punt.

BeDELEaIs, 40 [46]. The office or jurisdiction of a beadle.

BeDripa. Service rendered by customary tenants, viz., to reap the lord's corn in harvest.

BENDA, 79 [31]. A clamp or band of iron.

Bercaria, Bercharia. A sheepfold (a permanent structnre).

Bercarius. A shepherd.

Berebertus (i.e. Horderarius). One employed to look after the barley and other grain.

Bertona, Berthona (cf. Coria), 21 [37,58] a farmyard; also a secondary manor or steading, 66 [41]

Besca, Bescha. A spade or shovel, bnt also means a hoe.

Bica. A light cart with two wheels. See Carros and CABETA.

BrLs, 54 $[8,17]$. The hail, i.e., the arched support of the upper millstone.
BLADUM. All kinds of standing corn in the blade and ear.

Bozettus, 82 [30]. A bucket.

BORDA. A thick plank.

Bordellus, 64 [7,8]. A hut or cabin with a small holding attached, just as the 'cotaginm' was the bouse and holding of the cottar.

Bosco. 'Pro besco,' for 'pro forisfacto bosci,' a fine for some trespass on the lord's wood.

Botelaria, 21 [42]. 'T'he bnttery.

Bovaria, Boveria. An ox shed or ox stall.

Bovertus and Bovicusus. A ballock of one, two, or three years of age.

Boviculus. See Bovetrus.

Baschis ROTx. The spokes of a mill wheel that supported the radial blades or 'buckets.

Braciatura, Brasiatura. A browing; materials for brewing, 69 [29].

Brasiom, BraseUM. Malt.

Brecc A, $60[32,33,39]$. A breach in a river bank.

BrenNius. Bran.

Breve, 60 [26]. A letter or writ.

Brokra, Brogre, 48 [54]. Ling, heath, furze, etc.

BuKe, 30 [43, 47], 32 [21]. A 'bnck' a he-goat.

BÜRanULU, 77 [54-59]. Honse-gafol in contradiatinction to land-gafol.

BURGAGIUM. A house or other property held by burgage tennre.

Borochium, 67 [55]. A fish trap, or 'buck.'

Busca. Brushwood, or nuderwood.

BotrRus. Butter.

Calcea, 21 [5]]; Calcia, 42 [35]. The canseway of a mill-dam or fish-stew.

Cauibs. Steel.

Camera. The chamber.

Caminds, 53 [65]. A chimney.

Canevacium, Canevaz, 49 [22]. Canvas.

Capelia, 6 [4] a cloak ; 6 [36] a chapel.

CAPELL, 71 [65]. Iron caps, or morions.

Capistrom. A halter.

Caprenius, Capriolos. A kid.

Capot calcise. See Calcia.

Capur. Equivalent to the 'pondus.'

Captr jejunil, 2 [57]; 54 [5]. Ash Wednesday.

OARBo, 17 [52]. Fuel prepared from wood ; oharcoal.

Carcare, 31 [24]. To load.

Carita, Caretta. A cart or wagon ( $f f$. Biga, Carkus, Piaustrom).

Canetarios, Caretrarius. A carter; also a cart horse. Carinare, 12 [20]. To clean the floece, probably by the action of combing.

CARRO8. A kind of two-wheeled oart or truck.

CAsEUR. A cheese, of varying size.

OAtelús, 42 [23]. See Catulus.

CAtUles, 18 [28]. A young dog, or whelp.

Uedule, 35 [53]. Apparently for 'cendn]æ '-8trips of wood used to cover the roof of a house.

Ceavisia. Ale.

Cheniset. Originally an offering of corn at Martinmas. Afterwards this due was rendered in all kinds of produce.

Cellarium. The cellar.

Chiphus, 24. [49]. Perhaps for 'cipha,' a sieve.

Cicera, Sicera. Cider.

Cimentarius, $68[40,41]$ (for Cementarius). A mason. Cimiteriom, 74. [36]. A churchyard.

Cingoldm, 74 [46]. A girth.

Cippus, 50 [33]. The stocks.

Civeros, 60 [21]. A shallow wooden tab, locally 'kiver.' 
Cramor parsus. A false plaint for want of justice in the lord's court.

Cuatus. A wattled hardle made of stakes interwoven with oziers.

Cuarus. A weight of seven pounds ased for weighing wool.

Crostura (for Olanstnra), 24 [45] an enclosure or fence 61 [39], 82 [36] brashwood for making the same.

Cuuta. A plate of iron, especially on some part of the plough or axletree.

Corinus, 79 [20]. A coffer.

Condmaria. A pigeon houge.

Com $[$ A, 24 [45]. Perhaps for CoNs, a fork.

Companagiom, Cumpanagiom. A relish eaten with bread.

Compotes. An account.

ConCeramentuM. A concealment from the cognizance of the lord's officers.

Concordia. An agreement made between two or more persons by permission of the lord's court.

Consuetudo. Customary payment, or an allowance in kind.

Contrabraseux, $64[30,34]$. Malt of an inferior quality.

Co-opertura, 72 [42]; 73 [42]. Brushwood for loofing, etc. See also Clostrura.

Coperona, 26 [22]. The loppings of trees.

Coquina. The kitchen.

C'orRelis, 42 [4]. A basket used for carrying corn.

Correvium. An allowance of food.

Cottagios, 13 [7]. A cottar.

Crepestre (sc. molindinum). Perbaps a mill for grinding 'crap'; the description of Prior's Barton mill, Winchester.

Crirra, Cribla, 31 [4] ; Crinula, 38 [51]. A aieve.

CRopr. A tenement of meadow land.

CuILLARDI. Wethers drafted from the flock to be fattened for the market.

Cumulingum, 84 [19]. An animal that attaches itself to a place of its own accord ( $c f$. Fonturtu INVENTU8.)

Comolos, 53 [57]. The pitch of the roof.

Corailidu, Curation. Small or refuse corn. (See Introduction.)

Cunia. A court-yard, probably the harton of a manor house.

Corteliagiom, Cortiliagium. A curtilage or courtyard.

Custos, Costamentum. Outlay, cost.

Cutellaries, 77 [64]. Knife-maker, cutler.

Cuva lavatoria, 2 [47]. A washing tub.

DAERIA. A dairy.

DaIA. A dairy-woman.

Dealbare, to 'raw' or tan hides; 54 [2] to whitewash.

DEPECTUS. A deficiency in an estimate of revenue for which allowance has to be made to the accountant.

Defensa. Fence or forbidden.

Deporcia RI, $79[50]$. To keep back anything by force from the rightful owner.

Dignaridu, Digneridu, 24 [49]. A dinner.

Dispensaria. The 'spens' or pantry.

DouIuM, 9 [31]. A cask or tun.

Dominicum, Dominidu. The demesne.

DrasCa. Grains or draff chiefly used as food for pigs.

Emendatio. Repair, mending.

Entus, 74 [51]. A young tree, shoot, or cutting.

Escambidm, Excambiom. An exchange.

Eschina, Schina. A chine (of pork).

EsPerductum (sc. Fis perductum), 68, 71. A portion of steel drawn to a certain length and weighing a little over $4 \mathrm{lb}$.; 30 esperducts or gads made the garb or sheaf.

Estik A, Stika, 71 [47]. A stick of eels each containing twenty-five; ten such sticks making a bind.

Estrica, Estrixa, Strica. The 'rased' bushel or 'strike.'

Estuvariv M, 58 [37]. Timber necessary to keep the ploughs in repair.

ExITUS, DE Exitu. The prodnce or issue of crops or stock during the year. Also the issue of anything from stock or store.
Exitus MANERII. That part of the charge of the bailiff on his account which deals with the profits from the produce of the manor.

ExonIs, 21 [61]. Probably a variant or miscopying of ExenNIA, presents, e.g. Christmas gifts.

Falgare. To cut or mow down.

Farasa. Oatmeal.

Fauana, 71 [66]. Bills, or pikes.

Feodru. A fee or holding.

Ferramentum. Ironwork of any kind.

FERRURA. Horse-shoeing.

Ficussiez, 75 [37]. The novus ficussiez appears in the Knoyle account in distinction to the vetus campus. 76 acres being sown in the latter and 119 in the former.

Frrma, A rent of a fixed yearly value, as opposed to one varying in amount from year to year. Also the contract by which land was let on a stock and seed lease.

Firmarids. One who rents or has a lease of anything, or who cultivates land for the owner.

Fiftedai, 66 [16]. The fifth court-day.

Frado, 4[45]. A flawn; here apparently resembling a cheese-cake.

Forago, 67 [2]. For Furagium.

Forpenous, 20 [50]. A fine for buying or recovering property before the lord's officers were satisfied.

Forispa ctum. Forfeiture upon transgression.

Fortutru inventus. Cattle regarded as bona vacantia.

Fordia, 68 [24]. Fur for ormumenting and embroidering robes.

FoveA, 68 [43]. A limekiln.

Frimentum. Wheat.

Frustum ACEri. A small picce or fragment of steel.

Furagicm, Foragium. Forage, fodder.

Furlingus, 63 [50]. Probably equivalent to the fourth part of a plongh-land.

ForRatura agnella, 18 [4]. Fur of lamo's wool, an article much used for the trimming and lining of ecclesiastical vestments.

Furlebaticum molindindm. A fulling mill.

Fosa. The spindle of a niill which passes through the fixed nether mill-stone.

Fusta, 60 [46]. The ridge tree of a roof.

Gabuldu (assisum). Land-gafol, a rent paid to the loril from customary holdings.

GAGIUM, 56 !19]. A pawn or pledge.

Gaholla (i.e. Gaolla), 68 [56]. A gaol.

GaRBA. A sheaf (of steel or of oats).

Garderora, 17 [60]. A wardrobe or closet.

Gardinum. A garden.

Genicula (O.F. genice). A heifer or young cow.

Gercis. A young ewe or 'gimmer.'

Girpaucon, $39[9$ j. A girfalcon.

Graneia. A grange or granary.

GRDELLOM. Fine flour or meal.

Gungus, Gunnus. The hooks or hinges of a door ; probably the hooks, the 'vertevellæ' which are mentioned with them, being the hinges.

Gurla (i.e. Gorma), 68 [6]. A strong, round, twisted thread.

Gutteria, Goteria, 53 [57]. A gutter.

Harnesiom, Hemnasidu, 7 [31], 6 [ยl]. Harness, baggage.

Harta, 13 [43], 15 [30]. A halter.

Haspus, 71 [60]. 'The hasp or latch of a door.

$\mathrm{H}_{\Delta \mathrm{TH}}, \gamma_{1}[66]$. Possibly a misscript for Halb[ergetum], a haubert or coat of mail.

HAYWARD. A manorial officer whose duty it was to overlook the growing crops and meadow-land.

HERBAGIUM. The pasturage of a manor that was the separate property of the lord.

Hercia. Probably a large rake for covering seed, etc.

Heriet, Heriot. The best live beast, etc. (or its commuted value) of which the tenant died possessed, which according to the custom of the manor became the property of the lord.

Hos, Hois (cf. Ligo and Picois). A mattock.

Hoccts, 68 [64]. An instrument for excavation.

Hockedare. The second Tuesday after Easter Sunday. 
Hogettus, Hoggetrus. A sheep or pig of the second year, heing apparently equivalent to the later term ' hoggaster.'

Hopa. A corn measurc, eight being equal to a quarter.

Hospitalis, 65 [19]. A hospital or hostel.

Hospitare. To receive gnosts.

Hostium, Ostivm. a door.

Hostokivs, 38 [64]. A goshawk as opposed to a falcon.

HUNDREDPENI, 64 [41]. A levy prohably connectod with the Anxilium Vicecomitis or Aid in support of the offico of the sheriff.

HUNDREDEM. The Hundred Court.

Inclusa, 38 [62]. An unchorcss, recluse.

INCREMENTUM. A permanent advance in rent or othe payment.

Inносе, 52 [22]. An jnclosure made in a commos field for the purpose of separate tillage.

INKA, $54[8,17]$. The socket of a mill-spindle. According to some authorities the 'rind.'

Instauramentur, Instatrom. Everything belonging to the stocking or sowing of land.

Jonccos (i.e. Juncus), 1 [14]. Rushos.

Karruca, 67 [37]. See OARruCa.

Karrus. See Carrde.

Kinelius, 67 [54]. A fish-weir or kettle-net.

Kiviluos, 53 [55]. A tile pin.

I ACTEN8 (sc. pullus). An nnweaned colt.

LAIA. A ride or clearing in a thicket.

Langabulum. See GabULUM.

LARDARIUM. A larder or storehouse for salted meat.

LATTA. A lath.

LATTAKE. To fix laths.

LAVATOR. One who washes (sheep).

LEPORARIU⿴. A greyhonnd.

Leprosus, 19 [62], 38 [62]. A leper.

LEVARE. To cart.

Lex. The ordeal.

Liberatio. Payment or livery. Also the payments made into or out of the Bishop's exchequer.

Liberatum. A payment or delivery.

Licentia. Licence.

LIOARE. To cooper; to bind with iron.

LINUM, 47 [8]. Flax.

LovakIUM, $68[10]$. A laundry.

MAellus (sc. porcus). A boar pig.

Makremiu, Mairemium, Mairemuj. 'Timber.

Mairardus. A weight equal to $32 \mathrm{lb}$.

Maxconsus. 'Mang-corn' or mixed corn; wheat and rye sown in the same field, and afterwards ground together into an inferior sort of flour.

Manutenementum. Maintenance-money paid to the lord by the tenant for help in a lawsuit.

Marca, a (silver) marc, equal to $138.4 d$.

Miarescaluus. (1) The Marshall of the Bishop's house and exchequer. (2) The Marshall of the King's Exchequer.

Mariscos. A marsh.

Maritaciom. Merchet, the sign of villeinage; a fine paid on giving a daughter or sister in marriage.

MARITARE. To give in marriage.

Maria. Marl.

MARLARE. To marl, drese land with marl.

Marlator. One who marls.

- Marscalleta. Farrier's work.

Marteluus. A hammer.

M a TRIX (sc. ovis). A owe sheep.

MedIa BLADI. The lord had the right to purchase half the crop at a price below the ordinary market price, commuted for a money payment.

Medsipe, Messepe, Mrssipe, Messupe. The custom of turning loose a wether-sheep for the haymakers to catch.

MELLEA. "Ficht-wite" or fine for breach of the peace.

Mensura. A measure.

Merces. Wages.

Mesaerum (for Messuaginm). A messuage.
Messor, 59 [37]. The title sometimes given to the villein who by leason of his skill ncted as "ganger or "leading hand."

Mestello, Mestilo. Mesline, wheat and rye sown together ( $c f$. MI ANCORNus).

Metere. To reap.

Miliarum. A thousand.

Minator, 68 [63]. A miner.

MISERICORDIA. Geuerally in the phrase promisaricordia, ' at the mercy of the lord,' and so liable to an as. sessed tine.

Mrur[A],2 [18]. Possibly a misscript for 'moltura.'

Mola. A mill-stone.

Molens (sc. molindinum). A corn mill.

Molindinarios. A miller.

Molindinua. A mill. (See Introduction.)

Multura. A payment for not having corn giound at the lord's mill.

Morbors. Dead wood.

Motura. See Moltura.

Mullo, 73 [40]. A hayrick.

Multo. A mutton, wether sheep.

Muta. A mew (of hawks).

NАmím. A pledge.

Navata. A ship-load.

NuCEare, 64 [48]. To gather nuts in the lord's wood.

OBLacio. An offering or gift.

Orouvs. A halfpenny.

Ociosus, Oriosus. Store cattle as opposed to draught oxen or breeding stack.

Oula. A jar or pot.

Operari, 26 [12]. To be worked, i.e. to bo in cultivation.

OrERARIUS. A customary tenant occasionally working for a daily wage.

Optorate, 54 [16] (for Obdurare). To puddle.

Ordeum. Barley.

Palfaedus. A palfrey.

PaLiciom. A paling.

Panella. A saddle-cloth.

Pania. A pan.

Pannagium. Pannage, payment to the lord for driving swine into his woods.

Paramentum, 6 [32]. An equipment.

Parcaries. A parker.

Parcus. A park.

Parmentarius. A tailor.

Paronellus, Paronus. ? Leather harness.

Passagium, 80 [4]. A river-toll on shipping.

Pastura. Pasturage.

Pastus. Food snpplied by parveyance or custom.

Pela, 68 [47]. A woolen shovel.

Perdonum. A discharge or remisgion.

Perendinare, Perhendinare. To remain behind, delay; to spend time.

Pericere, 33 [10]. To complete, i.6. to make full weight.

remgamen. Parchment.

Prrtica, a perch of land; a measure used for piece-work

Petra. A stone, 14 lb. aroirdnpois.

Pichera. A pitcher.

Picoision, Picois. A pickaxe.

Pilare, 47 [35]. To drive piles.

Pincerna. A butler.

Pis s. The pea crop.

Piscare. To fish.

Prscatio. Fishing.

Piscator. A fisherman.

Piscatoria, 55 [54]. A fishery.

Piscatura, 79 [21]. Fishing gear.

Pisciculi, 13 [58]. Yonng fish for atocking.

Pistor. A baker.

Placitum. A plea.

Prana. A beam.

Puanca, Prancha. A plank.

Planchere. To cat planks.

Plantacio, 22 [29], 42 [8]. Planting or 'dibbling.'

Plantare. To plant uut, 'dibble.'

Pleasium. Pledge, suretyship.

Plevina. Replevin, bail.

Plombum. Lead.

Porium, 47 [35] (for Podium). A pile. 
Pochia. A ponch.

Ponderare. To weigh.

Pondos. A wey, or weigh, a measure used for wool and cheese iff. CAPUT).

Porcaria. A pig-stje.

Porcanivs. A swineherd.

Porcellus, Purcellus. A young pig. (See Introduction.)

Portahios. A porter.

Potagium. A 'drinking.'

Potralda. 'The custom of enfolding cattle in the lord's fold or making payment in default.

Porra. An earthenware pot.

Pranenda. Provender for cattle; food allowance.

PRACEPTUM. An order or writ.

PrApositus. The reeve, or bailiff of a manor.

Prasepe. A cattle yard.

Pravidere. To review, survey.

Precarta. Harvest work or other gervices performed by cnstomary tenante at the lord's request and at his cost of entertainment.

Preciari. To be worth.

Pressorium, Pressura. A cheese-pregr.

Priso. A prisoner.

Prolirior. More advanced in age (of cattle). (See Introduction.)

Provection. Older (of cattle). (See Introduction.)

Proventus, Ventus. Produced or issuing from.

Pullana. A filly.

Pullandos. A two-year-old colt.

Poulos. A yearling colt.

Punralda. The pound or pinfold

Purcellus. See Porcelius.

Porcrasium. Something acquired or received; sc. the issues of the manor court payable to the lord.

Purprestura. A purpresturo or encroachment, generally on the lord's waste.

Punnoc (for Parroc). A paddock.

Quadragesima. Lent; a fortieth part.

Qoadrass. A farthing or fourth part.

QUaRellUs. A cross-bow bolt.

QUABTERIOM. A quarter (of grain).

QUIETANCIA. An acquittance, an item written off an account.

QUietarI. To be 'quit,' owe nothing.

Quietus. 'Quit,' owing nothing.

Recepta. The receipt or credit side of an account.

Rendirilis (sc. summa), 72 [11]. The amount remaining to be paid in after expenses have been met.

ReDDitos. Rent.

REA RDUM. A regard or inspection of a foresc by a verderer.

Retaxacio. Remission, release from.

Relevom, 58 [18], for Relevium. A relief.

REManens. Á remainder.

Rerlaivm. Arrears of an account.

REsIDUUM. A remainder (of money).

REspeCUM, Respectus. Respite; a fine paid for respite.

REBPONDERE. To account for.

RogUs, 18, 68 [42]. A furnace or beacon.

RotUlare. To enrol.

Rotolds Magnos. The technical term for the Great Roll of the Royal Excheqner, on which the Bishop's exchequer was apparently modelled.

RUNGUS, 60 [49]. A rung (of a mill-whecl).

Sabratum. Saturday, used hore to denote the special plough-work performed on that day.

SACCUS. A sack.

SAGENA, $61[46,48]$. A seine net.

SAGIMEN, 58 [41]. Fat, lard, used with sulphnr as a remedy for sheep.

SaginatUs (sc. porcus). A pig fattened for killing.

Saisina, Seisina. Seigin of land.

SALARE. To salt or piokle.

Salciga, 23 [48]. Saugage.

Salsarids. The officer in charge of the Saucery.

Sarcina, for Saistra.

Sarclare, Sarculare, to hoe or weed laud.

SARPELER, SARPLLERIUM. A earpler or canvas bale to hold wool; hence a measure of wool.

Sartane. To make an assart, to enclose forest land.

Sıvonuy. Soap, grease.
Scaprom, 13 [36]. Perhape for Escapium, i.e. the fine for allowing a prisoner to escape; or, perhaps, a windfall.

ScApLIARE, 68 [52j. A pparently to square timber.

Schina. See Eschina.

Sclat, Schata. A roof.glate.

Scotalle, Scotallum, 20 [30]. A ' scot-gle' or drinking bout at the tenants' expense.

Secare. To reap.

SECTA HundREDI, 66 [17]. Suit of the Hundred (yourt. Senler, for Seevleep. A seed basket (local ' sellop').

Selere, 31 [23]. To seal.

SelLa. A eaddle.

Semella. A leather harness strap.

Senescaluus. The seneschal or ateward of a liberty or manor.

Seraratio (sc. a matre). Weaning.

SEPIS. A hedge.

Septimana. A week.

SePUM. Tallow.

Sera. A padlock.

Serrura. A bolt or lock.

SERVATORIUM. A figh-stew.

Servicium. Service or duty.

Serviens, A serjeant or manorial officur; a farm servant.

Sexтавidm. A sextary.

SicCarE. To dry or 'jerk' muat.

Siccitas. Drought. (See Introduction.)

Sicera. Cider.

Sigaldi, Silligo. Rye ( $f f$. Manconnub and Mestelifo).

Silvestris Equos. A native forest horse.

Soccus. The iron 'shoe' of a ploughshare.

Bounve, Soudus. A shilling; payment iu money.

SplotgaBdLUM, 83 [33]. A payment, perhaps for pasturage.

Stakula. A stable.

Stagnum. A mill pond.

Stalo. A stallion.

StaUrum. See Instaurum.

Sternere. To fell trees.

Stipendium. Wages.

Sripula. Straw, stubble.

STRINGERE. To fasten up.

Stoliloquidm. Probably 'miskenning, to vary evi. dence given before.

Sur-serviens. An under-serjeant or under-bailiff.

Surtiuss. Delicate (of young cattle); fine (of wool)

Sorlua. A young bow. (See Introduction.)

Sulcitivm, Sulcinidm (i.e. Salsitiv m, Fr, salisoun). The work of cleaning carcases; offal.

Suhlevare (i.e. Sublevare). To raise.

Sommarios. A sumpter or pack horse.

Summonitio. A summons.

Superannalis, Superannatus. More thail one and lese than two years old. (See Introduction.)

SupERDEM ANDA. Excese of claim.

Suprodiare. To support, nnder-pin.

Supravenditus. The balance sold.

SuRsisa. A sursize or penalty for contempt of court.

Taberna. A tavern.

Talllagium, Tahiagium. A tallage; here apparently the levy in respect of scutage.

Tallia, Taillia. A tally.

Talevaz, 71 [65]. A kind of buckler or shield.

TABGA, 71 [66]. A target or small round shield.

TA8CA, TASCHA. Task-work as oppoeed to day-work.

Tassare. To put hay into cocks, to heap.

TAssator, 47 [55]. A hay-maker.

TAssum, Tassus. A herp or mow of corn.

Tecron. A roofer or thatcher.

'I'EcTuRA. A roofing or thatch.

Teloneum, Theloneum, Thelonidu. A toll.

Terminus. A term of the fieca] year.

Terragium, 78 [25]. A plot of ground in or adjoining a town.

Thadamos. A withdrawing room.

Theninga, Tredinxis. A tithing.

Tiva. A tub or cask.

Tonelud Moneluds. A cask, a tun.

Tonsio. Sheep-shearing.

Tonsura. Clipping or lopping branches.

'l'iancileia, Trenchea. A trench.

Transitus, Sc. via. 80 [33] a toll.

Tripis, 'Tripos. A threo-legged stool. 
Thiturake. To thresh.

Thituratio. Threshing.

Trubla. A hoop-net for calching fish in a stew.

UnCTUM. Lard.

UnCTURA. Grease.

ULNa. An ell.

Vaccaria, $28\left[26_{\lrcorner}\right.$. A cow-house.

VAccariús. A cow-herd.

Vaccinos. Of or belonging to a cow.

VANARI. To winnow.

Venacio, Vexatio, 4 [59], 71 [45]. Venibon.

VFATILARE. 'To winnow.

Ventokivi. A winnowing fan.

Veredictu.I. A verdict.

VerTeVELLA. A hinge-hook.

Vrscl 1. 'T'he vetch crop.

VigiLia. A vigil or ere.

Viluanus. A villein.

VilLata. The people of a vill or tow uship.

VIRGA. A wand or stake; tenure by delivery of a wand.
ViRgata. A virgate of land.

VikgatakiUs. A 'yard-mau,' a villoin with the hormal holding of a virgate.

Vimidarios. A verderer.

VisElia, 71 [65] (for Visenia). A vizor.

Visor, Viscor, 39 [14], 75 [7] (sc. foresta), one who holds a 'view' of the forest.

VIVARIUM. A fishpond.

VoloA, 47 [30]. Perhaps a windlass.

WalluRA, $3530 j$. A wall, walling.

WARANTUM, $66[18]$. A. warrant.

WARDA. A watch.

Warecta, Waretum. Fallow-land.

WARENARIUS. A warrener.

W $а$ вRA, 31 [19]. A 'yaro' or fish-trap.

W ATERLETe, 67, 69 . 'i'he custom so called, apparently of digging a water-rourse.

Willata. See Villata.

IViscare. To wattle.

Wiscator. One who wattles.

Wiscatoka. Wattle-work.

Wlpes (i.e. VulPes). Foxes. (See Introduction.) 


\section{NDEX OF PLACES.}

Alebroc (Altbrook in Twyford), 50 [14], 52 [13].

Alresforde (Old Alresford, Hants), 36 [25], 41, 42, 60-63, 81 [24], 84 .

Alta Clera (High Clere, Hants), 5-7.

Ambresbiria (Ambresbury, Wilts), 46 [60].

Awoltone (Alton, Hants), 43 [27].

Bageberge (Bagborough West in Taunton Deane), 66 [12].

Bartona (Abbot's Barton), 79

Bellum Locum (Beaulieu, Hants), 42 [14], 54 [1]

Benetlegs (Bentley, Hants), 40 [36].

Bereforda (Barford in Downton), 20 [20 $\mathrm{j}$.

Beworda, Bewrda (Beauworth, Hants), 42 [53], 81-83.

Biketona (? Bighton in Sutton), 41 [29].

Biterne (Bitterne, Hants), 3, 4 [21], 7 [65], 9 [31], 25, $30-32,36[29], 38[59], 43[9], 54$ [36].

Boterham (Bodonham in Downton), 21 [10].

Bradeforda (in Taunton Deane), 66.

Brantmede (in Bishop's Stoke), 56 [50].

Brictewella, Brichtewolla, Brictwella (Brightwell, Berke), 13-16, 19 [50].

Bristollia (Briatol), 68, 75 [1], 76 [48].

Brombrigge (Brambridge in Twyford), 52.

Bruges (Bridgewater), 63 [51], 64 [14], 68 [37].

Burclera (Burghclere, Hants), 5, 6, 7, 8 [38], 9 [59].

Bursedona (Bursledon in Bishop's Waltham), 4 [57].

Cardif (Cardiff), 17 [50].

Cedena. See Ordena.

Cheritona (Cheriton, Hants), 4 [65], 42 [53], 43, 62 [19], 80-83.

Cherletona (Charlton in Downton), 21 [9].

Chert (Chart, Surrey), 37 [40].

Chippelega (Chipleigh in Ninehead F'lorey), 66 [7].

Olarendona (Clarendon, Wilts), 21 [62].

Clera, Clara (High Clere with Burghelere, Hants), 5, 8

12,23 [36], 32 [11], 40 [54], 43 [42], 75 [48].

Cliuara (Clewer, Berks), 29.

Onoel, Choel (Knoyle, Wilts), 7 [44], 22 [24], 23, 73-76.

Corf (Corfe, Somerset), 63 [54], 64, 65 [20],66 [43], 69, 70 [46].

Cranebutne (Cranborne, Wilts), 22 [42], 23 [32].

Craweleia (Crawley, Hants), 35 [60], 36, 50-52.

Credebrige (Curbridge in Witney), 17 [5].

Crich (Oreech in Heathfield), 68 [1].

Cudeluest (Cothelstone in Taunton Deane), 66 [24].

Culnhame, Culnam (Culham in Wargrave), 28, 29.

Cumba (in East Meon), 51 [12].

Cumtona, Comtona (Compton in Twyford), 52 [38], 54.

Dene (Deane in Overton), 24 [20].

Diris[æ] (Devizes, Wilts), 54 [54]

Dorkinges (Dorking, Surrey), 60 [47].

Drokeneforda (Droxford in Bishop's Waltham), 2 [40].

Dantona (Downton, Wilts), $8[44], 15[40], 16[25], 20$

$23,25[57], 32[37], 35[61], 37[13], 75[35], 76$.

Eblesburna (Bishopston in Downton), 22 [58].

Eboracum ( York), 18 [52]

Echeneswella, Echeneswelle (Itchingswell, Hants), 7, 8, $11,12$.

Edborbiria, Edborebiria (Adderbury, Oxford), 19, 20, 57$60,74,[49], 75$ [47].

Elestede (Elsted in Farnham), 37 [29].

Esmeresworthe, Esmeresworde (Ashniansworth, Hants), 7-11.

Euere (Iver, Bucks), $3[49$ ].

Exonia (Exeter), 68 [38], 72 [2].
Falaleis (Favoley, Hants), 31 [64], 79, 80.

Ferendona (Farrington, Hants), 41 [14].

Ferham, Fernham, Farham, Farrs, Ferram (Fareham. Hants), $2[59], 3[30], 4,25-28,49,53[60], 56[51]$ $57,61,62$.

Fernham, Farnham (Farnham, Surrey), 3, 7 [45], 32 [38], 36 [30], 37-40,45[59], 46[46].

Fidoc (Fidick in Bishop's Hull), 64 [13].

Fluri (Combs Florey, Somerset), 66 [20].

Forrefelda (in Bishop's Waltham), 3 [29].

Froxfelda (Froxfield, Hants), 46, 47.

Fulfords (Frulford in Taunton Deane), 64, 67 [27], 69, $71[51]$.

Funtel (Bishop's Fonthill, Wilts), 80.

Gillingeham (Gillingham, Wilts), 76.

Glocestria (Glowcester), 6, 7 .

Hache (Oake in Taunton Deans), 66 [21]

Haiwode (in Alresford), 60, 61 .

Hameledona (Hambledon, Hants), 3, 4 [66], 27 [11], 4648,50 .

Hamptona. See Sudbamptona.

Harewella, Herewella (Harwell, Berks), 7 [66], 11 [14!, $14-16,23$ [36].

Haselegge (Hazely in Twyford), 53 [39].

Hauocleia (East Oakley, Hants), 5 [33]

Hed [felde] (Heath field in Taunton Deane), 66 [22].

Hevenstigge (in Twyford), 53 [43].

Holweia (Holway in Taunton Deane), 63, 64, 66 [32], $69,70$.

Hulle (Bishop's Hull in Taunton Deane), 63-65, 67-70.

Hungerhulle (in Brightwell), 13 [32].

Huppam (Upham in Bishopstoke), 52 [14].

Huppelanda (in Farnham), 37 [28].

Ichulla (Itchel in Farnham), 38 [62].

lvingeho (Ivinghoe, Bucks), 31, 34.

Kaldecote (Calcot in Bishop's Waltham), 1 [30].

Kantoch (Quantock Hill, Somorset), 68 [43].

Kingestona (Kingston, Surrey). 60 [48]

Kingestona (Kingston in Taunton Deane), 64, 65 [57], $67[34], 69$.

Kneppe (Knapp in Westover), 45 [4]].

Langeforda (Langford in Norton Fitzwarren), 64 [26], $66[15], 70$.

Lidiardns, Puncherdona (Lydiard Pincherton in Taunton Deane), 66 .

Lidiardus Sancti Laurenci (Lydiard St. Lawrence in Taunton Deane), 66.

Lincolnia (Lincoln), 17 [54]

Lindeseia (Lindsey, Lincoln), 8 [4], 22 [56], 23, 40, 76 [17].

Londonia (London), 13 [51], 15 [39], 30, 34 [40], 40.

Luvegerstona (Laverstoke in Overton), 24 [43].

Matykegforda (Mattock's Ford in Bishop's Waltham), 1 [31].

Medbroc (in Bishop's Hull), 67 [7]

Menes (West Meon, Hants), 3 [27], 4, 32 [39], 38-40, 44$46,50[4], 54[43]$.

Mener, Ecclesia de (East Meon, Hants), 46-48.

Menes, Capella, 44 [13]

Meredona (Mardon, Kants), 21-23, 32 [34], 34-38, 40 $[53], 50,51,84[20]$. 
Merewella (Marwell, Hants), 3, 5 [12], 26 [23], 31 [40], $45[42], 47[43], 48[6], 52-55,57,55$, , 2

Merleberga (Marlborough, Wilts), 7-9

Muleland, La (in Taunton Deane), 63 [51], 66, 67.

Nailesburna (Nailesbourne in Taunton Deane), 63-65, $67[20], 69,71$.

Nidgeheda, Nigehida Monachornm (Ninehead Florey in

Taunton Deane), 66

Norhamptona (Northampton), 17 [43].

Nortmedo (in Brightwell), 13, 14.

Nortona (Norton Fitzwarren in Taunton Deane), 66 [21].

Nortwiforda (in Twyfurd), 52 [40], 54 [17].

Norwaltham (North Waltham, Hant8), 36 [25].

Nontona (Nunton in Downton), 20, 21, 23 [12].

Occumba (Hockham in Iydiard St. Lawrence), 66 [24].

Ora (Ower, Hants), 79, 80.

Ordena (i.e. Cedena) (Cheddon Fitz Payne in Taunton Deane), 66 [23].

Otriforda (Otterford in Taunton Deane), 64, 66, 67, 6971.

Overtona (Overton, Hants), 23-25, 31, 32.

Oreneburne (in West Meon), 44 [35].

Oxenford (Oxford), 17 [53].

Pictavia (Poitiers), 68 [33], 70 [1].

Pikestona (in Otterford, Taunton), 64 [12].

Pillingebere (Billingbear, Berks), 28 [42].

Pipmerastre (Pitminster in Taunton Deane), 64 [20].

Pondesforda, Pundlesforda (Poundisford in Taunton

Deane), 63 [55], 65-69.

Portesmutha (Portsmouth), 2 [59]

Prevet (Privet, Hants), 60-63, 83, 84.

Radingia (Reading), 29 [40].

Ricchercombe (in West Meon), 44 $\ulcorner 137$.

Rimtons (Rimpton, Somerset), 70 [51], 72, 73.

Rodenham (in West Meon), 44 [35].

Rycroft (in High Clere), 6 [43].

Sancti Leonardi, Foreata de, 60 [45].

Stanforda (in Bishop's Sutton), 40, 41.

Sandelforda (in King's Clere), 5 [26].

Scaldeforda (Shawford in Twyford), 52 [36], 54.

Scocia, 15 [43].

Segrim, Molendinum de (in Sparkford), 79 [34].

Sela, Sele (Seal in Farnham), 37-40.

Senellinges (in Farnham), 37 [39].

Sidemantona (Sidmonton in Burghclere), 9 [60].

Sperkeforda (Sparkford), 77, 79.

Stanlinch (Trafalgar in Downton), 20 [63].

Staple (Staplegrove in Taunton Deane), 63-65, 67 [12]. 69,70 .

Stepe (Steep, Hants), 46, 47.

Stokes (Bishop's Stoke), 3 [3], 4 [66], 25 [21], 54 [24], $55-57,75[48]$

Stokes (Stoke in St. Mary Bourne), 10, 11.

Stokes (in Hambledon), 49 [10].

Stokes (in Twyford), 52 14.

Sudhamptona Hsmptona (Southamptom), 2 [60], 18 [5], $21[57], 26[49], 28[12], 31,42[24], 53,54,78,79$.

Suttona, Suttane (Bishop's Sutton, Harts), 4 [68], 31, $32,40-44,62[13], 81,82,84$ [14].

Suwerke (Southwark), 60

Tabrigge (in Taunton Deane), 64 [31], 70 [21].

Talanda (Tolland in Taunton Deane), 66 [12].
Tantona (Manor of Taunton Doane, Somerset), 4, 63-72, 73 [16]; burgus de, 64 [19]; molendinum de burgo, 67 53]; molendinom sab castello, 64 [26], 70 [12]

Thædelega (Tadley in Overton), 24 [41].

Theukesbiria, Teokesbiria (Terolesbury), 18 [3].

Tileforda (Tilford in Farnham), 37 [40]

Trindle (Trendle in Pitminster), 63-66, 69, 70 .

Tuiforda (Twoyford, Hants), 4 [65], 52-55, 57 .

Uppelanda (in Witney), 16, 17.

Uppelanda (in Taunton Deane), 63 [49], 67 [607.

Usseburne (Hurstbourne Tarrant, Hants), 10 [43].

Walde (Wield, Hants), 42 [54], 62, 63, 83, 84.

Wallia, $7[45], 36[29], 68[36], 71[67]$

Wallingefordia (Wallingford), i3 [5].

Waltham (Bishop's Waltham, Hants), 1-5, 27 [22], 31, $32,36[28], 41[22], 45[37], 47-50,54-57,81,82$

Woltham (North Waltham in Overton), 24 [21], 36 [25]

Woltham (Waltham St Laurence), 28, 29.

Warefelde (Warfield, Berks), 28 [24].

Watelega, molindinum de (in Otterford, Taunton), 64.

Weregrave (Wargrave, Berks), 28-30, 57, 60 [26].

Westfolda (in Bishop's Stoke), 55 [50].

Westwidehaia (West Woodhay, Berks), 9 [13]

Wicumba (Wycombe, Bucks), 30 [23], 32-34.

Widehaia (East Woodhay, Hants), 7-11

Widegate (in Wargrave), 28 [50].

Widintona (Witherington is Downton), 20, 21.

Wika, Wike (Wick in Downton), 20, 21.

Wintonia (Winchester, Hants), 5 [17], 17 [49], 30, 31 $38,39,47[43], 51[63], 54[28], 58,59,81$ [8], $8:$ [10]

Ministerinm de, 76-78.

Backestrete (Buck Street, now Busket Lane), 78 [29].

Colbrokestrete (Colebrook Street), 77 [43].

Flesmangereatrete (Fleshmonger Street, now $S t$. Peter's Street), 77 [33].

Garstrete, Grastrete (Gar Street), 77.

Magnus Vicas (High Street), 77 [38]

Paillardestwichene (Canon Street), 77 [17].

Seftwichene (Canon Street), 77 [13].

Tanberestrete (Tanner's Street, now the Lower Brook), 77 .

Wenegerestrete (Wongar Street, now the Middle Brook), 77.

Hospitalis (Domas) Sanctas Crucis (St. Cross), 77 78.

Ecclesia Sancti Michaelis (St. Michael's Church), 77 [9].

Neubrigge, domns de, 77 [42].

Terra Templarioram apud, $7 \overrightarrow{7}$ [36].

Porta Anstralis (South Gate), 77, 78.

Poria Borealis (North Gate), 77, 78.

Porta Castelli (Castle Gate), 77 [23].

Kingate (King's Gate), 77, 78.

Porta Occidentalis (West Gute), 77 [57].

Porta Orientalis (East Gate), 76-78.

Fossatum et barra, 77 [2]

Mons Sancti Egidii (St. Giles' Hill), 78

Mons Sancte Katerine (St. Catherine's Mount), 82 [17].

Molindina de, i8, 79.

novum molindinum, 78 [49].

molindinum de Flodstoc, 79 [35]

de Fonte Segrim (Wharf Mill), 78 [41].

Sparkeforda, 79 .

Bartona (Crepestre), 79

Witteneia (Witney, Oxford) $5-8,14[63], 16-20,59,60$.

WIveseia (Wolvesey, Hants), 4, 5, 7, 8, 10-12, 27 [16],

$30[25], 35-37,39[11], 41-43,45,46,48-52,54,55$, $57,62,63,82[6], 84[42]$. 


\section{INDEX OF PERSONS.}

Abrabam Judæus, 60 [15].

Acardus, 78 [12]

Ace, 10 [23], 12 [10].

- - Nic., 17 [59], 18 [48].

Petrus, 27 [20], 48 [5], 51 [20], 57 [13], 60 [39], $62[13]$

Acelinus, 1 [38].

Acere, Acerre, $\overrightarrow{5}$ [61], 6 .

- Will., 10 [27]. de Helle, 6 [1.9].

Achardns, $55[46$ ].

Aclanda, Aucland, G. de, $6[60], 17,18[54], 27[24]$, $33[60], 39,42,45[33], 58[45], 59[16], 60[42$ $74[63], 75[42]$

Acsete, Ric. de, $45[11]$

Adam, Ada, $52[47], 65$ [48], 79 [48].

- caretarins, 78 [3]

clericus, $64[22], 65[60]$

de Ecclesia, 44 [39].

haiwardns, 24 [13].

præpositus, 1 [1], 11 [39], 81 [63].

warennarius, 2 [51].

Walteri, 3 [45]

Adan fil. Avicio, 41 [39].

- - Hngenis, $66[4]$

Leviva, 65 [25].

_- Malgeri de Stanfords, 41 [57].

- - Sewari, 38 [24].

- Willelmi, 65 [2l]

_- - vidux, 78 [37]; 79 [21].

Agatha vidua, 52 [44], 53 [9].

Agene, 7 ; $[50]$

Agnes, 17 [17]

Ailet, 20.

Ailmeri, v. Galfridus.

Ailricus, 52 [57]

Ailwardi, v. Willelmns.

Ailwardus, 83 [32]

-_- herebrettus, 20 [9].

- n magister, $39[57], 47\lceil 39]$.

Ailwinns, 41 [55], 43 [8], 45 [11], 58 [20], 81 [32].

- fil. Willolmi 58 [21].

—- molindinarins, 80 [40]. Simonis, 6 [8]

Ainolfus, 41 [49], 52 [51].

Aiwardns, 17 [18]

_- magister, 39 [57].

Akinges, Galf. de, 35 [50], 36 [ $\angle 0]$.

Alani, v. Willemus Alani.

A] anus, 82 [23].

- copellanus, $45[1]$

- nagister, $2[54], 3,5,17[58], 18,19,20[3]$.

Albrea, $v$ sireforda.

Albreda, $v$. Ohipelega.

Albury, canens of, $v$. Novo Loce.

Albus, v. Azo, Ingo, Nicolans, Osbertus, Thomas. Willelmus.

Ald' ridna, 6 [6].

A llire[dns], 44 [49].

Aldulfis, 78 [5].

Alebrec, Galfr. de, 56 [31]

Alexander, 38 [22]

_ allutarins, 78 [ 42$]$

- prxpositus, 16 [31] 17 [2]

Alf redns, $12[10], 49[8]$.

- fil. Boie, 77 [20]

Algar vidua, 65 .

Algerus gardiner, 56 [23].

Alicia, 58 [21].

_- do Sancta Maria, 78 [17].

Allutarius, v. Alexander.

A]nathus 74 [55].

Alresforde, Osbertus de, 24 [23]

Alricus fil. Ingmonis, 49 [2].

Alvet vidua $\mathrm{T}^{\mathrm{a}} \mathrm{arneri,} 33$ [16].
Alvredus, 66 [3].

pistor, $78[57]$.

- præpositns, 54 [26].

Alvricus, 33 [14].

A]winus, 6, 10 [26].

Andreas clerions de Cancellaria, $17[64], 18[50]$. capellir, 77 [66].

de Wallingfordia, 13 [12].

Anfeld, Wido de, 35 [12].

Augerus presbyterus, 77 [5].

Ang[evin], Rog., 64 $\{60$ j

Anketillus, 20 [47], 26 [11], 45 [5].

A psle, Ric. de, 65 [49.

Aqua, Joh. de, 2 [16]

Arnaldus, 13 [31].

Arnoldns prapositns, 50 [9], 54 [26]

Arnulfus vinitarius, 60 [18].

Artifex, v. Galfridns.

Aucland, v. Aclanda.

Audelini, v. Galfridus.

Andelinus serviens, 46 [63], 47, 45 [33].

A urifaber, v. Ricardus.

Avice, 41.

Azo, 1 [9], 77 [13].

- albus, 31 [63].

—— præpositus, 30 [32].

B. fil. Galfridi, 64 [12]

Babileia, Passor de, 18 [25].

Bacon, Ric., 1 [9]

Bacsete, Edm. de, 38 [17]

Galfr. de, 38 [18]

Baldewini, v. Ricardus Baldewini.

Baldicot, Will., 24 [17].

Baldwinus, Baldewiuns, 17 [5], 28, 77 :3].

Balistarius, v. Henricus, Thedbaldus.

Ballinge, Edw., 56 [8].

- Thom., 52 [62].

Balun, Ric. de, 78 [33].

Banne, 44. [63], $45[6]$.

Thom., 44 [59].

Barat, Ric., 53 [13].

Walt., 38 [1].

Baratte, Will., 60 [26]

Barda, Adam, 64 [56].

Bartolomaus de Walingeforda, 78 [2].

Bas, Edw., 30 [55].

Basilla, 1 [44].

Basset, Rob., 2, 3, 5 [5], 13 [32], 21 [62], 22 [21], 23, 26

$[46], 29,31[29], 35[51], 36[20], 34,40[31], 42,43$

[38], $45[45], 47[23], 50[60], 51[21], 53,54,56$ [52], 57 [14], $60[44], 75,76$.

Bastardns, Rad., 17, 18, 39 [10], 75 [5], 76 [41].

Will., 6, 7, 39 [55].

Bans, Symon, 21 [3]

Belami, Ric., 53 [5].

Belengirus, 78 [14].

Belfiz, Rog., 20 [5:3].

Bello Monte, Ric. de, 26 [12].

Bellus, v. Ricardus.

Benedictus, 78 [8]

- fil. Edwini, 61 [23].

Bere, Alfr., 24.

Bercarius, v. Galfridus, Gilibertus, Hago, Willelmus.

Berebrectus, v. Ailwardus, Ricardus, Willelmus.

Beringer, Adam, 65 [15].

Bernardus faber, 52 61?

Bertona, Rob. de, $56[30]$.

Bertramus speciarius, 77 [19].

Beupeine, Will., 78 [29].

Bigedena, Alex. de, 47 [15].

Bikebroc, Algerus de, 56 [17]

Wa]t. de, 56 [25].

Bilcdona (Bindon), Abbss de, 39 [11]. 
Biliz, Edw., 20 [56].

Billinge, Ric., $6[4]$.

Biretmar, Will,, $20[60]$.

Bissop, Adam, 65 [44].

Hugo, 68 [23].

Blakeman, Blakemannus, 74 [3].

Godwin, 9 [11].

— Rob., 41 [4].

- Rog., 9 [16].

Steph., 44.

- Will., 74 [55].

Blakewine, Galfr., 44 [46]

Blankardus, 49 [3]

Bleche, Galfr., 36 [24].

Blundus, Joh., 10

- Rog., 6 [18].

Thom., 44 [32].

Will., 12 [2].

Boia, 77 [20].

Boilat, Hugo, 38 [8].

Bole, Ric., 41 [32].

- Steph., $45[9]$.

Bolonia, Rob. de, 78 [16].

Bolt, Will., 12 [2].

Bor, Rog., 1 [47].

Bord[ene], Gocelinus de, 44 [31].

Bosco, Rob. de, 38 [27]

Will. de, 11 [47], 44 [59].

Bovarius, v. Johandes.

Brandus, 1 [42].

Bren, 20 [48]

Bresa, Ric., 66 [3]

Brea, Ernaldus, 21 [?]

Breuile, Helia de, 39 [12].

Brianus, 79 [47].

- de Garstrete, 78 [9].

Brichtewella, R. de, 63 [6].

- Rob., 83 [13].

Bricwini, v. Ricardus.

Brienuus, 79 [41].

Briwerre, Briwerus, Will., 39 [4], 64 [15].

Briwes, Joh. de, $45[45], 46$ [8].

Broche, Rob., 64 [56].

Brombrigge, Gilibertus de, 56 [6].

Gunhilda de, 56 [8].

Joh. de, 53 [15]

Thom de 56 .

Will. de, 56 [12].

Brun, Ric., 21, 5.

- Symon, 6 [3].

Walt., 10 [30]

Brung', Jocelinus, 45 [13].

Bubhe, Hugo, 6 [22]

luudde, Gauterus, 65 [29].

Bule, Adam, 78 [36].

- Ric., $65[52]$

Rog., 5 [42].

Bundi, v. Willelmus.

Burel, Walt., 21 [5].

Buric, Will., $6[9]$

Buriman, Symon, 77 [65].

Rus, Will., $2[2]$

Bux, Galfr., 65 [15].

Caie, 45 [2]

Cake, Rad., 32 [52]

Calceto, Cauz, Galfr. de, 7 [29], 17, 18, 33 [61], 39, $42[58], 43[53], 60,74[63]$.

Cale, Nich., 77 [6].

Oalvus, v. Ricardus.

Oamera, $v$. Dionisius

Cantoc, W. de, 28 [19], 19 [9].

Cantuariæ, Hen. Archidiaconus, 77 [11].

Capellanus, Alanus, 4.5 [1].

Geroldus, 65 [58]

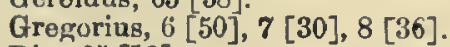

Ric., 35 [12].

Capellir, Capillir, v. Andreas, Ricardus.

Oapistrarius, v. Walterus.

Cappe, Will., 2 [17].

Capra, Steph., 20 [59].

Caprarius, $v$. Willelmas.

Oardevilla, Hen. de, 76 [57]

- magister Г.. de, 78 [23]

Caretarius, Carettarius, v. Adam, Lifing, Radulfus, Willelmus.
Oarpentarius, v. Martinus, Hugo, Ricardus.

Carter, Rob., 44 [35].

- Will., 44.

Carucarius, v. Erualdus, Robertus.

Casse, Adam, 66 [4]

Castoh., 66 [1]

Castello, Joh. de, 32 [55].

- Ric. de, 53 [13].

Cattesbert, 49 [10]

Cauz, v. Calceto.

Cave, Bernardus, 20 [56].

Galfr. de, 17 [66].

Cerarius, v. Radulfus.

Ohacheis, 44[60].

Cheffinges, Blakemannus, 5 [38].

Cherda, Ohelm', Rog., 65.

Cherdesleia, Walt. de, 17 [11].

Chert, Andreas de, 38 [28].

Chet, Walt. 64 [59].

Chicapadere, Chicledere, Chilcapadere, Adam de, 65 [37] 74 [27]

Will. de, 65 [24].

Chilwinus, 64, [57].

Chipelega, Albreda ridua de, 66 [17].

Thom. de, 66 [?]

Chnihtebrigge, Edm. de, 11 [61].

Walt. de, 12 [3].

Chus, Will., $48[6 \mathrm{C}]$.

Cimentarius, v. Ricardus.

Cittel, Citte, Ric., 6 [11].

- Will., 5 [62].

Clara, Comes de, 39, 40.

Clatera, 74. [2].

Olemens, 10 [28]

Clera, Clara, Will. de, 39 [21], 40.

Clericus, v. Adam, Andreas, Jordanus, Osbertus, Ricardus, Symon, Walterus, Willelmus.

Clopparde, Will., 60 [17].

Onavehalle, vidua de, 56 .

Onihtebrigge, Walt. de, 12 [3].

Cnolle, Will. de, 56 [22]

Cobbe, Herebertus, 56 [21].

- Reg., 77 [9], 78 [6]

Will., 41 [51].

Coc, Rob., 52 [16].

Coch, Iro, 50 [35].

Cochage, Phil. de, 65 [40].

Cochel, Rog., 64 [55].

Cocus, Nich., 23.

Cocus, Nich., 72 [48].

Ric., 1 [48], 2

- Will., 74 [55]

Cokerel, Joh., '24 [12, 26].

Cole, Ric., 65 [1].

- Walt., 6 [10].

Oolebroc, Ósbertus de, 56 [26].

- Rad. de, 56 [16]

Colegrime, Rad., 41 [47]

Colesneni, Rob., 2 [18]

Uoleswinus, 1 [37].

Colvedene, Arnoldas de, 52 [58].

Combe, Hunfredus de, 32 [46].

Comes (i.e. Earle), 20 [45].

- Gilibertus, $2[6]$

- Will., 41 [49].

Constabularius, v. Osmundus.

Coppc, Rob., 21 [2l], 22 [36].

Coquina, v. Nicholaus, Walterus.

Coquus, v. Willelmus.

Corfe, Herman de, 65 [23].

- Simon de, 65 [23]

Corkieu, de, $65[20]$

Cornhulla, R[eginaldus] de, 39 .

Cotere, vidua, 2 [3]

Covenant, Will., 41 [63].

Craft, Will., 52 [59].

Crassus, Adam, 49 [2]

Ores, Hen., 28 [48]

Orikelade, Kic. de, 78 [25].

Cristina filia Belengiri, 78 [13].

— vidua Edrici, 65 [5].

- Ruffi, 33 [15].

Croperie, Phil. de, 5 [3], 43 [62].

Cruce, Godfridus de, 41 [ 53$]$.

Cruce, Godfridus de, $41[53]$
Rob. de, $41[31], 66[6]$

- Steph, de, 28 [12] 
Cruce, Will, de, 21 [3].

-Wulwinus de, 5 [35].

Cruda, 52 [15].

Orulle, Rob., 48 [59].

Orune, Ernaldus, 21 [4].

Onderigge, Curderigge, Gunnilda de, 2 [9].

Osbertus de, 1 [41], 2

Cumba, Cumbe, Galfr. de, 44:

Gilebertus de, 44.

Jord. de, 65 [31].

- Reg. de, 9 [10], 44 [43].

Rog. de, 44 [56].

Ric. de, 44

Cumhicham, Walt., 21 [13].

Ouminora, Baldwinus de, 17 [55], 18 [45], 19 [59], 20 [3].

Cunan, Will., 38 [2]

Cune, Adam, 2.

Cupere, Walt., 6 [12].

Cupping, $6[20]$.

Curderigge, v. Cuderigge.

Cutellarius, v. Galfridus.

Dal, Will., 20 [55].

Damartin, v. Danmartin.

Daniel pincerna, 5 [19], 28 [12], 31 [22], 42 [58].

Danmartin, Damartin, Will. de, 25 [63]; 26 [47].

David, 47 [40].

Dccanas, v. Jobannes decanus, Rogerus decanus.

Dene, Petrus de, 9 [14].

Deneforde, Rob. de, 51 [13].

Depeforda, Rob. de, 65 [41]

Derewinus, Dereuin, 32.

Dionisius de Camera, 18, 38 [34].

Dipres, Rog., 5 [62].

Dispensa, $\boldsymbol{v}$. Jobannes.

Diva. Alicia de, $77[29,40]$.

Dodde, Will., 52 [54].

Doddehnlle, Adam de, 65 [51].

Dokenesfelda, Phil. de, 38 [26].

Dolbohe, Reg., 41 [59].

Dolfin, Rog., 2 [11].

Dom, Rob., 56 [18].

—, Will., 56 [15]

Done, Wa]t. de, $41[34 j$.

Dorsete, Vicecomes de. 70 [3].

Drake, Hunfridns, 41 [45]

Duc, Ric, 4 [5]

—-Will., 1 [38].

Duddingwoda, Algerus de, 52 [16].

Dudelestona, Almar de, 65 [28].

- Hen. de, 65 [35].

Dugel, Bernardus, 21 [16].

Dnlere, Ric., 17 [18].

Duna, Hug. de, 83 [32].

Dunlee, Algerus de, 34 [2].

Dunna, Eva, 17 [8].

Durc, Hen., 72 [29], 73 [16].

- Rog., 64 [59].

Dust, Osmundus, 5 [41].

Eblesburna, Thom. de, 80 [7]

Echeneswella, Godfridus de, 12 [3].

Ed. . ., Fuloo de, 46 [59]

Edgarus faber, 12 [7], 77 [28].

- niger, 12 [7].

Edit, 33 [17].

Editha, 20 [48].

Edmundus, 10, 37 [25, 38 [19], 4I [33], 52 [46], 53 [6].

— de molindino, 1 [49]

- præpositus, 38 [20].

Eiulfi, 10

Edredus, 56

Edricus, 55 [51]; 56 [15], 65.

Edwardus, 1 [43]

grossus, $30[5,4]$

molindarius, 24 [18]

de molindino, 64 [24].

Edwi, 7 [50].

Edwinus 17 [13], 38, 40 [2], 61 [25], 82 [32]. filius Godwini, 38. novus, 6 [9].

Ffforda, Will. de, 44 [40].

Eilricus, 38 [17].
Eiìns, 5 [64].

Eli, Heli, Thom. de, 6 [51], 7 [31], 39 [13].

Elias præpositus, 75 [ə]

Ellestede, Elestede, Rob. de, 2 [56], 3, 5, 21, 31, 45, $46[9]$.

Elviat Geroldi, 44 [51].

Emma, 20 [48]

- vidua de Halnake, 65 [7].

Emnolt, Nicolaus de, 35 [13].

- Walt. de, 36 [36]

Ernaldus, 61 [25].

- carncarius, 21 [8]

- fil. Comitis, 20 [45].

Ernolfus, 28 [47]

Eslac, Eadmundus, 75 [47].

Esmundus, Estmundus, 53.

Estons, Paganus de, 1 [52].

Estord', Randulfns de, 44 [55].

Estwichus, Nicolaus de, 9 [15].

Dverardus, 48 [41], 78 ! 15 ].

Faber, v. Bernardus, Edgarus, Godefridus, Johannes, Ricardns, Rogerus, Stephanus, Walterus, Willelmus.

Faccumba, Alwinus de, $5[60]$. Walt. de, 5 [61]

Faleleia, Will. de, 79 [39].

Fatte, Adam, 65 [28].

Felda, Rad. de, 2 [14].

- Walt. de, 35 [19]

Fent, Hugo, 17 [1].

Ferhem, Will. de, 68.

Fernham, leprosi de, 38.

Ferliche, Godmundus, 41 [47].

Fichet, Jord., 35 [17].

Eidoc, Jord. de, 64 [14].

Fis, Elias, 21 [19].

Fitil, 49 [10]

- Will. 49 [9].

Flandrensis, v. Gilebertus.

Fleca, Rog., 65 [1]

Flode, Rad. de, 41 [46].

Fluri, 38 [13].

- Simon de, 68 [67].

Thom. de, 68 [27]

Fohel, Ric., 52 [43].

Foleforda, Rog. de, 65 [35].

Fonte, Godwin de, 41 [58].

- Ivo de, 53 [3].

Ric. de, $41[60]$

vidus de, 2 [20]

Forde, Alwinus de, 6 [17?

Forestarius, v. Gilebertus, Osbertus, Waltcrus, Willelmus.

Fox, Wineman, 35 [14].

Foxcote, Rob. de, 51 [2]

Franciscus, Galfr., 56 [24].

Frawine, Galfr. 21 [6].

Fraxine, Walt. de, 35 [9].

Fraxino, Rio. de, 21 [18].

Freke, Galfr., 74 [25].

- Rad., junior, 52 [55].

Frere, Awinus, 48 [61].

Frewini, v. Willelmus.

Frida[m], Rad. de, 28

-_ Ranulfus, 28 [49]

Fride, Thoma, 70 [34]

Frithe, Ernisius de, 2 [13]

Frogge, Edw., 1 [31], 4 [4].

Froxfelda, vidua de, 44 [55]

Fngellus, 65 [34].

F'ulco, 17 [45], $18[40], 28$ [10].

Fulforda, Hegia' de, 65 [38].

Fungh', Edw. de, 44 [44].

Galfridi, v. B.

Galfridus, 32 [50], 40 [47], 44 [52], 48 [41].

$\rightarrow$ Ailmeri, 56 [20].

_- artifex, 53 [1]

artifex, $53[1]$; 60$]$.

cutellarins, 77 [64]

filius Edmundi, 52 [46].

- Petri. See Jnsticiarins.

molindinarins, 17 [4], 38 .

de Porta, 26 [4].

præpositus, 10 [8], 25 [63], 26 [7].

- serviens, $40[58], 46[63]$. 
Galinant, Thom, de, 64 [62].

Galmatons, Edricus de, 70 [45]

Gardinarius, Algerus, Ricardus.

Gardre, Ivo, 35 [10].

Garlieo, Rad., 52 [49].

Gartrete, Brianns de, 78 [9].

Hen. de, 78 [27].

Gaterigge, Walt. de, 41 [39].

Ganbert, 78 [33].

Geile, Galfr., 17.

G[e]ldun, 45 [7]. 17 [14].

Gcrardi, v. Willelmns.

Gerardns, 17.

Germ[anus], 45 [38].

Geroldi, v. Elvist.

Geroldne capellanns, 65 [58].

Gerstona, Ric. de. 6 [13].

Gerstone, Ric. de la, 6 [14?

Gervasius, 9 [15], 35 [51], 39 [16], 41 [37], 58 [20], 76 [63], 84.

-v. Johannes.

- venator, 3 [43].

Gible, t:.

Gilbertus, Gilibertus, Giilebertus, Gilebertus, 9 [18], 20 $[9], 21[63], 24,52[47], 65[10]$

- bercarius, 28 [44], 56 [6].

- filius Agathro, 52 [44].

- Flandrensis, $77[7]$.

- torestarius, 39 [12].

- præpositus, 82 [10].

- tegulator, 2 [52].

- vaccarius, 41 [45]

Girardus, 32 [49].

Gitte, Rob., 79 [47]

Glide, Ric., 20 [57].

Gocelinus, $9[13], 24[22], 38[6], 78[39], 79$ [22].

- propositus, 20 [9]

Goda, $28[46], 44[60], 49[5]$. Waldw, 45 .

\section{-v. Osbertus.}

Godefridus Episcopns, v. Wintoniensis Episcopus, faber, 49 [9].

præpositus, 13 [2], 15 [10].
Godester, Margarets de, 77 [38].

Goding, 39 [18]

Godira, 17 [19]

Godwin, 38 [5], 49 [4], 65 [11].

Goler, Guber, 73, 74 [1], 75 [2].

Gonnilda, 58 [29].

Gore, Ric., $65[46], 70$ [47].

Gos, Galfr., 24 [23].

Gosecrei, Galfr., 44 [54].

Gosei, Rob., 2 [13].

Gosse, Edw., 31 [63].

Govilda, 17 [16].

Grandel, Ric., 49 [5].

Grangarius, v. Willelmus.

Grant, Arnaldus, 17 [14.

- Ric., $17[3,9]$.

- Rob., 21 [6]

-... Symon, $17[10,20$

Grava, Galfr, de, 74 [8]

Gray, Ric. de, 58 [44], 59 [9].

Grede, Ric., $45[6]$.

Gredere, Godefridus, 44 [63].

Gregorins, 12 [5], 13 [2].

capellanus, $6[50], 7[30], 8[36]$.

Greb, Ric., 53 [8].

Groinvilla, Grenvilja, Eustachius de, 3 [48], 4 [47],

$[9], 7[29], 17[66], 18,19,45[46], 48,62[25], 75$ Greta, 52 [63].

Grey, Ric. de, 59 [9], 60 [10].

Grime, Ric., 2] [4].

Grom, Hugo, 38 [8].

Grossns, v. Edwardus.

Gule, Galfr., 44 [45].

Gunnilda, 65 [25].

Gunterus, $2[21], 56[9]$.

Gute, Will., 79 [49].

Gutte, - de Faleleia, 31 [64].

Hacbe, Thom. de, 66 [21].

Hadewolf, $7[50]$.

Will. 6.
Hadhewis, Ric., 44 [52].

Haidona, Edw. de, 65 [13].

Haistona, Walt. de, 52 [43].

Haiwardus, v. Adam, Willelmus.

Haiwode, Guibot de, 61 [26].

Hnle, Galfr. de, 74 [6]

Walt., $21[15$

Hales, Rob. de, 5 [7], 8 [37].

Halnake, Emma vidua do, 65 [7]

Halstede, Hngo de, 44 [44].

Hamo, cocas domini Regis, 17 [62], 18 [41].

Hamptesira, Will. de, 52 [56].

Hardekil, 17.

Harefangus, 60 [44].

Haringe, Nic., 38 [65], 39 [5]

Harold, 61 [1].

Ric., 56 [18].

Hasardus, 52 [17].

Hestiler, Hen., 41 [30]

Hatelinca, Hen. de, 41 [38].

Hattingelega, N. de, 68, 69 [65].

Haver, Rio., 20 [54]

- Wnlvrious, 21 [1]

Havercampe, Havercop, Hen. de, 41 [42]. Joh. de, 41 [61].

Rog. de, $40[62], 41$ [43], 43 [65].

Hawisia, Hawis, 26 [13].

- vidua, 52 [48]

Heche, Steph, de, 2 [15]

Hedewolf, 6 [21].

Helewisa, vidna, 83 [18].

Heli, Thom. de, 6 [51], 7 [31].

Helias, Palmer, 65 [16]

Helle, Aoerre de, 6 [19].

Henricus, 24 [27], 55 [53], 56, 84.

- balistarius, 54 [29], 55 [37]

flins Acelini, 1 [38].

- Azonis, 1 [9].

- Hugonis, 17 [12].

Radalfi, $1[46 \mathrm{j}$.

- de Garstrete, 78 [27].

- pistor, $3[40], 56[60]$.

præpositus, 55 [42].

- villatæ, 1 [2].

Herebertus, Herbertus, 24 [26].

- præpositus, 50, $52[5]$.

— Ric., 20 [57].

Herefordia, v. Petronella.

Hereveus, 26 [3.

Herevi, 65 [42].

Herfast, Arnoldus, 77 [45].

Herinodesvintho, Gervasius de, 84 [24].

Hernebi, 6 [21].

Herneburgus, 32 [52]

Hersent, 44 [58].

Ric., 52 [53].

Hervi, 41 [37].

Hervious, 2 [21].

Hestona, Thom. de, 1 [50].

Hevede, vidua, 38 [3]

Hilda, 17 [6].

Hiltonebrich, Adam de, 35 [19].

Hocs, Hen. de, 48 [62].

Hocre, 44 [61].

Hoke, Thom. de, 44 [50].

Holweis, Godwinus de, 70 [34].

Hola, Osbcrtus de, 66 [13].

Holemore, Walt. de, 1 [49].

Holewei, Walt. de, 44 [40].

Holeweiæ, Rog., 74 [4].

Holeweim, Rog., 74 [4].
Holme, Ric. de, 12 [9].

Warinus de, 33 [17].

Hoppere, Nic., 35 [15].

Hordrun, Will., 44 [63].

Hose, Galfr., 41 [6].

Hotot, $\mathbf{R}[\mathrm{ob}] \mathrm{de}, \mathbf{7 4}[64], 76[48]$.

Hou, Thom. de, $1[51]$.

Hud, Hudi, Alwin, 2 [2].

- Michael, 1 [3].

- Reg., 41 [51].

Serlo, 41 .

Hago, $17[12], 18,37,38,40[58], 49[2], 53[10], 66[4]$ $72[17], 77[10]$

albus, 9 [12].

- bercarins, 54 [49]

carpentarius, 62 [17]

flius Natricis, 41 [41]

- longus, 65 [49]. 
Hugo magister, 77 [10].

- medicus, 77 [8]

— piscator, 27 [35].

- propositus, 37 [25], 40 [58], 82 [52]. de Putea, $41[40]$

de Tribus Aulis, 79 [50].

Hulla, Hulle, Eustachius de, 16 [41].

- Rob. de, 38 [14]

Hundesher, Rob., 41 [52].

Huntingdoniæ, Hen. Archidiaconus, 18, 19.

Hnnwini, v. Nicholsus, Robertus.

Huppehulle, Warinus de, 66 [2]

Hurlega, Osbertus de, 28 [50].

Ildulf, 53 [2].

Ilestan, Hen. de, 45 [3].

Inglesham, Alanns de, 77 [35].

Ingulf, Rob., 53 [4].

- Will., 55 [13]

Ingulfus, 26 [11].

Insula, I vo de, 52 [58]

Rob. de, 50 [38]

_ Rog. de, 77 [6].

Isp ania, milites de, 60 [42].

Iver, Reg., 33 [16]

Ivingeho, Joh. de, 38 [2].

Iro, $24[26], 52[55], 53[7]$.

J. præpositus, 80 [23]

Jacubus, 17 [7], 20 [49], 80 [20].

Jaile, Eudo de la, 18 [49].

Wido de la, 17 [61].

Janitor, v. R.

Jocelinus filius Godæ, 49 [5].

frater Reginæ, 77 [61].

pistor, 77 [17].

Johannes, 2 [20], $11[6], 38[16], 74[7]$.

- bovarius, 52 [52].

- decanns, $2[24], 4[61], 6$ [27], 7 [38], 8, $9[21], 10$ $11,12,13[39], 15[26], 17[27], 21[25], 24[29], 25$ $[58], 26[35], 27[16], 28[54], 30,33[21], 35,[23]$ $37[19], 38[32], 41[66], 42[1], 43,45[19]$ $46,47[23], 48[3], 49[13], 50[43], 51,53,54[46$ $56[35], 57,58[33], 61[29], 72[30], 74[31], 78$ $79,80,82,83$ [35]

- de Dispensa, 4 [46], 22 [4]

faber, $74[267$

de Wintonis, 5 [15].

flius Govildæ, 17 [15]

Hugonis, 18, 38 [15].

Normanni, $20[60]$.

Petri, 77.

Ricardi Baldewini, 30 [53]

Udelini, $56[20]$.

Gervasii, 24 [16].

- juvenis, $6[10]$.

- Leterich, 41 [48]

de Londonia, 38 [33]

mercenarius, 77 [4]

molindinarius, 53 [16]

præpositus, 32 [42]; 8 [16].

- Rex Angliæ, 3 [16], 8 [36], 13 [12] 17, 18, 19 [43] 21 [61], $22[19], 23,39,40[42], 42[26], 43,54,55$, $58,68,70,71,72[4], 76,77[63], 78[28]$.

erviens, $6[46], 30[32], 31[20], 60$

—_ de Biterna, $79[39]$

Jordanus clericus, 38 [39].

_- filius Normanni, 20 [61]

Judaus, Abraham.

Juliana vidua Noreis, 80 [21].

Juonet, $20[51]$

Justiciarins, $39^{\circ}[4], 40[39,51]$.

Juvenis, v. Johannes, Petras, Ricardus, Rogerus, Symon.

Kemelif, Symon, 28 [43]

Kena, Kene, David, 17 [16]

Rio., 17 [11]

Kepping, Rob., 77 [32]

Kier', Will., 52 [55].

Kine, Edwinus, 38 [23].

Kinedoit, Rog., 13.

Kinge, Will., 56 [28].

Kingestona, Wlvricus de, 65 [22].
Kingwde, Osbertus, 74 [5].

Kitier, Hugo, 41 [31].

Kunlle, Kiville, Nic. de, 72, 80 .

Kokus, 49 [1].

Kulneham, Rog. de, 13 [37].

Laci, domina Iseut de, 38 [32].

Lake, Osbertus de la, 28 [45].

Lane, Rob. de, 20 [58].

Langelega, Osbertus de, 1 「8].

Laurencius, 35 [17], 77 [13].

Le, Hugo de la, 41 [42].

Lega, Oswardus de, 65.

Lega, Oswardus de,

Rob. de, 63 [48], 64 [7], 65 [9].

Legha, Adam de, 56 [14].

Leghe, Ric. de, 48 [62], 49 [6].

Leo, Rog., 79 [21].

Lep, Baldwinus, 29 [57].

- Rad., 28 [48].

Symon, 28 [41]

Lepus, Fulco, 77 [42]

Leticia, 47 [16].

Lenera, 33 [14].

Leverich, v. Johannes.

Leverici, v. Petrus.

Leviva, 65.

Lewinus, 58 [27]

Lidiardo Sancti Laurencii, Tho. de, 66 [27].

Liede, Joh., 53 [3]

Lifi[n]g, $65[43]$.

Lilleb[urna], Rog. de, 79 [51].

Linche, Ric. de, 24 [19]

Locarde, Walt., 21 [56].

Londonia $v$. Johannes, Ricardns.

Longus, Hugo, 65 [49].

- Rob., 50 [36], 65 [2], 70 [45].

Lorteburne, Ernaldns de, 33 [13].

Loveraz, Hen. de, 77 [47]

Lucas, 38 [7]

- pictor, 78 [15].

Luche, Thom: 65.

Lncia, vidua, 38 [25].

Lukitale, Ric , 44 [40].

Lurdona, Lourdune, Rob. de, 21 [59], 22 [25].

Lnwini, $v$. Ricardus.

Lya, Phil. de, 75 [6].

Mabon, Rog., 21 [20].

Mai, Ric., 20 [46].

Makene, Thom. de, 13 [34].

Malherbe, Nic., 78 [4].

-Will., 2 [17].

Malin, 44 [58]

Mallori, Edw., 1 [48].

Malpetit, Rog., $5[37]$.

Mansellus, Rob., 64 [10].

Mara, Mares, Cecilia de, 45 [5].

$\longrightarrow \mathrm{J} . \mathrm{de}, 46$ [S]

Joh. de, 3 [49], $4[47], 5[9], 18,39[54], 45,48$. Osbertus de, 45 [4].

- Sam. de, 49 [7].

Margareta, 38 [21].

Mariscallus, $v$ Osbertus.

Marisco, Alueva de, 38 [27].

Julianus de, $65[59]$.

R[ic.] de, 68 [33].

Ric. de, $22[22], 34[55], 70[4], 71[38], 72[3]$.

Rob. de, 72 [3]

Will de, 72 [28]

Martinus, 38 [13], 74 [55].

- oarpentarius, 78 [3].

- flius Ernaldi, 61 [24]

- la Martre, 77 [39].

de Widiela, 66 [26, 28].

Martre, Hen. la, $60[54], 61,63[6], 80,81$ [3], 82 [10], $83[13]$.

- Martinus la, 77 [39].

Mathæus, 49.

Mathias, 39 .

Matildis, $6[8], 47[18], 48[18]$.

Mandut, Rob., 77 [30].

Manlinn, Savaricus, 39

Mauricius serviens, 73 [56], 74 [61].

Meddere, Osbertus, 77 [30]

Medestede, Rob. de, ôl [23]. 
Medicus, v. Hugo, Ricardus.

Mellane, Will. de, 78 [1].

Mensel, Rog., 41 [44].

Mentor, Will., 13 [35.

Mercer, Will., 56 [29.

Merefreing, Mich. 58 [24].

- Randulfus, 58 [25]

Mercwella, Hen. de, 57 [47].

- præpositus de, 48 [5].

Merre, Hen. de, 33 [12]

Mertona, Canonici de, 41 [6].

Merwon, Alanus, 24, [15]

Michaelis præpositus, 5 [23]

Miles, v. Robertus, Rogerus.

Milo, 15 [23].

Mobnn, Will. de, $77\lceil 62]$.

Moises, 1 [39].

Molinarius, Molindinarius, de Molindino, v. Edmandus, Edwardus, Galfridus, Johannes, Philippus, Randulfus, Reginaldus, Ricardus, Rogerus, 'Thomas, Walterus, Willelmus.

Moneta, G. de, 37.

G. de, 38 [57], $39[32,60], 45$ [59]

Monte, Anketillus de, 78 [5].

Mop, Rob., $11[60], 12[6]$.

Mora, Benedictu de, $64[61]$

- Rad. de, 5 [63?

More, Alvredus, 9 [1].

Morellus, Ric., 32 [42].

Mortona, Randulfus de, 13 [36].

N. janitor, 68 [19].

Neuelin, Neuelus, Petrus, 8 [20], 55 [22].

Nicholaus, 4, 5 [3], 8 [61], 55 [52], 83 [55], 84 [13].

- fil. Godæ, $44[60]$

Nioolaus albus, 21 [1].

cocus, $23[40,41]$

de coquina, 5 [14], 43 [52], 57 [53], 76 [28], 82.

Hunwini, 52 [61].

præpositus, 8 [61].

qui fait occisus, 1 [28], 4 [19].

Nidgehida, Thon, de, 66 [19].

Vigellus, 77 r51].

Niger, v. Edgarns.

Rog., v. Rogerus.

Niwem[an], Hugo, 50 [36].

Nodwaltham, Cristinus de, 24 [13].

Noreis, 80 [21, 41]

- R., 80 [26].

Normannus, $20[61], 21$ [17].

Nortons, $v$. Radulfus

Germanus de, 66 [9]

Notte, Godwin, 6.

Novo Loco (Albury), Canonici de, 44 [13], 46 [59].

Novus, v. Edwinus.

Nuncius, $v$. Robertus.

Nuntona, Ailwardus de, 21 [15].

- Walkelinus de, 21 [14].

Natrix, $41[41]$

\section{Odo, 21 [19], 29, 50 [37], 79 [45]. \\ Odonis vidua, 79 [48].}

v. Willelmus.

Oisel, Jacobus, 20 [13ј.

- Steph., 26 [2].

Oisun, Thom., 77 [23].

Oliver, $20[7], 41$ [55], 60 [12].

Ordrich, Gilbertus, 64 [ 57 ].

- Rob., 65 [16].

Ordricus, 65 [17].

Orgarus, 76 [64].

Osbertas, 38 [12, 77 [50].

- albus, 2 [16].

clericus, 58 [17].

filius Baldwini, 28.

- Edwini, 38 [4].

forestarins, 39 [13].

Godæ2, 53 [7]

mariscallus, 77 [18]

molindinarius, 38 [4].

_- præpositus, 60 [54].

Osgod, 21 [13]

Osmundus, 9 [18], 53 [12].

- constabularius, 6 [20].

- serviens, 52 [46].

S 2422.
Othinus. $45[3]$.

Oute, Uupping, 35 [16]

Ov [er Jgig', Baldwinus, 29 [5i].

Pacbe, Rob., 76 [61]

Paganus, 17;11], 77 [2].

Pagel, Will., 52 [54]

Pallet, Rob., $24[15]$

Palmerins, Palmer, 65 [47].

- Absalon, 53 [1]

Altredus, 6 [5]

Helias, 65 [16].

Randulfus, 13 [34].

Ric., 35 [18].

Rob., $45[4]$.

-... Will., 41 [36], 50 [34].

Parco, Rad. de, 2 [5].

Parfit, Walt. de, $1[1], 45[54], 46,47[57], 48[\dot{\zeta}]$.

Parkehurste, Parkenrste, Godwiu de, $1[39], 2[\overline{19}$

- Moyses de, 2 [14].

Parmenter, Parmentarius, v. Ricardus, Thomas, Willelmus.

Parvus, v. Reginaldus, Willelmus.

Patricius, 45 [2]

præpositus, $44[61], 46[63]$

Pavia, 1 [39]

Pavilli, Paveilli, R[ic.] de, 17, 18, 19, 20 [3].

Rob. de, 54 [46].

Peche, Ailwardus, 65 [53].

- Godwin, 21 [18].

- Oshertus, 20 [46]

Peins, Alvredus, 56 [10].

Pelehaste, 6 : 66 ].

Pell [iparius], v. Reginaldus.

Peni, Gallr., 24 [14]

Pep, vidua, 21 [17].

Peruin, Mathæus, 29.

Petronella de Herefordia, 81 [2].

Petrus, 6 [48], 77.

- cocus, 72 [48].

- Episcopus, v. Wintoniensis, Epis. de.

filius Palmerii, 65 [46]

- Reimundi, $77[1,4-1]$

- juvenis, 6 [52], 7 [33], 8 [38], 28 [1], 76 [28]

Leverici, 1 [45]

nepos Episcopi, v. Wintoniensi

Philippus, $34[55], 41[31], 74[6]$

molindinarius 41 .

- serviens, $42[16]$

Picardus, 77 [49]

Pichemora, Rog. de, 35 [10?.

l'icouus, $55[51], 56[7]$.

Pictur, v. Lucas.

Pig, Gilebertus, 20 [62].

Nic., 2 [18]

Pila, Hugo de, 56 [9].

Pilvorkus, $23[60]$.

Pincerna, v. Daniel.

Pincernaria, $v$. Thomns.

Piscator, v. Hugo. Walterus, Willelmus.

Pistor, $v$. Alvredus, Henricus, Jocelinns.

Plumbarius, v. Walterus.

Poche, 45 [8].

- Ingulf, 53 [11].

Rob., 2 [3]

Poda, Rog., 65 [12]

Pola, Durandus de, 64 [61].

- Rad. de, 65 [4].

Ponte, Rad. de, 45 [8].

Porcarins, v. Walkelinus.

Porta, Gralfr. de, 26 [4].

- Geraldus de, 65 [29]

Portereve, Ric., 1 [40].

Porth, Ric., 52 [60j

Pote, Godefridus, 56 [11].

- Hen., 56 [22]

Serlo, 56 [14].

Potere, Rob., 53 [5].

Præpositus, v. Adam, Alexander, Alvredus, Arnoldus, Azo, Edmundus, Elias, Galfridus, Gillebertas, Goderiche Michael, Nicolaus, Osbertus, Patrieins, Radulfus, Ricardas, Robertus, Rogeras, Thomes, Willelmus.

Prat, Walt., 24 [16].

Prato, Will. de, 41. 
Presbyter, v. Angerns.

Prestes[man]. Walt., I [40].

Prinne, Ric., 1 52].

Will., 2 [1].

Privet, Will. de, 44 [33]

Proche, vidua, 42 [48].

Procus, Will., 48 [60], 49 [8].

Puddinge, 47.

Walt., 44 [62].

Pulcellns, 78 [19]

Pand, Fadwinus de, 5 [40?.

Punfalda, Will. de, 38 [24].

Pur, Galfr., 26 [3]

Parcel, Ric., $12[1,5]$

Pute, Seman. 21 ilg].

Puteo, Hugo de, 1.140$]$

Quabbe, Galfrida de, 56 [30].

Quercn, Kic. de, 38 [13]

Quinci. Alrredus de, 52 [5], 53 [48], 55 [42], 60 [42].

R. berebrettus, 80 [16]

- cerarins, 39 [54].

- janitor, $68[20]$.

salserius, $19[63], 75[5]$

Rabbere, Rog.. 35 [11].

Kadulfus, $4: 7]$ ] $53[2], 60[17]$

- Bustard, 17 [48].39 [10].

cartttarius, $65[6]$.

cerurius, $39[54], 60[51]$.

filius Agnetis, 17 [16].

—- Ailici, 52 [57].

— Edithæ, 20 [49]

— Edwini, 17 [1:3].

Gocelini. 38 [6]

Godæ, 28 [46].

Godwini, 49 [4].

Haiwardi de Alresforde, 41 [52]

Hardekil, 17 [6].

junior Freke, 52 [55.

præpositus de Nortona, 66 [18]

salserius, 51 [64].

Ramescumba, Will de 41 [41].

Ramesd[ene, Randulfus de, 4.4 [4]

Rob. de, $44[36]$.

Theobsidus de, 44 [48].

vidua de, 45 [12]

Randulfus molindinarius, 17 [17?.

Redingen, Rob. de, 78 [37], 79 [36.

Reginaldus, 34 [54], 58, 64 [57]. 77 .

- dominus, 8 [36].

- moliudinarins, de molindino, 11.[59], $12[8], 65$.

parvus, $5[64], 6[2]$.

- pelt[iparius], 47 [18]

- salsarius, sauserins, 4[44], $17[47], 80[59], 81$ $[7], 82[35]$.

Reimundns, $76[61], 77$.

Reinerns, 58 [44], $59[9], 60[10 j, 61[1]$.

Ret, Will., 12 [8].

Rethereiate, Bretellus de. 27 [35].

Rex, Jobannes (i.e. John King), 33 [13].

Ricardus, 13, 38 [7].

- anrifaber, 77 [22].

- Baldewini, 30 [53?

bellus, $41\left[60^{\circ}\right.$

berebrettns, 82 [10].

Bricwini, 35 [15].

calvas, 55 [44]

cepellanus, 35 [12]

capillir, 78 [19]

carpentarius, 44 [51]

de Castello, $53[13]$

cimentarius, 38.

clericns, $47[39]$

_- Episcopus, v. Wintoniensis Episcopus.

faber, 49 [47]

filius Edwini, 38 [14].

Gonnildæ, 58 [29]

__ _ Heliæ Palmer, 65 [16].

_- Hngonis, 38 [22].

- Ivonis, 53 [7].

—— Reginaldi, 58 [26].

- Roberti, 41 .

- Wlvrici, 53 [11]

— do Fonte, 41 [60].
Ricardns gardinarins, 2 [10].

Giliberti, 1 pr

- heiwardus, 58 r27?

_ juvenis, 55 [53].

- de Lond[onia], 37 [39], 38 [6].

-- Luwini, 53 [12].

medicus, $77[13$

molindinarius, 12 [4], 58 [22].

palmentarius. 20 [62].

piscator, 61 [2]

præpositus, $32[12], 37,57[60\rfloor, 73\ulcorner 56], 44[7$ de Scotia, 77 [25].

erviens, $13,14,15[10], 16,17[41], 33[40], 34$ 47], $50[9], 51[67], 58[44]$.

Sewardi, 56 [13].

- Simonis, 52

subserviens, 51 [67].

taverner, $41\left[30^{\circ}\right.$

telarius, $41 ; 62]$.

Ridere, Enstachins, 65 [14]

Ristoua, Ordrich de, 64 [59]

Rohertus, $24[6], 33[15], 40[3], 41,52[62], 66[36]$.

- de Bolonia, 78 [16]

-.. carncarins, $10[26]$. de Cruce, 41 .

-.. - de Cruce, 41 [31], 66 [6].

frater, $24[6]$.

filius Ailwini, 43 [8].

- Alfiedi, 49 [8].

- Briani, 79 [47].

- Edivæ, 65 [8].

- Edwini, $82[22]$.

-

- Lenevæ, 33 [14].

[- Lewini, $58 \mathrm{r} 27]$

- Ricardi, 38 [7].

- Sewardi, 44 [45].

Willelmi carter, 44 [35].

Hunwini, 15 [22]

longas, 50 [36], 65 [2], 70 [47].

miles, 50 [39].

molindinarius, 74 [27].

nuncius, 17 [50].

Ordrich, 65 [16]

- prepositus, 34 [47], 46 [63].

- Radulfi, 20 [55].

serviens, 33 [40].

Stigandi, 44 $[32,47]$

Rogerus, 58 [8].

decanus, 77 [3]

faber, $41[29], 48[61]$

filins Adam. 79 [49].

- - Ailwini, $41[55]$.

- Gileberti, 65 [10]

Hildæ, $17[6]$

juvenis, 17 [2:]

- de Lilleb[urna], 79 [52].

- miles, 41 [4].

- molindinarins, 44 [36]

- jiger, 49 [5].

- prepositns, 43 [59], 44 [1]

- de Bradeforda, 66 [19]

- serviens, $57[60]$.

Tessie, 21

Roies, $77[6]$

Rolandus, 38 [63]

Romanus, Walt. 52 [18], 54 [30].

Roterns, 72 [46].

Roxi, Adam de, 20 [49].

Ruffins, 33 [15]

- Elias, 73 [59], 74 [25], 76.

- Godwin 6 [19]

- Nic., 6 [18].

- Rad., 28 [45]

- Ric., 44 [38].

- Rob, 13 [32]

- Will, $]$

Rundet, Joh., 35 [16].

Runewika, Willekin de, 38 [19].

Ruscel, Joh., 74 [55

Russinol, Petrus, 7 [36], 15, 16 [27], 18 [44], 31 [22], 59 $[7], 83[10]$.

Sage, 5 [41].

Saia, Gocelinus, 45 [2]

Salsarius, Salserius, Sauser, v. Radulfus, Reginaldus. 
Samson, Rob., 64 [24].

Sancta Cruce, Gilbertus de, 77 [48].

Sancta Maria, Alicia de, 78 [17].

Sancta Maria Magdalena, leprosis de, 43 [51].

Sancto Albano, Hen. de, 60 [15].

Sancto Maxentio, W[ill.] de, $99[6], 63[34], 70[4], 71$ $[38], 72[3]$

Sanforda, Reg. de. 16 [41]

Saus[erius], 47 [19].

- v. Salsarins.

scalc, Adam, 20 [58].

Segar, Adam, $52[50]$.

Seiva, vidıa, 9 [17].

Seiwa, $34[55]$.

Seles, Joh. de, 24 [49].

Selida, $20[47]$

Serescallus, 4 [46], 6, 7 [28], $8[36], 13[51], 14,15,16$ $[27] .17[40], 18,19,20[4], 21[57], 22[59], 24[42]$ $25[8], 2722], 30,31,32,33,34,42[30], 45[37]$, $46,48,49[21], 50[59], 53[60], 55[25], 57 \quad 40]$, $58[43], 59$ [16]. $61[47], 62[15], 73[59], 75,76$.

Scrich, Rog. 27 [38].

Ser'lo, 48 [41].

- filius Pagani, 77 [2]

Serubege, Sernege, Algarns de, $65[7] .70^{r} 45^{-7}$

Servicns, v. Audeliuus, Galfridus, Johannes, Mauricins, Osmundus, Philippus, Ricardus, Robertus, Korerus, Willelmus.

Sevaras, Sewarus, 38, 56 [10].

Sevenhac, Steph. de, 65 [27]

Sewade, Aco, 52 [59].

Sowalus. Sewale, $21[60], 50[37$.

Sewardus, 44.45$]$

- Ric., 56 [13].

Sewoldus de Torra, 65 [56].

Sidegiva, 24 [27].

Silvester, $47[40]$.

- clericus, 42 [27].

Sinion, Symon, 10 [31].

- clericus, 5 [39], 77 [33].

- juvenis, $6 \mathrm{I}, 62$

v. Ricardus.

Sireforda, Albrea vidua de, 65 [17].

- Rob. de, 70 [34].

Siveken, Adam, 58 [28]

Siwa]dus, 20 [52].

Siwardıs, 30 [54], 44 45$]$

Skegge, Alexander, 1 [41]

Sleate, Edw. de, 56 [21].

- Galfr. de, $56[19$

Smalbroch, Rob., 65 [32].

Simar". Hugo, 44 ; 50$\}$.

Sociebeche, Tebaldns de, 455 [12]

Sole. Ric. de la, 41 [33].

Solis, Thom. de, $26-4$

Soredich Gilibertus de, 65 [4].

Solewella, Sorewilla, Sorevilla, Will. de, 3, 39, $63[45]$ $72[7], 73[49], 75[1], 76[-18]$.

Speciarius, v. Bertramns.

Spelemau, Rogerus, 2 r]

Sperkelorda. Nigellus de, 77 [66].

Walkelinas de, 77 [67].

Suraculi, Will., 52 [51].

spiringe, Will., 1 [4t].

Sprot, Ric., 21 [2]

Spure, Ailwardus, 55 [7].

Herebertus. 77 [67].

Stanere, Ailwardus, 53 [4].

Stana, Rail. de, 65 48].

Stanforde, Malgerus de, 41 [57].

- Reginalöus de, 41 [58]

Stanbulle, Osmundus de, 9 [17].

Stanlinch, Will. Ruffus de. 20 [63].

St:tple, Hugo de, 65 ? 47

Steorre, Will., 24 [19]

Stede, Rob., $74-247$

Stephanus, 6 [48], 15 [24].

firber, $40^{\circ}[01], 41[50$ ].

Stucespunda, Levericus de, 35 [13].

Stoches, Mlauricius de, 65 [2]

Godefridus de, 64 [5s], 65 [2].

Stoct [er], Osbertus, 49 [7]

Stukes, Hen. de, 55 [45].

- Manricius de, 65 [13].

- Randulfus de, $6[1]$.

Stonbam, Joh. de, 54 [36].

Strode, Gocelinus de, 44 [37].

Will. de, 28 [47].
Strong'. 50 [13].

Stut, Will., 38 [3].

Sumersete, vicecomes, 68 [34], 70 [2], 72 [3].

Sumeter, Ric., 74 [62].

Surlonde, Agnes de, 40 [62].

Sutere, Osmundus, 5 [32.

Swafham, Swofham, IVill. de, 64 [21], 65 [22], 68.

Swanemere, Walt. de, $1[10]$.

Swede, Adam, 2 ;]

Swein. $40[7$

--. Walt., 10 [25], 70 [42].

Swenge, Rob., 35 [14].

Hen., 78 [38].

Sweta, 65 [53].

Swift, 64 [6]

Suno Will., 76 [64]

'Tal,rigge, Randulfus de, 65 [18].

Talou, Talun, Osbertus, $78[57]$

Tanculfus, 50 [38].

Tantona, Prior de, 63 [47].

Tapinare, Will., 78 [1]

Taverner, Ric., 41 [30]

'l'ebaldus. T'edbaldus, $41[35,45[1]$.

- de Sodebech, 45 [12].

lee, Rog. de $l_{n}, 41$ [48].

Tegulator, v. Gilibertus.

Telarius, $v$. Ricardus.

Teler, Will., 24, [14].

Templarii, 7 ? [36].

Tessit, v. Rogerns.

Tetta, 74 [3].

Thadelega. Ric. de, $24[21]$

Rob. de, 24 [22]

T[h]edba]dus, Teodbaldus, balistarius, 17 [4 4 , 18 [39].

Thedricus, 34 [7]

Thomas. $13[31,15[55], 32[5 \dot{1} 1], 46[63]$

- albus, $45[10]$.

- filins Baldwini, 17 [j]

- molindarius, 24 '18].

- parmenter, $5[38$ ?

- de Pincernariu, 15 [35]

- præpositus, $44[7], 46$ [63].

- sacerdos de Funtel, 80 r 8$]$.

Ticheburua, Rogerus de, 77 [21]

'lichere, Walt., 76 [64].

I'ole, Will., 50 [35]

Toli, Rad., 47.

Torra, Matildis de, 65 ;55].

Sewoldus de, $65[56]$.

Tragis, Will., $\check{5} 8$ [19].

Trai, Rad., 65 [14].

Trapel, Rad., 20 [54].

Trenchem[an], Gilibertus, 48 [5 $55^{\circ}$.

Trewe, Godwin, 56 [11].

Tribus Aulis, Hugo de, 79 [51].

Trilest[er], Hen. de, 47 [19].

Trindele, Lifig de, 65 [41].

Triwe, Edwardus, 52 [49].

- Nicholaus, 52 [42].

Tuars, vicecomes de, 39 .

Turbertas, 38 [9], 52 [15].

Turbin, Rob., 2. [7].

Turg [isius], 40 [3].

T'urigge, Will., 32 [54].

Turneham, Torneham, Rob. de, 2 [59], 28, 6s [32] 70 [1], 72 [2]

Tarnur, Reg., 12 [1].

Rog., 12 [9].

Turri, Will., 81 [32]

'Turricus, 9 [15].

Turvi:e, Turvilla, M. de, 45 [45], 46 [9?.

Tut, Will., 58 [25].

Udelini, Joh.. 2 [12].

Udelinns, $56\left[20^{\circ}\right.$

Upham, Hen. de, 1 [4l].

Vaccarius, v. Gilibertus.

Valletorta, Reg. de, 39 [5].

Veggel, Ric., 56 [23].

Veneis, Rog. de. 65, [45]

Vilur, Hen., 1 [43]

Vinea, Ric. de, 50 [39].

Vinitarius, Arnulfns. 
Vis, Vise, Will., 2 [1], 63 [3].

Voxgrave, Joh., 65.

Vule, Emma de, 2 [10].

Wacelinus, Rog., 19 [58], 21 [61], 22 [20], 23 [27] 39 $54], 43[47], 57[48], 75[3], \gamma 6\left[40^{-}\right.$

Waite, $68[21,23]$

Walingefordia, Bartolomæus de, 78 [2]

Walkelinus, 6 [22], 44 [57].

porcarins, 17 [24].

Wallingfordia, Andreas de, 13 [12].

Walterus, 20 [51] 38 [11].

capistrarius, $52[60]$.

-.. clericus, $48[5 ? 7,79[46]$.

- de coquina, 17 [47].

- faber, $17[7]$.

- filius Avice, 41 [32].

—- Galfr. $44[52]$.

_- Reginaldi, 58 [28]

- forestarius, 6 [12]

Wrater, 22 [57], 42, 53 [48], 75 [39].

- molindarins, 20 [59].

_- piscator, $31[25], 39$ [15].

— plumbarius, $76[61]$

_._. Tedgari, 44 [46], 45 [97.

- warennarius, $2[5 \mathrm{I}]$

- Wlvrici, 12.

Waltham, Rob. de, $28[40]$.

Warennarius, $v$. Adam.

Warinus, $7 \div$ [57]

Warnerus, 33 [16], 52 [52].

Watel[ega], Will. de, 66 [2]

Watte, Adam, 65 [33].

Weltona, $v$. Woltons.

Wibbe, Galfr., 11 [61], 12 [6].

Wica, Walt. de, 17 [20].

Wicham, Nic. de, 58 [23].

Wichtona, Thom. de, 60 [61].

Widecoc, Will. $2\left[51_{\text {j, }} 3[14], 39[16], 54\right.$ [28].

Widehaie, Ric. de, 43.

Widiela, Martinus de, 66 .

Widintona, Will. de, 20 [12].

Wika, Helias de, 20 [53], 21 [12]

Ingelram de, 20 [52]. Rad. de, 21 [11].

Wikewano, Rad. de, 59 [15].

_... Ric. de, 17 [55].

Wilde, Edm, 52 [63]

Wile, Will. de, 38 [21]

Wilidonæ, Rad., 32 45]

Willa, Ric. de, 66 [16, 27].

IVillelmus, 5, 39 [1], 40,45[Ii], $55[51], 58[21], 62$ $[44], 64[21,65[21], 75[26], 77[19]$

Alanı, $20[6: 3]$

—_ albus, $52[51]$.

- Ailwardi, 74 [26].

- bercarins, 10, 21 [16].

- berebrettus, 80 [41].

_ Bundi, 74 [9].

- caprarius, 41 [56]

- caretarius, $36[9], 39$ [17], 45 [13].

- clericus, 58 [22]

- de Cnolle, 56 [22]

- cocus, $74[55]$

- concilius, 72 [44].

- coquus, 68 [18, 27], 69 [66]. Edwini Kinc, 38 [23]

foker, $65[37]$

- filiaster Willelmi Proci, 48 [60]. filius Ainolf, 41 [49].

- - Ailwini, 41 [๘6], 58 [20].
Willelmus filius Alrrici, 33 [14].

aketilli, 45 [5].

- - Edit, $33[177$

— Edmundi, 53 [6].

- Eilrici, 38 [17].

- - Gerardi, 6 [16].

- Giliberti, 20 [9], $21\left\lceil 63_{\lrcorner}, 52\right.$ [47].

Matildis, 58 ['18].

Osberti clerici, 58 [17].

—_ Osgod, 21 [13].

_ _ Reginaldi, $34[54], 64[57], 77$ [29], 78 [41].

- Selidæ, 20 [47].

- - Sirmarius de Rimptona, 73.

_- forestarius, 49 [3].

- frater, $39[56], 44$ [34]:

- Frewini, 53 [14]

- fugitivas, 36 [35]

- Gerardi, $6[16]$

- grangarius, $25[61]$

- haiwardus, 9 [12]

- de Hamptesira, 52 [56]

- miles, $77[47]$.

molindinarius, de molindino, $1[50], 38[11], 41$ [35].

_. Odonis, 56 [26].

- parmentarius, $2[19], 52$ [45].

- parvas, 78 [11].

piscator, 21 [7]

præpositus, 23 [52], $28[19], 40[58]$.

- Ricardi, 56 [24]

- serviens, 24 [53], 78 [23], 80, $81[6], 82$ [10].

Wimarkus, 35 [11]

Winterhulla, Matillis de, 4 [5]

Wintonia, Joh. de, 5 [15].

Simon de, $2[8]$

Sanctimonialcs de, 39 [33].

Wintoniæ, Horderus, 17 [43].

Wintoniensis, Episcopns, ] [34], 2, 3, 4, 5, 6, 7, 8 [58] $9[47,11,14[9], 15,17,18,19,20[2], 21[60], 22$, $23,25[7], 27,28,29,30[29], 31,33[58], 35[52]$, 36,37 [ะ3], 38, $: 9,40,41\lceil 61], 42,43,44[54], 46$ $48,50[36], 51-20], 52[24], 53[34] .54,55,56,57$ $58,59,60,61[25], 62[24], 64[51], 65,68,69,70$ $[5], 71$ [39], 72 [4], 75, 76,77, 78, 80 [49]. 81, 82, 83. Godefridus, 41 [7], 76,77 .

—- Petrus, $18[44], 4 \dot{3}[64], 52$ [18].

$-\longrightarrow$ nepos, 17,19

milites, $15,58,59$

-- Ricardus, 64, 75 [63], 76 [62], 77.

Witemor, Will. de, 65 [50].

Withonde, Thom., 27 [39].

IVitteneia, Baldwinus de, 23 [52].

Wixi, Rob., 5 [36

Wlfus, Walt., 12 [4].

Wlgar, Rog., 21 [Q].

Wlvricus, Wolvricus, 53 [11], 83 [31].

de Kingestona, 65 [22]

Wodeforde, Unfridus. Hunfridus de, 61 [24], 62 [27].

Woltona, Weltona, Woeltona, Joh. de, 5 [23], 8 [6I], 10 [8], 11 [39]

Wolvricus, $12[7]$

Worvesur. Hngo, 41 [46].

Writh', Thom. de, 44 [ร3].

Wudeman, $2[4] 44$ [61]

Wudesman, Will., 37 [22]

Wulwini, Adam, 2 [15].

Ylebertus, 65 [24]

Ypre, Rad. de, 52 [18].

Y sabc serviens, 78 [24]. 


\title{
LONDON SCHOOL OF ECONOMICS AND POLITICAL SCIENCE.
}

\author{
UNIYERSITY OFIIONDON. \\ CLARE MARKET, LONDON, W.C.
}

THe special aim of the London School of Economics and Political Science is the study and investigation of Economic and Political Institutions.

Most of the teaching in the Faculty of Economics and Political Science (including Commerce and Industry), uewly created by the reorganised University of London, is provided by the School of Economics, whose curriculum is arranged as a three years' course of study for students desirous of proceeding to the degree of B.Sc. in Economics.

Many of the Lectures and Classes are designed to promote a wider knowledge of modern commercial conditions and to meet the needs of those engaged in the civil and municipal services, journalism, teaching, and public work. The School provides training in methods of investigation, including Palæography and Diplomatic, and affords facilities for original work in Economics, History, and Political Science.

The fee for the full course is $£ 10$ a year or $£ 310$ s. a term, but students other than University students are admitted to individual courses of lectures at fees varying according to the number of lectures in the course.

Lectures are held on Economics, Statistics, Commercial and Political History and Geography, Law (International, Constitutional, and Commercial), Public Administration, Public Finance, Accountancy, Banking, Insurance, Transport, Foreign Trade, etc., when possible, in the evening between 6 and 9 .

The School is open to men and women.

The Academic year begins in October, and is divided into three terms of ten weeks each, viz.: (1) October to December; (2) January to March; (3) April to July. Students may join the School at any time.

Scholarships are given to enable students of ability to pursue their studies at the School.

Full particulars may be obtained from the Director at the School. 
"A permanent and invaluable contribution to the sum of human knowledge. . . . We commend to the publie a book which is a monument of researeh and full of candour. . . . Indispensable to cvery publicist and politician."Tïmes, 4 th January, 1898 (day of publication).

Post 8vo, Two Vols, xxix. and 929 pp., with Two Diagrams.

Price 25s. net.

\section{N D U S T R I A L DEMOCRACY.}

\section{By SIDNEY and BEATRICE WEBB}

(Authors of "The History of Trade Unionism.")

\section{A D VERTISEMENT.}

In this work the authors of "The History of Trade Unionism" deal, not with the past, but with the present. They describe, with the systematic detail of the scientific observer, and in the same objective spirit, all the forms of Trade Unionism, Factory Legislation, and other regulations of industry to be found in the British Isles. The whole structure and function of Labour Organisation and Restrictive Legislation in every industry is analysed and criticised in a manner never before attempted. The employer in difficulties with his workman, the trade unionist confronted with a fresh assault upon his standard rate, the politician troubled about a new project for Factory Legislation, the public-spirited citizen concerned as to the real issues of a labour dispute, will find elucidated in this work the very problems on which they are thinking. It is a storehouse of authenticated facts about every branch of the "Labour Question," gathered from six years' personal investigation into every industry in all parts of the kingdom; systematically classified and made accessible by an unusually elaborate Index.

But the Book is more than an Encyclopædia on the Labour Question. Scientific examination of trade union structure reveals, in these thousand self-governing republics, a remarkable evolution in democratic constitutions, which throws light on political problems in a larger sphere. The century-long experience of these workingclass organisations affords unique evidence as to the actual working of such expedients as the Referendum, the Initiative, Government by Mass Meeting, Annual Elections, Proportional Representation, Payment of Members, and, generally, the relation between the Citizen-elector, the chosen Representative, and the Executive Officer. The intricate relations of trade with trade have an interesting bearing upon such problems as Local Government, Federation, and Home Rule. Those who regard the participation of a working-class electorate in the affairs of government as the distinctive, if not the dangerous, feature in modern politics, will here find the phenomenon isolated, and may learn how the British workman actually deals with similar issues in his own sphere.

The analysis of the working of Trade Unionism and Factory Legislation in the various industries of the United Kingdom has involved a reconsideration of the conclusions of Political Economy. The authors give a new and original description of the working of industrial competition in the business world of to-day; and they are led to important modifications of the views currently held upon Capital, Interest, Profits, Wages, Women's Labour, the Population Question, Foreign Competition, Froe Trade, \&c. The latter part of the work is, in fact, a treatise upon Economics.

Finally, it should be stated that the authors, in the Preface, describing their investigations, propound a new view as to the scope and method of Political Science.

\section{O N G M A N S, G R E E N, \& C O.,} LONDON, NEW YORK, AND BOMBAY. 


\title{
OTHER WORKS BY THE SAME AUTHORS.
}

\author{
Published Dy SWAN SONNENSCHEIN \& CO.
}

THE CO-OPERATIVE MOVEMENT IN GREAT BRITAIN. By Bratrice Potter (Mrs. Sidney Webb). Second Edition, 1893. Crown 8vo, with Coloured Map showing Distribution of the Co-operative Movement, Appendices, and Index. Price 2s. 6d.

"Miss Beatrice Potter's luminous and suggestive volume is not a mere bald, historical outline, hut a thoughtful and pregnant study of tendencies, causes, and effeets."-Times.

"The whole volume is full of suggestion, both to eo-operators and politicians. . . . It is without doukt the ablest and most philosophical analysis of the eo-operative movement which has yet been produced."-Speaker.

SOCIALism IN ENGLAND. By Sidney Webb, LL.B. Second Edition, 1893. With New Introductory Chapter. Crown 8vo. Price 2s. 6d.

"The hest general view of the subject from the moderate Socialist side."-Athenceum.

THE LONDON PROGRAMME. By Sinney Webb, LL.B. Second Edition, 1894. With New Introductory Chapter. Crown 8vo. Price 2s. 6d.

"Brimful of excellent ideas."-Anti-Jacobin.

\section{Published by WALTER SCOTT.}

THE EIGHT-HOURS DAY. By Sidney Webi, LL.B., and Harold Cox, B.A. Crown 8vo, 280 pp., with Bibliography. Paper Covers. Price 1s.

"The unique value of this little book lies in its collection of facts. It is likely, for some time, to hold the field as the handbook to one of the chicf items in the social politics of the immediate future."-Pall Mall Gazetle.

\section{Published by GRANT RICHARDS.}

LABOUR IN THE LONGEST REIGN, I837-1897. By SIDNEY WeBb, LL.B. Small 8vo, Cloth. Price 1s.

Published by LONGMANS, GREEN, \& CO.

THE HISTORY OF TRADE UNIONISM. By SIDNey and Beatrice WebB. Second Edition, 1896. Revised (completing 2,500 copies). Post 8vo, 547 pp., Cloth, with Coloured Map. Price 18s.

"A masterly piece of work."-Times.

"To the politician . . . an invaluable guide."-Observer.

PROBLEMS OF MODERN INDUSTRY. By Sidney and Beatrice Webb. Now Ready. Post 8vo, 286 pp. Price 7s. 6d. 



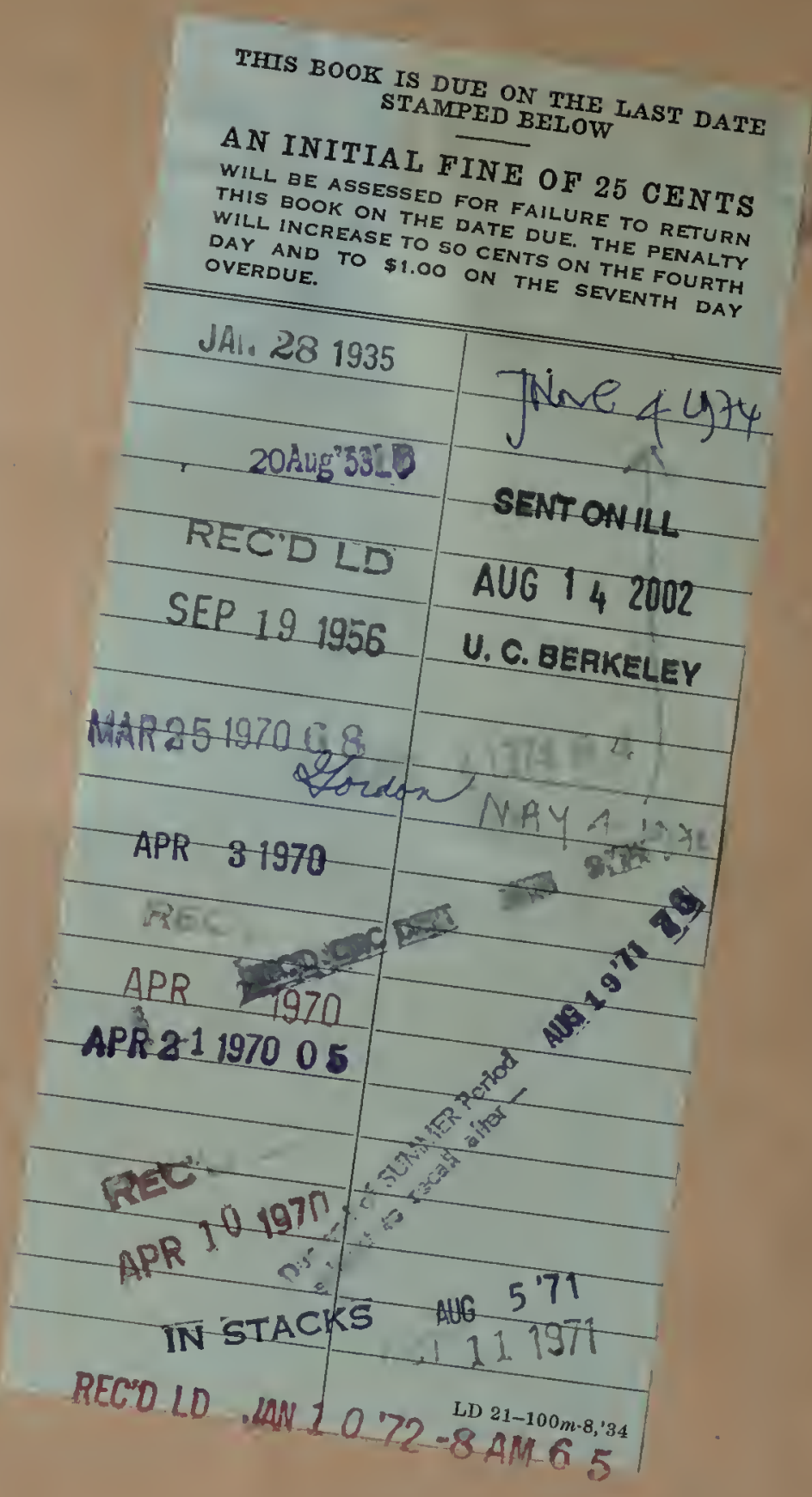




\section{$3075 \div 1$}

RETURN TO the circulation desk of any or to the

NORTHER

NORTHERN REGIONAL LIBRARY FACILITY

University of California

Richmond California

Richmond, CA 94804-4698

ALL BOOKS MAY BE RECALLED AFTER 7 DAYS

2-month loans may be renewed by calling
(510)642-6753

- 1-year loans may

books to NRLF

- Renewals and recha

4 days prior to due date may be made

\section{DUE AS STAMPED BELOW}

\section{'JAN 222004}


